\title{
Search for New Heavy Charged Gauge Bosons
}

\section{Dissertation}

\section{Carsten Magaß}

\section{Physikalisches Institut A RWWNAACHEN}

Additional gauge bosons are introduced in many theoretical extensions to the Standard Model. A search for a new heavy charged gauge boson $W^{\prime}$ decaying into an electron and a neutrino is presented. The data used in this analysis was taken with the $D \varnothing$ detector at the Fermilab proton-antiproton collider at a center-of-mass energy of $1.96 \mathrm{TeV}$ and corresponds to an integrated luminosity of about $1 \mathrm{fb}^{-1}$. Since no significant excess is observed in the data, an upper limit is set on the production cross section times branching fraction $\sigma_{W^{\prime}} \times \operatorname{Br}\left(W^{\prime} \rightarrow e \nu\right)$. Using this limit, a $W^{\prime}$ boson with mass below $\sim 1 \mathrm{TeV}$ can be excluded at the $95 \%$ confidence level assuming that the new boson has the same couplings to fermions as the Standard Model $W$ boson. 



\title{
Search for New Heavy Charged Gauge Bosons
}

\author{
Von der Fakultät für \\ Mathematik, Informatik und Naturwissenschaften der \\ Rheinisch-Westfälischen Technischen Hochschule Aachen zur \\ Erlangung des akademischen Grades eines Doktors der \\ Naturwissenschaften genehmigte Dissertation
} vorgelegt von Diplom-Physiker
Carsten Martin Magaß aus Übach-Palenberg

Berichter: Univ.-Prof. Dr. T. Hebbeker Univ.-Prof. Dr. A. Stahl

Tag der mündlichen Prüfung: 02.11.2007

Diese Dissertation ist auf den Internetseiten der Hochschulbibliothek online verfügbar. 



\section{Abstract}

The TEVATRon proton-antiproton collider at Fermilab (near Chicago/USA) is operating at a center-of-mass energy of $\sqrt{s}=1.96 \mathrm{TeV}$ since March 2001. This analysis uses data taken with the $\mathrm{D} \varnothing$ detector until February 2006 corresponding to an integrated luminosity of about $\int \mathcal{L} \mathrm{d} t=1 \mathrm{fb}^{-1}$. Using this dataset, a search for a new heavy charged gauge boson $W^{\prime}$ and its subsequent decay into an electron and a neutrino is performed:

$$
p \bar{p} \rightarrow W^{\prime}+X \rightarrow e \nu+X .
$$

Additional gauge bosons (including the equivalent to the $Z$, the $Z^{\prime}$ ) are introduced in many extensions to the Standard Model of particle physics. Assuming the most general case, the new gauge group can comprise a new mixing angle and new couplings. Here, the Altarelli Reference Model is considered which represents a generalization of the Manifest Left-Right Symmetric Model with light right-handed neutrinos. This model makes the assumptions that the new gauge boson $W^{\prime}$ has the same couplings as the Standard Model $W$ boson and that there is no mixing. Hence, the $W^{\prime}$ is a heavy copy of the Standard Model $W$ boson.

The clear decay signature (in analogy to the decay of the $W$ ) contains an isolated electron with extreme high energy which is important for triggering. The neutrino can not be detected, but it gives rise to missing energy in the detector. The Jacobian peak in the transverse mass distribution stemming from the $W$ decay is used for calibration, whereas the tail of the transverse mass distribution is searched for a possible $W^{\prime}$ signal.

The data agrees with the expectation from background processes. For instance, in the data 37 events are reconstructed with transverse masses above $300 \mathrm{GeV}$ compared to a prediction of $37.1 \pm 2.1$ (stat) ${ }_{-3.7}^{+6.0}$ (sys) background events.

Since no significant excess is found in the data, an upper limit is set on the production cross section for heavy charged gauge bosons decaying into electron and neutrino, $\sigma_{W^{\prime}} \times$ $\operatorname{Br}\left(W^{\prime} \rightarrow e \nu\right)$. Using this limit, a lower bound on the mass of the new gauge boson can be derived at the $95 \%$ confidence level,

$$
m_{W^{\prime}}>1002 \mathrm{GeV} \text {. }
$$





\section{Zusammenfassung}

Seit März 2001 werden am Tevatron-Beschleuniger auf dem Forschungsgelände FermiLAB (nahe Chicago/USA) Protonen und Antiprotonen bei einer Schwerpunktsenergie von $\sqrt{s}=1.96 \mathrm{TeV}$ zur Kollision gebracht. Die vorliegende Analyse verwendet Daten, die mit dem DØ-Detektor bis zum Februar 2006 aufgenommen wurden und einer integrierten Luminosität von etwa $\int \mathcal{L} \mathrm{d} t=1 \mathrm{fb}^{-1}$ entsprechen. In diesem Datensatz wird nach einem neuen schweren geladenen Eichbosonen $W^{\prime}$ und dessen Zerfall in ein Elektron und ein Neutrino gesucht:

$$
p \bar{p} \rightarrow W^{\prime}+X \rightarrow e \nu+X
$$

Zusätzliche Eichbosonen (das Pendant zum $Z$-Boson, das $Z^{\prime}$, eingeschlossen) kommen in zahlreichen Erweiterungen des Standardmodells der Teilchenphysik vor. Im Allgemeinen können durch die neue Eichgruppe Mischungswinkel und neue Kopplungen auftreten. In dieser Analyse wird das Referenzmodell von Altarelli - eine Verallgemeinerung des manifesten links-rechts-symmetrischen Modells mit leichten rechtshändigen Neutrinos herangezogen.

Hier werden die Annahmen gemacht, dass das neue Eichboson $W^{\prime}$ dieselben Kopplungen wie das $W$-Boson im Standardmodell hat und dass es keine Mischungen enthält. Somit stellt das $W^{\prime}$ eine schwere Kopie des $W$-Bosons aus dem Standardmodell dar.

Die klare Zerfallssignatur (identisch zum $W$-Zerfall) enthält ein isoliertes, hochenergetisches Elektron, welches den Trigger auslöst. Das Neutrino kann nicht detektiert werden; es zeigt sich jedoch durch fehlende Energie im Detektor. Der vom $W$-Zerfall stammende Jacobi-Peak im Spektrum der transversalen Masse wird zur Kalibration verwendet, während der Bereich höchster transversaler Massen auf Anwesenheit eines $W^{\prime}$-Signals untersucht wird.

Die in den Daten gemessenen Ereignisse sind verträglich mit der Erwartung von Untergrundereignissen. So werden beispielsweise für transversale Massen oberhalb von 300 $\mathrm{GeV}$ in den Daten 37 Ereignisse rekonstruiert, während $37.1 \pm 2.1$ (stat) ${ }_{-3.7}^{+6.0}$ (sys) Untergrundereignisse vorhergesagt werden.

Da kein signifikanter Überschuss in den Daten erkennbar ist, können obere Ausschlussgrenzen auf den Produktionswirkungsquerschnitt von schweren geladenen Eichbosonen und deren Zerfall in Elektron und Neutrino, $\sigma_{W^{\prime}} \times \operatorname{Br}\left(W^{\prime} \rightarrow e \nu\right)$, angegeben werden. Daraus ergibt sich bei einem Vertrauensniveau von $95 \%$ eine untere Grenze auf die Masse des neuen Eichbosons von

$$
m_{W^{\prime}}>1002 \mathrm{GeV} \text {. }
$$





\section{Contents}

1 Introduction $\quad 1$

2 Theoretical Foundations $\quad 5$

2.1 Measures and Conventions . . . . . . . . . . . . . . 5

2.2 Aspects of the Standard Model . . . . . . . . . . . . . . . . . . 8

2.2 .1 The Gauge Principle . . . . . . . . . . . . . . . . . . . 8

2.2 .2 The Electroweak Interaction . . . . . . . . . . . . . . 10

2.2 .3 The Strong Interaction . . . . . . . . . . . . . . . . . . . . 14

2.2.4 The Higgs Mechanism . . . . . . . . . . . . . . . . . . 16

2.2.5 The Running of the Coupling Constants . . . . . . . . . . . . . . 21

2.2.6 Parton Distribution Functions ～. . . . . . . . . . . . . 22

2.2.7 The $W$ Production Cross Section . . . . . . . . . . . . . . . . . . 24

2.3 New Charged Gauge Bosons beyond the Standard Model . . . . . . . . . . 26

2.3.1 Left-Right Symmetric Extension of the Standard Model . . . . . . . 27

2.3.2 The $W^{\prime}$ Reference Model as a General Approach . . . . . . . . . . . 31

2.3.3 Bounds on the $W^{\prime}$ Mass . . . . . . . . . . . . . . . . . . . 32

3 Tevatron and the DØ Detector $\quad 35$

3.1 The Tevatron Collider Complex . . . . . . . . . . . . . . . . . . 37

3.2 Luminosity and Multiple Interactions . . . . . . . . . . . . . . . . . 43

3.3 The $\mathrm{D} \varnothing$ Detector . . . . . . . . . . . . . . . . . . . . . 45

3.3 .1 Tracking System . . . . . . . . . . . . . . . . . . . 48

3.3.2 Calorimeter . . . . . . . . . . . . . . . . . . 51

3.3.3 Muon System . . . . . . . . . . . . . . . . . . . . . . . 55

3.3.4 Luminosity Monitor . . . . . . . . . . . . . . . . . . 58

3.4 Trigger and Data Acquisition . . . . . . . . . . . . . . . 60 
3.4 .1 Level 1 Trigger . . . . . . . . . . . . . . . . . . 60

3.4.2 Level 2 Trigger . . . . . . . . . . . . . . . . . . 61

3.4 .3 Level 3 Trigger . . . . . . . . . . . . . . . . . . 61

3.4.4 Trigger and Luminosity . . . . . . . . . . . . . . . . 64

3.4 .5 Data Acquisition . . . . . . . . . . . . . . . 65

3.5 Data Reconstruction and Data Quality . . . . . . . . . . . . . . 67

4 Object Identification $\quad 71$

4.1 Electron Reconstruction . . . . . . . . . . . . . . . . . . . . 71

4.2 Jet Reconstruction . . . . . . . . . . . . . . . . . . . . . . . . . 79

4.3 Neutrino Reconstruction . . . . . . . . . . . . . . . . . . . . . . 82

5 Simulation of Background and Signal Events 85

5.1 Simulation Chain . . . . . . . . . . . . . . . . . . . . 86

5.2 Standard Model Backgrounds . . . . . . . . . . . . . . . . . . 88

5.3 The $W^{\prime}$ Signal and its Properties . . . . . . . . . . . . . . . . . . 91

6 Data Selection and QCD Background Estimation 101

6.1 Data Sample, Triggers and Luminosity . . . . . . . . . . . . . . . . . . . 101

6.2 Event Selection Criteria _ . . . . . . . . . . . . . . . . . 105

6.3 QCD Background . . . . . . . . . . . . . . . . . . 112

6.4 Effective Luminosity $\mathcal{L}_{W} \ldots \ldots \ldots \ldots \ldots \ldots$

7 Efficiencies, Corrections and Uncertainties 117

7.1 Electron Energy Resolution and Scale . . . . . . . . . . . . . . . 117

7.2 Efficiencies . . . . . . . . . . . . . . . . . . 120

7.2.1 Electron Reconstruction Efficiencies . . . . . . . . . . . . . 120

7.2 .2 Trigger Efficiency . . . . . . . . . . . . . . . . . . . . 124

7.3 Reweighting of the $p_{T}$ Spectrum of the $W$ Boson $\ldots \ldots \ldots$. . . . . 129

7.4 Systematic Uncertainties . . . . . . . . . . . . . . . . . . . . . . 143

7.4.1 Normalization Uncertainties . . . . . . . . . . . . . . . . . . . . . 144

7.4.2 Shape Changing Uncertainties . . . . . . . . . . . . . . . . . 144 
8.1 Distributions and Numbers for the $W$ Sample . . . . . . . . . . . . . . . . 151

8.2 Distributions and Numbers for the Search Sample . . . . . . . . . . . 158

8.3 Extraction of the Limit . . . . . . . . . . . . . . . . . 170

8.3.1 Binned Likelihood Method _. . . . . . . . . . . . 170

8.3.2 Limits on the Production Cross Section and the Mass . . . . . . . 173

9 Conclusion and Outlook $\quad 179$

$\begin{array}{ll}\text { A Abbreviations } & 181\end{array}$

B Rapidity and Pseudorapidity 185

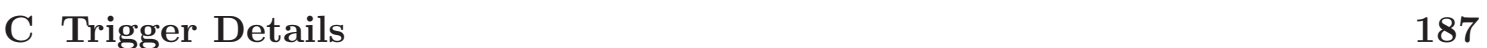

$\begin{array}{ll}\text { D } \text { Modified } W^{\prime} \text { Signal } & 189\end{array}$ 



\section{Chapter 1}

\section{Introduction}

Now hear the fourfold roots of everything:

Enlivening Hera, Hades, shining Zeus

And Nestis, moistening mortal springs with tears.

"Doctrine of the Four Elements", Empedocles

The desire of breaking down the basics of nature into fundamental pieces started a long time ago. According to the Greek philosophy, everything is made of the four classical elements fire, earth, air and water. The scientist and philosopher Empedocles (5th century B.C.) associated these elements not only with physical manifestations or material substances, but also with spiritual essences. Assigning the elements to Greek gods and goddesses (see above) suggests that their further decomposition may be unseizable for mankind.

The first important step in the history of particle physics can be granted to Democritus (4th centure B. C.). He brought up the idea that nature consists of imperishable, indivisible elements which he called ' $a$ tomos'. He already mentioned that they are not all the same, as they differ in shape. According to him, it is even possible to split each of the four elements into tiny basic pieces. Although being controversial to other theories appearing at the same time, his atomic model was valid until the beginning of the 19th century.

A renaissance of the early atomic theory appeared more than 2,000 years later. During the 18th century, Lavoisier managed to determine the constituents of water, one of the previous classical elements. In 1803 Dalton published his atomic model which was still based on Democritus' picture of atoms. One century later, Mendeleev arranged the known elements according to their chemical and physical properties, and made predictions for the elements that were missing. It seemed that the description of nature's basic ingredients got more complicated (from four to more than 60 elements), but the periodicity and ordinal structure were strong hints for an underlying substructure.

During the beginning of the 20th century, when Rutherford made his scattering experiments and quantum mechanics was established, the picture of the irreducible atom in Democritus' sense broke down, and the predicted substructure was revealed: the atom consists of a nucleus containing protons and neutrons, and a shell containing electrons. From Heisenberg's uncertainty relation $\Delta x \cdot \Delta p_{x} \geq \hbar / 2$ it was clear that in order to resolve 
the structure $\Delta x$ of the subatomic particles - and to determine wether those are fundamental or not - highly energetic probe particles have to be used. For example, Rutherford used $\alpha$ particles with an energy of $E \sim \mathrm{MeV}$ to resolve the atomic structure $\left(r \approx 10^{-13} \mathrm{~m}\right)$.

The era of fixed target and collider experiments (linear, circular) starting in the 1950s should solve the question of a possible substructure of the subatomic particles - and more mysterious particles like the pion or muon found in cosmic radiation. But first of all, new questions were brought up due to the huge amount of new particles discovered at the colliders. Hence, history repeated itself and likewise the solution to the so-called particle zoo: the hadrons (neutron, proton, pion, ... ) have a substructure and consist of quarks.

Figure 1.1: History of the basics of nature: from the classical four elements, to Mendeleev's periodic table up to today's picture of elementary particles [1], [2].
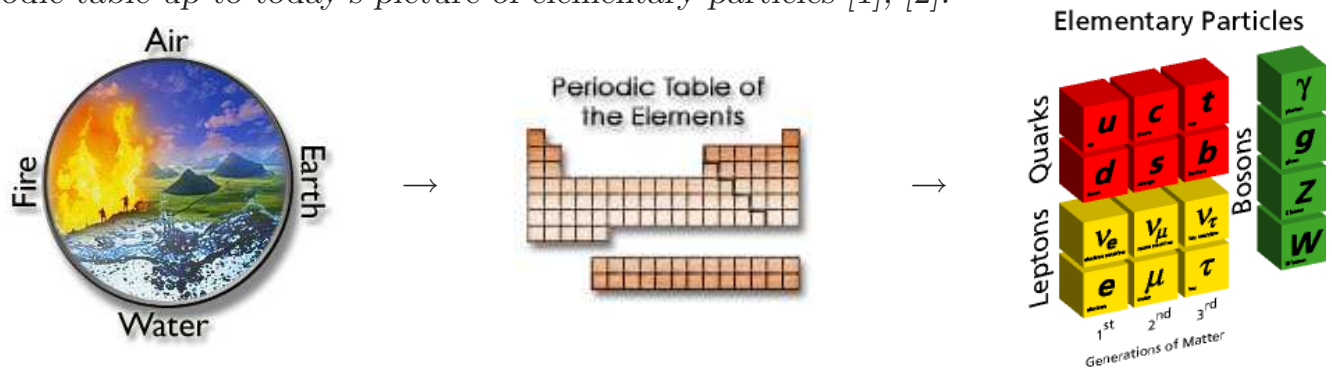

Finally, after more than 2,000 years, nature can be decomposed into fundamental particles defined as pointlike and irreducible at the current experimental level. Today's theoretical picture - the Standard Model - based on S. L. Glashow, A. Salam and S. Weinberg describes nature, i.e. matter and interactions of matter, at a high level of accuracy. The underlying symmetry group is $\mathrm{SU}(3)_{C} \otimes \mathrm{SU}(2)_{L} \otimes \mathrm{U}(1)_{Y}$ describing Quantum Chromodynamics (QCD, strong interaction) in the color space $\mathrm{SU}(3)_{C}$ of the quarks, and the unification of the weak and electromagnetic interactions. The latter ones act on doublets of left-handed fermions and singlets of the hypercharge $Y$. This model includes a rather small amount of elementary particles compared to the periodic table or the particle zoo of hadrons (see Tab. 1.1): Matter is made up of fermions, namely quarks and leptons, and interactions are conveyed by gauge bosons. The number of free parameters in the Standard Model is 28 (19 assuming massless neutrinos). The question marks in Tab. 1.1 indicate that ingredients of the Standard Model are still missing. The existence of a Higgs

Table 1.1: Overview of elementary particles. The Higgs boson is introduced in order to provide massive particles in the Standard Model (see Sec. 2.2), but it has not yet been observed.

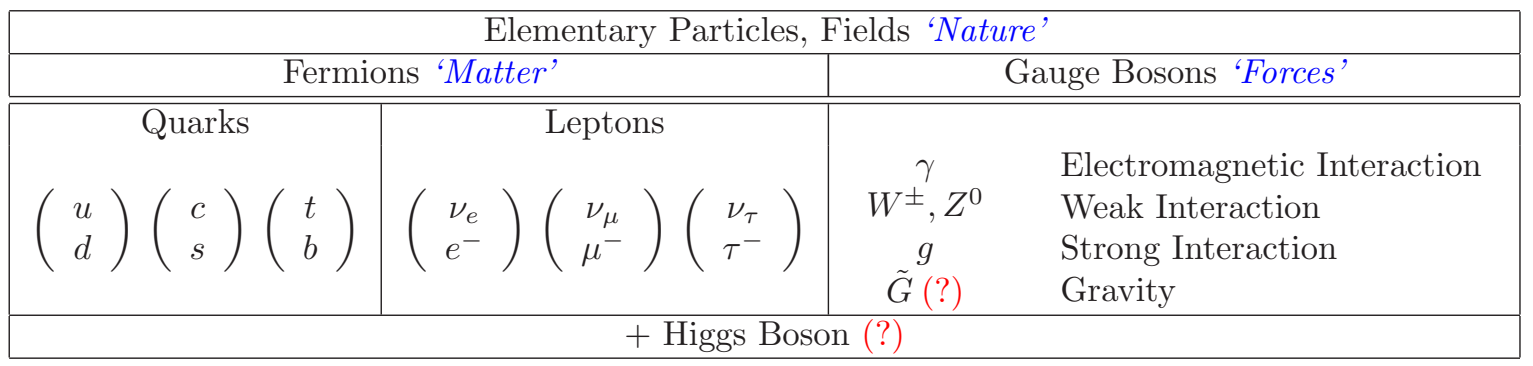


boson is required in order to generate massive particles. Further, the graviton $\tilde{G}$ which mediates gravity is a hypothetical spin-2-particle. Since a renormalizable quantum field theory for the gravity does not exist, this interaction is not contained in the Standard Model.

The fact that two of four forces are unified can be seen as a hint for a further unification. Indeed, the electroweak and the strong interaction can be unified at extremely high energies in extensions to the Standard Model. One promising approach is the introduction of supersymmetric partner particles to the Standard Model particles. Already the minimal extension, the Minimal Supersymmetric Standard Model (MSSM), makes it possible that the coupling constants of the electromagnetic, weak and strong interactions meet at one point at the energy of $\approx 10^{16} \mathrm{GeV}$, in contrast to the Standard Model, see Fig. 1.2.

Figure 1.2: Evolution of the inverse gauge couplings $\alpha^{-1}(Q)$ in the Standard Model (dashed lines) and the MSSM (solid lines) [with $\alpha_{i}=\frac{g_{i}^{2}}{4 \pi}, e=g \sin \theta_{w}=g^{\prime} \cos \theta_{w}, g_{2} \equiv g$ and $g_{1}=\sqrt{5 / 3} g^{\prime}$, see Sec. 2.2]. In the MSSM case, the masses of the supersymmetric particles are varied between $250 \mathrm{GeV}$ and $1 \mathrm{TeV}$, and $\alpha_{3}\left(m_{Z}\right)$ between 0.113 and 0.123 (taken from [3]).

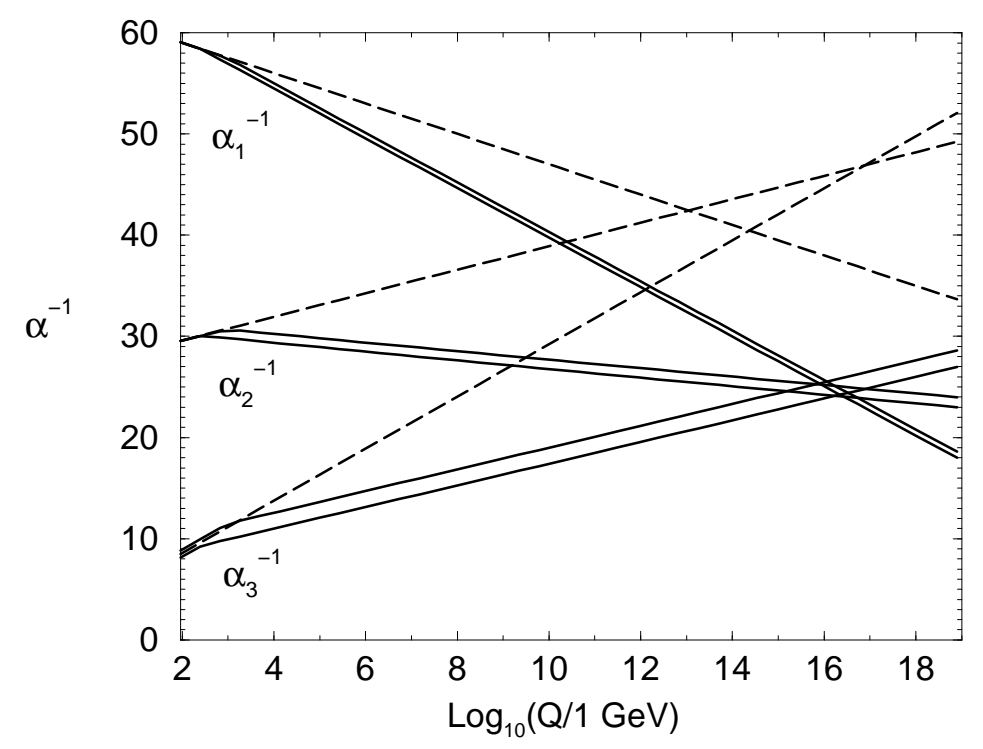

Although affecting many aspects of the everyday life, the remaining force, the gravitational force, still escapes from a description by a quantum field theory due to the perturbative non-renormalizability of the Lagrangian density. Gravity does not play a significant role in today's collider experiments due to its weakness compared to the other forces, but will become important at the Planck scale $\left(\approx 10^{19} \mathrm{GeV}\right)$. However, a quantum field theory with only one force and the Standard Model as the low energy limit, the Theory of Everything, is still not in sight.

There are lots of open questions in conjunction with the Standard Model: Why do we observe a family structure both in the lepton and quark sectors? What about the number of parameters? What about the hierarchy of masses? There are also problems in the profundities of the theory concerning the fine-tuning of the mass of the Higgs boson. All this inspired theorists to extend the Standard Model so that one or more of the open questions can be solved, like the unification of the couplings and the solution to the problem of the fine-tuning of the Higgs boson mass in supersymmetric models. 
The extensions usually predict new particles and parameters. These new particles and their decays can be looked for at a collider experiment like the proton-antiproton collider TEVATRON which operates at a center-of-mass energy of $\sqrt{s}=1.96 \mathrm{TeV}$ or the upcoming LHC proton-proton collider at $\sqrt{s}=14 \mathrm{TeV}$. At these colliders, it is possible to produce particles with extremely high masses that could never be explored before.

In the Standard Model the charged current of the weak interaction is restricted to left-handed particles only. Therefore the extension by a right-handed sector has not only aesthetic reasons, but it provides for example a mechanism for parity violation which has to be implemented by hand in the Standard Model. The left-right symmetric gauge group is now $\mathrm{SU}(2)_{L} \otimes \mathrm{SU}(2)_{R} \otimes \mathrm{U}(1)_{\tilde{Y}}$ introducing a modified hypercharge $\tilde{Y}$. New gauge bosons denoted as $W^{\prime}$ and $Z^{\prime}$ which can be searched for at colliders arise in these models. In order to get the $\mathrm{SU}(2)_{L} \otimes \mathrm{U}(1)_{Y}$ structure at energies around $100 \mathrm{GeV}$, the introduced left-right symmetry has to be broken by a scalar Higgs field.

In this thesis a search for a new heavy charged gauge boson $W^{\prime}$ and its subsequent decay into electron and neutrino is performed using data taken at the TEVATRON collider corresponding to an integrated luminosity of about $\int \mathcal{L} \mathrm{d} t=1 \mathrm{fb}^{-1}$. First, the Standard Model is reviewed together with a discussion of the Left-Right Symmetric Model. This is followed by a description of the experimental setup and the object identification. The simulation of background processes and the expected signal as well as its properties are outlined in the next chapter. After this the data selection criteria are described, followed by the discussion of efficiencies, corrections and the corresponding uncertainties. Finally, the results are presented and discussed. 


\section{Chapter 2}

\section{Theoretical Foundations}

God used beautiful mathematics in creating the world.

Paul Dirac

\subsection{Measures and Conventions}

\section{Units}

Throughout this analysis natural units are used with

$$
\hbar=c=1 .
$$

Then the dimension of basic quantities can be written in terms of the energy

$$
[\text { energy }]=[\text { mass }]=[\text { momentum }]=[\text { time }]^{-1}=[\text { length }]^{-1}=[\text { cross section }]^{-2} .
$$

In particle physics it is convenient to measure the energy (and thus all other quantities) in units of 'electronvolt' (eV). One electronvolt is the amount of kinetic energy gained by a single unbound electron when it passes through an electrostatic potential difference (in vacuum) of one volt. The conversion to $\mathrm{SI}^{1}$ units which makes use of the charge of the electron $e$ with $e=1.60217653(14) \cdot 10^{-19} \mathrm{C}[4]$ is as follows

$$
1 \mathrm{eV}=1 \cdot\left(1.602 \cdot 10^{-19} \mathrm{C}\right) \cdot 1 \mathrm{~V}=1.602 \cdot 10^{-19} \mathrm{~J}=1.602 \cdot 10^{-19} \mathrm{~kg} \mathrm{~m}{ }^{2} / \mathrm{s}^{2} .
$$

A quantity given in natural units can unambigously be transformed into SI units by multiplying with powers of $\hbar$ and $c$.

\section{Notation}

In the following, a simplified notation is used:

- The symbol $W$ (for the $W$ boson) refers to $W^{+}$and $W^{-}$;

- $Z$ stands for $Z^{0}$;

\footnotetext{
${ }^{1}$ Système International d'Unités; International System of Units.

Within this system it is $\hbar=1.05457168(18) \cdot 10^{-34} \mathrm{~kg} \mathrm{~m}{ }^{2} / \mathrm{s}$ and $c=2.99792458 \cdot 10^{8} \mathrm{~m} / \mathrm{s} \mathrm{[4]}$.
} 
- $e$ stands for $e^{+}$or $e^{-}$; the term 'electron' is used for the electron itself as well as for the positron;

- $\nu$ stands for $\nu_{\ell}$ or $\bar{\nu}_{\ell}(\ell=e, \mu, \tau)$.

Hence, $Z \rightarrow e e$ replaces $Z^{0} \rightarrow e^{+} e^{-}$, and $W \rightarrow e \nu$ is used instead of $W^{+} \rightarrow e^{+} \nu_{e}$ and $W^{-} \rightarrow e^{-} \bar{\nu}_{e}$, respectively. $\tau^{-} \rightarrow e^{-} \bar{\nu}_{e} \nu_{\tau}$ is reduced to $\tau \rightarrow e \nu \nu$.

Further, Einstein's summation convention is applied.

\section{Kinematics}

The coordinate system (Fig. 2.1) is right-handed with the $z$-axis pointing in direction of the proton beam; the $y$-axis increases with height of the $\mathrm{D} \varnothing$ detector. The $x$-and $y$-axes build up the transverse plane (with respect to the beam). The origin of the coordinate system is located in the geometric center of the D $\varnothing$ detector. All quantities are calculated with respect to the vertex of the hard collision $\left(v_{x}, v_{y}, v_{z}\right)$. Nevertheless, in some cases certain variables are given with respect to the origin $(0,0,0)$, too, in order to reflect geometric properties of the detector (see Fig. 2.1).

The momentum vectors are defined as follows

$$
\begin{gathered}
\vec{p}=\left(\begin{array}{c}
p_{x} \\
p_{y} \\
p_{z}
\end{array}\right)=\left(\begin{array}{c}
p \cos \phi \sin \theta \\
p \sin \phi \sin \theta \\
p \cos \theta
\end{array}\right) \quad \Rightarrow \quad p=|\vec{p}|=\sqrt{p_{x}^{2}+p_{y}^{2}+p_{z}^{2}} \\
\vec{p}_{T}=\left(\begin{array}{c}
p_{x} \\
p_{y} \\
0
\end{array}\right)=\left(\begin{array}{c}
p_{T} \cos \phi \\
p_{T} \sin \phi \\
0
\end{array}\right) \Rightarrow p_{T}=\left|\vec{p}_{T}\right|=\sqrt{p_{x}^{2}+p_{y}^{2}}=p \sin \theta .
\end{gathered}
$$

Apart from cartesian $\left\{p_{x}, p_{y}, p_{z}\right\}$ or spherical coordinates $\{p, \phi, \theta\}$, modified spherical coordinates $\{p, \phi, \eta\}$ are used. It is convenient to replace the polar angle $\theta$ by the pseudorapidity $\eta$ (see App. B). In the high energy limit, $\eta$ can be calculated from the polar angle $\theta$ via

$$
\eta=-\ln \tan \frac{\theta}{2}
$$

Figure 2.1: Coordinate system with the definition of the momentum $\vec{p}$ and transverse momentum $\vec{p}_{T}$ (left-hand sketch); a comparison between $\eta$ and $\eta_{\text {det }}$ is displayed in the right-hand figure.
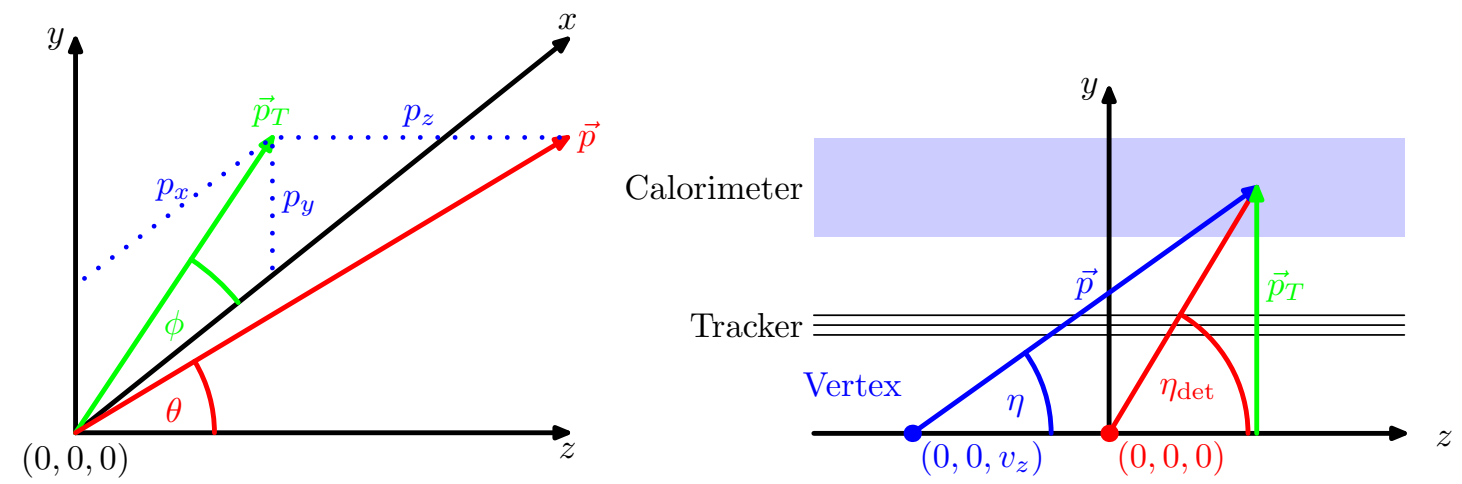
Momentum 4-vectors $p^{\mu}$ with energy $E$ and 3-momentum $\vec{p}$ are used

$$
p^{\mu}=\left(\begin{array}{c}
E \\
\vec{p}
\end{array}\right)=\left(\begin{array}{c}
E \\
p_{x} \\
p_{y} \\
p_{z}
\end{array}\right)
$$

Collisions of protons and antiprotons are analyzed in which each of the two particles carries an energy of $E=980 \mathrm{GeV}$. Because of $E \gg m_{p}$ (limit of high energies), the 4-vectors (Fig. 2.2) of the proton $p_{p}^{\mu}$ and the antiproton $p_{\bar{p}}^{\mu}$ can be written as

$$
p_{p}^{\mu}=\left(\begin{array}{c}
E \\
0 \\
0 \\
E
\end{array}\right) \quad \text { and } \quad p_{\bar{p}}^{\mu}=\left(\begin{array}{c}
E \\
0 \\
0 \\
-E
\end{array}\right)
$$

resulting in a center-of-mass energy $\sqrt{s}$ of

$$
s=\left(p_{p}+p_{\bar{p}}\right)^{2}=4 E^{2} \quad \Rightarrow \quad \sqrt{s}=2 E=1.96 \mathrm{TeV} .
$$

Partons (sea quarks, valence quarks and gluons) which are the constituents of hadrons are involved in the collisions at a hadron collider; they participate in the hard scattering process. Fig. 2.2 shows this for the process $p \bar{p} \rightarrow W+X \rightarrow e \nu+X$. In the picture of Feynman's infinite momentum frame a highly energetic hadron (here: proton or antiproton) consists of collinear quarks carrying a certain momentum fraction $x$. In the infinite momentum frame transverse momenta of the partons are neglected so that

$$
p_{d}^{\mu}=x_{p} \cdot p_{p}^{\mu}=\left(\begin{array}{c}
x_{p} E \\
0 \\
0 \\
x_{p} E
\end{array}\right) \quad \text { and } \quad p_{\bar{u}}^{\mu}=x_{\bar{p}} \cdot p_{\bar{p}}^{\mu}=\left(\begin{array}{c}
x_{\bar{p}} E \\
0 \\
0 \\
-x_{\bar{p}} E
\end{array}\right)
$$

The center-of-mass energy in the hard scattering process $\sqrt{\hat{s}}$ is thus reduced

$$
\hat{s}=\left(p_{d}+p_{\bar{u}}\right)^{2}=4 x_{p} x_{\bar{p}} E^{2}=x_{p} x_{\bar{p}} \cdot s \quad \Rightarrow \quad \sqrt{\hat{s}}=\sqrt{x_{p} x_{\bar{p}}} \cdot \sqrt{s} .
$$

Figure 2.2: Kinematics in proton-antiproton collisions.

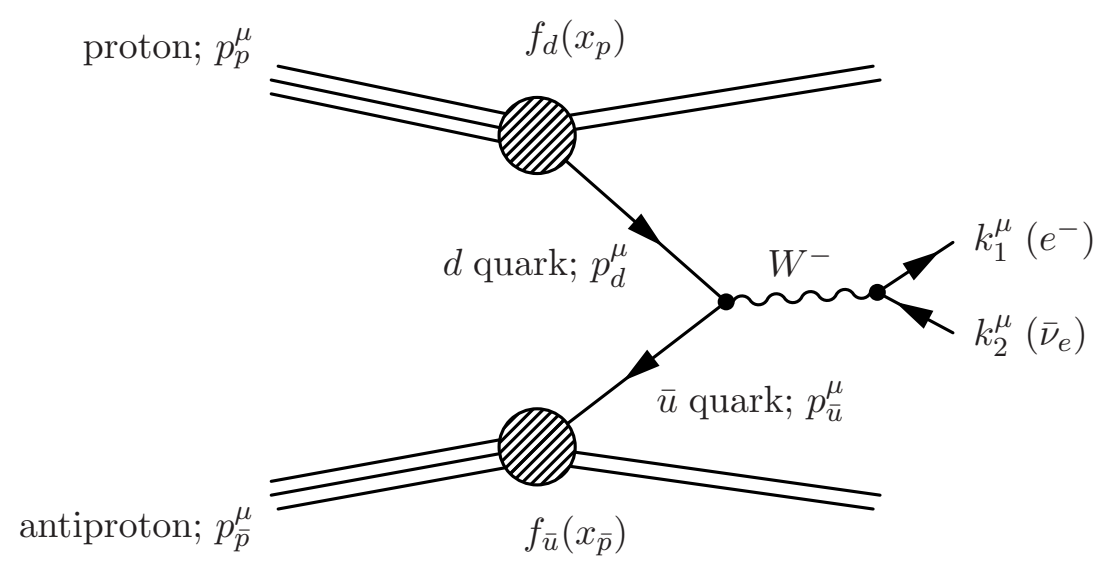


Since the partonic energy fractions $x(0<x<1)$ follow certain distributions $f(x)$ (parton distribution function, parton density function, see Sec. 2.2.6), the initial state of the parton collision is not well defined. In general, the partonic system is boosted in the direction of the $z$-axis. Since differential rapidity distributions are invariant under Lorentz boosts along the $z$-axis $\left(\mathrm{d} y=\mathrm{d} y^{\prime}\right.$, see App. B), the rapidity is used instead of the polar angle.

The transverse momenta of the incoming partons (nearly) vanish so that at least in the transverse plane energy conservation can be applied

$$
\sum_{\text {initial state }} \vec{p}_{T}^{i}=\sum_{\text {final state }} \vec{p}_{T}^{f}=\overrightarrow{0}
$$

This relation is important for the reconstruction of particles that escape detection like neutrinos. One can rewrite Eq. 2.12 separating visible and invisible objects in the final state

$$
\sum_{\text {final state }} \vec{p}_{T}^{f}=\overrightarrow{0}=\sum_{\text {visible }} \vec{p}_{T}^{v}+\sum_{\text {invisible }} \vec{p}_{T}^{i} \Rightarrow \vec{E}_{T} \equiv \sum_{\text {invisible }} \vec{p}_{T}^{i}=-\sum_{\text {visible }} \vec{p}_{T}^{v}
$$

One can only reconstruct the sum of all missing particles in the final state whose energy is denoted as missing transverse energy $\vec{E}_{T}$ or MET. Of course, particles going into noninstrumented parts of the detector contribute to the missing transverse energy as well as very weakly interacting particles like neutrinos - or other not yet discovered particles.

\subsection{Aspects of the Standard Model}

The basic constituents of nature have already been summarized in the introduction. Now, a more detailed discussion of the underlying interactions is presented (for a complete review see e.g. [5] or [6]). Quarks and leptons are fermions with spin $1 / 2$. Their wave function $\psi(x)$ - a spinor with four components - satisfies the Dirac equation

$$
\left(i \gamma^{\mu} \partial_{\mu}-m\right) \psi(x)=0
$$

with the spacetime index $\mu(\mu=0,1,2,3)$, the $4 \times 4$ gamma matrices $\gamma^{\mu}$, the fermion mass $m$ and the 4 -gradient $\partial_{\mu}$ with $\partial_{\mu}=\left(\partial_{0}, \nabla\right)$. Interactions are conveyed by spin-1-particles ${ }^{2}$ (bosons) which fulfill

$$
\left(\partial^{\mu} \partial_{\mu}+M^{2}\right) V^{\nu}(x)-\partial^{\nu}\left(\partial_{\mu} V^{\mu}(x)\right)=j^{\nu}(x)
$$

with the gauge field $V^{\mu}(x)$, the mass of the gauge boson $M$ and the corresponding 4-current $j^{\mu}(x)$.

\subsubsection{The Gauge Principle}

The presence of gauge bosons which mediate interactions between fermionic matter fields is a consequence of the gauge principle:

\footnotetext{
${ }^{2}$ except for the gauge boson of the gravitation (graviton, $\tilde{G}$ ) which has to be a spin-2-particle. The gravitational interaction as a quantum field theory is not contained in the Standard Model.
} 
The Dirac equation is postulated to be invariant under arbitrary local (i.e. spacetime-dependent) transformations $U(x)$ :

$$
\psi(x) \quad \rightarrow \quad \psi^{\prime}(x)=U(x) \psi(x) .
$$

This is not possible in a field-free environment; one has to require the existence of an external spin-1-field (vector boson) $V^{\mu}(x)$ which has also to be transformed.

This introduces the gauge bosons which couple to Dirac spinors and to itself if the corresponding self-couplings are not zero. In Sec. 2.2.2 it is shown that local transformations of the weak isospin and the hypercharge lead to the existence of the $W$ and $Z$ bosons as well as the photon. Local transformations in the color space of the quarks lead to the existence of the gluons (Sec. 2.2.3).

A single symmetry is described by the set of all unitary gauge transformations $\left(U^{\dagger} U=\right.$ 1) and can generally be written as

$$
U(x)=\exp \left\{i g \cdot \alpha_{i}(x) \mathcal{G}_{i}\right\}, \quad i=1, \ldots, n
$$

with a coupling constant $g$, parameters $\alpha_{i}(x)$ specifying the local transformation and $n$ generators $\mathcal{G}_{i}$ determining the algebra of the underlying symmetry. If the generators fulfill the commutator relation

$$
\left[\mathcal{G}_{i}, \mathcal{G}_{j}\right] \equiv \mathcal{G}_{i} \mathcal{G}_{j}-\mathcal{G}_{j} \mathcal{G}_{i}=i \cdot c_{i j k} \mathcal{G}_{k}
$$

the corresponding group is referred to as a Lie group. The $c_{i j k}$ are called structure constants of the group. If the structure constant vanishes, the group is called Abelian. This is the case for the $\mathrm{U}(1)_{Y}$ symmetry group. The $\mathrm{SU}(2)_{L}$ and $\mathrm{SU}(3)_{C}$ symmetry groups are non-Abelian.

In order to guarantee invariance of the equations of motion (Eq. 2.14 and 2.15), the fields and derivatives have to be transformed as well

$$
\begin{aligned}
\partial^{\mu} & \rightarrow \quad D^{\mu}=\partial^{\mu}+i g \cdot \mathcal{G}_{i} V_{i}^{\mu}(x), \\
V_{i}^{\mu}(x) & \rightarrow \quad V_{i}^{\prime \mu}=V_{i}^{\mu}(x)-\partial^{\mu} \alpha_{i}(x)-g \cdot c_{i j k} \alpha_{j}(x) V_{k}^{\mu}(x) .
\end{aligned}
$$

One can see that the external field $V^{\mu}(x)$ enters the Dirac equation via the modified derivative (covariant derivative). The field strength tensor $T_{i}^{\mu \nu}$ of the gauge field $V^{\mu}(x)$ which is used to describe the energy density of the gauge boson field is defined as follows

$$
T_{i}^{\mu \nu}(x)=\partial^{\mu} V_{i}^{\nu}(x)-\partial^{\nu} V_{i}^{\mu}(x)-g \cdot c_{i j k} V_{j}^{\mu}(x) V_{k}^{\nu}(x) .
$$

The last term is responsible for the self-interaction of the gauge boson associated to the field $V^{\mu}(x)$. This term vanishes in Abelian theories. The field strength tensor transforms as follows

$$
T_{i}^{\mu \nu}(x) \quad \rightarrow \quad T_{i}^{\prime \mu \nu}(x)=T_{i}^{\mu \nu}(x)-g \cdot c_{i j k} \alpha_{j}(x) T_{k}^{\mu \nu}(x) .
$$

The 4-current in the wave equation Eq. 2.15 is

$$
j_{i}^{\nu}(x)=g \cdot c_{i j k} V_{\mu, j} T_{k}^{\mu \nu} .
$$


The Lagrangian density contains all relevant information of the Standard Model. In the next sections the Lagrangian density of the fermions in a field-free environment $\mathcal{L}_{\text {free }}$ will consecutively be extended with the observed interactions, namely the electromagnetic, weak and strong interaction. In general, the underlying symmetry has to be established, the derivative replaced by the covariant derivative and the energy density of the new gauge field to be added to the Lagrangian $\mathcal{L}_{\text {int }}$

$$
\mathcal{L}_{\text {free }}=i \cdot \sum_{\text {fermions } f} \bar{\psi}_{f} \gamma_{\mu} \partial^{\mu} \psi_{f} \quad \rightarrow \quad \mathcal{L}_{\text {int }}=i \cdot \sum_{\text {fermions } f} \bar{\psi}_{f} \gamma_{\mu} D^{\mu} \psi_{f}-\frac{1}{4} T_{\mu \nu}^{i} T_{i}^{\mu \nu}
$$

with $\bar{\psi}=\psi^{\dagger} \gamma^{0}$. Since the Standard Model in this form is only valid for massless particles, finally the Higgs mechanism is discussed which takes care of the masses (Sec. 2.2.4).

\subsubsection{The Electroweak Interaction}

The left-handed fermions are grouped in doublets with respect to the weak isospin $I$ ( $I=1 / 2$ with $I_{3}= \pm 1 / 2$ ), whereas the right-handed fermions form singlets $(I=0)$. The weak hypercharge $Y$ is connected with the electrical charge $Q$ and the third component of the weak isospin $I_{3}$ via the Gell-Mann-Nishijima relation

$$
Q=I_{3}+\frac{Y}{2}
$$

Since there is no experimental evidence for right-handed neutrinos, they are not contained in the Standard Model Tab. 2.1. Right-handed neutrinos are difficult to detect because they do not interact with other particles of the Standard Model (not charged, singlets with respect to the weak interaction). However, the presence of right-handed neutrinos together with the Higgs mechanism is able to endow mass to the observed lefthanded neutrinos (see Sec. 2.3.1).

With the notation introduced in Tab. 2.1, the Lagrangian of free massless fermions can be written as

$$
\mathcal{L}_{\text {free }}=i \cdot \sum_{\ell=1}^{3} \bar{L}_{\ell} \gamma_{\mu} \partial^{\mu} L_{\ell}+i \cdot \sum_{\ell=1}^{3} \bar{R}_{\ell} \gamma_{\mu} \partial^{\mu} R_{\ell}+i \cdot \sum_{q=1}^{3} \bar{L}_{q} \gamma_{\mu} \partial^{\mu} L_{q}+i \cdot \sum_{q=1}^{6} \bar{R}_{q} \gamma_{\mu} \partial^{\mu} R_{q}
$$

Table 2.1: Properties of the fermions ( $L=$ lepton number, $B=$ baryon number).

\begin{tabular}{|c|c|c|c|c|c|c|c|c|}
\hline & $1^{\text {st }}$ Generation & $2^{\text {nd }}$ Generation & $3^{\text {rd }}$ Generation & $I_{3}$ & $Q$ & $Y$ & $L$ & $B$ \\
\hline \hline$L_{\ell}$ & $L_{1}=\left(\begin{array}{c}\nu_{e} \\
e^{-}\end{array}\right)_{L}$ & $L_{2}=\left(\begin{array}{c}\nu_{\mu} \\
\mu^{-}\end{array}\right)_{L}$ & $L_{3}=\left(\begin{array}{c}\nu_{\tau} \\
\tau^{-}\end{array}\right)_{L}$ & $\begin{array}{c}1 / 2 \\
-1 / 2\end{array}$ & $\begin{array}{c}0 \\
-1\end{array}$ & -1 & 1 & 0 \\
\hline$R_{\ell}$ & $R_{1}=e_{R}^{-}$ & $R_{2}=\mu_{R}^{-}$ & $R_{3}=\tau_{R}^{-}$ & 0 & -1 & -2 & 1 & 0 \\
\hline \hline$L_{q}$ & $L_{1}=\left(\begin{array}{c}u \\
d^{\prime}\end{array}\right)_{L}$ & $L_{2}=\left(\begin{array}{c}c \\
s^{\prime}\end{array}\right)_{L}$ & $L_{3}=\left(\begin{array}{c}t \\
b^{\prime}\end{array}\right)_{L}$ & $\begin{array}{c}1 / 2 \\
-1 / 2\end{array}$ & $\begin{array}{c}2 / 3 \\
-1 / 3\end{array}$ & $1 / 3$ & 0 & $1 / 3$ \\
\hline$R_{q}$ & $R_{1}=u_{R}$ & $R_{2}=c_{R}$ & $R_{3}=t_{R}$ & 0 & $2 / 3$ & $4 / 3$ & 0 & $1 / 3$ \\
\hline$R_{q}$ & $R_{4}=d_{R}$ & $R_{5}=s_{R}$ & $R_{6}=b_{R}$ & 0 & $-1 / 3$ & $-2 / 3$ & 0 & $1 / 3$ \\
\hline
\end{tabular}


taking into account that left-handed and right-handed particles do not couple to each other. Left- and right-handed spinors can be obtained using projection operators

$$
\begin{aligned}
L & =P_{L} \psi(x)=\frac{1-\gamma^{5}}{2} \psi(x) \quad \text { and } \\
R & =P_{R} \psi(x)=\frac{1+\gamma^{5}}{2} \psi(x) .
\end{aligned}
$$

Mass eigenstates $q$ of the left-handed quarks with $I_{3}=-1 / 2$ are different from the eigenstates regarding the weak interaction, $q^{\prime}$. Both sets of eigenstates are connected via the Cabbibo-Kobayashi-Maskawa matrix (CKM matrix) $V_{C K M}$

$$
\left(\begin{array}{c}
d^{\prime} \\
s^{\prime} \\
b^{\prime}
\end{array}\right)_{L}=V_{C K M} \cdot\left(\begin{array}{c}
d \\
s \\
b
\end{array}\right)=\left(\begin{array}{ccc}
V_{u d} & V_{u s} & V_{u b} \\
V_{c d} & V_{c s} & V_{c b} \\
V_{t d} & V_{t s} & V_{t b}
\end{array}\right) \cdot\left(\begin{array}{c}
d \\
s \\
b
\end{array}\right) .
$$

The unitary matrix $V_{C K M}$ depends in general on three angles and six phases. Five phases can be removed by redefining the phases of the quark eigenstates leaving one single physical phase. This phase is responsible for the $\mathcal{C P}$ violation in meson decays in the Standard Model. The elements $V_{i j}$ can not be calculated in the framework of the Standard Model; they are free parameters which have to be determined by measurements.

Observations indicate that neutrinos are able to change their flavor (atmospheric neutrino oscillation $\nu_{\mu} \rightarrow \nu_{\tau}$ [7], solar neutrino oscillations $\nu_{e} \rightarrow \nu_{\mu}$ [8], confirmed by reactor experiments [9], [10]). The Maki-Nakagawa-Sakata matrix $U_{M N S}$ connects mass eigenstates $\nu_{1}, \nu_{2}$ and $\nu_{3}$ with the flavor eigenstates $\nu_{e}, \nu_{\mu}$ and $\nu_{\tau}$

$$
\begin{gathered}
\left(\begin{array}{c}
\nu_{1} \\
\nu_{2} \\
\nu_{3}
\end{array}\right)=U_{M N S}\left(\begin{array}{c}
\nu_{e} \\
\nu_{\mu} \\
\nu_{\tau}
\end{array}\right)=\tilde{U}_{M N S}\left(\begin{array}{ccc}
e^{i \alpha_{1} / 2} & 0 & 0 \\
0 & e^{i \alpha_{2} / 2} & 0 \\
0 & 0 & 1
\end{array}\right)\left(\begin{array}{c}
\nu_{e} \\
\nu_{\mu} \\
\nu_{\tau}
\end{array}\right) \\
\tilde{U}_{M N S}=\left(\begin{array}{ccc}
c_{12} c_{13} & s_{12} c_{13} & s_{13} e^{-i \delta} \\
-s_{12} c_{23}-c_{12} s_{23} s_{13} e^{i \delta} & c_{12} c_{23}-s_{12} s_{23} s_{13} e^{i \delta} & s_{23} c_{13} \\
s_{12} s_{23}-c_{12} c_{23} s_{13} e^{i \delta} & -c_{12} s_{23}-s_{12} c_{23} s_{13} e^{i \delta} & c_{23} s_{13}
\end{array}\right)
\end{gathered}
$$

with $s_{i j}=\sin \theta_{i j}$ and $c_{i j}=\cos \theta_{i j}$. This matrix contains three mixing angles $\theta_{i j}$, one $\mathcal{C P}$ phase $\delta$ and two Majorana phases $\alpha_{i}$. These phases are relevant if the neutrinos are Majorana particles, identical to their antiparticles. Since quarks are Dirac particles, these phases are not contained in $V_{C K M}$. The nature of neutrinos (Dirac or Majorana) is not yet revealed by experiments. The neutrino sector introduces - together with the neutrino masses - nine additional parameters in the Standard Model.

The weak hypercharge is connected with a one-dimensional phase transformation $\mathrm{U}(1)_{Y}$

$$
L^{\prime}=\exp \left\{i g^{\prime} \cdot \frac{Y}{2} \cdot \chi(x)\right\} L \quad \text { and } \quad R^{\prime}=\exp \left\{i g^{\prime} \cdot \frac{Y}{2} \cdot \chi(x)\right\} R
$$

whereas the underlying symmetry group for the transformation of the left-handed fermions is $\mathrm{SU}(2)_{L}$ with

$$
L^{\prime}=\exp \left\{i g \cdot \frac{\sigma_{i}}{2} \cdot \beta_{i}(x)\right\} L
$$

The transformations introduce coupling constants $g^{\prime}$ and $g$ and the generators of the symmetry, namely the three Pauli matrices $\sigma_{i}(i=1,2,3)$ for $\mathrm{SU}(2)_{L}$ and the $1 \times 1$ unit 
matrix 1 for $\mathrm{U}(1)_{Y}$. The terms $\chi(x)$ und $\beta_{i}(x)\left(i=1,2,3 ; x=x^{\mu}\right)$ are spacetime dependent functions. In order to satisfy the gauge principle, that is to keep $\mathcal{L}$ invariant under local transformations $\mathrm{U}(1)_{Y}$ and $\mathrm{SU}(2)_{L}$, gauge fields have to be introduced. The hypercharge is connected with a vector field $B^{\mu}$, the invariance under $\mathrm{SU}(2)_{L}$ transformations requires three vector fields $W_{i}^{\mu}(i=1,2,3)$. The vector fields transform as follows

$$
\begin{aligned}
B^{\prime \mu} & =B^{\mu}-\partial^{\mu} \chi(x), \\
W_{i}^{\prime \mu} & =W_{i}^{\mu}-\partial^{\mu} \beta_{i}(x)-g \cdot \varepsilon_{i j k} \beta_{j}(x) W_{k}^{\mu} .
\end{aligned}
$$

The term $\varepsilon_{i j k}{ }^{3}$ denotes the structure constant of $\mathrm{SU}(2)_{L}$; the structure constant of $\mathrm{U}(1)_{Y}$ is zero. The commutator relation for the $\mathrm{SU}(2)_{L}$ reads

$$
\left[\tau_{i}, \tau_{j}\right]=i \cdot \varepsilon_{i j k} \tau_{k} \quad \text { with } \quad \tau_{i}=\frac{\sigma_{i}}{2} .
$$

The last missing ingredient is the covariant derivative which replaces the 4-gradient $\partial^{\mu}$

$$
D_{E W}^{\mu}=\partial^{\mu}+i g \cdot \tau_{i} \cdot W_{i}^{\mu}+i g^{\prime} \cdot \frac{Y}{2} \cdot B^{\mu} .
$$

Right-handed particles are singlets under $\mathrm{SU}(2)_{L}\left(I=0 \Rightarrow \tau_{i}=0\right)$ so that $\tau_{i} \cdot W_{i}^{\mu}=0$. Making use of the ladder operators $\tau_{ \pm}=\frac{1}{2}\left(\sigma_{1} \pm i \sigma_{2}\right)=\tau_{1} \pm i \tau_{2}$ it follows

$$
\tau_{i} \cdot W_{i}^{\mu}=\frac{1}{\sqrt{2}}\left(\tau_{+} W^{(-) \mu}+\tau_{-} W^{(+) \mu}\right)+\tau_{3} W_{3}^{\mu} .
$$

Now, the fields of the charged $W$ bosons can be extracted

$$
W^{ \pm} \quad \leftrightarrow \quad W^{( \pm) \mu}=\frac{1}{\sqrt{2}}\left(W_{1}^{\mu} \pm i W_{2}^{\mu}\right)
$$

so that the covariant derivative can be written in the following form

$$
D_{E W}^{\mu}=\partial^{\mu}+i \frac{g}{\sqrt{2}} \cdot\left(\tau_{+} W^{(-) \mu}+\tau_{-} W^{(+) \mu}\right)+i g \cdot \tau_{3} \cdot W_{3}^{\mu}+i g^{\prime} \cdot \frac{Y}{2} \cdot B^{\mu} .
$$

The remaining gauge fields for the photon $\gamma$ and the $Z$ boson are linear combinations of the fields $W_{3}^{\mu}$ und $B^{\mu}$

$$
\left(\begin{array}{c}
\gamma \\
Z
\end{array}\right) \leftrightarrow\left(\begin{array}{c}
A^{\mu} \\
Z^{\mu}
\end{array}\right)=\left(\begin{array}{rr}
\cos \theta_{w} & \sin \theta_{w} \\
-\sin \theta_{w} & \cos \theta_{w}
\end{array}\right)\left(\begin{array}{c}
B^{\mu} \\
W_{3}^{\mu}
\end{array}\right)
$$

The Weinberg angle $\theta_{w}$ (or weak mixing angle) is connected with the coupling constants $g$ and $g^{\prime}$ via

$$
\sin \theta_{w}=\frac{g^{\prime}}{\sqrt{g^{\prime 2}+g^{2}}} \quad \text { and } \quad \cos \theta_{w}=\frac{g}{\sqrt{g^{\prime 2}+g^{2}}}
$$

and is measured to $[4]$

$$
\sin ^{2} \theta_{w}=0.23122 \pm 0.00015
$$

\footnotetext{
${ }^{3} \varepsilon_{i j k}$ indicates the total antisymmetric tensor of covariant rank three. It is $\varepsilon_{123}=\varepsilon_{231}=\varepsilon_{312}=+1$, $\varepsilon_{132}=\varepsilon_{213}=\varepsilon_{321}=-1$. For all other combinations of (123) $\varepsilon_{i j k}$ vanishes. In three dimensions one does not distinguish between upper and lower indices, i. e. $\varepsilon_{i j k}=\varepsilon^{i j k}$.
} 
Further, the elementary charge $e$ is related to $g, g^{\prime}$ and $\theta_{w}$

$$
e=g^{\prime} \cos \theta_{w}=g \sin \theta_{w} .
$$

The fields $B^{\mu}$ and $W_{3}^{\mu}$ can be replaced by the physical fields $A^{\mu}$ and $Z^{\mu}$

$$
\begin{aligned}
D_{E W}^{\mu}= & \partial^{\mu}+i e\left(\tau_{3}+\frac{Y}{2}\right) A^{\mu}+i \frac{g}{\sqrt{2}} \cdot\left(\tau_{+} W^{(-) \mu}+\tau_{-} W^{(+) \mu}\right)+ \\
& +i \frac{g}{\cos \theta_{w}}\left(\tau_{3} \cos ^{2} \theta_{w}-\frac{Y}{2} \sin ^{2} \theta_{w}\right) Z^{\mu} .
\end{aligned}
$$

For right-handed fermions the covariant derivative is reduced to $(Q=Y / 2)$

$$
D_{E W, R}^{\mu}=\partial^{\mu}+i e Q A^{\mu}-i \underbrace{\frac{g}{\cos \theta_{w}} \sin ^{2} \theta_{w}}_{=g^{\prime} \sin \theta_{w}} Q Z^{\mu} .
$$

Hence, the Lagrangian for (massless) fermions including electroweak interactions can be written as follows

$$
\begin{aligned}
\mathcal{L}= & i \cdot \sum_{\ell=1}^{3} \bar{L}_{\ell} \gamma_{\mu} D_{E W}^{\mu} L_{\ell}+i \cdot \sum_{\ell=1}^{3} \bar{R}_{\ell} \gamma_{\mu} D_{E W, R}^{\mu} R_{\ell}+ \\
& +i \cdot \sum_{q=1}^{3} \bar{L}_{q} \gamma_{\mu} D_{E W}^{\mu} L_{q}+i \cdot \sum_{q=1}^{6} \bar{R}_{q} \gamma_{\mu} D_{E W, R}^{\mu} R_{q}-\frac{1}{4} F_{\mu \nu}^{i} F_{i}^{\mu \nu}-\frac{1}{4} f_{\mu \nu} f^{\mu \nu}(2.4
\end{aligned}
$$

with the field strength tensors

$$
\begin{aligned}
F_{i}^{\mu \nu} & =\partial^{\mu} W_{i}^{\nu}-\partial^{\nu} W_{i}^{\mu}-g \cdot \varepsilon_{i j k} W_{j}^{\mu} W_{k}^{\nu} \quad \text { and } \\
f^{\mu \nu} & =\partial^{\mu} B^{\nu}-\partial^{\nu} B^{\mu} .
\end{aligned}
$$

The examination of Eq. 2.47, more precisely the terms $\propto \bar{\psi} V^{\mu} \psi$ where the external gauge field (thus the interaction) enters, yields all possible vertices and couplings in the electroweak theory for massless particles:

- The coupling of the photon field to all fermions (left- and right-handed) is always proportional to the charge of the fermion $Q_{f}$

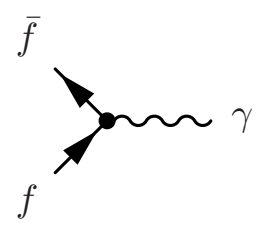

$$
\begin{aligned}
& \sim-i e \bar{\psi}(\bar{f}) \gamma_{\mu}\left(\tau_{3}+\frac{Y}{2}\right) \psi(f) A^{\mu} \\
& =-i e \bar{\psi}(\bar{f}) \gamma_{\mu}\left(I_{3}+\frac{Y}{2}\right) \psi(f) A^{\mu} \\
& =-i e Q_{f} \bar{\psi}(\bar{f}) \gamma_{\mu} \psi(f) A^{\mu}
\end{aligned}
$$

- Due to the ladder operator the charged current $W^{+}$or $W^{-}$changes the third component of the weak isospin at the vertex, hence right-handed fermions can not interact with the charged gauge boson. There is only the following graph for the $e^{-}-W^{-}$ coupling which involves left-handed electrons and right-handed antineutrinos:

$$
\bar{\nu}_{e} \sim W^{-} \sim-i \frac{g}{\sqrt{2}} \bar{\psi}\left(\bar{\nu}_{e}\right) \gamma_{\mu} \frac{1-\gamma^{5}}{2} \tau_{+} \psi\left(e^{-}\right) W^{(-) \mu}
$$


- The coupling of fermions to the $Z$ boson is derived making use of the vector $g_{V}$ and axialvector coupling $g_{A}$ which are defined as follows

$$
\begin{aligned}
& \sim-i \frac{g}{\cos \theta_{w}} \bar{\psi}(\bar{f}) \gamma_{\mu}\left[c_{L} \frac{1-\gamma^{5}}{2}+c_{R} \frac{1+\gamma^{5}}{2}\right] \psi(f) Z^{\mu} \\
& =-i \frac{g}{2 \cos \theta_{w}} \bar{\psi}(\bar{f}) \gamma_{\mu}\left[\left(c_{L}+c_{R}\right)-\left(c_{L}-c_{R}\right) \gamma^{5}\right] \psi(f) Z^{\mu} \\
& =-i \frac{g}{2 \cos \theta_{w}} \bar{\psi}(\bar{f}) \gamma_{\mu}\left[g_{V}-g_{A} \gamma^{5}\right] \psi(f) Z^{\mu} .
\end{aligned}
$$

$c_{L}$ and $c_{R}$ can be determined using Eq. 2.45 and 2.46

$$
\begin{aligned}
c_{L}: & -i \frac{g}{\cos \theta_{w}} \bar{\psi}(\bar{f}) \gamma_{\mu}\left(\tau_{3} \cos ^{2} \theta_{w}-\frac{Y}{2} \sin ^{2} \theta_{w}\right) \frac{1-\gamma^{5}}{2} \psi(f) Z^{\mu} \\
& -i \frac{g}{\cos \theta_{w}} \bar{\psi}(\bar{f}) \gamma_{\mu} \underbrace{\left(I_{3}-Q_{f} \sin ^{2} \theta_{w}\right)}_{=c_{L}} \frac{1-\gamma^{5}}{2} \psi(f) Z^{\mu} \\
c_{R} \quad: \quad & -i \frac{g}{\cos \theta_{w}} \bar{\psi}(\bar{f}) \gamma_{\mu}\left(\tau_{3} \cos ^{2} \theta_{w}-\frac{Y}{2} \sin ^{2} \theta_{w}\right) \frac{1+\gamma^{5}}{2} \psi(f) Z^{\mu} \\
& -i \frac{g}{\cos \theta_{w}} \bar{\psi}(\bar{f}) \gamma_{\mu} \underbrace{\left(-Q_{f} \sin ^{2} \theta_{w}\right)}_{=c_{R}} \frac{1+\gamma^{5}}{2} \psi(f) Z^{\mu}
\end{aligned}
$$

so that the couplings can be written as

$$
\begin{aligned}
g_{V} & =c_{L}+c_{R}=I_{3}-2 Q_{f} \sin ^{2} \theta_{w}, \\
g_{A} & =c_{L}-c_{R}=I_{3} .
\end{aligned}
$$

In 1934 Enrico Fermi accomplished a description of the radioactive $\beta$ decay assuming a four-fermion interaction with a vertex factor $G_{F}=1.16637(1) \cdot 10^{-5} \mathrm{GeV}^{-2}$ [4], the Fermi constant. This theory is the low-energy approximation of the weak theory introduced in this section. The conversion between the coupling constant $g$ and the Fermi constant is as follows (at leading order)

$$
\frac{G_{F}}{\sqrt{2}}=\frac{g^{2}}{8 m_{W}^{2}},
$$

and including electroweak corrections $\Delta r=f$ ( Higgs boson mass, top quark mass, ...)

$$
G_{F}=\frac{\sqrt{2} g^{2}}{8 m_{W}^{2}}(1+\Delta r)
$$

Since $G_{F}$ can be measured very precisely via the lifetime of muons, it is possible to probe the Standard Model and to derive constraints on the Higgs boson mass, although the correction $\Delta r$ depends only logarithmic on the Higgs mass.

\subsubsection{The Strong Interaction}

The strong interaction is mediated by gauge fields with spin 1, namely the gluons. In order to quantify the underlying symmetry, a new degree of freedom of the fermions (like the hypercharge and isospin in the electroweak theory) is required. The presence of a new 
"hidden" quantum number can be derived from the examination of the wave function of the $\Omega^{-}$baryon. This particle consists of three strange quarks with a total spin of $3 / 2$, thus a fermion, but the wave function can only be constructed symmetrically. This contradicts the Pauli principle. Hence, a new degree of freedom of the quarks - the color charge or just color - with the three different values $R$ (red), $G$ (green) and $B$ (blue) is introduced to solve the problem

$$
\begin{aligned}
\left|\Omega^{-}\right\rangle & =\left|s^{\uparrow} s^{\uparrow} s^{\uparrow}\right\rangle \\
\Rightarrow\left|\Omega^{-}\right\rangle & =\frac{1}{\sqrt{6}}\left|s_{R}^{\uparrow} s_{G}^{\uparrow} s_{B}^{\uparrow}-s_{R}^{\uparrow} s_{B}^{\uparrow} s_{G}^{\uparrow}+s_{B}^{\uparrow} s_{R}^{\uparrow} s_{G}^{\uparrow}-s_{B}^{\uparrow} s_{G}^{\uparrow} s_{R}^{\uparrow}+s_{G}^{\uparrow} s_{B}^{\uparrow} s_{R}^{\uparrow}-s_{G}^{\uparrow} s_{R}^{\uparrow} s_{B}^{\uparrow}\right\rangle .
\end{aligned}
$$

Individual quarks which carry the color charge can not be observed. Just "color-neutral" combinations, like mesons (consisting of a quark and an antiquark) or baryons (consisting of three quarks or three antiquarks), are realized in nature and can be measured. This phenomenon is referred to as confinement. The symmetry group that reflects this behaviour is $\mathrm{SU}(3)_{C}$. This symmetry acts in the color space of the quarks; leptons are singlets with respect to the color-SU(3). The theoretical description of the interaction of gluons and quarks is referred to as quantum chromodynamics $Q C D$.

Starting with the gauge principle, the transformation acting in the color space on a quark spinor $\psi$ under $\mathrm{SU}(3)_{C}$ can be written in the following way

$$
\psi^{\alpha}=\exp \left\{i g_{s} \cdot \frac{\lambda_{i}^{\alpha \beta}}{2} \cdot \omega_{i}(x)\right\} \psi^{\beta}
$$

with a new coupling, the strong coupling constant $g_{s}$, eight spacetime dependent functions $\omega_{i}(x)(i=1, \ldots, 8)$ and finally the generators of the symmetry, namely the eight $3 \times 3$ Gell-Mann matrices $\lambda_{i}^{\alpha \beta}(i=1, \ldots, 8)$. The indices $\alpha, \beta$ denote the color $(\alpha, \beta=1,2,3$ $=R, G, B)$. For the $\mathrm{SU}(3)_{C}$ gauge group eight vector boson fields $G_{i}^{\mu}$ are required which can be identified with the eight gluons $g_{i}$. The boson fields transform as follows

$$
g_{i} \leftrightarrow G_{i}^{\prime \mu}=G_{i}^{\mu}-\partial^{\mu} \omega_{i}(x)-g_{s} \cdot f_{i j k} \omega_{j}(x) G_{k}^{\mu} .
$$

The structure constants $f_{i j k}$ are totally antisymmetric $\left(f_{i j k}=f_{j k i}=f_{k i j}=-f_{i k j}\right)$ with the following non-zero values

$$
\begin{aligned}
1 & =f_{123}, \\
\frac{1}{2} & =f_{147}=f_{165}=f_{246}=f_{257}=f_{345}=f_{376}, \\
\frac{\sqrt{3}}{2} & =f_{458}=f_{678} .
\end{aligned}
$$

The vector fields enter the Lagrangian via the covariant derivative, but only in the quark sector $\left(D_{\text {leptons }}^{\mu}=D_{E W}^{\mu}\right)$ and only in the color space (using $\delta_{\alpha \beta}=1$ for $\alpha=\beta, \delta_{\alpha \beta}=0$ for $\alpha \neq \beta$ )

$$
\left(D_{\text {quarks }}^{\mu}\right)^{\alpha \beta}=\delta_{\alpha \beta} \cdot D_{E W}^{\mu}+\left(D_{Q C D}^{\mu}\right)^{\alpha \beta} \quad \text { with } \quad\left(D_{Q C D}^{\mu}\right)^{\alpha \beta}=i g_{s} \cdot \frac{\lambda_{i}^{\alpha \beta}}{2} \cdot G_{i}^{\mu} .
$$

The field strength tensor $H_{i}^{\mu \nu}$ has the following form

$$
H_{i}^{\mu \nu}=\partial^{\mu} G_{i}^{\nu}-\partial^{\nu} G_{i}^{\mu}-g_{s} \cdot f_{i j k} G_{j}^{\mu} G_{k}^{\nu} .
$$


The Lagrangian can now be extended including the strong interaction

$$
\begin{aligned}
\mathcal{L}= & i \cdot \sum_{\ell=1}^{3} \bar{L}_{\ell} \gamma_{\mu} D_{E W}^{\mu} L_{\ell}+i \cdot \sum_{\ell=1}^{3} \bar{R}_{\ell} \gamma_{\mu} D_{E W, R}^{\mu} R_{\ell}+ \\
& +i \cdot \sum_{q=1}^{3} \bar{L}_{q} \gamma_{\mu} D_{E W}^{\mu} L_{q}+i \cdot \sum_{q=1}^{6} \bar{R}_{q} \gamma_{\mu} D_{E W, R}^{\mu} R_{q}+ \\
& +i \cdot \sum_{q=1}^{6} \bar{\psi}_{q}^{\alpha} \gamma_{\mu}\left(D_{Q C D}^{\mu}\right)^{\alpha \beta} \psi_{q}^{\beta}-\frac{1}{4} F_{\mu \nu}^{i} F_{i}^{\mu \nu}-\frac{1}{4} f_{\mu \nu} f^{\mu \nu}-\frac{1}{4} H_{\mu \nu}^{i} H_{i}^{\mu \nu}
\end{aligned}
$$

Note, that the strong interaction acts on mass eigenstates $\psi_{q}$.

Experiments do not indicate any $\mathcal{C P}$ violation in the QCD sector, although the QCD Lagrangian can be extended with terms that are able to violate $\mathcal{C P}$ symmetry (strong $\mathcal{C P}$ problem). This phase, $\theta_{Q C D}$, is a free parameter in the Standard Model. One implication of a non-zero phase would be an observable electrical dipole moment of the neutron which is not realized $\left(d_{n}<0.63 \cdot 10^{-25} e \mathrm{~cm}, 90 \% \mathrm{CL}[4]\right)$. The special choice of the phase $\left(\theta_{Q C D}=0\right)$ is an example of fine-tuning in particle physics.

\subsubsection{The Higgs Mechanism}

All previous deliberations - including the final Lagrangian Eq. 2.65 - only apply if all fermions and gauge bosons are massless. This contradicts reality: the $W$ and $Z$ bosons are massive, as well as all fermions. In the Standard Model massive particles and thus the symmetry breaking of $\mathrm{SU}(2)_{L} \otimes \mathrm{U}(1)_{Y}$ to the observed $\mathrm{U}(1)_{E M}$ symmetry are realized within the Higgs mechanism which describes the interaction of fermions and bosons with an external, massive, scalar (spin-0) field, the Higgs field. The couplings between fermions and gauge bosons derived in the previous sections have to remain unchanged. In the simplest case (minimal Higgs model) the Higgs field $\phi$ consists of a doublet $(I=1 / 2$, $Y=1$ ) of complex fields

$$
\phi=\left(\begin{array}{c}
\phi^{+} \\
\phi^{0}
\end{array}\right)
$$

The Lagrangian density of the Higgs field is as follows

$$
\mathcal{L}_{H}=\left(D_{E W}^{\mu} \phi\right)^{\dagger}\left(D_{\mu, E W} \phi\right)-V\left(\phi^{\dagger}, \phi\right)
$$

with the Higgs potential $V$ ( "Mexican hat" potential [11])

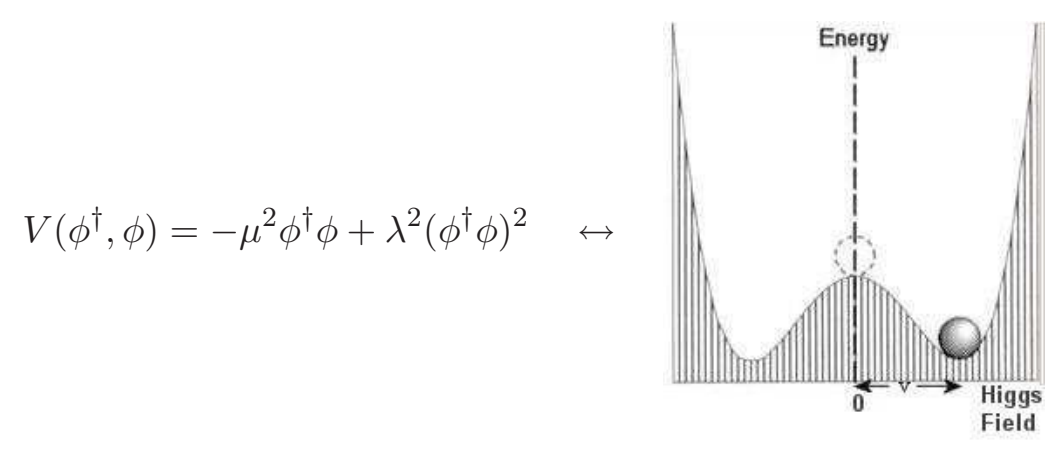


The scalar spin-0-Higgs field fulfills the Klein-Gordon equation

$$
\left(\partial^{\mu} \partial_{\mu}+m_{H}^{2}\right) \phi=0
$$

In order to derive the masses of the $W$ and $Z$ bosons, it is sufficient to consider only the part of the full Lagrangian which contains the Higgs field, namely $\mathcal{L}_{H}$ from Eq. 2.67. This Lagrangian is gauge-invariant under local $\mathrm{U}(1)_{Y}$ and $\mathrm{SU}(2)_{L}$ transformations. The Higgs potential is minimal at

$$
V\left(\phi^{\dagger}, \phi\right) \stackrel{!}{=} \min \quad \Leftrightarrow \quad \phi^{\dagger} \phi=\left|\phi^{\dagger} \phi\right|^{2}=\frac{\mu^{2}}{2 \lambda^{2}} \equiv \frac{v^{2}}{2} .
$$

This state with minimal energy can be identified with the "vacuum". A possible choice of the vacuum state is

$$
\phi_{0}=\frac{1}{\sqrt{2}}\left(\begin{array}{l}
0 \\
v
\end{array}\right) .
$$

This state obviously breaks the $\mathrm{SU}(2)_{L} \otimes \mathrm{U}(1)_{Y}$ symmetry. The spontaneous symmetry breaking can only appear if $\left|\phi_{0}\right| \neq 0$ holds

$$
S U(2)_{L} \otimes U(1)_{Y} \quad \stackrel{v}{\longrightarrow} U(1)_{E M} .
$$

The vacuum $\phi_{0}$ is invariant under a transformation connected with a generator $\mathcal{G}_{i}$ if

$$
\exp \left\{i g \cdot \alpha_{i} \mathcal{G}_{i}\right\} \phi_{0} \approx\left(1+i g \cdot \alpha_{i} \mathcal{G}_{i}\right) \phi_{0}=\phi_{0} \quad \Leftrightarrow \quad \mathcal{G}_{i} \phi_{0}=0 .
$$

The vacuum remains invariant only under $\mathrm{U}(1)_{E M}(Y=1)$ :

$$
\begin{aligned}
\tau_{1} \phi_{0} & =\frac{1}{2 \sqrt{2}}\left(\begin{array}{c}
v \\
0
\end{array}\right) \neq 0, \quad \tau_{2} \phi_{0}=\frac{1}{2 \sqrt{2}}\left(\begin{array}{c}
-i v \\
0
\end{array}\right) \neq 0, \\
\tau_{3} \phi_{0} & =\frac{1}{2 \sqrt{2}}\left(\begin{array}{c}
0 \\
-v
\end{array}\right) \neq 0, \quad Y \phi_{0}=\frac{1}{\sqrt{2}}\left(\begin{array}{c}
0 \\
v
\end{array}\right) \neq 0 \\
\Rightarrow Q \phi_{0} & =\left(\tau_{3}+\frac{Y}{2}\right) \phi_{0}=0 .
\end{aligned}
$$

Now, $\phi$ is expanded around the vacuum expectation value

$$
\phi(x)=\frac{1}{\sqrt{2}}\left(\begin{array}{c}
0 \\
v+H(x)+i \zeta(x)
\end{array}\right) .
$$

Putting this state into $\mathcal{L}_{H}$ and neglecting higher order contributions yields

$$
\begin{aligned}
\mathcal{L}_{H}= & \frac{1}{2}\left[\left(\partial^{\mu} H\right)\left(\partial_{\mu} H\right)-2 \mu^{2} H^{2}\right]+\frac{1}{2}\left[\left(\partial^{\mu} \zeta\right)\left(\partial_{\mu} \zeta\right)\right] \\
& +\frac{1}{2} \cdot \frac{g^{2} v^{2}}{4}\left(\left|W_{\mu}^{(+)}\right|^{2}+\left|W_{\mu}^{(-)}\right|^{2}\right)+\frac{1}{2} \cdot \frac{g^{2} v^{2}}{4 \cos ^{2} \theta_{w}}\left|Z_{\mu}\right|^{2} .
\end{aligned}
$$

The (minimal) Higgs mechanism results in

- one massive, neutral Higgs boson (particle connected with the $H$ field) with a mass of $m_{H}=\sqrt{2} \mu$;

- one massless, neutral (thus not measurable) Goldstone boson (particle connected with the $\zeta$ field); 
- massive $W$ bosons, whereas $W^{+}$and $W^{-}$get the same mass of

$$
m_{W}=\frac{g v}{2}
$$

- one massive $Z$ boson with a mass of

$$
m_{Z}=\frac{g v}{2 \cos \theta_{w}}=\frac{m_{W}}{\cos \theta_{w}}
$$

- still a massless photon since $\mathcal{L}_{H}$ does not contain a term $\propto\left|A_{\mu}\right|^{2}$.

The relation between the Weinberg angle and the $W$ and $Z$ boson masses can be written in a more general way

$$
\rho=\left(\frac{m_{W}}{m_{Z} \cos \theta_{w}}\right)^{2}
$$

with $\rho=1$ in the minimal Higgs model. This value is confirmed by precision measurements. Using Eq. 2.57 and 2.79 the vacuum expectation value of the Higgs field is determined as

$$
v=\frac{1}{\sqrt{G_{F} \sqrt{2}}} \approx 246 \mathrm{GeV} .
$$

The Higgs field can also attribute masses to the fermions if the following term (Yukawa interaction ) - which preserves the $\mathrm{SU}(2)_{L} \otimes \mathrm{U}(1)_{Y}$ gauge invariance - is added to the Lagrangian

$$
\mathcal{L}_{\text {Yukawa }}=\sum_{\text {fermions }}-g_{f}\left[(\bar{L} \phi) R+\bar{R}\left(\phi^{\dagger} L\right)\right] .
$$

With $\phi$ expanded around the vacuum expectation value from Eq. 2.77, one gets for e. g. the first quark family

$$
\mathcal{L}_{\text {Yukawa }}=-\frac{\tilde{g}_{d} v}{\sqrt{2}}\left(\bar{d}_{L} d_{R}+\bar{d}_{R} d_{L}\right)-\frac{\tilde{g}_{d}}{\sqrt{2}}\left(\bar{d}_{L} d_{R}+\bar{d}_{R} d_{L}\right) H .
$$

Here, mass eigenstates are used. One can rewrite the equation above using the eigenstate with respect to the weak interaction, $d_{L}^{\prime}$, compensating the corresponding CKM elements into a modified coupling $g_{d}$.

$$
\begin{aligned}
\mathcal{L}_{\text {Yukawa }} & =-\frac{g_{d} v}{\sqrt{2}}\left(\bar{d}_{L}^{\prime} d_{R}+\bar{d}_{R} d_{L}^{\prime}\right)-\frac{g_{d}}{\sqrt{2}}\left(\bar{d}_{L}^{\prime} d_{R}+\bar{d}_{R} d_{L}^{\prime}\right) H \\
& =-m_{d}\left(\bar{d}_{L}^{\prime} d_{R}+\bar{d}_{R} d_{L}^{\prime}\right)-\frac{m_{d}}{v}\left(\bar{d}_{L}^{\prime} d_{R}+\bar{d}_{R} d_{L}^{\prime}\right) H
\end{aligned}
$$

The last terms containing $H$ describe the coupling of fermions to the Higgs boson. In order to generate masses for fermions with $I_{3}=1 / 2$, a new Higgs doublet is required. The charge conjugation operator $\mathcal{C}$ provides the appropriate doublet with $Y=-1$

$$
\phi_{\mathcal{C}}=-i \sigma_{2} \phi^{*}=\left(\begin{array}{c}
-\bar{\phi}^{0} \\
\phi^{-}
\end{array}\right) \quad \stackrel{\text { breaking }}{\longrightarrow} \frac{1}{\sqrt{2}}\left(\begin{array}{c}
v+H(x)+i \zeta(x) \\
0
\end{array}\right) .
$$

The Lagrangian which assigns masses to all fermions and contains the fermion-Higgs interaction is as follows

$$
\mathcal{L}_{\text {Yukawa }}=\sum_{\text {fermions }}-g_{f}\left\{\left[(\bar{L} \phi) R+\bar{R}\left(\phi^{\dagger} L\right)\right]+\left[\left(\bar{L} \phi_{\mathcal{C}}\right) R+\bar{R}\left(\phi_{\mathcal{C}}^{\dagger} L\right)\right]\right\} .
$$


The charge conjugated doublet can not only be used for the up-type quarks, but also for neutrinos. Within the Higgs mechanism it is possible to generate massive neutrinos if right-handed neutrinos exist. In the following, the origin of the neutrino masses is briefly outlined (cf. [12]).

\section{Dirac Neutrinos}

According to this approach, the reason that right-handed neutrinos have escaped detection so far is that their interactions are at least 26 orders of magnitude weaker than left-handed neutrinos. The idea of the Dirac neutrino works in the sense that neutrino masses can be generated via the Higgs mechanism. However, it also suggests that neutrinos should have similar masses to the other particles in the Standard Model. To avoid this problem, the strength of neutrino interactions with the Higgs boson has to be set at least 12 orders of magnitude weaker than that of the top quark.

\section{Majorana Neutrinos}

In this scheme, it is possible for right-handed neutrinos to have a mass of their own without relying on the Higgs boson. Unlike other quarks and leptons, the mass of the right-handed neutrino, $M$, is not tied to the mass scale of the Higgs boson. Rather, it can be much heavier than other particles. The seesaw mechanism [13] can naturally give rise to light neutrinos. When a left-handed neutrino interacts with the Higgs boson, it acquires a mass, $m$, which is comparable to the mass of other quarks and leptons. At the same time it transforms into a right-handed neutrino with a mass $M$, which is much heavier than energy conservation would normally allow. However, the Heisenberg uncertainty principle allows this state to exist for a short time interval, $\Delta t$, given by $\Delta t \sim 1 / M$, after which the particle transforms back into a left-handed neutrino with mass $m$ by interacting with the Higgs boson again. In this picture, the observed left-handed neutrino has a mass of $m^{2} / M$ averaged over time.

Hence, the Higgs mechanism is not only able to assign masses to the $W$ and $Z$ bosons, but also capable to generate massive fermions

$$
m_{f}=g_{f} \frac{v}{\sqrt{2}} .
$$

This implies that as many new couplings have to be introduced as massive fermions exist. The fermion-Higgs coupling $g_{f}$ is referred to as Yukawa coupling. The Yukawa couplings are proportional to the mass of the corresponding fermion, $g_{f} \propto m_{f}$. Thus, the top quark couples the most strongly to the Higgs boson. Note, that a process like $H \rightarrow \gamma \gamma$ occurs only due to higher-order effects, like loop diagrams containing a top quark.

The Higgs boson has not yet been discovered, but the mass can indirectly be estimated fitting the electroweak parameters [14]. Fig. 2.3 shows the $\Delta \chi^{2}=\chi^{2}-\chi_{\min }^{2}$ derived from high- $Q^{2}$ precision measurements of the electroweak parameters, performed at LEP and by SLD, CDF, and D $\varnothing$, as a function of the Higgs mass assuming the Standard Model to be the correct theory of nature. The preferred value for its mass, corresponding to the minimum of the curve, is $m_{H}=76_{-24}^{+33} \mathrm{GeV}$ (at $68 \%$ confidence level derived from $\Delta \chi^{2}=1$ for the black line, thus not taking the theoretical uncertainty shown as the blue band into account). This result is only little affected by the low- $Q^{2}$ results such as the $\mathrm{NuTeV}$ measurement. With the one-sided $95 \%$ confidence level upper limit derived from $\Delta \chi^{2}=2.7$ for the blue band (thus including both the experimental and the theoretical uncertainty) the Higgs mass should be lower than $144 \mathrm{GeV}$. Including the direct limit of $m_{H}>114 \mathrm{GeV}$ the upper limit on the Higgs mass increases to $m_{H}<182 \mathrm{GeV}$. 
Figure 2.3: $\Delta \chi^{2}=\chi^{2}-\chi_{\min }^{2}$ derived from high- $Q^{2}$ precision measurements of the electroweak parameters, performed at LEP and by SLD, CDF, and DØ, as a function of the Higgs mass assuming the Standard Model to be the correct theory of nature [14].

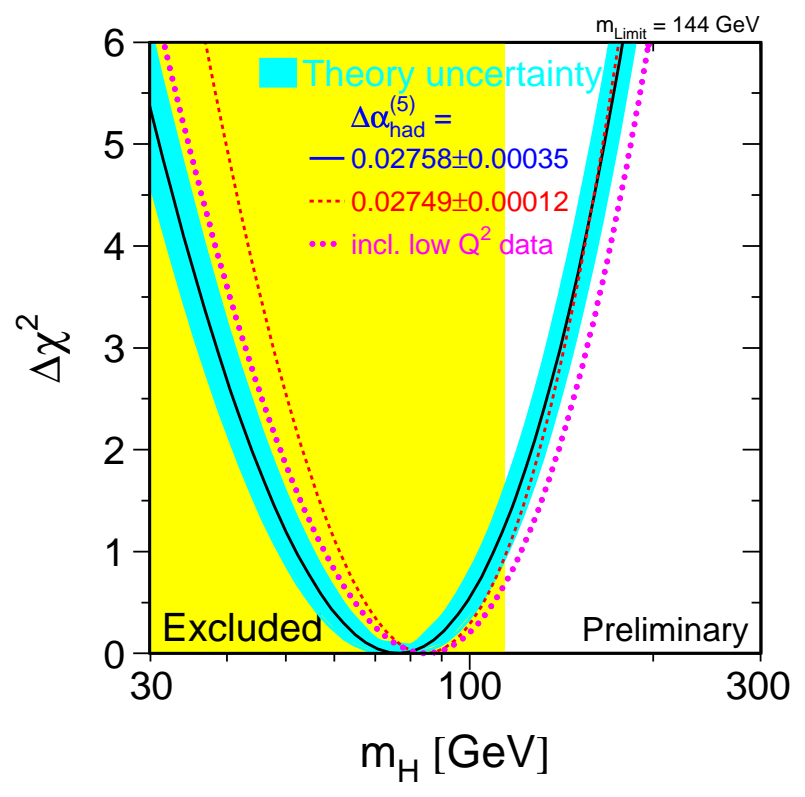

\section{Summary}

All results derived in the preceding sections can be summarized in the final Lagrangian of the Standard Model

$$
\begin{aligned}
\mathcal{L}_{S M} & =\sum_{\text {fermions }} \bar{\psi}_{f}\left(i \gamma_{\mu} \partial^{\mu}-m_{f}-\frac{g m_{f}}{2 m_{W}} H\right) \psi_{f}-\frac{g_{s}}{2} \sum_{\text {quarks }} \bar{\psi}_{q}^{\alpha} \gamma_{\mu} \lambda_{i}^{\alpha \beta} G_{i}^{\mu} \psi_{q}^{\beta} \\
& -\frac{g}{2 \sqrt{2}} \sum_{\text {fermions }} \bar{\psi}_{f} \gamma_{\mu}\left(1-\gamma^{5}\right)\left(\tau_{+} W^{(-) \mu}+\tau_{-} W^{(+) \mu}\right) \psi_{f} \\
& -e \sum_{\text {fermions }} Q_{f} \bar{\psi}_{f} \gamma_{\mu} \psi_{f} A^{\mu}-\frac{g}{2 \cos \theta_{w}} \sum_{\text {fermions }} \bar{\psi}_{f} \gamma_{\mu}\left(g_{V}^{f}-g_{A}^{f} \gamma^{5}\right) \psi_{f} Z^{\mu} \\
& -\frac{1}{4} F_{\mu \nu}^{i} F_{i}^{\mu \nu}-\frac{1}{4} f_{\mu \nu} f^{\mu \nu}-\frac{1}{4} H_{\mu \nu}^{i} H_{i}^{\mu \nu} \\
& +\frac{1}{2}\left[\left(\partial^{\mu} H\right)\left(\partial_{\mu} H\right)-m_{H}^{2} H^{2}\right]+\frac{1}{2}\left[\left(\partial^{\mu} \zeta\right)\left(\partial_{\mu} \zeta\right)\right]-V\left(\phi^{\dagger}, \phi\right) \\
& +\frac{m_{W}^{2}}{2}\left(\left|W_{\mu}^{(+)}\right|^{2}+\left|W_{\mu}^{(-)}\right|^{2}\right)+\frac{m_{Z}^{2}}{2}\left|Z_{\mu}\right|^{2} .
\end{aligned}
$$

Note, that the strong interaction acts on mass eigenstates which are related to the eigenstates of the weak interaction via the CKM matrix. The underlying symmetry group is $\mathrm{SU}(3)_{C} \otimes \mathrm{SU}(2)_{L} \otimes \mathrm{U}(1)_{Y}$, whereas the electroweak part is spontaneously broken by a non-zero vacuum expectation value $v$ of the Higgs field

$$
S U(2)_{L} \otimes U(1)_{Y} \quad \stackrel{v}{\longrightarrow} U(1)_{E M} .
$$


The Standard Model contains $19+9$ free parameters:

- six quark masses and four CKM parameters (three angles and one $\mathcal{C} \mathcal{P}$-violating phase);

- three masses from the charged leptons;

- three couplings $g, g^{\prime}$ and $g_{s}$, or equivalently $\alpha_{E M}, \sin ^{2} \theta_{w}$ and $\alpha_{s}$;

- two parameters from the Higgs mechanism $v$ and $\lambda$, or equivalently $m_{Z}$ and $m_{H}$;

- the strong $\mathcal{C P}$-violation phase $\theta_{Q C D}$ and

- nine neutrino parameters (three masses, three mixing angles, one $\mathcal{C P}$-violating phase and two Majorana phases).

\subsubsection{The Running of the Coupling Constants}

The couplings in the electroweak and strong interaction have been introduced as "constants". As a result of the renormalizability of the theory, the couplings in fact depend on the momentum transfer $Q^{2}$ of a given process. The running of the coupling constant is determined by the following differential equation, the Renormalization Group Equation (RGE)

$$
2 Q^{2} \frac{\partial g\left(Q^{2}\right)}{\partial Q^{2}}=\beta\left(g\left(Q^{2}\right)\right)
$$

The beta-function $\beta$ and the running coupling for the electromagnetic interaction is as follows $\left(\alpha_{E M}=e^{2} / 4 \pi\right)$

$$
\beta\left(\alpha_{E M}\left(Q^{2}\right)\right)=\frac{2 \alpha_{E M}\left(Q^{2}\right)^{2}}{3 \pi} \Rightarrow \alpha_{E M}\left(Q^{2}\right)=\frac{\alpha_{E M}\left(Q_{0}^{2}\right)}{1-\frac{\alpha_{E M}\left(Q_{0}^{2}\right)}{3 \pi} \ln \left(\frac{Q^{2}}{Q_{0}^{2}}\right)}
$$

with the coupling at a specific momentum transfer $Q_{0}^{2}$. At $Q_{0}^{2} \approx m_{W}^{2}=(80.4 \mathrm{GeV})^{2}$ the coupling is $\alpha_{E M}\left(m_{W}^{2}\right)=1 / 128$.

The strong coupling exhibits a completely different scaling behaviour because the betafunction is negative in the Standard Model with six flavors of quarks $\left(n_{f}=6, \alpha_{s}=g_{s}^{2} / 4 \pi\right)$

$$
\beta\left(\alpha_{s}\left(Q^{2}\right)\right)=-\left(11-\frac{2 n_{f}}{3}\right) \frac{\alpha_{s}\left(Q^{2}\right)^{2}}{2 \pi} \Rightarrow \alpha_{s}\left(Q^{2}\right)=\frac{\alpha_{s}\left(Q_{0}^{2}\right)}{1+\frac{\alpha_{s}\left(Q_{0}^{2}\right)\left(33-2 n_{f}\right)}{12 \pi} \ln \left(\frac{Q^{2}}{Q_{0}^{2}}\right)} .
$$

The coupling decreases with increasing momentum transfer; in the limit of highest energies the coupling even vanishes leading to the asymptotic freedom of QCD. On the other hand, $\alpha_{s}$ becomes huge at low energies. One can rewrite Eq. 2.93 introducing a cut-off parameter $\Lambda$. At $Q^{2}=\Lambda^{2}$ the denominator of Eq. 2.93 vanishes so that $\alpha_{s}\left(\Lambda^{2}\right) \rightarrow \infty$

$$
\alpha_{s}\left(Q^{2}\right)=\frac{12 \pi}{\left(33-2 n_{f}\right) \ln \left(\frac{Q^{2}}{\Lambda^{2}}\right)} .
$$


The value of the cut-off parameter $\Lambda$ can not be predicted, but is determined from experiments to $\Lambda \approx 200 \mathrm{MeV}$. For $Q^{2}<\Lambda^{2}$, i. e. $\Delta x>1 \mathrm{fm}$ (radius of a nucleon), perturbative QCD is no longer applicable.

The evolution of the three coupling constants as a function of the momentum transfer is shown in Chap. 1, Fig. 1.2. Within the Standard Model the couplings do not unify.

\subsubsection{Parton Distribution Functions}

In order to calculate a hadronic cross section $\sigma(p \bar{p} \rightarrow V+X)$ (e.g. $\left.V=W, W^{\prime}, Z, \gamma\right)$, one has to evaluate the partonic cross section $\hat{\sigma}\left(q \bar{q}^{\prime} \rightarrow V\right)$ first. The hadronic cross section is obtained by the summation of all contributing quark-antiquark combinations $q_{i}, \bar{q}_{j}$ and integration over the momentum fractions

$$
\sigma(p \bar{p} \rightarrow V+X) \propto \int_{0}^{1} \mathrm{~d} x_{p} \int_{0}^{1} \mathrm{~d} x_{\bar{p}} \sum_{i, j} f_{q_{i}}\left(x_{p}, Q^{2}\right) f_{\bar{q}_{j}}\left(x_{\bar{p}}, Q^{2}\right) \hat{\sigma}\left(q_{i} \bar{q}_{j} \rightarrow V\right) .
$$

The probability to find a parton (valence quark, sea quark, gluon) in the proton with momentum fraction $x$ is given by the corresponding Parton Distribution (Density) Function, PDF, $f_{\text {parton }}\left(x, Q^{2}\right)$. All partons build up the proton, hence the sum of all parton momenta equals the proton momentum

$$
\sum_{\text {partons }} \int_{0}^{1} x \cdot f_{\text {parton }}(x) \mathrm{d} x=1 .
$$

The PDFs are connected with the (electromagnetic) proton structure functions $F_{1}$ and $F_{2}$ via $\left(Q_{q}\right.$ denotes the charge of the quark)

$$
2 x F_{1}(x)=F_{2}(x)=x \cdot \sum_{\text {quarks }} Q_{q}^{2} f_{q}(x) .
$$

Since the proton does not only consist of quarks, one also has to consider the gluon contributions, like $g \rightarrow q \bar{q}, q \rightarrow q g$ and $g \rightarrow g g$, as well. This correction introduces the dependency of the structure functions on $Q^{2}$

$$
F_{2}\left(x, Q^{2}\right)=x \cdot \sum_{\text {quarks }} Q_{q}^{2}\left(f_{q}(x)+\Delta f_{q}\left(x, Q^{2}\right)\right) \rightarrow x \cdot \sum_{\text {quarks }} Q_{q}^{2} f_{q}\left(x, Q^{2}\right),
$$

which is referred to as scaling violation. It is possible to calculate the evolution of the density functions with $Q^{2}$, but the absolute value can only be determined from experimental data. The following integro-differential equations for the quark and gluon distribution functions were compiled by Dokshitzer, Gribov, Lipatov, Altarelli and Parisi [15] (DGLAP equations)

$$
\begin{aligned}
& \frac{\mathrm{d} f_{q_{i}}\left(x, Q^{2}\right)}{\mathrm{d} \ln Q^{2}}=\frac{\alpha_{s}\left(Q^{2}\right)}{2 \pi} \int_{x}^{1} \frac{\mathrm{d} y}{y}\left(f_{q_{i}}\left(y, Q^{2}\right) P_{q q}\left(\frac{x}{y}\right)+f_{g}\left(y, Q^{2}\right) P_{q g}\left(\frac{x}{y}\right)\right), \\
& \frac{\mathrm{d} f_{g}\left(x, Q^{2}\right)}{\mathrm{d} \ln Q^{2}}=\frac{\alpha_{s}\left(Q^{2}\right)}{2 \pi} \int_{x}^{1} \frac{\mathrm{d} y}{y}\left(\sum_{i} f_{q_{i}}\left(y, Q^{2}\right) P_{g q}\left(\frac{x}{y}\right)+f_{g}\left(y, Q^{2}\right) P_{g g}\left(\frac{x}{y}\right)\right) .
\end{aligned}
$$


Figure 2.4: Shown is the structure function $F_{2}\left(x, Q^{2}\right)$ measured by the HERA experiments H1 and ZEUS, including measurements from other experiments [16].

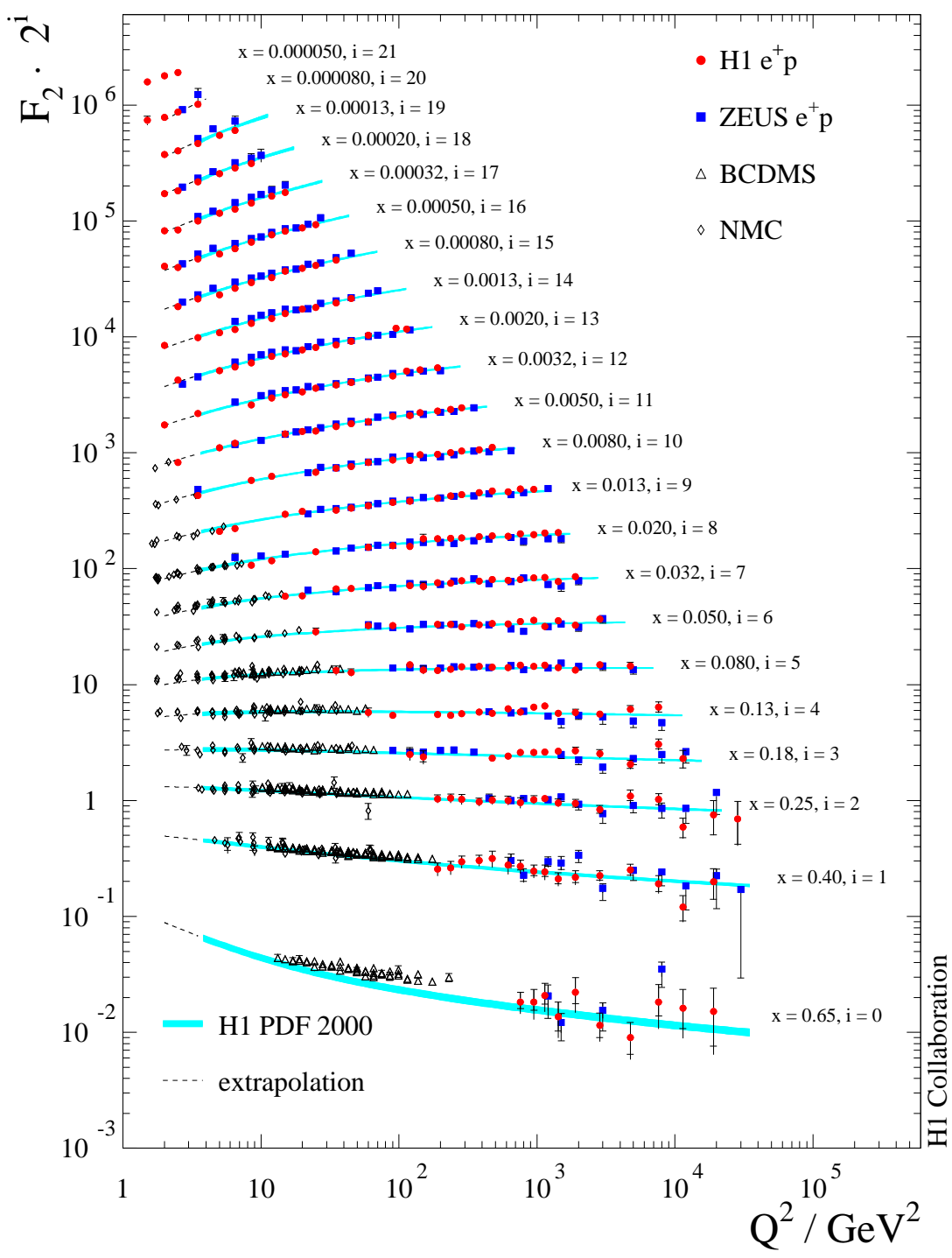

Figure 2.5: Parton density functions for the gluons, sea and valence quarks derived from fits to the experimental data of the $\mathrm{H} 1$ and ZEUS experiment at the HERA collider, compared to the distributions obtained from the CTEQ6.1M parameterization of the parton density functions [16].

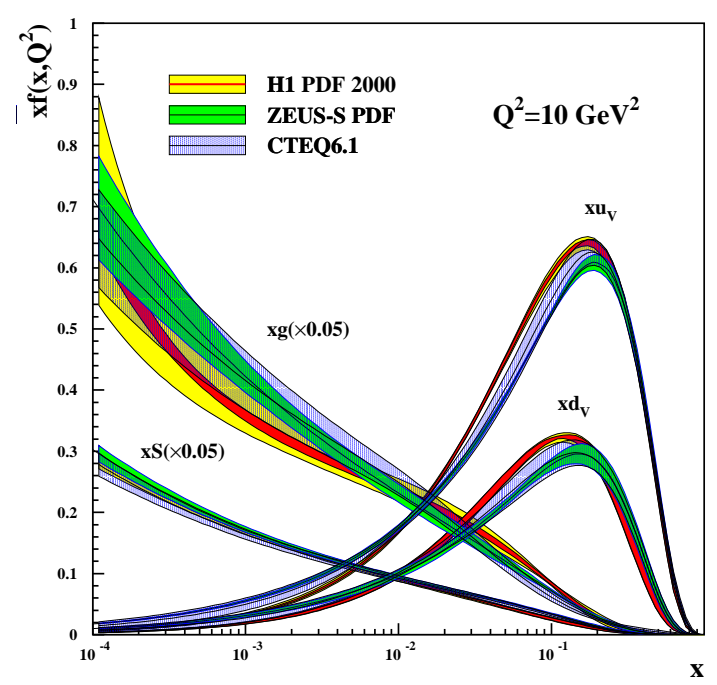


Eq. 2.99 expresses the fact that a quark with momentum fraction $x$ could have come from a parent quark with a larger momentum $y$ which has radiated a gluon. The probability of this process is proportional to $\alpha_{s} P_{q q}(x / y)$. The second term considers the possibility that a quark with momentum fraction $x$ could have been produced by a gluon with momentum fraction $y>x$ via $g \rightarrow q \bar{q}$. The probability of this process is proportional to $\alpha_{s} P_{q g}(x / y)$. The integral is the sum over all possible momentum fractions $y>x$. The first term of Eq. 2.100 corresponds to the process $q_{i} \rightarrow q_{i} g$. Here, a quark could have radiated the gluon, whereas the second term expresses the probability that the gluon stems from $g \rightarrow g g$.

The PDFs have for example been determined by exploring the structure of the proton in deep inelastic electron-proton collisions at the HERA ${ }^{4}$ collider. The measured structure function $F_{2}\left(x, Q^{2}\right)$ is shown in Fig. 2.4. Fig. 2.5 displays the individual contributions $x f\left(x, Q^{2}\right)$ from the valence quarks in the proton $\left(x u_{V}\right.$ and $\left.x d_{V}\right)$, as well as from the sea quarks $(x S)$ and gluons $(x g)$, both multiplied by 0.05 . The PDFs are derived from fits to the experimental data of the H1 and ZEUS experiments at the HERA collider. One possible parameterization of the PDFs, namely CTEQ6.1M [17] provided by the CTEQ ${ }^{5}$ [18] collaboration, is also displayed.

\subsubsection{The $W$ Production Cross Section}

As the last aspect of the Standard Model discussion, the production of $W$ bosons is addressed due to the close affinity to the expected $W^{\prime}$ signal (see Sec. 2.3.2).

The cross section for the inclusive $W$ production $p \bar{p} \rightarrow W+X$ (without taking into account the decay of the $W$ boson) is derived from the quark subprocess $q \bar{q}^{\prime} \rightarrow W$. This cross section can be calculated in leading order applying the Feyman rules, the summation over the spins of the quarks and the polarization of the $W$ boson

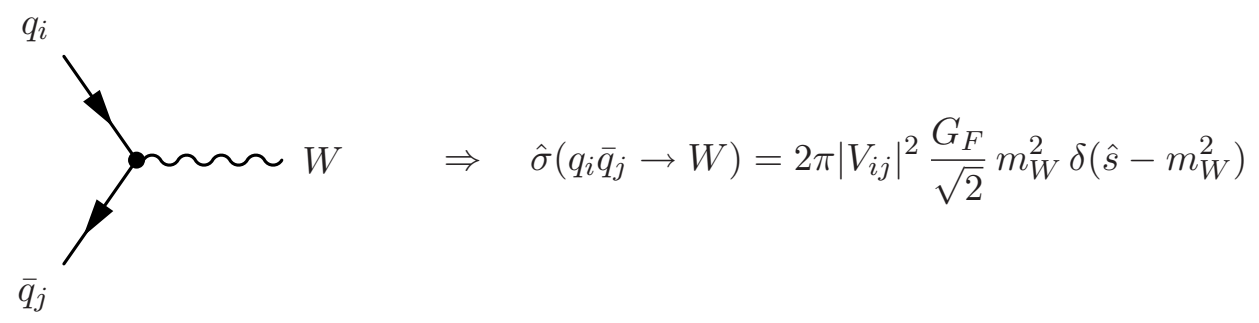

with the Fermi constant $G_{F}$, the center-of-mass energy in the partonic system $\sqrt{\hat{s}}$ (see Sec. 2.1), the mass of the $W$ boson $m_{W}$ and the CKM matrix element $V_{i j}$. The hadronic cross section is

$$
\sigma(p \bar{p} \rightarrow W+X)=\frac{1}{3} \int_{0}^{1} \mathrm{~d} x_{p} \int_{0}^{1} \mathrm{~d} x_{\bar{p}} \sum_{i, j} f_{q_{i}}\left(x_{p}, Q^{2}\right) f_{\bar{q}_{j}}\left(x_{\bar{p}}, Q^{2}\right) \hat{\sigma}\left(q_{i} \bar{q}_{j} \rightarrow W\right) .
$$

The factor $1 / 3$ is a consequence of the color: the quark and antiquark that annihilate to a $W$ boson must have the same color (note, that colors are not distinguished when measuring

\footnotetext{
${ }^{4}$ HERA = Hadron-Elektron-Ring-Anlage at DESY; DESY $=$ Deutsches Elektronen-SYnchroton, Hamburg, Germany

${ }^{5} \mathrm{CTEQ}=$ Coordinated Theoretical-Experimental Project on QCD
} 
the PDF in the deeply inelastic electron-proton collisions). The PDFs are evaluated at $Q^{2}=\hat{s}$.

It is convenient to transform the momentum fraction variables using the rapidity $y$ defined in Eq. B.2 (following [19], [20])

$$
\begin{aligned}
\hat{s} & =x_{p} x_{\bar{p}} s \Rightarrow \frac{\mathrm{d} \hat{s}}{\mathrm{~d} x_{\bar{p}}}=x_{p} s \\
x_{p} & =\frac{m_{W}}{\sqrt{s}} e^{y} \Rightarrow \frac{\mathrm{d} x_{p}}{\mathrm{~d} y}=x_{p} \\
x_{\bar{p}} & =\frac{m_{W}}{\sqrt{s}} e^{-y} \\
\Rightarrow \mathrm{d} x_{p} \mathrm{~d} x_{\bar{p}} & =\frac{1}{s} \mathrm{~d} \hat{s} \mathrm{~d} y
\end{aligned}
$$

so that the $\delta$-distribution can be integrated constraining $\hat{s}=m_{W}^{2}$. This transformation results in

$$
\sigma(p \bar{p} \rightarrow W+X)=\frac{2 \pi m_{W}^{2} G_{F}}{3 \sqrt{2} s} \int_{-\ln \frac{\sqrt{s}}{m_{W}}}^{\ln \frac{\sqrt{s}}{m_{W}}} \sum_{i, j}\left|V_{i j}\right|^{2} f_{q_{i}}\left(\frac{m_{W}}{\sqrt{s}} e^{y}, m_{W}^{2}\right) f_{\bar{q}_{j}}\left(\frac{m_{W}}{\sqrt{s}} e^{-y}, m_{W}^{2}\right) \mathrm{d} y .
$$

The rapidity limits are obtained from the constraints $x_{p} \leq 1$ and $x_{\bar{p}} \leq 1$. The integration using the CTEQ6.1M [17] PDFs and a center-of-mass energy of $\sqrt{s}=1.96 \mathrm{TeV}$ results in $\sigma(p \bar{p} \rightarrow W+X)=19.6 \mathrm{nb}$ [21]. Higher-order contributions to the Leading Order (LO, Born cross section) calculation are summarized in $K$ factors. The next-to-next-to-leading order correction, defined as

$$
K_{N N L O}=\frac{\sigma_{N N L O}}{\sigma_{L O}}
$$

is $K_{N N L O}=1.23$ yielding an inclusive cross section of $\sigma(p \bar{p} \rightarrow W+X)=24.2 \mathrm{nb}$ [21], [22] (see also Sec. 5.2).

Now, also the decay of the $W$ is included. The total cross section for the scattering process $p \bar{p} \rightarrow W+X \rightarrow e \nu+X$ can be determined by the multiplication of the production cross section with the branching fraction,

$$
\begin{aligned}
\sigma(p \bar{p} \rightarrow W+X \rightarrow e \nu+X) & =\sigma(p \bar{p} \rightarrow W+X) \times \operatorname{Br}(W \rightarrow e \nu), \\
\operatorname{Br}(W \rightarrow e \nu) & =\frac{\Gamma(W \rightarrow e \nu)}{\Gamma_{W}}
\end{aligned}
$$

using the partial and total decay width, $\Gamma(W \rightarrow e \nu)$ and $\Gamma_{W}$. The leading order calculation of the partial width into fermion pairs results in

$$
\Gamma\left(W \rightarrow f_{i} \bar{f}_{j}\right)=\frac{N_{C} G_{F} m_{W}^{3}}{6 \pi \sqrt{2}} \quad \text { with } \quad N_{C}=\left\{\begin{array}{ll}
3 \cdot\left|V_{i j}\right|^{2} & \text { for quarks } \\
1 & \text { for leptons }
\end{array} .\right.
$$

Note, that the decay $W \rightarrow t b$ is kinematically not possible. The total width is approximately $\Gamma_{W} \approx 9 \cdot \Gamma(W \rightarrow e \nu)\left(V_{i j} \approx \delta_{i j}\right)$. The values for the branching fraction and total width obtained from measurements are [4]

$$
\begin{aligned}
\operatorname{Br}(W \rightarrow e \nu) & =0.1080 \pm 0.0009 \\
\Gamma_{W} & =2.141 \pm 0.041 \mathrm{GeV} .
\end{aligned}
$$


The cross section for $W$ production and the subsequent decay into an electron and a neutrino can be directly calculated evaluating the matrix element for the scattering process

$$
\overbrace{W} \overbrace{\bar{\nu}}^{q_{i}} \Rightarrow \hat{\sigma}\left(q_{i} \bar{q}_{j} \rightarrow W \rightarrow e \nu\right)=\frac{\left|V_{i j}\right|^{2}}{3 \pi}\left(\frac{G_{F} m_{W}^{2}}{\sqrt{2}}\right)^{2} \frac{\hat{s}}{\left(\hat{s}-m_{W}^{2}\right)^{2}+\left(\Gamma_{W} m_{W}\right)^{2}} .
$$

Here, the $\delta$-distribution is replaced by the relativistic Breit-Wigner-function which takes the finite lifetime of the intermediate gauge boson into account. The total hadronic cross section is computed evaluating Eq. 2.102.

\subsection{New Charged Gauge Bosons beyond the Standard Model}

The Standard Model as outlined in the previous section agrees excellently with a high precision with the current experimental data. Deviations from the predictions of the Standard Model and measurements of precision observables are below the $3 \sigma$ level, see Fig. 2.6. Nevertheless, there are hints that the Standard Model is not the ultimate theory

\begin{tabular}{|c|c|c|c|}
\hline & Measurement & Fit & $\mathrm{O}^{\text {meas }} \mathrm{O}^{\mathrm{fit}} \mid / \sigma^{\text {meas }}$ \\
\hline$\overline{\Delta \alpha_{\text {had }}^{(5)}\left(m_{z}\right)}$ & $0.02758 \pm 0.00035$ & 0.02768 & 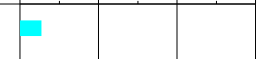 \\
\hline$m_{z}[\mathrm{GeV}]$ & $91.1875 \pm 0.0021$ & 91.1875 & \\
\hline$\Gamma_{Z}[\mathrm{GeV}]$ & $2.4952 \pm 0.0023$ & 2.4957 & b \\
\hline$\sigma_{\text {had }}^{0}[n b]$ & $41.540 \pm 0.037$ & 41.477 & \\
\hline $\mathrm{R}_{\mathrm{I}}$ & $20.767 \pm 0.025$ & 20.744 & \\
\hline$A_{\mathrm{fb}}^{0,1}$ & $0.01714 \pm 0.00095$ & 0.01645 & \\
\hline$A_{1}\left(P_{\tau}\right)$ & $0.1465 \pm 0.0032$ & 0.1481 & - \\
\hline $\mathrm{R}_{\mathrm{b}}$ & $0.21629 \pm 0.00066$ & 0.21586 & \\
\hline $\mathrm{R}_{\mathrm{c}}$ & $0.1721 \pm 0.0030$ & 0.1722 & \\
\hline$A_{f b}^{0, b}$ & $0.0992 \pm 0.0016$ & 0.1038 & \\
\hline$A_{f b}^{0, c}$ & $0.0707 \pm 0.0035$ & 0.0742 & \\
\hline$A_{b}$ & $0.923 \pm 0.020$ & 0.935 & \\
\hline$A_{c}$ & $0.670 \pm 0.027$ & 0.668 & \\
\hline$A_{1}(S L D)$ & $0.1513 \pm 0.0021$ & 0.1481 & \\
\hline $\sin ^{2} \theta_{\text {eff }}^{\text {lept }}\left(Q_{\mathrm{fb}}\right)$ & $0.2324 \pm 0.0012$ & 0.2314 & \\
\hline $\mathrm{m}_{\mathrm{W}}[\mathrm{GeV}]$ & $80.398 \pm 0.025$ & 80.374 & \\
\hline$\Gamma_{\mathrm{W}}[\mathrm{GeV}]$ & $2.140 \pm 0.060$ & 2.091 & 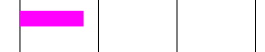 \\
\hline$m_{t}[G e V]$ & $170.9 \pm 1.8$ & 171.3 & 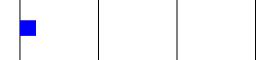 \\
\hline & & & 2 \\
\hline
\end{tabular}
due to several unresolved questions and problems:

Figure 2.6: Comparison of measurements of precision observables with Standard Model fits [14]. 
- Why does the weak force distinguish left and right?

- Why does the Standard Model contain a rather large number of free parameters?

- The Standard Model does not provide the unification of the electroweak and strong force.

- The nature of the neutrinos (Dirac $\leftrightarrow$ Majorana) is unknown as well as the mechanism of mass generation and the reason for the smallness of the masses compared to the charged leptons in the same doublet $\left(m_{\ell} \gg m_{\nu_{\ell}}\right)$.

- The Standard Model does not explain the hierarchy of masses $\left(m_{t}>m_{b}>m_{c}>\right.$ $\left.m_{s}>m_{d}>m_{u}, m_{\tau}>m_{\mu}>m_{e}\right)$.

- In the Standard Model the Higgs mass is extremely sensitive to radiative corrections so that a fine-tuning ${ }^{6}$ of the Higgs mass is required.

- The Standard Model does not comprise gravity due to the fact that it is not renormalizable.

In order to comply with current measurements, extensions have to be constructed so that the Standard Model is embedded or at least contained as a low-energy theory.

\subsubsection{Left-Right Symmetric Extension of the Standard Model}

In the Standard Model parity violation is introduced by hand. Therefore the extension by a right-handed sector has not only aesthetic reasons, but it provides a mechanism for parity violation. The left-right symmetry group $\mathrm{SU}(2)_{L} \otimes \mathrm{SU}(2)_{R} \otimes \mathrm{U}(1)_{\tilde{Y}}$ has to be broken in order to reflect the Standard Model structure

$$
S U(2)_{L} \otimes S U(2)_{R} \otimes U(1)_{\tilde{Y}} \quad \rightarrow \quad S U(2)_{L} \otimes U(1)_{Y} \quad \rightarrow \quad U(1)_{E M} .
$$

This can be accomplished by extending the minimal Higgs mechanism outlined in Sec. 2.2.4. The new $\mathrm{SU}(2)_{R}$ symmetry leads to additional gauge bosons $\left(W^{\prime}\right.$ and $Z^{\prime}$ ) which can be looked for at collider experiments. For a detailed discussion of the Left-Right Symmetric Model consider e. g. [23], [24], [25], [26], [27], [28].

The Left-Right Symmetric Model is constructed by placing the singlets of right-handed fermions into doublets regarding $\mathrm{SU}(2)_{R}$. This requires the introduction of right-handed neutrinos $\nu_{R}$

$$
u_{R}, d_{R} \rightarrow\left(\begin{array}{c}
u \\
d
\end{array}\right)_{R} \text { and } \nu_{R}, \ell_{R} \rightarrow\left(\begin{array}{c}
\nu \\
\ell
\end{array}\right)_{R} .
$$

The hypercharge has to be modified so that this quantum number is identical for leftand right-handed particles. Using the third component of the weak isospin for left- and right-handed particles, the following relation is derived

$$
Q=I_{3 L}+I_{3 R}+\frac{1}{2} \tilde{Y}=I_{3 L}+I_{3 R}+\frac{1}{2}(B-L) \quad \Rightarrow \quad \tilde{Y}=B-L .
$$

\footnotetext{
${ }^{6}$ very precise adjusting of parameters
} 
Table 2.2: Quantum numbers for fermions in the Left-Right Symmetric Model (cf. Tab. 2.1).

\begin{tabular}{|c|cc|cc|c|c|c|}
\hline fermion doublet & $I_{L}$ & $I_{3 L}$ & $I_{R}$ & $I_{3 R}$ & $B$ & $L$ & $\tilde{Y}=B-L$ \\
\hline \hline$\left(\begin{array}{c}\nu_{\ell} \\
\ell\end{array}\right)_{L}$ & $1 / 2$ & $\begin{array}{c}1 / 2 \\
-1 / 2\end{array}$ & 0 & 0 & 0 & 1 & -1 \\
\hline$\left(\begin{array}{c}\nu_{\ell} \\
\ell\end{array}\right)_{R}$ & 0 & 0 & $1 / 2$ & $\begin{array}{c}1 / 2 \\
-1 / 2\end{array}$ & 0 & 1 & -1 \\
\hline \hline$\left(\begin{array}{c}u \\
d^{\prime}\end{array}\right)_{L}$ & $1 / 2$ & $\begin{array}{c}1 / 2 \\
-1 / 2\end{array}$ & 0 & 0 & $1 / 3$ & 0 & $1 / 3$ \\
\hline$\left(\begin{array}{c}u \\
d^{\prime}\end{array}\right)_{R}$ & 0 & 0 & $1 / 2$ & $\begin{array}{c}1 / 2 \\
-1 / 2\end{array}$ & $1 / 3$ & 0 & $1 / 3$ \\
\hline
\end{tabular}

The modified hypercharge $\tilde{Y}$ can be calculated from the lepton number $L$ and the baryon number $B$ of the fermions. The relevant quantum numbers are summarized in Tab. 2.2 (cf. Tab. 2.1). The notation $F(L, R, \tilde{Y})$ expresses the transformation behaviour of a fermion doublet $F$, e.g. the right-handed quarks $q_{R}$ with $q_{R}(1,2,1 / 3)$ are doublets under $\mathrm{SU}(2)_{R}$, and singlets under $\mathrm{SU}(2)_{L}$ and $\mathrm{U}(1)$ with a hypercharge of $1 / 3$.

The covariant derivative from the electroweak theory Eq. 2.37 has to be modified and the right-handed part to be extended

$$
\begin{aligned}
& D_{E W, L}^{\mu}=\partial^{\mu}+i g_{L} \cdot \tau_{i} \cdot W_{i, L}^{\mu}+i g^{\prime} \cdot \frac{B-L}{2} \cdot B^{\mu}, \\
& D_{E W, R}^{\mu}=\partial^{\mu}+i g_{R} \cdot \tau_{i} \cdot W_{i, R}^{\mu}+i g^{\prime} \cdot \frac{B-L}{2} \cdot B^{\mu} .
\end{aligned}
$$

This assures the invariance under the exchange $L \leftrightarrow R$. Particles acquire masses via the Yukawa term $\propto \bar{\psi}_{L} \phi \psi_{R}$ in the Lagrangian. Hence, the Higgs fields $\phi$ and $\tilde{\phi}=\sigma_{2} \phi^{*} \sigma_{2}$ are required to be $2 \times 2$ matrices of scalar fields

$$
\phi=\left(\begin{array}{cc}
\phi_{1}^{0} & \phi_{1}^{+} \\
\phi_{2}^{-} & \phi_{2}^{0}
\end{array}\right) \quad \text { with } \quad \phi(2,2,0)
$$

The upper index denotes the charge of the field. The most general coupling of the fermions to the Higgs fields can be written as

$$
\mathcal{L}_{\text {Yukawa }}=-\sum_{\text {quarks, leptons }}\left\{\bar{\psi}_{i, L} \Gamma_{i j} \phi \psi_{j, R}+\bar{\psi}_{i, L} \Omega_{i j} \tilde{\phi} \psi_{j, R}+\text { h.c. }\right\}
$$

with $i, j=1,2,3$ and coupling constants $\Gamma_{i j}$ and $\Omega_{i j}$.

The Left-Right Symmetric Model is defined in such a way that every left-handed field has a right-handed counterpart. The transition from left to right can be interpreted as a parity transformation $\mathcal{P}$ with

$$
\begin{aligned}
W_{i, L / R}^{\mu}(x) & \rightarrow \varepsilon(\mu) W_{i, R / L}^{\mu}(\hat{x}), \\
B^{\mu}(x) & \rightarrow \varepsilon(\mu) B^{\mu}(\hat{x}), \\
\psi_{L / R}(x) & \rightarrow U_{R / L} \gamma^{0} \psi_{R / L}(\hat{x}), \\
\phi(x) & \rightarrow \phi^{\dagger}(\hat{x}) .
\end{aligned}
$$


using the conventions

$$
\hat{x}=\left(\begin{array}{c}
x^{0} \\
-\vec{x}
\end{array}\right) \text { and } \varepsilon(\mu)=\left\{\begin{array}{r}
1 \\
-1
\end{array} \text { for } \begin{array}{l}
\mu=0 \\
\mu=1,2,3
\end{array} .\right.
$$

The Lagrangian is required to be invariant under this transformation. This leads to the following relations for the coupling constants and the (arbitrary) unitary matrices $U$

$$
g_{L}=g_{R}, \quad U_{R}^{\dagger} \Gamma U_{L}=\Gamma^{\dagger}, \quad U_{R}^{\dagger} \Omega U_{L}=\Omega^{\dagger} .
$$

In the simplest case one assumes $U_{L / R}=\mathbf{1}$ which implies $\Gamma=\Gamma^{\dagger}$ and $\Omega=\Omega^{\dagger}$.

In general, a new CKM mixing matrix can appear in the right-handed quark sector which differs from the left-handed matrix. In the Manifest Left-Right Symmetric Model the left-handed CKM matrix equals the right-handed, $V_{C K M}^{L}=V_{C K M}^{R}$. Assuming not only $\mathcal{P}$ invariance, but also $\mathcal{C} \mathcal{P}$ invariance, the quark Yukawa couplings can be further constrained to be real and symmetric

$$
\begin{aligned}
\Gamma_{i j} & =\Gamma_{j i}, & & \Gamma_{i j}=\Gamma_{i j}^{*}, \\
\Omega_{i j} & =\Omega_{j i}, & & \Omega_{i j}=\Omega_{i j}^{*} .
\end{aligned}
$$

In this case, known as Pseudo-Manifest Left-Right Symmetric Model, $V_{C K M}^{L}=\left(V_{C K M}^{R}\right)^{*}$ holds.

The Higgs field $\phi$ is able to assign masses to the fermions, but it can not be used for the symmetry breaking Eq. 2.115. Because of this, the Higgs sector has to be enlarged by two scalar triplets: $\Delta_{L}(3,1,2)$ and $\Delta_{R}(1,3,2)$. This extension is referred to as Minimal Left-Right Symmetric Model. The triplets with hypercharge $\tilde{Y}=2$ (the lepton number is $L=-2$ ) are constructed using the Pauli matrices $\sigma_{i}$

$$
\Delta_{L / R}=\frac{1}{\sqrt{2}} \sigma_{i} \delta_{i, L / R}=\frac{1}{\sqrt{2}}\left(\begin{array}{cc}
\delta_{3} & \delta_{1}-i \delta_{2} \\
\delta_{1}+i \delta_{2} & -\delta_{3}
\end{array}\right)_{L / R} \equiv\left(\begin{array}{cc}
\delta^{+} / \sqrt{2} & \delta^{++} \\
\delta^{0} & -\delta^{+} / \sqrt{2}
\end{array}\right)_{L / R}
$$

containing neutral, single and doubly charged Higgs bosons. These fields transform under parity as follows

$$
\Delta_{L / R}(x) \rightarrow \Delta_{R / L}(\hat{x}) .
$$

The Yukawa couplings of the triplet are given by

$$
\mathcal{L}_{\text {Yukawa }}=\sum_{\text {leptons }}\left\{\psi_{i, L}^{T} G_{L, i j} \mathcal{C}^{-1} i \sigma_{2} \Delta_{L} \psi_{j, L}+\psi_{i, R}^{T} G_{R, i j} \mathcal{C}^{-1} i \sigma_{2} \Delta_{R} \psi_{j, R}+\text { h.c. }\right\}
$$

with the charge conjugation operator $\mathcal{C}=i \gamma_{2}$ and coupling constants $G_{L / R, i j}$ which transform as follows under parity transformation

$$
G_{L / R}=\left(U_{L / R}\right)^{T} G_{R / L} U_{L / R}
$$

The invariance leads to $G_{L}=G_{R}$ (assuming $U_{L / R}=1$ ). The vacuum expectation values are chosen so that the photon remains massless

$$
\phi_{0}=\frac{1}{\sqrt{2}}\left(\begin{array}{cc}
v_{1} & 0 \\
0 & v_{2}
\end{array}\right) \quad \text { and } \quad \Delta_{L / R, 0}=\left(\begin{array}{cc}
0 & 0 \\
w_{L / R} & 0
\end{array}\right) .
$$


Using the following assumption regarding the relative size of the vacuum expectation values

$$
\left|w_{L}\right|^{2} \ll\left|v_{1}\right|^{2}+\left|v_{2}\right|^{2} \ll\left|w_{R}\right|^{2},
$$

one can assign the symmetry breaking $\mathrm{SU}(2)_{R} \otimes \mathrm{U}(1)_{B-L}$ to the presence of $w_{R}$. The $\phi$ field takes care of the symmetry breaking to the observed $\mathrm{U}(1)_{E M}$ symmetry

$$
S U(2)_{L} \otimes S U(2)_{R} \otimes U(1)_{B-L} \otimes \mathcal{P} \stackrel{w_{R}}{\longrightarrow} S U(2)_{L} \otimes U(1)_{Y} \stackrel{v_{1}, v_{2}}{\longrightarrow} U(1)_{E M} .
$$

The symbol $\mathcal{P}$ denotes the symmetry under parity transformation which is broken by $w_{R}$. The relation $\left|w_{L}\right|^{2} \ll\left|w_{R}\right|^{2}$ directly translates into the masses of the left- and righthanded gauge bosons. Hence, the physical eigenstates $W_{L / R}$ do not correspond to the mass eigenstates $W_{1 / 2}$. This mixing is specified in the following way

$$
\left(\begin{array}{l}
W_{1} \\
W_{2}
\end{array}\right)=\left(\begin{array}{cc}
\cos \xi & -\sin \xi e^{i \lambda} \\
\sin \xi e^{-i \lambda} & \cos \xi
\end{array}\right)\left(\begin{array}{l}
W_{L} \\
W_{R}
\end{array}\right)
$$

introducing a mixing angle $\xi$ and a phase $\lambda$. Using the vacuum expectation values and Eq. 2.135 one gets

$$
e^{i \lambda}=-\frac{v_{1} v_{2}^{*}}{\left|v_{1} v_{2}\right|}, \quad \xi \simeq \frac{2\left|v_{1}\right|\left|v_{2}\right|}{\left|v_{1}\right|^{2}+\left|v_{2}\right|^{2}}\left(\frac{m_{1}}{m_{2}}\right)^{2}, \quad\left|v_{1}\right|^{2}+\left|v_{2}\right|^{2} \simeq \frac{1}{\sqrt{2} G_{F}}
$$

and

$$
m_{1}^{2} \simeq \frac{1}{4} g^{2}\left(\left|v_{1}\right|^{2}+\left|v_{2}\right|^{2}\right) \simeq m_{W}^{2}, \quad m_{2}^{2} \simeq \frac{1}{4} g^{2}\left(2\left|w_{R}\right|^{2}+\left|v_{1}\right|^{2}+\left|v_{2}\right|^{2}\right) \simeq \frac{1}{2} g^{2}\left|w_{R}\right|^{2} .
$$

Hence, $W_{1}$ corresponds to the $W$ boson from the Standard Model with $m_{1} \approx m_{W}$, whereas the mass $m_{2}$ of the new gauge boson $W_{2}$ is determined by the breaking scale $w_{R}$ of $\mathrm{SU}(2)_{R}$. Since this scale has not yet been observed, $\left|w_{R}\right|$ and thus $m_{2} \approx m_{W^{\prime}}$ have to be large $\left(m_{W^{\prime}} \gg m_{W}\right)$. This observation justifies the assumption Eq. 2.135. Due to the huge mass difference, the mixing is negligible

$$
\xi \propto\left(\frac{m_{W}}{m_{W^{\prime}}}\right)^{2} \approx 0
$$

so that $W_{1} \approx W_{L}=W$ and $W_{2} \approx W_{R}=W^{\prime}$.

The Left-Right Symmetric Model provides small standard neutrino masses because it incorporates the seesaw mechanism. The symmetry breaking assigns masses to Majorana neutrinos,

$$
\mathcal{L} \approx \frac{1}{2}\left(\nu_{L}\left(\nu_{R}\right)_{\mathcal{C}}\right) \mathcal{C}^{-1}\left(\begin{array}{cc}
0 & m_{\ell} \\
m_{\ell} & w_{R}
\end{array}\right)\left(\begin{array}{c}
\nu_{L} \\
\left(\nu_{R}\right)_{\mathcal{C}}
\end{array}\right)+\text { h.c. }
$$

resulting in three heavy $\left(m_{N}\right)$ and three light neutrinos $m_{\nu}$. The order of magnitude of the heavy neutrino is again determined by the scale $w_{R}$

$$
m_{N} \sim w_{R} \sim m_{W^{\prime}}, \quad m_{\nu} \sim m_{\ell}^{2} / m_{N} .
$$

However, it is possible to generate small neutrino masses even if the right-handed neutrino is light. These modifications of the Left-Right Symmetric Model are discussed in [29]. 


\subsubsection{The $W^{\prime}$ Reference Model as a General Approach}

Additional charged (and neutral) gauge bosons arise in many other extensions to the Standard Model. Already the smallest unification group SU(5) predicts heavy bosons $X$ and $Y$. However, this model is ruled out because the anticipated proton decay is not observed (lifetime limit: $\tau>10^{31}-10^{33}$ years [4]). Another extension predicting extra gauge bosons, $\mathrm{SO}(10)$, is consistent with experimental data. Due to the large variety of models (other unification groups like $E_{6}$, models with Supersymmetry, Little Higgs Models, Extra Dimensions) a more general approach is considered, namely the Reference Model which has been discussed first by Altarelli et al. [30].

In this model the new charged gauge boson $W^{\prime}$ appears as a heavier version of the (lefthanded) Standard Model $W$ boson. The couplings of the $W^{\prime}$ boson to quarks, leptons and gauge bosons of the electroweak interaction are assumed to be identical to the Standard Model couplings. As a consequence of this approach, the new decay channel $W^{\prime} \rightarrow W Z$ is dominant for high masses $m_{W^{\prime}}>2 m_{Z}$ because of

$$
\Gamma\left(W^{\prime} \rightarrow W Z\right) \propto m_{W^{\prime}} \cdot \frac{m_{W^{\prime}}^{4}}{m_{Z}^{2} m_{W}^{2}} .
$$

This leads to widths of the $W^{\prime}$ boson which are larger than the mass for $m_{W^{\prime}}>500 \mathrm{GeV}$. Considering extended gauge models, like the Left-Right Symmetric Model, this channel is suppressed by the mixing angle $\xi$ (even $\xi^{2}[30]$ ), which is small due to the huge breaking scale (see Eq. 2.140). In the Reference Model this suppression has to be built in by hand.

With these basic prerequisites, cross sections and branching fractions can be calculated. Due to the close affinity to the Standard Model $W$ boson, the formulae derived in Sec. 2.2.7 are subject to merely marginal modifications. The partial width into fermion pairs is as follows

$$
\begin{aligned}
\Gamma\left(W^{\prime} \rightarrow f_{i} \bar{f}_{j}\right) & =\frac{N_{C} G_{F} m_{W}^{2} m_{W^{\prime}}}{6 \pi \sqrt{2}} \text { with } \quad N_{C}= \begin{cases}3 \cdot\left|V_{i j}\right|^{2} & \text { for quarks } \\
1 & \text { for leptons }\end{cases} \\
& =\frac{N_{C} g^{2} m_{W^{\prime}}}{48 \pi}=\Gamma\left(W \rightarrow f_{i} \bar{f}_{j}\right) \cdot \frac{m_{W^{\prime}}}{m_{W}}
\end{aligned}
$$

This behaviour translates also into the total width. One has to take into account that the decay channel into the third quark family, $W^{\prime} \rightarrow t b$, opens for masses beyond the kinematical threshold $\left(m_{t}+m_{b} \approx 180 \mathrm{GeV}\right)$ for this process. With

$$
\sum_{\text {decay channels }} N_{C} \simeq\left\{\begin{array}{c}
9 \\
12
\end{array} \text { for } \quad \begin{array}{l}
m_{W^{\prime}} \ll 180 \mathrm{GeV} \\
m_{W^{\prime}} \gg 180 \mathrm{GeV}
\end{array}\right.
$$

the total width of the heavy gauge boson is approximately given by

$$
\Gamma_{W^{\prime}} \simeq\left\{\begin{array}{rlrl}
\Gamma_{W} \cdot \frac{m_{W^{\prime}}}{m_{W}} & \text { for } & m_{W^{\prime}} \ll 180 \mathrm{GeV} \\
\frac{4}{3} \cdot \Gamma_{W} \cdot \frac{m_{W^{\prime}}}{m_{W}} & & m_{W^{\prime}} \gg 180 \mathrm{GeV}
\end{array} .\right.
$$

The cross sections for the inclusive $W^{\prime}$ production $p \bar{p} \rightarrow W^{\prime}+X$ and for the process $p \bar{p} \rightarrow W^{\prime}+X \rightarrow e \nu+X$ are derived from the partonic cross sections Eq. 2.101 and 2.114 
by just replacing the mass and the width

$$
\begin{aligned}
\hat{\sigma}\left(q_{i} \bar{q}_{j} \rightarrow W^{\prime}\right) & =\frac{\pi\left|V_{i j}\right|^{2}}{4} g^{2} \delta\left(\hat{s}-m_{W^{\prime}}^{2}\right) \\
\hat{\sigma}\left(q_{i} \bar{q}_{j} \rightarrow W^{\prime} \rightarrow e \nu\right) & =\frac{\left|V_{i j}\right|^{2}}{192 \pi} g^{4} \frac{\hat{s}}{\left(\hat{s}-m_{W^{\prime}}^{2}\right)^{2}+\left(\Gamma_{W^{\prime}} m_{W^{\prime}}\right)^{2}} .
\end{aligned}
$$

The total hadronic cross section is obtained by the integration over the momentum fractions according to Eq. 2.102 which incorporates the PDFs.

Note, that Eq. 2.144, 2.148 and 2.149 hold not only for the left-handed $(V-A)$, but also for the right-handed $(V+A)$ charged current. The $W e \nu$ vertex Eq. 2.51 can be written in the following form introducing vector $g_{V}^{c}$ and axialvector couplings $g_{A}^{c}$ for the charged current

$$
-i \frac{g}{2 \sqrt{2}} \bar{\psi}\left(\bar{\nu}_{e}\right) \gamma_{\mu}\left(g_{V}^{c}+g_{A}^{c} \gamma^{5}\right) \tau_{+} \psi\left(e^{-}\right) W^{(-) \mu} .
$$

In the Standard Model it is $g_{V}^{c}=1$ and $g_{A}^{c}=-1$ which is referred to as $V-A$ coupling, whereas the right-handed $W^{\prime}$ introduced in the Left-Right Symmetric Model couples with $g_{V}^{c}=1$ and $g_{A}^{c}=1(V+A)$. Right-handed neutrinos are assumed to be light, $m_{\nu_{R}} \ll m_{W^{\prime}}$, so that the process $W^{\prime} \rightarrow e \nu_{R}$ can occur. Due to the fact that the couplings enter the calculations only quadratically, e.g.

$$
\Gamma\left(W^{\prime} \rightarrow f_{i} \bar{f}_{j}\right)=\frac{\left(g_{V}^{c}\right)^{2}+\left(g_{A}^{c}\right)^{2}}{2} \frac{N_{C} g^{2} m_{W^{\prime}}}{48 \pi},
$$

the $W^{\prime}$ bosons in Altarelli's sense can be either left- or right-handed ${ }^{7}$ with $\left|g_{V}^{c}\right|=\left|g_{A}^{c}\right|=$ 1. In summary, the Reference Model is the generalization of the Manifest Left-Right Symmetric Model with light right-handed neutrinos.

The analysis presented in this thesis is based on the Reference Model - and thus the Manifest Left-Right Symmetric Model with light right-handed neutrinos. The production and subsequent decay of such a new gauge boson $W^{\prime}\left(W^{\prime+}, W^{\prime-}\right.$, left- or right-handed) into an electron and a light, non-detectable neutrino is searched for. This channel provides a clean final state containing a highly energetic electron which is important for triggering. It is possible to reconstruct electrons up to highest energies with reasonable precision, unlike the muon channel $\left(W^{\prime} \rightarrow \mu \nu\right)$. In this case one suffers from the momentum resolution which deteriorates with increasing muon momentum.

\subsubsection{Bounds on the $W^{\prime}$ Mass}

There are two basic strategies for testing a new model: the direct search for the production (and decay) of new particles, and the indirect search. In the latter case, the new physics is identified via additional contributions in Feynman diagrams. These may enhance or suppress a given process. In direct searches the new particles are produced for example at colliders. In the resonant process the center-of-mass energy is required to be equal to the production threshold for on-shell production, $\sqrt{\hat{s}} \approx m_{W^{\prime}}$.

\footnotetext{
${ }^{7}$ Note, that it is not possible to distinguish between left- and right-handed $W$ bosons in protonantiproton (or in proton-proton [31]) collisions without a polarization measurement of e.g. the electrons in the final state.
} 
Most of the previous searches consider the Manifest or Pseudo-Manifest Left-Right Symmetric Model with the phase $\lambda=0$ or $\lambda=\pi$. The Particle Data Group [32] quotes roughly 50 different results from various searches which can not be discussed in detail in this thesis. Only selected results are presented here. A full review of searches for new heavy charged gauge bosons can be found in [4].

\section{Direct Searches}

The most stringent direct limit to date for the production of Standard-Model-like charged gauge bosons is derived from the analysis of proton-antiproton collisions at the TEVATRON collider during Run I $(1992-1995)$ at a center-of-mass energy of $\sqrt{s}=1.8 \mathrm{TeV}$. The data was taken with the $\mathrm{D} \varnothing$ detector and corresponds to an integrated luminosity of $\int \mathcal{L} \mathrm{d} t=109 \mathrm{pb}^{-1}[33]$. Events with two jets are analyzed looking for an enhancement in the invariant mass spectrum of the two jets which is assumed to stem from the process $W^{\prime} \rightarrow q_{i} \bar{q}_{j}$. Since the data is consistent with the Standard Model prediction, additional charged gauge bosons can be excluded up to masses of $800 \mathrm{GeV}$

$$
m_{W^{\prime}}>800 \mathrm{GeV} @ 95 \% \text { C.L. . }
$$

The decay channel $W^{\prime} \rightarrow e \nu$ has recently been analyzed at the TEvatron collider in Run II by the CDF collaboration at the increased center-of-mass energy of $\sqrt{s}=1.96 \mathrm{TeV}$. The data taken in $2002-2003$ corresponds to an integrated luminosity of $\int \mathcal{L} \mathrm{d} t=205 \mathrm{pb}^{-1}$ [34]. The distribution of the transverse mass reconstructed from the electron and missing transverse energy ( $\simeq$ neutrino) is shown in the left-hand plot of Fig. 2.7. This distribution is tested for a possible signal contribution by making use of a binned maximum likelihoood fit. Since no significant excess over the background expectation is observed, an upper limit on the production cross section times branching fraction can be set, see the right-hand plot of Fig. 2.7. This limit can be converted to a limit on the mass, excluding new charged gauge bosons up to $788 \mathrm{GeV}$

$$
m_{W^{\prime}}>788 \mathrm{GeV} \text { @ } 95 \% \text { C.L. . }
$$

Since this analysis assumes the Manifest Left-Right Symmetric Model with a light and stable right-handed neutrino, the limit is directly comparable to the di-jet search $(W \rightarrow$ $\left.q_{i} \bar{q}_{j}\right)$ from $\mathrm{D} \varnothing$ and the analysis presented in this thesis $\left(W^{\prime} \rightarrow e \nu\right)$.

\section{Indirect Searches}

The mixing angle $\xi$ can be constrained by analyzing Kaon decays, $K \rightarrow \pi \pi$ and $K \rightarrow \pi \pi \pi$, because those are sensitive to admixtures of right-handed currents. The current limit is $|\xi| \leq 0.004[35]$ assuming that the right-handed boson is much heavier than the left-handed.

The kaon sector can as well be consulted for indirectly restricting the mass of the new gauge boson, $W^{\prime}$. In the case of the $K_{L}-K_{S}$ mixing, additional contributions have to be considered, see the left-hand Feynman diagram in Fig. 2.8. In the Manifest and Pseudo-Manifest Left-Right Symmetric Model a limit of $m_{W^{\prime}}>1.6 \mathrm{TeV}$ can be set which is independent of the mixing angle $\xi[36]$.

Another limit on the mass of the $W^{\prime}$ boson can be derived analyzing the neutrinoless double $\beta$ decay. If the neutrinos have Majorana masses, the process shown in the righthand Feynman diagram Fig. 2.8 occurs. The amplitude $\mathcal{M}$ is strongly enhanced if righthanded gauge bosons are involved. The neutrinoless double $\beta$ decay has not been observed up to now. Hence, a limit of $m_{W^{\prime}} \geq 1.1 \mathrm{TeV}$ is obtained, if the experimental limit from the decay of ${ }^{76} \mathrm{Ge}$ is combined with arguments of vacuum stability [37]. 
Figure 2.7: Results from the search for the process $W^{\prime} \rightarrow e \nu$ with the CDF detector [34]. Shown is the distribution of the transverse mass reconstructed from the electron and missing transverse energy (left-hand plot). The right-hand plot shows the resulting limit on the cross section.
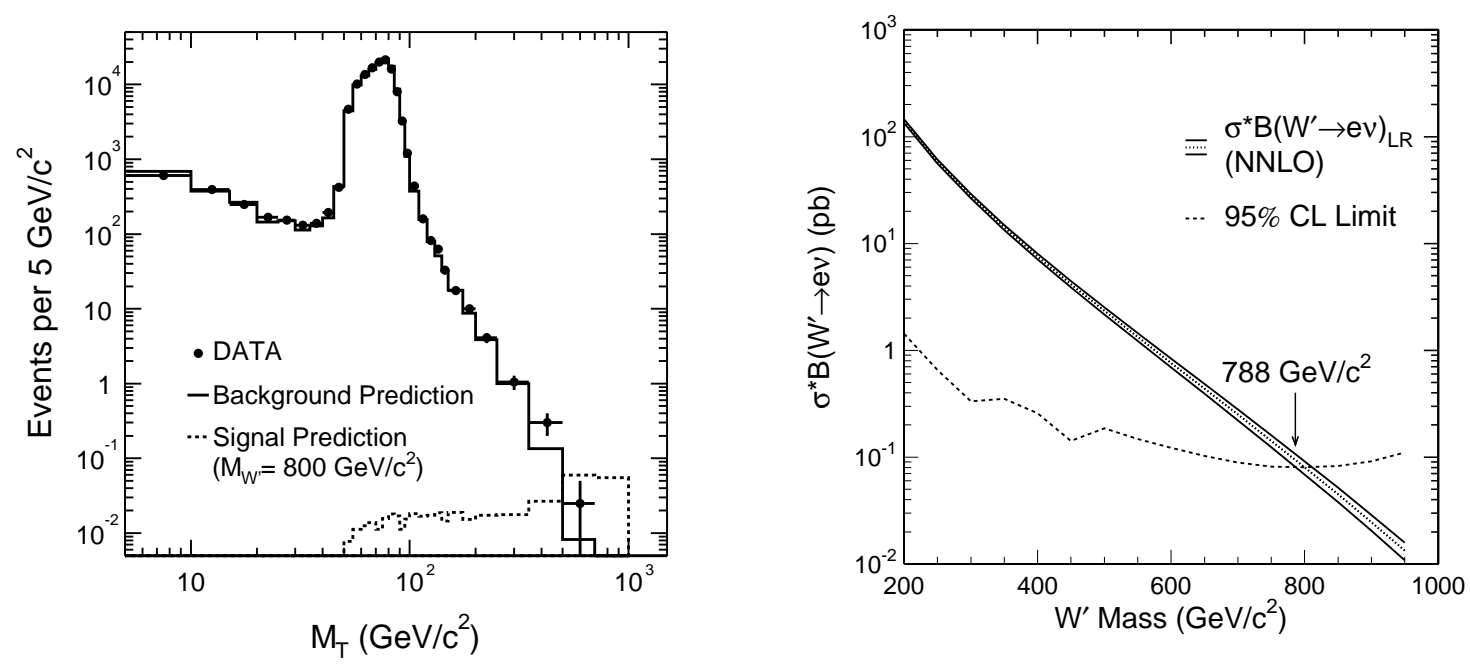

Figure 2.8: Contributions from right-handed $W$ bosons in the $K_{L}-K_{S}$ mixing (left-hand Feynman diagram) and the neutrinoless double $\beta$ decay (right-hand Feynman diagram).
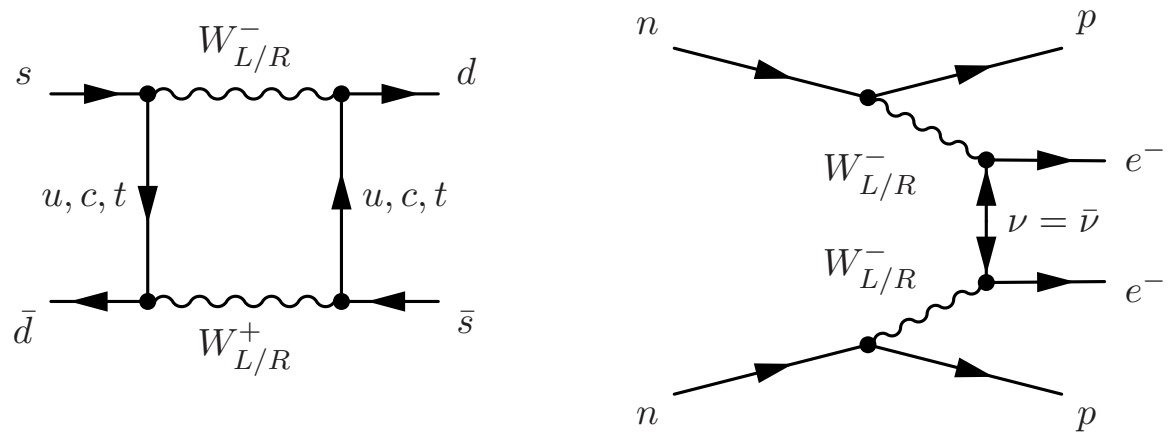


\title{
Chapter 3
}

\section{Tevatron and the DØ Detector}

\author{
What we observe is not nature itself, \\ but nature exposed to our method of questioning. \\ Werner Heisenberg
}

The data analyzed in this thesis was taken with the $\mathrm{D} \varnothing$ detector [38] at the TEvatron proton-antiproton collider. Two multi-purpose detectors, D $\varnothing$ as well as the second experiment, $\mathrm{CDF}^{1}$ [39], are designed for recording particles produced in the collisions of protons and antiprotons, whereas each of the colliding hadrons carries an energy of $E=980 \mathrm{GeV}$. The collider complex with the experiments, accelerators and preaccelerators, as well as several other facilities (e.g. SiDet, Minos, MiniBooNE) are located at the Fermilab site [40] (FNAL, Fermi National Accelerator Laboratory) in Batavia/Illinois (USA), covering an area of $27.5 \mathrm{~km}^{2}$ roughly $50 \mathrm{~km}$ west of Chicago. Fig. 3.1 shows an aerial view of the accelerator complex with the detectors, including the Feynman Computing Center (location of the data storage devices and computing farms of the experiments D $\varnothing$ and CDF) and the main building Wilson Hall (headquarter for the laboratory's administrative staff, theoretical physics department, CMS department, ...).

Fermilab was established in 1967 and is part of the U.S. Department of Energy [41] (DOE). Milestones in the history of research at FERMILAB are the discoveries [42] of the bottom quark (1977), the top quark (1995) and the tau neutrino $\nu_{\tau}$ (2000). The top quark was discovered by DØ [43] and CDF [44] in proton-antiproton collisions at a centerof-mass energy of $\sqrt{s}=1.8 \mathrm{TeV}$. The TEVATRON collider operated at this energy from 1992 to 1996 (Run I), accumulating an integrated luminosity of $\int \mathcal{L} \mathrm{d} t=110 \mathrm{pb}^{-1}$ per experiment. After upgrading both detectors and the accelerator, the Run II data taking period at the increased center-of-mass energy of $\sqrt{s}=1.96 \mathrm{TeV}$ started in 2002, after one year dedicated to the commissioning of the detector. Until February 2006, after four years of running, the TEVATRON delivered roughly $\int \mathcal{L} \mathrm{d} t=1.6 \mathrm{fb}^{-1}$ per experiment, from which $\int \mathcal{L} \mathrm{d} t=1.35 \mathrm{fb}^{-1}$ were recorded to tape by the $\mathrm{D} \varnothing$ experiment, see Fig. 3.2. This data, referred to as Run IIa dataset, is analyzed in this thesis. During a shutdown of three months, the detectors and the accelerators were again upgraded. The D $\varnothing$ experiment has already accumulated more than $1 \mathrm{fb}^{-1}$ since the start of Run IIb in June 2006, see Fig. 3.2 .

\footnotetext{
${ }^{1} \mathrm{CDF}=$ Collider Detector at Fermilab
} 
Figure 3.1: Aerial view of the Tevatron collider at the Fermilab site [45]. Proton-antiproton collisions are recorded with two multi-purpose detectors, CDF and DØ.

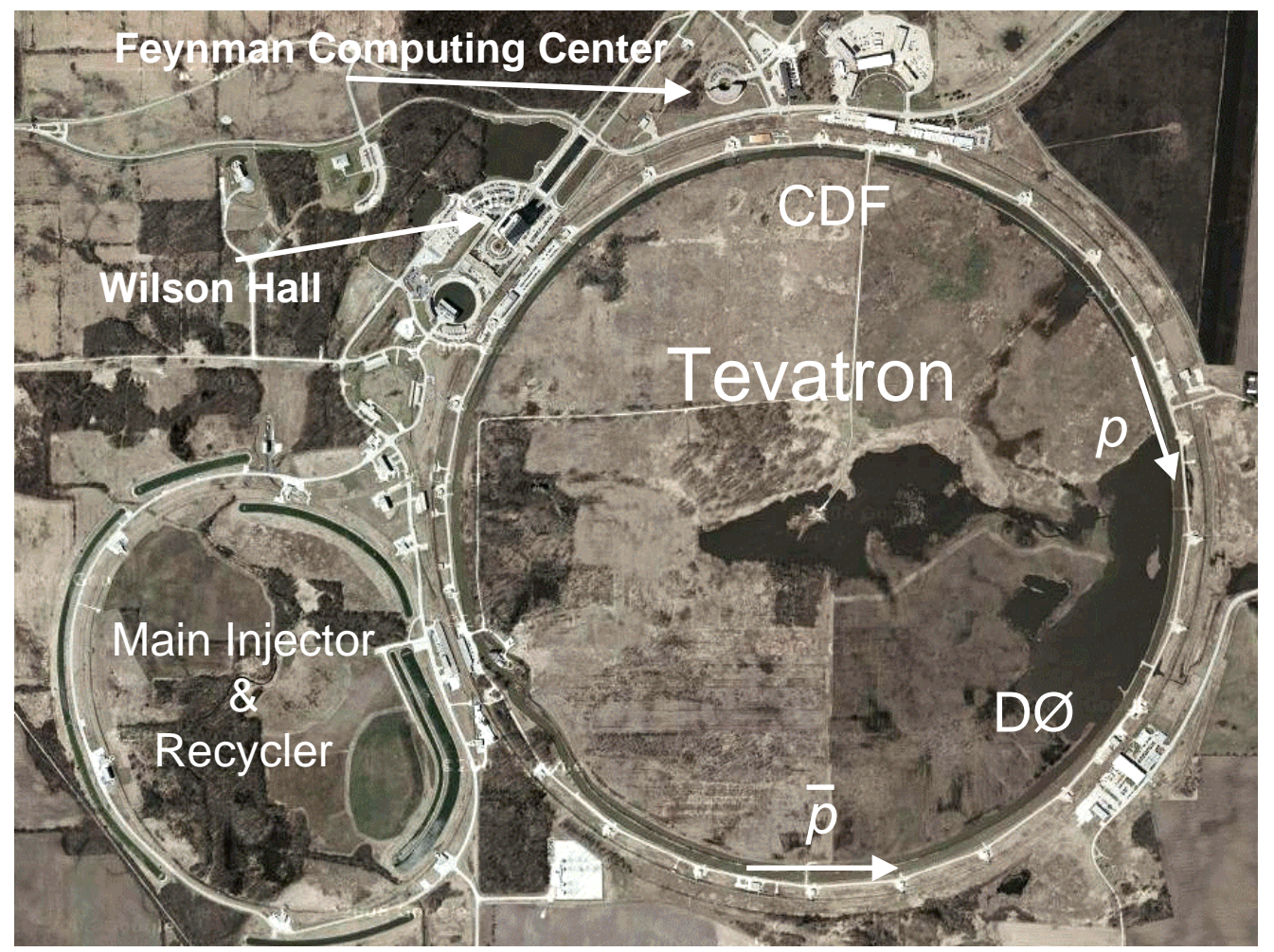

Figure 3.2: Shown is the progression of luminosity delivered by the TEVATRon (red) and recorded by the DØ experiment (blue) during Run II until August 2007 [46].

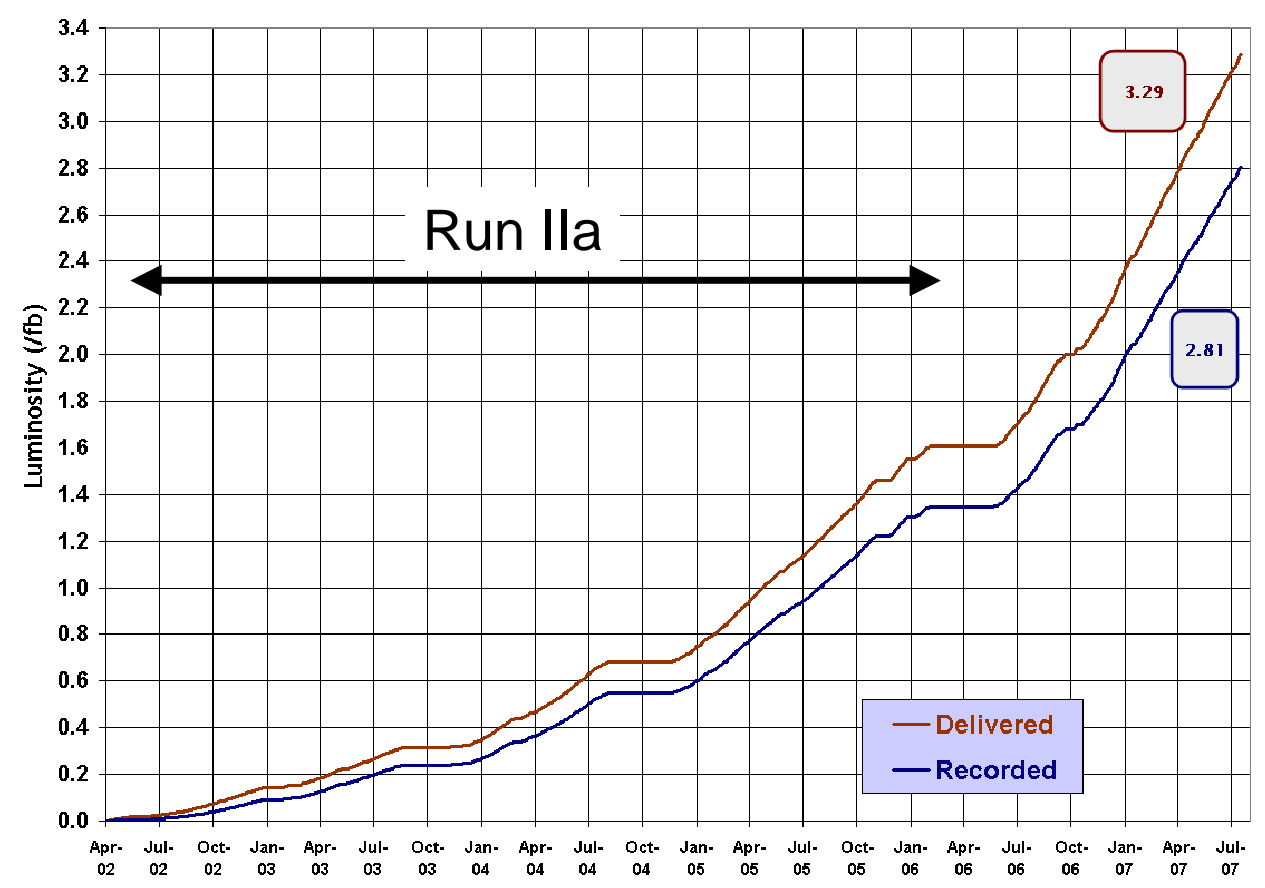




\subsection{The Tevatron Collider Complex}

The Tevatron collider is currently (Run II) operating at a center-of-mass energy of $\sqrt{s}=1.96 \mathrm{TeV}$, thus being the world's most energetic particle collider until the startup of the Large Hadron Collider LHC which will collide protons with protons at a centerof-mass energy of $\sqrt{s}=14 \mathrm{TeV}$. FermilaB's protons and antiprotons are accelerated to the energy of $980 \mathrm{GeV}$ in different steps (see Fig. 3.3) which are briefly outlined in the following. A full description can be found in [47], [48].

Starting point of the chain of accelerators is a bottle containing 6.9 liters $^{2}$ of compressed hydrogen gas $\left(H_{2}\right)$. A Magnetron creates negatively charged hydrogen ions, $H^{-}$. These ions come from a plasma formed near a metal surface, see left-hand sketch of Fig. 3.4: Hydrogen is fed into a volume between two molybdenum electrodes (a matchbox-sized, oval-shaped cathode and a surrounding anode). An external magnet provides a $0.1-0.15 \mathrm{~T}$ magnetic field parallel to the cathode surface. Electrons efficiently ionize the gas so that a dense plasma of $\mathrm{H}^{+}$ions and electrons is formed in the gap. $\mathrm{H}^{+}$ions strike the cathode and occasionally pick up two electrons. These $H^{-}$ions are now repelled from the cathode and charge-exchange with neutral hydrogen atoms at the plasma boundary producing $H^{-}$ ions with a smaller energy spread. A pulsed electrostatic extractor accelerates the negative ions out of the source. The production of negative ions by the cathode is enhanced by introducing cesium vapor into the source which coats the electrodes and lowers the surface work function. There are actually two of these sources called $H^{-}$source and $I^{-}$source, but only one of these is used at a time. The names do not refer to the type of ion accelerated, as both machines are configured to deliver $\mathrm{H}^{-}$ions, which are accelerated up to $750 \mathrm{keV}$ by a Cockcroft-Walton Accelerator. The ion source and the Cockcroft-Walton Accelerator are referred to as Pre-Accelerator.

The Linear Accelerator (Linac) represents the next level of acceleration for the negatively charged hydrogen ions. It takes the ions with an energy of $750 \mathrm{keV}$ and accelerates them to an energy of $400 \mathrm{MeV}$.

The Linac is followed by the Booster. First, the beam of $H^{-}$ions is passed through a thin carbon foil so that the weakly bound electrons are stripped off leaving only protons which are then accelerated to $8 \mathrm{GeV}$. The Booster is the first circular accelerator (synchrotron with a radius of $75 \mathrm{~m}$ ) in the chain of accelerators. The Pre-Accelerator, the Linac and the Booster comprise the Proton Source.

The Main Injector (MI) provides the next step of acceleration. The circumference of this circular synchroton is $3.32 \mathrm{~km}$, hence roughly half the circumference of the TEVATRON. Depending on the usage of the protons, they can be accelerated to either $120 \mathrm{GeV}$ or $150 \mathrm{GeV}$. Protons with $150 \mathrm{GeV}$ are transfered to the TEVATRON for further acceleration. The Main Injector can as well accept antiprotons of $8 \mathrm{GeV}$ from the Accumulator or Recycler (see below) which then circle in opposite direction to the protons; they are accelerated to $150 \mathrm{GeV}$ and injected into the Tevatron.

In order to produce antiprotons, protons with an energy of $120 \mathrm{GeV}$ stemming from the Main Injector strike a nickel target and produce a spray of secondary particles as illustrated

\footnotetext{
${ }^{2}$ This corresponds to $5 \cdot 10^{25}$ hydrogen atoms. Operating 365 days, 24 hours a day, the gas bottle could last 500,000 years. In fact, the bottle is replaced semi-annually, mainly due to inefficiencies in the ion source.
} 
Figure 3.3: Schematic view of the different components of the Tevatron collider complex [49]. The name of the $D \varnothing$ detector is based on the naming scheme of the TEvatron collider [50] (lower plot). The $D \varnothing$ detector is located in section $D$, precisely at the point ' $D 0$ ', whereas the CDF detector for example can be found in section B (at the point 'B0'). The Main Injector is connected to the TeVATron in section $F$ (at the point ' $F 0$ ').

\section{FERMILAB'S ACCELERATOR CHAIN}

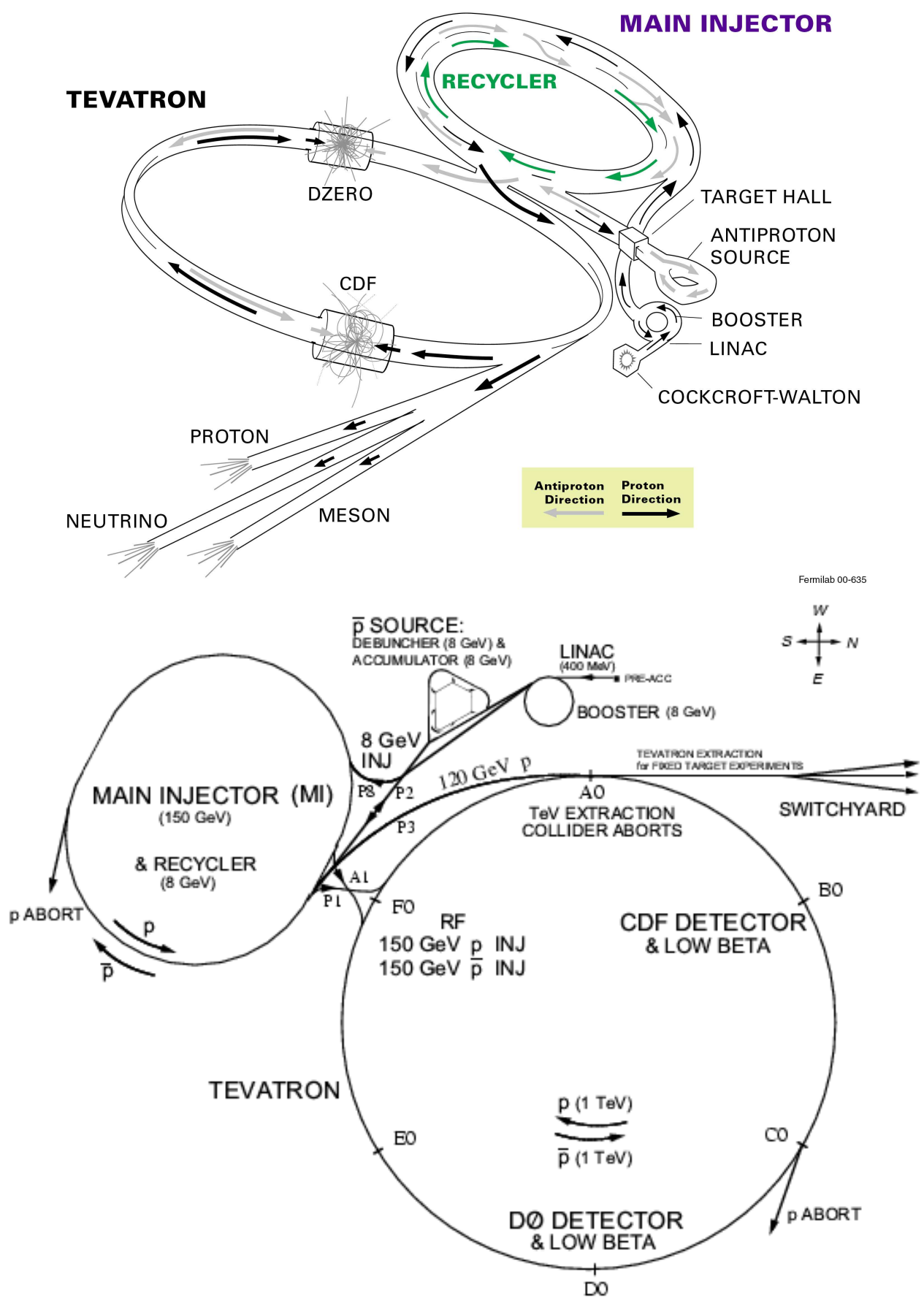


Figure 3.4: Sketches of the Magnetron [47] (ion source, left) and the antiproton source [51] (right).
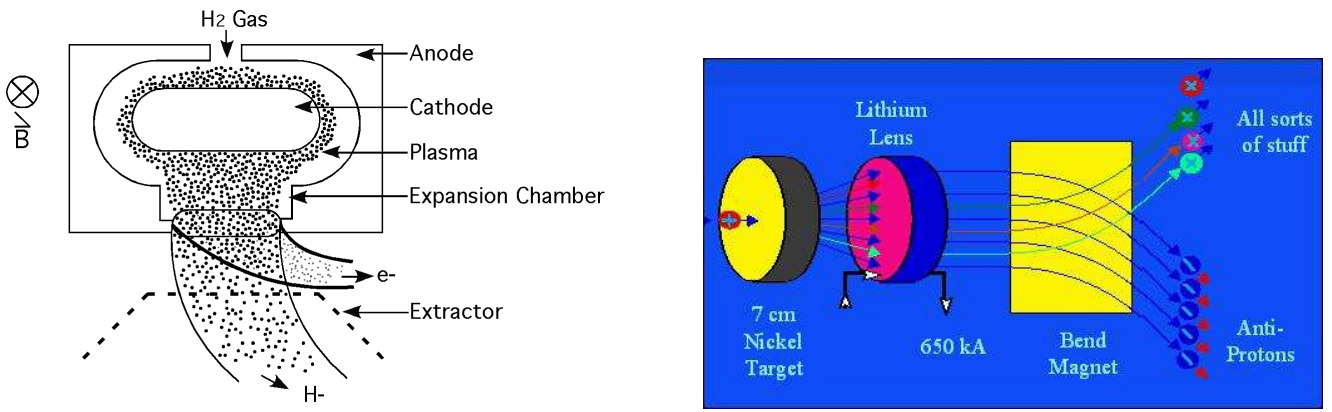

in the right-hand sketch in Fig. 3.4. This particle spray coming off the target is focused with a lithium lens, but it still contains many different particles besides antiprotons. A magnet which acts as charge-momentum spectrometer is utilized to filter antiprotons with an energy of $8 \mathrm{GeV}$. The antiprotons are fed into the Debuncher and Accumulator in order to establish a manageable beam.

The Debuncher, which is a rounded triangular-shaped synchroton with a mean radius of $90 \mathrm{~m}$, does not accelerate the antiprotons, but maintains the energy of $8 \mathrm{GeV}$, and is used to efficiently capture the high momentum spread antiprotons. The $120 \mathrm{GeV}$ protons that arrive at the target station are bunched in time and space. The antiprotons coming off the target also have a narrow time spread, but the energy spread is large, see Fig. 3.5 (middle). Moreover, antiprotons with different energies are located on different orbits. The Debuncher is used to exchange the large energy spread and narrow time spread into a narrow energy spread and large time spread. This process is known as bunch rotation. Antiprotons with low energy see a different phase of the radio frequency than antiprotons with higher energy. The phase difference causes the less energetic particles to be accelerated, and the more energetic particles to be decelerated.

Finally, the prepared antiprotons are stored in the Accumulator $(8 \mathrm{GeV})$ which is also a triangular-shaped synchroton with a mean radius of $75 \mathrm{~m}$. Both, Debuncher and

Figure 3.5: The Debuncher is used to exchange the large energy spread and narrow time spread into a narrow energy spread and large time spread [51] (left and middle). The right-hand plot indicates the operation mode of stochastic cooling [47].
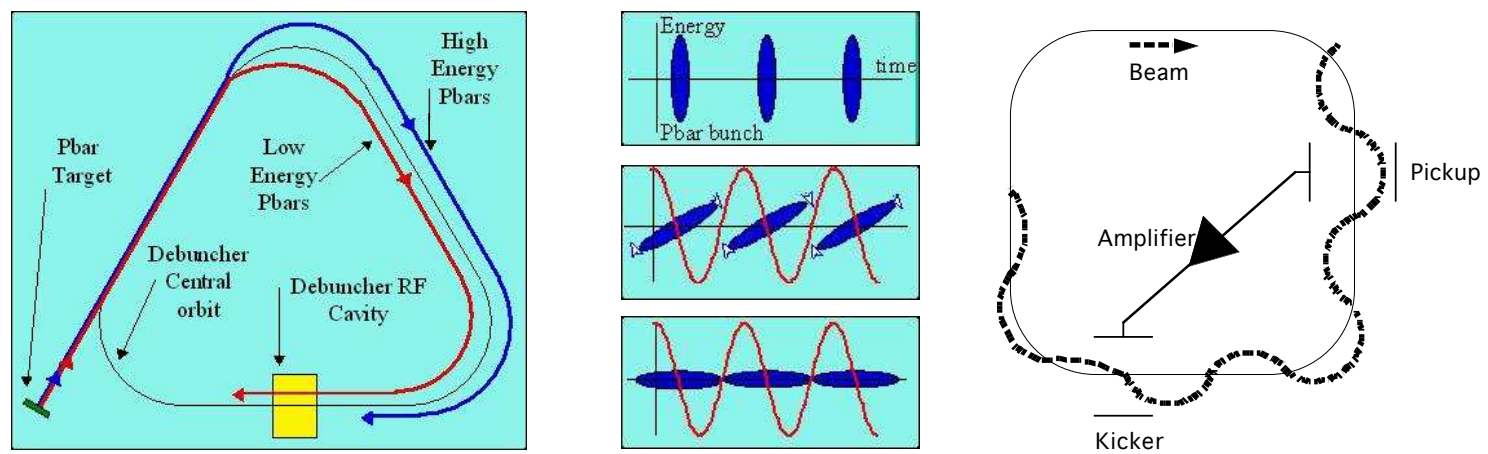
Table 3.1: In order to convert hydrogen into high energy protons and antiprotons several different accelerators are used. The accelerators are connected with each other via transfer lines.

\begin{tabular}{|l|l|l|}
\hline Particles and their origin & Accelerator & Final energy \\
\hline \hline$H_{2} \rightarrow H^{-}$[ion source] & Cockcroft-Walton Accelerator & $750 \mathrm{keV}$ \\
& Linear Accelerator (Linac) & $400 \mathrm{MeV}$ \\
\hline$H^{-} \rightarrow p[$ carbon foil] & Booster & $8 \mathrm{GeV}$ \\
\hline$p(8 \mathrm{GeV})$ & Main Injector (MI) & $150 \mathrm{GeV}$ \\
& Tevatron & $\mathbf{9 8 0 ~ G e V}$ \\
\hline$p(8 \mathrm{GeV})$ & Main Injector (MI) & $120 \mathrm{GeV}$ \\
$p(120 \mathrm{GeV}) \rightarrow \bar{p}(8 \mathrm{GeV})[$ nickel target] & Debuncher & $8 \mathrm{GeV}$ \\
& Accumulator & $8 \mathrm{GeV}$ \\
& Main Injector (MI) & $150 \mathrm{GeV}$ \\
& Tevatron & $\mathbf{9 8 0 ~ G e V}$ \\
\hline $\bar{p}(8 \mathrm{GeV})$ & Recycler & $8 \mathrm{GeV}$ \\
\hline
\end{tabular}

Accumulater, make use of the stochastic cooling ${ }^{3}$ (invented by S. van der Meer [52]). As indicated in the right-hand plot of Fig. 3.5, an 'error' signal from the circulating antiprotons is picked up on one side of the ring. This signal can be the particle's energy or transverse momentum (horizontal and vertical). After processing and amplifying this signal, it is used to steer a kicker magnet at another part of the ring so that the orbit of the circulating antiprotons can be corrected if necessary. The complex of the Target, Debuncher and Accumulator is referred to as Antiproton Source. From one million protons that hit the target, only 20 antiprotons with $8 \mathrm{GeV}$ arrive at the Accumulator. The Antiproton Source is able to produce $2 \cdot 10^{11}$ antiprotons per hour.

The largest of FermilaB's accelerators is the TEvatron collider and storage ring with a circumference of $6.28 \mathrm{~km}$. Here, protons and antiprotons can be accelerated from the injection energy of $150 \mathrm{GeV}$ to the nominal collision energy of $980 \mathrm{GeV}$. The TevATRON is the only cryogenically cooled accelerator at FERMILAB. The magnets used in the TEVATRON are made up of a superconducting niobium/titanium alloy that needs to be kept extremely cold $(\sim 4 \mathrm{~K})$ to remain superconducting. The magnet cavities operate at a radio frequency $(\mathrm{RF})$ of $f_{R F}=53.104 \mathrm{MHz}$.

The Recycler operating at $8 \mathrm{GeV}$ is a storage ring for antiprotons. The ring is located along the ceiling of the Main Injector tunnel. The main task ${ }^{4}$ is to accept antiprotons from the Antiproton Source and cool them further than the Accumulator is capable. The Recycler does not only utilize stochastic cooling, but also electron cooling because stochastic cooling starts losing its effectiveness if more than $2 \cdot 10^{12}$ antiprotons are stored in the ring. More detailed information about the electron cooling can be found in [53] and [54]. Tab. 3.1 summarizes the properties of the various accelerator and storage rings.

The protons and antiprotons circulating in the TEVATRON are arranged in bunches. A bunch is a collection of particles revolving together inside an accelerator in the same RF

\footnotetext{
${ }^{3}$ Cooling is a reduction in the phase space of the beam which is equivalent to a reduction of the random motion of the beam. Since the random motion can be described as a temperature, beam cooling can be described as a reduction of the beam temperature.

${ }^{4}$ The Recycler was originally proposed to 'recycle' antiprotons from a TEVATRON store, cooling and storing them alongside the antiprotons from the Accumulator. Due to early problems in Run II, this project was abandoned.
} 
Figure 3.6: Shown is the beam structure of the Tevatron in Run II [55], [47].

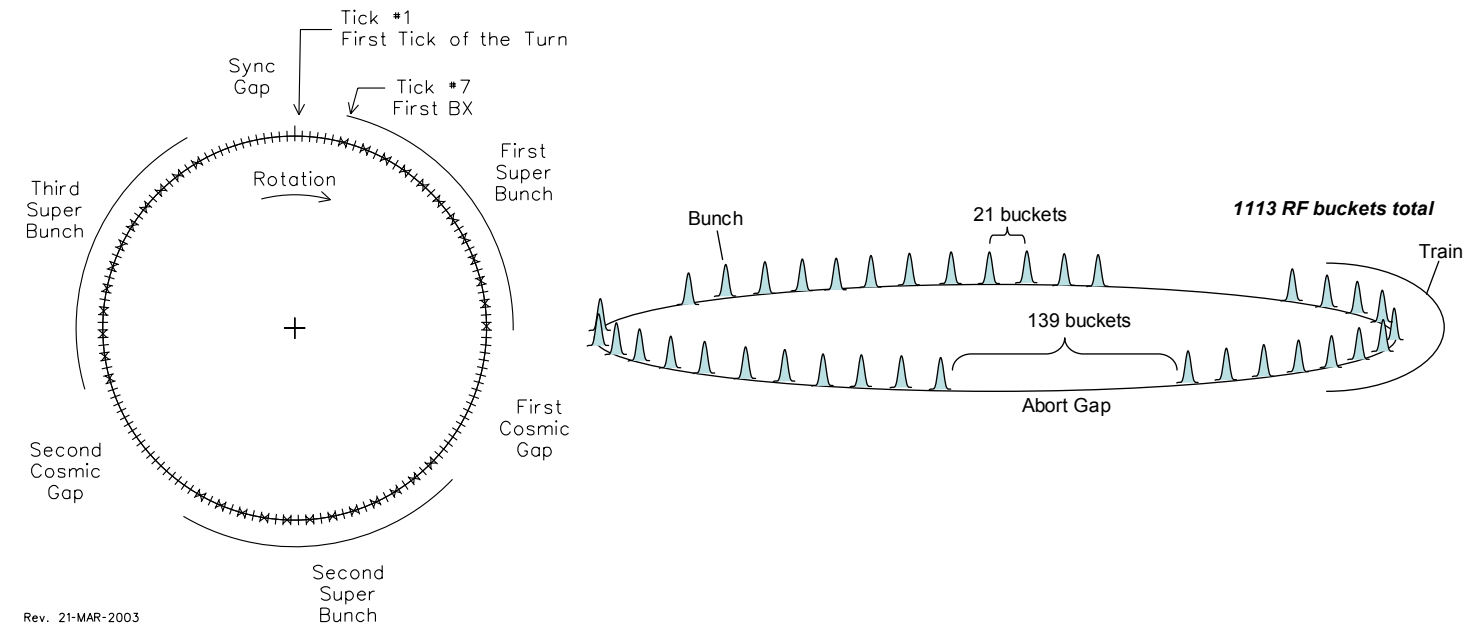

bucket. The minimal time unit of the TEVATRON is determined by the distance of two RF buckets $t_{\min }=1 / f_{R F}$. The maximal number $n$ of buckets in the ring (harmonic number) can be calculated from the time $T$ that a particle needs for one turn if it is moving with the velocity of light $c$, and the circumference of the ring $s=6.28 \mathrm{~km}$

$$
T=\frac{s}{c}=n \cdot t_{\min }=\frac{n}{f_{R F}} \quad \Rightarrow \quad T=21 \mu \mathrm{s}, \quad n=1113, \quad t_{\min }=18.8 \mathrm{~ns} .
$$

For technical reasons, the beam crossing can not be closer than once every seven RF buckets, so the minimal bunch spacing is one tick corresponding to a gap of $7 \cdot t_{\min }=132 \mathrm{~ns}$ (7.59 MHz), see Fig. 3.6. Hence, one turn consists of 159 ticks (= 1113 RF buckets). In Run II there are 36 bunch crossings per turn; the bunches are subdivided into three symmetrically spaced superbunches (trains) due to the threefold symmetry of the collider ring (see Figs. 3.3 and 3.6)

$$
\{F 0 \leftrightarrow B 0 \leftrightarrow D 0\} \quad \Leftrightarrow \quad\{\text { Injection } \leftrightarrow \mathrm{CDF} \text { detector } \leftrightarrow \mathrm{D} \varnothing \text { detector }\}
$$

For each train consisting of 12 bunches a number of 159 : $3=53$ ticks are available, but not all are filled. The bunches are only filled into every third tick according to an operational bunch spacing of $396 \mathrm{~ns}=3$ ticks $=21 \mathrm{RF}$ buckets. The remaining empty ticks $(53-(12-1) \cdot 3=20)$ correspond to a gap of $2.6 \mu$ s which is required by the beam abort system. The bunch spacing of $396 \mathrm{~ns}$ implies a collision rate of $2.5 \mathrm{MHz}$. Due to the fact that $17(=53-3 \cdot 12)$ ticks are not filled, an effective rate of $1.7 \mathrm{MHz}(=36 / 53$. $2.5 \mathrm{MHz}$ ) is usually quoted. The first bunch is filled into the seventh tick of a turn (see Fig. 3.6). One of the gaps between superbunches, the Synchronization Gap, is used by DØ for internal resets of the front-end and readout crates; the other two gaps are referred to as Cosmic Gaps. The antiprotons exhibit the same structure, just that they are rotating in the opposite direction of the protons. The bunch length is about $37 \mathrm{~cm}$ for both, protons and antiprotons [48]. The transverse emittance ${ }^{5}$ for the proton beam is $20 \pi \mathrm{mm} \cdot \mathrm{mrad}$ and $15 \pi \mathrm{mm} \cdot \mathrm{mrad}$ for the antiproton beam, respectively [48]. Both beams intersect with a crossing angle of zero.

\footnotetext{
${ }^{5}$ The emittance is defined as the area in phase space occupied by a particle beam. The units are $\mathrm{mm}$ times milliradians for the transverse emittance.
} 
If protons and antiprotons from the Accumulator or Recycler have passed through the various acceleration processes, reached the final energy of $980 \mathrm{GeV}$ in the TeVATRON and exhibit stable conditions, collisions are initiated in the two interaction points, B0 (CDF) and D0 (DØ). The beam preparation procedure is referred to as shot setup. In the two interaction regions, the low beta regions, the colliding beams feature a small longitudinal width. The term 'low beta' stems from the (accelerator) beta function which describes in detail the behaviour of the beam at each point of the collider. The nominal value of the beta function at the interaction point, denoted as $\beta^{*}$, is $35 \mathrm{~cm} \mathrm{[48].}$

Once the collisions are initiated and the beams stabilized, a store is declared and the experiments start taking data. A store lasts for 30 hours at most - depending on the initial luminosity. The progression of a typical Run IIa store (store \#4571, December 28 30, 2005) is shown in Fig. 3.7. At the beginning, $85 \cdot 10^{11}$ protons and $20 \cdot 10^{11}$ antiprotons are stored in the collider. The luminosity and the number of protons/antiprotons drop continuously due to losses caused by interactions of the beams with each other, the beam gas or the beam pipe. Once the luminosity is too small, the store is ended and the shot setup for the next store begins. Of course, a store can be prematurely ended due to failures of accelerator components or instable beam conditions leading to a beam abort. The beam abort can not only be issued by the accelerator, but also by the experiments which continuously monitor the halo of the beams. This is necessary to protect the subdetectors close to the beam pipe from damage.

The luminosities measured by CDF and D $\varnothing$ (see Fig. 3.7, red: CDF, yellow: DØ) differ because the actual values of the beta function differ at both interaction points. Further, the experiments do not see the same proton-antiproton collisions: When the first proton bunch arrives at $\mathrm{D} \varnothing$, it collides with the thirteenth antiproton bunch. On the other side, when the first proton bunch arrives at CDF, it collides with the twenty-fifth antiproton bunch.

Figure 3.7: Luminosity and number of protons/antiprotons during store \#4571. This plot is created reading the ACNET (Accelerator Controls NETwork) via a web interface [56].

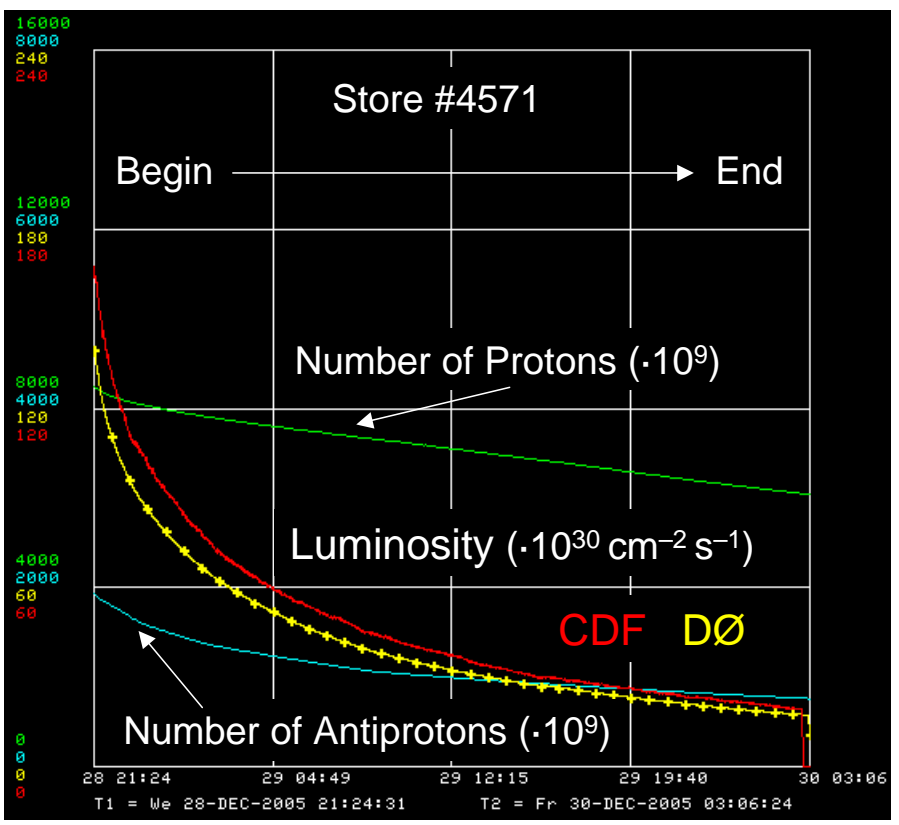




\subsection{Luminosity and Multiple Interactions}

The luminosity relates the rate $\dot{N}$ for a particular process to occur at a collider and the cross section $\sigma$ for this process,

$$
\dot{N} \propto \sigma \Rightarrow \dot{N}=\frac{\mathrm{d} N}{\mathrm{~d} t}=\sigma \cdot \mathcal{L}(t) \quad \Rightarrow \quad N=\sigma \cdot \int \mathcal{L}(t) \mathrm{d} t
$$

The overall number of produced events is proportional to the integrated luminosity, $\int \mathcal{L}(t) \mathrm{d} t$, which is measured in units of inverse (pico/femto) barns, $[\mathcal{L}]=[\sigma]^{-1}=$ barn $^{-1}=$ $\mathrm{b}^{-1}$. Thus, the unit for the instantaneous luminosity $\mathcal{L}(t)$ is $(\mathrm{b} \cdot \mathrm{s})^{-1}$. Often ' $\mathrm{cm}^{2}$ ' is used instead of 'barn' with the conversion

$$
1 \cdot 10^{30} \frac{1}{\mathrm{~cm}^{2} \cdot \mathrm{s}}=1 \frac{1}{\mu \mathrm{b} \cdot \mathrm{s}} .
$$

The peak values of the instantaneous luminosity for all Run IIa stores are displayed in Fig. 3.8. For instance, the $\mathrm{D} \varnothing$ experiment measured an initial (peak) luminosity of $140 \cdot 10^{30}$ $\mathrm{cm}^{-2} \mathrm{~s}^{-1}=140 \mu \mathrm{b}^{-1} \mathrm{~s}^{-1}$ for the particular store \#4571. The left-hand plot of Fig. 3.9 shows cross sections $\sigma$ (left-hand axis) and event rates $\dot{N}$ (right-hand axis) for selected processes at a given luminosity of $10^{33} \mathrm{~cm}^{-2} \mathrm{~s}^{-1}=1 \mathrm{nb}^{-1} \mathrm{~s}^{-1}$. Hence, 60 million events from the inelastic scattering $\left(\sigma_{\text {inel }} \approx 60 \mathrm{mb}\right)$ and 0.007 top-antitop events $\left(\sigma_{t \bar{t}} \approx 7 \mathrm{pb}\right)$ are produced per second at this luminosity in proton-antiproton collisions at a center-of-mass energy of $\sqrt{s}=1.96 \mathrm{TeV}$.

The instantaneous luminosity $\mathcal{L}(t)$ can be derived from properties of the colliding beams using

$$
\mathcal{L}(t)=\frac{f_{R F} N_{p} N_{\bar{p}} B}{2 \pi\left(\sigma_{p}^{2}+\sigma_{\bar{p}}^{2}\right)} \cdot F\left(\frac{\sigma_{l}}{\beta^{*}}\right)
$$

with the TEVATRON radio frequency $f_{R F}=53.104 \mathrm{MHz}$, the number of bunches in each beam $B=36, N_{p} / N_{\bar{p}}$ the number of protons/antiprotons in a bunch and the transverse width of the proton/antiproton bunch $\sigma_{p} / \sigma_{\bar{p}}$. The form factor $F$ depends on the beta function at the interaction point, $\beta^{*}$, and the longitudinal bunch length $\sigma_{l}$ (design: $\sigma_{l}=$ $37 \mathrm{~cm} \mathrm{[48]).}$

Since a detailed knowledge of the full beam characteristics in the interaction point is not feasible, reference processes with known cross sections are utilized in order to measure the luminosity. At D $\varnothing$ the luminosity is determined by counting the rate of inelastic protonantiproton collisions which are recorded by the luminosity monitors (see Sec. 3.3.4). The luminosity is then defined as

$$
\mathcal{L}(t) \equiv \frac{1}{\sigma_{\text {inel }}} \frac{\mathrm{d} N}{\mathrm{~d} t} \quad \text { with } \quad \sigma_{\text {inel }}=60.7 \pm 2.34 \mathrm{mb}[57] .
$$

Since more than $10^{10}$ particles are contained in each bunch, more than one hard protonantiproton collision can happen. The number of interactions per crossing $n$ of the proton and antiproton beam is given by the Poisson distribution

$$
P(n)=\frac{\mu^{n}}{n !} \cdot e^{-\mu}
$$


Figure 3.8: Peak luminosities during Run II until August 2007 [46].

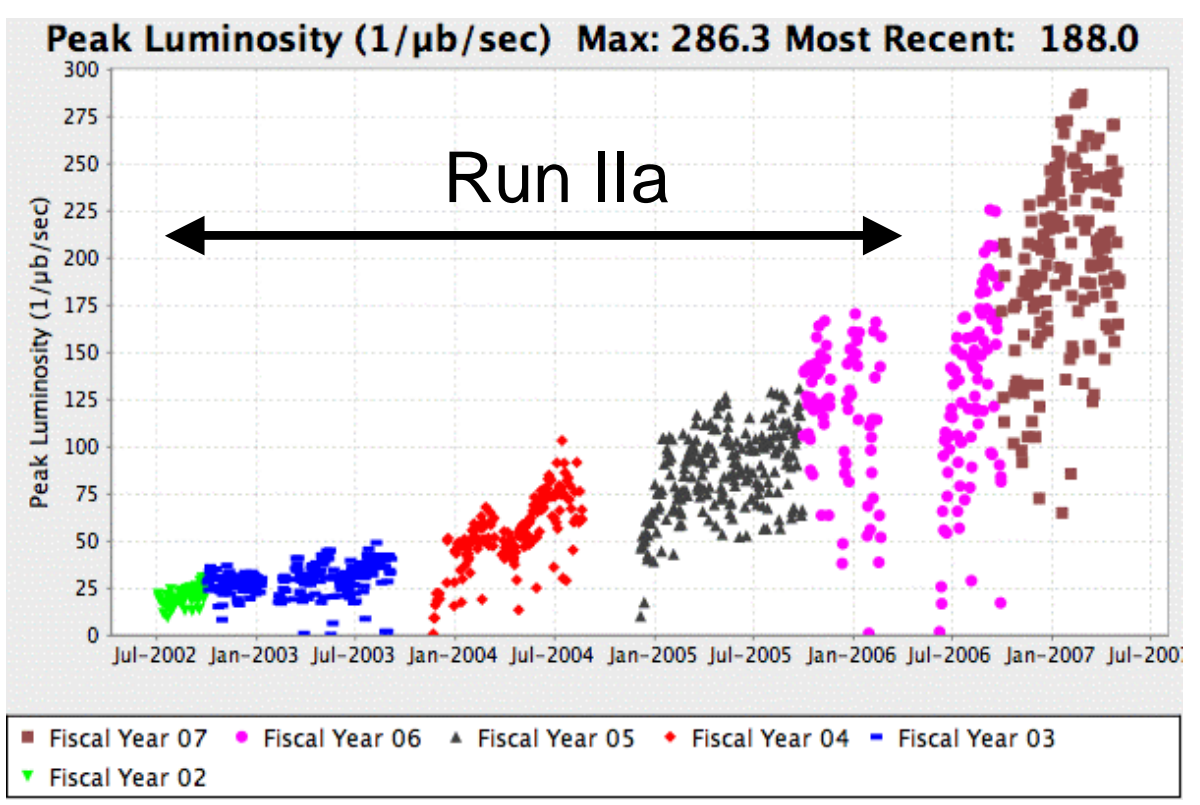

Figure 3.9: Left: Cross sections and event rates for selected processes as a function of the centerof-mass energy $\sqrt{s}$ [58]. Right: Number of interactions per crossing for different instantaneous luminosities [59].

proton - (anti)proton cross sections

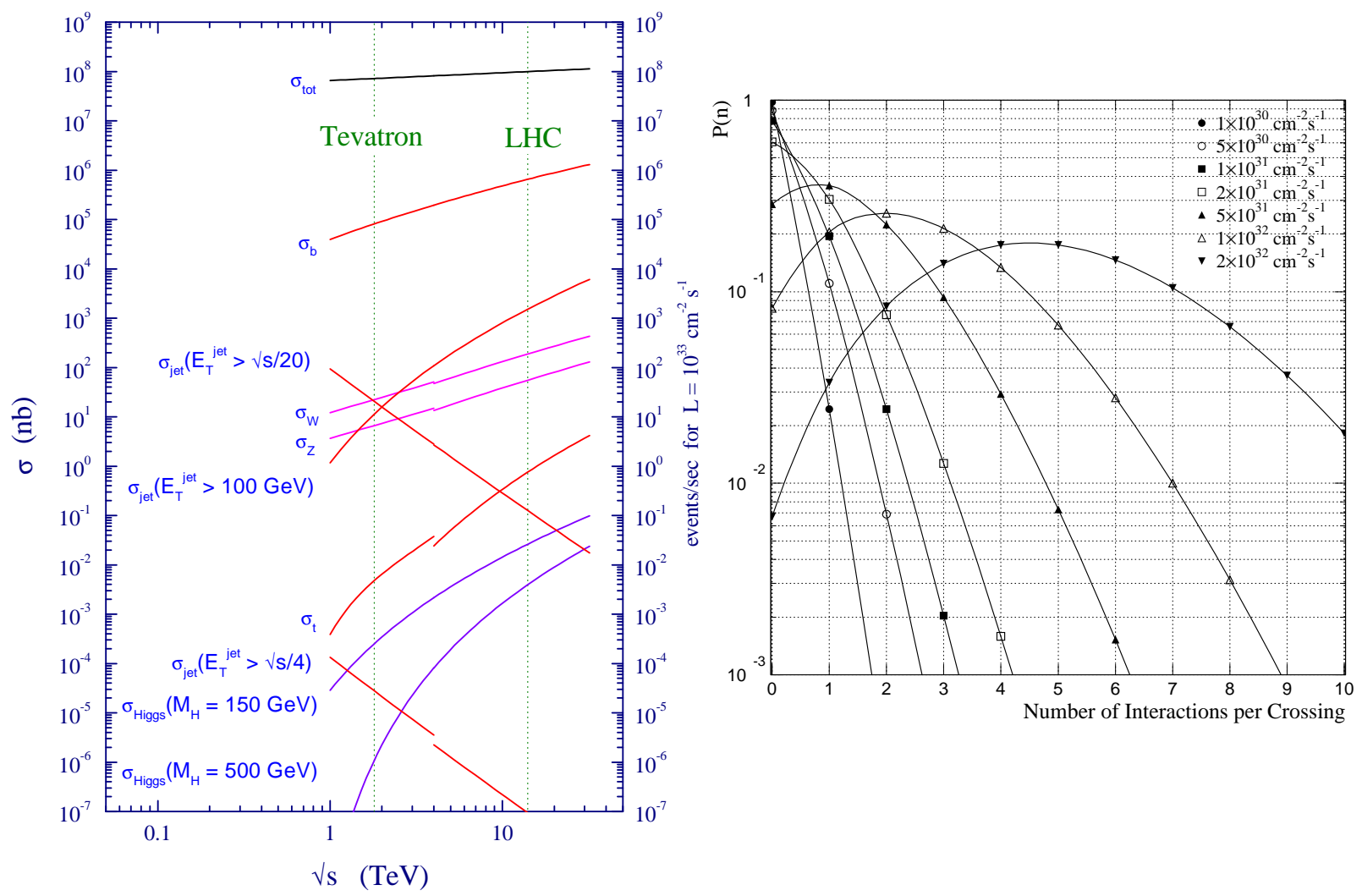


with $\mu$ being the average number of interactions per crossing. For the determination of the instantaneous luminosity the number of empty crossings, thus $P(n=0)=e^{-\mu}$, is measured so that

$$
\mathcal{L}(t)=\frac{1}{\sigma_{\text {inel }}} \cdot \mu f_{R F}=-\frac{f_{R F}}{\sigma_{\text {inel }}} \cdot \ln (P(n=0)) .
$$

The probability distributions $P(n)$ are displayed in Fig. 3.9 (right-hand plot) for different luminosities $\left(\mu=\bar{n} \approx 0.8\right.$ for $50 \cdot 10^{30} \mathrm{~cm}^{-2} \mathrm{~s}^{-1}, \mu=\bar{n} \approx 2$ for $\left.100 \cdot 10^{30} \mathrm{~cm}^{-2} \mathrm{~s}^{-1}\right)$.

\subsection{The DØ Detector}

The $\mathrm{D} \varnothing$ detector exhibits all properties of a multi-purpose detector in high energy physics. It is hermetic with a solid angle coverage of almost $4 \pi$. The layers of the individual subdetectors are arranged around the geometric center of the detector (nominal interaction point) like the shells of an onion, see Fig. 3.10. Each layer is designed to identify and measure (or confirm/remeasure) the energy of particles that are not measured by the previous layer because no single detector can determine identity and measure energy or momentum of all particles.

The Run IIa configuration of the DØ detector (length: $20 \mathrm{~m}$, height: $13 \mathrm{~m}$ ) is shown in Fig. 3.11 (top: schematic, bottom: picture of the detector located in the assembly hall). The innermost part is displayed in Fig. 3.12 (left) at a larger scale. The coordinate system has already been discussed in Sec. $2.1 ; \eta$ refers to $\eta_{\text {det }}$ throughout this section (cf. Fig. 2.1).

Figure 3.10: The left-hand sketch displays the onion-like structure of a common multi-purpose detector in high energy physics [60]. The DØ detector (right-hand picture) represents such a detector [49]. One can see various components: the beam pipe in the center, enclosed by the calorimeter within a cryostat, followed by the innermost layer of the muon system, toroid magnet (red) and outer layers of the muon detector. The picture was taken in 1999.
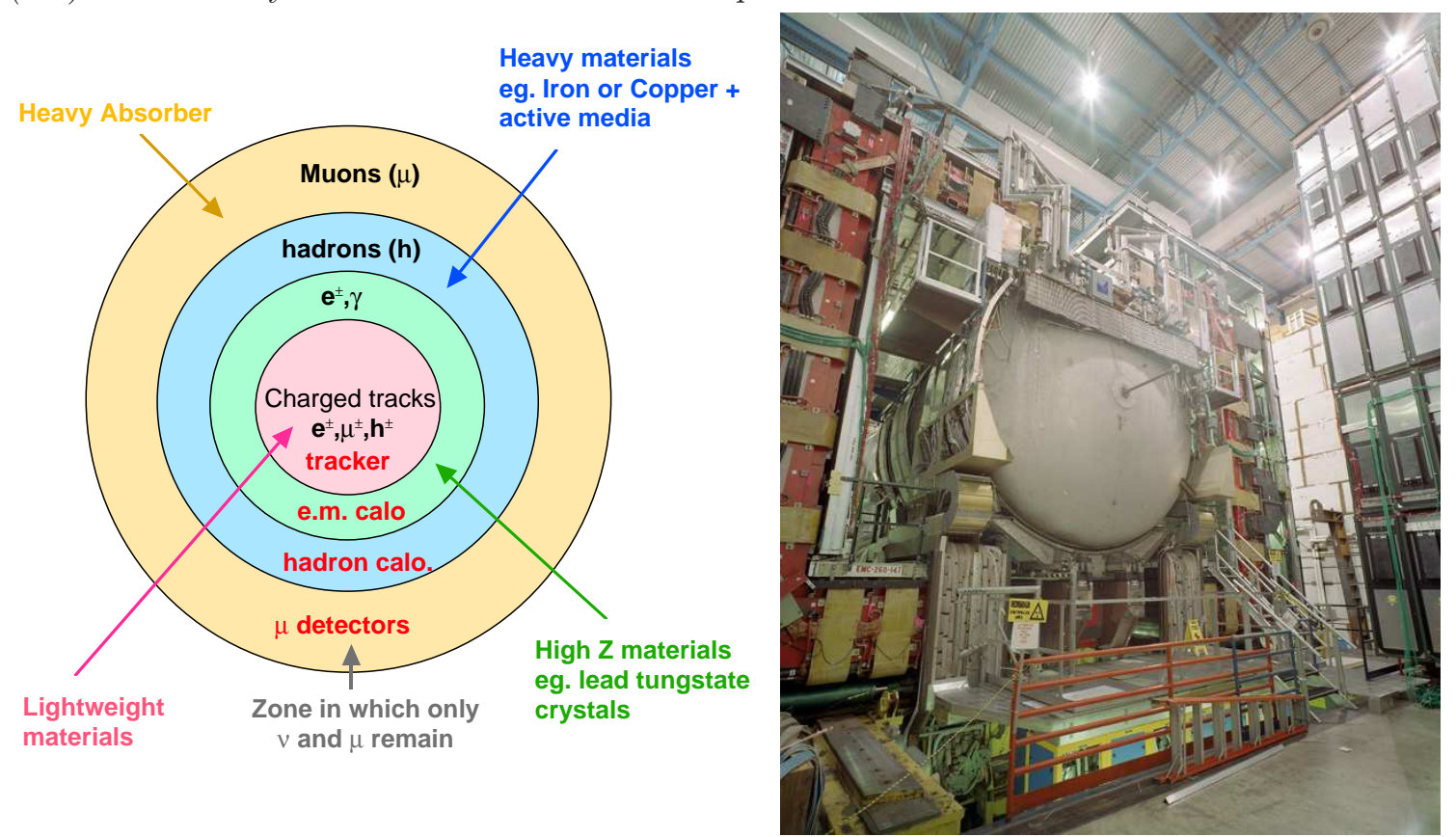
Figure 3.11: Schematic view of the $D \varnothing$ detector from inside the Tevatron ring [61]. The picture at the bottom shows the $D \varnothing$ detector after the assembly in the collision hall [62] (view from outside the ring).
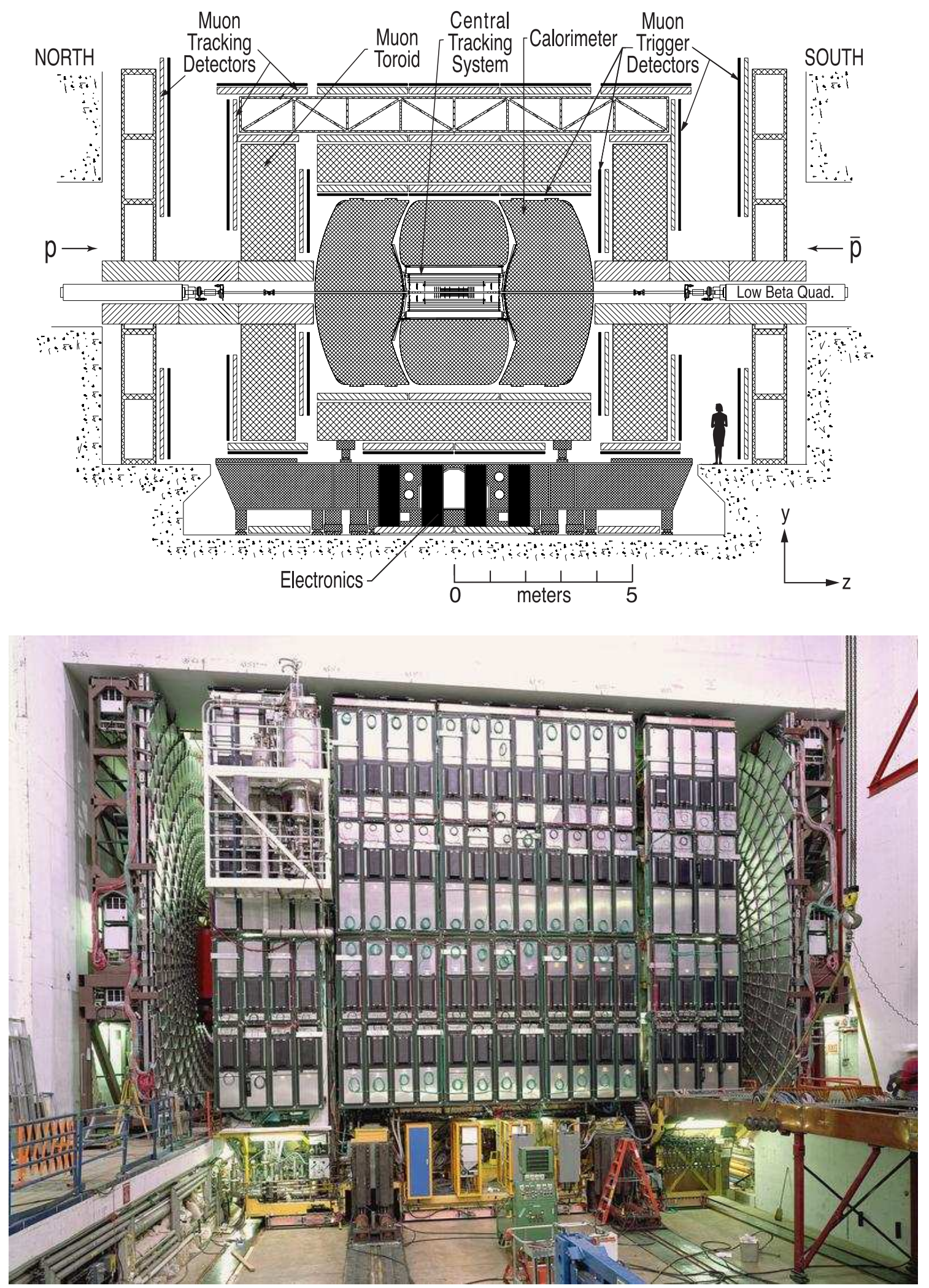
Figure 3.12: Left: Cross-sectional view of the tracking system [61]. Right: Insertion of the central fiber tracker into the magnet bore; the solenoid itself is located within a hole in the central calorimeter cryostat [63].

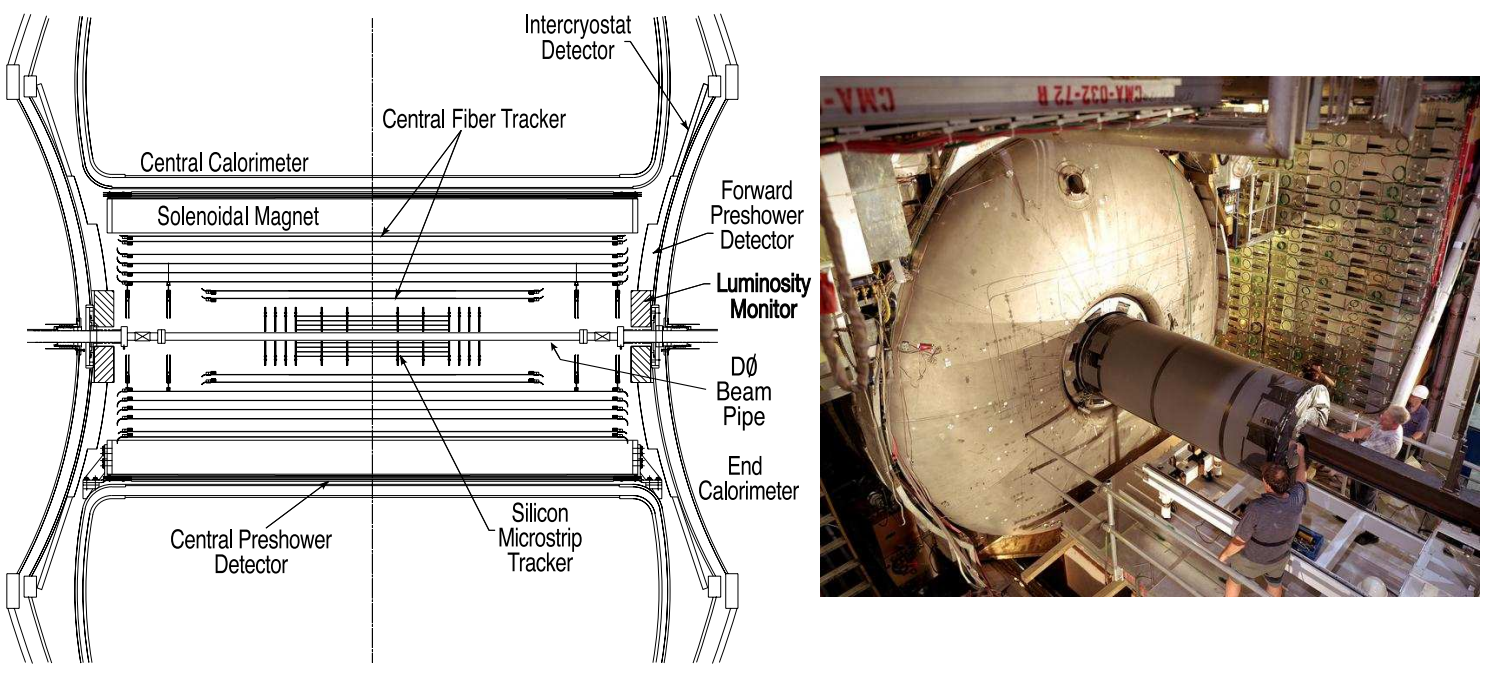

At D $\varnothing$ momenta of charged particles can be measured with two independent tracking devices, the Silicon Microstrip Tracker, SMT, and the Central Fiber Tracker, CFT. Both detectors are situated within a $2 \mathrm{~T}$ axial field ${ }^{6}$ so that charged particles are bent in the transverse $(x-y)$ plane. The solenoidal magnet extends over a length of $2.73 \mathrm{~m}$ and has an inner [outer] diameter of $1.07 \mathrm{~m}$ [1.42 m]. The innermost detectors surround the beryllium beam pipe, which has an outer diameter of $38.1 \mathrm{~mm}$, a wall thickness of $0.508 \mathrm{~mm}$ and a length of $2.37 \mathrm{~m}$.

The calorimeter - a sampling calorimeter with liquid argon acting as active medium and uranium/copper absorbers - is housed in three cryostats in order to maintain a temperature of $90 \mathrm{~K}$. The Central Calorimeter, CC, covers $|\eta|<1$ and the two calorimeter vessels in the forward direction (Endcap Calorimeter, EC) extend the coverage to $|\eta| \approx 4$.

The Central PreShower detector (CPS) with a coverage $|\eta|<1.3$ is located within the small gap of $5 \mathrm{~cm}$ between the solenoid and the central calorimeter vessel. The Forward PreShower detectors (FPS) cover $1.5<|\eta|<2.5$ and are located in front of the two forward calorimeter vessels. Both preshower detectors are made from triangular strips of scintillator. The light signal from the scintillator is transmitted via fibers to Visible Light Photon Counters, VLPCs, which transform the light into electrical signals. The preshower detectors are instrumented with 22,564 channels of VLPC readout. Another subdetector, the InterCryostatic Detector, ICD, allows an energy measurement between the two forward and the central calorimeter vessels. The ICD provided by scintillator sampling covers the region $1.1<|\eta|<1.4$ and consists of tiles $(\Delta \eta \times \Delta \phi \approx 0.3 \times 0.4)$ which are divided into subtiles $(\Delta \eta \times \Delta \phi \approx 0.1 \times 0.1)$. The 378 signal channels are read out via fibers which terminate at photomultiplier tubes located in a low-magnetic-field region.

\footnotetext{
${ }^{6}$ The current of $I=4750$ A corresponding to a magnetic field strength of $2 \mathrm{~T}$ could not be sustained after the detector shutdown in autumn 2004. A warm-up of the (superconducting) magnet was inevitable to fix a vapor cooled current lead leak. The solenoid kept quenching during the cooling process so that the current had to be reduced to $I=4550$ A yielding a slightly lower magnetic field strength of $1.9 \mathrm{~T}$.
} 
The muon system is the outermost detector device. The central system covers $|\eta|<1$, whereas the forward spectrometer covers $1<|\eta|<2$. The muon detector system consists of three layers (denoted as $A, B$ and $C$ ) of drift tubes and layers of scintillation counters, two in the central and three in the forward region. A $1.8 \mathrm{~T}$ toroidal magnet field between layers $A$ and $B$ of the drift tubes - one layer of scintillation counters is as well placed in front of the toroid - allows a separate stand-alone momentum measurement of muons. In addition, the wire chambers (as well as the scintillation counters) are used for triggering.

The detector is not described in detail, but rather the properties of the main components (tracking, calorimeter, muon system, luminosity monitors) are outlined in the following. For a full review consult [64], [61] and references therein.

\subsubsection{Tracking System}

The tracking system is crucial for momentum measurements and vertexing. The two detectors, SMT and CFT, are able to locate the primary interaction vertex with a resolution of $\sigma_{P V z}=35 \mu \mathrm{m}$ along the beamline. The impact parameter resolution for tagging $b$ quark jets is better than $15 \mu \mathrm{m}$ in $r-\phi$ direction (for particles with transverse momentum $p_{T}>10 \mathrm{GeV}$ at $|\eta|=0$ ). The momentum resolution $\Delta p_{T}$ of the entire tracking system within the solenoidal magnet field is

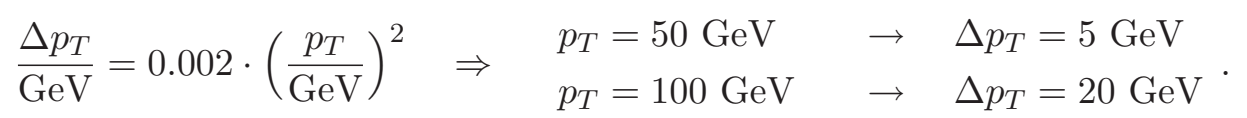

Tracking and vertexing over nearly the full $\eta$ coverage $(|\eta|<4)$ is provided by the SMT detector which consists of barrel and disk modules: barrels which are interspersed by disks in the central region, and disks (only) in the forward region. The barrel section ranges from $-38.2 \mathrm{~cm}$ to $38.2 \mathrm{~cm}$, thus roughly corresponding to the length of the interaction region with $\sigma_{z}=26 \mathrm{~cm}$ (see Fig. 3.25). The $r-\phi$ coordinate is primarily measured by the barrel detectors, while the disks measure $r-z$ as well as $r-\phi$. Vertices in the very forward direction are reconstructed in three dimensions by the disks, whereas in the central region the barrels as well as the CFT are utilized.

Fig. 3.13 (left) shows an isometric view of the whole SMT detector surrounding the beam pipe. The SMT is composed of six barrels with a length of $12 \mathrm{~cm}$, each with four

Figure 3.13: Left: The silicon microstrip tracker is made up of disks and barrels [61]. On the right-hand side a cross sectional $(r-\phi)$ view of the barrel detector is shown [65].
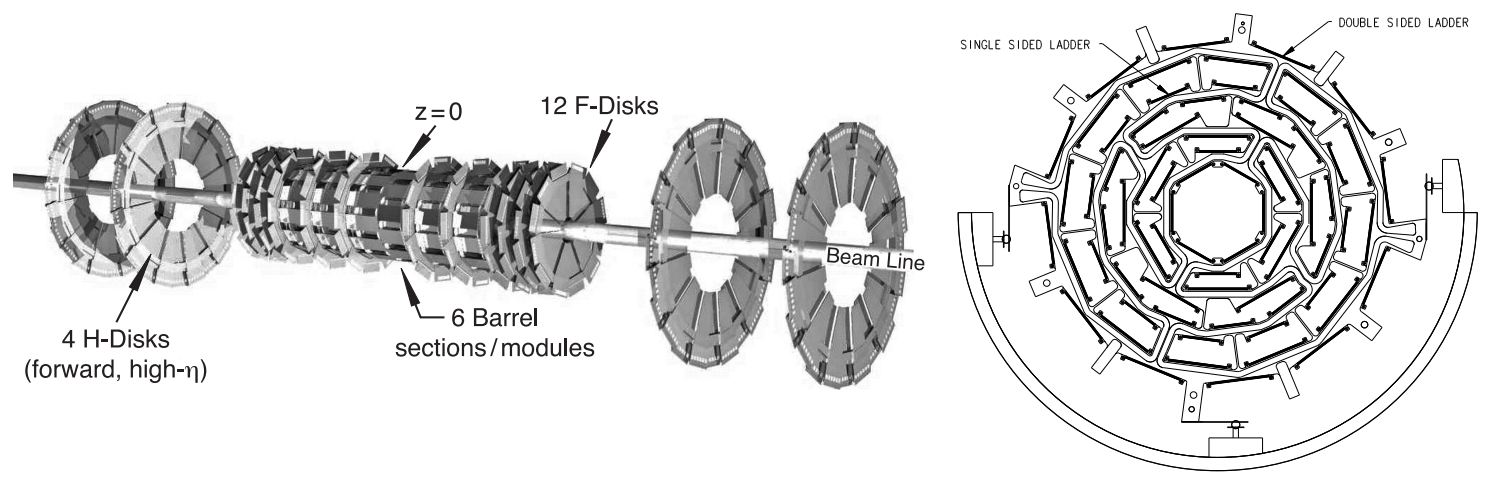
Table 3.2: Characteristics of the SMT ( $i / o$ : inner/outer radius, $c$ : center of the barrel).

\begin{tabular}{|l|c|l|l|l|}
\hline Module & Layer & Type & Radius $[\mathrm{cm}]$ & $|z|[\mathrm{cm}]$ \\
\hline \hline F-disks (12) & - & double-sided & $2.57^{i}$ & $12.5,25.3,38.2,43.1,48.1,53.1$ \\
& & & $9.96^{\circ}$ & \\
\hline H-disk (4) & - & single-sided & $9.5^{i}$ & $100.4,121.0$ \\
& & & $26^{\circ}$ & \\
\hline Central barrels (4) & 1 & double-sided & 2.715 & $6.2^{c}, 19.0^{c}$ \\
& 2 & double-sided & 4.55 & \\
& 3 & double-sided & 7.582 & \\
& 4 & double-sided & 10.51 & \\
\hline Outer barrels (2) & 1 & single-sided & 2.715 & $31.8^{c}$ \\
& 2 & double-sided & 4.55 & \\
& 3 & single-sided & 7.582 & \\
& 4 & double-sided & 10.51 & \\
& & & & \\
\end{tabular}

silicon readout layers. Individual modules which are called ladders build up a layer. The first two layers consist of twelve, the last two layers of twenty-four ladders, totaling 432 ladders. The right-hand plot of Fig. 3.13 shows a cross sectional view of the barrel detector. The disks are made up of wedges, twelve wedges form an F-disk leading to a total amount of $144 \mathrm{~F}$-type wedges. The H-disks on the other hand consist of twenty-four "full" wedges (made of two back-to-back "half" wedges) yielding a total of $96 \mathrm{H}$-type wedges. The readout strips on the wedges are arranged parallel to the long edge of the device so that a stereo angle of $30^{\circ}$ for the F-disks and $15^{\circ}$ for the H-disks is provided. Different types of modules are used, single-sided and double-sided. The SMT detector has an overall number of 792,675 readout channels. Tab. 3.2 summarizes properties of the detector elements. Fig. 3.14 shows pictures from the assembly of the SMT.

The detector has to be cooled in order to reduce radiation damage to the silicon. Heat generated by e.g. the readout chips (SVXIIe [66]) which are mounted on the ladders may lead to type inversion and increas the depletion voltage. The coolant supplied at $-10^{\circ} \mathrm{C}$ is used to keep the temperature of the modules below $10^{\circ} \mathrm{C}$.

Figure 3.14: Left: F-disk made of twelve double-sided wedges [62]. Middle: Assembly of the SMT [62]. The barrel detectors are mounted inside two carbon fiber half-cylinders (black). Right: Two ladders mounted on a beryllium bulkhead [67]. One can also see the cooling channel, cf. Fig. 3.13 .
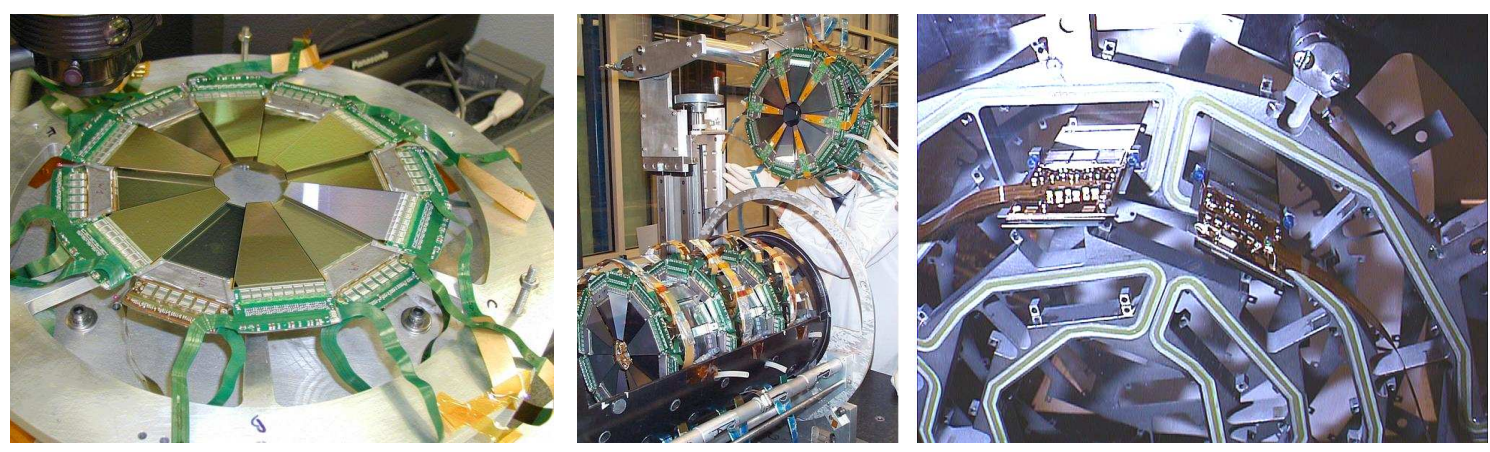
The second tracking device, the CFT, covers the central detector region with $|\eta|<1.6$ and consists of scintillating fibers mounted on eight concentric cylinders. The radii of the cylinders range from $20 \mathrm{~cm}$ to $52 \mathrm{~cm}$; the length is $2.52 \mathrm{~m}$, except for the two inner cylinders with a length of $1.66 \mathrm{~m}$ so that the H-disks can be housed. Each of the eight cylinders accommodates a doublet layer of fibers oriented along the $z$-axis denoted as axial layer, and another doublet at a stereo angle of $\pm 3^{\circ}$ called stereo layer. The fiber diameter of $835 \mu \mathrm{m}$ results in a double layer resolution of $100 \mu \mathrm{m}$. The stereo angle alternates from cylinder to cylinder. Tab. 3.3 summarizes the properties of the CFT detector. The picture on the right-hand side shows a front view of the CFT during the assembly. The ends of the eight cylinders are clearly visible.

The scintillating light coming out of the 76,800 fibers is coupled to fiber waveguides and carried to the VLPCs [68] that are also used for the preshower readout. The waveguides which range in length from $7.8 \mathrm{~m}$ to $11.9 \mathrm{~m}$ are only mounted on one end of the fibers; the opposite end is mirrored. The VLPCs are impurity-band silicon avalanche photodetectors with the following specifications:

- $9 \mathrm{~K}$ operating temperature;

- excellent quantum efficiency $\geq 75 \%$;

- fast response;

- high gain of $22 \mathrm{k}$ to $65 \mathrm{k}$;

- low gain dispersion;

- ability to work in high background environment.

The cooling is accomplished by mounting the VLPC cassettes which also accommodate the readout electronics on Analog Front End boards (AFE) in cryostat slots. A bundle of 128 fibers terminates in one of the eight modules on the top (warm) end of a cassette. The VLPCs are mounted in the lower (cold) end. Ninety-nine cassettes providing readout for $99 \times 8 \times 128=101,376$ channels are contained in the cryobox on the D $\varnothing$ platform beneath the central calorimeter so that the fiber detectors (CFT, CPS and FPS with

Table 3.3: Characteristics of the CFT. Each layer consists of one doublet of axial and one doublet of stereo layers. Right: Front view of the central fiber tracker during the assembly [63]. One can see the eight cylinders, cf. Fig. 3.12 .

\begin{tabular}{|l|l|l|l|l|}
\hline Cylinder & Stereo Angle & $\begin{array}{l}\text { Radius } \\
{[\mathrm{cm}]}\end{array}$ & $\begin{array}{l}\text { Fibers per } \\
\text { Cylinder }\end{array}$ & $\begin{array}{l}\text { Length } \\
{[\mathrm{m}]}\end{array}$ \\
\hline \hline 1 & $+3^{\circ}$ & 20 & $2 \times 2 \times 1280$ & 1.66 \\
2 & $-3^{\circ}$ & 25 & $2 \times 2 \times 1600$ & 1.66 \\
\hline 3 & $+3^{\circ}$ & 30 & $2 \times 2 \times 1920$ & 2.52 \\
4 & $-3^{\circ}$ & 35 & $2 \times 2 \times 2240$ & 2.52 \\
5 & $+3^{\circ}$ & 40 & $2 \times 2 \times 2560$ & 2.52 \\
6 & $-3^{\circ}$ & 45 & $2 \times 2 \times 2880$ & 2.52 \\
7 & $+3^{\circ}$ & 50 & $2 \times 2 \times 3200$ & 2.52 \\
8 & $-3^{\circ}$ & 52 & $2 \times 2 \times 3520$ & 2.52 \\
\hline
\end{tabular}

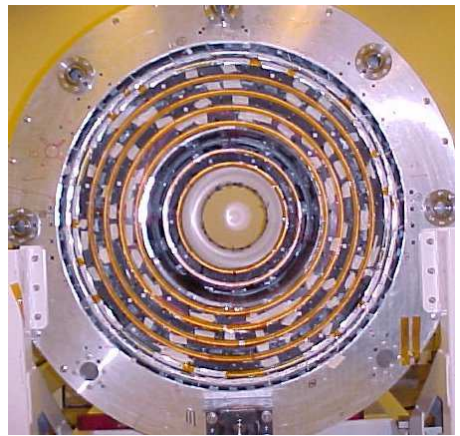


Figure 3.15: Left: Waveguides coming out of the CFT, ICD and FPS [62]. Middle: DØ platform beneath the central calorimeter showing fiber waveguides ending in the readout bords (AFE and VLPC). At the bottom the cryo cassette is visible [62]. Right: LED calibration spectrum for a single VLPC for an axial CFT fiber [61].
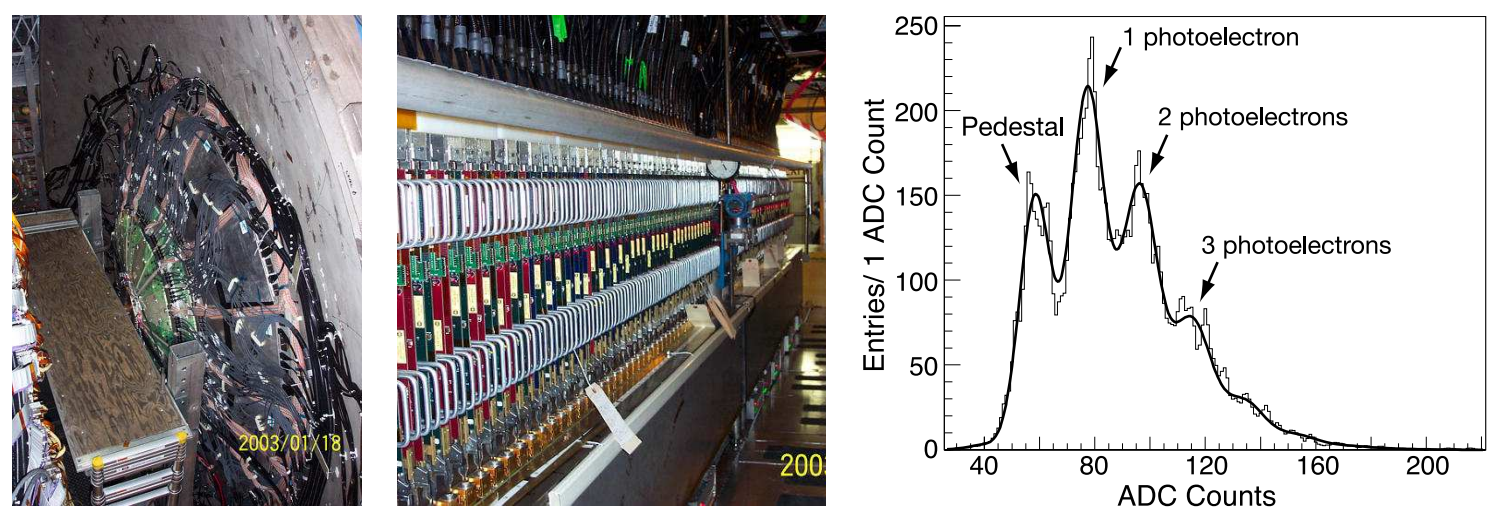

a total of 99,364 channels) can be instrumented with VLPC readout. Fig. 3.15 shows the fiber waveguides coming out of the fiber detectors (left) and arriving at the VLPC cassettes (middle) on the platform. As for the SMT, the SVXIIe chip is used for the signal processing on the AFE board.

The small-diameter scintillating fibers on one hand and the long waveguides on the other hand imply very small signals of $\approx 10$ photoelectrons out of one VLPC. Channel thresholds must be set between 1.5 and 2 photoelectrons (pe) so that an acceptable efficiency is assured. The analog signal is required to be digitized with a noise less than 0.4 pe (according to a charge of roughly $2 \mathrm{fC}$ ) so that not only the stability of the low threshold can be maintained, but also individual photoelectron peaks can be distinguished during calibration, see right-hand plot in Fig. 3.15. In fact, the front-end electronics is capable to fulfill or exceed these requirements. The LED calibration ${ }^{7}$ spectra of all axial fibers result in a mean pedestal width of 0.24 pe $(1.6 \mathrm{fC})$. Discriminator thresholds can be set below $10 \mathrm{fC}$ because these have similar noise $(1.2 \mathrm{fC})$ and offsets less than $2 \mathrm{fC}$.

\subsubsection{Calorimeter}

The calorimeter (Fig. 3.16, left) is divided into three sections: the electromagnetic section $(\mathrm{EM})$ closest to the interaction region, followed by the fine $(\mathrm{FH})$ and coarse $(\mathrm{CH})$ hadronic sections. The active medium, liquid argon, requires cooling of the calorimeter to maintain the temperature of $90 \mathrm{~K}$. Three separate cryostats, one in the center (CC) and two in the forward regions (EC, referred to as 'north' and 'south', ECN and ECS) contain the active material and absorber plates that facilitate the showering of particles. In the electromagnetic section nearly depleted uranium plates with thicknesses of $3 \mathrm{~mm}(\mathrm{CC})$ and $4 \mathrm{~mm}$ (EC) are used. The fine hadronic section is equipped with $6 \mathrm{~mm}$ uranium-niobium

\footnotetext{
${ }^{7}$ The CFT, CPS and FPS detectors are equipped with blue-emitting LED pulsers. They are used for testing the connectivity, monitoring the stability of the VLPC readout over time and channel-to-channel energy calibration of the fiber-VLPC system. Calibration runs are taken between stores or during detector commissioning.
} 
Figure 3.16: Left: Isometric view of the calorimeter [69]. The picture on the right-hand side shows the opened detector [62]. One can see the central calorimeter cryostat, followed by the innermost layer of scintillation counters and the toroid magnet (red). The white boxes upon the (disassembled) endcap calorimeter accommodate preamplifiers for the calorimeter.
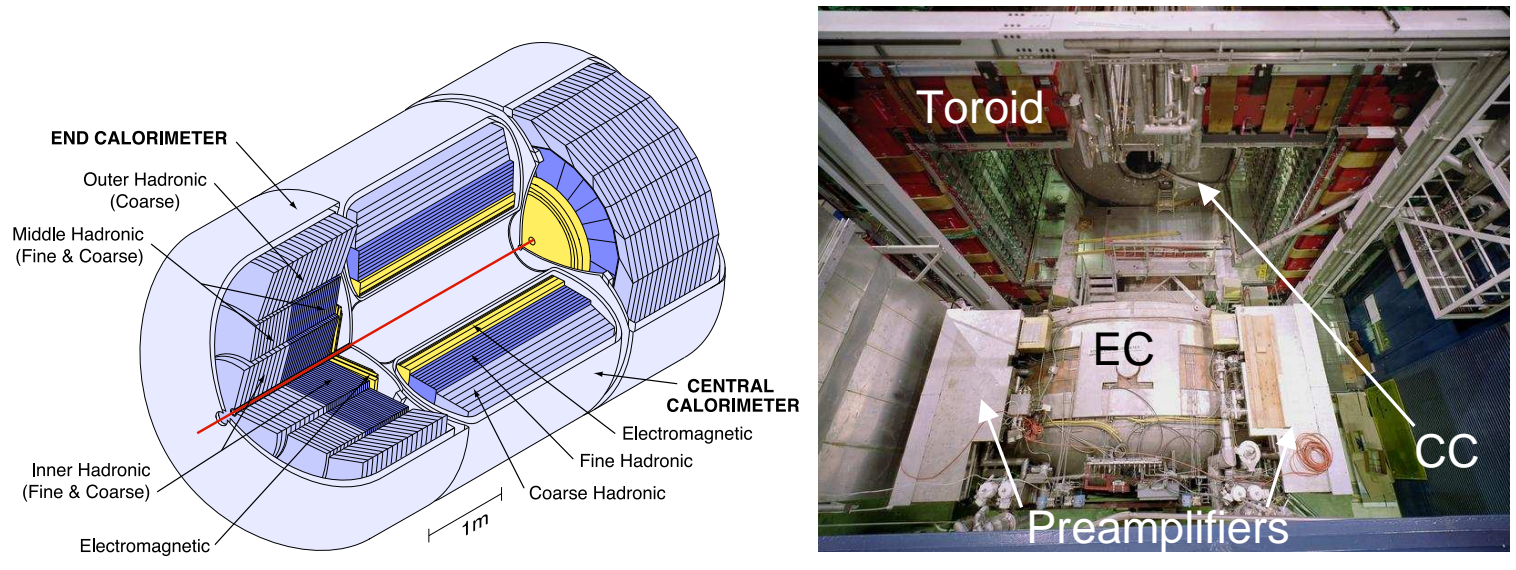

alloy, whereas $46.4 \mathrm{~mm}$ thick copper [stainless steel] plates are used in the central [endcap] coarse hadronic calorimeter.

A schematic view of a calorimeter unit cell is shown in Fig. 3.17 (left). A cell consists of alternating layers of absorber and signal boards. All signal boards, except for the EM and small-angle hadronic modules in the EC, are composed of $0.5 \mathrm{~mm}$ plastic (G-10) sheets, coated with carbon-loaded epoxy on the outside, and with copper on the inside. A voltage of $+2 \mathrm{kV}$ is impressed upon the resistive surface of the signal boards (the absorber plates are grounded) leading to an electron drift time of $450 \mathrm{~ns}$ across the liquid argon gap of $2.3 \mathrm{~mm}$. The copper pads are split to allow a segmented readout. For the signal boards located in the EM and small-angle hadronic EC multilayer printed circuit boards are used. The outer surfaces are coated with carbon-loaded epoxy as well, but here etched pads on the interior surface yield the segmentation.

The calorimeter pads at approximately the same $\eta$ and $\phi$ are combined to a readout cell, and the cells build up pseudo-projective towers which are subdivided in depth, see Fig. 3.18. The term "pseudo-projective" stems from the fact that the centers of the towers point to the nominal interaction point (= geometric center of the detector, cf. Fig. 2.1). The cell boundaries are arranged perpendicular to the absorber plates. The

Figure 3.17: Left: Schematic view of a calorimeter unit cell. Right: Readout chain of the calorimeter [61].
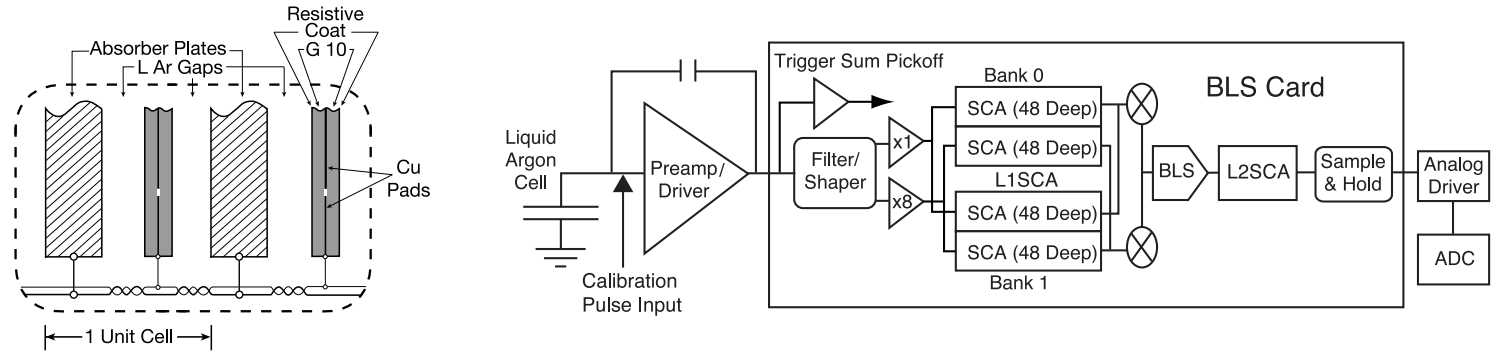
Figure 3.18: Schematic view of the calorimeter demonstrating the segmentation into pseudoprojective towers [61]. The shading pattern indicates that groups of cells are combined for signal readout.

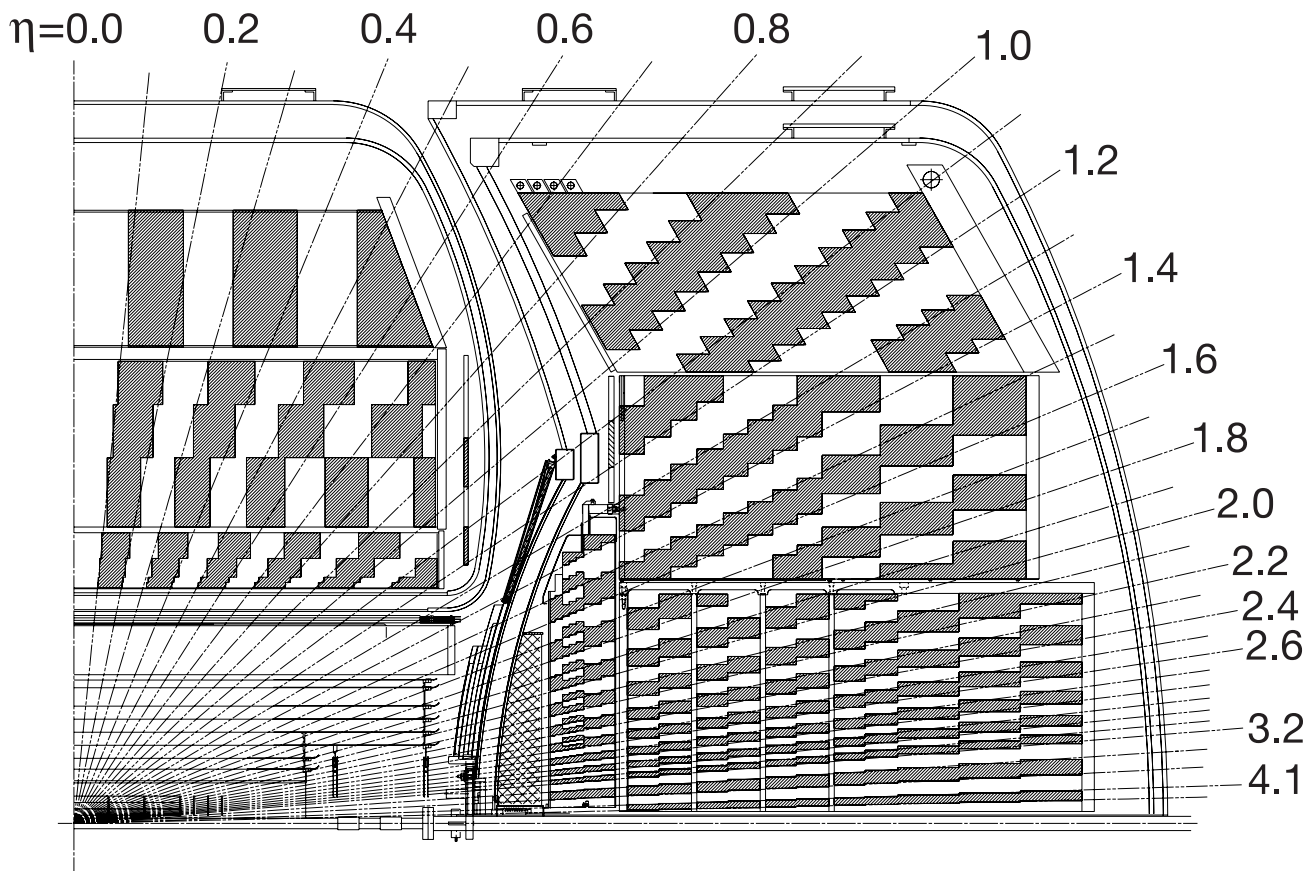

readout towers are segmented into $\Delta \eta=0.1$ and $\Delta \phi=2 \pi / 64 \approx 0.1$. The third EM layer, where the electromagnetic showers are expected to develop their maximum, exhibits a finer segmentation of $\Delta \eta \times \Delta \phi=0.05 \times 0.05$. This allows a precise location of an electromagnetic interacting particle. The segmentation in depth is different for the electromagnetic and hadronic sections in the CC and EC, see Tab. 3.4. At $\eta=0$ the EM part of the calorimeter consists of four layers adding up to a thickness of $20 X_{0}$ (measured in units of the radiation length $X_{0}$, this corresponds to $\approx 20 \mathrm{~cm}$ ), the hadronic part consists of three $\mathrm{FH}$ layers with a thickness of $3.06 \lambda_{a}$ (measured in units of the absorption length $\lambda_{a}$, corresponding to $\approx 60 \mathrm{~cm})$ and one $\mathrm{CH}$ layer with $3.2 \lambda_{a}(\approx 50 \mathrm{~cm})$. All detector components between the interaction region and the first active liquid argon gap sum up to a total amount of $4 X_{0}$ of material.

The readout of 47,032 modules $^{8}$ starts with the preamplification of the signal, see righthand sketch of Fig. 3.17. The preamplifiers are located on top of the calorimeter vessels, see pictures in Fig. 3.16 (right) and 3.19 (left). Then the signal is routed to the BaseLine Subtractor (BLS) cards, located below the cryostats. A fast energy measurement (for Level 1 and Level 2 triggers $^{9}$ ) is provided by making sums via resistor packs on the BLS board. In the meantime the signal is stored and held by Switched Capacitor Arrays (SCA) until the trigger decision is made. The baseline subtraction which removes any low frequency noise or pile-up ${ }^{10}$ from the signal is also accomplished by the SCAs. Only

\footnotetext{
${ }^{8} 55,296$ channels exist, but only 47,032 channels are connected

${ }^{9}$ The calorimeter trigger towers have a size of $\Delta \eta \times \Delta \phi=0.2 \times 0.2$.

${ }^{10}$ If energy deposits from more than one event are contained in the detector due to previous interactions in a beam crossing or due to interactions in multiple beam crossings, this is referred to as pile-up.
} 
Table 3.4: Characteristics of the calorimeter. The values given for the first EM layer include the material from the outer warm walls to the first active liquid argon gap.

\begin{tabular}{|c|c|c||c|c|c|c|c|}
\hline \multicolumn{2}{|c||}{ Electromagnetic Calorimeter $\left[X_{0}\right]$} & \multicolumn{5}{|c|}{ Hadronic Calorimeter $\left[\lambda_{a}\right]$} \\
\hline Layer & CC & EC & Layer & CC & \multicolumn{3}{|c|}{ EC } \\
& & & & & inner & middle & outer \\
\hline \hline EM1 & 1.4 & 1.6 & FH1 & 1.3 & 1.1 & 0.9 & - \\
EM2 & 2.0 & 2.6 & FH2 & 1.0 & 1.1 & 0.9 & - \\
EM3 & 6.8 & 7.9 & FH3 & 0.76 & 1.1 & 0.9 & - \\
EM4 & 9.8 & 9.3 & FH4 & - & 1.1 & 0.9 & - \\
& & & CH & 3.2 & 4.1 & 4.4 & 6.0 \\
\hline EM & $\mathbf{2 0 . 0}$ & $\mathbf{2 1 . 4}$ & HAD & $\mathbf{6 . 2 6}$ & $\mathbf{8 . 5}$ & $\mathbf{8 . 0}$ & $\mathbf{6 . 0}$ \\
\hline
\end{tabular}

two-thirds of the charge (hence $260 \mathrm{~ns}$ of signal collection) are used in order to minimize the effect of pile-up. Since the SCA is not capable to simultaneously read and write, two banks are required. In order to extend the ADC (Analog to Digital Converter) readout range, two gain paths, $\times 1$ and $\times 8$, are utilized. After a positive Level 1 trigger decision, the baseline is subtracted and the signal stored (L2SCA) until the Level 2 information is available. Finally, after a positive Level 2 decision, the signal is sent to the ADC.

The (theoretical) amount of 55,296 readout channels translates into 1,152 BLS boards. Signals from forty-eight channels from four pseudo-projective towers are processed on one BLS board, whereas each tower corresponds to up to twelve preamplifier signals. Hence, one BLS board carries four daughtercards (one per tower) with five SCAs, totaling 23,040 SCA chips. For calibration purposes, a pulser signal can be fed into the readout chain instead of a signal from a liquid argon cell.

The DØ sampling calorimeter allows identification and energy measurement for electrons, photons (see Sec. 4.1) and jets (see Sec. 4.2). It further assists in identifying muons (as minimum-ionizing particles) and provides a measurement of the transverse energy bal-

Figure 3.19: Pictures of the calorimeter preamplifiers on top of the endcap calorimeter vessel [62] (left) and BLS boards located on the DØ platform beneath the calorimeter [70] (right).
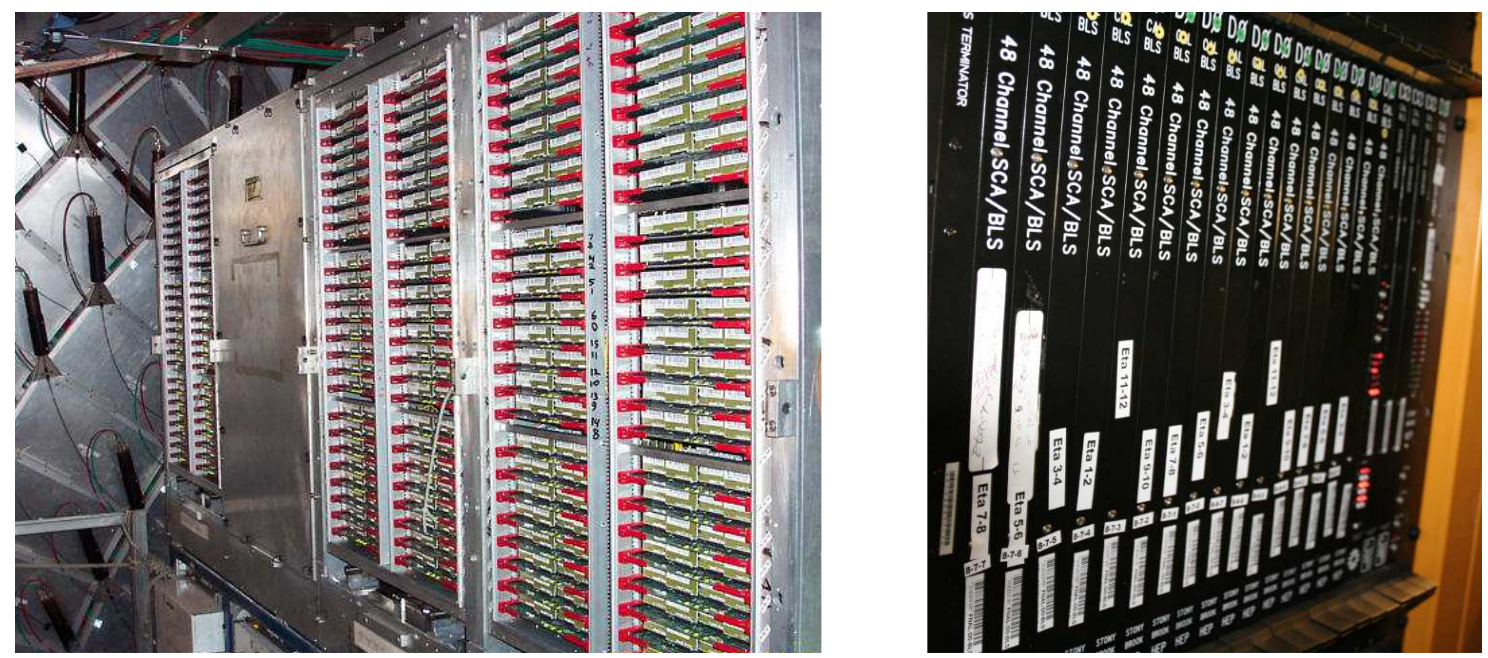
ance and hence the missing transverse energy ( Neutrino, see Sec. 4.3). The energy resolution of the calorimeter can be parameterized in the following way

$$
\frac{\Delta E}{E}=\frac{S}{\sqrt{E / \mathrm{GeV}}} \oplus \frac{N}{E / \mathrm{GeV}} \oplus C \Rightarrow\left(\frac{\Delta E}{E}\right)^{2}=\left(\frac{S}{\sqrt{E / \mathrm{GeV}}}\right)^{2}+\left(\frac{N}{E / \mathrm{GeV}}\right)^{2}+C^{2} .
$$

This description incorporates three contributions:

- Sampling term: $\Delta E / E \propto 1 / \sqrt{E}$

The first term describes fluctuations in the energy deposition because the basic phenomena in showers are statistical processes. The accuracy of a calorimeter is intrinsically limited, but improves with energy.

- Noise term: $\Delta E / E \propto 1 / E$

This contribution which does not depend on the energy $(\Delta E \propto N)$ is due to instrumental effects, like uranium noise or pedestal subtraction, and is dominant at low energies.

- Constant term: $\Delta E / E \propto C$

The last term (systematic term) takes uncertainties from the calibration, nonuniformities and non-linearities of the calorimeter devices into account. The resolution at high energies is limited by this contribution.

Table 3.5: Energy resolution parameters (see Eq. 3.10) for the central calorimeter measured from data (electromagnetic [71], hadronic [72]).

\begin{tabular}{|l|c|c|c||c|c|}
\hline & $S$ & $N$ & $C$ & \multicolumn{2}{|c|}{$\Delta E[\mathrm{GeV}]$} \\
& & & & $E=50 \mathrm{GeV}$ & $E=100 \mathrm{GeV}$ \\
\hline \hline Electrons, photons & 0.15 & 0.29 & 0.041 & $2.3(4.6 \%)$ & $4.3(4.3 \%)$ \\
\hline Jets & 1.05 & 2.13 & 0.036 & $7.9(16 \%)$ & $11(11 \%)$ \\
\hline
\end{tabular}

\subsubsection{Muon System}

The central muon system which covers $|\eta|<1$ consists of three layers of Proportional Drift Tubes (PDT), see left-hand drawing in Fig. 3.20. The first layer, the $A$ layer which is closest to the interaction region, is placed in front of the toroidal magnet, and two further layers, referred to as $B$ and $C$ layers, are located outside the magnet. Roughly $90 \%$ of the central detector region is covered by at least two layers; $55 \%$ is covered by three layers. Losses of the hermeticity stem from support structures at the bottom of the detector.

The drift chambers, which have dimensions of $2.8 \times 5.6 \mathrm{~m}^{2}$, are made of aluminum tubes. The $B$ and $C$ layers are made of three decks of drift cells; the $A$ layer PDTs have four decks (except for the bottom $A$ layer with three decks). Twenty-four cells, each with a width of $10.1 \mathrm{~cm}$, build up one deck so that one chamber typically contains 72 (96) cells. One cell consists of vernier cathode pads at the top and bottom $(+2.3 \mathrm{kV})$ and an anode wire $(+4.7 \mathrm{kV})$. Wires from adjacent cells are ganged together and read out at one end 
Figure 3.20: The muon detector system consists of layers of drift tubes (left) and scintillation counters (right) [61].

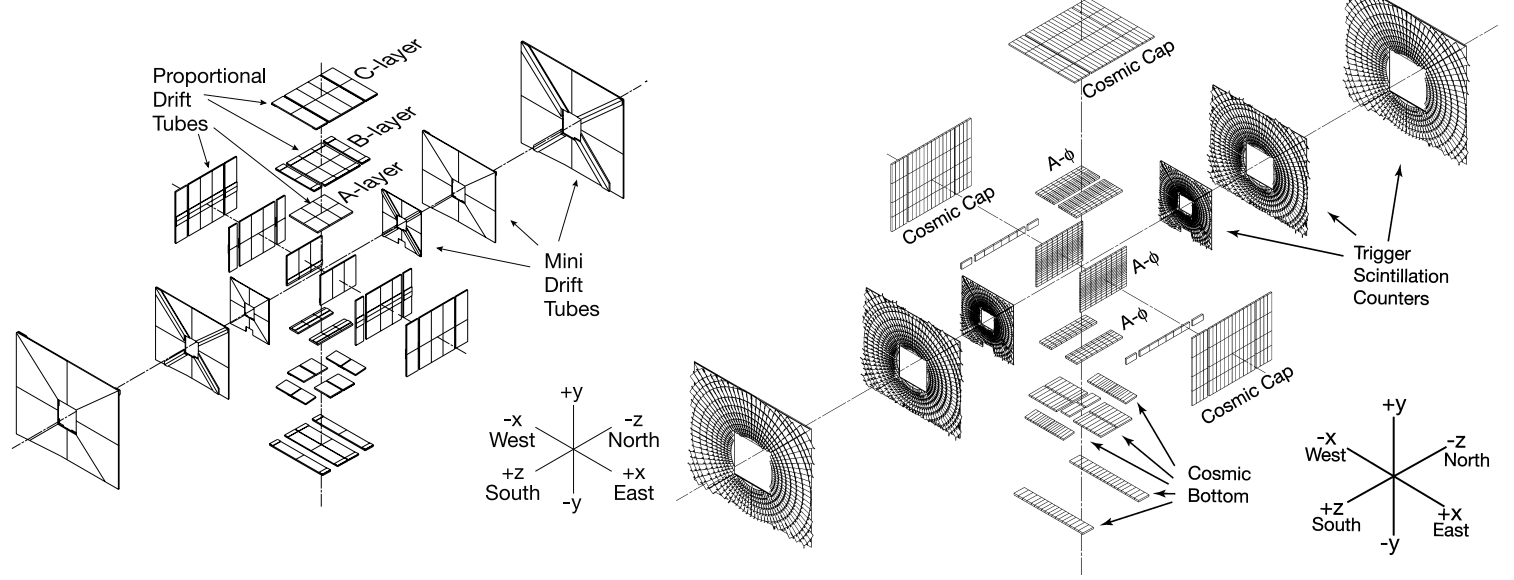

of each chamber. For each PDT hit the electron drift time, the charge deposition on the cathode pads and the difference in the arrival times of signal pulses on the two combined wires are recorded. The drift distance in the gas mixture of $84 \%$ argon, $8 \%$ methane and $8 \% \mathrm{CF}_{4}$ can be measured with a resolution of $1 \mathrm{~mm}$. The maximum drift time at a drift velocity of $10 \mathrm{~cm} / \mu \mathrm{s}$ is $500 \mathrm{~ns}$.

Scintillation counters build up the second part of the central muon system, see righthand sketch in Fig. 3.20. Due to their fast timing signal which is used to associate a muon found in the PDTs with the appropriate bunch crossing, they allow a discrimination against muons from cosmic ray background. The sets on the top and upper sides of the PDT $C$ layer (totaling 240 counters) are referred to as cosmic cap. Due to the calorimeter

Figure 3.21: Pictures of the muon detector [62]. The left-hand picture was taken with the toroid opened. One can see the three PDT layers. The right-hand picture shows the A layer forward scintillation counters, together with the beam pipe and shielding (red block).
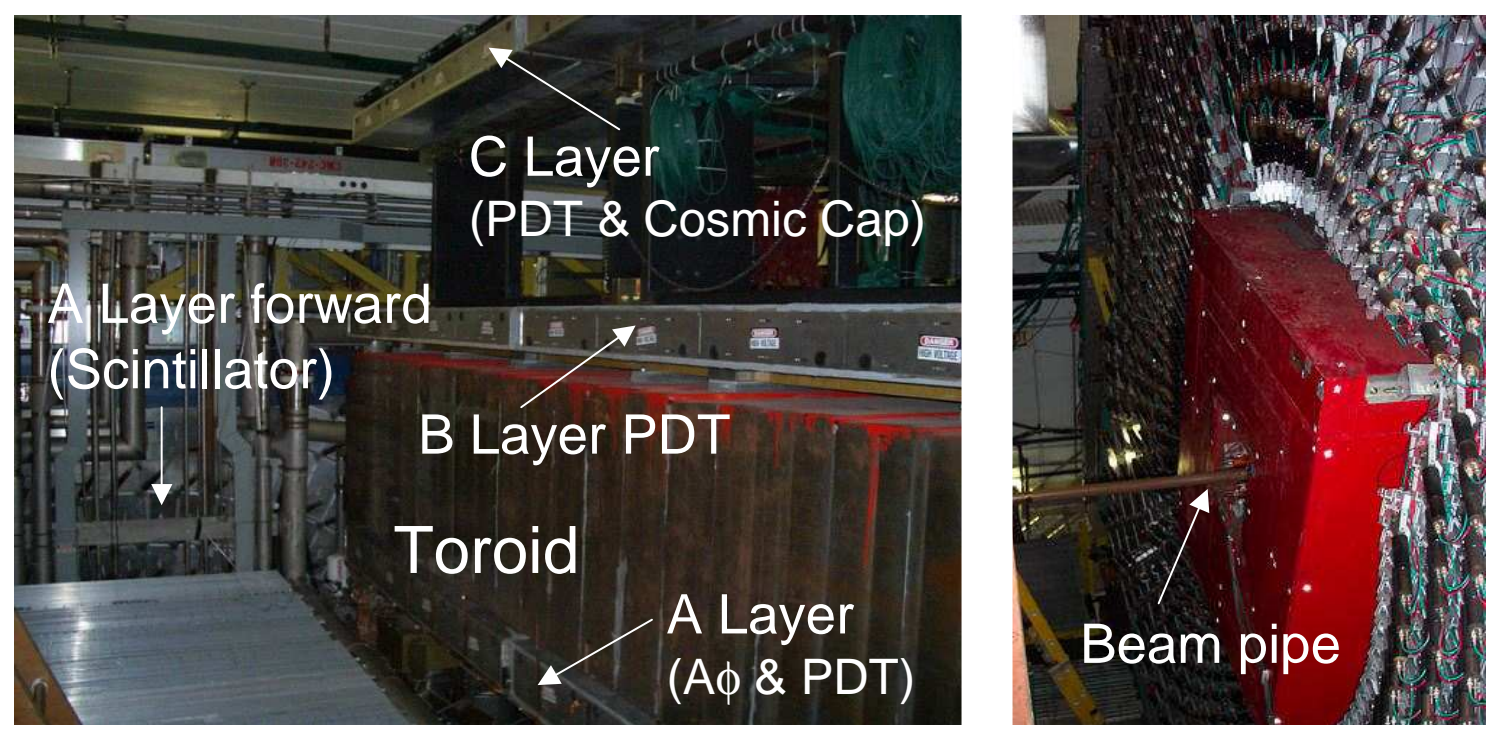
Figure 3.22: Pictures of the muon MDTs during the assembly [62].
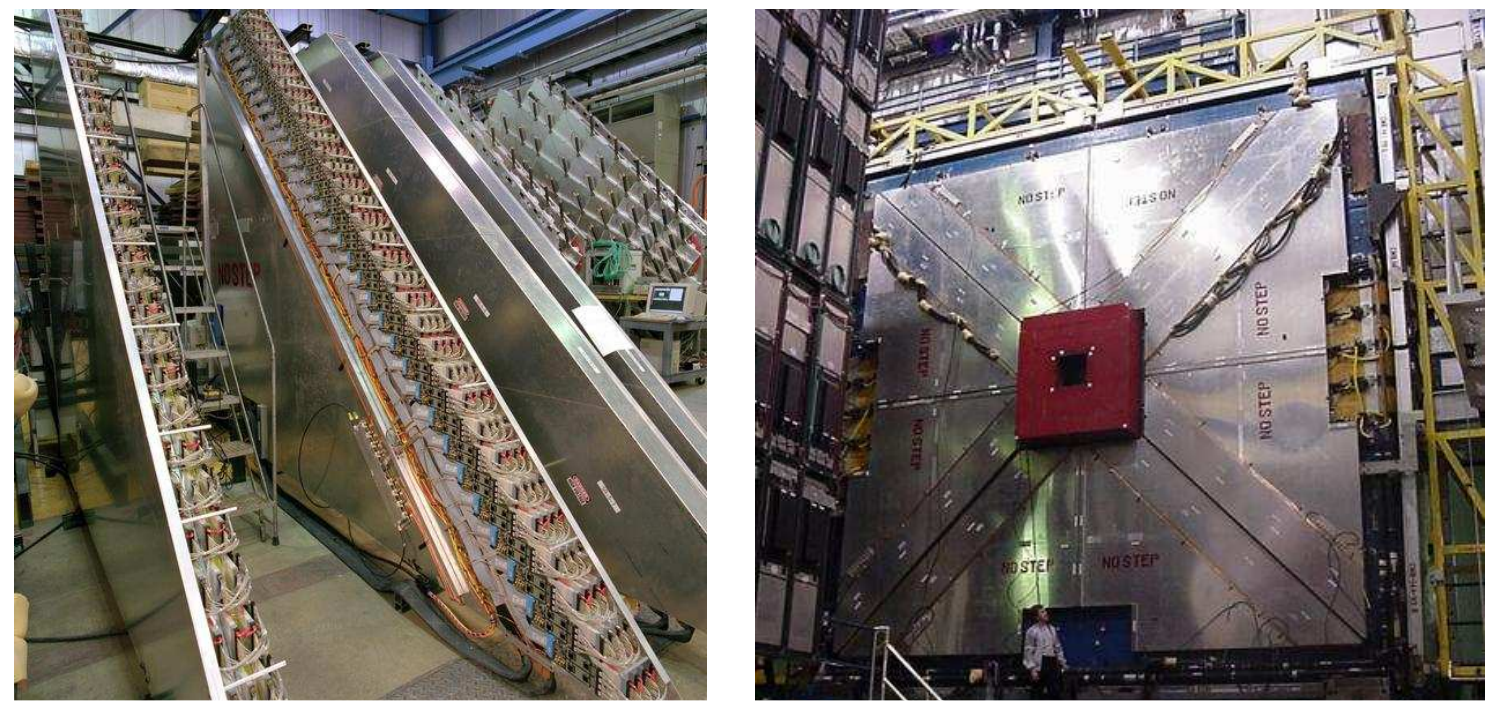

support structures at the bottom, this region, the cosmic bottom, is only instrumented partially with 132 counters. Photomultipliers are used for the readout. The light yield ranges from eighteen to thirty photoelectrons per hit, depending on the distance from the phototube. Further, an additional layer of 630 scintillators ( $A \phi$ counters) is mounted between the calorimeter and the $A$ layer PDTs. Again, the bottom part is not fully instrumented. A muon signal in the $A \phi$ counters with a timing resolution of about $2 \mathrm{~ns}$ induces $50-60$ photoelectrons. The left-hand picture in Fig. 3.21 (taken during the shutdown in 2004) shows the opened toroidal magnet with the different components of the central muon system.

The forward muon system $(1<|\eta|<2)$ consists of three layers of Mini Drift Tubes (MDT) and scintillation counters. The MDTs in each layer are divided into eight octants, see Fig. 3.20. Eight cells $\left(9.4 \times 9.4 \mathrm{~mm}^{2}\right)$ build up an MDT tube. The $A[B$ and $C]$ layer consists of four [three] planes of tubes which are mounted along the magnetic field lines. The outer tubes in the $C$ layer exhibit the maximum tube length of $5.83 \mathrm{~m}$. In the MDTs a gas mixture of $\mathrm{CF}_{4}-\mathrm{CH}_{4}$ (90\%-10\%) with a maximum drift time of $40 \mathrm{~ns}$ (for perpendicular tracks) is used. The anode wire is grounded, whereas a voltage of $-3.2 \mathrm{kV}$ is applied to the cathode. A total amount of 48,640 MDT wires is read out. The forward muon system has a hit resolution of $0.7 \mathrm{~mm}$ per hit. The standalone momentum resolution is approximately $20 \%\left(p_{T}<20 \mathrm{GeV}\right)$. The pictures in Fig. 3.22 show the MDTs during the assembly.

Three layers of trigger scintillation counters, one in front of the magnet and two outside, build up the second device of the forward muon spectrometer. The layers are divided into octants and contain ninety-six counters. The right-hand picture in Fig. 3.21 shows the $A$ layer forward scintillation counters which surround the beam pipe and the shielding (red block). A total amount of nearly 5,000 scintillator plates with a trapezoidal shape is assembled. The largest counters located in the outer rows of the $C$ layer have dimensions of $60 \times 110 \mathrm{~cm}^{2}$. All muon scintillators can be calibrated and monitored by an LED-based pulser system. 
The shielding (layers of iron, polyethylene and lead) provides a significant reduction of backgrounds from beam halo interactions and protons/antiprotons interacting with the beam pipe, low beta quadrupoles and ends of the calorimeter.

\subsubsection{Luminosity Monitor}

The luminosity is determined by a measurement of the counting rate of the inelastic proton-antiproton interaction, see Sec. 3.2. The DØ luminosity monitors are not only able to measure $P(n>0)$ or $P(n=0)$ (cf. Eq. 3.8), but as well allow a measurement of the $z$ coordinate of the interaction vertex and the beam halo rates (shown in Fig. 3.7). The monitors are mounted in front of the endcap calorimeters at $z= \pm 140 \mathrm{~cm}$ and cover $2.7<|\eta|<4.4$ (between the beam pipe and the FPS). The location is displayed in the left-hand sketch of Fig. 3.23. The right-hand drawing shows the geometry of one of the monitors which consists of twenty-four plastic scintillation counters with photomultipliers. The diameter of one monitor is $371 \mathrm{~mm}$, each counter has a length of $15 \mathrm{~cm}$.

Interactions from beam halo particles are the main background for the luminosity measurement. These processes can be separated by a precise time-of-flight measurement. Assuming that particles that hit the luminosity monitors stem from a proton-antiproton collision, one can reconstruct the $z$ coordinate of the vertex via

$$
z_{v}=\frac{c}{2}\left(t_{-}-t_{+}\right)
$$

with the time-of-flight $t_{ \pm}$measured by the luminosity monitor placed at $z= \pm 140 \mathrm{~cm}$. Proton-antiproton collisions are required to fulfill $\left|z_{v}\right|<100 \mathrm{~cm}$. This corresponds to $4 \sigma_{z}$, whereas $\sigma_{z}=26 \mathrm{~cm}$ denotes the length of the interaction region. Fig. 3.25 displays the distribution of the $z$ position of the vertex measured from data using tracks reconstructed in the central tracking system. Beam halo particles reactions are eliminated by the cut on $z_{v}$ because these exhibit $z_{v} \approx \pm 140 \mathrm{~cm}$.

The Luminosity Block Number, LBN, is a fundamental unit for the luminosity measurement. The LBN is increased

- after 60 seconds have elapsed;

- after run or store transitions;

- after an initialization of the trigger framework or the $\mathrm{SCL}^{11}$;

- by request.

The maximal size of 60 seconds is chosen so that the instantaneous luminosity is effectively constant during an LBN.

The luminosity is calculated with respect to a given trigger because deadtime and losses in the data acquisition system are taken into account. This is technically accomplished by grouping Level 1 triggers together so that they share common sources of "enable", "disable" and readout. More information about the luminosity is compiled in [59].

\footnotetext{
${ }^{11} \mathrm{SCL}=$ Serial Command Link. This denotes a common path that carries information about timing information, results of Level 1/Level 2 trigger decisions or initialization of detector components.
} 
Figure 3.23: Left: Location of the luminosity detectors. Right: Schematic drawing displaying the geometry. The red dots indicate the location of the photomultipliers [61].
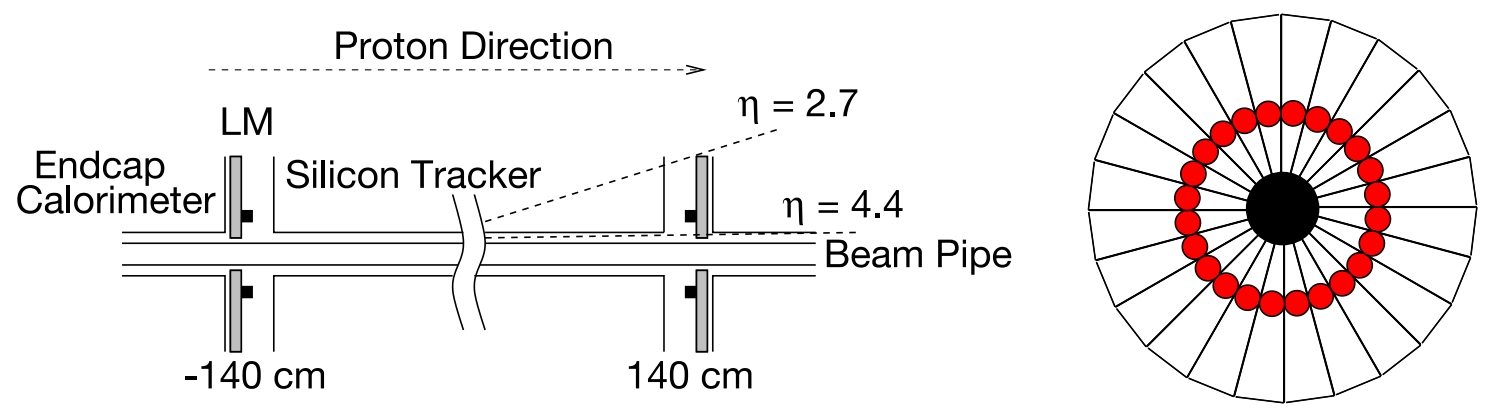

Figure 3.24: Picture of a luminosity monitor (left). The right-hand picture shows one of the twenty-four scintillation counters with a photomultiplier on the top [73].
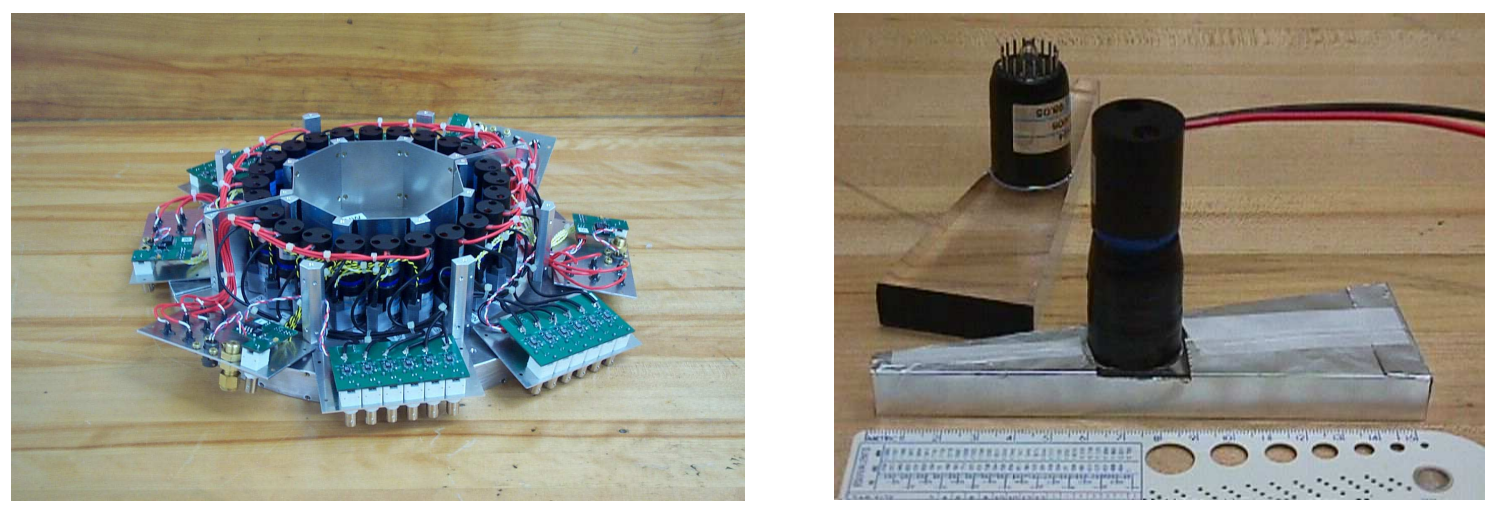

Figure 3.25: Distribution of the $z$ position of the reconstructed primary vertex from data.

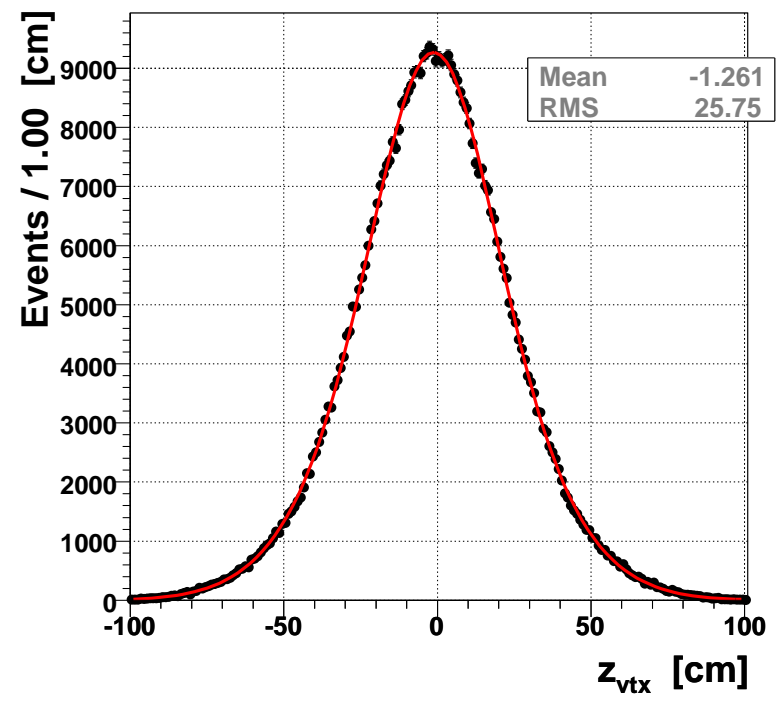




\subsection{Trigger and Data Acquisition}

A sophisticated system of triggers is inevitable in order to select interesting physics events out of 1.7 million collisions per second. At DØ trigger decisions are made in three steps. Each of the levels is able to significantly reduce the rate so that the next, more complex level can examine the smaller number of events in greater detail. The selection of triggers is referred to as trigger list, and each trigger consists of a Level 1, Level 2 and Level 3 trigger bit. The trigger list history, together with the full description of all triggers can be found at [74]. In the following the data flow from the detector to the storage tapes (see left-hand sketch in Fig. 3.26) is discussed.

Figure 3.26: Left: Overview of the trigger and data acquisition system. Right: Flow of triggerrelated data between the detector, Level 1, Level 2 and the trigger framework [61].

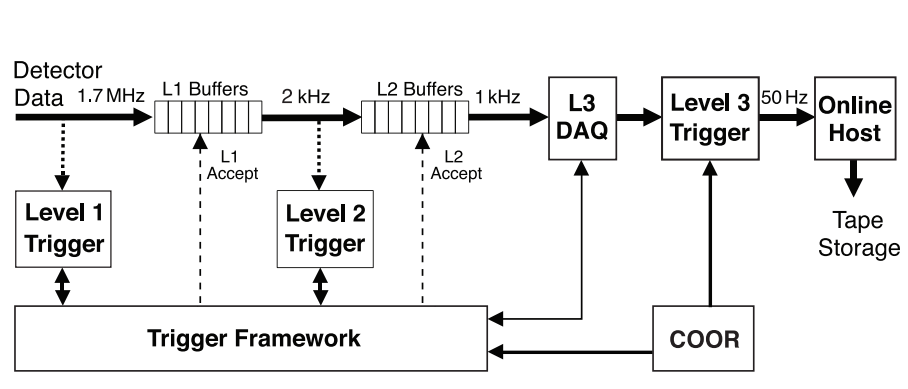

Detector Level1 Level2

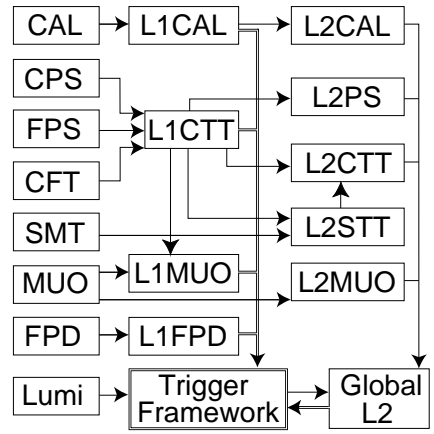

\subsubsection{Level 1 Trigger}

The Level 1 trigger (see right-hand plot in Fig. 3.26) consists of hardware and firmware trigger elements. The calorimeter (L1CAL) is searched for energy depositions, the central track trigger (L1CTT) checks for track patterns above a given threshold in the CPS, FPS and CFT. The muon trigger (L1MUO) looks for patterns consistent with muons evaluating the muon scintillation counters, drift tubes and tracks from L1CTT. The Forward Proton Detectors (FPDs) are checked for diffractively-produced events (L1FPD). Several individual terms form a Level 1 script, for example $\operatorname{TTK}(1,10) \operatorname{CEM}(2,3) \operatorname{CEM}(1,9)$. The Level 1 trigger bit for this particular trigger is set to 'true', if the event contains one CFT track with $p_{T}>10 \mathrm{GeV}$ and two EM calorimeter trigger towers with $E_{T}>3 \mathrm{GeV}$, whereas one of these towers must have $E_{T}>9 \mathrm{GeV}$.

During the processing the events are buffered (sixteen Level 1 buffers are available) so that the deadtime ( $5 \%$ on average, see Fig. 3.30) is minimized. The Level 1 decision must arrive at the Trigger FrameWork (TFW) in $3.5 \mu$ s or less. The TFW collects the information from the individual Level 1 trigger systems and decides to accept or reject the event. It further communicates with the front-end electronics and the trigger control computer. The prescaling of individual triggers is governed by the TFW as well. Prescales are applied on triggers in order to reduce the rate without switching off the trigger. If a trigger is prescaled with $n$, only one out of $n$ events that passed the trigger is not rejected. 


\subsubsection{Level 2 Trigger}

The output of Level 1 (rate: $2 \mathrm{kHz}$ ) is then analyzed at the next level, Level 2, which reduces the trigger rate by a factor of two. Hardware engines and embedded microprocessors, denoted as preprocessors, analyze signals from the front-ends of individual subdetectors as well as the Level 1 trigger system and form physics objects from the calorimeter (L2CAL), the preshower detectors (L2PS), the muon system (L2MUO) and the CFT (L2CTT). In addition, information from the SMT can be accessed at Level 2 (L2STT). It is possible to check for correlations in physics signatures because a global Level 2 processor can perform a combination of individual physics objects that come out of the preprocessors. The global processor checks the Level 2 trigger condition (Level 2 script) for each Level 1 trigger bit that fired. The script itself evaluates if a minimum number of physics objects pass one or more filters, whereas a filter itself can consist of multiple filters. The physics objects are produced by tools $(\mathrm{C}++$ classes). For example, the script L2CALTRK $(1,3,5, \mathrm{TTK})$ selects events containing one calorimeter tower of $E_{T}>3 \mathrm{GeV}$ that has been matched with an L1CTT track of $p_{T}>5 \mathrm{GeV}$ within $|\eta|<2$. A trigger list can at most comprise 128 Level $1 /$ Level 2 trigger bits.

\subsubsection{Level 3 Trigger}

Events are buffered (eight Level 2 buffers are available) until the TFW issues the Level 2 accept. The Level 3 trigger, a high level, fully programmable software trigger, provides a limited reconstruction of events (similar to offline algorithms) and thus reduces the Level 2 output rate of $1 \mathrm{kHz}$ to the desired $50 \mathrm{~Hz}$ rate-to-tape. The Level 3 software is executed on the Level 3 computer farm which consists of more than 300 PC. The ScriptRunner (see

Figure 3.27: Level 3 execution tree [75]. Trig-n: Level 1/Level 2 trigger bit; Filt-n: Level 3 script; orange box: filter; turquoise box: tool.

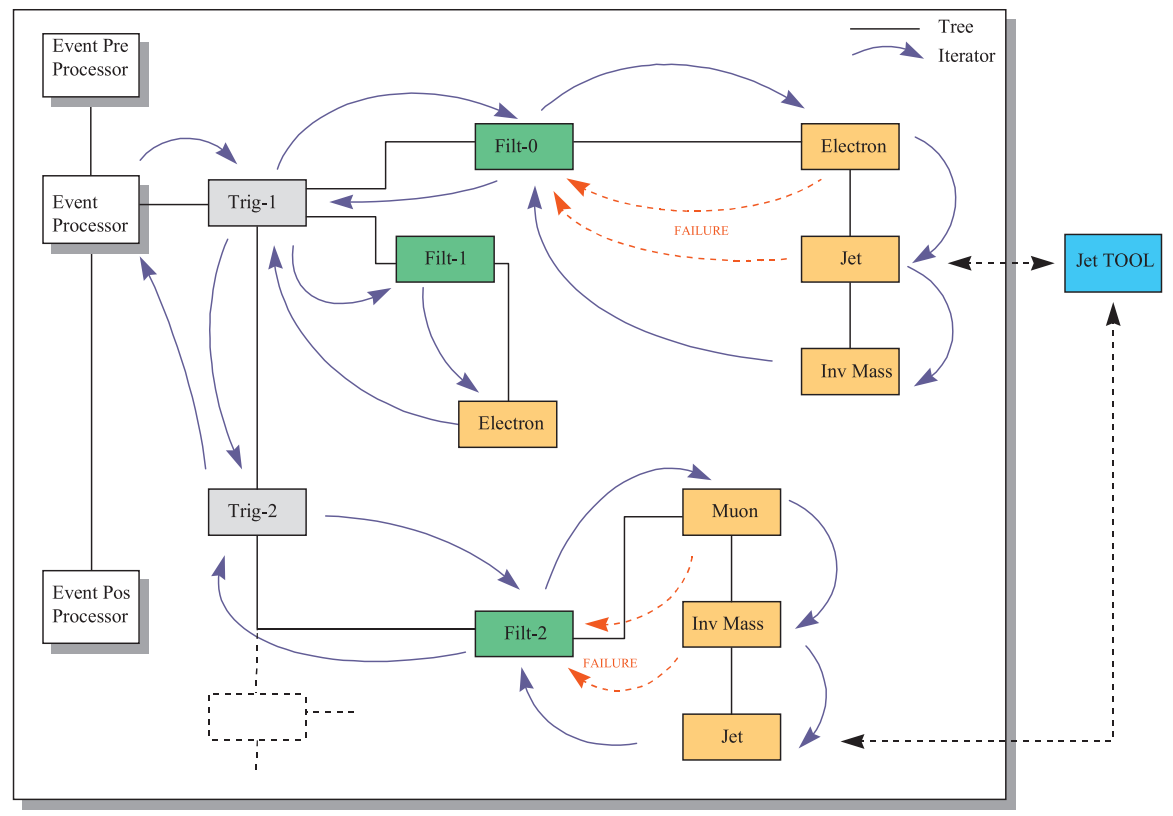


execution tree in Fig. 3.27), which is the driver of the Level 3 trigger, parses a given trigger list and executes the Level 3 scripts that are associated with each of the Level 1/Level 2 trigger bits that fired. Each Level 3 script is associated with a Level 1/Level 2 trigger bit, whereas more than one Level 3 script, but at most 256, can be associated with one Level 1/Level 2 bit. In the figure, two Level 3 scripts (Filt-0 and Filt-1) are associated with the Level 1/Level 2 bit Trig-1. The scripts call - similar to Level 2 - filters and tools. One Level 3 script consists of logical ANDs of one or more filters. If one individual filter fails, the execution of a Level 3 script is terminated, and the next Level 3 script is processed. The physics objects are reconstructed within tools. The results of the tools are cached because they are usually called multiple times, and the major part of the processing time is spent in the tools. The filters call results of the tools and check if these fulfill certain sets of parameters.

The sketch in Fig. 3.28 shows the design of an advanced Level 3 tool, the isolation tool [76], [77], [78]. In the last Run IIa trigger list (June 2005 - February 2006) more than 100 triggers (25\% of the trigger list) made use of this tool which allows to trigger on a given "source" object (electron, photon, muon or track) that is isolated. The isolation is defined via energy depositions in a hollow cone around the source object, whereas the hollow cone is defined in distances of $\eta$ and $\phi$. The distance $\Delta R_{i}$ of an object $i$ with respect to the source object is calculated in the following way

$$
\Delta R_{i}=\sqrt{(\Delta \eta)^{2}+(\Delta \phi)^{2}}=\sqrt{\left(\eta_{\mathrm{src}}-\eta_{i}\right)^{2}+\left(\phi_{\mathrm{src}}-\phi_{i}\right)^{2}} .
$$

In the calculation of isolation, which can be defined with respect to tracks and calorimeter cells, the momentum/energy of an object $i$ is summed up, if it is contained in the hollow cone, $R_{\text {inner }}<\Delta R_{i}<R_{\text {outer }}$

$$
\begin{aligned}
\text { track isolation } & \left.\Leftrightarrow \quad \sum_{\text {tracks }} p_{T}^{i}\right|_{R_{\text {inner }, \mathrm{t}}<\Delta R_{i}<R_{\text {outer }, \mathrm{t}}}<P_{\text {max }} \\
\text { calorimeter isolation } & \left.\Leftrightarrow \quad \sum_{\text {calorimeter cells }} E_{T}^{i}\right|_{R_{\text {inner }, \mathrm{c}}<\Delta R_{i}<R_{\text {outer }, \mathrm{c}}}<E_{\text {max }} .
\end{aligned}
$$

All parameters (radii for calorimeter and track cones, maximal sums of momenta/energies, source objects) can be set individually.

Figure 3.28: Design of the Level 3 isolation tool.

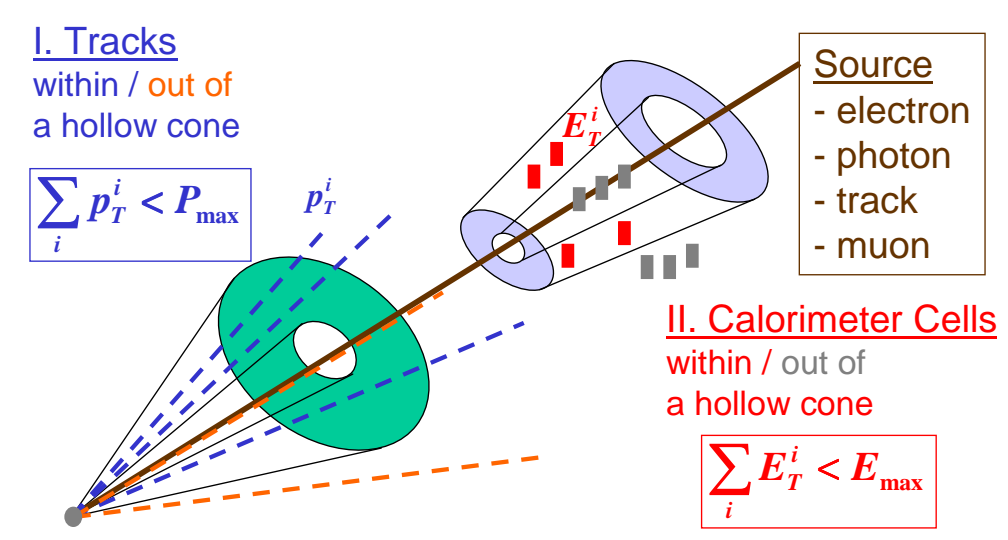


Figure 3.29: Performance of the Level 3 isolation tool [79]. Left: Efficiency and rejection for data and Monte Carlo $Z \rightarrow e e$ events. Right: Sum of track momenta in the hollow cone for $Z \rightarrow e e$ Monte Carlo events and data (arbitrary run).
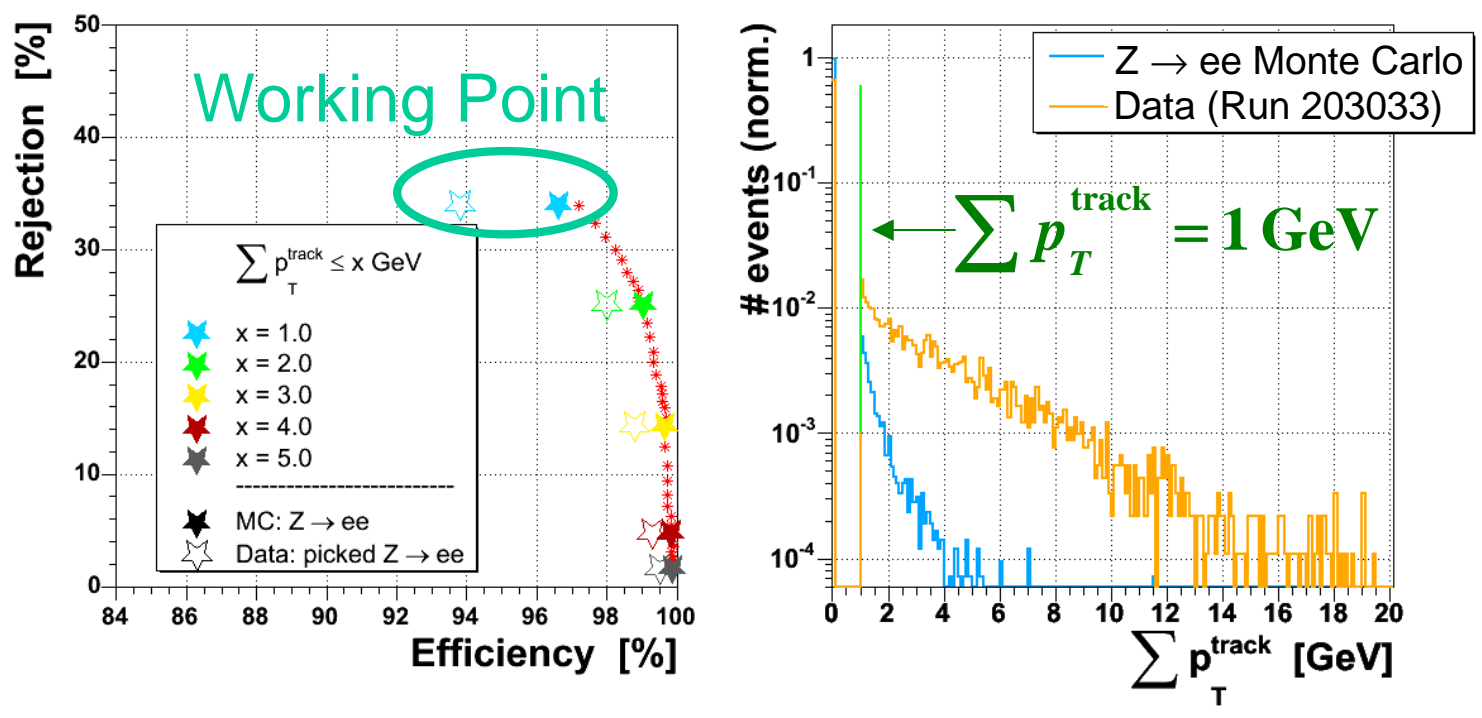

The plots in Fig. 3.29 display the performance of the track isolation for electrons which is derived from selected $Z \rightarrow e e$ data events, simulated $Z \rightarrow e e$ Monte Carlo events and an "unbiased" (= not restricted to a particular physics process) dataset. The efficiency and rejection are defined as follows

$$
\begin{aligned}
\text { Efficiency (Data) } & =\frac{\# \text { selected } Z \rightarrow e e \text { data events with } \sum p_{T}^{\text {tracks }}<P_{\max }}{\# \text { selected } Z \rightarrow e e \text { data events }} \\
\text { Efficiency (Monte Carlo) } & =\frac{\# Z \rightarrow e e \text { Monte Carlo events with } \sum p_{T}^{\text {tracks }}<P_{\max }}{\# Z \rightarrow e e \text { Monte Carlo events }} \\
\text { Rejection } & =\frac{\# \text { data events (unbiased) with } \sum p_{T}^{\text {tracks }}>P_{\max }}{\# \text { data events (unbiased) }} .
\end{aligned}
$$

The unbiased data events for the determination of the rejection are taken from an arbitrary run (here: run \#203033). The left-hand plot shows the efficiency and rejection for different cuts $x$ on the maximal sum of track momenta in the hollow cone, $P_{\max }$. The working point for track isolation of electrons is set to $\sum p_{T}^{\text {tracks }}<1 \mathrm{GeV}$ and exhibits a high efficiency of $94 \%$ with a rejection of $34 \%$. The efficiency derived from Monte Carlo is slightly better because effects like "fake" tracks that appear in data are underestimated in the simulation. However, deviations are small (Monte Carlo efficiency: 96.6\%, data efficiency: 93.8\%). As a consequence, Monte Carlo efficiencies have to be corrected by applying event weights in order to describe the data, see Sec. 7.2.1. The right-hand plot in Fig. 3.29 shows the sum of the track momenta in the hollow cone for $Z \rightarrow e e$ Monte Carlo events and data events from run \#203033. The sum is either zero (no tracks found in the hollow cone), or greater than one because the Level 3 tracking tools have a minimal threshold on the track momenta of $p_{T}^{\min }=1 \mathrm{GeV}$. 


\subsubsection{Trigger and Luminosity}

The instantaneous luminosity and the number of protons/antiprotons steadily decrease during a store, see Fig. 3.7. This behaviour translates into the trigger rates. Fig. 3.30 displays the progression of luminosity (purple) and the Level 1 (black), Level 2 (blue) and Level 3 (red) rates during a typical store. The numbers from one to ten indicate the beginning of a new run. A run is a period of data taking with a well-defined detector and trigger configuration, and usually lasts between two and four hours, depending on the instantaneous luminosity. During Run IIa the Level 1 [Level 2] rate was required not to exceed $1.4 \mathrm{kHz}$ [850 Hz] (dashed lines) so that the Front End Busy (FEB, deadtime associated with the readout; green and yellow dashed lines) is well below $10 \%$. One can clearly see the impact of the Level 1 prescales: With decreasing trigger rates (thus luminosity) prescales are loosened at the beginning of a new run so that the rate-to-tape is maximized.

Figure 3.30: Progression of luminosity and trigger rates during store \#4571 [80] (cf. Fig. 3.7).

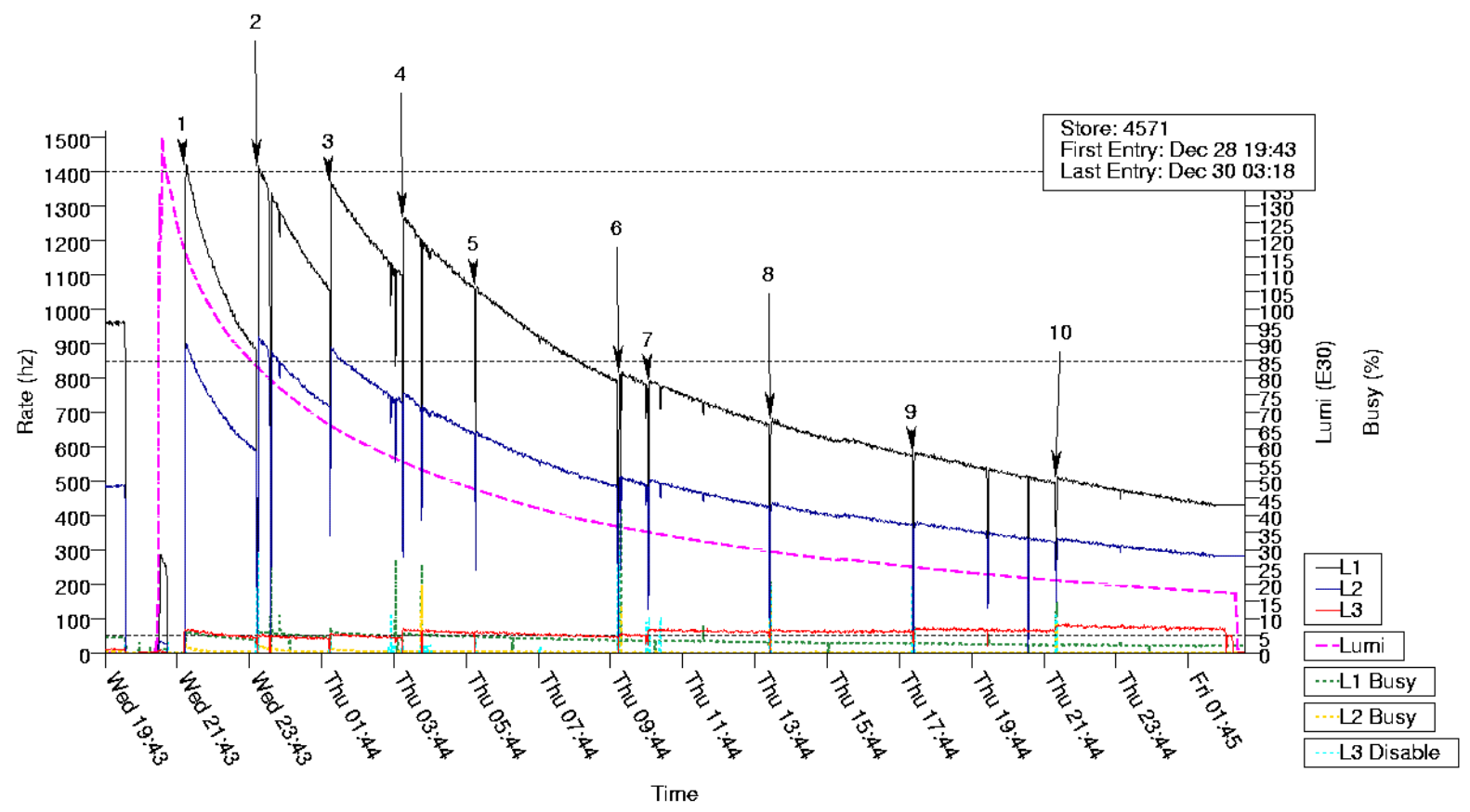

Prescales represent a powerful tool for rate control without modifications of the trigger list. Since the luminosity is determined with respect to a given (Level 1) trigger (see Sec. 3.3.4), the effect of a prescale applied to a trigger is automatically taken into account in the luminosity calculation. For example, the luminosity of a trigger with prescale $n_{1}=1$ is reported to be $100 \mathrm{pb}^{-1}$ for a fixed data taking period. Then, another trigger with $n_{2}=2$ records a luminosity of $100 \mathrm{pb}^{-1} \cdot n_{1} / n_{2}=50 \mathrm{pb}^{-1}$ during the same data taking period.

In practice, the logical OR of multiple triggers is used in physics analyses [81]. The correct treatment of trigger efficiencies, prescales and luminosity is complicated. Details of combining triggers are discussed in [82], [83] and [84]. This analysis uses the OR of several calorimeter triggers [85] with $(n \neq 1)$ and without $(n \equiv 1)$ prescale, see Sec. 6.1. The 
effect of the prescale is included in the trigger efficiency, and the luminosity is calculated with respect to the unprescaled trigger.

\subsubsection{Data Acquisition}

Once a Level 2 accept is issued, the Data AcQuisition system (DAQ) accomplishes the transport of detector data from the sixty-three $\mathrm{VME}^{12}$ readout crates to the Level 3 computer farm which consists of more than 300 PCs. This process is controlled and coordinated by COOR (DØ run control) which interacts with the TFW and the DAQ supervising system, and is responsible for coordinating changes in states of software and hardware components including the DAQ. Data from each detector readout crate is collected in Single Board Computers (SBCs) and sent to a node on the filter farm through a commercial ethernet switch, see left-hand sketch in Fig. 3.31. The flow of information from the readout crates to the filter nodes is controlled by the Routing Master. The trigger programming is loaded onto the filter nodes by the supervisor node. On each farm node runs the event builder process, which assembles a complete event from the event fragments received from the SBCs, and makes it available for the Level 3 filter process.

Figure 3.31: Left: Illustration of the data flow through the DAQ system. The sketch on the right-hand side shows the architecture of the online host system [61].
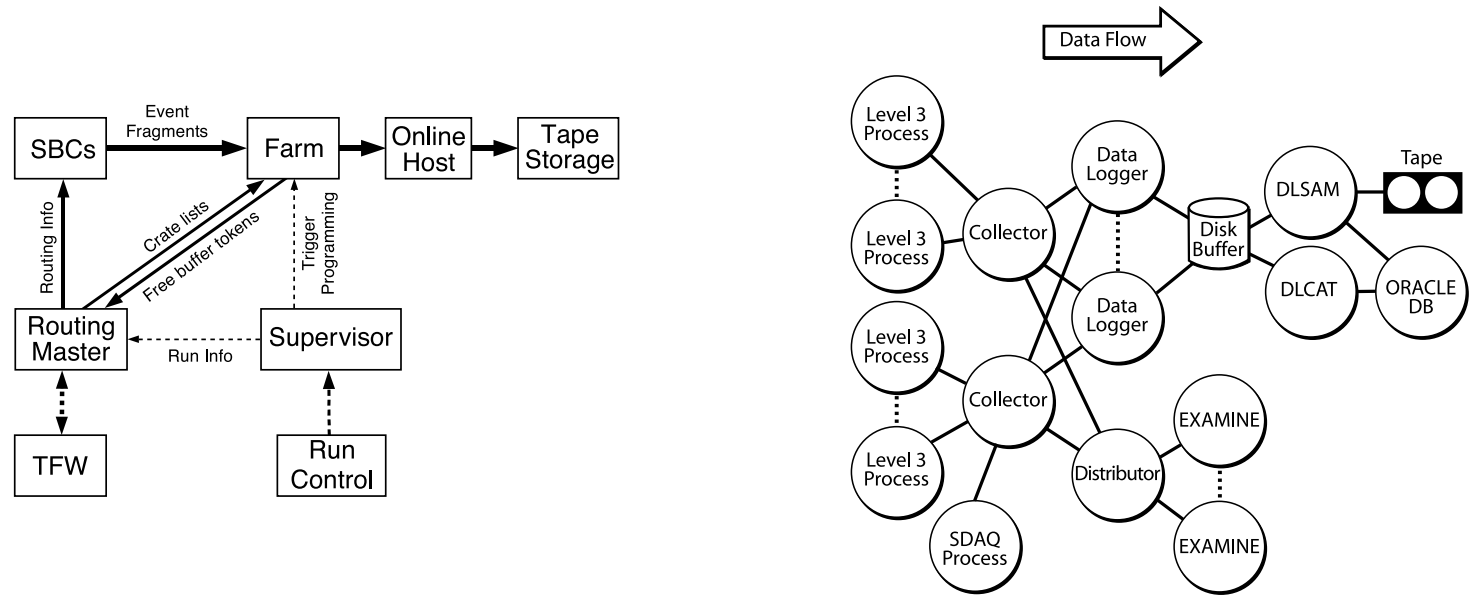

The online host system collects the data from the Level 3 nodes (see right-hand sketch in Fig. 3.31) and routes each event to the appropriate data logger. It is possible to take more than one run in parallel (e.g. SMT and CFT can take calibration runs at the same time). Further, the system is capable to write multiple streams simultaneously. These streams contain special events marked by the Level 3 trigger [86]. An event can only be assigned to one (data) stream. However, it is possible to assign an event additionally to a special monitor stream. This method allows for example a quick offline access to the calibration trigger data which are written to the monitor stream. The final data storage devices are located at the Feynman Computing Center, $3 \mathrm{~km}$ away from the detector (see Fig. 3.1). Since the remote tape drive nodes do not have an intermediate disk buffer, the $\mathrm{D} \varnothing$ online system provides a disk buffer which also fills in if the tape robot is temporarily

\footnotetext{
${ }^{12} \mathrm{VME}=$ VERSAModule Eurocard. The VME bus is an industrial open standard system designed to be plugged into the backplane of a crate that has up to 21 slots.
} 
unavailable. The data streams are saved with the Sequential Access via Metadata [87] (SAM) data handling system which allows access to the data in a flexible and transparent manner. The DLSAM process, which represents the interface between the data loggers and SAM, is responsible for monitoring the local data buffer disks and storing the data files in the mass storage system (ENSTORE). The bookkeeping is performed by DLCAT (Data Logger event CATaloguer) which checks and fixes metadata (= information about the data) missing in the SAM ORACLE database - if necessary. A part of the data $(1 \%-100 \%$, configurably) is sent to the distributor process which supplies between ten and twenty monitoring clients (examines, e.g. physics examine, see Fig. 3.32) allowing immediate data quality checks in the $\mathrm{D} \varnothing$ control room. The distributor provides data events in near real-time via an event queueing system. The Level 3 and DAQ system can be bypassed using a secondary data path, SDAQ. This mechanism is used by the SMT, CFT, CPS, FPS and FDP subdetector systems for calibration and monitoring purposes.

Figure 3.32: Physics examine plots for tracks displaying the total number of hits in the tracker and the number of SMT and CFT hits for run \#232662 (black markers) compared to a reference run (red histogram) [88].
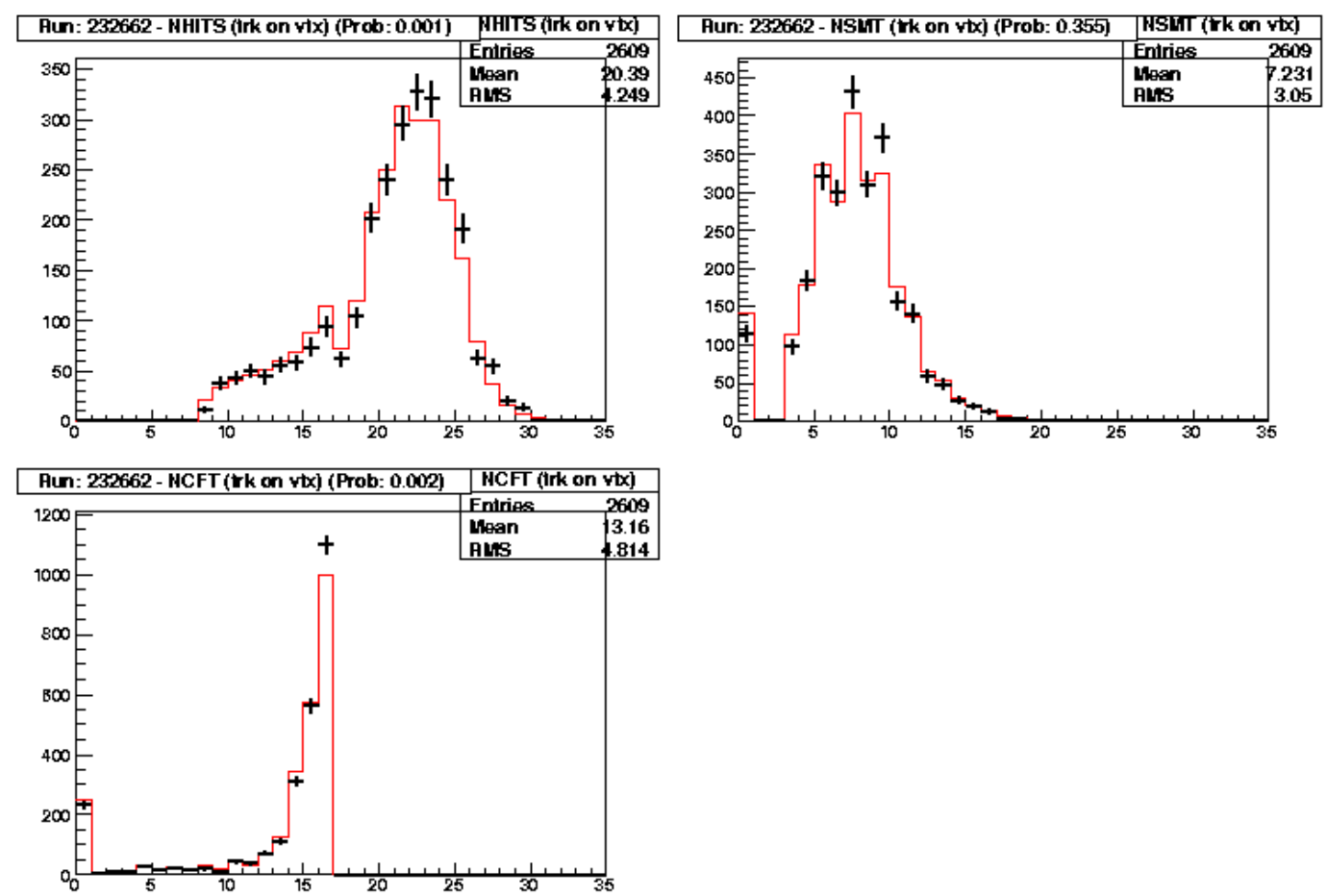

The DØ experiment uses EPICS (Experimental Physics and Industrial Control System) in order to monitor the slow control system of the detector. EPICS consists of nodes running application programs, and Input/Output Controller (IOC) nodes which interface with the detector hardware. The SES, the Significant Event System, collects and distributes all changes of states of the entire detector. Sender clients (IOC, COOR) connect to the SES server and report any changes, like alarm transitions, to this server. This information is distributed to three standard receiving clients: A user can access the current state of the detector via the alarm display; the alarm watcher is capable to pause the data taking process if an alarm condition compromises data quality (e.g. a high volt- 
age problem affecting an individual detector component often causes efficiency losses); the logger, the third of the SES clients, writes all SES messages to a disk file. The DØ detector is configured using a single server program, COMICS, which manages all parts of the detector that can be accessed by EPICS.

\subsection{Data Reconstruction and Data Quality}

The reconstruction software relies on the Event Data Model, EDM, which consists of a library of $\mathrm{C}++$ classes and templates. The results of a single beam crossing are stored in the event container which manages not only the raw output of the detector and the results of the trigger processing, but also the results of different reconstruction processes. Since detailed information about the configuration of the reconstruction processes are contained as well, it is possible to run multiple instances of a single algorithm based on different configuration information. The access to the stored reconstructed objects is also provided by the EDM. The D $\varnothing$ Object Model, DØOM, handles the conversion of the $\mathrm{C}++$ objects to a persistent format. Input/Output (I/O) packages facilitate the translation between $\mathrm{C}++$ objects and the persistent format.

The offline reconstruction is accomplished by the package dØreco [89] which can not only handle data events, but also simulated Monte Carlo events (see Sec. 5.1). The results of this CPU-intensive program running on the offline production computer farm are saved in SAM. Information and results, managed by the EDM, are contained in blocks (chunks). For example, the Raw Data Chunk, RDC, is created by a Level 3 processor (or Monte Carlo) and contains the raw detector signals.

The reconstruction is performed in the following hierarchical steps:

I. Detector specific processing

$\rightarrow$ the detector data blocks are unpacked;

$\rightarrow$ the raw information is decoded;

$\rightarrow$ readout channels are associated with physical detector elements;

$\rightarrow$ calibration constants are applied

II. Pre-reconstruction

$\rightarrow$ cluster reconstruction for the calorimeter and preshower detectors;

$\rightarrow$ hit reconstruction for the tracking systems

III. Tracking

$\rightarrow$ reconstruction of global tracks from hits in the SMT and CFT incorporating different algorithms

IV. Vertexing

$\rightarrow$ reconstruction of primary vertex candidates;

$\rightarrow$ identification of displaced secondary vertices 


\section{Particle identification}

$\rightarrow$ reconstruction of "simple" objects: electrons, photons, muons and jets;

$\rightarrow$ identification of heavy-quark ( $b$ and $c$ ) jets, as well as $\tau$ candidates;

$\rightarrow$ reconstruction of missing transverse energy $\mathbb{E}_{T}$

The output is stored in the ThumbNail format [90] which constitutes a way of keeping the relevant information in a compact format omitting redundant information. The information from the physics objects chunk in the dØreco output is packed in the ThumbNailChunk, together with reduced trigger chunks and selected pieces of the RDC.

The Common Samples Group [91] (CSG) takes care of reprocessing of the data (if necessary), and provides preselected subsamples (skims) in the ThumbNail format. This analysis makes use of the EMinclusive skim which is required to contain at least one electron candidate. The Common Samples Group is as well in charge of the central production of Root [92] trees (via tmb_analyze [93]) in the Common Analysis Format, CAF [94]. During the production the package dØcorrect [95] is applied which calls the postprocessing codes (corrections, certifications) for offline objects. This analysis is performed in the Common Analysis Format Environment (CAFE) because many software tools, like event and object selectors, efficiency corrections and the data quality processor, are provided for CAF trees. Not only the raw data, but also the ThumbNails out of dØreco, the skimmed ThumbNails and the CAF Root trees are stored in SAM.

Already during data taking the shifters in the D $\varnothing$ control room (see Fig. 3.33) monitor detector subsystems and report the quality of a run for the calorimeter, muon system, SMT and STT, CFT and CTT. A run can be declared as 'good', 'reasonable' or 'bad'. Any incidents, like disabled muon chambers, hot cells in the calorimeter or broken sectors in the CFT, as well as discrepancies observed in the examine plots are entered into the logbook [96] and run summaries [97]. If no report is available, the run quality is marked as 'unknown'. Some 'special' runs can not be used for regular physics analyses because they are taken with a special detector configuration (special trigger lists or subdetectors/magnets switched off) or even without beam (cosmic muon runs). The quality of a run is stored in the Offline Run Quality Database [98].

Figure 3.33: Pictures of the DØ control room [49]. The right-hand picture shows the DAQ console.
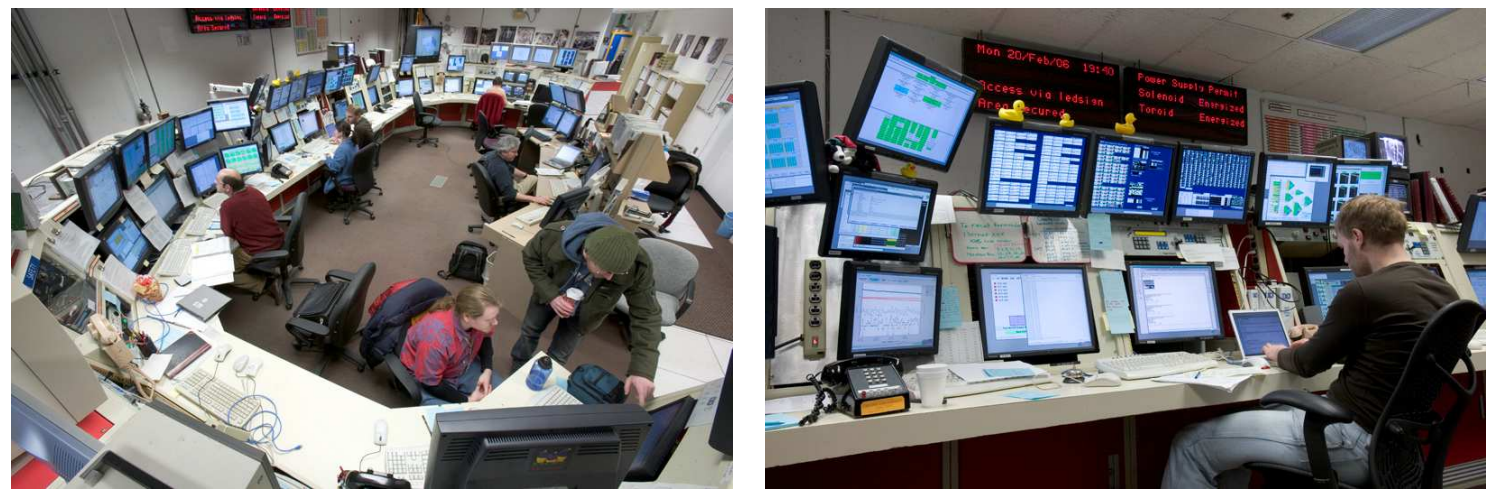
The quality of the luminosity measurement is determined offline and not classified on a run-by-run basis, but rather based on luminosity blocks (LBNs, see Sec. 3.3.4). An LBN can be declared as 'bad' due to downtimes of the luminosity system, online losses, incomplete events, Level 3 losses and unreadable tapes. Information about the status of an LBN is stored in the Luminosity Database [99].

For the calorimeter advanced certification procedures are applied which determine the data quality not only on a run-by-run basis, but also for individual LBNs and even for particular events. This reduces the amount of rejected data compared to whole 'bad runs' because certain noise features are sometimes only present during a short period of time and not persistent during an entire run. A documentation of the 'bad calorimeter LBN' selection can be found at [100]. Fig. 3.34 (left) shows the "ring of fire" noise in the electromagnetic calorimeter. Due to limited space in the intercryostat region, the high voltage distribution layout for the electromagnetic layers is built as a ring electrode. Hence, external noise can be picked up by this electrode and affects all cells in $\phi$ direction at a fixed value of $\eta$. This results in rings where more than $90 \%$ of the cells measure a signal. The impact of bad runs, noisy events and cells with readout problems ('bad' cells) on the missing transverse energy is shown in the right-hand plot in Fig. 3.34; they all lead to an excess in the tail of this distribution. The Data Quality Group [101] provides lists of bad runs and LBNs together with related software tools.

Figure 3.34: Left: Energy deposits in the calorimeter cells as a function of $\eta$ and $\phi$. This event shows the typical "ring of fire" characteristics [102]. Right: Bad runs, calorimeter cells and noisy events contribute to "fake" missing transverse energy [103].
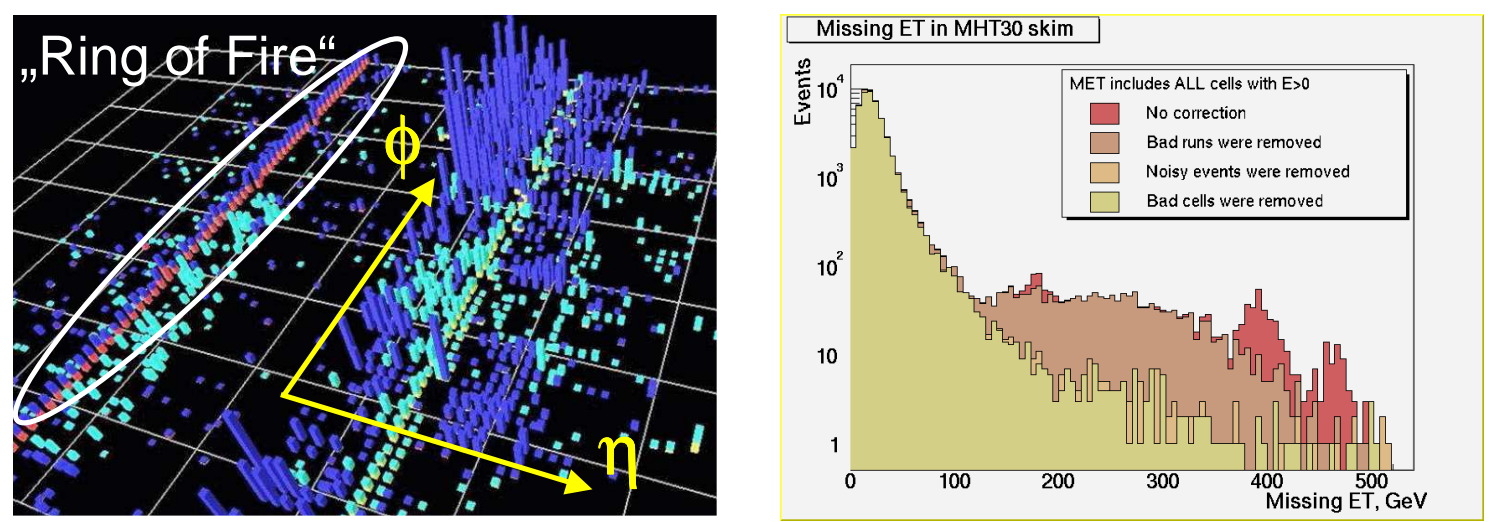



\title{
Chapter 4
}

\section{Object Identification}

\begin{abstract}
Nature uses only the longest threads to weave her patterns, so that each small piece of her fabric reveals the organization of the entire tapestry.
\end{abstract}

Richard Feynman

The $\mathrm{D} \varnothing$ detector is built with an onion-like structure (see Fig. 3.10) with various different types of devices which are optimized for the identification and energy/momentum measurement of the Standard Model particles (electrons, muons, taus, photons, quarks, gluons and neutrinos). Quarks and gluons can not be observed directly, but they can be identified as "jets" (except for the $t$ quark) after fragmentation and hadronization (see Sec. 5.1). The neutrino as a very weakly interacting particle escapes detection, but it gives rise to missing energy in the transverse plane with respect to the proton/antiproton beam (see Eq. 2.13). Unstable objects ( $t$ quark, $\tau$ lepton, $W$ and $Z$ bosons) can be identified consulting known decay channels. Tab. 4.1 summarizes the identification of Standard Model particles. Fig. 4.1 shows a $t \bar{t}$ candidate event where both $W$ bosons from the top quark $t \rightarrow b W$ decay into lepton + neutrino ("dilepton channel"). In order to detect such complex events, sophisticated object reconstruction algorithms with both high efficiency and background rejection are inevitable. Since this analysis relies on electrons, missing transverse energy and jets (marginally), the identification of taus, muons and heavy quarks is not discussed. Details can be found at [104] (top quark), [105] (heavy flavor identification), [106] (tau identification) and [107] (muon identification). Information about the reconstruction of the primary vertex (= location of the hard interaction) and secondary vertices stemming from decays of long-lived particles like mesons containing $b$ quarks is compiled at [108], together with a discussion of the various tracking algorithms.

\subsection{Electron Reconstruction}

The reconstruction of electron candidates starts with clusters in the electromagnetic calorimeter which are defined as a set of towers in a cone of radius $R_{\text {cone }}=0.2$ around 
Table 4.1: Identification of Standard Model particles.

\begin{tabular}{|c|c|}
\hline Particle & Identification with the $\mathrm{D} \varnothing$ detector \\
\hline electron & $\begin{array}{l}\text { track, electromagnetic shower, } \\
\text { almost no energy deposition in the hadronic calorimeter }\end{array}$ \\
\hline photon & $\begin{array}{l}\text { no track, electromagnetic shower, } \\
\text { almost no energy deposition in the hadronic calorimeter }\end{array}$ \\
\hline muon & $\begin{array}{l}\text { track, hits in the muon scintillation counters and drift chambers, } \\
\text { energy deposition in the calorimeter (minimal ionizing particle) }\end{array}$ \\
\hline tau & $\begin{array}{l}\text { unstable, depends on the decay signature: } \\
\tau \rightarrow e \nu \nu: \text { see electron and neutrino } \\
\tau \rightarrow \mu \nu \nu: \text { see muon and neutrino } \\
\tau \rightarrow \text { hadrons: track(s) and calorimeter energy deposition } \\
\quad \text { (narrow isolated jets with low track multiplicity) }\end{array}$ \\
\hline neutrino & $\begin{array}{l}\text { invisible, use energy balance in the transverse plane } \\
\left.\quad \text { (missing transverse energy, } M E T, E_{T}\right)\end{array}$ \\
\hline light quarks and gluons & $\begin{array}{l}\text { visible as jet: } \\
\text { tracks, energy deposition in the calorimeter }\end{array}$ \\
\hline$b$ quark & $\begin{array}{l}\text { visible as } b \text { jet: } \\
\text { tracks stemming from a displaced secondary vertex, } \\
\text { energy deposition in the calorimeter, } \\
\text { exclusive } B \text { hadron decays, } \\
\text { (low energetic muons from } B \text { meson decays) }\end{array}$ \\
\hline$t$ quark & $\begin{array}{l}\text { unstable, } t \rightarrow b W: \\
b \text { jet, decay products of the } W \text { boson } \\
\quad(\text { electron/muon/tau }+ \text { neutrino or } 2 \text { light quarks })\end{array}$ \\
\hline
\end{tabular}

Figure 4.1: $\quad$ Measured $t \bar{t} \rightarrow b \bar{b} e \mu \nu \nu$ candidate event (left-hand plot: $X Y$ view of the event display, right-hand plot: facsimile). The event display shows reconstructed tracks in the center, and calorimeter towers with energy depositions in the electromagnetic (red) and hadronic layers (blue). Hits in the wire chambers of the muon system are drawn as colored boxes outside the calorimeter towers. The yellow bar indicates the missing transverse energy. Two $b$ jets are identified using secondary vertices (marked as circles in the facsimile). One of the $b$ jets contains a muon from a $B$ meson decay.

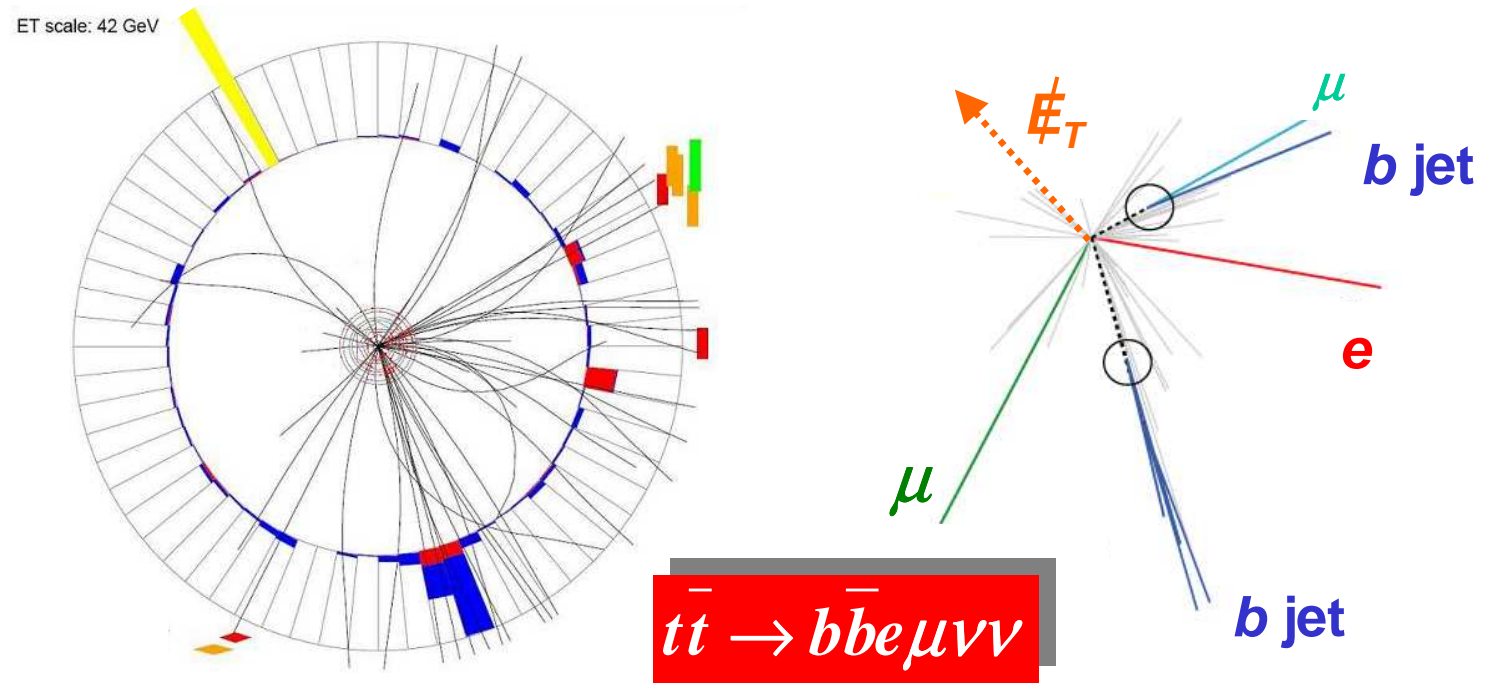


a seed. The seed (initial tower) is required to exceed a certain energy of $E_{T}>0.5 \mathrm{GeV}$. The cone is defined in distances of $\eta$ and $\phi$. The distance $\Delta R_{t}$ of a tower $t$ with respect to the initial tower $i$ is calculated in the following way

$$
\Delta R_{t}=\sqrt{(\Delta \eta)^{2}+(\Delta \phi)^{2}}=\sqrt{\left(\eta_{i}-\eta_{t}\right)^{2}+\left(\phi_{i}-\phi_{t}\right)^{2}} .
$$

Energies are summed up if $\Delta R_{t}<R_{\text {cone }}=0.2$. The cluster is only considered as a possible electron candidate if the total energy is greater than $1.5 \mathrm{GeV}$, and if most of its energy $(>90 \%)$ is deposited in the electromagnetic part of the calorimeter (layers EM1, EM2, $E M 3, E M 4)$. This fraction is referred to as electromagnetic energy fraction emf

$$
e m f=\frac{E_{E M 1}+E_{E M 2}+E_{E M 3}+E_{E M 4}}{E_{E M 1}+E_{E M 2}+E_{E M 3}+E_{E M 4}+E_{F H 1}}=\frac{E_{E M}}{E_{\mathrm{tot}}}>0.9 .
$$

whereas $E_{F H 1}$ denotes the energy deposited in the first fine hadronic layer $(F H 1)$ of the calorimeter. If a track can be assigned to the electromagnetic cluster, it is tagged as $I D= \pm 11$ (depending on the charge of the track). Otherwise, $I D=10$ is assigned to the cluster.

Furthermore, the electron shower is required to be isolated in the following way (see also Fig. 4.2)

$$
\text { iso } \equiv \frac{E_{\text {tot }}\left(\Delta R_{t}<0.4\right)-E_{E M}\left(\Delta R_{t}<0.2\right)}{E_{E M}\left(\Delta R_{t}<0.2\right)}<0.2 .
$$

This criterion selects showers that are narrow and concentrated in the EM part of the calorimeter.

A cut on the $\chi^{2}$ value of the seven-dimensional "HMatrix" $(H M x 7)$ is applied to separate electromagnetic from hadronic showers. The seven input variables are:

- energy fractions in the four EM layers,

- logarithm of the total energy,

- vertex position and

- transverse shower width in $\phi$ direction in the third EM layer.

In case of electrons reconstructed in the endcaps of the calorimeter, the longitudinal shower width in $z$ direction is also taken into account $(H M x 8)$

$$
\begin{array}{rll}
\left|\eta_{\text {det }}\right|<1.1 & : & \chi_{\mathrm{HMx} 7}^{2}<12, \\
1.5<\left|\eta_{\text {det }}\right|<2.5 & : & \chi_{\mathrm{HMx} 8}^{2}<20 .
\end{array}
$$

Figure 4.2: Definition of isolation iso for electromagnetic showers.

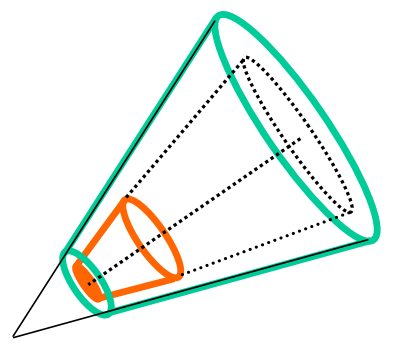

$$
\text { iso }=\frac{E_{0.4}^{t o t}-E_{0.2}^{E M}}{E_{0.2}^{E M}} \equiv \square \square / \square<0.2
$$


Both variables are calculated using a covariance matrix $M_{i j}(i, j=1, \ldots, 7$ or 8$)$

$$
M_{i j}=\sum_{n=1}^{N}\left(x_{i}^{n}-\bar{x}_{i}^{n}\right)\left(x_{j}^{n}-\bar{x}_{j}^{n}\right)
$$

which is determined from $N$ Monte Carlo electrons. The parameters $x_{i}$ denote one of the seven/eight input variables with corresponding mean values $\bar{x}_{i}$. The HMatrix is calculated comparing the variables measured from data with the mean values derived from Monte Carlo electrons incorporating the inverse covariance matrix

$$
\chi_{\mathrm{HMx} 7 / 8}^{2}=\sum_{i, j=1}^{7 / 8}\left(x_{i}^{\mathrm{data}}-\bar{x}_{i}^{\mathrm{MC}}\right) M_{i j}^{-1}\left(x_{j}^{\mathrm{data}}-\bar{x}_{j}^{\mathrm{MC}}\right) .
$$

Note, that the $\chi_{\mathrm{HMx} 7 / 8}^{2}$ distributions do not follow true $\chi^{2}$ distributions because in general the observables $x_{i}$ do not follow Gaussian distributions. However, the HMatrix variable is useful to separate electromagnetic from hadronic showers.

The electron is required to have a track matched in $z$ and $\phi$ direction (spatial track match) with the probability

$$
P\left(\chi_{\text {spatial }}^{2}\right)>0.01 \quad \text { with } \quad \chi_{\text {spatial }}^{2}=\left(\frac{\Delta z}{\sigma_{z}}\right)^{2}+\left(\frac{\Delta \phi}{\sigma_{\phi}}\right)^{2} .
$$

The variable $\Delta x=x_{E M}-x_{\text {track }}(x=\phi, z)$ denotes the difference between the electron variable $x_{E M}$ and the track variable $x_{\text {track }}$ extrapolated into the third EM layer. The corresponding uncertainties are given by $\sigma_{x}$. A full track match

$$
P\left(\chi^{2}\right)>0.01 \quad \text { with } \quad \chi^{2}=\left(\frac{\Delta z}{\sigma_{z}}\right)^{2}+\left(\frac{\Delta \phi}{\sigma_{\phi}}\right)^{2}+\left(\frac{E_{T} / p_{T}-1}{\sigma_{E_{T} / p_{T}}}\right)^{2}
$$

including $E / p$ matching is not applied due to the steeply decreasing resolution of the tracker with increasing transverse momenta/energies, see left-hand plot in Fig. 4.3 (compare tracker resolution $\Delta p_{T} \propto p_{T}^{2}$ to calorimeter resolution $\Delta E \propto E$ for $\left.E \gg 50 \mathrm{GeV}\right)$. The right-hand plot in Fig. 4.3 indicates the precise match in $\phi$ direction. Due to the fact that the efficiency for the full track match is considerably lower than for the spatial track match and that the full track match does not improve the identification of high energy electrons, only a cut on $P\left(\chi_{\text {spatial }}^{2}\right)$ is considered.

In the present analysis electrons reconstructed in the central calorimeter $\left(\left|\eta_{\text {det }}\right|<1.1\right)$ are considered. Cuts on the electromagnetic fraction, isolation, HMatrix7 and spatial track match probability are applied in order to select well defined electron candidates. Efficiencies of the identification criteria and differences observed in data and Monte Carlo reconstruction efficiencies are discussed in Sec. 7.2.1.

The electron identification criteria introduced above also hold for photon identification, except for the track match condition. In this case, a veto is usually applied, e. g. $P\left(\chi^{2}\right)<$ 0.001 [109]. A more sophisticated photon identification is used in [110]. In order to discriminate between electrons, photons and hadronic objects in the calorimeter, electron likelihood variables have been designed which use different numbers and types of variables. Details can be found in [111]. 
Figure 4.3: Transverse energy of the electron $E_{T}$ from $W^{\prime}$ decays $(M C)$ vs. difference between the transverse energy/momentum measured with the calorimeter $E_{T}$ and tracker $p_{T}^{\text {track }}$ (left-hand plot). The right-hand plot indicates the precise matching in $\phi$ direction.
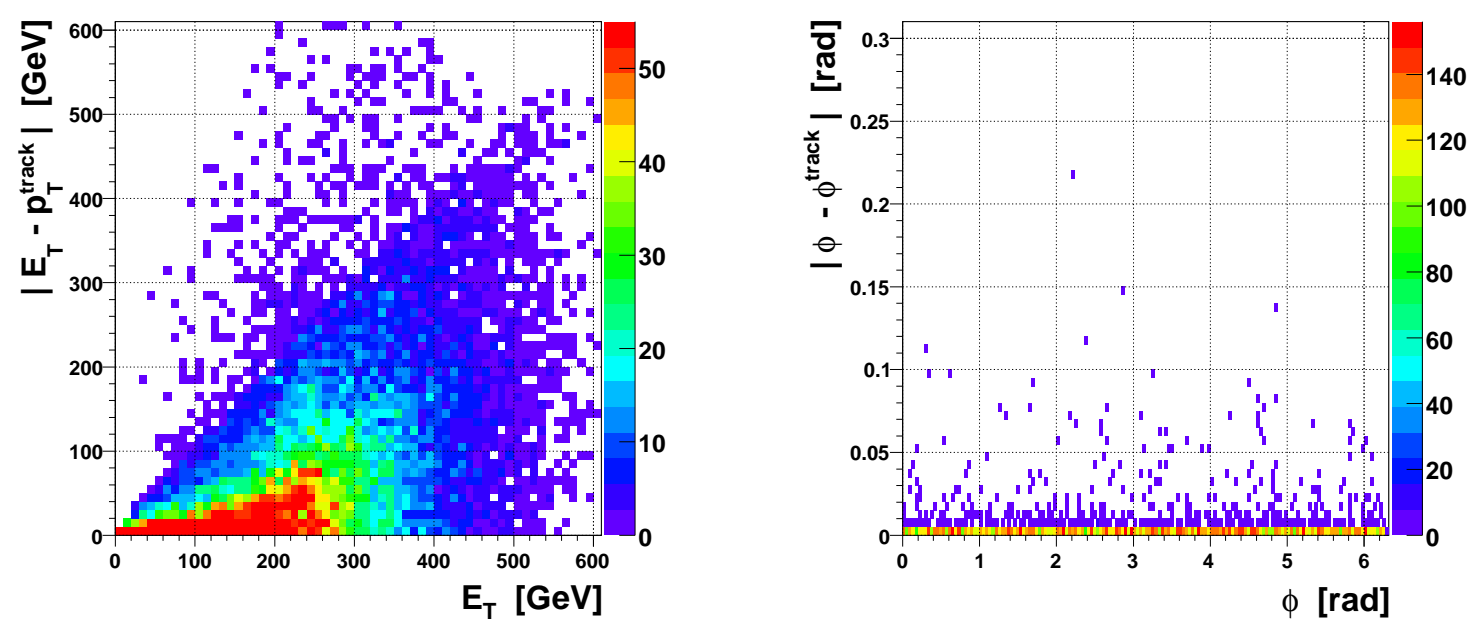

Figure 4.4: Width of the electromagnetic shower in $\phi$ direction in the third EM layer $\left(\sigma_{\phi}^{E M 3}\right)$ as a function of the electron transverse energy $E_{T}$ (left-hand plot). In the right-hand plot the mean values of the $\sigma_{\phi}^{E M 3}$ distribution (extracted from the scatter plot) as a function of $E_{T}$ are shown.
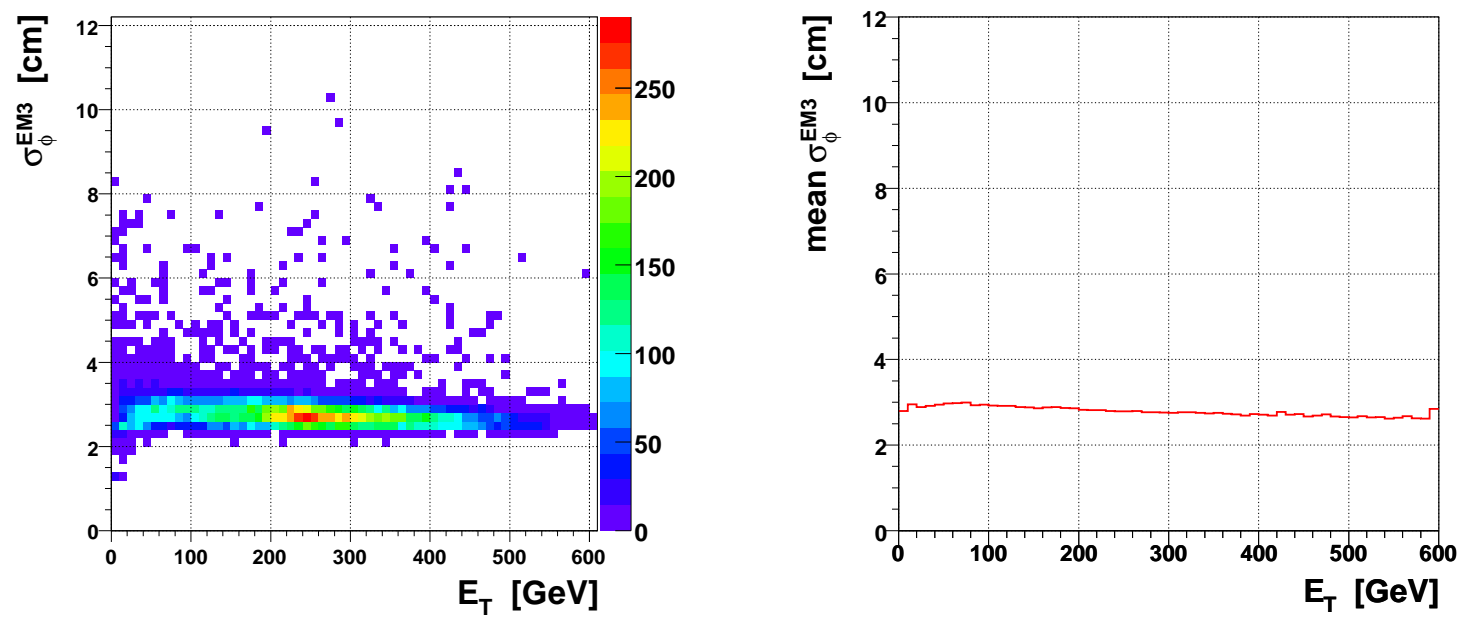
Figure 4.5: Variable iso as a function of the electron transverse energy $E_{T}$ (left-hand plot). In the right-hand plot the mean values of the iso distribution (extracted from the scatter plot) as a function of $E_{T}$ are shown. The green line indicates the cut iso $<0.2$.
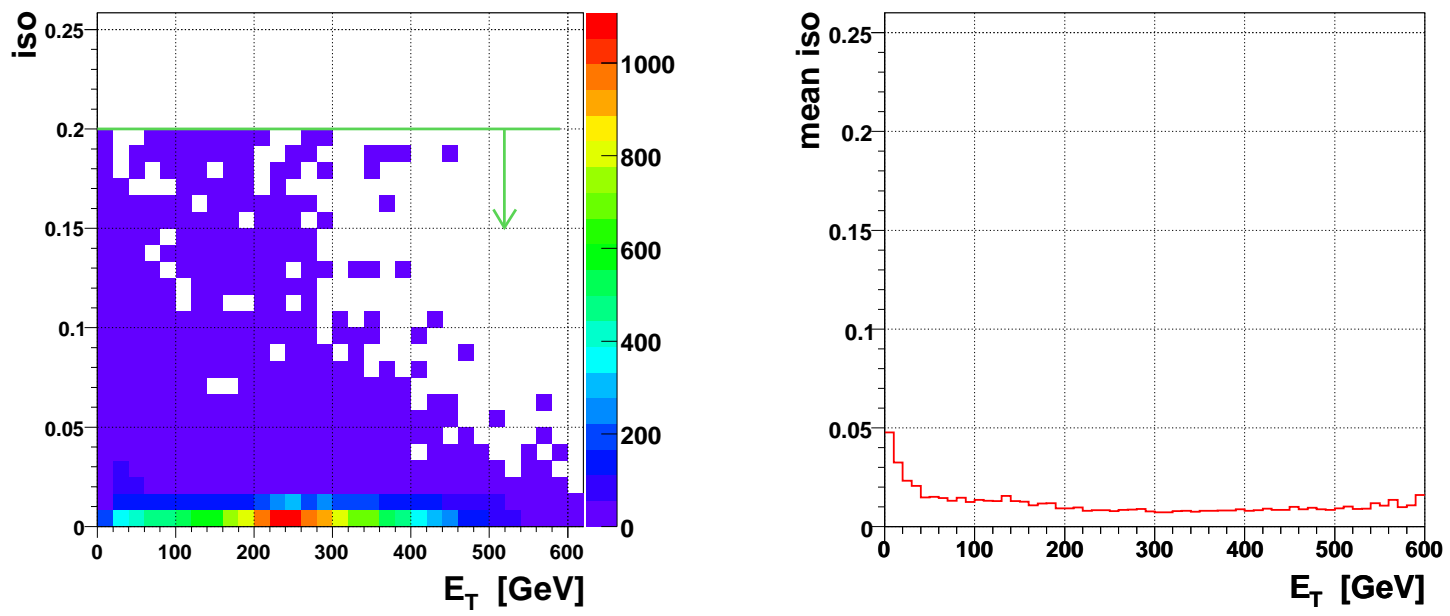

Figure 4.6: Variable emf as a function of the electron transverse energy $E_{T}$ (left-hand plot). In the right-hand plot the mean values of the emf distribution (extracted from the scatter plot) as a function of $E_{T}$ are shown. The green line indicates the cut emf $>0.9$.
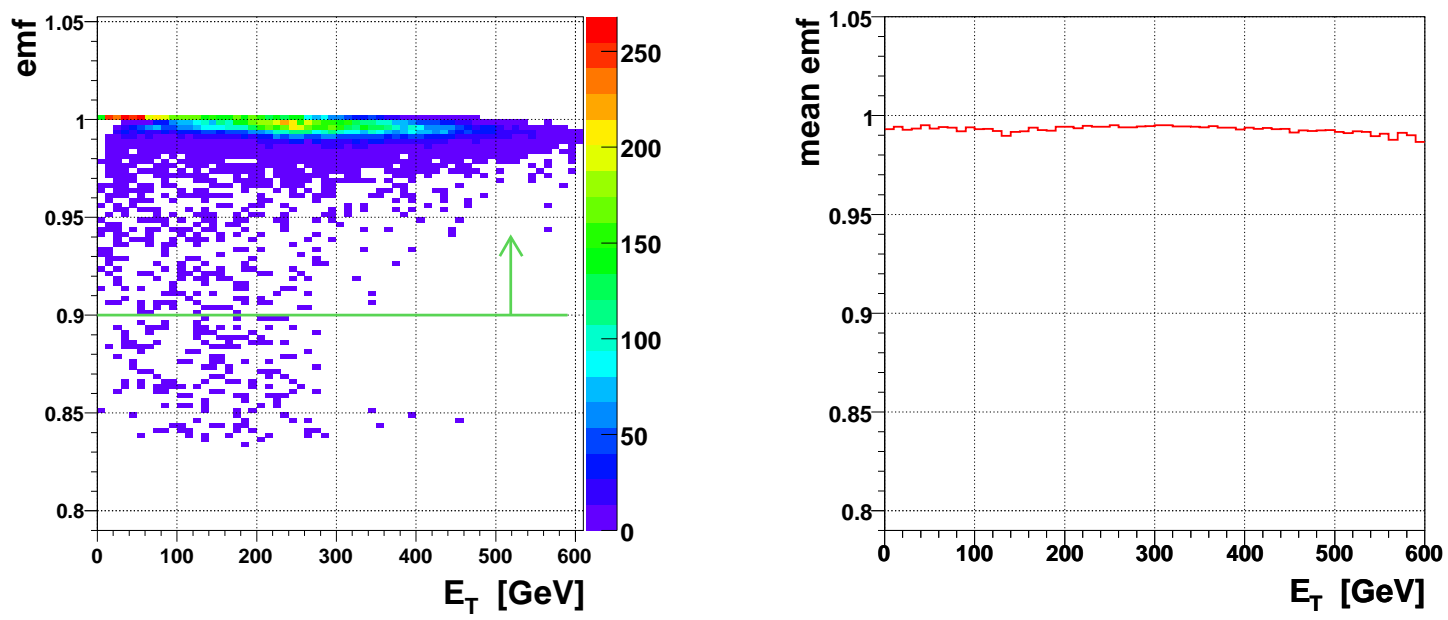
Figure 4.7: Relative energy fractions in the four EM layers as a function of the electron transverse energy $E_{T}$ (upper plots). In plot at the bottom the mean values of the energy distributions (extracted from the scatter plots) as a function of $E_{T}$ are shown. In addition to the EM layers, the dependency of the energy fraction deposited in FH1 is displayed (see also Fig. 4.6).
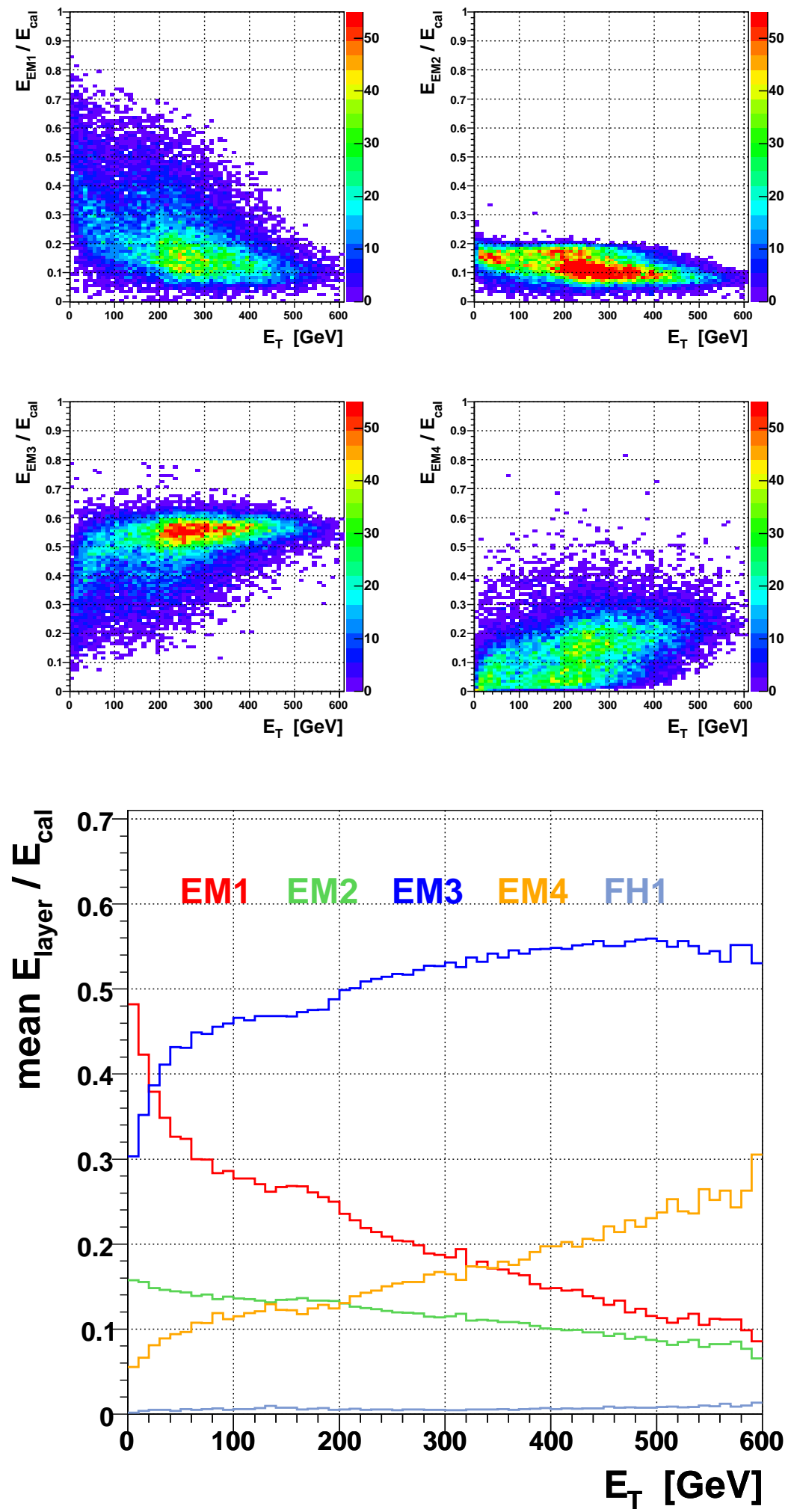
Figure 4.8: Variable $\chi_{\mathrm{HMx}}^{2}$ as a function of the electron transverse energy $E_{T}$ (left-hand plot). In the right-hand plot the mean values of the $\chi_{\mathrm{HMx} 7}^{2}$ distribution (extracted from the scatter plot) as a function of $E_{T}$ are shown. The green line indicates the cut $\chi_{\mathrm{HMx} 7}^{2}<12$.
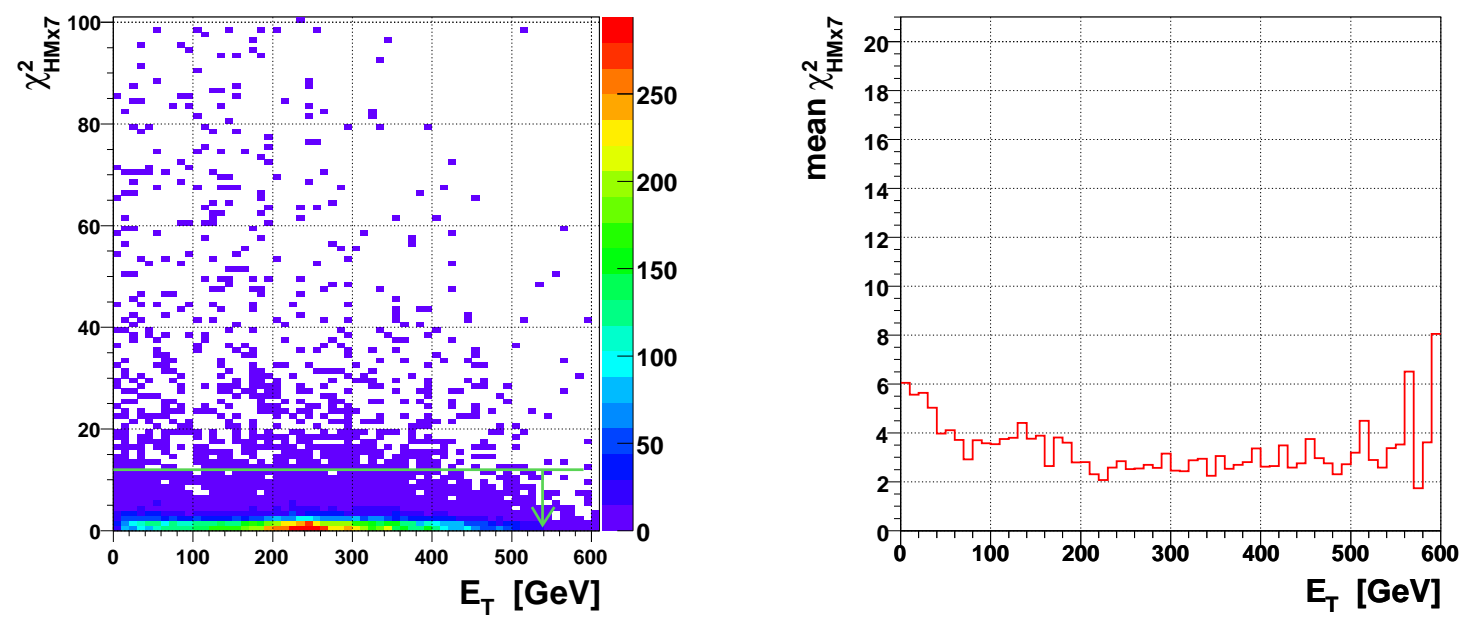

The energy dependence on the basic electron reconstruction criteria has been studied with Monte Carlo electrons from $W^{\prime}$ decays because they span a large energy range (see Fig. 5.10). The plots in Figs. $4.4-4.8$ show various shower and identification variables as a function of the transverse energy of the electron $E_{T}$ reconstructed in the central calorimeter, $\left|\eta_{\text {det }}\right|<1.1$. The results of these studies are:

- The width of the electromagnetic shower in $\phi$ direction $\sigma_{\phi}^{E M 3}$ in the third EM layer does not show a strong dependence on the transverse energy of the electron (see Fig. 4.4).

- Also the isolation does not depend on the transverse energy of the electron (see Fig. $4.5)^{1}$.

- With increasing energy a small increase of energy deposited in the first fine hadronic layer FH1 can be observed. This leads to a small decrease of the mean of the electromagetic fraction emf (see Fig. 4.6 and Fig. 4.7). The efficiency using the 'standard' cut (emf $>0.9)$ is not affected.

- The relative energy fractions in the four EM layers change. With increasing electron energy the shower tends to deposit more energy in the third and fourth layer, and less energy in the first two layers (see Fig. 4.7).

- The $\chi^{2}$ distribution of the HMatrix (Fig. 4.8) does not depend on the electron energy, in spite of making use of the (energy dependent) shower variables. This is due to the fact that the HMatrix is tuned resulting in an energy independent variable ${ }^{2}$.

In summary, the standard cuts on electron reconstruction variables can be used, since there is no evidence for a strong energy dependence.

\footnotetext{
${ }^{1}$ The selection $|I D|=10$ or 11 contains an implicit cut on $i s o<0.2$.

${ }^{2}$ This was not the case in older software releases [112].
} 


\subsection{Jet Reconstruction}

Quarks and gluons (parton jets, see Fig. 4.9) can only be observed after fragmentation and hadronization, see detailed discussion in Sec. 5.1. The resulting particle jets containing hadrons deposit most of their energy in the calorimeter. It is possible to reconstruct jets in the tracker, but the best energy resolution is obtained from the calorimeter (calorimeter jets). However, tracking information is consulted to identify $b$ quark jets which originate from secondary vertices.

Jets are not part of the electron + missing transverse energy final state, but may be present in an event and contribute to missing energy. This section is dedicated to a discussion of the jet reconstruction algorithm, the jet identification criteria and finally the jet energy scale.
Figure 4.9: From partons to jets in the calorimeter [113].

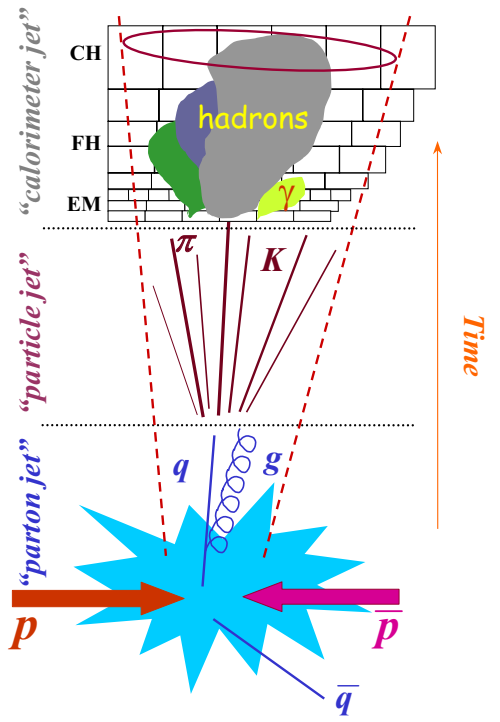

Jets are reconstructed using a cone algorithm [114]: all calorimeter energies contained in a cone with radius $R_{\text {cone }}=0.5$ are summed up. The cone is defined in the $\eta-\phi$ plane in order to ensure invariance under boosts along the $z$-axis. Ideally, the energy of the primary parton is given by the jet energy, and the cone axis corresponds to the jet direction which is defined as the transverse energy weighted centroid of the particles within the cone. The algorithm starts with the most energetic calorimeter tower above a given threshold $E_{T}^{\min }=1 \mathrm{GeV}$ ( seed). Pre-clusters are built by adding neighboring towers within $\Delta R=\sqrt{(\Delta \eta)^{2}+(\Delta \phi)^{2}}<0.3$ of the seed towers. The pre-clustering is necessary in order to reduce the number of seeds passed to the main algorithm keeping the calculation computationally feasible. A cone of radius $R_{\text {cone }}=0.5$ with center at the centroid of the pre-cluster is formed around each pre-cluster. Then, the new jet center is determined by adding all calorimeter towers within $R_{\text {cone }}$ around each pre-cluster. This procedure is repeated iteratively for each of the seeds until the jet center is stable. The resulting objects of this procedure are called proto-jets and sorted in descending order of transverse energy. Note, that two proto-jets may overlap $\left(\Delta R<2 R_{\text {cone }}\right)$ and share calorimeter towers. If this is not the case, the proto-jet is considered as final. Otherwise, neighboring proto-jets are merged into a single jet if an energy fraction of more than $f=0.5$ of the lower-energy jet is contained in the overlap region. If the fraction is smaller, the energy in the shared towers is assigned to the nearest jet. In both cases, energy and momentum have to be recomputed. Jets with $E_{T}<6 \mathrm{GeV}$ are discarded.

It has to be checked if the jet algorithm introduced above exhibits the following dependencies [114]:

\section{- Detector sensitivity}

There should not be any dependence on boosts in longitudinal directions, the luminosity, on the detector performance and detector region. 
Figure 4.10: Seed collinear (left), $E_{T}$ ordering (middle) and infrared (right) sensitivity of the jet cone algorithm [114].
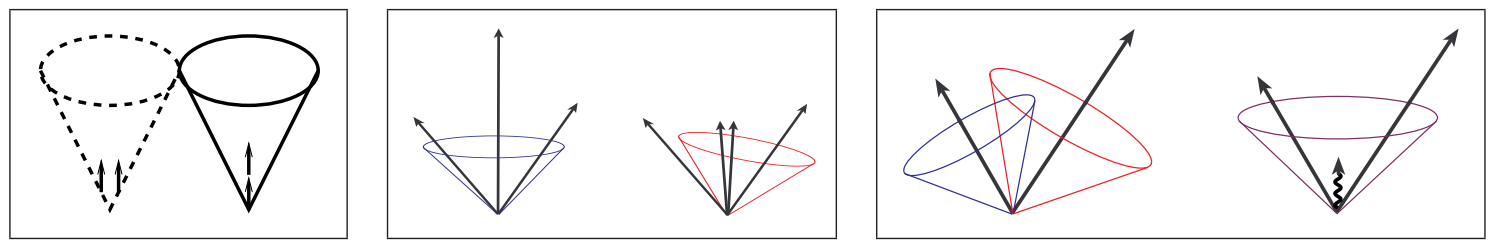

- Seed collinear sensitivity (left sketch in Fig. 4.10)

Individual towers might fail to produce a seed because the energy is split among several towers. The jet algorithm used by $\mathrm{D} \varnothing$ is found to be fully collinear safe for seed thresholds of $E_{T}^{\min }=1 \mathrm{GeV}$.

- $E_{T}$ ordering sensitivity (middle sketch in Fig. 4.10)

If the jet algorithm depends on the $E_{T}$ ordering of towers, another collinear problem appears as shown in the middle sketch. The separation between the most distant partons be more than $R_{\text {cone }}$ but less than $2 R_{\text {cone }}$ so that the three partons are reconstructed within one jet (blue cone). If the central (hardest) parton is split, the right parton has the largest energy. Hence this seed is looked at first, and a jet may be found containing only the right and two central partons (red cone). The left parton is a jet by itself. This can be avoided by making the ordering of seeds independent of $E_{T}$.

- Infrared sensitivity (right sketch in Fig. 4.10)

If a jet algorithm considers only seeds exceeding a minimum amount of energy, it is not infrared safe. In this case, if a particle radiates a soft gluon which is considered as a seed, two clearly separated towers can be merged into one cone.

The problems outlined above are introduced by seed based algorithms. Since only seedless clustering is infrared safe and insensitive to collinear radiation, a set of unbiased seeds has to be provided. This can be accomplished by considering each tower as a possible seed. This would translate into $\mathcal{O}\left(10^{3}\right)$ towers which is technically unfeasible, but can be avoided using pre-clustered objects as outlined above. Further, the infrared sensitivity is removed by adding midpoints between pairs of proto-jets with $\Delta R<2 R_{\text {cone. }}$. The midpoint jet algorithm is referred to as Improved Legacy Cone Algorithm [114].

Jets reconstructed using the midpoint cone algorithm with $R_{\text {cone }}=0.5$ are only looked at if they have transverse energies above $15 \mathrm{GeV}$, are located within $|\eta|<2.5$ and pass the following standard jet identification criteria [115]. The fraction of energy deposited in the EM layers of the calorimeter is required to be less than $95 \%$

$$
e m f<0.95 \text {. }
$$

However, jets are expected to deposit a minimal amount of energy in the EM layers of the calorimeter. This cut depends on the location of the jet with respect to the center of the detector $\left(\eta_{\text {det }}\right)$ and to the primary vertex $(\eta)$, and on the width $\sigma_{\eta}$ of the jet in $\eta$ direction 


$$
\begin{aligned}
& \quad \text { emf }>0.05 \\
& \text { or } \quad\left(\text { emf }>0.03 \text { and } 1.1<\left|\eta_{\text {det }}\right|<1.4\right) \\
& \text { or } \quad\left(\text { no emf cut and } 1.3>|| \eta_{\text {det }}|-1.25|+\max \left\{0,40 \cdot\left(\sigma_{\eta}-0.1\right)\right\}\right) .
\end{aligned}
$$

Furthermore, jets are required not to deposit a significant amount of energy outside the EM and fine hadronic layers. Hence, a cut on the coarse hadronic fraction chf is applied

$$
\begin{array}{ll} 
& \operatorname{chf}<0.4 \\
\text { or } & \left(\operatorname{chf}<0.6 \text { and } 0.85<\left|\eta_{\operatorname{det}}\right|<1.25 \text { and } n 90<20\right) \\
\text { or } \quad(\operatorname{chf}<0.44 \text { and } \quad|\eta|<0.8) \\
\text { or } \quad(\operatorname{chf}<0.46 \text { and } 1.5<|\eta|<2.5) .
\end{array}
$$

$n 90$ denotes the number of towers containing $90 \%$ of the jet energy. This cut is necessary in order to remove jets which are dominated by noise originating in the coarse hadronic calorimeter, especially around $\left|\eta_{\text {det }}\right| \approx 1$. The jet should be confirmed by the Level 1 readout [116]. Because of this, a cut on the Level 1 ratio

$$
L 1_{\text {ratio }}=\frac{E_{T}(\text { from Level } 1 \text { readout })}{E_{T}(\text { from precision readout })}
$$

is applied with

$$
\begin{array}{ll} 
& L 1_{\text {ratio }}>0.5 \\
\text { or } & \left(L 1_{\text {ratio }}>0.35 \text { and } E_{T}<15 \mathrm{GeV} \text { and }|\eta|>1.4\right) \\
\text { or } & \left(E_{T}(\text { from Level } 1 \text { readout })>55 \mathrm{GeV}\right) .
\end{array}
$$

The jet selection introduced above exhibits an overall efficiency of $\sim 99 \%$ [115].

The Jet Energy Scale (JES) is responsible for the translation from measured jet energies $E^{\text {reco }}$ to particle level energies $E^{\text {corr }}$ (see Fig. 4.9). Despite the fact that the calorimeter is a very powerful device for absorbing electromagnetic and hadronic energies of jets, several mechanisms can cause deviations of parton and measured jet energies. The corrected jet energy can be derived from the measured energy via

$$
E^{\mathrm{corr}}=\frac{E^{\mathrm{reco}}-O}{R \cdot S}
$$

The following relevant contributions are taken into account:

\section{- Calorimeter response $R$}

The response of the calorimeters depends not only on calorimeter regions ( $\eta$ dependency, inhomogeneous instrumentation, dead material), but can also be distorted for different partons. $R$ is measured using $q g \rightarrow q \gamma$ events making use of the electromagnetic energy scale for the photon which is known at high accuracy from $Z \rightarrow e e$ decays.

\section{- Energy offset $O$}

Additional energy in the calorimeter stemming from the underlying event, pile-up and noise from the uranium absorber material or from the electronics causes an energy offset. This contribution which can be determined in events without any hard interaction has to be subtracted. 


\section{- Showering correction $S$}

This correction describes energy losses due to particles which are not contained in the cone, and is measured from jet energy profiles.

The JES is parameterized as a function of the transverse energy $E_{T}$ and the location $\eta$. Due to differences observed in data and Monte Carlo events, a separate correction is applied on simulated events. The total uncertainty on the JES which contains statistical and systematic contributions from both, data and Monte Carlo, is given by

$$
\sigma_{\mathrm{JES}}=\sqrt{\sigma_{\mathrm{stat}, \mathrm{data}}^{2}+\sigma_{\mathrm{sys}, \mathrm{data}}^{2}+\sigma_{\mathrm{stat}, \mathrm{MC}}^{2}+\sigma_{\mathrm{sys}, \mathrm{MC}}^{2}} .
$$

Finally, in order to compensate for differences between data and Monte Carlo, further corrections are applied to the Monte Carlo jets (referred to as Jet-Shifting-SmearingRemoval procedure [117]). Details regarding the jet efficiency measurement and the Monte Carlo corrections are compiled in [118].

\subsection{Neutrino Reconstruction}

The neutrino is the only stable particle in the Standard Model that does not interact with the detector material and is hence not directly measurable. However, it can be identified indirectly using the momentum conservation in the transverse plane with respect to the beam axis (z-axis), see Eq. 2.12. In this case, the transverse energy of the neutrino $E_{T}^{\nu}$ corresponds to the missing transverse energy $\mathbb{E}_{T}$ (or "MET") in the event. This can clearly be seen in $W^{\prime} \rightarrow e \nu$ Monte Carlo events containing one neutrino, see left-hand plot in Fig. 4.11. Note, that also other neutral, very weakly interacting particles introduced in models beyond the Standard Model, like the lightest neutralino $\tilde{\chi}_{1}^{0}$ in $R$ parity conserving SUSY, would give rise to missing energy. Further, in case of more than one "invisible" particle in the final state (e.g. $\tau \rightarrow e \nu \nu$ ) one can only access the vector sum

$$
\vec{E}_{T}=\sum_{\mathrm{SM}} \vec{E}_{T}^{i}(\nu)+\sum_{\text {non SM }} \vec{E}_{T}^{i}(\text { invisible }) .
$$

In addition, since MET is calculated from other measured visible objects in the event

$$
\vec{E}_{T}=-\sum_{\text {visible }} \vec{E}_{T}^{v}
$$

missing energy may emerge from the limited detector resolution, even in events without neutrinos ("fake" MET). The impact of detector failures and noise on the missing energy is shown in the right-hand plot in Fig. 3.34 (Sec. 3.5).

The missing transverse energy is a quantity which depends not only on the entire detector, but also on all object corrections, like the electromagnetic and jet energy scale. Because of this, the MET is calculated in several steps [119], [120]:

- The raw MET is derived from all cells located in the electromagnetic, fine hadronic calorimeter and intercryostat detector. All cells with positive energy deposits are 
summed up

$$
\begin{aligned}
& E_{x}^{\text {raw }}=-\sum_{i \in\{\text { cells with } E>0\}} E_{x}^{i}
\end{aligned}
$$

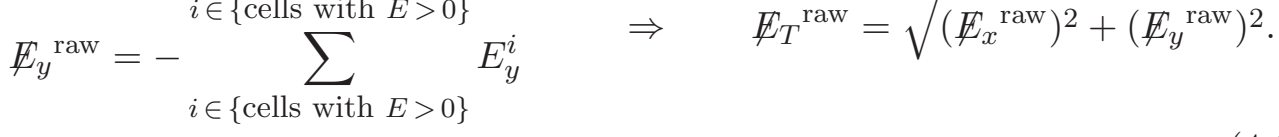

Note, that the contribution from the coarse hadronic cells is not taken into account due to the fact that these cells are too noisy (exception: coarse hadronic cells within "good" jets, see below). The raw MET is calculated whithin dØreco with respect to the primary vertex.

- Jets, electrons, photons and muons are reconstructed as well within dØreco, but corrections to the object identification are applied within dØcorrect. Because of this, the raw MET has to be corrected

- for the EM scale $d \vec{E}_{T}{ }^{\mathrm{EM}}$,

- for the jet energy scale $d \vec{E}_{T}$ JES

- taking into account the coarse hadronic contribution of jets passing the standard identification criteria outlined in the previous section, $d \vec{E}_{T}{ }_{T}$ and

- for the muon momentum and muon energy deposition in the calorimeter.

$$
d \vec{E}_{T}^{\mathrm{MU}}=-\sum_{\text {muons }} \vec{p}_{T}+\sum_{\text {muons }} \vec{E}_{T}^{\text {mip }} .
$$

The energy deposition in the calorimeter $\vec{E}_{T}^{\text {mip }}$ is already contained in $\vec{E}_{T}^{\text {raw }}$ and has therefore to be removed for the full muon correction.

- The final MET is given as follows

$$
\vec{E}_{T}=\vec{E}_{T}^{\text {raw }}+d \vec{E}_{T}^{\mathrm{EM}}+d \vec{E}_{T}^{\mathrm{JES}}+d \vec{E}_{T}^{\mathrm{CH}}+d \vec{E}_{T}^{\mathrm{MU}} .
$$

In case of final updates of any of the corrections, it is possible to recalculate the $\mathrm{MET}$ within $\mathrm{CAFE}^{3}$.

In this analysis the muon correction is not taken into account $\left(d \vec{E}_{T}{ }^{\mathrm{MU}}=0\right)$ because neither the signal nor the dominant backgrounds are expected to contain muons. Further, Monte Carlo simulations do not describe the fully corrected MET very well. This is likely due to the fact that the transverse momentum resolution of high- $p_{T}$ muons is underestimated so that badly measured high- $p_{T}$ muons enter the full muon correction and produce fake MET [121].

The resolution of the missing transverse energy can be derived using $W^{\prime} \rightarrow e \nu$ Monte Carlo events ( $E_{T}^{\nu}$ Monte Carlo neutrino transverse energy, $E_{T}$ missing transverse energy). For energies $\mathbb{E}_{T}=(0.9 \ldots 1.1) \cdot E_{T}^{\nu}$ the resolution distribution $\left(E_{T}^{\nu}-E_{T}\right) / E_{T}^{\nu}$ is fitted using a Gaussian distribution, see right-hand plot in Fig. 4.11. The core resolution of $5.1 \%$ is determined by the electron energy resolution of $4.1 \%$ (see Sec. 7.1), slightly deteriorated by additional energy deposits in the calorimeter due to noise and multiple interactions, while the non-Gaussian tails are mostly due to a fraction of events containing additional jets and due to cells which are not assigned to any reconstructed objects (unclustered energy [120]).

\footnotetext{
${ }^{3}$ The full processing chain $(\mathrm{d} \emptyset$ reco, $\mathrm{d} \emptyset$ correct, ...) is shown in Fig. 6.1 in Sec. 6.1.
} 
Figure 4.11: Resolution of the missing transverse energy derived from $W^{\prime} \rightarrow$ ev Monte Carlo events. The plot on the left-hand side shows the missing transverse energy $\mathbb{E}_{T}$ as a function of the true neutrino transverse energy $E_{T}^{\nu}$. The right-hand plot displays the resolution. The dotted blue line indicates a fit with a Gaussian distribution (mean: 0, width: 0.051).
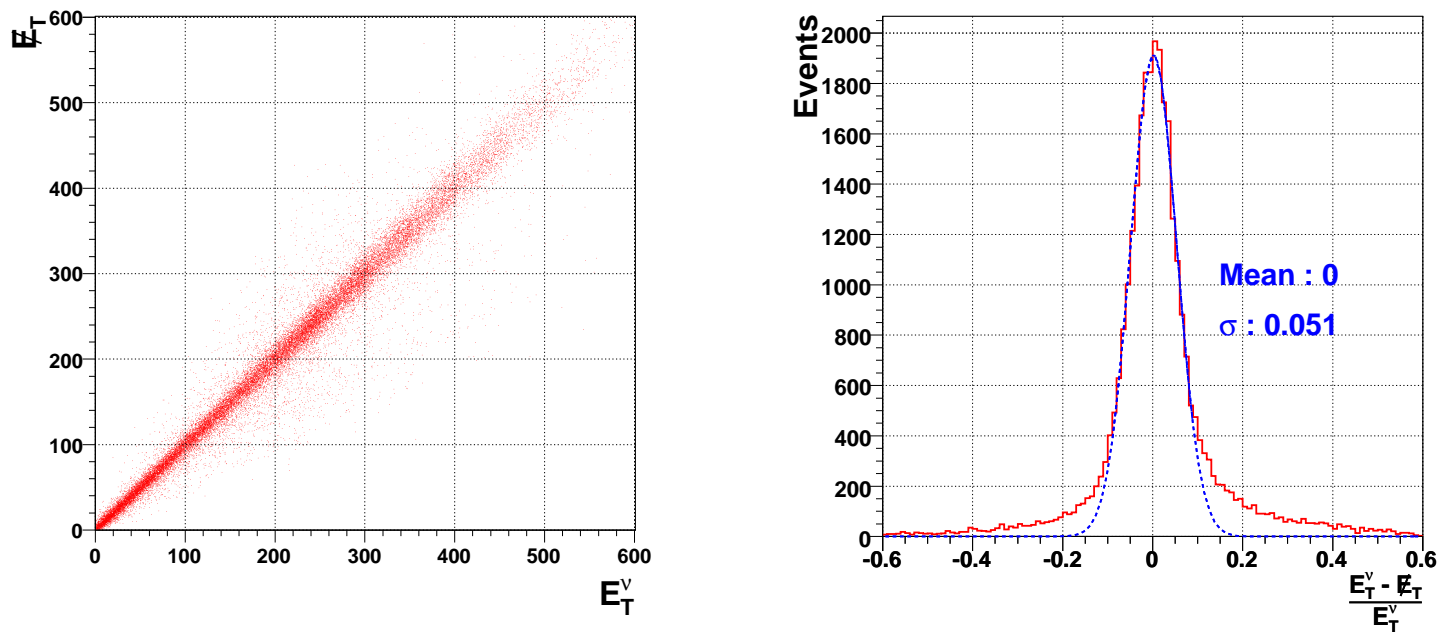


\title{
Chapter 5
}

\section{Simulation of Background and Signal Events}

\author{
Nothing is too wonderful to be true \\ if it be consistent with the laws of nature. \\ Michael Faraday
}

In the present analysis proton-antiproton collisions are searched for new gauge bosons decaying into an electron and an electron neutrino. The neutrino can not be detected, but arises via missing transverse energy $\mathbb{E}_{T}$ in the detector. No further objects are required, but may be present in the inclusive $\left(e+\mathbb{E}_{T}+X\right)$ final state, like jets stemming from quarks, other neutrinos or leptons. The search is accomplished by comparing the data with simulated events from the expected signal process and Standard Model processes (background processes) that contribute to the inclusive final state.

This chapter is dedicated to the simulation of events at DØ. First, the simulation chain is reviewed, followed by a discussion of the Standard Model backgrounds (see Fig. 5.1). The third section deals with properties of the signal process.
Figure 5.1: Cross sections and event rates for the signal and background processes at a center-of-mass energy of $\sqrt{s}=1.96 \mathrm{TeV}$.

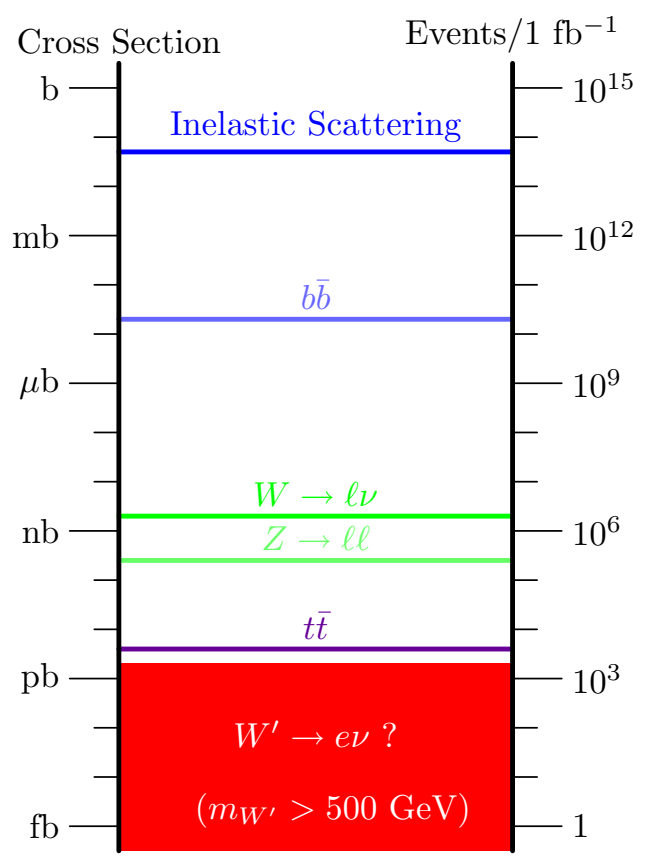




\subsection{Simulation Chain}

The full simulation chain which consists of several steps is shown in Fig. 5.2 for the process $p \bar{p} \rightarrow Z+X \rightarrow q^{\prime} \bar{q}^{\prime}+X$. The first major part (event generation, fragmentation and hadronization) does not depend on the detection device, whereas the second part involves detector related software. Information about Monte Carlo simulation at the D $\varnothing$ experiment can be found at [122].

Standard Model background events as well as signal events are generated with the parton shower Monte Carlo generator Pyтнia [123]. After configuring the initial state (1), the parton distribution functions (2) incorporate the transition from the initial proton and antiproton to (anti)quarks that participate in the hard interaction (3), here: $q \bar{q} \rightarrow Z$. If a resonance is produced, the subsequent decay is counted among the hard process $(2 \rightarrow 1 \rightarrow 2$ process $\hat{=} 2 \rightarrow 2$ process $)$. In addition, PyтHIA does not only take care of the beam remnants (relics of the colliding proton/antiproton), but also of the simulation of initial- and final-state radiation (e.g. $q \rightarrow q g, e \rightarrow e \gamma$ ).

As outlined in Sec. 2.2.3 and 2.2.5, QCD as a perturbative theory is only valid at short distances. At long distances, perturbation theory breaks down, and partons become strongly interacting with the result of the confinement of partons. The transition from colored partons into colorless hadrons is mediated by fragmentation (4), whereas hadronization (5) itself denotes the combination of fragmentation and the subsequent de-

Figure 5.2: Simulation Chain: from the proton-antiproton collision to reconstructed jets.

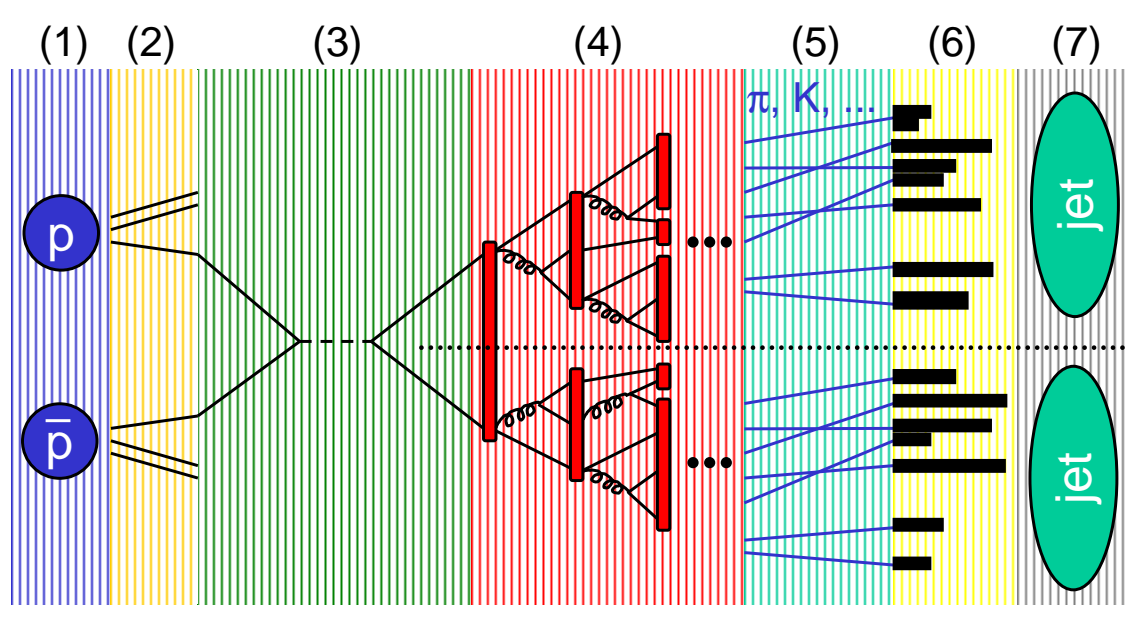

\begin{tabular}{|c|c|c|}
\hline $\begin{array}{l}(3) \\
(4) \\
(5)\end{array}$ & $\begin{array}{l}\text { initial state, experimental setup, e.g. } p \rightarrow \leftarrow \bar{p} \\
\text { parton distribution functions (PDFs) incorporate the } \\
\text { transition from (anti)protons to (anti)quarks } \\
\text { matrix element from the theory, e.g. } q \bar{q} \rightarrow Z \rightarrow q^{\prime} \bar{q}^{\prime} \\
\text { fragmentation according to the Lund string model } \\
\text { strings split up into hadrons, decays of hadrons } \\
\text { (hadronization = fragmentation + decays) }\end{array}$ & PYтhiA \\
\hline (6) & $\begin{array}{l}\text { simulated detector response and } \\
\text { digitization of the detector signal } \\
\text { reconstructed objects: jets in the calorimeter }\end{array}$ & $\begin{array}{l}\text { dØgstar } \\
\mathrm{d} \emptyset \text { sim } \\
\mathrm{d} \emptyset \text { reco }\end{array}$ \\
\hline
\end{tabular}


cay of unstable particles (according to [123]). Since the fragmentation process is not yet understood from first principles (i.e. the QCD Lagrangian), phenomenological models have to be consulted. PythiA makes use of the Lund string model [124] which belongs to the group of string fragmentation models ${ }^{1}$. Fig. 5.3 (left) displays the principle of the fragmentation of a color-singlet $q \bar{q}$. In this case, the energy is stored in a linear color dipole field (yellow band) which increases with the separation. If enough energy is stored, the string breaks and produces a new $q^{\prime} \bar{q}^{\prime}$ pair resulting in two color-singlet systems $q \bar{q}^{\prime}$ and $q^{\prime} \bar{q}$. The fragmentation process ends if on-mass-shell hadrons remain. The right-hand plot in Fig. 5.3 illustrates this for the fragmentation of a $c \bar{c}$ system into hadrons.

Figure 5.3: Left: Fragmentation of a $q \bar{q}$ system. Right: The fragmentation of a $c \bar{c}$ system ends if on-mass-shell hadrons remain [127].
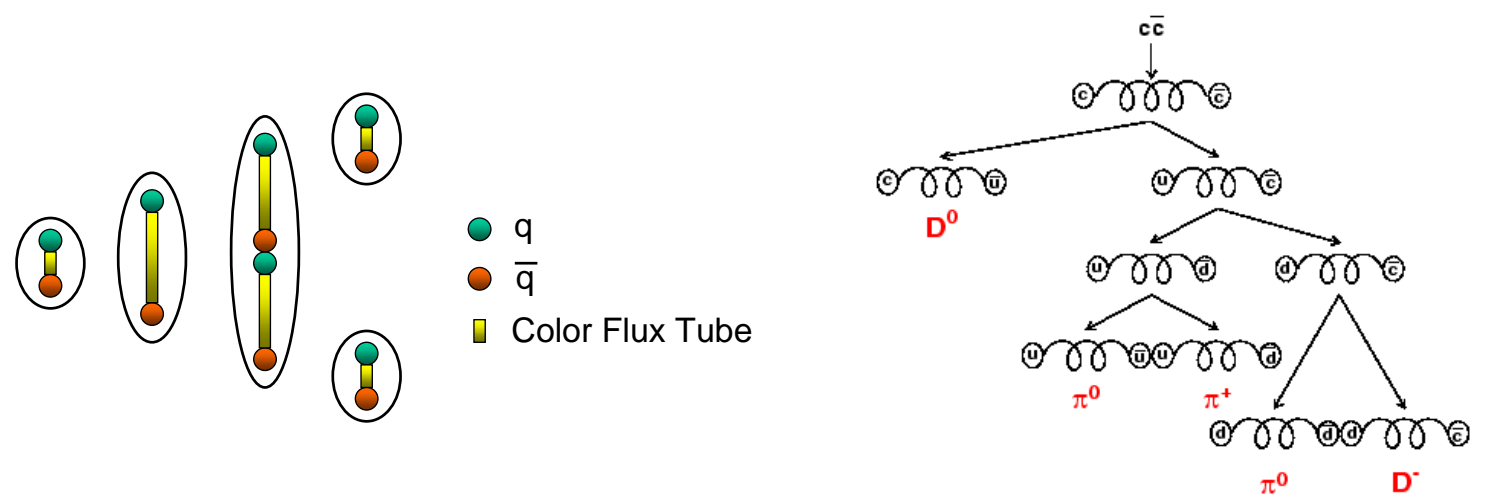

The detector related parts of the simulation chain (6), (7) consist of three programs: $\mathrm{d} \emptyset$ gstar, $\mathrm{d} \emptyset$ sim and $\mathrm{d} \emptyset$ reco. $\mathrm{d} \emptyset \mathrm{gstar}(\mathbf{D} \emptyset$ GeANT Simulation of the Total Apparatus Response) is the full simulation of the D $\varnothing$ detector and uses GEANT [128] in order to describe the passage of elementary particles through matter and thus to determine how much energy is deposited in the active areas of the detector. The output file is processed through $\mathrm{d} \emptyset$ sim which simulates the electronics (digitization and raw data simulation), manages the handling of multiple interactions (see below) and creates the RDC. Furthermore, several sources of noise in the detector components and inefficiencies are included in this step. Finally, dØreco (see Sec. 3.5) reconstructs the events and creates output files in the ThumbNail format which are then stored in SAM. The CSG is again responsible for the centralized production of CAF Root trees (using tmb_analyze [93]; the postprocessing corrections are accomplished within dØcorrect [95]). Information about the available Monte Carlo samples is summarized at [129] and [130].

It is possible to simulate multiple interactions in a beam crossing (pile-up, see footnote on p. 53 and Fig. 5.4), but the agreement between data and Monte Carlo prediction is not satisfying. Hence, real data events prepared with the program dØraw2sim [131] are used and included in the simulation chain. These events - referred to as zero bias events - are recorded using a special trigger condition ${ }^{2}$. The plot in Fig. 5.5 shows a comparison between selected $Z \rightarrow \mu \mu$ data events (blue) and a Monte Carlo sample including simulated

\footnotetext{
${ }^{1}$ The most common models are string, independent and cluster fragmentation [125], [126].

${ }^{2}$ The zero bias trigger uses the live accelerator beam crossing condition (ALiveBX) which marks a window of time within a beam crossing (= collision) may occur. The prescale is set to $1-2$ times TEvatron's radio frequency so that a constant rate of $\approx 1 \mathrm{~Hz}$ containing unbiased events is written to tape.
} 
Figure 5.4: The sketch displays an event with multiple interactions in a beam crossing [132].

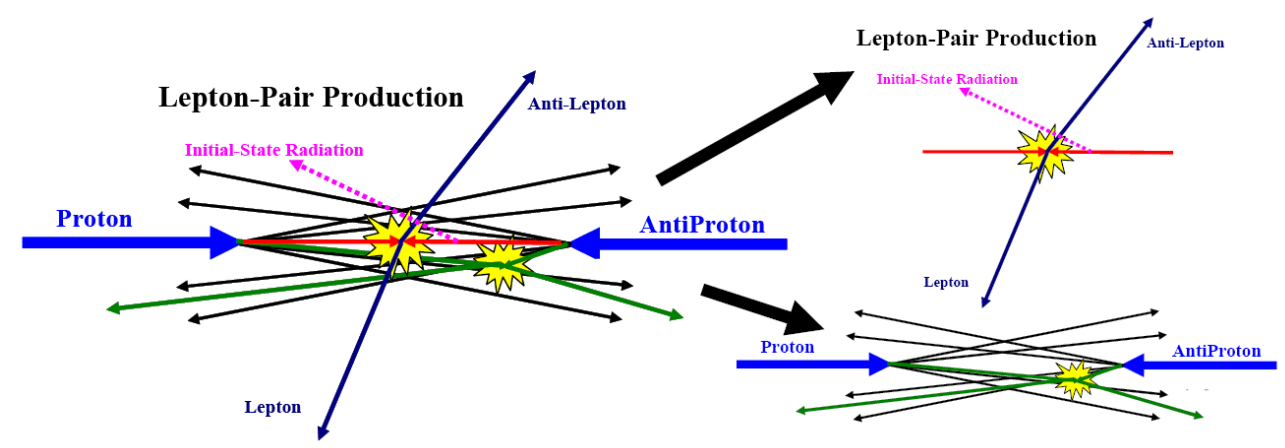

Figure 5.5: The plot shows the invariant mass distribution for $Z \rightarrow \mu \mu$ data events (blue line) which are compared with Monte Carlo events using pile-up from Monte Carlo (dashed purple line) and data (red line) [133].

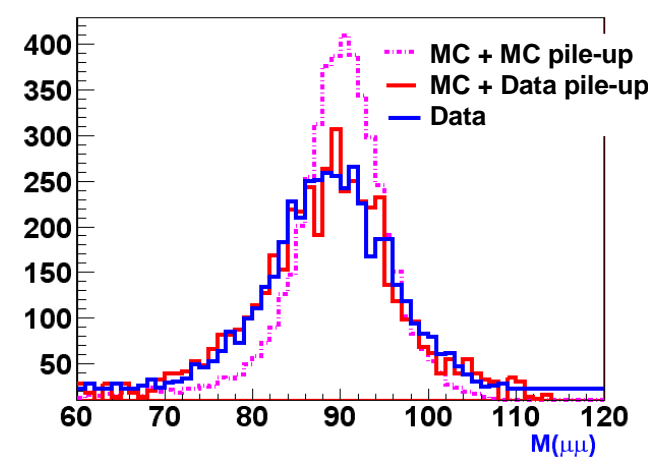

pile-up events (dashed purple). The agreement is considerably better using Monte Carlo events with pile-up from data (red).

\subsection{Standard Model Backgrounds}

The following Standard Model processes which can contribute to the inclusive final state under investigation are considered ( $Y=$ one or more jets from initial-state radiation; $X$ $=$ leptons, neutrinos, jets):

- $W \rightarrow e \nu Y$,

- $W \rightarrow \tau \nu Y \rightarrow e \nu X$,

- $Z \rightarrow \tau \tau Y \rightarrow e \nu X$,

- $W W \rightarrow e \nu X$,

- $W Z \rightarrow e \nu X$,

- $Z Z \rightarrow e \nu X$,

- $t \bar{t} \rightarrow e \nu X$. 
Due to the close affinity to the signal process and the high cross section (see Fig. 5.1), the background from $W \rightarrow e \nu$ events is the most important one. However, electrons from the expected signal tend to have high energies (see next section). Because of this, it is necessary to generate a sufficient large sample of $W$ events - especially at high energies for a meaningful interpretation of the data. Due to the fact that more than $99 \%$ of the electrons from $W$ decays have transverse energies $E_{T}<100 \mathrm{GeV}$, a special Monte Carlo sample is generated in order to enrich the statistics in the tail of the electron spectrum. This is accomplished by the application of a cut on the generator electron transverse energy via the package $\mathrm{d} \emptyset$ mess [134]: only events with $E_{T}^{\text {gen }}>100 \mathrm{GeV}$ are passed. In order to obtain 250 events with electron energies above $100 \mathrm{GeV}$, more than 430,000 events have to be generated by the Monte Carlo generator PYTHIA. Later in the analysis, both samples containing $W \rightarrow e \nu$ events are combined.

In all processes quoted above the missing transverse energy is produced by one (or more) neutrinos. In addition, there are two sources of instrumental background:

- $Z \rightarrow e e Y$

This can lead to a final state with missing transverse energy if one electron is lost, e. g. going into non-instrumented sections of the calorimeter. Further, misreconstruction of the electron and additional jets contribute to missing transverse energy.

- $q_{i} q_{j} \rightarrow q_{i} q_{j}, q_{i} \bar{q}_{i} \rightarrow q_{k} \bar{q}_{k}, q_{i} \bar{q}_{i} \rightarrow g g, q_{i} g \rightarrow q_{i} g, g g \rightarrow q_{k} \bar{q}_{k}, g g \rightarrow g g$

All these processes, referred to as QCD multijet production, result in at least two jets in the final state, whereas additional jets stem from initial-/final-state radiation. These QCD processes have to be considered due to the high cross sections at a hadron collider, see Fig. 5.1 or Fig. 3.9, although the probability of a jet mimicking an electron is fairly low $(2 \%-3 \%$ if a track is matched to the electron [135]). The misidentification of the jet in addition to energy mismeasurement from the jet in the opposite direction leads to the electron + missing transverse energy final state.

The latter kind of processes are not only difficult to model due to large theoretical uncertainties, but also suffer from a lack of statistics: The event selection for this analysis (Sec. 6.2) requires a high energetic electron and a significant amount of missing transverse energy. The steeply decreasing spectrum of the transverse momenta of QCD jets, together with the small probability that a jet mimics an electron makes the use of QCD Monte Carlo samples in this analysis unfeasible. However, it is possible to estimate the QCD multijet contribution from data. This is discussed in detail in Sec. 6.3.

All Standard Model processes (except for the QCD multijet production) have been simulated with PYTHIA using the CTEQ6L1 [17] parton distribution functions (PDF). Since Pythia does not contain full higher-order matrix elements ${ }^{3}$, the leading order PDF CTEQ6L1 (with leading order $\alpha_{s}$ ) is used for the event generation. The events which have been combined with zero bias events from data were passed through the full detector simulation. Tab. 5.1 summarizes for all processes the cross sections and the numbers of generated events.

Following common guidelines [136], the available highest-order calculations for the total cross sections are used. The cross sections in Tab. 5.1 are given at NNLO except for

\footnotetext{
${ }^{3}$ One exception is the inclusive $W$ production which contains $q \bar{q}^{\prime} \rightarrow W$ and the two first-order processes $q g \rightarrow W q^{\prime}$ and $q \bar{q}^{\prime} \rightarrow W g$, see Fig. 7.13 .
} 
Table 5.1: Summary of background Monte Carlo sets used in the analysis. The quoted cross sections for $Z / \gamma^{*}$ production are in LO only. In the analysis the cross section is multiplied by the corresponding $K\left(m_{Z / \gamma^{*}}\right)$ factor (see Fig. 5.6) on an event-by-event basis.

\begin{tabular}{|l|l|l|l|r|}
\hline Process & Remarks & Order & $\sigma \times \mathrm{Br}[\mathrm{pb}]$ & Events \\
\hline \hline$W \rightarrow e \nu Y$ & & NNLO & $2583_{-84}^{+94}(\mathrm{PDF}) \pm 5$ (scale) & 4543690 \\
$W \rightarrow e \nu Y$ & electron $E_{T}^{\text {gen }}>100 \mathrm{GeV}$ & NNLO & $1.498_{-0.045}^{+0.055}(\mathrm{PDF}) \pm 0.003$ (scale) & 11843 \\
$W \rightarrow \tau \nu Y$ & & NNLO & $2583_{-84}^{+94}(\mathrm{PDF}) \pm 5$ (scale) & 5236279 \\
\hline$Z / \gamma^{*} \rightarrow e e Y$ & $15-60 \mathrm{GeV}$ & LO & 330 & 818188 \\
$Z / \gamma^{*} \rightarrow e e Y$ & $60-130 \mathrm{GeV}$ & $\mathrm{LO}$ & 181 & 1812151 \\
$Z / \gamma^{*} \rightarrow e e Y$ & $130-250 \mathrm{GeV}$ & $\mathrm{LO}$ & 1.37 & 76720 \\
$Z / \gamma^{*} \rightarrow e e Y$ & $250-500 \mathrm{GeV}$ & $\mathrm{LO}$ & 0.115 & 25774 \\
$Z / \gamma^{*} \rightarrow e e Y$ & $>500 \mathrm{GeV}$ & LO & 0.0046 & 34737 \\
\hline$Z / \gamma^{*} \rightarrow \tau \tau Y$ & $15-60 \mathrm{GeV}$ & OO & 330 & 864974 \\
$Z / \gamma^{*} \rightarrow \tau \tau Y$ & $60-130 \mathrm{GeV}$ & LO & 181 & 1809302 \\
$Z / \gamma^{*} \rightarrow \tau \tau Y$ & $130-250 \mathrm{GeV}$ & LO & 1.37 & 149757 \\
$Z / \gamma^{*} \rightarrow \tau \tau Y$ & $250-500 \mathrm{GeV}$ & LO & 0.115 & 46209 \\
$Z / \gamma^{*} \rightarrow \tau \tau Y$ & $>500 \mathrm{GeV}$ & OO & 0.0046 & 37165 \\
\hline$W W$ & inclusive & NLO & $12.0 \pm 0.6(\mathrm{PDF}) \pm 0.3$ (scale) & 258729 \\
$W Z$ & inclusive & NLO & $3.68 \pm 0.22(\mathrm{PDF}) \pm 0.12$ (scale) & 95609 \\
$Z Z$ & inclusive & NLO & $1.42 \pm 0.06(\mathrm{PDF}) \pm 0.05$ (scale) & 94676 \\
\hline$t \bar{t}$ & inclusive & NNLO & $6.77 \pm 0.42$ & 104559 \\
\hline
\end{tabular}

the diboson samples (NLO) and the Drell-Yan processes $Z / \gamma^{*}$ which are LO. In the latter case, a $K$ factor depending on the momentum transfer $\sqrt{Q^{2}}=m_{Z / \gamma^{*}}$ is applied [22]. In this special case, the $K$ factor is defined as follows (cf. Eq. 2.108)

$$
K_{N N L O}=\frac{\sigma(\mathrm{NNLO} \text { with } \mathrm{NLO} \mathrm{PDF})}{\sigma(\mathrm{LO} \text { with LO PDF })}
$$

because the events are generated with CTEQ6L1 (LO with LO PDF). The $K$ factor together with its uncertainties is shown in Fig. 5.6 (right). The left-hand plot shows the composition of the $Z$ Monte Carlo sample. The separation into intervals regarding the momentum transfer $\sqrt{Q^{2}}=m_{Z / \gamma^{*}}$ is necessary to accumulate reasonable statistics in the steeply falling spectrum. Samples with $m_{Z / \gamma^{*}}<15 \mathrm{GeV}$ are omitted since the electron does not pass the event selection $\left(E_{T}>30 \mathrm{GeV}\right)$. The quoted cross sections for $W$ samples and $K$ factors for the Drell-Yan samples are calculated with the NLO PDFs CTEQ6.1M [17] and include estimates of the PDF and scale uncertainties. The PDF uncertainties are determined using the 40 error functions (CTEQ6.1M. $x x, x x=1, \ldots, 40$ [17]). Due to missing higher order terms, theoretical calculations of cross sections depend on both the factorization and renormalization scale. An estimate of this contribution can be derived by independent variations of the two scales. The scale variation uncertainty - if available - is also quoted in Tab. 5.1. The $t \bar{t}$ cross section is extracted from [137], all other cross sections and uncertainty estimates are summarized in [21]. 
Figure 5.6: Left: Composition of the $Z$ sample. Right: $N N L O$ correction ( $K$ factor) as a function of the momentum transfer $\sqrt{Q^{2}}$ (extracted from [22]).
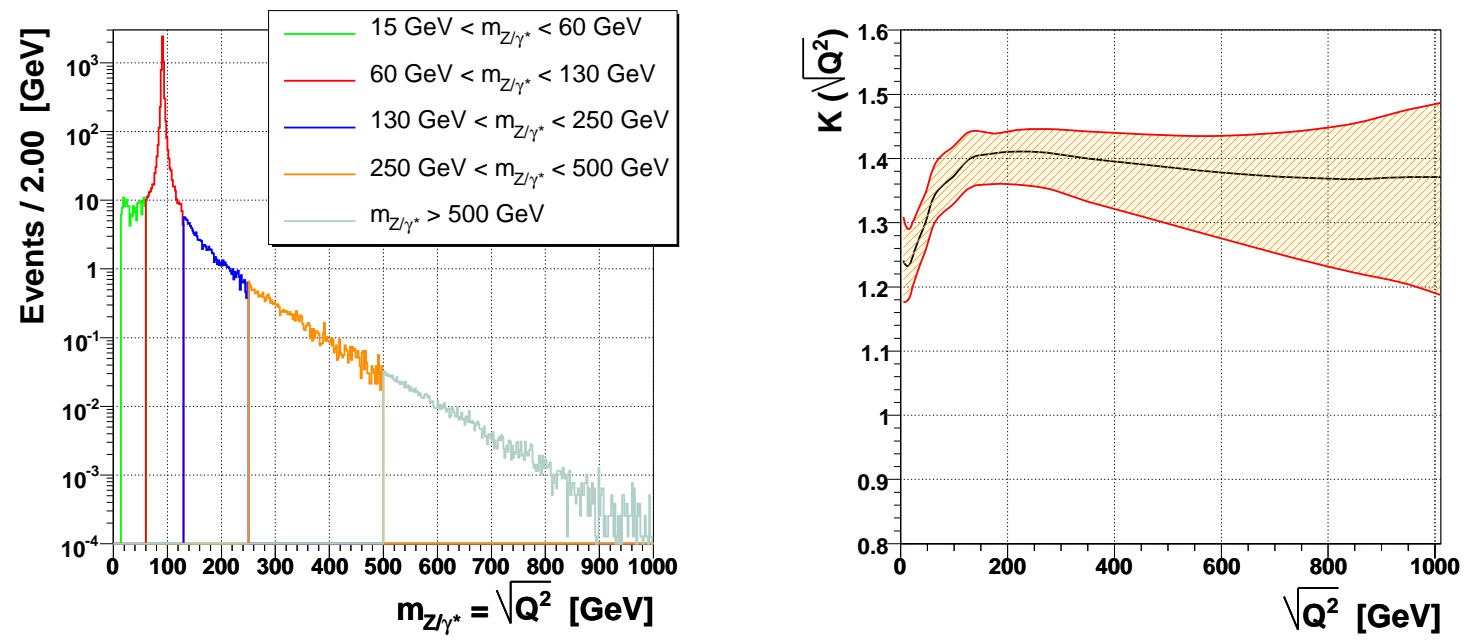

\subsection{The $W^{\prime}$ Signal and its Properties}

Signal events (5000 events for each mass point) for the channel $p \bar{p} \rightarrow W^{\prime}+X \rightarrow e \nu+X$ have been generated with the Monte Carlo generator PyTHIA [123] using the CTEQ6L1 [17] PDF set. The parameters in PyтнIA are set according to the Altarelli Reference Model discussed in Sec. 2.3.2 with the assumptions

- that the new charged gauge boson $W^{\prime}$ couples to fermions as the charged gauge boson $W$ in the Standard Model does;

- that the total width $\Gamma_{W^{\prime}}$ scales with its mass $m_{W^{\prime}}$ (see Eq. 2.147);

- and that the channel $W^{\prime} \rightarrow W Z$ is suppressed by the mixing angle $\xi^{2}$ (see Eq. 2.140).

Tab. 5.2 summarizes the main parameters in PүтніA, together with their settings and explanations.

The generated events have been combined with zero bias events from data and processed through the full detector simulation. Next-to-next-to leading order corrections on the Pyтнia leading order cross sections ( $K$ factor, see Fig. 5.6) have been applied. The $K$ factors and errors due to PDF uncertainties for the signal are extracted from [22] since they are derived for the process $p \bar{p} \rightarrow V+X$ with $V$ being a vector boson $\left(V=W, Z, W^{\prime}\right.$, $\left.Z^{\prime}\right)$. The NNLO cross sections and errors, together with the total width $\Gamma_{W^{\prime}}$ and numbers of events (after applying the data quality criteria [see Sec. 6.1] on the zero bias event) are summarized in Tab. 5.3. The cross section as a function of the $W^{\prime}$ mass is displayed in Fig. 5.7 (red lines, including uncertainties).

From the plot and the numbers in the table one can see that the cross section falls steeply with increasing mass of the $W^{\prime}$ boson. Another effect of the heavier bosons is shown in Fig. 5.8: large tails towards very low masses occur. For $m_{W^{\prime}}=1200 \mathrm{GeV}$ more 
Table 5.2: PYThIA parameters for $W^{\prime}$ production.

\begin{tabular}{|c|c|c|}
\hline Parameter & Value & Explanation \\
\hline MSEL & 22 & switch for $W^{\prime}$ production via ISUB $=142\left(f_{i} \bar{f}_{j} \rightarrow W^{\prime}\right)$ \\
\hline $\operatorname{PMAS}(34,1)$ & $m_{W^{\prime}}$ & mass of the $W^{\prime}$ boson \\
\hline $\operatorname{PMAS}(34,2)$ & $\Gamma_{W^{\prime}}$ & total width of the $W^{\prime}$ boson, see Eq. 2.147 \\
\hline PARU (131) & 1 & vector coupling of the $W^{\prime}$ to $q \bar{q}^{\prime}$ \\
\hline PARU (132) & -1 & axialvector coupling of the $W^{\prime}$ to $q \bar{q}^{\prime}$ \\
\hline PARU (133) & 1 & vector coupling of the $W^{\prime}$ to $\ell \nu$ \\
\hline PARU (134) & -1 & axialvector coupling of the $W^{\prime}$ to $\ell \nu$ \\
\hline PARU (135) & 1 & decay $W^{\prime} \rightarrow W Z$ is allowed, but suppressed with $\xi^{2}$, see Eq. 2.140 \\
\hline PARU (136) & 0 & $\begin{array}{l}\text { mixture in the decay chain } W^{\prime} \rightarrow W Z \rightarrow 4 \text { fermions: } \\
\text { 1-PARU (136) corresponds to the same angular distribution } \\
\text { between the four final fermions as in } W \rightarrow Z W \\
\text { (mixture of transverse and longitudinal } W / Z \text { ); } \\
\text { PARU(136) corresponds to } H \rightarrow Z W \text { the same way } \\
\text { (longitudinal } W / Z \text { ) }\end{array}$ \\
\hline
\end{tabular}

than $90 \%$ of the generated events are outside the peak region. First of all, the relative cross sections for small masses exactly reflect the shape of the Breit-Wigner curve. The ratio can be estimated from the partonic cross section in Eq. 2.149. Assuming $\sqrt{\hat{s}} \ll m_{W^{\prime}}$ the terms in the denominator can be simplified using $\left(\hat{s}-m_{W^{\prime}}^{2}\right)^{2} \approx m_{W^{\prime}}^{4} \ll \Gamma_{W^{\prime}}^{2} m_{W^{\prime}}^{2}$ so that

$$
\hat{\sigma} \propto \frac{\hat{s}}{\left(\hat{s}-m_{W^{\prime}}^{2}\right)^{2}+\Gamma_{W^{\prime}}^{2} m_{W^{\prime}}^{2}} \quad \Rightarrow \quad \hat{\sigma} \propto \frac{1}{m_{W^{\prime}}^{4}} .
$$

The tails towards low masses $(\sqrt{\hat{s}}=m \rightarrow 0)$ show the expected cross section ratio for all $m_{W^{\prime}}$, see Fig. 5.8:

$$
\begin{aligned}
& \sigma_{W^{\prime}}(1100 \mathrm{GeV}): \sigma_{W^{\prime}}(500 \mathrm{GeV})=500^{4}: 1100^{4} \sim 1: 23 \\
& \sigma_{W^{\prime}}(1100 \mathrm{GeV}): \sigma_{W^{\prime}}(700 \mathrm{GeV})=700^{4}: 1100^{4} \sim 1: 6, \\
& \sigma_{W^{\prime}}(1100 \mathrm{GeV}): \sigma_{W^{\prime}}(900 \mathrm{GeV})=900^{4}: 1100^{4} \sim 1: 2 .
\end{aligned}
$$

The reason for the enhancement of the absolute signal cross sections for all nominal $m_{W^{\prime}}$ at low masses $\left(\sqrt{\hat{s}}=m \ll m_{W^{\prime}}\right)$ lies in the PDFs [138]. In order to produce heavy bosons on-shell with $\sqrt{\hat{s}}=m \approx m_{W^{\prime}}$, the quarks are required to carry a significant amount of the momentum of the (anti)proton. With the assumption $x \approx x_{p} \approx x_{\bar{p}}$ and $\sqrt{\hat{s}} \approx m_{W^{\prime}}=$ $(500 \ldots 1200) \mathrm{GeV}$, the required momentum fractions $x$ for on-shell production can be estimated as follows (cf. Eq. 2.11)

$$
\sqrt{\hat{s}}=\sqrt{x_{p} x_{\bar{p}}} \cdot \sqrt{s} \Rightarrow x \approx \frac{\sqrt{\hat{s}}}{\sqrt{s}}=0.26 \ldots 0.61 .
$$

From Fig. 2.5 one can see that high momentum fractions $x$ are strongly suppressed, and thus the production of heavy on-shell bosons. Fig. 5.9 shows the momentum fractions of the (anti)quarks that participate in the hard collision $q \bar{q}^{\prime} \rightarrow W^{\prime}$. In both distributions the mean values are $\bar{x}_{p}=\bar{x}_{\bar{p}}=0.27$ (green stars). For the lightest generated boson with $m_{W^{\prime}}=500 \mathrm{GeV}(\hat{=} x=0.26 \approx \bar{x})$ more than $90 \%$ of the events are contained in the 
Table 5.3: Cross sections and errors due to PDF uncertainties, total width $\Gamma_{W^{\prime}}$ and event numbers for different masses of the $W^{\prime}$ boson (the mass cut is explained later in the text).

\begin{tabular}{|c|c|c|c|c|}
\hline \multirow{2}{*}{$\begin{array}{l}m_{W^{\prime}} \\
{[\mathrm{GeV}]}\end{array}$} & \multirow{2}{*}{$\begin{array}{l}\Gamma_{W^{\prime}} \\
{[\mathrm{GeV}]}\end{array}$} & \multirow{2}{*}{$\begin{array}{c}\sigma_{W^{\prime}} \times \operatorname{Br}\left(W^{\prime} \rightarrow e \nu\right) \\
{[\mathrm{pb}]}\end{array}$} & \multicolumn{2}{|c|}{ Events } \\
\hline & & & without mass cut & with mass cut \\
\hline 500 & 17.6 & $2.44_{-0.14}^{+0.08}$ & 4213 & 3821 \\
\hline 600 & 21.1 & $0.84_{-0.06}^{+0.03}$ & 4522 & 3977 \\
\hline 700 & 24.6 & $0.30_{-0.02}^{+0.02}$ & 3895 & 3174 \\
\hline 800 & 28.2 & $0.11_{-0.01}^{+0.01}$ & 3962 & 2840 \\
\hline 900 & 31.7 & $0.044_{-0.005}^{+0.002}$ & 3960 & 2294 \\
\hline 1000 & 35.2 & $0.019_{-0.002}^{+0.001}$ & 3944 & 1504 \\
\hline 1100 & 38.7 & $0.0095_{-0.0011}^{+0.0008}$ & 4485 & 906 \\
\hline 1200 & 42.3 & $0.0056_{-0.0007}^{+0.0004}$ & 4068 & 334 \\
\hline
\end{tabular}

Figure 5.7: Signal cross sections as a function of the $W^{\prime}$ mass (the mass cut is explained later in the text).

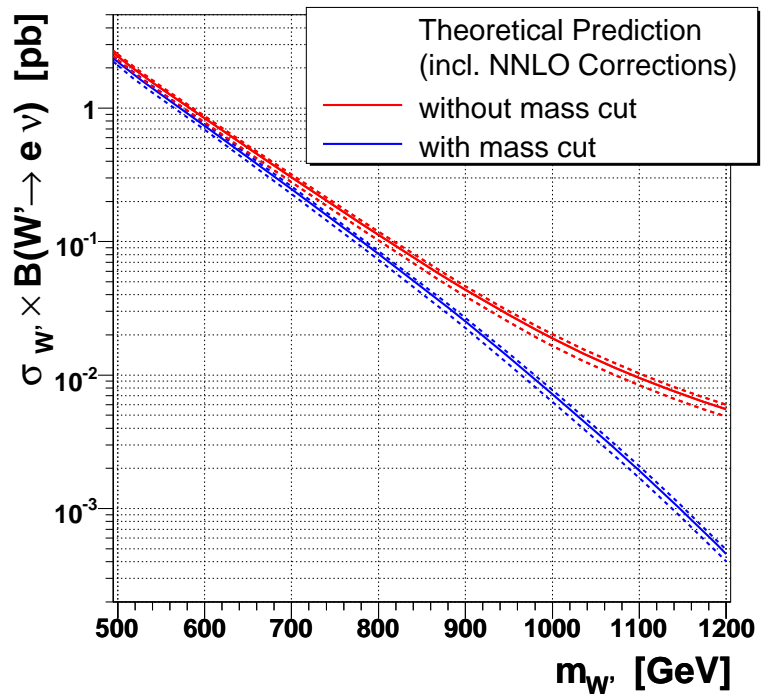

Figure 5.8: Mass distributions of the generated $W^{\prime}$ bosons.

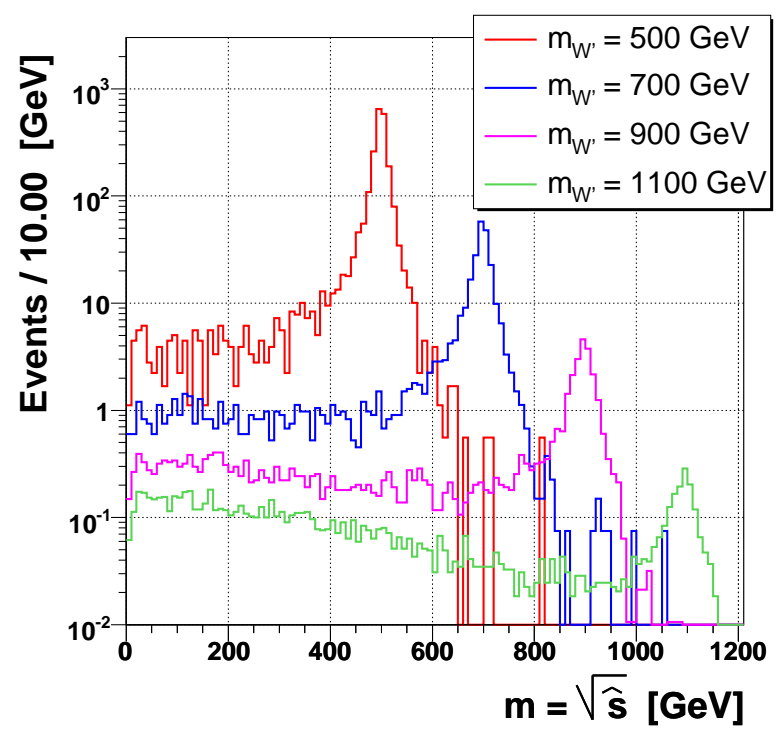


Figure 5.9: Momentum fractions $x_{i}$ of the two quarks that annihilate and produce the $W^{\prime}$ boson. The blue shaded areas indicate the immediate peak region of the $W^{\prime}( \pm 100 \mathrm{GeV})$. The green stars display the mean values $\bar{x}$ of the histograms.
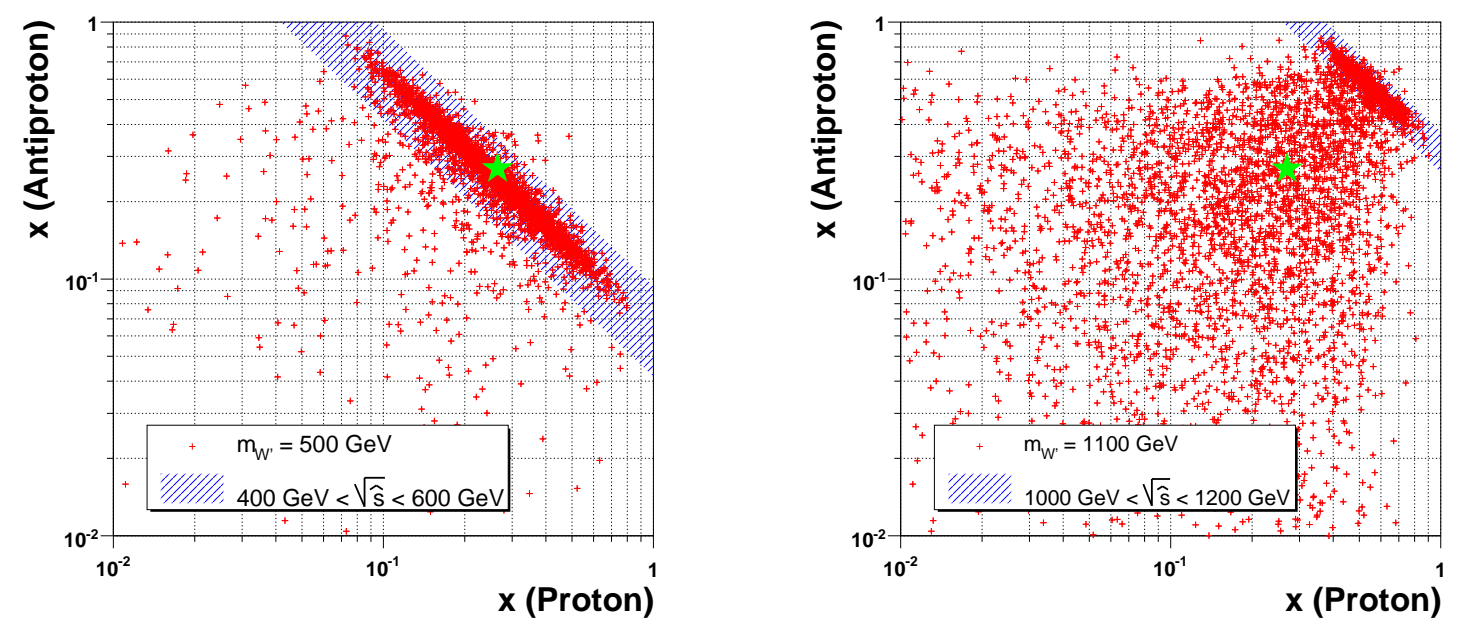

immediate peak region, whereas in case of $m_{W^{\prime}}=1100 \mathrm{GeV}(\hat{=} x=0.56>\bar{x})$ the peak region contains less than $20 \%$ of the events. To conclude, the parton distribution functions suppress the on-shell production of extremely heavy gauge bosons.

Since this behaviour has yet not been confirmed by measurements ${ }^{4}$, one could consider a scenario rejecting the events outside the resonance region [139]. This can be implemented by applying a cut on the $W^{\prime}$ mass

$$
m>m_{W^{\prime}}-5 \cdot \Gamma_{W^{\prime}}
$$

One should keep in mind that in this scenario the cross sections from Tab. 5.3 have to be corrected for the generator mass cut via

$$
\tilde{\sigma}_{W^{\prime}}=\sigma_{W^{\prime}} \cdot \frac{\# \text { events with mass cut }}{\# \text { events without mass cut }}
$$

Event numbers after the generator mass cut are quoted in Tab. 5.3; the blue lines in Fig. 5.7 show the modified cross sections.

Fig. 5.10 shows distributions of generator variables without (left-hand plots) and with (right-hand plots) the cut on the generator mass. The invariant mass distribution $m$ is not accessible in the measurement due to the unknown momentum of the neutrino in $z$ direction. Only the transverse mass $m_{T}$ can be measured and is calculated from the electron and the neutrino (= missing transverse energy, MET, $\mathbb{E}_{T}$ ) momenta in the

\footnotetext{
4"The secondary peak at small $\tau\left[\tau=x_{p} x_{\bar{p}}=\hat{s} / s\right]$ may give a rather high cross section, which can even rival that of the ordinary peak around the nominal mass. This is the case, for instance, with $W$ production. Such a peak has never been observed experimentally, but this is not surprising, since the background from other processes is overwhelming at low $\hat{s}$. [...] When resonance production is studied, it is therefore important to set limits on the mass of the resonance [...]. If not, cross-section information given by the program may be very confusing." [123]
} 
Figure 5.10: Left-hand plots: Mass distributions of the generated $W^{\prime}$ bosons, transverse mass $m_{T}$ distributions and electron transverse energy $E_{T}$ distributions for different masses of the $W^{\prime}$ boson (generator level). The plots on the right-hand side show the distributions for the same variables after applying the cut on the generator mass. All distributions are normalized to 1.
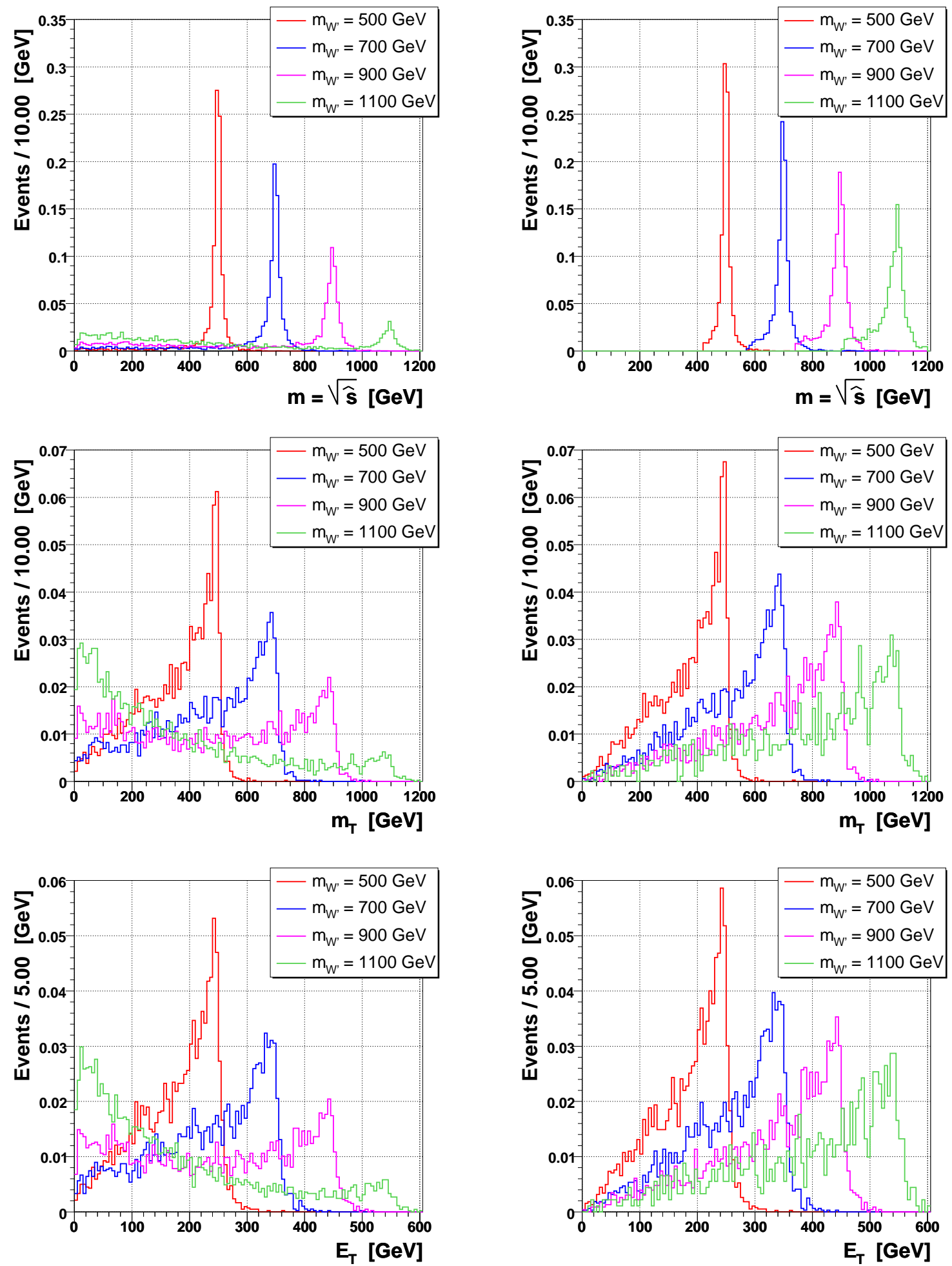
following way

$$
\begin{aligned}
m_{T}^{2} & =\left[\left(\begin{array}{c}
E_{T} \\
E_{x} \\
E_{y} \\
0
\end{array}\right)+\left(\begin{array}{c}
E_{T} \\
E_{x} \\
E_{y} \\
0
\end{array}\right)\right]^{2} \\
& =\left(E_{T}+E_{T}\right)^{2}-\left(E_{x}+E_{x}\right)^{2}-\left(E_{y}+E_{y}\right)^{2} \\
& =2\left(E_{T} E_{T}-E_{x} E_{x}-E_{y} E_{y}\right) \quad\left[E_{T}^{2}=E_{x}^{2}+E_{y}^{2}, \quad E_{T}^{2}=E_{x}^{2}+E_{y}^{2}\right] \\
& =2 E_{T} E_{T}\left(1-\frac{E_{x}}{E_{T}} \frac{E_{x}}{E_{T}}-\frac{E_{y}}{E_{T}} \frac{E_{y}}{E_{T}}\right) \\
& =2 E_{T} E_{T}\left(1-\left(\cos \phi_{e} \cos \phi_{m}+\sin \phi_{e} \sin \phi_{m}\right)\right) \\
& =2 E_{T} E_{T}(1-\cos \Delta \phi)
\end{aligned}
$$

using $\cos \Delta \phi=\cos \left(\phi_{e}-\phi_{m}\right)=\cos \phi_{e} \cos \phi_{m}+\sin \phi_{e} \sin \phi_{m}$ as well as

$$
\begin{aligned}
& E_{x}=E_{T} \cos \phi_{e} \\
& E_{y}=E_{T} \sin \phi_{e}
\end{aligned} \quad \text { and } \quad \begin{aligned}
& E_{x}=E_{T} \cos \phi_{m} \\
& E_{y}=E_{T} \sin \phi_{m}
\end{aligned} .
$$

One can see in Fig. 5.10 that in both scenarios the Jacobian peak in the transverse mass distribution is blurred with increasing mass of the new gauge boson. Due to the off-shell production of heavy bosons, the electrons tend to have relatively "smaller" (compared to the mass of the generated boson) transverse energies with flat tails towards high energies (in the samples without the generator mass cut). This behaviour - together with tiny cross sections - makes the detection of such heavy bosons difficult.

Fig. 5.11 and 5.12 show the following distributions after the full simulation and reconstruction (see Chap. 4): ratio of electron transverse energy $E_{T}$ and missing transverse energy $\mathbb{E}_{T}$ (MET), angular difference between electron and MET, number of jets (if present in the event), and the (maximal) angle between jets and electrons and MET, respectively. These variables are selected because they are used in the event selection later on, see Sec. 6.2. All distributions - again shown for the signal without and with the generator mass cut applied - are very similar in both scenarios. Further studies indicate that the major part $(\approx 85 \%)$ of the electrons stemming from the $W^{\prime}$ decays are emitted into the central detector region, namely in the central electromagnetic calorimeter, which covers the range $-1.1<\eta_{\text {det }}<1.1(\mathrm{CC})$, see Fig. 5.13. Because of this, only the CC region is studied. The region of low transverse masses $\left(m_{T}<140 \mathrm{GeV}\right.$, see plots in Fig. 5.14) contains a significant amount of electrons with $\left|\eta_{\text {det }}\right|>1.1$, but is rejected due to the extremely low signal-to-background ratio, see Sec. 8.2. In this region the overwhelming background from Standard Model $W$ production can not be reduced. Further, this part of the transverse mass spectrum can not be included in the search for the production of heavy charged gauge bosons because it is used for normalization (measurement of the effective luminosity, see Sec. 6.4) and calibration (extraction of the scaling factor for the QCD multijet background estimated from data, see Sec. 6.3).

In this analysis the signal Monte Carlo samples are used without the cut on the generator mass. It is assumed that the PDFs, which cause the enhancement of the cross sections at low masses, are still valid at the scale of the $W^{\prime}$ production process. A scenario where events outside the resonance region are rejected is discussed in App. D. 
Figure 5.11: Left-hand plots: Ratio of electron $E_{T}$ and MET, angle between electron and MET and number of jets in the event (after full simulation and reconstruction). The plots on the righthand side show the distributions for the same variables after applying the cut on the generator mass. All distributions are normalized to 1.
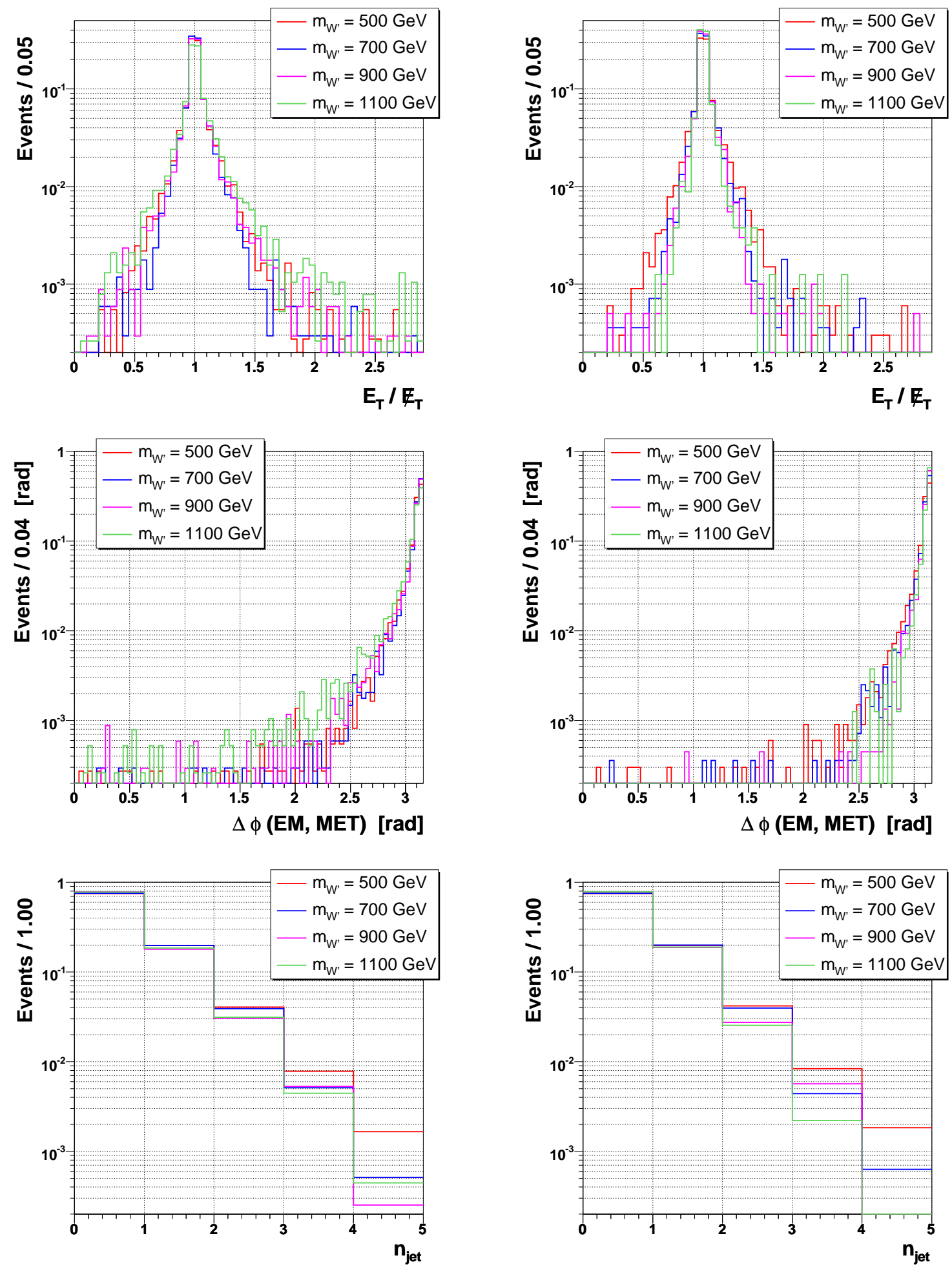
Figure 5.12: Left-hand plots: Maximal angle between the jet and electron or MET if jets are present if the event (after full simulation and reconstruction). The plots on the right-hand side show the distributions for the same variables after applying the cut on the generator mass. All distributions are normalized to 1 .
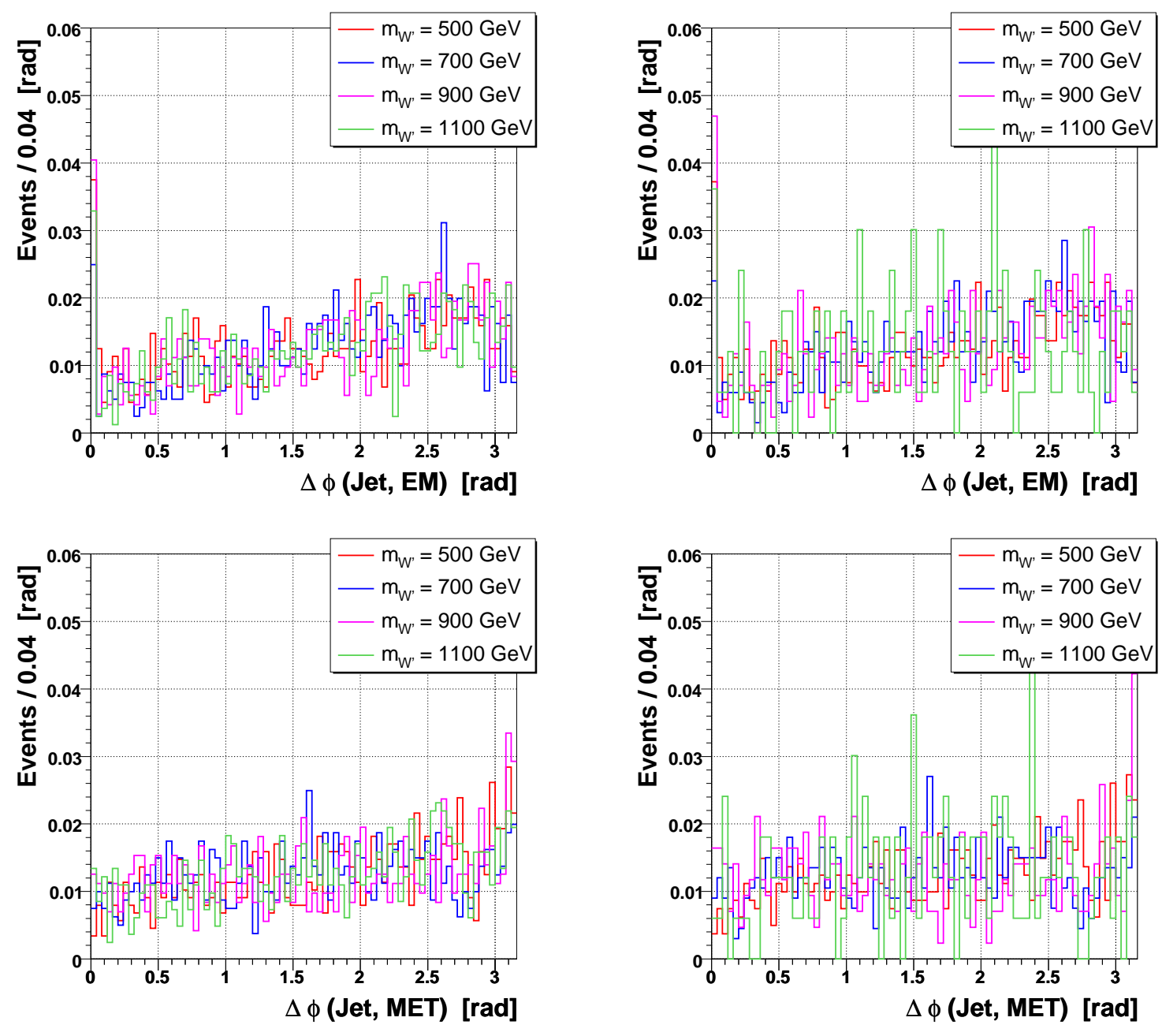
From the studies the following conclusions can be drawn regarding the characteristics of the generated $W^{\prime}$ signal:

- small cross sections ( $2 \mathrm{pb}$ for $m_{W^{\prime}}=500 \mathrm{GeV}$ to $6 \mathrm{fb}$ for $m_{W^{\prime}}=1200 \mathrm{GeV}$ );

- on-mass-shell production of $W^{\prime}$ bosons is heavily suppressed;

- flat transverse mass distributions;

- the event is balanced in terms of transverse energies, $E_{T} \sim E_{T}$;

- the electrons tend to be central and to have energies well above $100 \mathrm{GeV}$;

- the electron (EM) and missing transverse energy are back-to-back, $\Delta \phi(E M, M E T) \sim \pi$

- there is only marginal jet activity from initial-state radiation, more than $75 \%$ of the events do not contain a jet with transverse energy above $15 \mathrm{GeV}$;

- if jets are present, they are nearly uniformly distributed (in terms of distances with respect to the electron and MET).

Based on these properties, in the next chapter analysis cuts are introduced in order to efficiently reject the Standard Model backgrounds while keeping the signal efficiency as high as possible. Further, a search region has to be defined in order to separate the expected $W^{\prime} \rightarrow e \nu$ signal events from the irreducible background from $W \rightarrow e \nu$ events.

Figure 5.13: Electron $\eta_{\text {det }}$ distribution (without generator mass cut, normalized to 1). The "holes" at $\left|\eta_{\text {det }}\right| \approx 1.3$ are due to non-instrumented regions of the electromagnetic calorimeter.

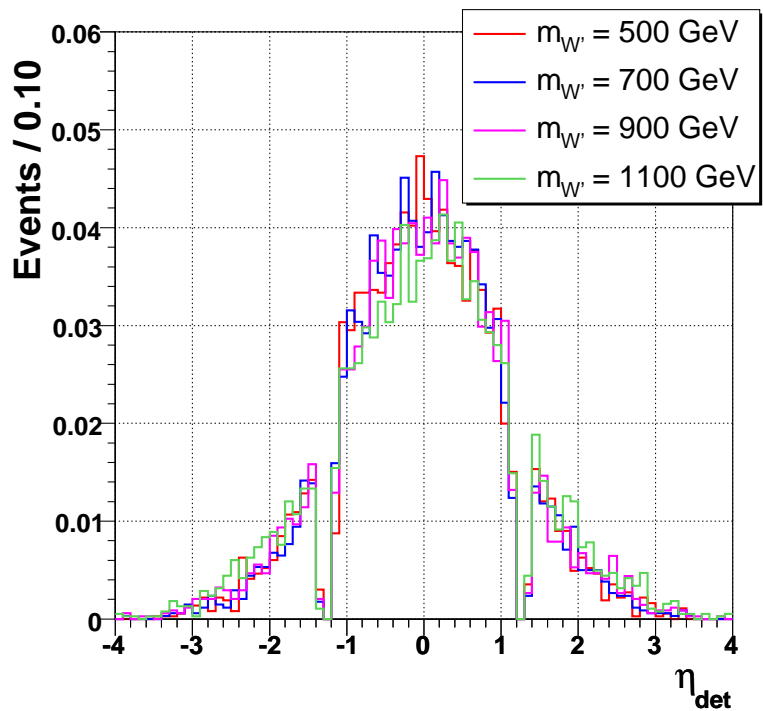


Figure 5.14: Electron $\eta_{\text {det }}$ distribution as a function of the reconstructed transverse mass $m_{T}$ for various masses of the $W^{\prime}$ boson $m_{W^{\prime}}$ (without generator mass cut). The green lines indicate analysis cuts (see Sec. 6.2).
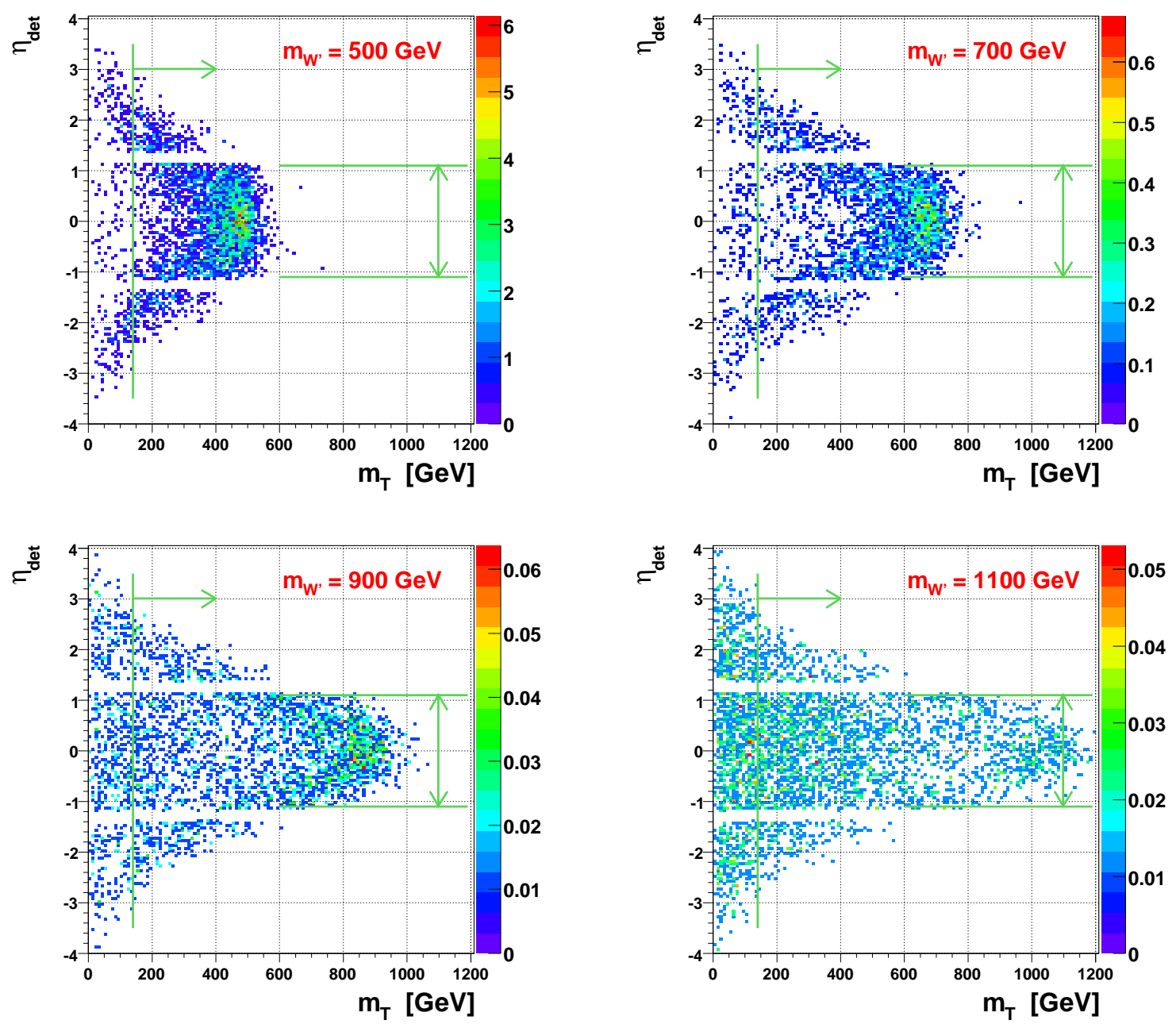


\section{Chapter 6}

\section{Data Selection and QCD Background Estimation}

The true inquirer seeks the truth everywhere.

Ernst Mach

This section is dedicated to issues in conjunction with the data. After discussing the data sample and related items (processing, data quality, triggers and corresponding luminosity), the selection criteria are presented. After this, the method for extracting the QCD multijet background from data is introduced. This is followed by a measurement of the effective luminosity using $W \rightarrow e \nu$ events from data.

\subsection{Data Sample, Triggers and Luminosity}

The full Run IIa dataset collected with the DØ detector between October 2002 (run number 166505/store \#1888) and February 2006 (run number 215670/store \#4666) is used in this analysis. A loose preselected subsample of the data (skim) provided by the CSG forms the basis of the selection. This subsample - the EMinclusive skim - contains various classes of tagged events which have in common that they all require at least one electron candidate with $|I D|=10$ or 11 , see Tab. 6.1. These events which are available in the Common Analysis Format (CAF) as Root trees have been analyzed within the Common Analysis Format Environment (CAFE), making use of standardized object identification criteria and corrections. The processing within CAFE applies the data quality, trigger selection and a tighter skimming: only events with electron candidates with transverse energy $E_{T}>20 \mathrm{GeV}$ and missing transverse energy $E_{T}>20 \mathrm{GeV}$ are passed and stored in customized Rоoт trees. Tab. 6.2 summarizes event numbers in the various steps of the processing (cuts and samples are defined later in the text). The entire processing chain is displayed in Fig. 6.1 (the program np_tmb_stream [140] is used for the skimming). Details on the CAFE packages (green box on the left-hand side) can be found at [94]; the packages needed for accessing the luminosity information (green box on the right-hand side) are outlined in [141]. 
Table 6.1: Event tags contained in the EMinclusive skim.

\begin{tabular}{|l|l|l|}
\hline Tag & Objects & Requirement \\
\hline \hline 1EMLOOSE & EM & $|I D|=10$ or $11, E_{T}>20 \mathrm{GeV}$ \\
\hline 1EM2JET & EM & $|I D|=10$ or $11, E_{T}>12 \mathrm{GeV}, \chi_{\mathrm{HMx}}^{2}<75$ \\
& track & $p_{T}>7 \mathrm{GeV}$ matched to EM object with $\Delta \phi<0.1$ \\
& $3 \mathrm{jets}$ & $E_{T}>8 \mathrm{GeV}$ \\
\hline 2EM & $2 \mathrm{EM}$ & $|I D|=10$ or $11, E_{T}>7 \mathrm{GeV}$ \\
\hline EM1TRK & EM & $|I D|=10$ or $11, E_{T}>8 \mathrm{GeV}$ \\
& track & $p_{T}>5 \mathrm{GeV}$ matched to EM object with $\Delta \phi<0.2$ \\
\hline JES_B & EM & $|I D|=10$ or $11, E_{T}>4 \mathrm{GeV}$ \\
& trigger & at least one electron trigger fired \\
\hline
\end{tabular}

Several types of bad events, runs and luminosity blocks have to be discarded (see Sec. $3.5)$ :

- runs marked with the flag 'bad' for the calorimeter, tracker and muon system in the Offline Run Quality Database;

- luminosity blocks declared as 'bad' (calorimeter offline certification, Luminosity Database);

- events containing calorimeter noise;

- runs in which the trigger used to determine the luminosity has a prescale $\neq 1$.

The bad runs and bad LBN lists go into the analysis and into the luminosity calculation as well. Since the analysis is almost entirely calorimeter based (tracking information is used for spatial track match only), the quality of an event from the calorimeter point of view is of particular concern. Events affected by the following known problems are detected and tagged offline [102]:

- "coherent noise": synchronous pedestal shift in the ADCs;

- "missing crate": information from one or several ADCs was not read in the event;

- "ring of fire" (see Fig. 3.34) and "noon noise": caused by external noise.

Following the recommendations, events marked with at least one of the tags are removed. This removal is performed on an event-by-event basis, but the luminosity can only be calculated for individual LBNs. In order to account for the loss of luminosity, the fraction of events removed by this cut has to be determined. This is accomplished by using an unbiased data sample delivered by the zero bias trigger (see footnote on p. 87) whose trigger condition is independent of the calorimeter noise. Counting events that pass/fail the calorimeter event flags in this sample results in a fraction of $2.9 \%( \pm 0.003 \%)$ of rejected events [142].

Only triggered events are considered in order to ensure the proper normalization. The trigger selection depends on trigger list versions (see Sec. 3.4) because the triggers 
Table 6.2: Data flow during processing.

\begin{tabular}{|l|r|l|}
\hline Selection & Events & Processing \\
\hline \hline EMinclusive Dataset & $316,285,298$ & CAF RoOT trees (SAM) \\
\hline Data Quality & $249,434,393$ & CAFE + cm_analyze \\
Trigger fired & $131,598,946$ & $\rightarrow$ customized RoOT trees \\
Electron $E_{T}>20 \mathrm{GeV}, E_{T}>20 \mathrm{GeV}$ & $2,065,987$ & (local disk) \\
\hline$W$ Sample & 471,938 & O(500) histograms in RoOT files \\
QCD Sample & 68,427 & (local disk) \\
\hline
\end{tabular}

Figure 6.1: Processing chain.

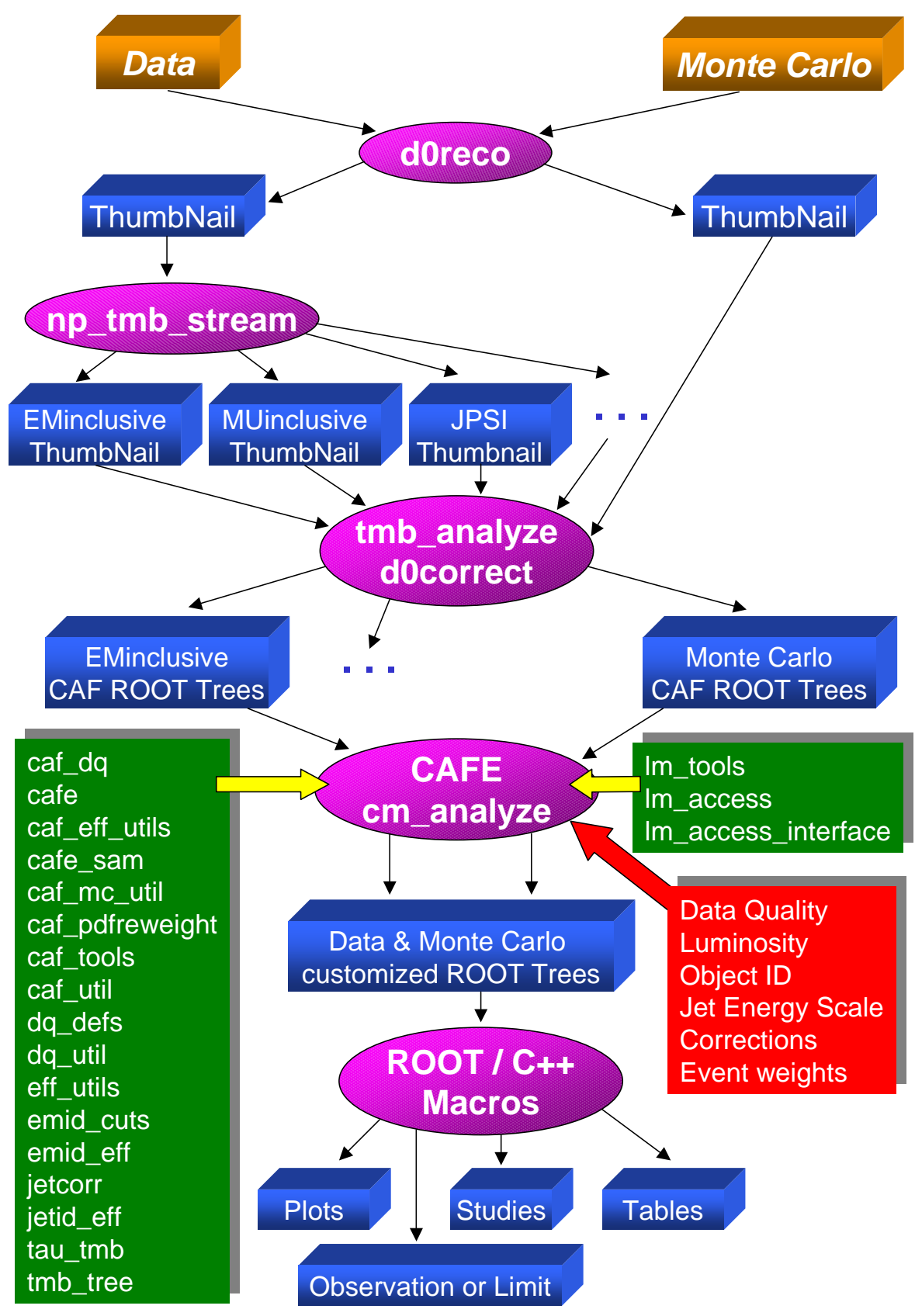


are repeatedly modified according to the improved detector performance (extension of $\eta$ coverage, new trigger tools) and increases in the instantaneous luminosity. Changes within a major trigger list version (e.g. $9.20 \rightarrow 9.30$ ) are usually marginal. In Tab. 6.3 the trigger selection and luminosity for each run range are given. The selected inclusive single electron triggers [143] have the following generic trigger level requirements:

Level 1: energy deposit in the electromagnetic trigger towers;

Level 2: electromagnetic object with minimum transverse energy (there is no Level 2 condition in some trigger lists);

Level 3: tightening of the electromagnetic object (shower shape, electromagnetic fraction).

Details of the triggers used in this analysis can be found in App. C; all trigger lists including a breakdown of the individual triggers are stored in the Trigger Database [74]. Using App. C one can assemble the full trigger requirements for e.g. EM_MX and EM_MX_EMFR8 in v11:

Level 1: one EM trigger tower with $E_{T}>15 \mathrm{GeV}$

Level 2: no additional requirement

Level 3: $\begin{cases}\text { EM_MX: } & \text { one loose electron with } E_{T}>30 \mathrm{GeV} \\ \text { EM_MX_EMFR8: } & \text { one very loose electron with } E_{T}>40 \mathrm{GeV}\end{cases}$

Table 6.3: Trigger selection and luminosity for the trigger list versions and run ranges used in the analysis. Five major periods are distinguished; the trigger conditions remain unchanged within each period.

\begin{tabular}{|c|c|c|c|}
\hline Trigger list version & Run number range & Trigger selection & $\begin{array}{l}\text { Luminosity } \\
{\left[\mathrm{pb}^{-1}\right]}\end{array}$ \\
\hline$\geq 8.41$ & $166505-167018$ & & 3.10 \\
\hline 9 & $167019-170246$ & EM_[i], EM_[i]_SH, EM_[i]_EMFR8 & 24.60 \\
\hline 10 & $170247-174844$ & $(\mathrm{i}=\mathrm{HI}, \mathrm{MX})$ & 10.11 \\
\hline 11 & $174845-178721$ & & 64.05 \\
\hline 12 & $178722-194566$ & $\begin{array}{l}\text { E[i]_SHT20, E1_SH30 }(\mathrm{i}=1,2,3), \\
\text { E1_L50, E1_VL70 }\end{array}$ & 230.78 \\
\hline$<13.20$ & $194567-195838$ & $\begin{array}{l}\text { E[i]_SHT20, E[i]_SH30 }(\mathrm{i}=1,2,3,4), \\
\text { E1_L50, E1_NC90 }\end{array}$ & 20.45 \\
\hline$\geq 13.20$ & $195839-208500$ & $\begin{array}{l}\text { E[i]_SHT22, E[i]_SH30 }(\mathrm{i}=1,2,3,4), \\
\text { E1_L70, E1_NC90 }\end{array}$ & 377.26 \\
\hline 14 & $208500-215670$ & $\begin{array}{l}\text { E[i]_SHT25, E[i]_SH35 (i=1, 3, 4), } \\
\text { E1_L70, } \\
\text { 2CEM12_E15_(SHT22, SH30), } \\
\text { 2CEM6_E15_(SHT22, SH30) }\end{array}$ & 332.95 \\
\hline $8.41-14$ & $166505-215670$ & OR of all triggers & 1023.30 \\
\hline
\end{tabular}


Figure 6.2: Trigger efficiency as a function of the electron transverse energy $E_{T}$ for EM_MX (left) and EM_MX_EMFR8 (right) in trigger lists v8 - v11 [85]. Due to the background subtraction method in the determination of the efficiencies, the efficiencies and corresponding uncertainties can exceed 1.
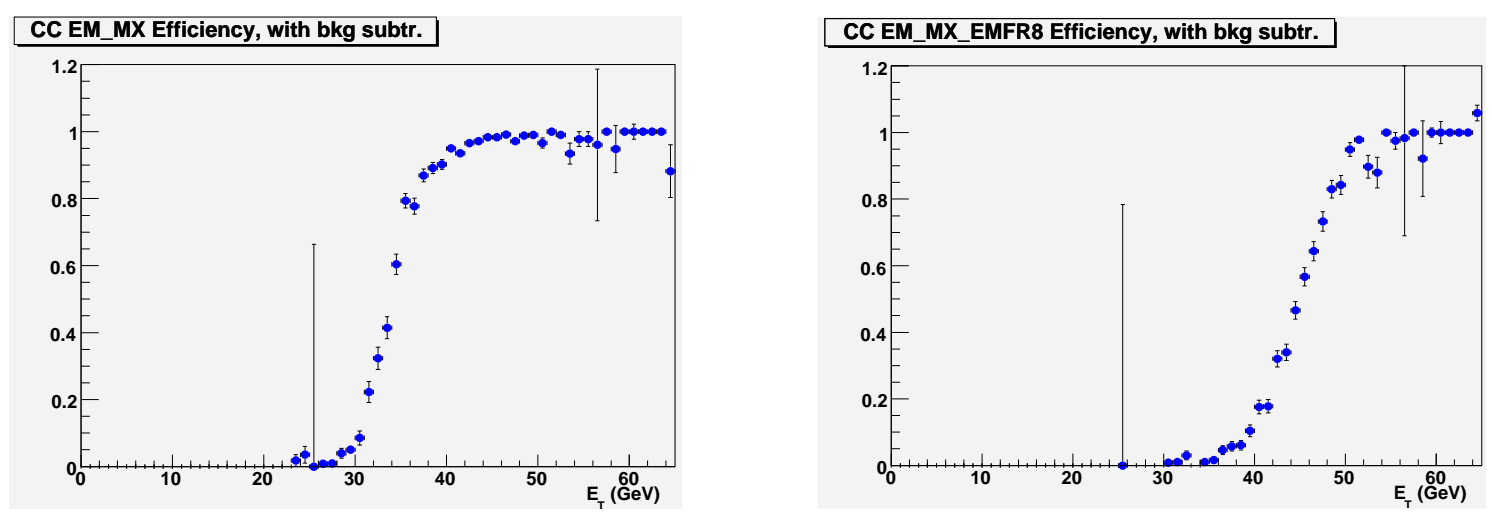

The tighter electron requirement comes along with a lower threshold. In order to maximize the trigger efficiency and minimize the total thresholds, the logical OR of several triggers of a trigger list is used [85]. Fig. 6.2 shows the turn-on curve (efficiency as a function of an offline variable, here: $E_{T}$ of the electron) for both triggers. The loose trigger with the higher threshold usually compensates for inefficiencies of a tighter trigger with lower threshold. However, for the particular triggers shown in Fig. 6.2 the tighter cut on the electromagnetic fraction $e m f>0.9$ for EM_MX (emf $>0.8$ for EM_MX_EMFR8) does not exhibit a strong inefficiency (see also Sec. 4.1, Fig. 4.6).

Not only the trigger ORing, but also the proper determination of the luminosity requires at least one unprescaled trigger (see Sec. 3.4.4). The following triggers satisfy this requirement: EM_MX for trigger lists v8 up to v11, E1_SH30 for trigger lists v12 and v13, and E1_SH35 for trigger list v14. Runs are rejected in which these triggers are prescaled.

The luminosity is calculated for the EMinclusive sample for the different run ranges using the luminosity interface packages (lm_tools [144], see also green box on the righthand side in Fig. 6.1) and yields an integrated luminosity of $1023 \mathrm{pb}^{-1}$. The uncertainty which is dominated by the error in the measurement of the inelastic $p \bar{p}$ cross section is $6.1 \%$ [145]. This luminosity has to be corrected for the bad calorimeter event fraction resulting in $\int \mathcal{L} \mathrm{d} t=(993 \pm 61) \mathrm{pb}^{-1}$.

\subsection{Event Selection Criteria}

The event selection of the inclusive electron + missing transverse energy final state is straightforward. Apart from basic constraints on the event quality (vertex) and the requirement for a high energetic electron and missing transverse energy $\left(\mathrm{MET}, \mathbb{E}_{T}\right.$ ), further efficient "cleaning" cuts are introduced. Starting with the customized Root trees discussed in the previous section, the following cuts (which are explained later in the text) are applied which define the preselection sample: 
- event contains at least one electron candidate with

$-|I D|=10$ or 11 ,

- transverse energy $E_{T}>30 \mathrm{GeV}$,

- pseudorapidity $\left|\eta_{\text {det }}\right|<1.1(\mathrm{CC})$,

- isolation iso $<0.2$,

- electromagnetic fraction emf $>0.9$,

- spatial track match probability $P\left(\chi_{\text {spatial }}^{2}\right)>0.01$;

- missing transverse energy $\mathbb{E}_{T}>30 \mathrm{GeV}$;

- vertex constraints

- primary vertex position $\left|z_{\mathrm{vtx}}\right|<60 \mathrm{~cm}$,

- number of tracks associated to the vertex $\geq 3$;

- further constraints on MET and the electron candidate

$-\left|z_{\mathrm{vtx}}-z_{E M}\right|<5 \mathrm{~cm}$ (electron from primary vertex),

- ratio of electron energy and missing transverse energy $0.6<E_{T} / \mathbb{E}_{T}<1.4$;

- jet veto: if jets with $E_{T}>15 \mathrm{GeV}$ are present

$-\Delta \phi($ jet, electron $)<2.8$,

$-\Delta \phi($ jet, MET $)<2.8$.

If the electron candidate fulfills also the shower shape cut $\chi_{\mathrm{HMx} 7}^{2}<12$, the sample is called $\boldsymbol{W}$ sample. Another subsample - the $\boldsymbol{Q} \boldsymbol{C D}$ sample (see Sec. 6.3) - is derived from the preselection sample. In this case the electron candidate fails the cut on the shower shape variable $\left(\chi_{\mathrm{HMx} 7}^{2}>12\right)$. The search for new heavy gauge bosons is performed in the tail of the transverse mass spectrum, $m_{T}>140 \mathrm{GeV}$ (see Sec. 8.2). This last cut on the $W$ sample defines the search sample. Fig. 6.3 shows an overview of the data samples used in the analysis. In the following the cuts introduced above are discussed (for the electron, jet and neutrino identification see Chap. 4).

Figure 6.3: Overview of the data samples used in the analysis.

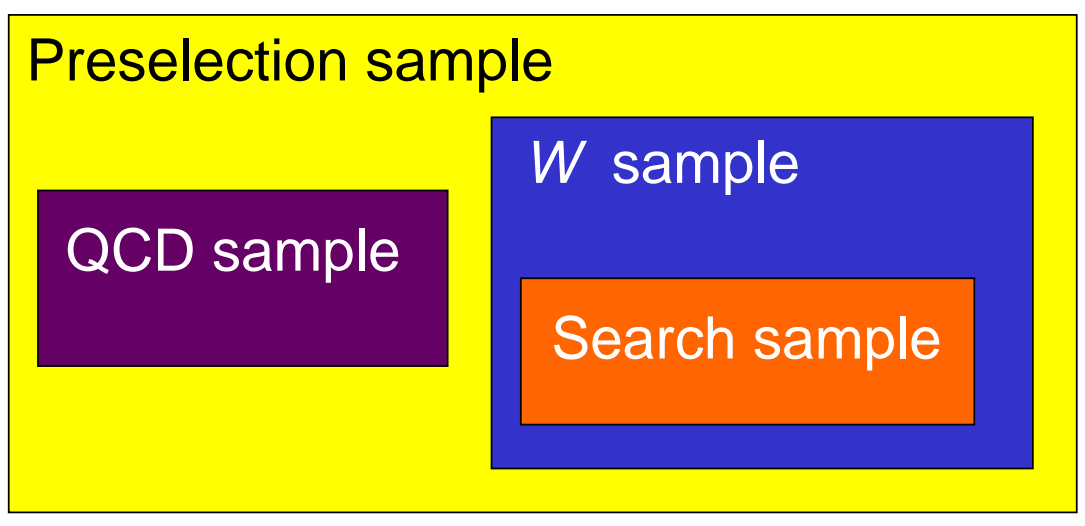


Figure 6.4: Control plots for the $W$ sample. Upper plot: before vertex cut; lower plots: before cut on difference between electron and vertex $z$ position.
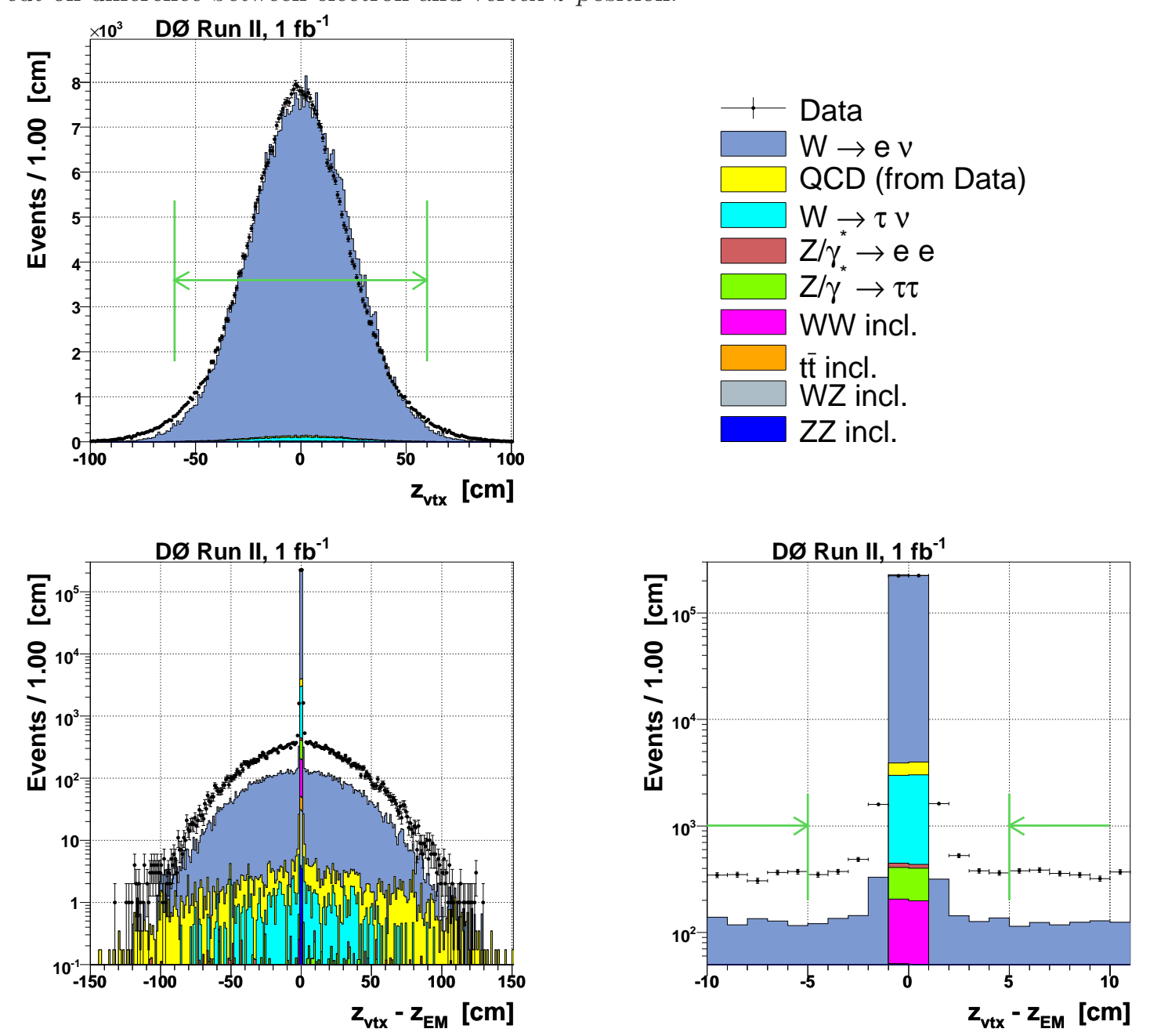

Constraints on the vertex are applied in order to suppress cosmics and backgrounds from beam-induced interactions. The vertex $z_{\mathrm{vtx}}$ with at least three associated tracks is required to be located in the central part of the tracking system. A rather loose cut is applied on the difference between the vertex and electron $z$ position because the Monte Carlo does not describe this variable properly, see lower right-hand plot in Fig. 6.4. In order not to cut in a steeply increasing (decreasing) area, the cut is placed where both, data and Monte Carlo, are flat. Since the missing transverse energy is calculated with respect to the primary vertex, it is important to ensure that the electron also stems from this particular vertex.

The cut on the ratio of the transverse energy of the electron and missing transverse energy is motivated by a Monte Carlo study, see Fig. 6.5. The signal (upper plots) is balanced in terms of transverse energies, $E_{T} \sim \mathbb{E}_{T}$, whereas the QCD and the $Z / \gamma^{*} \rightarrow e e$ samples tend to prefer $E_{T} \ll E_{T}$. In $W \rightarrow e \nu$ Monte Carlo events - which are the dominant background for the search - one observes that the energy imbalance is due to events with jets, see Fig. 6.6. In events with $\mathbb{E}_{T} \gg E_{T}$ the missing energy points (nearly) 
Figure 6.5: In the upper left-hand plot the transverse energy of the electron $E_{T}$ vs. ratio of electron energy $E_{T}$ and missing transverse energy $E_{T}$ is displayed for the $W^{\prime}$ Monte Carlo. The other plots show electron $E_{T}$ vs. $E_{T}$ (for signal, $W \rightarrow e \nu, Z / \gamma^{*} \rightarrow e e, Q C D$ and the $W$ sample). The green lines indicate the selected area.
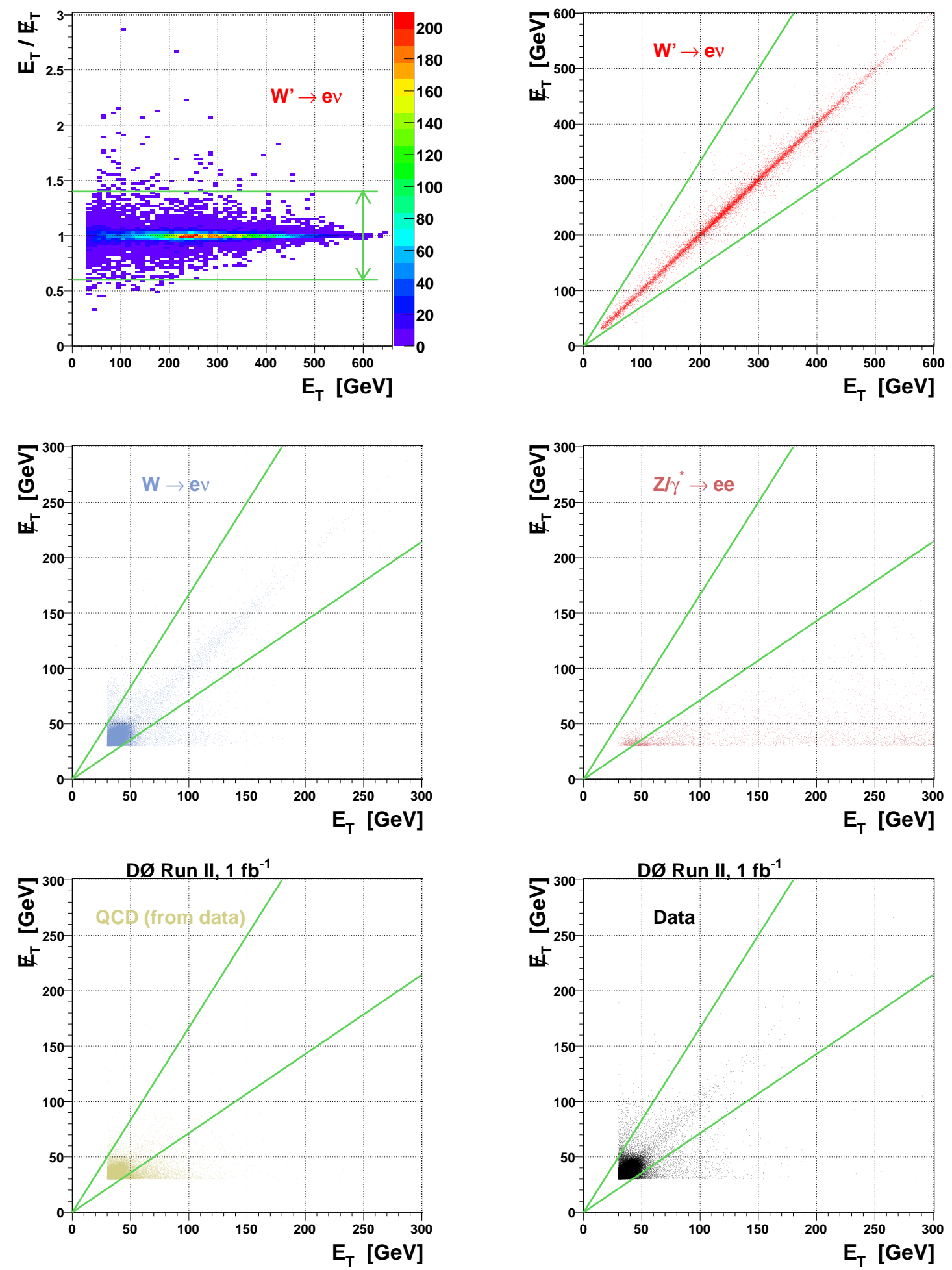
Figure 6.6: The left-hand [right-hand] plot displays the angular differences between jets and electron/MET in $W \rightarrow e \nu$ Monte Carlo events with $E_{T}<0.6 \cdot E_{T}\left[E_{T}>1.4 \cdot E_{T}\right]$ containing at least one jet.
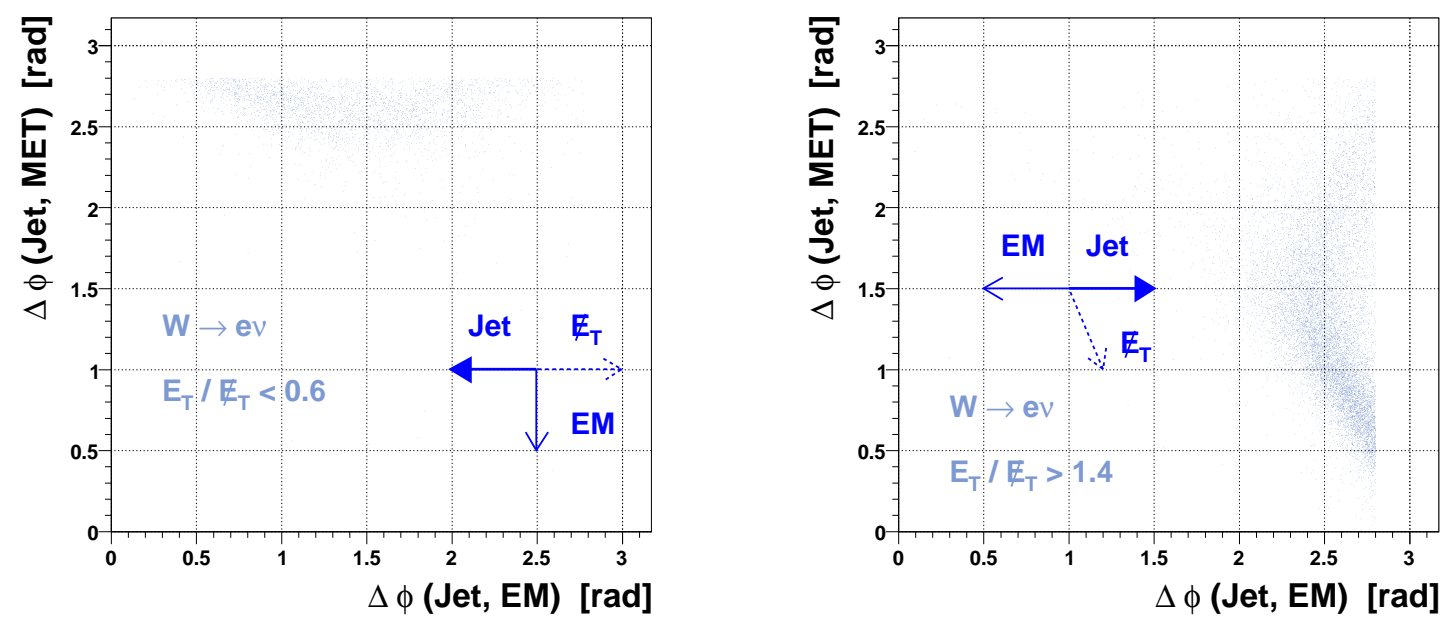

in the opposite direction of the jet, whereas the electron is emitted perpendicular to the jet. In events with $\mathbb{E}_{T} \ll E_{T}$ the electron and the jet are back-to-back $(\Delta \phi=\pi)$. Hence, the ratio cut provides a clean final state without explicitly removing jets.

Jets - if present - are required not to be back-to-back to electron and missing transverse energy in order to efficiently remove events from QCD multijet background. Fig. 6.7 shows 2-dimensional plots of the (maximal) angular differences in events with at least one jet for the $W$ and signal samples, as well as for the following background samples: QCD, $Z / \gamma^{*} \rightarrow e e, t \bar{t}$ and $W \rightarrow e \nu$. One can see that this cut reduces not only the QCD background significantly, but also the instrumental background from $Z / \gamma^{*} \rightarrow e e$ events and $t \bar{t}$ events which contain at least two jets from top quark decays $(t \rightarrow W b)$. The signal is nearly unaffected by this cut, see also Fig. 5.12.

The efficiencies for the selection criteria for the signal Monte Carlo samples are summarized in Tab. 6.4. The numbers - given in percent - show the evolution with the subsequent addition of the various cuts. Note, that the cut on the transverse mass (last row) is correlated with the cut on $\eta_{\text {det }}$, see plots in Fig. 5.14. Tab. 6.5 illustrates the inefficiency $f$ of an individual cut (in percent). $f$ is defined as follows

$$
f=1-\frac{\text { all cuts applied }}{\text { all cuts applied except for the cut under investigation }} .
$$

The electron identification exhibits an inefficiency of $6-8 \%$, whereas the region cut (CC) rejects $13-15 \%$. These numbers do not strongly depend on the $W^{\prime}$ boson mass. However, the cut on the transverse mass becomes more inefficient with increasing mass of the generated heavy bosons ( $1 \%$ for $m_{W^{\prime}}=500 \mathrm{GeV}$ to $16 \%$ for $m_{W^{\prime}}=1200 \mathrm{GeV}$, see also plots in Fig. 5.14). The last row quotes the inefficiency of the combination of region and transverse mass cuts. Due to the extremely low signal-to-background ratio of $1: 10^{5}$ (for $m_{W^{\prime}}=500 \mathrm{GeV}$ ), the low transverse mass region is not used for the search for $W^{\prime}$ bosons. 
Figure 6.7: The plots show the angular differences between jets and electron/MET for the signal, $Q C D, Z / \gamma^{*} \rightarrow e e, t \bar{t}, W \rightarrow e \nu$ and the $W$ sample. The green lines indicate the selected area.
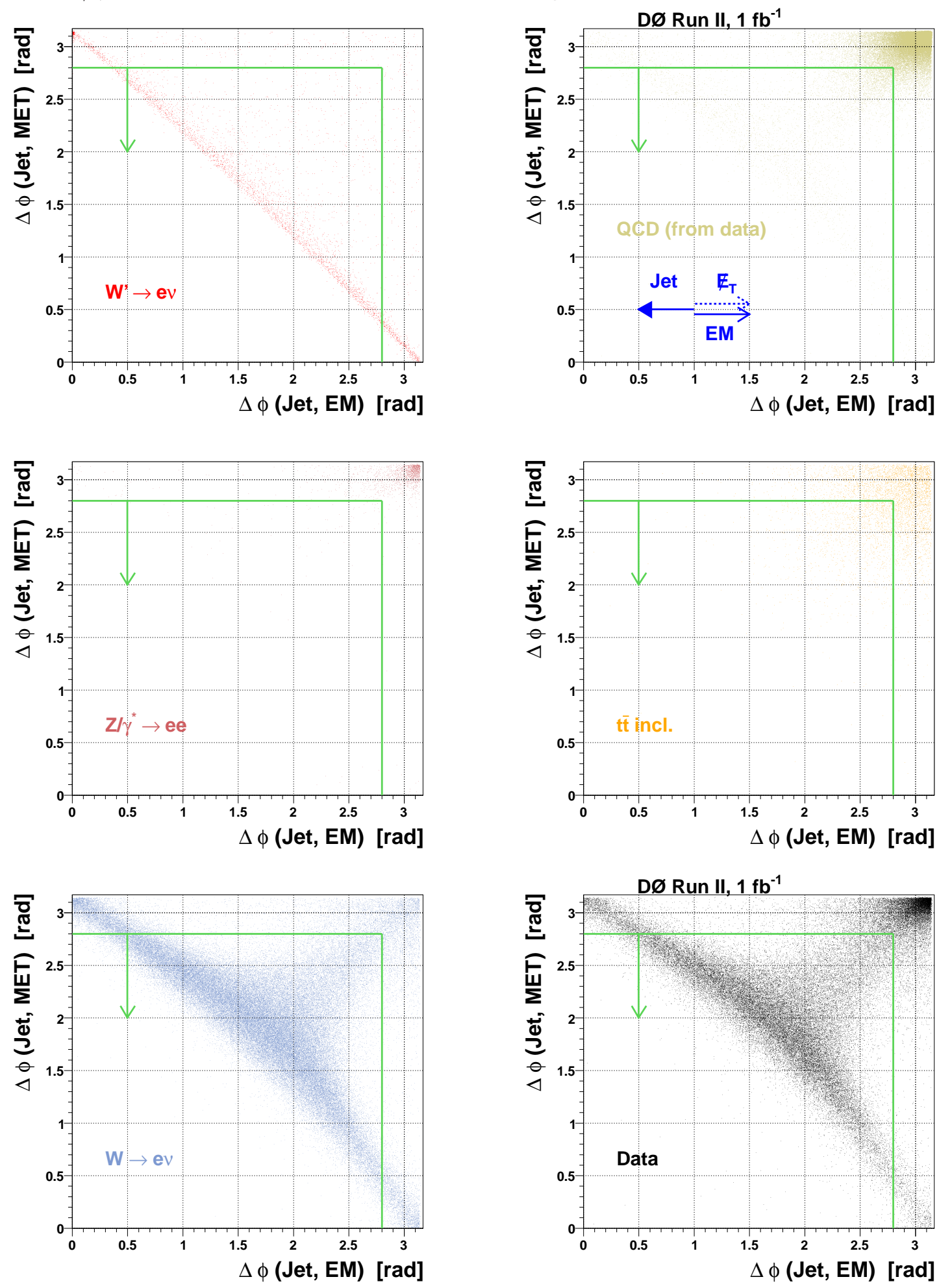
Table 6.4: Efficiencies (in \%) for the selection criteria for the signal Monte Carlo samples.

\begin{tabular}{|l|r|r|r|r|r|r|r|r|}
\hline$m_{W^{\prime}}(\mathrm{GeV})$ & 500 & 600 & 700 & 800 & 900 & 1000 & 1100 & 1200 \\
\hline \hline no cut & 100.0 & 100.0 & 100.0 & 100.0 & 100.0 & 100.0 & 100.0 & 100.0 \\
$+|I D|=10$ or 11 & 92.9 & 92.1 & 92.4 & 92.7 & 92.0 & 92.3 & 92.3 & 91.3 \\
$+E_{T}>30 \mathrm{GeV}$ & 87.2 & 86.8 & 86.6 & 84.5 & 82.2 & 79.5 & 76.1 & 71.9 \\
$+\left|\eta_{\text {det }}\right|<1.1$ & 68.7 & 67.6 & 68.7 & 66.8 & 65.1 & 62.2 & 58.0 & 54.5 \\
$+i s o<0.2$ & 68.7 & 67.6 & 68.7 & 66.8 & 65.1 & 62.2 & 58.0 & 54.5 \\
+ em $f>0.9$ & 67.9 & 67.1 & 68.2 & 66.2 & 64.4 & 61.6 & 57.5 & 53.9 \\
$+\chi_{\text {HMx }}^{2}<12$ & 65.3 & 63.5 & 65.4 & 62.6 & 60.9 & 58.9 & 54.8 & 51.4 \\
$+P\left(\chi_{\text {spatial }}^{2}>0.01\right.$ & 62.2 & 61.0 & 62.3 & 59.8 & 58.3 & 56.5 & 52.4 & 48.6 \\
\hline$+E_{T}>30 \mathrm{GeV}$ & 62.1 & 60.9 & 62.2 & 59.7 & 58.0 & 56.1 & 51.7 & 47.8 \\
\hline$+\left|z_{\text {vtx }}\right|<60 \mathrm{~cm}$ & 61.2 & 60.2 & 61.3 & 58.8 & 57.2 & 55.1 & 50.9 & 47.0 \\
$+n_{\text {tracks }} \geq 3$ & 60.2 & 58.9 & 60.4 & 57.6 & 56.0 & 53.8 & 49.9 & 45.9 \\
\hline$+\left|z_{\text {vtx }}-z_{E M}\right|<5 \mathrm{~cm}$ & 59.2 & 57.9 & 59.6 & 57.2 & 55.2 & 52.6 & 49.0 & 45.1 \\
$+0.6<E_{T} / E_{T}<1.4$ & 58.5 & 57.1 & 59.3 & 56.7 & 54.7 & 51.7 & 47.8 & 44.1 \\
+ jet veto & $\mathbf{5 5 . 0}$ & $\mathbf{5 4 . 1}$ & $\mathbf{5 6 . 4}$ & $\mathbf{5 3 . 6}$ & $\mathbf{5 1 . 9}$ & $\mathbf{4 9 . 7}$ & $\mathbf{4 5 . 8}$ & $\mathbf{4 2 . 0}$ \\
\hline$+m_{T}>140 \mathrm{GeV}$ & $\mathbf{5 4 . 3}$ & $\mathbf{5 3 . 1}$ & $\mathbf{5 4 . 7}$ & $\mathbf{5 1 . 8}$ & $\mathbf{4 8 . 6}$ & $\mathbf{4 4 . 7}$ & $\mathbf{4 0 . 3}$ & $\mathbf{3 5 . 1}$ \\
\hline
\end{tabular}

Table 6.5: Inefficiencies (in \%) for the individual selection criteria of the search sample. All selection criteria are applied except for the one under investigation.

\begin{tabular}{|c|c|c|c|c|c|c|c|c|}
\hline$m_{W^{\prime}}(\mathrm{GeV})$ & 500 & 600 & 700 & 800 & 900 & 1000 & 1100 & 1200 \\
\hline 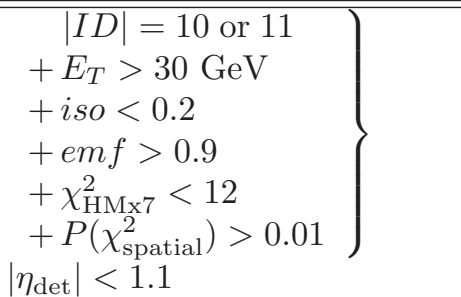 & 13.9 & 15.1 & 14.2 & 14.4 & 14.9 & 13.7 & 13.5 & 13.6 \\
\hline$E_{T}>30 \mathrm{GeV}$ & 0.0 & 0.0 & 0.0 & 0.0 & 0.0 & 0.0 & 0.0 & 0.0 \\
\hline$\left|z_{\mathrm{vtx}}\right|<60 \mathrm{~cm}$ & 1.2 & 0.9 & 1.3 & 1.1 & 1.0 & 1.3 & 1.5 & 1.4 \\
\hline$n_{\text {tracks }} \geq 3$ & 1.5 & 2.0 & 1.3 & 2.0 & 1.8 & 2.1 & 1.9 & 2.2 \\
\hline$\left|z_{\mathrm{vtx}}-z_{E M}\right|<5 \mathrm{~cm}$ & 1.6 & 1.8 & 1.3 & 0.8 & 1.5 & 2.2 & 1.7 & 1.9 \\
\hline $0.6<E_{T} / E_{T}<1.4$ & 0.5 & 0.5 & 0.1 & 0.3 & 0.7 & 0.8 & 0.8 & 0.8 \\
\hline jet veto & 5.9 & 5.3 & 5.0 & 5.5 & 5.3 & 4.1 & 4.3 & 5.2 \\
\hline$m_{T}>140 \mathrm{GeV}$ & 1.3 & 1.9 & 3.0 & 3.3 & 6.5 & 10.0 & 12.2 & 16.3 \\
\hline$\left|\eta_{\text {det }}\right|<1.1 \& m_{T}>140 \mathrm{GeV}$ & 17.9 & 19.3 & 18.9 & 19.1 & 22.2 & 25.1 & 28.1 & 32.0 \\
\hline
\end{tabular}


Figure 6.8: Consistency check for triggers and luminosity. The plots (left: $W$ sample, right: search sample) show the cross sections for each trigger list.
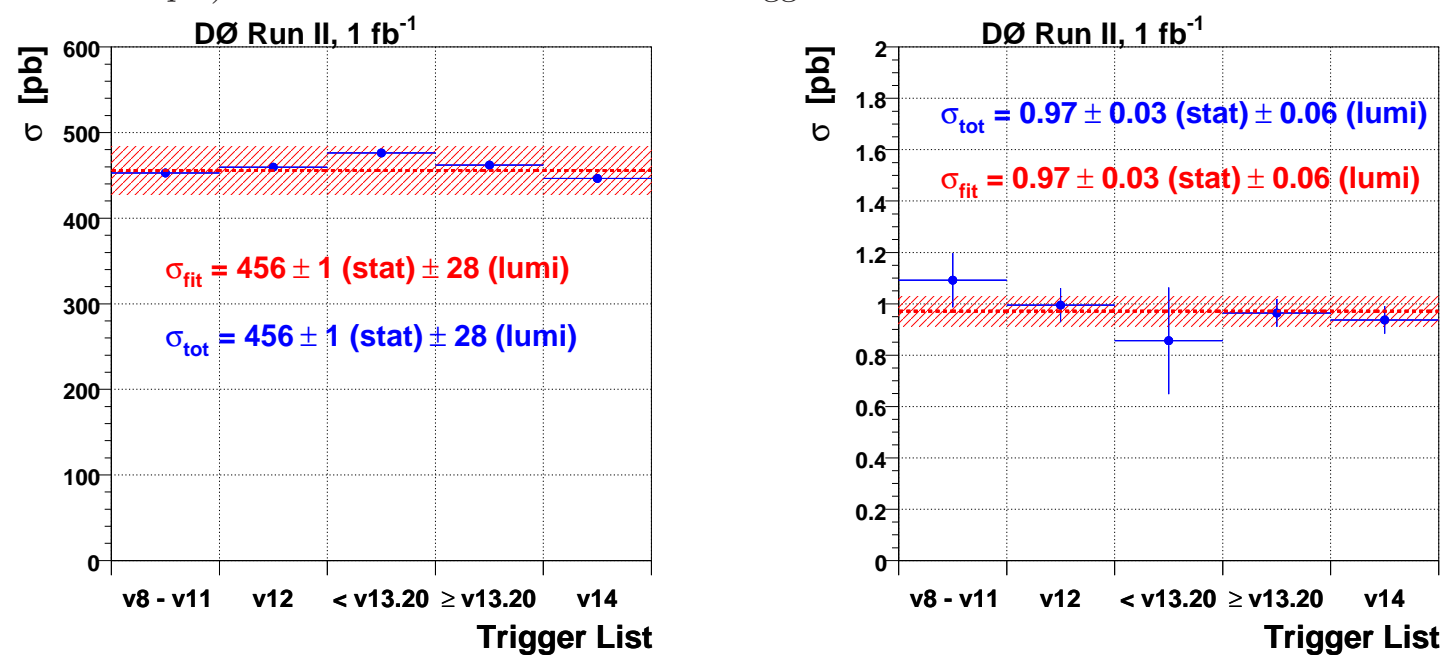

In order to check the consistency between triggers and luminosity, the total dataset is split into five subsamples with respect to the trigger lists that are used, see Tab. 6.3. Fig. 6.8 shows for each subset the corresponding cross section $\sigma_{i}(i=1, \ldots, 5)$ and the total cross section $\sigma_{\text {tot }}$ with

$$
\sigma_{i}=\frac{N_{i}}{\int \mathcal{L}_{i} \mathrm{~d} t} \quad \text { and } \quad \sigma_{\text {tot }}=\frac{N_{\text {tot }}}{\int \mathcal{L} \mathrm{d} t} .
$$

These numbers can be interpreted as the visible production cross section for the inclusive $e+E_{T}+X$ final state defined above. Note, that for the data points only statistical errors are drawn. The shaded areas show the luminosity uncertainty. The distributions are flat within the uncertainties indicating the consistency between event yields, triggers and the corresponding luminosity, as well as the good understanding of the detector performance over time.

\subsection{QCD Background}

Events from QCD multijet production can contribute to the final state under investigation: One jet can be misidentified as an electron, and additional energy mismeasurement in the event can cause large missing energy. A typical di-jet data event containing a hadronic and a (mainly) electromagnetic jet is shown in Fig. 6.9. The event display shows the tracker with hits (blue points) and reconstructed tracks, and calorimeter towers (64 $\phi$ segments corresponding to $\Delta \phi \approx 0.1$ in the $X Y$ view, $\eta$ is segmented with $\Delta \eta=0.1$ in the $R Z$ view) with energy depositions in the electromagnetic (red) and hadronic layers (blue). Hits in the wire chambers of the muon system are drawn as colored boxes outside the calorimeter towers. The yellow bars in the $X Y$ view and the lego plot indicate the missing transverse energy. The event contains a well reconstructed electron candidate with $E_{T}=265 \mathrm{GeV}$ and $\chi_{\mathrm{HMx} 7}^{2}=9$

In order to estimate this contribution, the $Q C D$ sample as defined in the previous section is utilized. In this sample, the electron candidate fails the shower shape cut. 
Figure 6.9: Schematic illustration of QCD multijet background with one jet misidentified (top) and example of a di-jet event observed in DØ data (bottom): XY view (left); RZ view (upper right); lego plot (lower right).
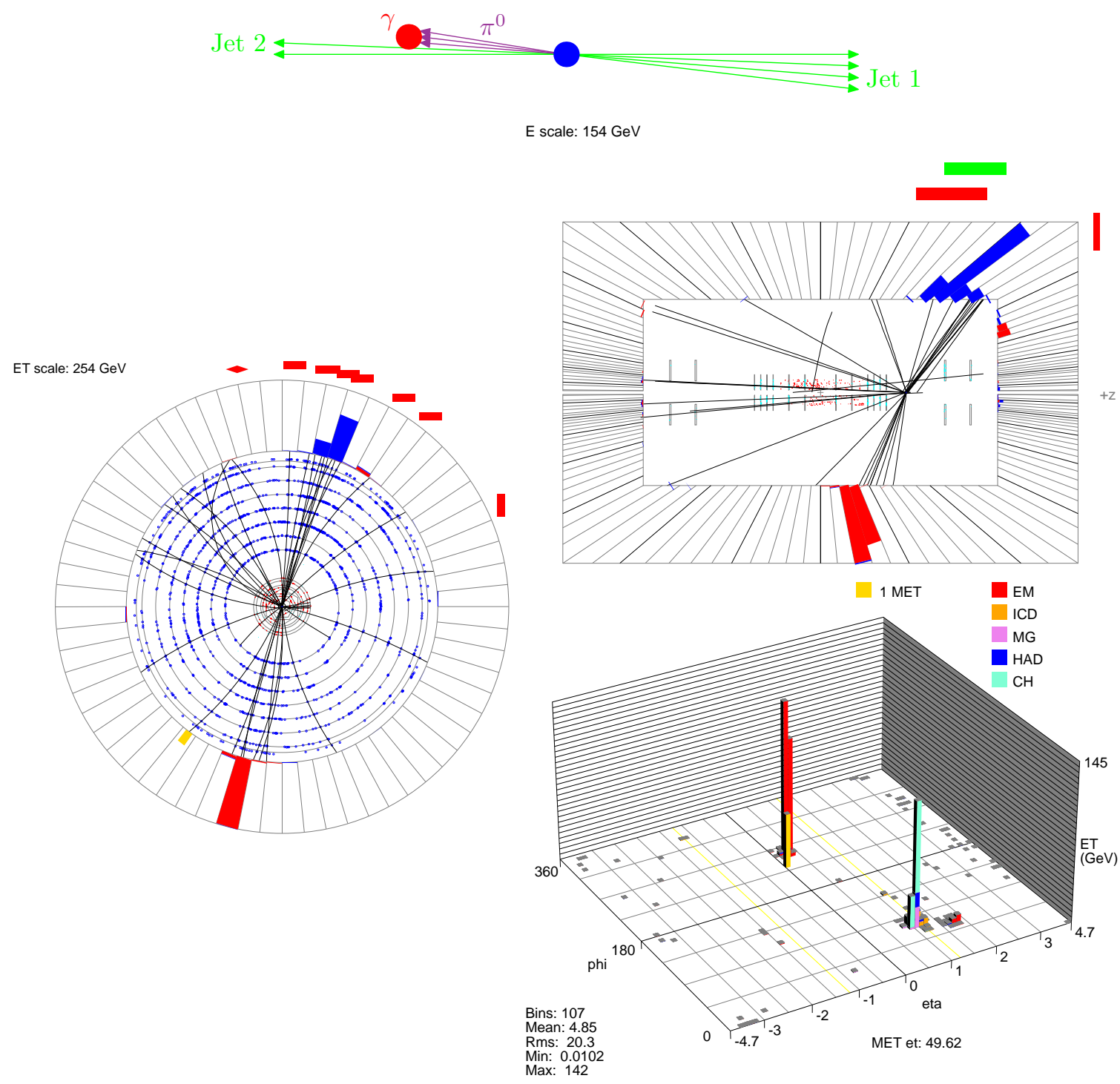

Although a huge amount of events of this kind is rejected by the cleaning cuts (energy ratio cut, jet veto), the sample still contains roughly 70,000 events. "Real" electrons having high values of $\chi_{\mathrm{HMx} 7}^{2}$ are taken into account by subtracting the badly reconstructed electrons in the background Monte Carlo samples from the QCD data sample. The plot on the left-hand side of Fig. 6.10 shows the distribution of $\chi_{\mathrm{HMx} 7}^{2}$ for data events and $W \rightarrow e \nu$ Monte Carlo events.

The resulting events are scaled to the $W$ sample, whereas the scale factor $f_{\mathrm{QCD}}$ is determined via minimizing the $\chi^{2}$ distribution

$$
\chi^{2}(f)=\sum_{m_{T}^{\min }}^{m_{T}^{\max }}\left(\frac{\text { Data }-(\text { PYTHIA background samples }+f \cdot \text { QCD sample })}{\text { Statistical uncertainty }}\right)^{2} .
$$


It is summed over $N_{\text {bins }}$ bins of a subset of the transverse mass distribution, $\left[m_{T}^{\min }, m_{T}^{\max }\right]$. The value of the scaling factor is extracted at the minimum of the $\chi^{2}$ distribution

$$
f=f_{\mathrm{QCD}} \quad \Leftrightarrow \quad \chi^{2}\left(f_{\mathrm{QCD}}\right)=\chi_{\min }^{2} .
$$

The value at the minimum provides information about the goodness of the fit, and should correspond to the number of degrees of freedom, $N_{\text {dof }}$,

$$
\chi_{\text {min }}^{2} \simeq N_{\text {dof }}=N_{\text {bins }}-1 \quad \Leftrightarrow \quad \frac{\chi_{\text {min }}^{2}}{N_{\text {bins }}-1} \simeq 1 .
$$

This procedure is conducted in a region dominated by QCD background events. Fig. 6.10 (right-hand plot) shows the QCD sample before the subtraction of Monte Carlo electrons with $\chi_{\mathrm{HMx} 7}^{2}>12$. One can clearly see the aggregation at transverse masses $m_{T}$ of $50-$ $90 \mathrm{GeV}$ which stems from "real", but badly reconstructed electrons from $W$ decays. The "real" QCD multijet background is not expected to peak at these masses. Hence, the region of low reconstructed transverse masses $\left(m_{T}^{\min }=0 \mathrm{GeV}, m_{T}^{\max }=20 \mathrm{GeV}\right)$ is consulted for the adjustment of $f_{\mathrm{QCD}}$. One can see from Fig. 6.11 that a discrepancy between data and background prediction (excluding the QCD contribution) would be observable if not filling up with events derived from the QCD sample. In order to estimate the uncertainty, the fit range (namely $\left.m_{T}^{\max }\right)$ has been varied. The resulting scale factor is $\left(\chi_{\min }^{2} / N_{\text {dof }}=0.985\right)$

$$
f_{\mathrm{QCD}}=0.173 \pm 0.012 \text {. }
$$

Since the transverse mass $m_{T}$ is connected with the angular difference between electron and missing transverse energy $\Delta \phi(E M, M E T)$ (cf. Eq. 5.7) via

$$
m_{T}=\sqrt{2 E_{T} E_{T}(1-\cos \Delta \phi(E M, M E T))},
$$

one can use this distribution as well for cross checks, see the right-hand plot in Fig. 6.11. $f_{\mathrm{QCD}}$ is fairly insensitive to the scaling with the correction factors that have to be applied to the PyтніA Monte Carlo samples (like the luminosity) because the contributions from these processes are small in the fit region.

Figure 6.10: Left: $\chi_{\mathrm{HMx} 7}^{2}$ distribution for the $W$ data sample (black) and $W \rightarrow e \nu$ (blue) Monte Carlo events (both normalized to 1). The plot on the right-hand side shows the angular difference between electron and MET, $\triangle \phi(E M, M E T)$, as a function of the transverse mass $m_{T}$ for the QCD sample before the subtraction of badly reconstructed electrons.
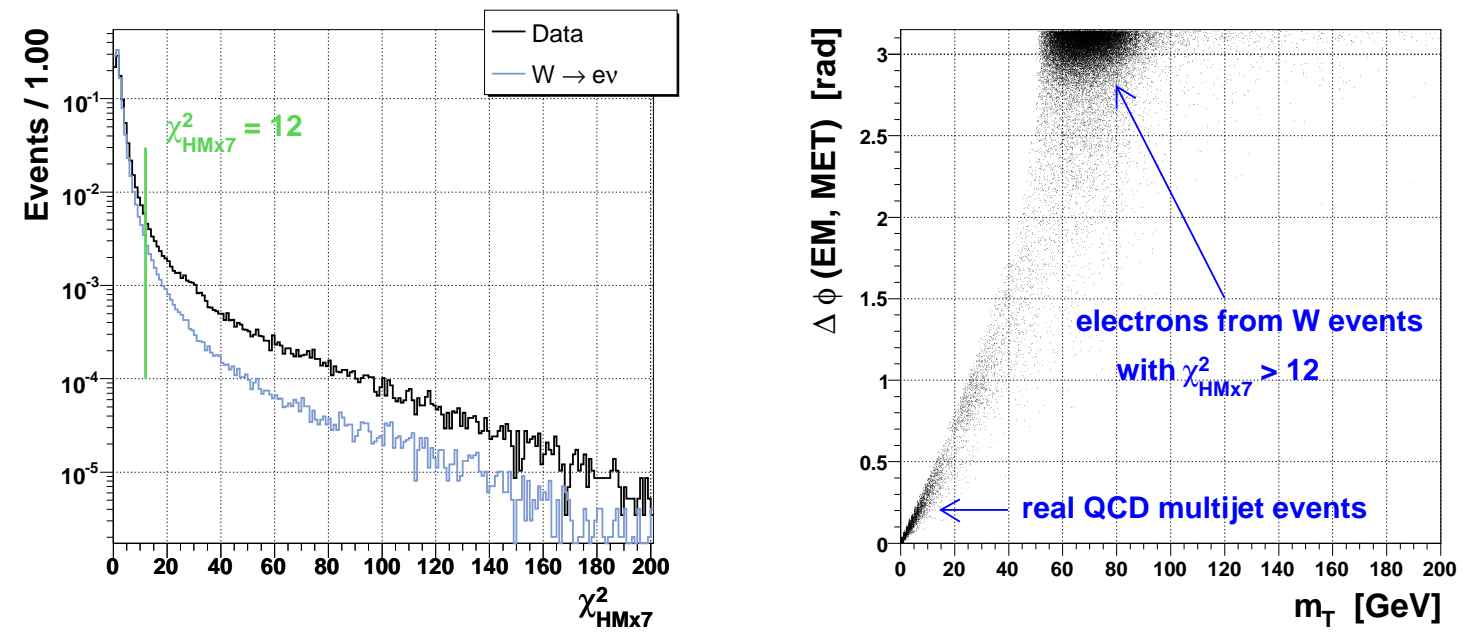
Figure 6.11: After scaling the $Q C D$ sample (yellow) with $f_{\mathrm{QCD}}$ to the $W$ sample, the agreement at low transverse masses (left-hand plot) is good. Low transverse masses correspond to small angular differences (cf. Eq. 6.7) as shown in the right-hand plot.
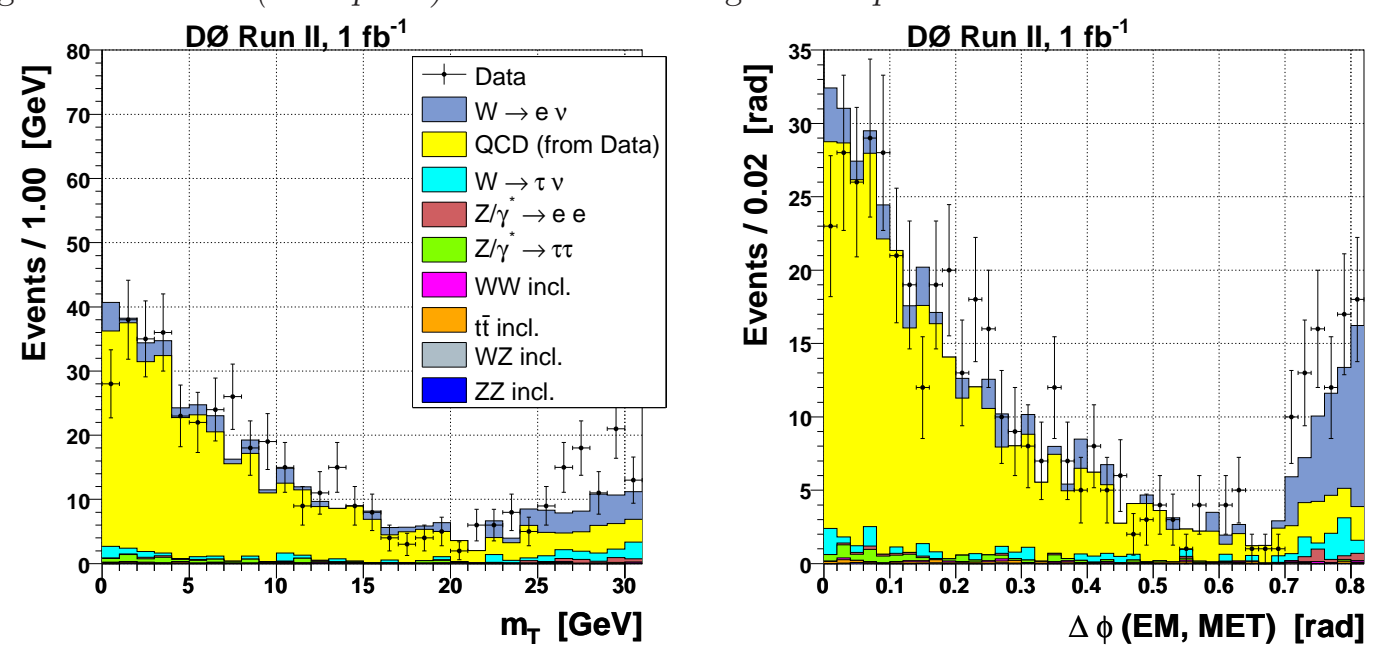

\subsection{Effective Luminosity $\mathcal{L}_{W}$}

The integrated luminosity $\mathcal{L}_{\text {Data }}=\int \mathcal{L} \mathrm{d} t$ can be treated as a free parameter which is determined by comparing the background expectation with the data in a known region. This is possible because the luminosity enters the (PyTHIA) Monte Carlo samples via global event weights $w$ according to

$$
\mathcal{L}_{M C}=\frac{N_{\text {gen }}}{\sigma} \quad \Rightarrow \quad w=\frac{\mathcal{L}_{\text {Data }}}{\mathcal{L}_{M C}}=\frac{\sigma}{N_{\text {gen }}} \cdot \mathcal{L}_{\text {Data }}
$$

The cross sections and numbers of generated events are summarized in Tab. 5.1. The normalization is performed in the $W$ peak region, thus $m_{T}^{\min }=60 \mathrm{GeV}$ and $m_{T}^{\max }=$ $120 \mathrm{GeV}$, shown in Fig. 6.12. Here, the corrections for differences in reconstruction efficiencies (see Sec. 7.2.1) are already taken into account. The free parameter $\mathcal{L}_{\text {Data }} \equiv \mathcal{L}_{W}$ is determined minimizing the $\chi^{2}$ distribution, Eq. 6.3. Errors on $\mathcal{L}_{W}$ are mainly due to the $W$ production cross section uncertainty of $\sim 4 \%$ (see Tab. 5.1). Other processes (and the corresponding uncertainties) - including the QCD background - can safely be ignored because more than $98 \%$ of the events in the $W$ peak region stem from $W \rightarrow e \nu$. The result is $\left(\chi_{\min }^{2} / N_{\text {dof }}=3.45\right)$

$$
\mathcal{L}_{W}=(963 \pm 39) \mathrm{pb}^{-1},
$$

which is $\sim 3 \%$ smaller than the luminosity calculated using the luminosity monitors and the luminosity tools $\left(\int \mathcal{L} \mathrm{d} t=993 \pm 61 \mathrm{pb}^{-1}\right)$, but consistent within the uncertainties. This analysis uses the effective luminosity $\mathcal{L}_{W}$ and its uncertainty. 
Figure 6.12: Distribution of the transverse mass $m_{T}$ for the $W$ sample after applying the luminosity scale factor $\mathcal{L}_{W}$ (top: logarithmic scale, bottom: linear scale).
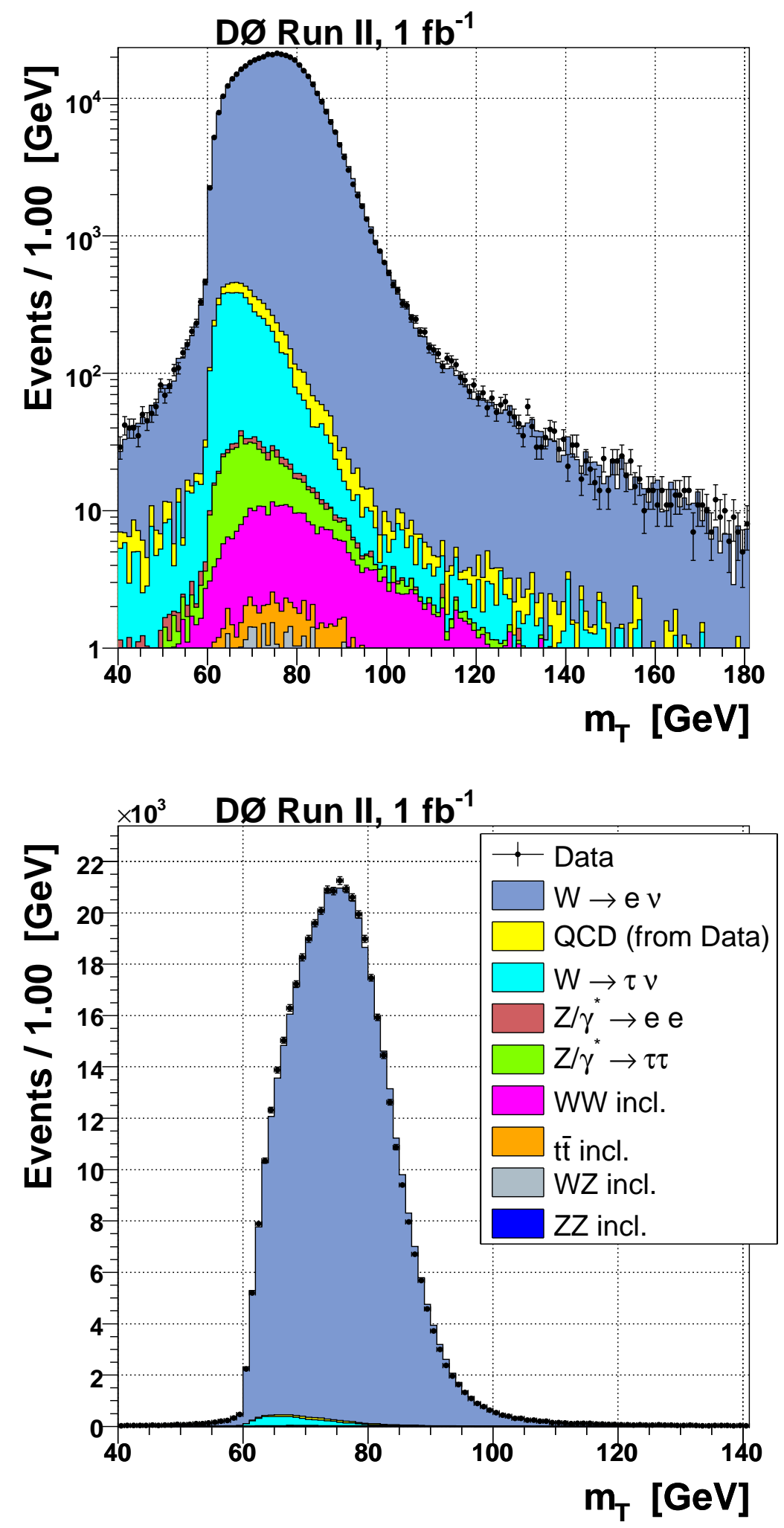


\section{Chapter 7}

\section{Efficiencies, Corrections and Uncertainties}

The first principle is that you must not fool yourself and you are the easiest person to fool.

Richard Feynman

The prediction of background events from generated PYTHIA Monte Carlo processes and the prepared QCD sample can only be compared to the data after proper normalization. Since certain characteristics are quite different for data and Monte Carlo events, the latter have to be corrected for effects that the full detector simulation is not capable to describe sufficiently or does not even include at all (like the trigger emulation). In the first part of this chapter the following items are addressed:

(1) Correction for electron energy resolution and scale (Sec. 7.1);

(2) Correction for the difference in reconstruction efficiencies (Sec. 7.2.1);

(3) Check for trigger inefficiencies (Sec. 7.2.2);

(4) Correction for higher order effects (jets in the $W$ Monte Carlo sample, Sec. 7.3).

The last part of this chapter (Sec. 7.4) deals with uncertainties related to the corrections.

\subsection{Electron Energy Resolution and Scale}

The calorimeter energy resolution is not perfectly modeled in the detector simulation due to for example missing material in the description of the detector geometry (dØgstar) [146]. In order to reproduce the resolution observed in data, basic quantities have to be smeared in the Monte Carlo events during the post-processing phase (døcorrect). The basic variable regarding the electron reconstruction is its total energy deposited in the calorimeter, E. All derived quantities are recalculated, including the missing transverse 
energy. The smearing parameters are optimized using clean di-electron data events on the $Z$ resonance, $Z \rightarrow e e$. All Monte Carlo samples used in this analysis are already corrected for the resolution observed in data.

In the following the electron resolution and scale are derived from Monte Carlo electrons, and compared to the nominal values determined from data. Due to the large energy spread in the electrons from $W^{\prime}$ decays, the signal Monte Carlo samples can be utilized for these cross checks. Fig. 7.1 displays the Monte Carlo electron energy resolution in the central calorimeter region derived from $W^{\prime} \rightarrow e \nu$ events. The blue line indicates the parameterization (cf. Eq. 3.10) which comprises the measured values of the parameters quoted in Tab. 3.5. Since the constant term $C=0.041 \pm 0.003$ [71] is dominant at energies above $100 \mathrm{GeV}$, only this uncertainty is shown (dashed blue lines). For the resolution study intervals in terms of the true electron energy are constructed (see upper left-hand plot in Fig. 7.2) with

$$
\text { Mean }=\frac{E_{\min }+E_{\max }}{2} \text { and } \sigma=\frac{E_{\max }-E_{\min }}{\sqrt{12}}=\frac{5 \mathrm{GeV}}{\sqrt{12}} \approx 1.4 \mathrm{GeV} .
$$

The Monte Carlo resolution is extracted from the reconstructed electron energy distributions that correspond to the true electron energy intervals, see upper right-hand plot in Fig. 7.2. The value is given by the standard deviation $\sigma$ which has to be corrected for the interval width of $5 \mathrm{GeV}$. The lower plot in Fig. 7.2 indicates that the reconstructed energy $E$ can be obtained using a Gaussian with mean $\mu=E^{\text {true }}$ and width $\sigma=0.041 \cdot E^{\text {true }}$ due to the dominance of the constant term

$$
\begin{aligned}
f\left(\frac{E^{\text {true }}-E}{E^{\text {true }}}\right) & \propto \operatorname{gauss}\left(\frac{E^{\text {true }}-E}{E^{\text {true }}} ; 0,0.041\right) \\
f(E) & \propto \operatorname{gauss}\left(E ; E^{\text {true }}, 0.041 \cdot E^{\text {true }}\right)
\end{aligned}
$$

Figure 7.1: Electron energy resolution $\Delta E$ as a function of the energy $E$ derived from $W^{\prime} \rightarrow e \nu$ Monte Carlo events. The blue line indicates the measured resolution according to Eq. 3.10 and Tab. 3.5, the dashed blue lines mark the uncertainty stemming from the constant term $C$.

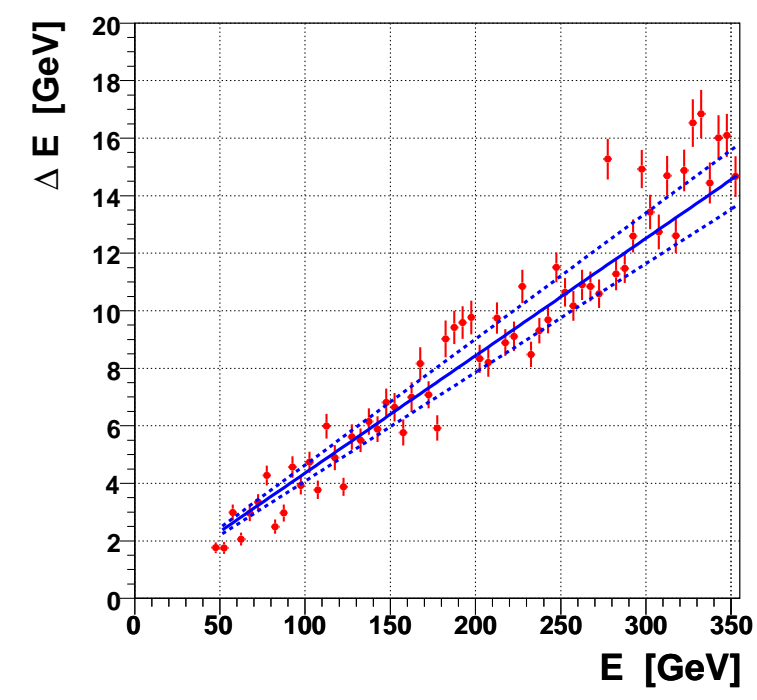


Figure 7.2: Electron energy resolution derived from $W^{\prime} \rightarrow e \nu$ Monte Carlo events. The upper plots show the true (left) and reconstructed (right) electron energy for two selected intervals. The lower plot shows another representation of the resolution.
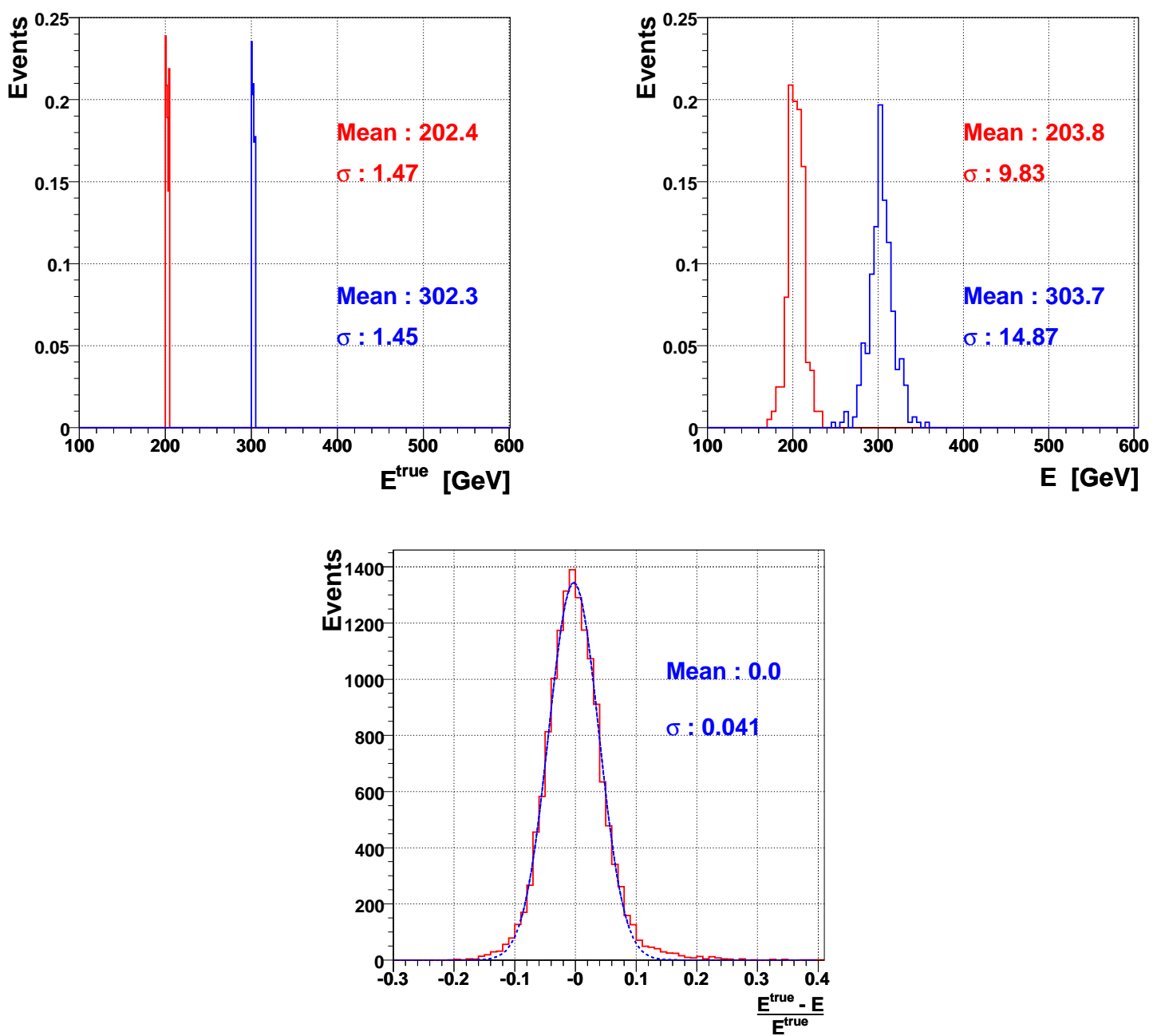

with

$$
\operatorname{gauss}(x ; \mu, \sigma)=\frac{1}{\sqrt{2 \pi} \sigma} \exp \left\{-\frac{(x-\mu)^{2}}{2 \sigma^{2}}\right\} .
$$

In summary, the reconstructed Monte Carlo electron energy $E$ follows a Gaussian distribution $(f(E)$ in Eq. 7.2) with the measured resolution. The small non-Gaussian tails are due to additional contributions to the resolution besides the constant term ( $N$ and $S$ at lower energies).

The electron energy scale can be measured very precisely (at the $0.2 \%$ level [71]) using di-electron data events from $Z$ decays

$$
E=(0.191 \pm 0.048) \mathrm{GeV}+(1.0054 \pm 0.0020) \cdot E^{\text {true }} .
$$

Since the energy scale has to be extrapolated to very high energies (see Fig. 5.10), a conservative estimate of the uncertainty of $2 \%$ is assumed [147]. The electron energy scale can as well be checked using signal Monte Carlo events. Instead of the standard deviation, 
Figure 7.3: Reconstructed electron energy $E$ as a function of the true energy $E^{\text {true }}$ derived from $W^{\prime} \rightarrow$ ev Monte Carlo events. The blue line indicates the measured scale Eq. 7.4, the dashed blue lines the uncertainty of $2 \%$.

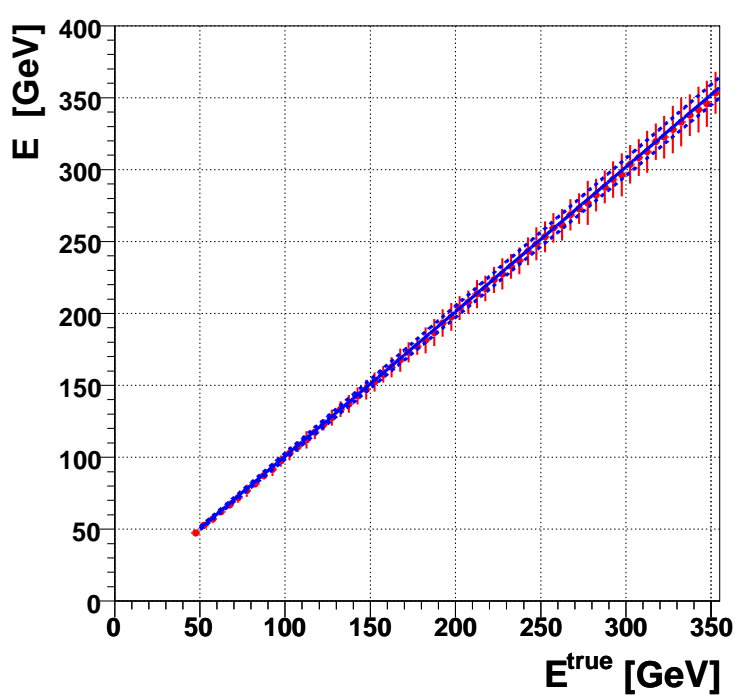

the means are here consulted, see Fig. 7.3. The plot also shows the measured energy scale (blue line) including the $2 \%$ uncertainty (dashed blue lines). In summary, the measured electron energy scale and resolution are reproduced by the tuned Monte Carlo samples.

\subsection{Efficiencies}

Although the Monte Carlo samples are adjusted to the resolution observed in data and the same reconstruction algorithms are applied to data and Monte Carlo events, different reconstruction efficiencies in both cases are observed. Hence, correction factors which depend on the location of the electron in the detector $\left(\eta_{\text {det }}\right.$ and $\left.\phi_{\text {det }}\right)$ have to be applied on an event-by-event basis.

Furthermore, the trigger simulation is not part of the full detector simulation so that in principle the turn-on behaviour of the triggers as a function of the electron transverse energy $E_{T}$ has to be taken into account. Due to the event selection and the choice of multiple highly-efficient triggers, this correction can be omitted. However, a check for possible trigger inefficiencies is performed. The correction factors as well as the turn-on curves are prepared by the DØ Electron and Photon Identification (EMID) Group [148]. In the spirit of a common framework, all corrections are contained in packages (see Fig. 6.1) and can be accessed within CAFE.

\subsubsection{Electron Reconstruction Efficiencies}

The efficiencies are measured using the tag and probe method which is applied to $Z \rightarrow e e$ Pythia Monte Carlo events and to a sample of di-electron data events from $Z$ decays, where the invariant mass of the two electron candidates is required to be consistent with 
the mass of the $Z$ boson. The following very tight cuts on a calorimeter electron candidate matched to a track define the tag electron

EM: $|I D|=10$ or 11 , iso $<0.2$ emf $>0.9,\left\{\begin{array}{ll}\left|\eta_{\operatorname{det}}\right|<1.1: & \chi_{\mathrm{HMx} 7}^{2}<12 \\ 1.5<\left|\eta_{\operatorname{det}}\right|<2.5: & \chi_{\mathrm{HMx} 8}^{2}<20\end{array}\right.$, $E_{T}>25 \mathrm{GeV}$,

Track: track matched to EM object (with $E / p$ ), $p_{T}^{\text {track }} / \Delta p_{T}^{\text {track }}>1,>0$ SMT hits;

Trigger: electron matched to trigger electron.

The other electron - the probe electron - is required to pass the cuts under study. The efficiency $\varepsilon$ is calculated by counting events in which the probe candidate passes $(P)$ or fails $(F)$ the cut under investigation

$$
\varepsilon=\frac{P}{P+F} .
$$

In order to remove any bias, the probe electron is as well checked for passing the tag cuts. Further, the efficiency is calculated per electron rather than per event. For example, an event with two electrons satisfying the tag conditions is counted twice. The efficiency is quoted as a function of one $\left(E_{T}, \eta_{\text {det }}, \phi_{\text {det }}\right)$ or two $\left(\eta_{\text {det }}\right.$ and $\left.\phi_{\text {det }}\right)$ specific electron variables. Although the tight identification cuts on the tag electron ensure purity, a certain amount of background events remain which can bias the efficiency measurement. Therefore the background is subtracted after fitting the invariant mass peak with a signal and background hypothesis. In case of the $Z$ Monte Carlo the background subtraction is omitted.

The electron reconstruction efficiencies for both, data and Monte Carlo, are determined in two steps: first for electrons satisfying loose cuts, then for electrons meeting the tighter cuts (used in the analysis) with

loose electron: $|I D|=10$ or 11 , iso $<0.2$, emf $>0.9, E_{T}>15 \mathrm{GeV}$;

tight electron: loose electron, $P\left(\chi_{\mathrm{spatial}}^{2}\right)>0.01, \begin{cases}\left|\eta_{\mathrm{det}}\right|<1.1: & \chi_{\mathrm{HMx} 7}^{2}<12 \\ 1.5<\left|\eta_{\mathrm{det}}\right|<2.5: & \chi_{\mathrm{HMx} 8}^{2}<20\end{cases}$

so that the total reconstruction efficiency corresponds to the efficiency for the loose identification multiplied by the tight identification efficiency based on loose probe electron candidates

$$
\varepsilon=\varepsilon(\text { loose }) \cdot \varepsilon(\text { tight } \mid \text { loose }) .
$$

Details about the method and the results can be found in [149] and references therein.

\section{Loose Electron Identification}

In this case an isolated track with the following specifications serves as probe electron candidate

- $p_{T}>12 \mathrm{GeV}$ in $\left|\eta_{\text {det }}\right|<1.1$ or $1.5<\left|\eta_{\text {det }}\right|<2.5$ with $>0$ SMT hits,

- distance of closest approach to the beam line in the transverse plane $|d c a|<1 \mathrm{~cm}$, 
- track is separated from a muon candidate $\Delta R$ (track, muon) $>0.2$,

- sum of track momenta $<3 \mathrm{GeV}$ in a cone of $R=0.4$ around the track candidate,

- $\mid \Delta z$ (track, tag electron) $\mid<2 \mathrm{~cm}$ (both objects from the same vertex),

- $\Delta \phi$ (track, tag electron) $>2$ (both objects back-to-back in $\phi)$,

- invariant mass $m_{\text {inv }}$ (track, tag electron) $>65 \mathrm{GeV}$.

The probe track passes the loose electron identification criteria if it can be matched to a loose electron candidate with $\Delta R$ (track, loose electron) $<0.1$. Fig. 7.4 (upper lefthand plot) shows the loose electron identification efficiency for data and Monte Carlo as a function of $\eta_{\text {det }}$. The efficiencies for data and Monte Carlo are very similar, but one observes increasing differences towards small values of $\left|\eta_{\text {det }}\right|$. The correction factor $f=\varepsilon_{\text {Data }} / \varepsilon_{\mathrm{MC}}$ varies in the central calorimeter region from 0.96 to 1 . The correction is constant as a function of the transverse energy for $E_{T}>30 \mathrm{GeV}$ in $\left|\eta_{\text {det }}\right|<1.1$ (upper right-hand plot). Note, that due to fluctuations in the background, the efficiency can be larger than one.

\section{Tight Electron Identification}

The tight electron identification efficiency is determined with respect to electrons that passed the loose identification cuts. Hence, loose electron candidates serve as probe electrons in this case. The plots in Fig. 7.4 (middle and bottom) show the efficiency as a function of miscellaneous variables. One can see that the efficiencies in data and Monte Carlo can differ substantially depending on the location $\left(\eta_{\text {det }}\right.$ and $\left.\phi_{\text {det }}\right)$ of the electron. The ratio $f=\varepsilon_{\text {Data }} / \varepsilon_{\text {MC }}$ as a function of the electron transverse energy (plot on the lower right-hand side) is flat for electrons with $E_{T}>30 \mathrm{GeV}$ reconstructed in the central detector region $\left(\left|\eta_{\text {det }}\right|<1.1\right)$.

In the analysis Monte Carlo events containing an electron that satisfies the tight electron identification criteria - and thus the electron selection in the analysis - are weighted with

$$
f=f\left(\text { loose } ; \eta_{\text {det }}\right) \cdot f\left(\text { tight } \mid \text { loose } ; \eta_{\text {det }}, \phi_{\text {det }}\right)
$$

in order to agree with efficiencies observed in data. The left-hand plot in Fig. 7.5 shows the overall correction factor as a function of $\eta_{\text {det }}$ and $\phi_{\text {det }}$ which varies from 0.72 to 1.08 . The corresponding uncertainty on the correction factor displayed in the right-hand plot is of the order of $3 \%$.

The reconstruction efficiencies and correction factors as a function of the transverse energy $E_{T}$ are found to be flat for electrons from $Z$ decays with energies $E_{T}<65 \mathrm{GeV}$ events. Since the signal is expected to have energies well above this threshold (see Fig. 5.10), the reconstruction efficiency has to be checked for a possible energy dependence. For this study the $W^{\prime} \rightarrow e \nu$ signal Monte Carlo sample is consulted. Fig. 7.6 shows efficiencies as a function of the transverse energy $E_{T}$ for the following identification criteria: emf, iso, $\chi_{\mathrm{HMx} 7}^{2}, P\left(\chi_{\mathrm{spatial}}^{2}\right)$. The plot on the bottom shows the overall reconstruction efficiency which is constant for all transverse energies. This justifies the application of correction factors which depend only on the location of the electron in the detector. 
Figure 7.4: The upper plots show the electron reconstruction efficiencies in data and Monte Carlo events as a function of $\eta_{\text {det }}$ (left) and $E_{T}$ (right, relative efficiency) for the loose identification. The plots in the middle and bottom show the electron reconstruction efficiencies in data and Monte Carlo events for the tight identification as a function of $\eta_{\operatorname{det}}$ and $\phi_{\operatorname{det}}$ (middle), and the relative efficiencies as a function of ( $\left.\eta_{\mathrm{det}}, \phi_{\mathrm{det}}\right)$ and $E_{T}$ (bottom).
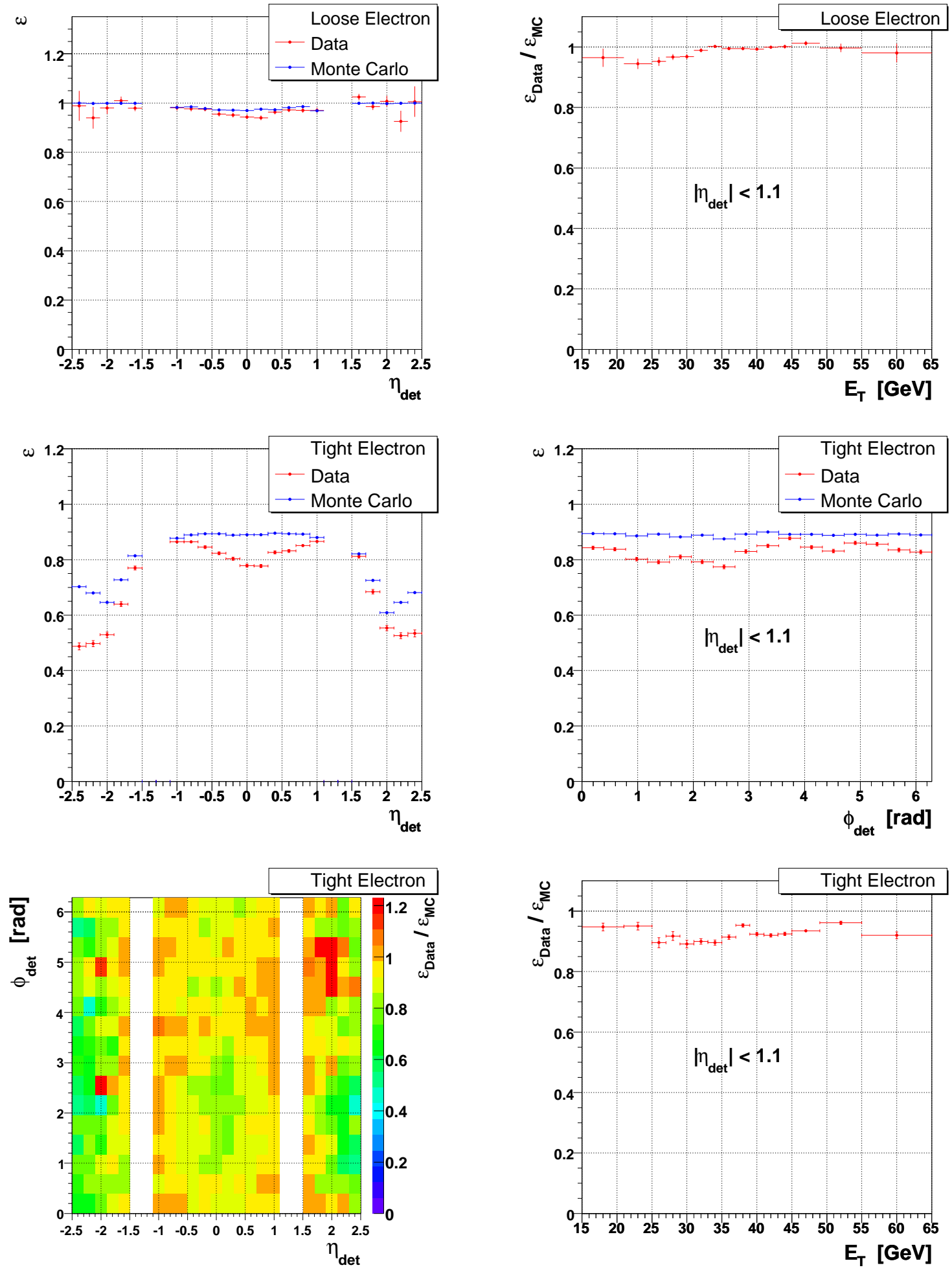
Figure 7.5: Overall efficiency correction factor (left-hand plot) and corresponding uncertainty (right-hand plot) which has to be applied to Monte Carlo events containing an electron that satisfies the tight analysis cuts.
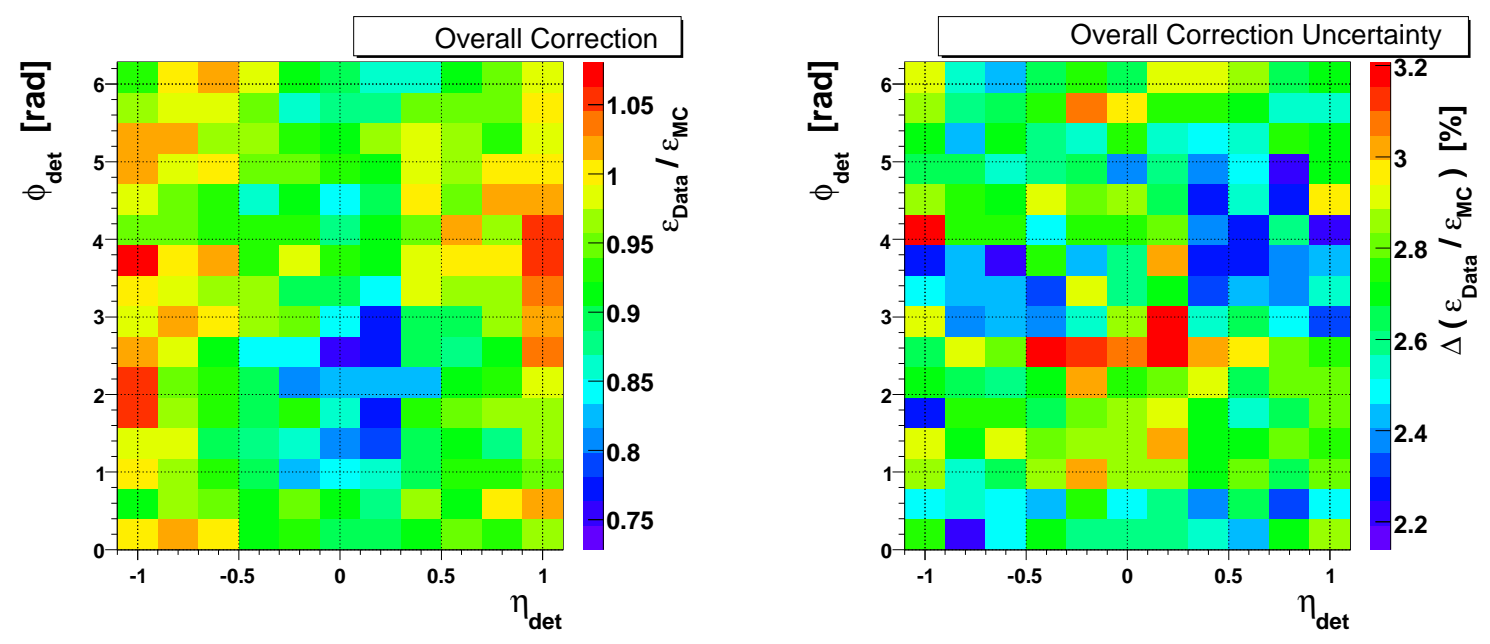

\subsubsection{Trigger Efficiency}

In the ideal case, an electron trigger should have an efficiency of $100 \%$ if the energy of the electron exceeds the threshold $S=\max ($ Level $1 / 2 / 3$ threshold) of a given trigger and if the offline identification criteria are more restricting than the trigger requirements, see the blue curve in Fig. 7.7. Hence, for the trigger EM_MX in trigger list v8 (see Tab. 6.3 and App. C) one would expect $S=\max (15 \mathrm{GeV}, 30 \mathrm{GeV})=30 \mathrm{GeV}$. Due to the fact that the triggers use simplified object reconstruction algorithms and that several object corrections can not be applied at the trigger level, the turn-on curve is blurred, displayed as the red curve in Fig. 7.7. Usually parameterized functions of the trigger turn-on curves are adopted, where some of them make use of the Gaussian error function Erf (equation in red) with

$$
\operatorname{Erf}(x)=\frac{2}{\sqrt{\pi}} \int_{0}^{x} e^{-t^{2}} \mathrm{~d} t .
$$

For example, for the trigger EM_MX with a nominal threshold of $30 \mathrm{GeV}$ the halfpoint is at $c_{0}=34 \mathrm{GeV}$, and the trigger is fully efficient $\left(c_{2}=1\right)$ for $E_{T}>45 \mathrm{GeV}$, see Fig. 6.2.

Trigger efficiencies can be determined for an individual trigger, for a specific Level 1, 2 or 3 condition or for a set of triggers. In all cases relevant here the tag and probe method is adopted for the efficiency measurement. Both, tag and probe electrons are required to pass stringent offline electron identification cuts. Further, the tag electron is postulated to be matched to a trigger electron at all trigger levels of an unprescaled trigger with $\Delta R$ (tag electron, trigger electron $)<0.4$. The probe electron passes the trigger condition if it can as well be matched to a trigger electron with $\Delta R$ (probe electron, trigger electron) $<0.4$ at all trigger levels to the trigger (or trigger set) under study. Due to the fact that the trigger efficiency is measured with respect to an unprescaled trigger, inefficiencies stemming from prescaled triggers are automatically taken into account. Detailed information about trigger efficiency measurements can be found in [85]. 
Figure 7.6: Monte Carlo reconstruction efficiencies for the different identification criteria. For this study electrons from $W^{\prime}$ decays are used. In the plot at the bottom one can see that the overall reconstruction efficiency does not show a strong dependency on the transverse energy of the electron $E_{T}$. The blue lines indicate fits with a constant.
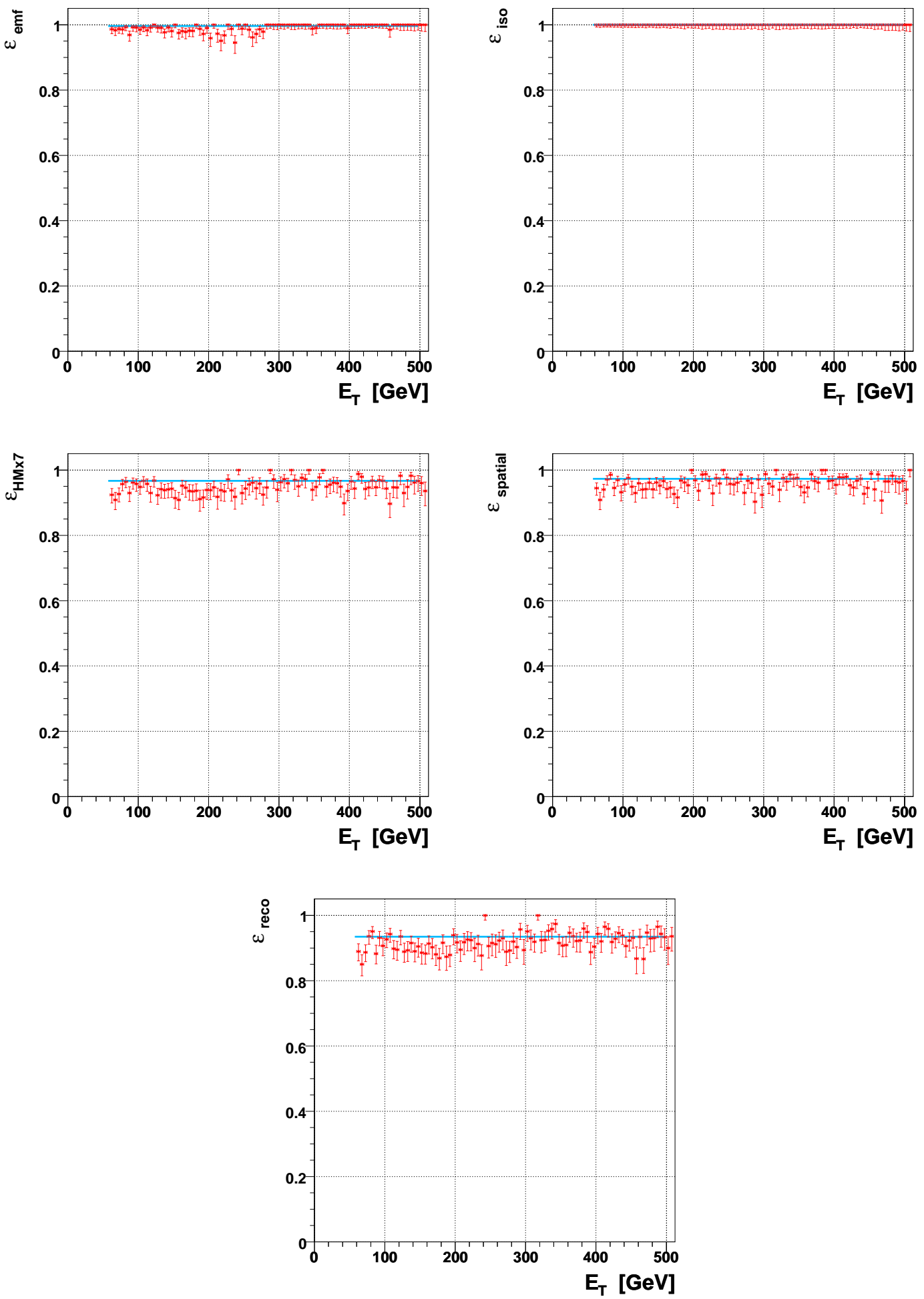
Figure 7.7: Sketch of a trigger turn-on curve (blue: ideal, red: real) as a function of the transverse energy $E_{T}$. The equation in red indicates a possible parameterization using the Gaussian error function Erf.

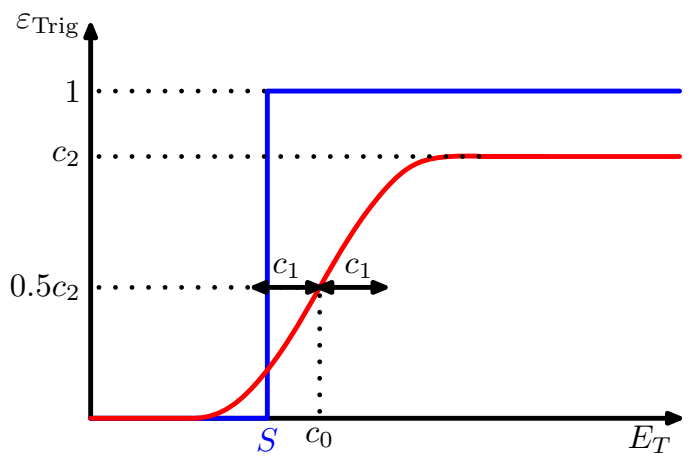

$$
\begin{gathered}
\varepsilon_{\operatorname{Trig}}\left(E_{T}\right)=\left\{\begin{array}{lll}
1 & \text { for } & E_{T}>S \\
0 & E_{T}<S
\end{array}\right. \\
\varepsilon_{\operatorname{Trig}}\left(E_{T}\right)=\frac{1}{2} c_{2}\left(1+\operatorname{Erf}\left(\frac{E_{T}-c_{0}}{\sqrt{2} c_{1}}\right)\right) \\
c_{0}: \text { Halfpoint }, \\
c_{1}: \text { Slope } \\
c_{2}: \text { Plateau. }
\end{gathered}
$$

The plots in Fig. 7.8 show measured trigger efficiencies for the trigger sets used in the analysis (see Tab. 6.3, the two v13 sets are merged into a single one) as a function of the transverse energy $E_{T}$ of electrons with $\left|\eta_{\text {det }}\right|<1.1$. One can clearly see the turn-on behaviour starting around $20 \mathrm{GeV}$. All trigger sets are fully efficient $\left(\varepsilon_{\text {Trig }}=1\right)$ for electron energies above $30 \mathrm{GeV}$. Nevertheless, the data sample ( $W$ sample, see Sec. 6.2) is checked for a possible trigger bias. For this purpose, the following ratio $r$ is explored

$$
r=\frac{\text { data }- \text { QCD background }}{\text { sum of all backgrounds }- \text { QCD background }}
$$

after weighting the Monte Carlo samples with the effective luminosity $\mathcal{L}_{W}$ and applying the reconstruction efficiency correction. The QCD multijet background is estimated from data and therefore subtracted. In order to remove any additional bias stemming from jets (see next section), only events without jets $\left(n_{\text {jet }}=0\right)$ are considered. The ratio $r$ as a function of the transverse energy $E_{T}$ of the electron is shown in the left-hand plot in Fig. 7.9. The ratio is compatible with one, especially in the critical region $E_{T}<40 \mathrm{GeV}$. However, a slight deviation from one is visible for $50 \mathrm{GeV}<E_{T}<60 \mathrm{GeV}$, but this region contains only $3 \%$ of the data, see the electron $E_{T}$ distribution in Fig. 7.15.

As a further cross check of the normalization procedure, the ratio Eq. 7.9 is fitted as a function of the transverse mass $m_{T}$ in events without any jets, see the right-hand plot in Fig. 7.9. Again, the ratio is compatible with one. Major fluctuations for $m_{T}<$ $40 \mathrm{GeV}$ stem from the QCD background contribution which is estimated from events with jets. Finally, Fig. 7.10 shows transverse mass distributions for different intervals of the electron transverse energy $E_{T}$. In general, data and Monte Carlo prediction agree very well. Numbers from the different fits are summarized in Tab. 7.1. In summary, the trigger sets are fully $(100 \%)$ efficient for the tight electron selection used in the analysis, and the uncertainties are covered by the uncertainty of the overall normalization with $\mathcal{L}_{W}$. 
Figure 7.8: Trigger efficiencies for the trigger sets used in the analysis as a function of the transverse energy $E_{T}$ of electrons reconstructed in $\left|\eta_{\text {det }}\right|<1.1$ [85].
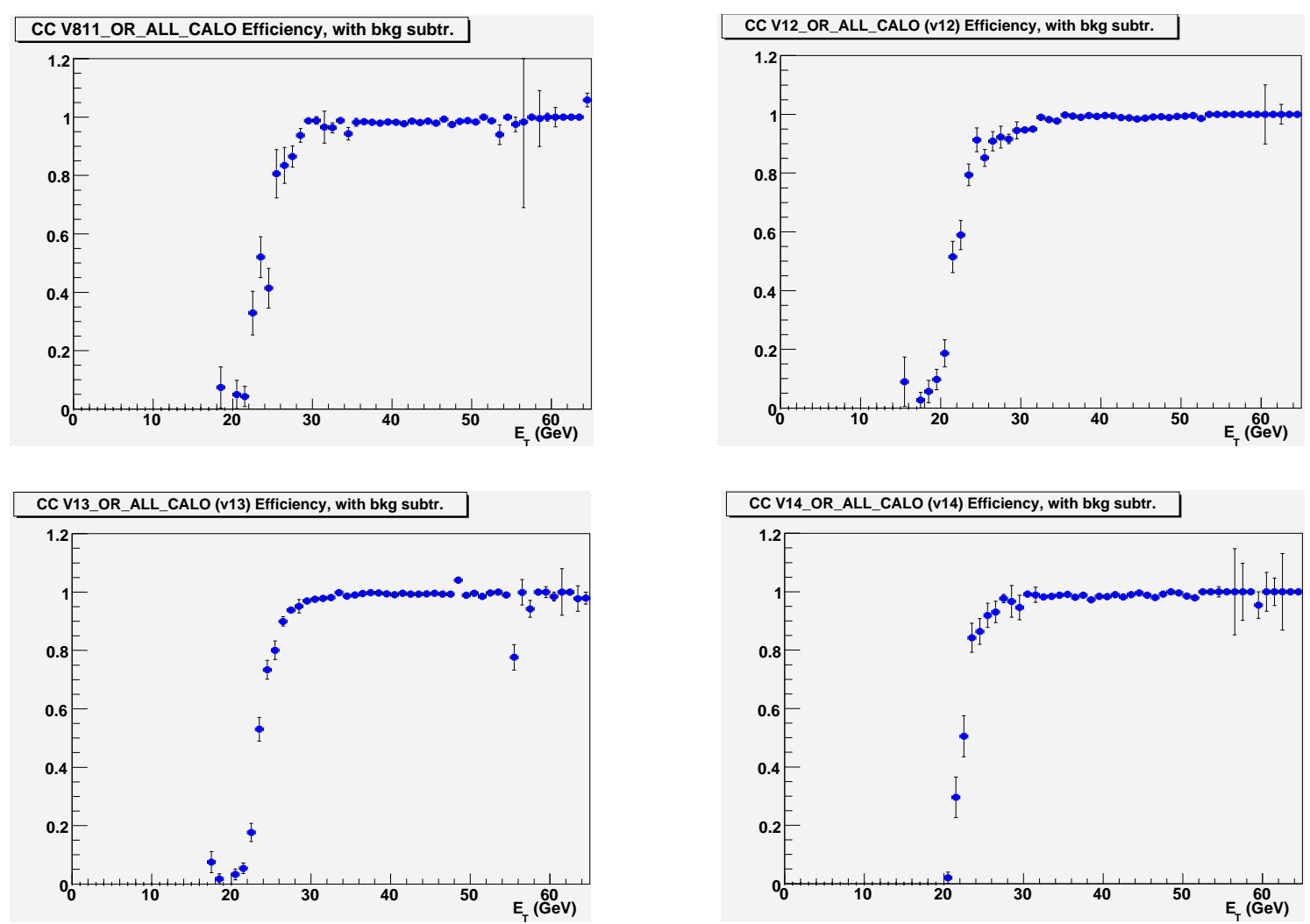

Figure 7.9: Data/Monte Carlo ratio $r$ as defined in Eq. 7.9 in events without any jets ( $W$ sample). The left-hand plot shows the ratio as a function of the transverse energy of the electron $E_{T}$, the right-hand plot as a function of the transverse mass $m_{T}$.
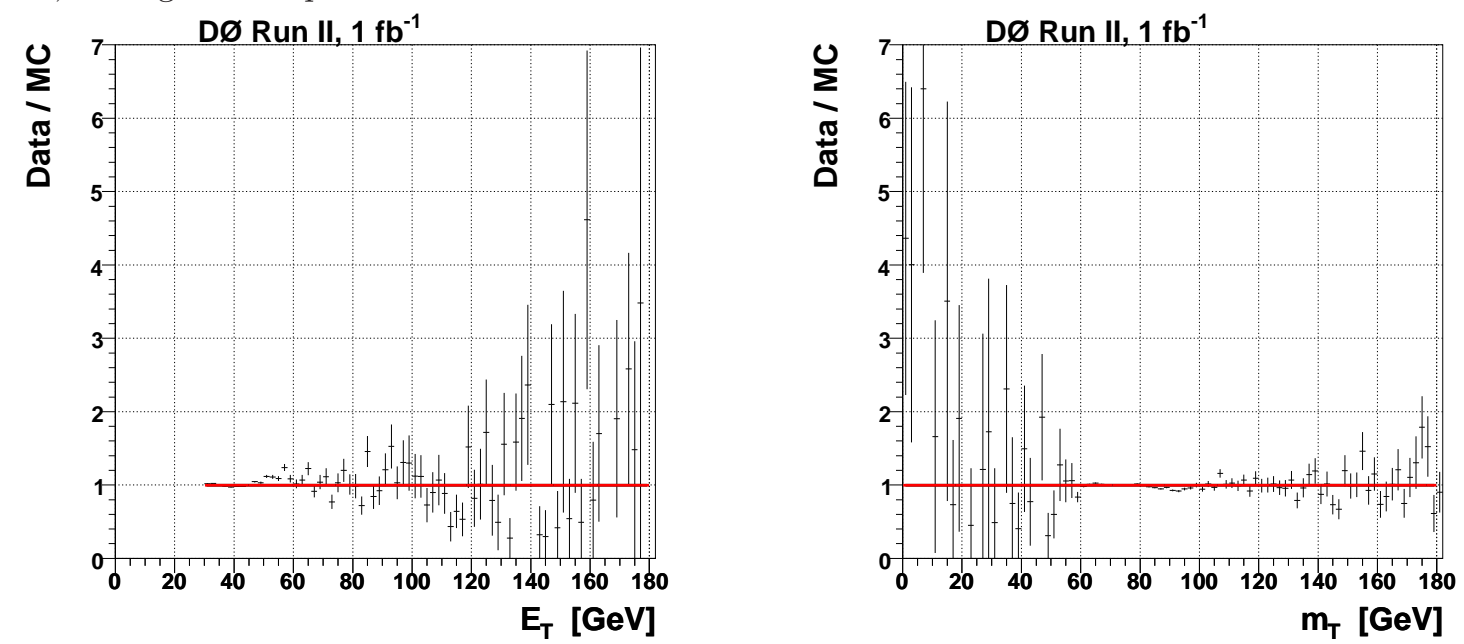
Figure 7.10: Distributions of the transverse mass $m_{T}$ in events without any jets ( $W$ sample) for different ranges of the electron transverse energy $E_{T}$.
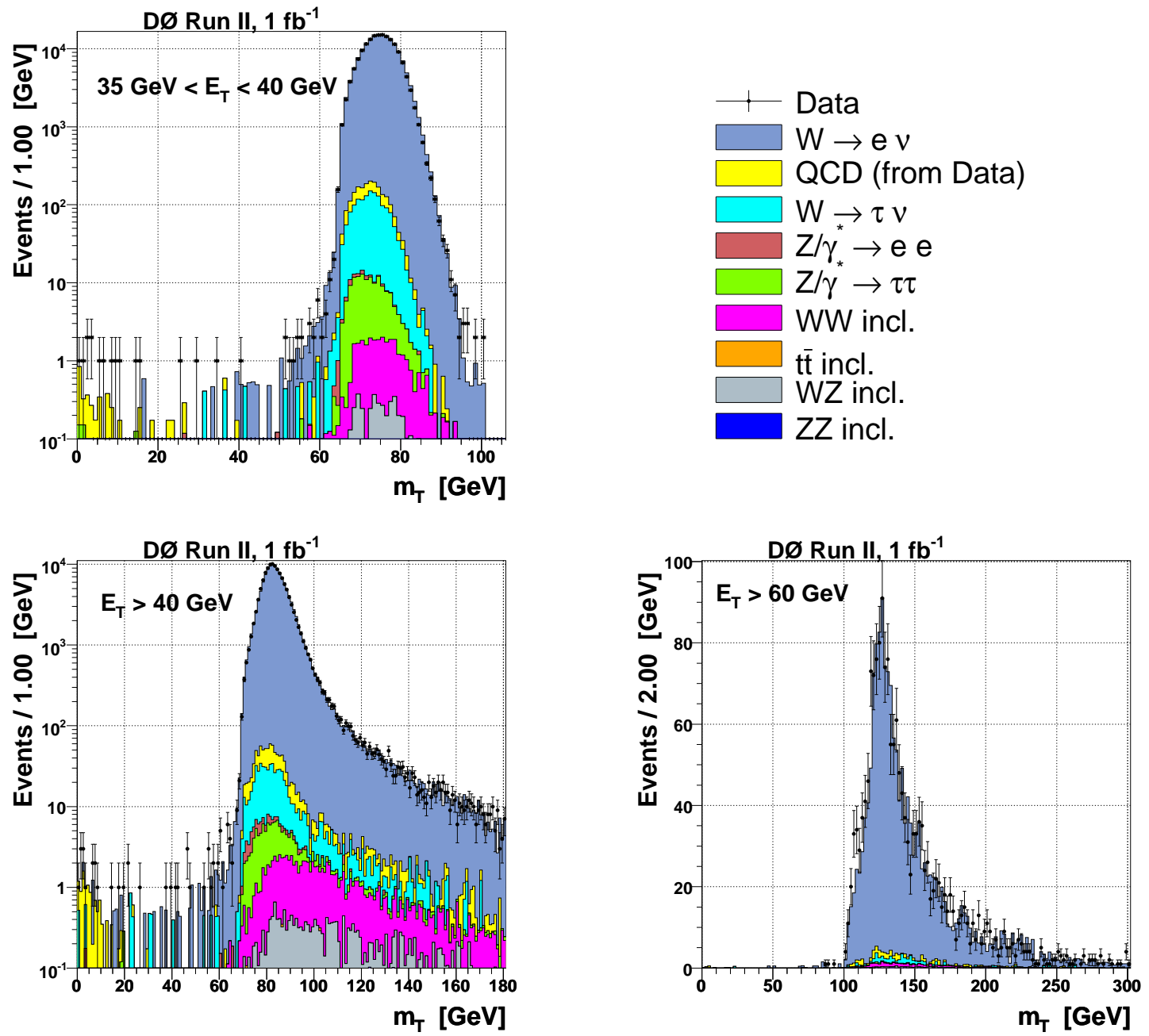

Table 7.1: Trigger efficiency and normalization check: Numbers from the various fits of the data/Monte Carlo ratio $r$ as defined in Eq. 7.9 (including statistical errors).

\begin{tabular}{|c|c|c|}
\hline electron $E_{T}$ range $[\mathrm{GeV}]$ & $m_{T}$ fit & $E_{T}$ fit \\
\hline \hline$>30$ & $0.995 \pm 0.002$ & $0.995 \pm 0.002$ \\
$>40$ & $0.996 \pm 0.003$ & $0.997 \pm 0.003$ \\
$>50$ & $1.003 \pm 0.027$ & $0.988 \pm 0.025$ \\
\hline $30-35$ & $1.013 \pm 0.003$ & $1.014 \pm 0.003$ \\
$35-40$ & $0.980 \pm 0.002$ & $0.982 \pm 0.002$ \\
$40-45$ & $0.975 \pm 0.003$ & $0.983 \pm 0.003$ \\
$45-50$ & $1.016 \pm 0.007$ & $1.031 \pm 0.007$ \\
\hline
\end{tabular}




\subsection{Reweighting of the $p_{T}$ Spectrum of the $W$ Boson}

If no further corrections are applied, discrepancies between data and background prediction can be observed, see Figs. 7.11 and 7.12. The following distributions are shown: transverse energy of the electron $E_{T}$; missing transverse energy $(\mathrm{MET}) \mathbb{E}_{T}$; angular difference between electron and MET, $\Delta \phi(E M, M E T)$; ratio of the electron transverse energy and MET, $E_{T} / E_{T}$; and the maximal angular differences between jets (if present in the event) and electron/MET $\Delta \phi($ Jet, $E M / M E T)$. Further, the transverse momentum $p_{T}^{E M+M E T}$ that can be calculated from the electron and MET is shown

$$
p_{T}^{E M+M E T}=\left|\vec{p}_{T}^{E M+M E T}\right| \quad \text { with } \quad \vec{p}_{T}^{E M+M E T}=\vec{E}_{T}+\vec{E}_{T}=\left(\begin{array}{c}
E_{x}+E_{x} \\
E_{y}+E_{y} \\
0
\end{array}\right) .
$$

In the case of $W \rightarrow e \nu$ events without initial state radiation this quantity corresponds to the transverse momentum of the $W$ boson, $p_{T}^{E M+M E T} \equiv p_{T}^{W}$. Another distribution of a compound variable shown in Fig. 7.12 is the event shape variable thrust $T$ which is defined in the transverse plane with respect to the beam axis as follows

$$
T=\max _{\varphi} \frac{\sum_{i}\left|\vec{p}_{T}^{i} \cdot \vec{e}_{\varphi}\right|}{\sum_{i}\left|\vec{p}_{T}^{i}\right|}, \quad \vec{e}_{\varphi}=\left(\begin{array}{c}
\cos \varphi \\
\sin \varphi \\
0
\end{array}\right) \quad \varphi \in[0, \pi]
$$

The index $i$ denotes all reconstructed physics objects in the final state (electron, MET, $1^{\text {st }}$ jet, $2^{\text {nd }}$ jet, ...). Although the agreement between data and background prediction for the transverse energy distributions of the electron and MET is reasonable, deviations are clearly visible in the distributions of event shape, ratio and angular difference variables.

The reason for the observed discrepancies is that the spectrum of the transverse momentum $p_{T}^{W}$ of the $W$ (and $Z[150]$ ) boson is not properly described by the PYTHIA Monte Carlo generator, especially when jets are present due to higher order contributions as shown in Fig. 7.13. Instead of using a matrix element generator for the $W+$ jets processes (like AlPGEN [151]), a simplified approach is pursued: The available inclusive Pүтніа $W \rightarrow e \nu$ samples containing events with higher jet multiplicities are reweighted. This procedure, which affects only $10 \%$ of all $W \rightarrow e \nu$ events, avoids the following issues related to Alpgen Monte Carlo samples ${ }^{1}$ :

- the proper matching between parton showers and matrix elements has to be implemented [153];

- the individual cross sections for all $W+n$ jets $(n=0,1,2, \ldots)$ processes need to be known;

- additional weighting factors have to be determined [154];

- the samples have to be corrected for errors in the DØ ALPGEN production [155].

\footnotetext{
${ }^{1}$ Details about the DØ ALPGEN $W+$ jets production can be found at [152].
} 
Figure 7.11: Comparison between data ( $W$ sample) and background prediction after applying all corrections except for the $W p_{T}$ reweighting (1). The QCD peak in the $p_{T}^{E M+M E T}$ distribution is discussed later in the text.
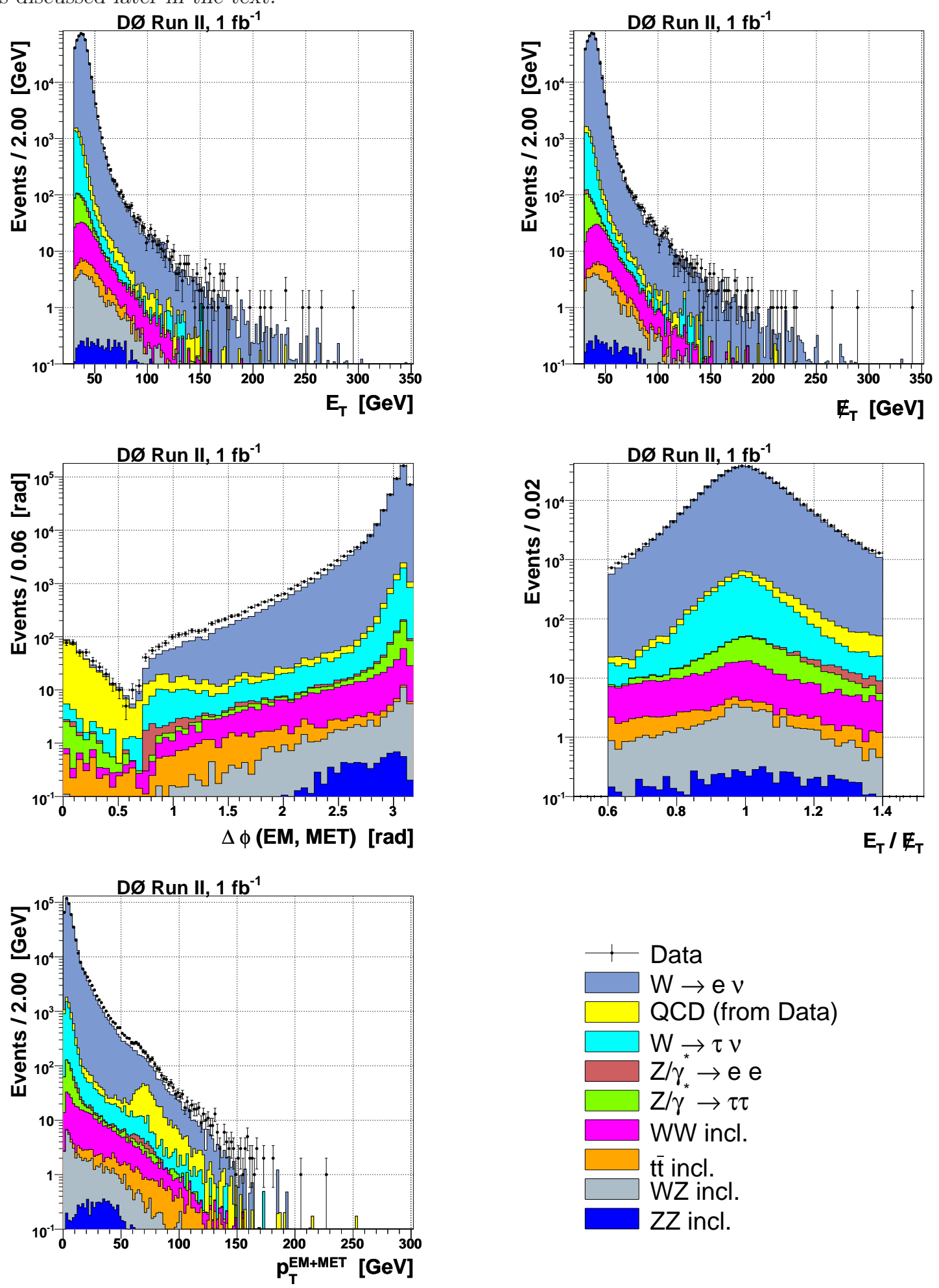
Figure 7.12: Comparison between data ( $W$ sample) and background prediction after applying all corrections except for the $W p_{T}$ reweighting (2).
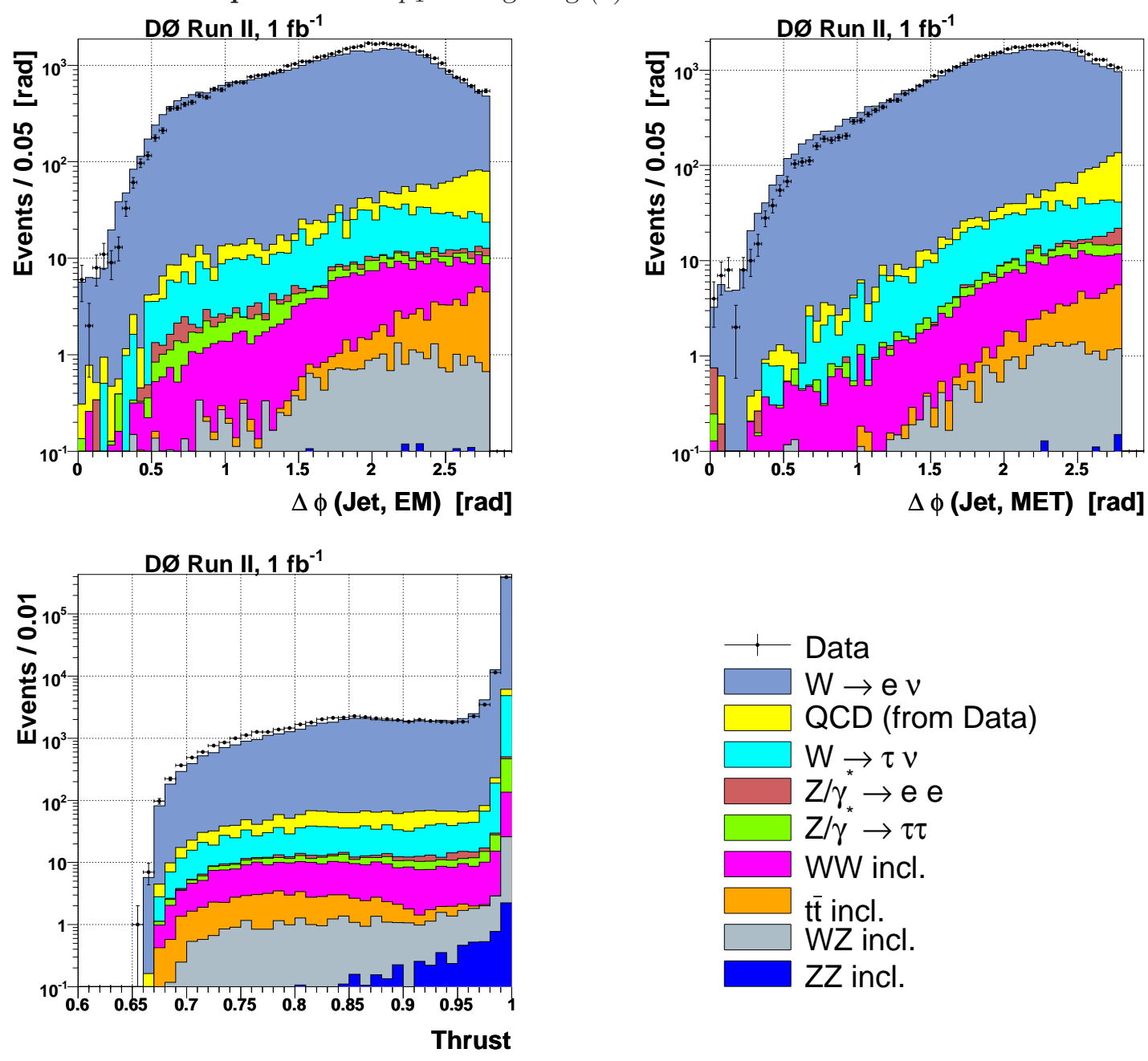

Figure 7.13: Higher order contributions to the process $q \bar{q}^{\prime} \rightarrow W$ leading to jets in the final state.
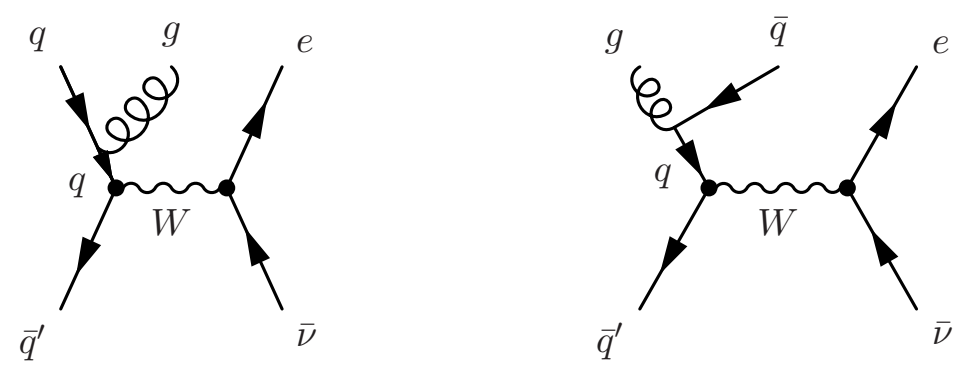
In events without any jets ('clean' electron + neutrino final state), data and background prediction agree (see Figs. 7.14 and 7.15). PүтніA is a leading order Monte Carlo generator and is expected to describe the final state without jets properly. The data/Monte Carlo ratio $R$ shown in the plot at the bottom of Fig. 7.14 is defined as follows

$$
\begin{gathered}
R\left(p_{T}^{E M+M E T}\right)=\frac{W \text { "data" }}{W \text { PyтhiA Monte Carlo }} \\
=\frac{\text { data }- \text { sum of all backgrounds except for } W}{W \text { Pythia Monte Carlo }} .
\end{gathered}
$$

The ratio is close to one with a small deviation from one for $15 \mathrm{GeV}<p_{T}^{E M+M E T}<$ $20 \mathrm{GeV}$. Note, that just a very small fraction of events is affected since this region is two orders of magnitude below the peak. Because of this, one observes a good agreement regarding shapes and overall normalization in all distributions; there is no need for reweighting events without any jets. Due to the reduced impact of higher order QCD effects, this sample ( $W$ sample with $n_{\text {jet }}=0$ ) has been used for the trigger and normalization cross checks in Sec. 7.2.2. Hence, the observed discrepancies in Figs. 7.11 and 7.12 are due to events with jets.

This can clearly be seen in events with exactly one jet, see Figs. 7.16 and 7.17. Inspired by the shape of the ratio plot in Fig. 7.16, the following function is fitted

$$
w_{W}\left(p_{T}^{E M+M E T}\right)=\frac{1}{2} \cdot\left[p_{2}+\operatorname{Erf}\left(\frac{p_{T}^{E M+M E T}-p_{0}}{\sqrt{2} p_{1}}\right)\right]
$$

which makes use of the Gaussian error function (see Eq. 7.8). The fit is performed in the region that is dominated by the contribution from the $W$ Monte Carlo $\left(p_{T}^{E M+M E T}<\right.$ $60 \mathrm{GeV}$ ). Reweighting the $p_{T}$ spectrum of the $W$ Monte Carlo - namely the reconstructed $p_{T}^{E M+M E T}$ from the electron and MET (= neutrino) - with this function results in a very good agreement in all relevant distributions, see Figs. 7.18 and 7.19.

The reweighting procedure can also be applied to events with higher jet multiplicities. The plots in Figs. 7.20 and 7.21 [Figs. 7.22 and 7.23] show the relevant distributions before [after] reweighting events with exactly two jets. Even in these events the distributions of the transverse momenta of jets $p_{T}^{\text {jet } i}$ and the transverse momentum of the Hadronic Final State, HFS, agree in a reasonable way after applying the reweighting. The HFS is defined as the sum of the jet momenta in the final state

$$
H F S=\sum_{\text {jets }} \vec{p} .
$$

Events with exactly three jets are reweighted using a constant; events with higher jet multiplicities $\left(n_{\text {jet }}>3\right)$ are not reweighted due to the lack of Monte Carlo statistics. The numbers from all fits including statistical uncertainties are given in Tab. 7.2. In summary, the reweighting of the $p_{T}$ spectrum of the $W$ boson is applied to events with one, two and three jets in the event. After the reweighting, which affects approximately $10 \%$ of the $W \rightarrow e \nu$ Pyтнia Monte Carlo events, a reasonable agreement between data and background prediction in all distributions is achieved. 
Figure 7.14: Comparison between data ( $W$ sample) and background prediction in events without any jets after applying all corrections (1). The data/Monte Carlo ratio $R$ is defined in Eq. 7.12.
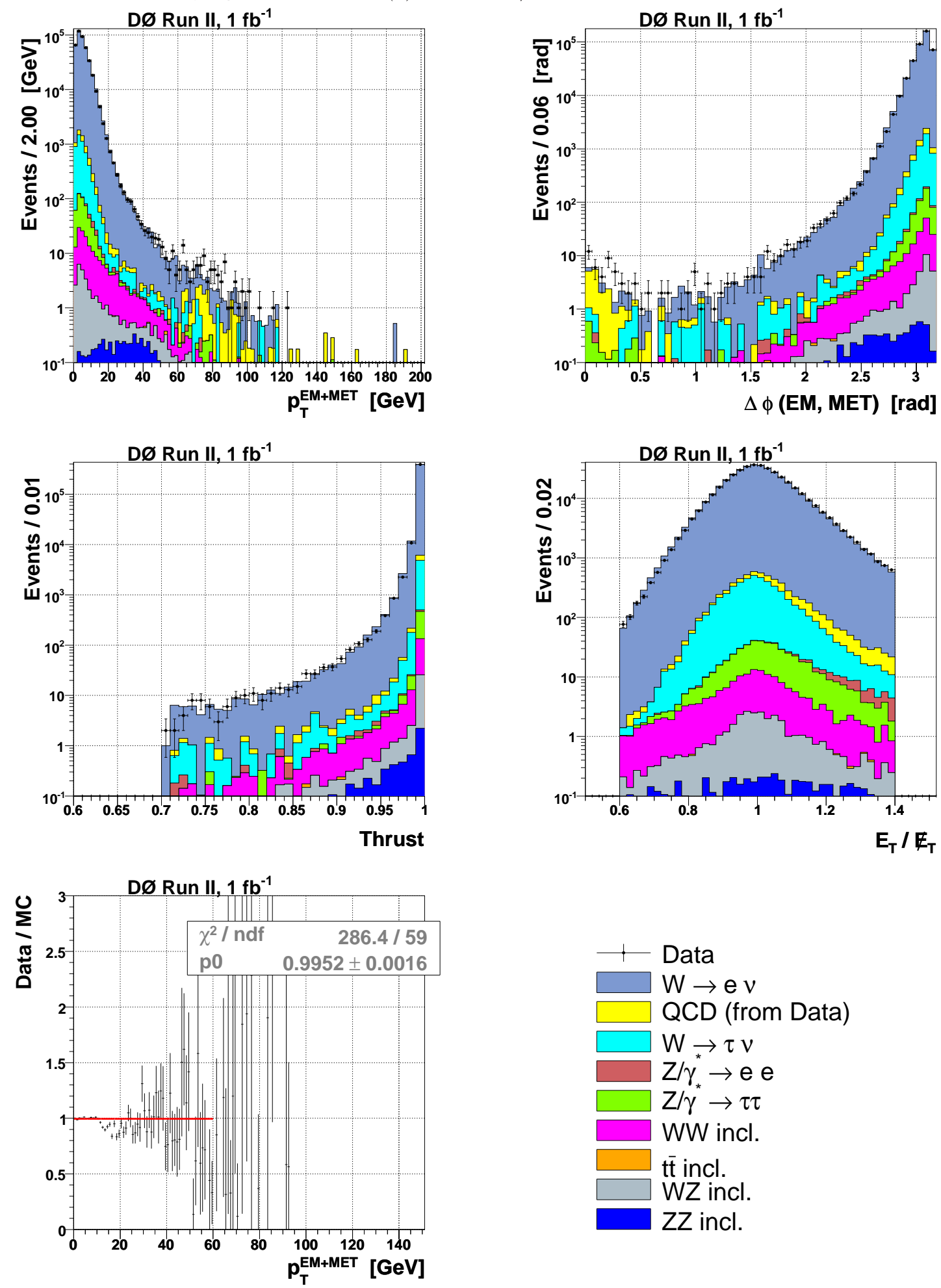
Figure 7.15: Comparison between data ( $W$ sample) and background prediction in events without any jets after applying all corrections (2).
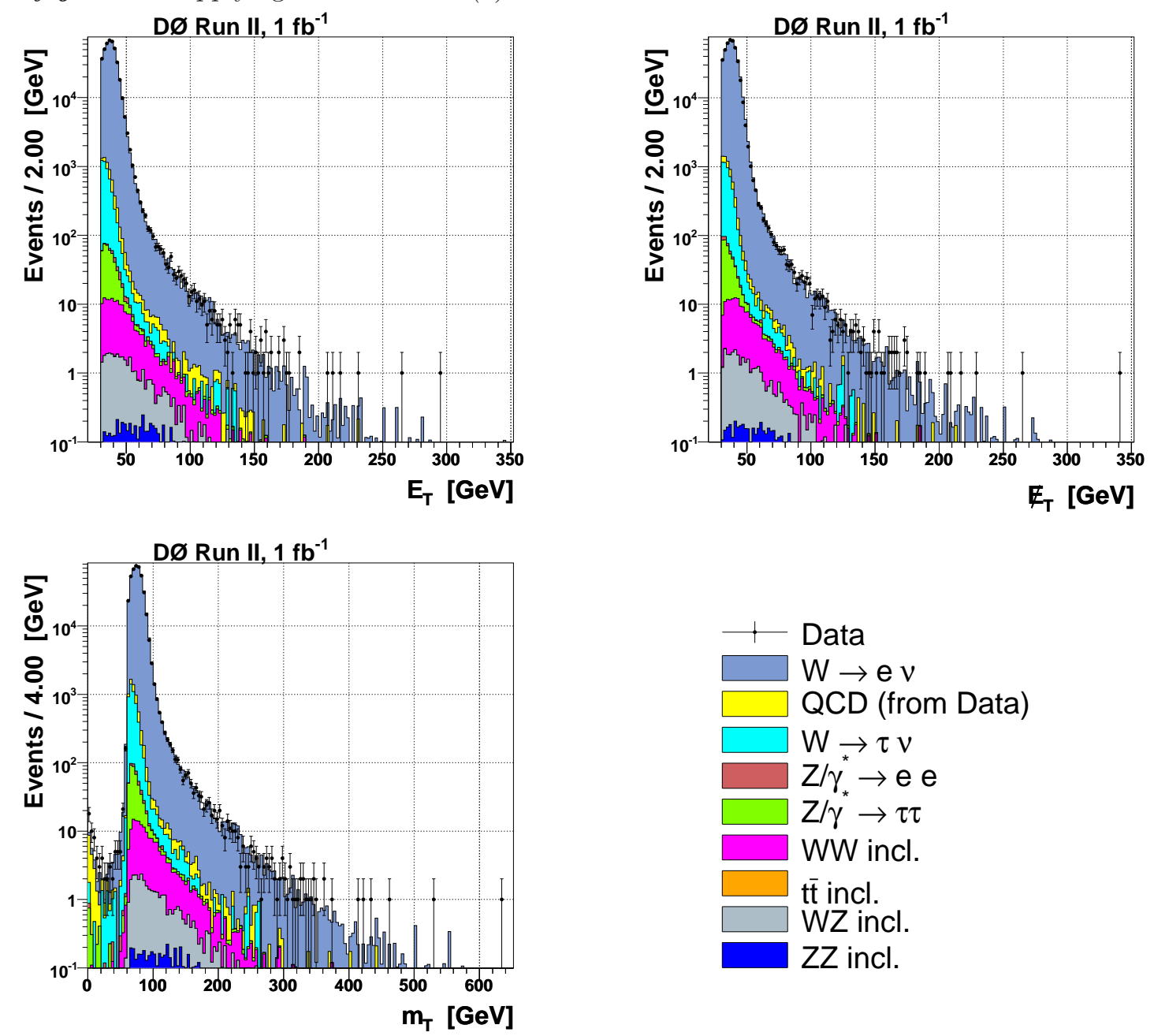

Table 7.2: Parameters from the fits of $R\left(p_{T}^{E M+M E T}\right)$. For $n_{\text {jet }}=3$ a constant, for $n_{\text {jet }}=1,2$ the function Eq. 7.13 is used for the fit. The reweighting is not applied in events without any jets.

\begin{tabular}{|c|c|c|c|}
\hline$n_{\text {jet }}$ & $p_{0}$ & $p_{1}$ & $p_{2}$ \\
\hline \hline 1 & $12.3 \pm 0.2$ & $5.48 \pm 0.17$ & $1.55 \pm 0.02$ \\
\hline 2 & $11.9 \pm 2.6$ & $13.0 \pm 1.9$ & $2.03 \pm 0.12$ \\
\hline 3 & $1.32 \pm 0.08$ & n/a & n/a \\
\hline
\end{tabular}


Figure 7.16: Comparison between data ( $W$ sample) and background prediction in events with one jet after applying all corrections except for the $W p_{T}$ reweighting (1).
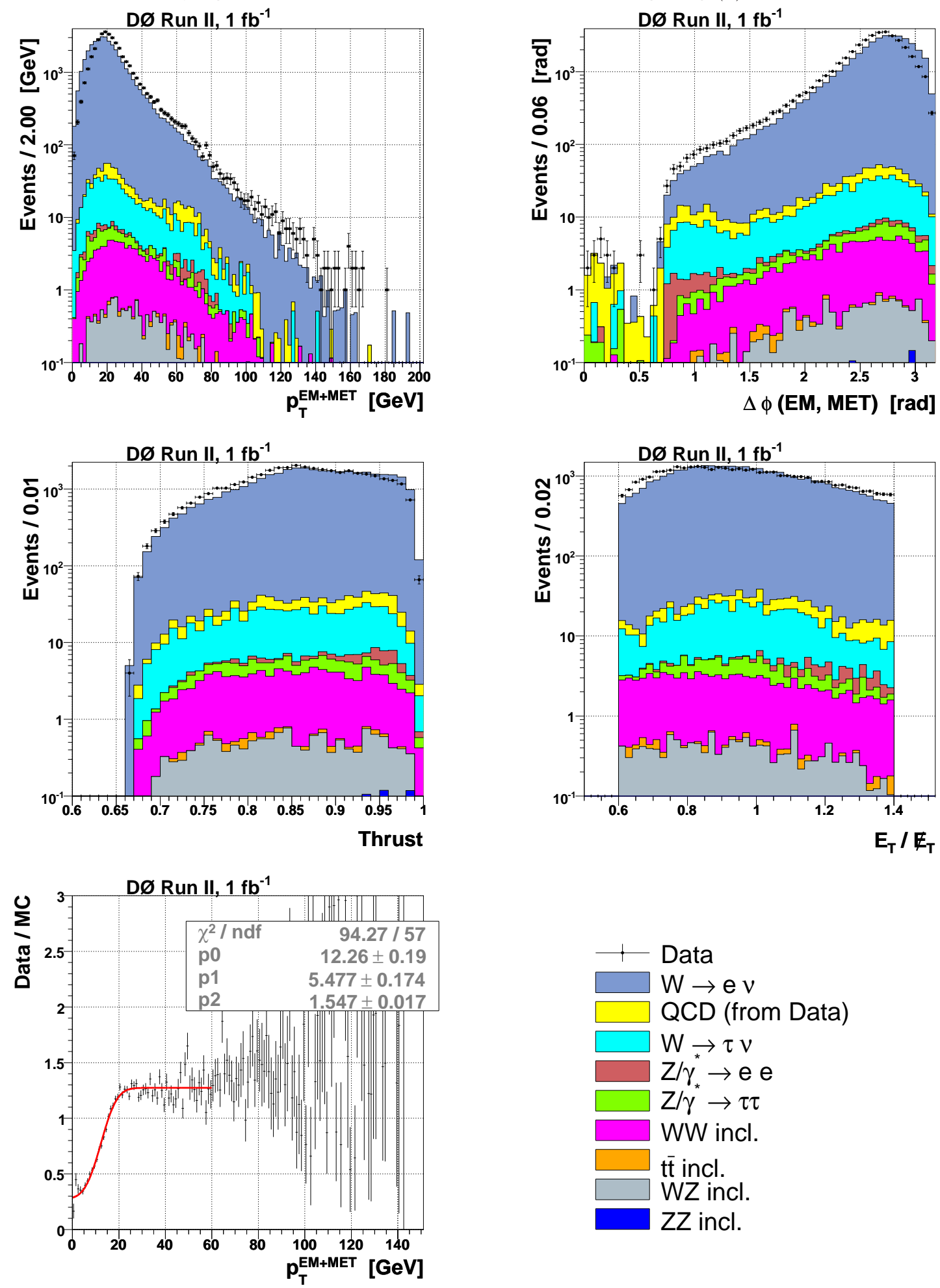
Figure 7.17: Comparison between data ( $W$ sample) and background prediction in events with one jet after applying all corrections except for the $W p_{T}$ reweighting (2).
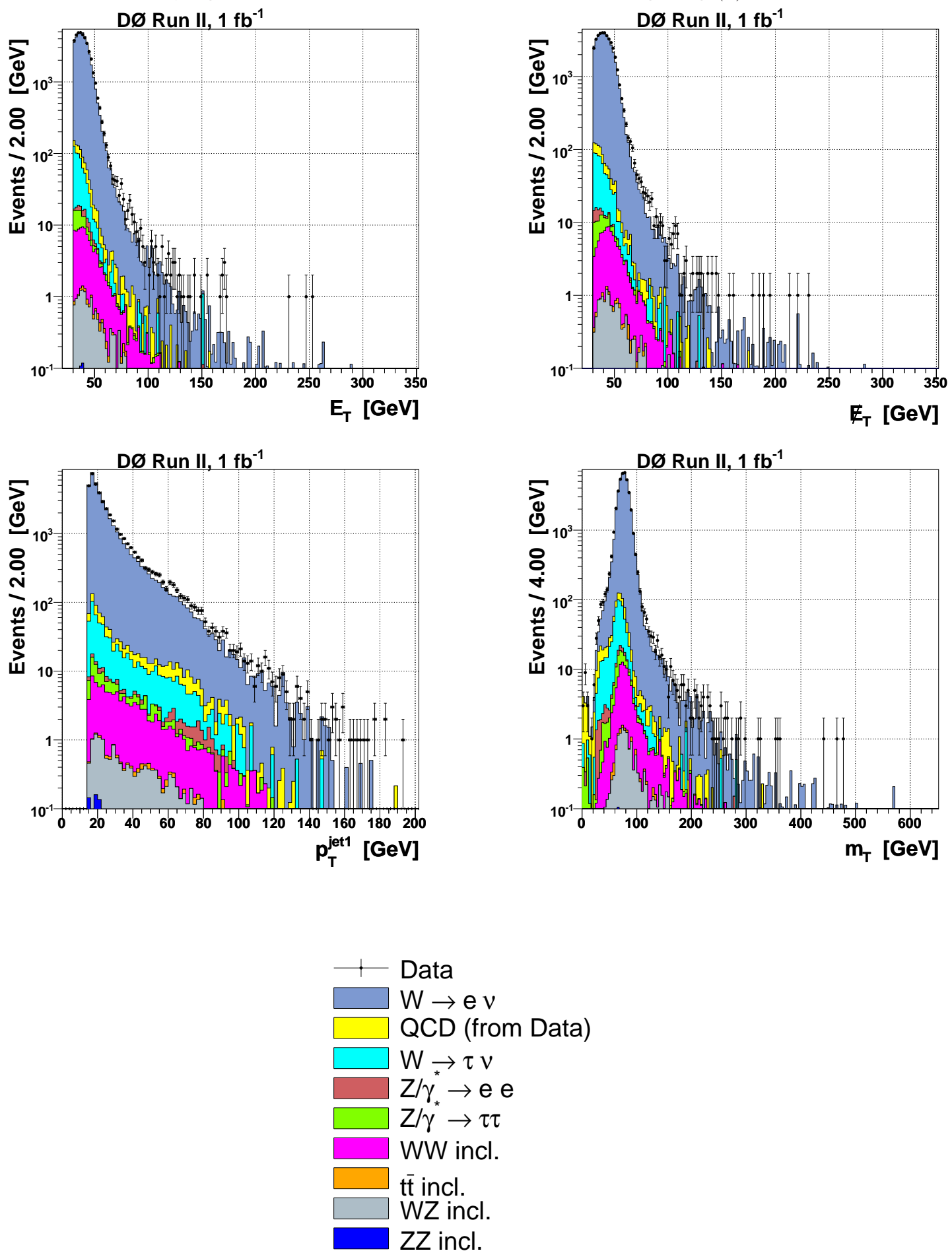
Figure 7.18: Comparison between data ( $W$ sample) and background prediction in events with one jet after applying the $W p_{T}$ reweighting (1).
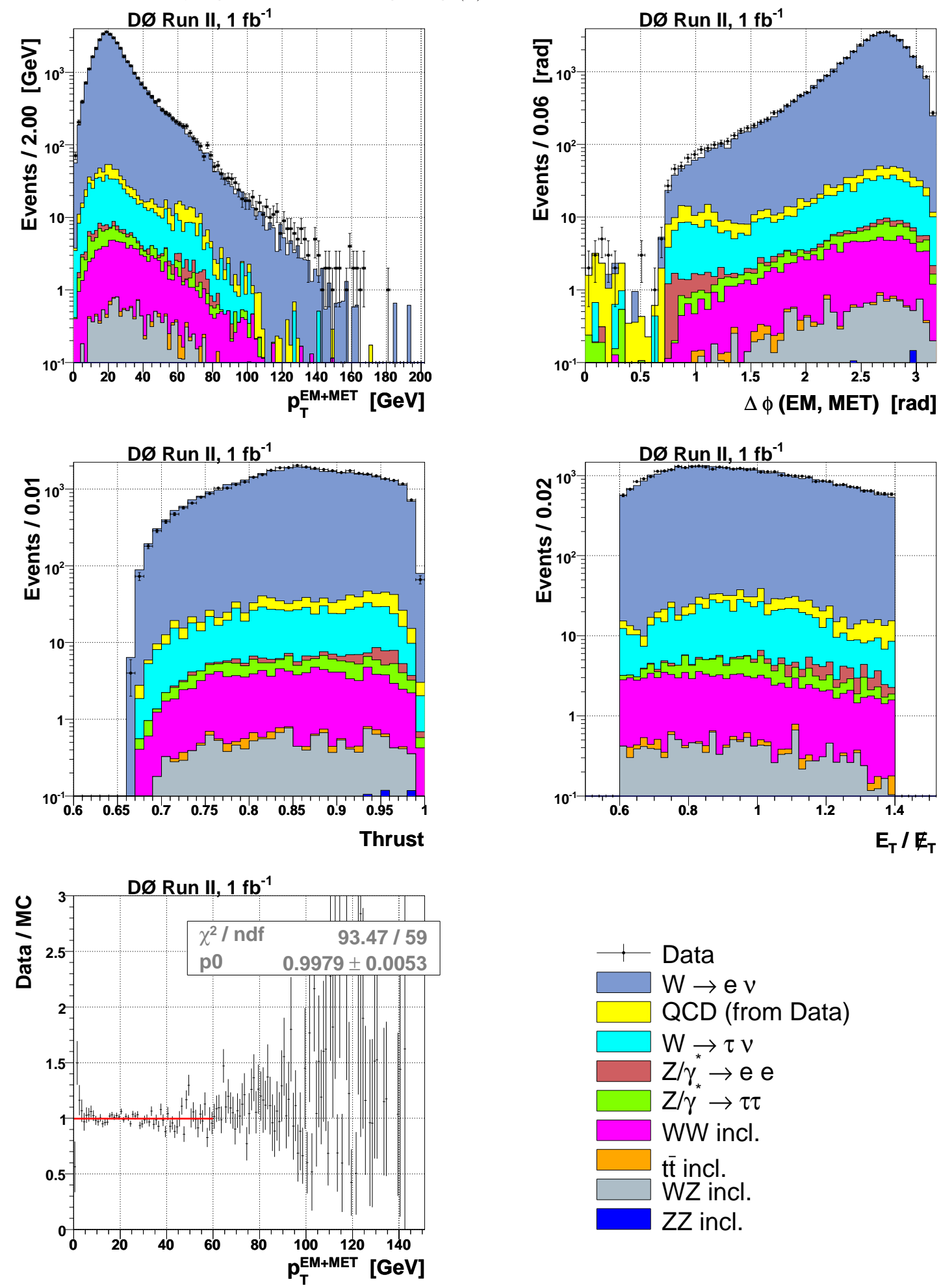
Figure 7.19: Comparison between data ( $W$ sample) and background prediction in events with one jet after applying the $W p_{T}$ reweighting (2).
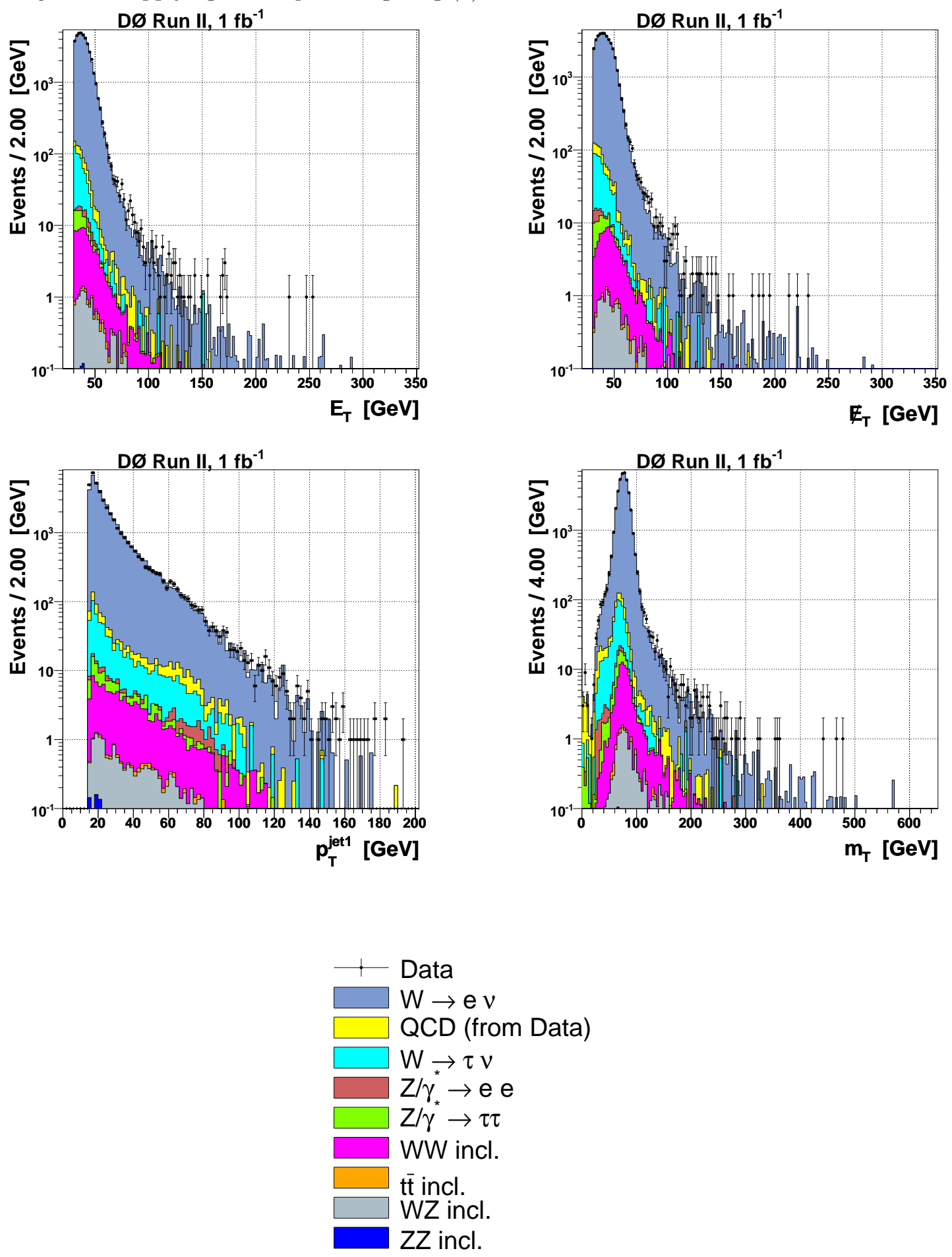
Figure 7.20: Comparison between data ( $W$ sample) and background prediction in events with two jets after applying all corrections except for the $W p_{T}$ reweighting (1). The QCD peak in the $p_{T}^{E M+M E T}$ distribution is discussed later in the text.
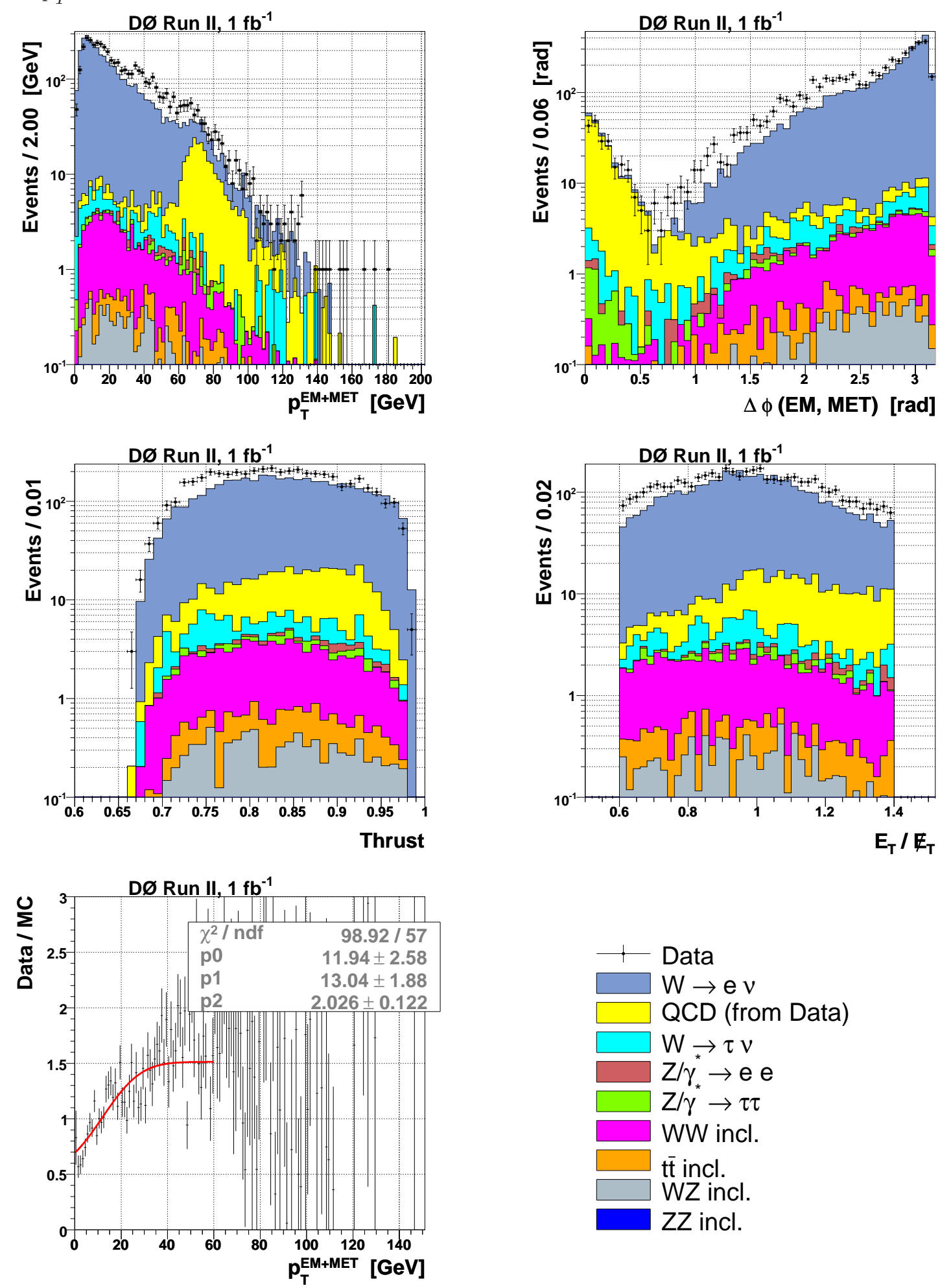
Figure 7.21: Comparison between data ( $W$ sample) and background prediction in events with two jets after applying all corrections except for the $W p_{T}$ reweighting (2). The QCD peak in the $p_{T}^{H F S}$ distribution is discussed later in the text.
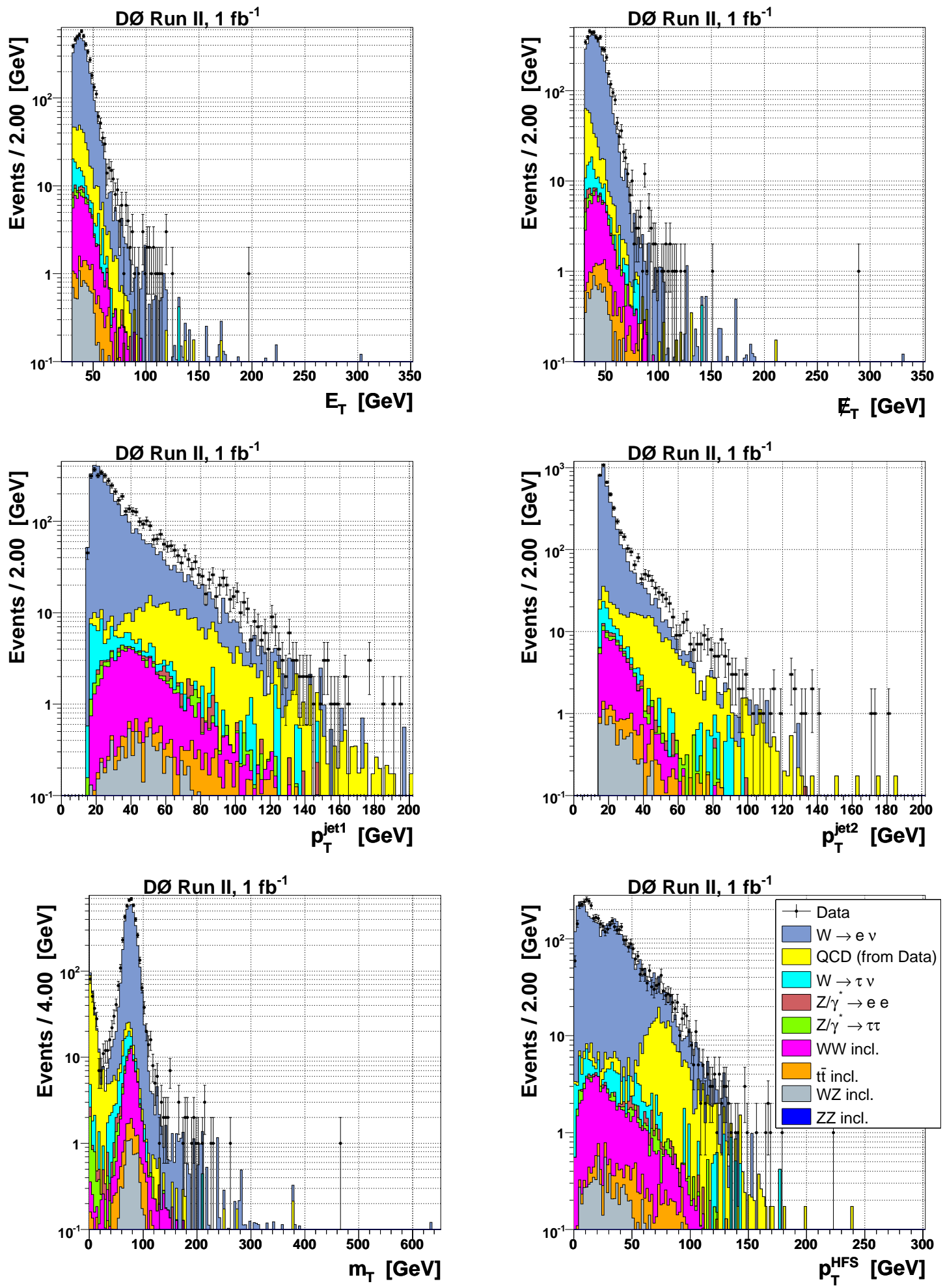
Figure 7.22: Comparison between data ( $W$ sample) and background prediction in events with two jets after applying the $W p_{T}$ reweighting (1). The $Q C D$ peak in the $p_{T}^{E M+M E T}$ distribution is discussed later in the text.
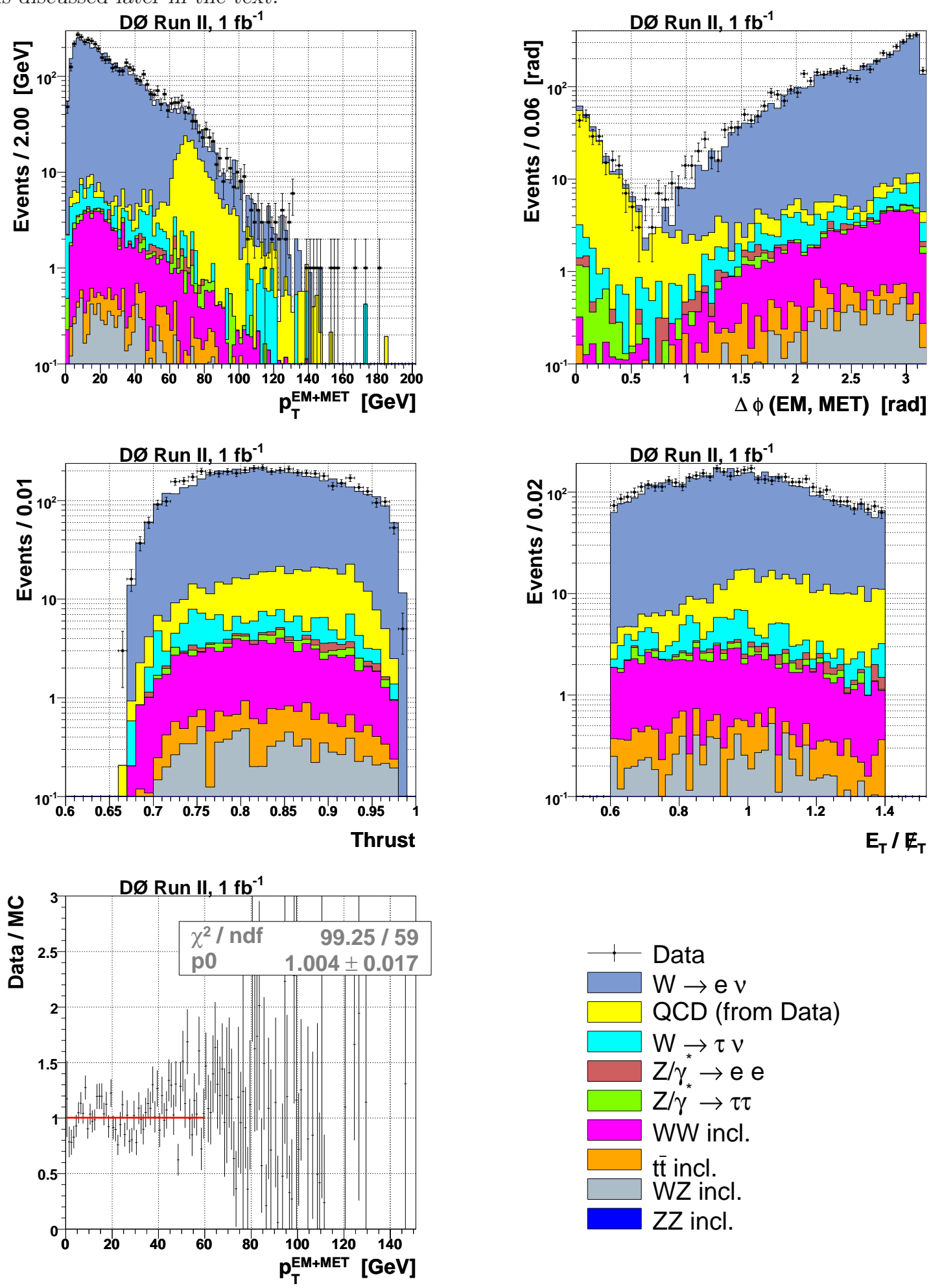
Figure 7.23: Comparison between data ( $W$ sample) and background prediction in events with two jets after applying the $W p_{T}$ reweighting (2). The QCD peak in the $p_{T}^{H F S}$ distribution is discussed later in the text.
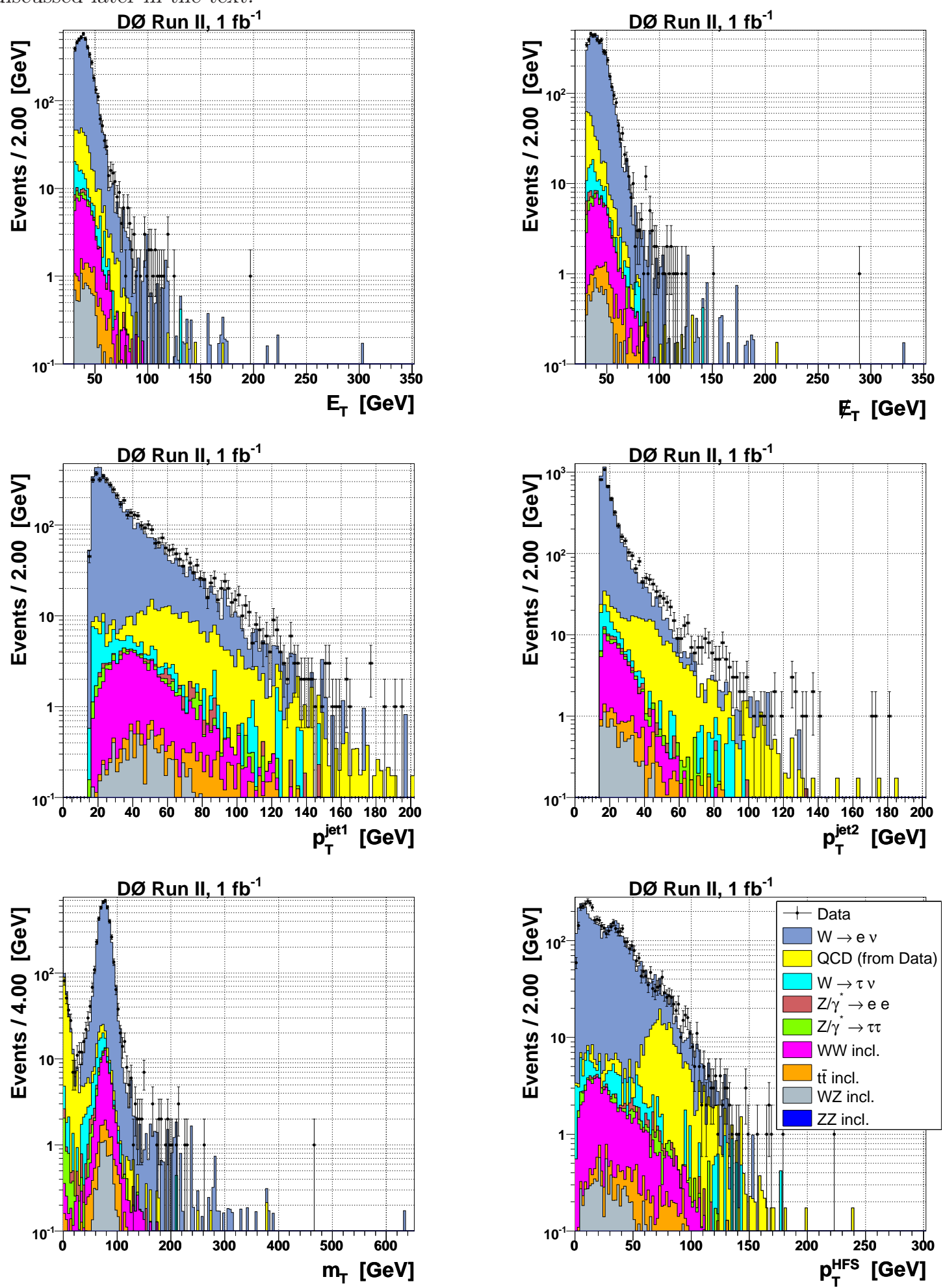
In the $p_{T}^{E M+M E T}$ and $p_{T}^{H F S}$ distributions the QCD background exhibits a peak structure, see Figs. 7.11 and $7.20-7.23$. The plots in Fig. 7.24 show the $p_{T}^{E M+M E T}$ (left) and $p_{T}^{H F S}$ (right) distributions only for the QCD sample in events with two jets. One can see that the peak region starts at $p_{T}^{H F S} \approx p_{T}^{E M+M E T} \approx 60 \mathrm{GeV}$. The reason for this accumulation lies in the event selection $\left(E_{T}>30 \mathrm{GeV}\right.$, electron $\left.E_{T}>30 \mathrm{GeV}\right)$. The events in the peak are in fact three-jet events $(q \bar{q} g)$ with one jet misidentified as electron, see sketch in the left-hand plot in Fig. 7.24. The missing transverse energy points into the same direction as the fake electron candidate so that

$$
p_{T}^{E M+M E T} \approx E_{T}+E_{T}>E_{T}^{\min }+E_{T}^{\min }=30 \mathrm{GeV}+30 \mathrm{GeV}=60 \mathrm{GeV} .
$$

The hadronic final state points in the opposite direction of the fake electron (and thus the missing energy) and exhibits therefore a similar behaviour

$$
-\vec{p}_{T}^{E M+M E T} \approx \vec{p}_{T}^{H F S} \Rightarrow p_{T}^{E M+M E T} \approx p_{T}^{H F S}>60 \mathrm{GeV} .
$$

Figure 7.24: Peak structure in the $Q C D$ sample $\left(n_{\text {jet }}=2\right)$.
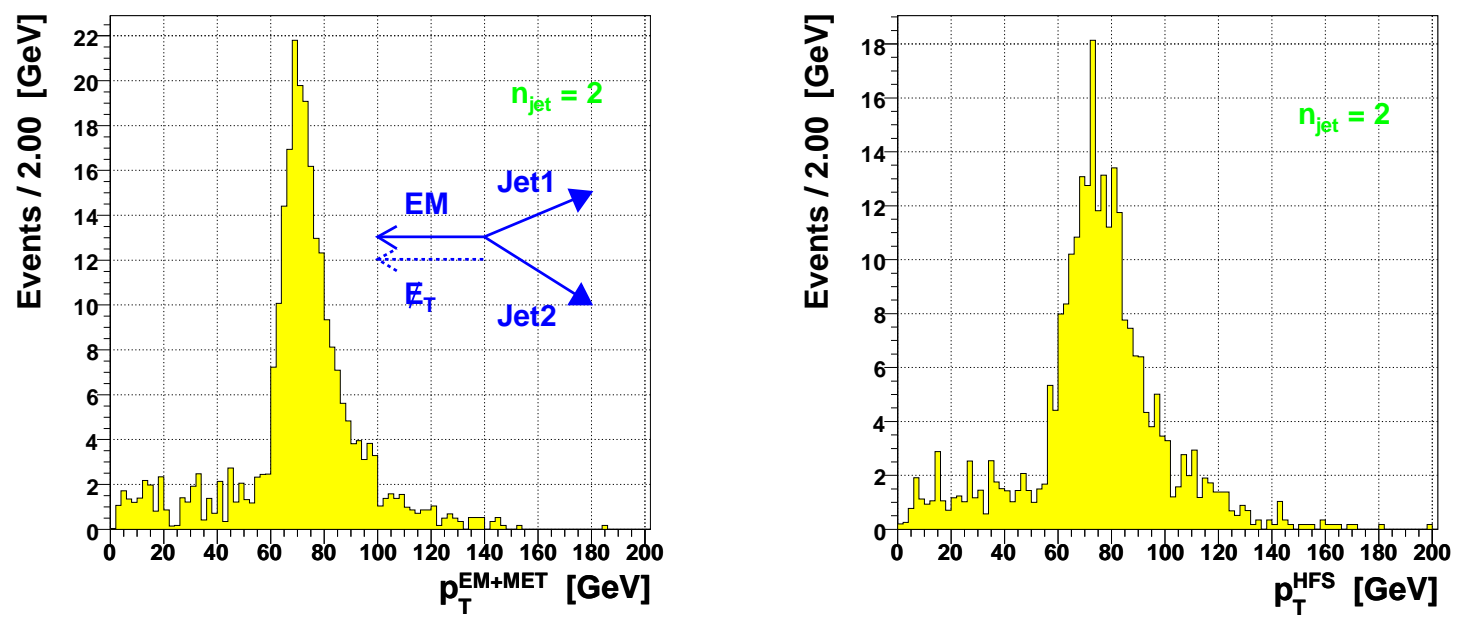

\subsection{Systematic Uncertainties}

Two kinds of systematic uncertainties contribute in this analysis. The uncertainties of the effective luminosity, the cross section, the electron reconstruction efficiency corrections and the QCD scale factor affect only the normalization. Uncertainties on the parton density functions (PDFs), electron energy scale and resolution, jet energy scale, decay width $\Gamma_{W}$ of the $W$ boson and the reweighting of the transverse momentum of the $W$ boson lead to changes of the shape of the distributions.

The statistical error for the data sample with $N$ events is given by $\sqrt{N}$. The statistical error for the QCD sample is reduced by the QCD scaling factor, $f_{\mathrm{QCD}} \cdot \sqrt{N}$. For the Pyтнia Monte Carlo samples the generated events $N^{\text {gen }}$ are weighted with global event weights $w$ (see Eq. 6.8). The statistical error is

$$
N^{\text {gen }} \pm \sqrt{N^{\text {gen }}} \Rightarrow w N^{\text {gen }} \pm w \sqrt{N^{\text {gen }}}=N \pm \sqrt{w N} .
$$




\subsubsection{Normalization Uncertainties}

Uncertainties on the cross sections of the background and signal samples are discussed in Sec. 5.2 and 5.3. The numbers in Tab. 5.1 and 5.3, respectively, include uncertainties stemming from the PDFs and from the factorization/normalization scale (if available) and range from $4-10 \%$. The scaling of the QCD background (Sec. 6.3) has an uncertainty of $7 \%$, whereas the effective luminosity $\mathcal{L}_{W}$ used for the overall normalization can be determined to a precision of $4 \%$, see Sec. 6.4. The uncertainty of efficiency correction factors is typically $3 \%$, see Fig. 7.5 in Sec. 7.2.1.

\subsubsection{Shape Changing Uncertainties}

\section{Electron energy scale and resolution}

In order to study the effect of the electron energy scale and resolution, the electron energies $E$ are varied with the uncertainties quoted in Sec. 7.1. The reconstructed electron energy $E$ is replaced as follows [156]

$$
\begin{array}{cl}
\text { EMscale: } & E \rightarrow E \cdot(1 \pm 0.02) \\
\text { EMresolution: } & E \rightarrow E^{\text {true }} \cdot(1+\mathrm{g}(0,0.041 \pm 0.003))
\end{array}
$$

with $g(\mu, \sigma)$ being a Gaussian random number with mean $\mu$ and width $\sigma$. For the resolution the true energy extracted from the Monte Carlo generator is used. Fig. 7.25 shows the resolution coming out of the signal Monte Carlo (upper left-hand plot), the smearing of the true (= generator) electron energy (upper right-hand plot) and variation of the electron resolution (lower plots). If no generator electron can be matched to a reconstructed electron or no generator information is available ( $<1 \%$ of the Monte Carlo events), the reconstructed electron energy is taken instead and just slighty shifted

$$
E \rightarrow E \cdot(1 \pm 0.003)
$$

The variation of scale and resolution are performed independently. The missing transverse energy is recalculated after varying the electron energy. The impact of the energy scale variation is shown in Fig. 7.26 for the $W$ Monte Carlo sample and for a hypothetical $W^{\prime}$ boson with a mass of $m_{W^{\prime}}=900 \mathrm{GeV}$. The overall uncertainty on the event number is large for samples with a steeply increasing or decreasing distribution of the transverse energy of the electron around the analysis cut of $E_{T}>30 \mathrm{GeV}$. For the $W$ Monte Carlo sample and heavy $W^{\prime}$ bosons an uncertainty of $4 \%$ on the overall event number is derived, whereas the scale uncertainty leads to a small uncertainty of $1 \%$ on the overall event number for light $W^{\prime}$ bosons. However, in some bins of the transverse mass distribution one can observe a huge effect (50\%). The uncertainty of the energy resolution is an order of magnitude smaller compared to the energy scale uncertainty. 
Figure 7.25: Plots showing the variation of the electron energy resolution using $W^{\prime} \rightarrow e \nu$ Monte Carlo events. The upper left-hand plot displays the resolution after the full simulation and reconstruction. In the upper right-hand plot the reconstructed energy is replaced by the smeared true Monte Carlo energy $(\sigma=0.041$, cf. Eq. 7.19). The lower plots show the impact of the varied resolution $(\sigma=0.041 \pm 0.003)$.
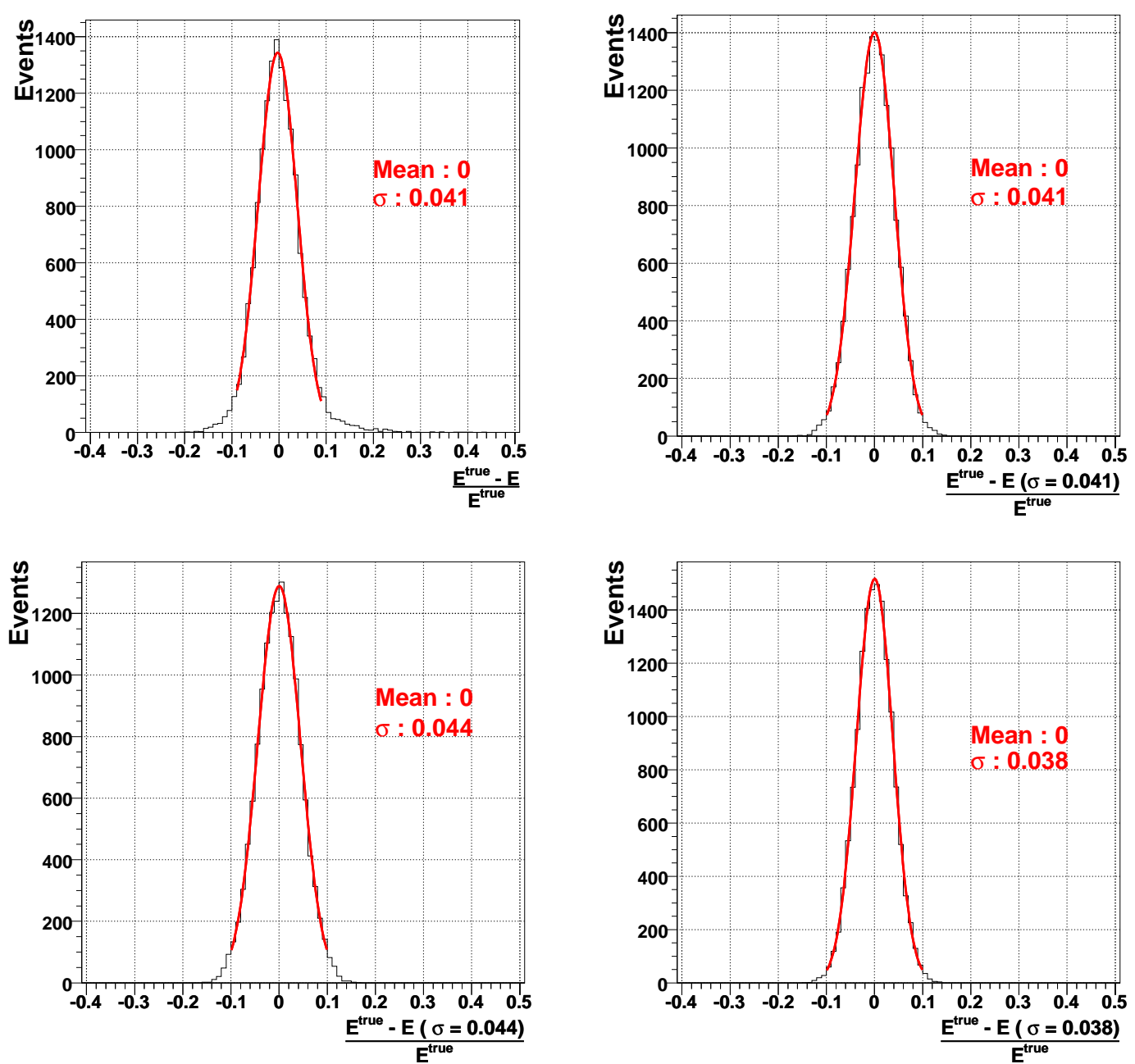
Figure 7.26: Impact of the electron energy scale variation on the $W$ Monte Carlo sample (left) and on the $W^{\prime}$ signal with $m_{W^{\prime}}=900 \mathrm{GeV}$ (right).
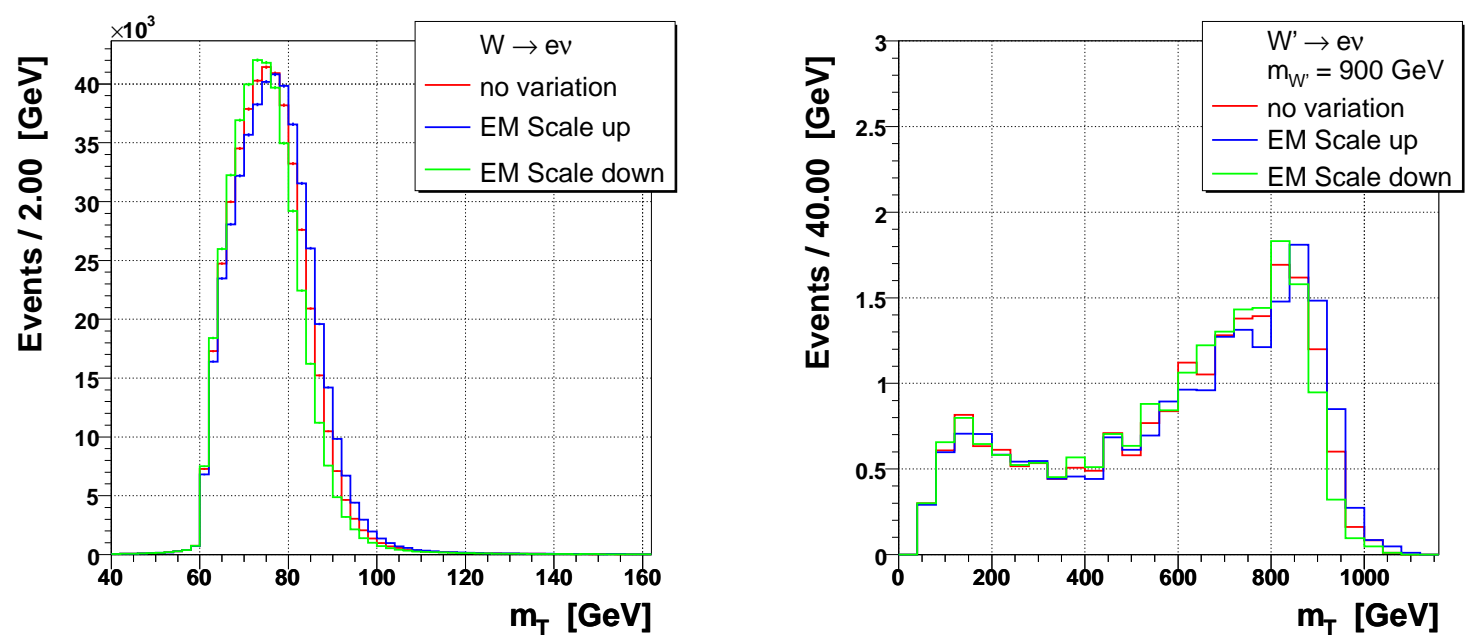

\section{PDF uncertainty}

The PDF uncertainty is studied using the CTEQ6.1M PDFs and the 40 error functions [17]. In order to avoid generating new events with the shifted PDFs, the available Monte Carlo events, which have been produced using CTEQ6L1, are reweighted [136] to CTEQ6.1M.x $x$ $(x x=0, \ldots, 40)$.

The PDFs are obtained from global fits to the experimental data using a $\chi^{2}$ approach, see Sec. 2.2.6. The $\chi^{2}$ function is characterized in the neighborhood of the global minimum providing a systematic method to assess the compatibility of the datasets and to estimate the uncertainties of the PDFs and their physical predictions within a certain practical tolerance. The basic idea (shown in Fig. 7.27) is to diagonalize the Hessian matrix

Figure 7.27: Implementation of the Hessian method to derive the sets of eigenvector PDFs [17].

\section{2-dim $(i, j)$ rendition of $d$-dim ( 20) PDF parameter space}

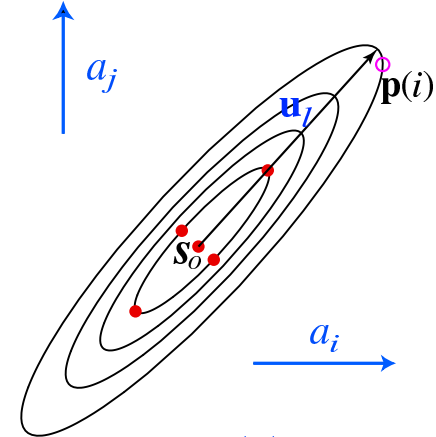

(a) contours of constant $\chi^{2}$ global

$\mathbf{u}_{l}$ : eigenvector in the l-direction

$\mathbf{p}(i)$ : point of largest $a_{i}$ with tolerance $T$

$\boldsymbol{s}_{0}$ : global minimum

$$
\begin{aligned}
& \stackrel{\text { diagonalization and }}{\longrightarrow} \\
& \text { rescaling by }
\end{aligned}
$$

- Hessian eigenvector basis sets

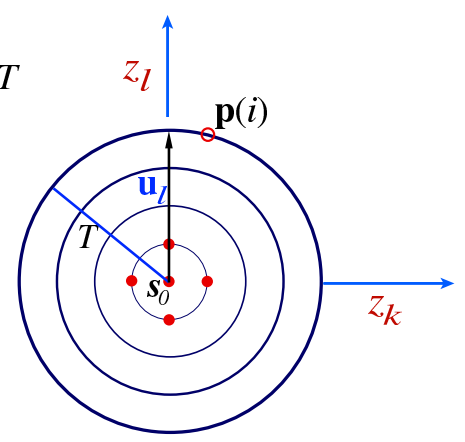

(b)

Original parameter basis

Orthonormal eigenvector basis 
iteratively resulting in a set of $2 \cdot 20(20=$ number of free PDF parameters, 2 : up and down variations) orthogonal eigenvectors. The full CTEQ6.1M PDF set consists of the best fit CTEQ6.1M.00 and the eigenvector basis set CTEQ6.1M. $x x(x x=1, \ldots, 40)$ in plus ( $x x$ odd) and minus ( $x x$ even) directions along each eigenvector. Details are given in [157].

For each of the 40 sets the analysis cuts are applied, and finally the total uncertainty $\Delta^{ \pm} m_{T}$ in the transverse mass distribution $m_{T}$ is calculated for each bin $i$ via

$$
\begin{aligned}
\Delta^{+} m_{T}^{i}= & \sqrt{\sum_{x x=01}^{40}\left|m_{T}^{i}(\mathrm{CTEQ6.1M} . x x)-m_{T}^{i}(\mathrm{CTEQ} 6.1 \mathrm{M} .00)\right|^{2}} \\
& \text { if } m_{T}^{i}(\mathrm{CTEQ6.1M} . x x)>m_{T}^{i}(\mathrm{CTEQ6} .1 \mathrm{M} .00)
\end{aligned}
$$

and

$$
\begin{aligned}
\Delta^{-} m_{T}^{i}= & \sqrt{\sum_{x x=01}^{40}\left|m_{T}^{i}(\mathrm{CTEQ6} .1 \mathrm{M} . x x)-m_{T}^{i}(\mathrm{CTEQ6.1M.00})\right|^{2}} \\
& \text { if } m_{T}^{i}(\mathrm{CTEQ6.1M} . x x)<m_{T}^{i}(\mathrm{CTEQ6.1M.00)}
\end{aligned}
$$

The uncertainty derived from the analysis of the CTEQ6.1M error functions is then applied to the CTEQ6L1 Monte Carlo sets. Fig. 7.28 shows a comparison between the CTEQ6L1, CTEQ6.1M.00 and the uncertainty derived from the error functions. One can clearly see differences between CTEQ6L1 (leading order) and CTEQ6.1M.00 (next-to-leading order), and that CTEQ6L1 is not covered by the error band. Nevertheless, lacking a consistent NLO Monte Carlo, the error extracted from the CTEQ6.1M PDFs is applied to the Monte Carlo samples generated with CTEQ6L1 in order to get an estimate of the uncertainties.

In Fig. 7.29 the impact of the PDF uncertainty on the signal is shown. The overall uncertainty varies from $3 \%\left(m_{W^{\prime}}=500 \mathrm{GeV}\right)$ to $9 \%\left(m_{W^{\prime}}=1200 \mathrm{GeV}\right)$. For the $W$ Monte Carlo sample an uncertainty of $2 \%$ is derived.

Figure 7.28: Comparison between CTEQ6L1, CTEQ6.1M.00 and the associated uncertainties.
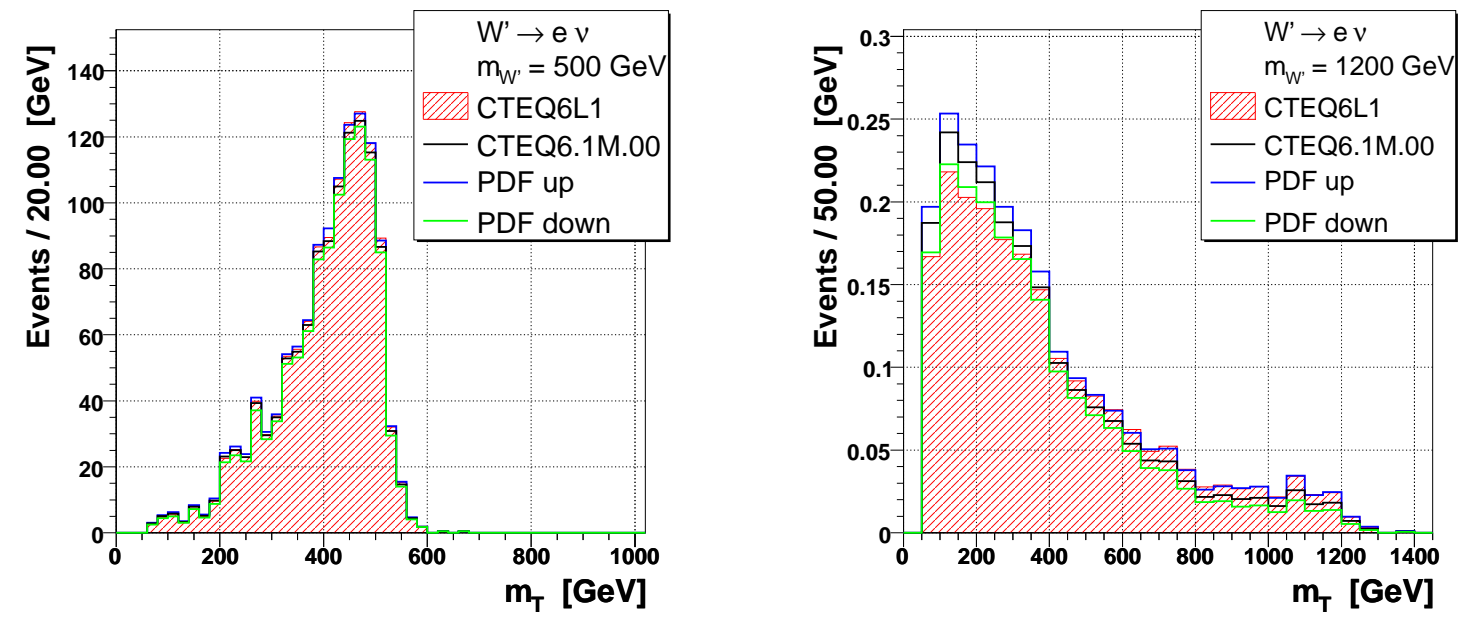
Figure 7.29: Impact of the PDF uncertainty on the $W^{\prime}$ signal. Left-hand plot: $m_{W^{\prime}}=500 \mathrm{GeV}$; right-hand plot: $m_{W^{\prime}}=1200 \mathrm{GeV}$.
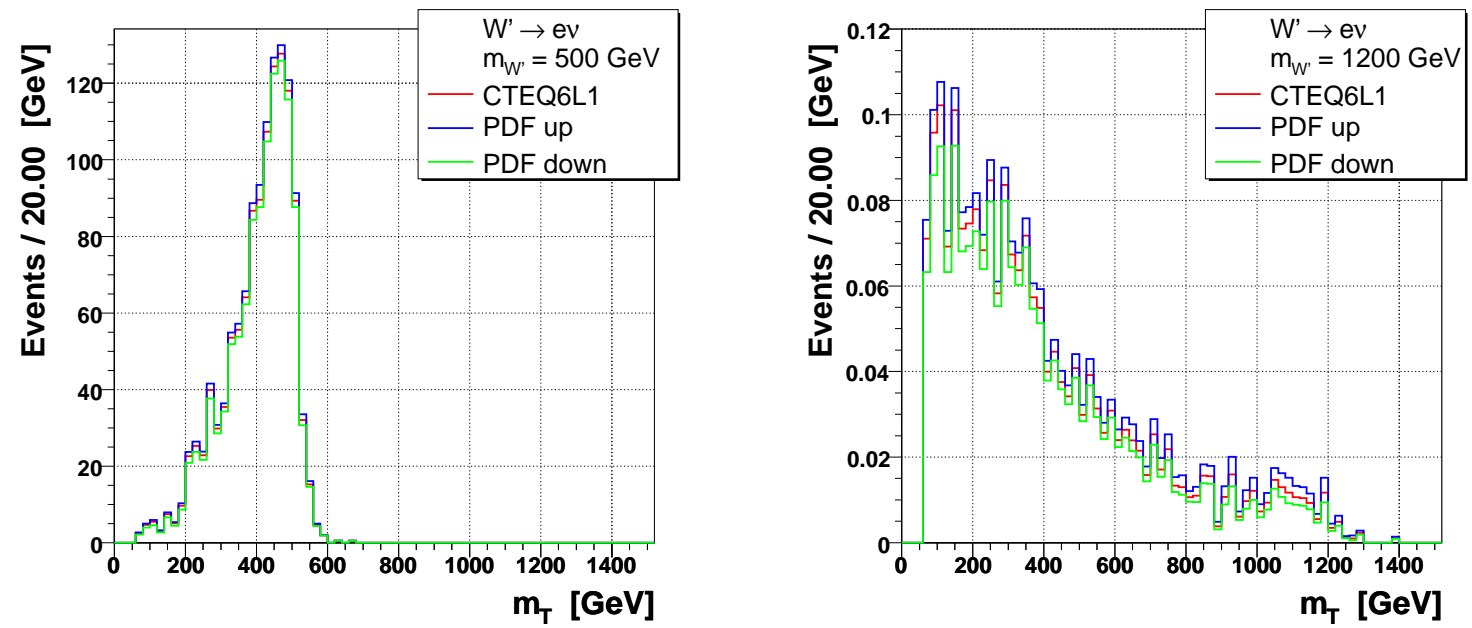

\section{Uncertainty of the width $\Gamma_{W}$ of the $W$ boson}

The width of the $W$ boson is known at the $2 \%$ level [4]. In order to estimate the impact of this uncertainty, events are reweighted, therefore avoiding the need for generating events with the shifted width, $\Gamma_{W} \pm \Delta \Gamma_{W}$. Each event gets a weight $w_{\Gamma}^{ \pm}$which depends on the mass $m$ of the $W$ boson $\left(m_{W}=80.4 \mathrm{GeV}\right)$

$$
w_{\Gamma}^{ \pm}(m)=\frac{B W\left(m, m_{W}, \Gamma_{W} \pm \Delta \Gamma_{W}\right)}{B W\left(m, m_{W}, \Gamma_{W}\right)}
$$

using the Breit-Wigner Distribution

$$
B W\left(m, m_{W}, \Gamma_{W}\right)=\frac{1}{2 \pi} \frac{\Gamma_{W}^{2}}{\left(m-m_{W}\right)^{2}+\frac{1}{4} \Gamma_{W}^{2}} .
$$

Fig. 7.30 shows the mass distribution of the decaying $W$ boson together with fits using the regular Breit-Wigner Eq. 7.24 and the relativistic Breit-Wigner function

$$
r B W\left(m, m_{W}, \Gamma_{W}\right)=\frac{1}{\left(m^{2}-m_{W}^{2}\right)^{2}+m_{W}^{2} \Gamma_{W}^{2}} .
$$

Both fits result in $m_{W}=80.4 \mathrm{GeV}$ and $\Gamma_{W}=2.1 \mathrm{GeV}$. The modified width causes a $2 \%$ shift in the peak region around $80 \mathrm{GeV}$ and a $4 \%$ shift in the tail of the transverse mass distribution of the $W$, see Fig. 7.31. The ratio $R_{\Gamma}$ is defined as follows

$$
R_{\Gamma}=\frac{\left(W \text { Monte Carlo with } \Gamma_{W}+\Delta \Gamma_{W}\right)-\left(W \text { Monte Carlo with } \Gamma_{W}\right)}{W \text { Monte Carlo with } \Gamma_{W}} .
$$

\section{Uncertainty from the reweighting of the $W p_{T}$ spectrum}

The $W \rightarrow e \nu$ PyThia Monte Carlo events with one and two jets have been reweighted with the function $w_{W}\left(p_{T}^{E M+M E T}\right)$ from Eq. 7.13, and events with three jets with a constant, see previous section. The fit parameters have been derived from data (Tab. 7.2). In order 
Figure 7.30: Mass distribution for the $W$ Monte Carlo sample. The spectrum is fitted with a regular Breit-Wigner (red) and the relativistic Breit-Wigner (blue) function.

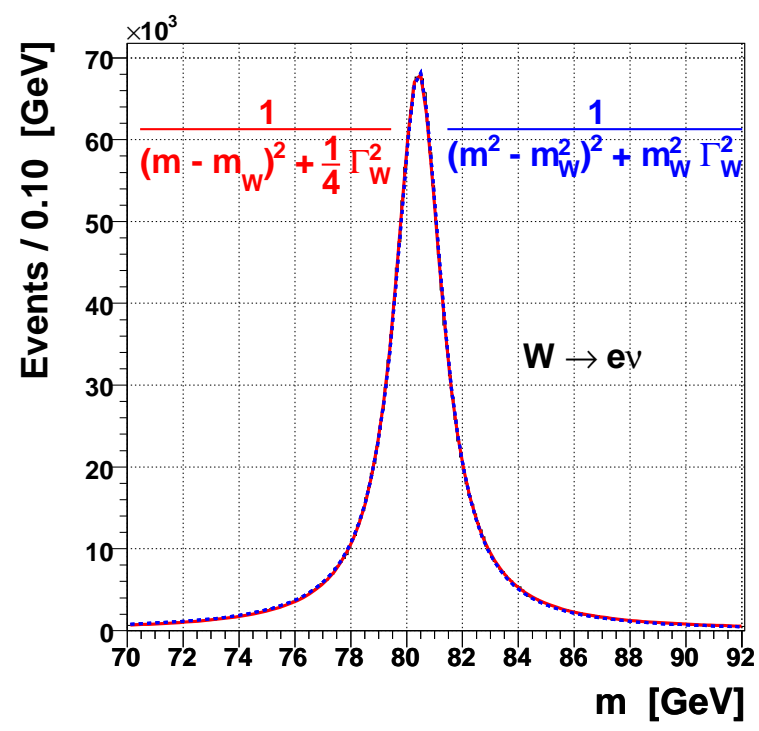

to estimate the uncertainty without performing a variation of the three fit parameters, the fit function is shifted upward and downward with $\varepsilon$

$$
w_{W}^{ \pm}\left(p_{T}^{E M+M E T}\right)=w_{W}\left(p_{T}^{E M+M E T}\right) \pm \varepsilon
$$

so that $68 \%$ of the data points are contained in the interval spaned by the two variations, $\left[w_{W}^{-}\left(p_{T}^{E M+M E T}\right), w_{W}^{+}\left(p_{T}^{E M+M E T}\right)\right]$. Fig. 7.32 displays the fit and the shifted fit functions for various jet multiplicities. Note, that the reweighting is not applied in events without jets. The resulting values for $\varepsilon$ are given in Tab. 7.3.

Since only a small fraction of events $(10 \%)$ is affected by the reweighting, the overall uncertainty is small $(\ll 1 \%)$.

Table 7.3: Numbers of the reweighting uncertainty for the different jet multiplicities $n_{\text {jet }}$.

\begin{tabular}{|c|c|}
\hline$n_{\text {jet }}$ & $\varepsilon$ \\
\hline \hline 1 & 0.027 \\
2 & 0.076 \\
3 & 0.264 \\
\hline
\end{tabular}

\section{Jet energy scale (JES)}

In order to estimate this contribution, the Pyтнia Monte Carlo samples are reprocessed. The JES (see Sec. 4.2) is shifted up and down with the total uncertainty $\sigma_{\text {JES }}$ from Eq. 4.16. After this, the missing transverse energy is recalculated. Although it causes just a minor uncertainty of $\ll 1 \%$, this effect is taken into account. 
Figure 7.31: Impact of the uncertainty of the width $\Gamma_{W}$ of the $W$ boson on the transverse mass spectrum.
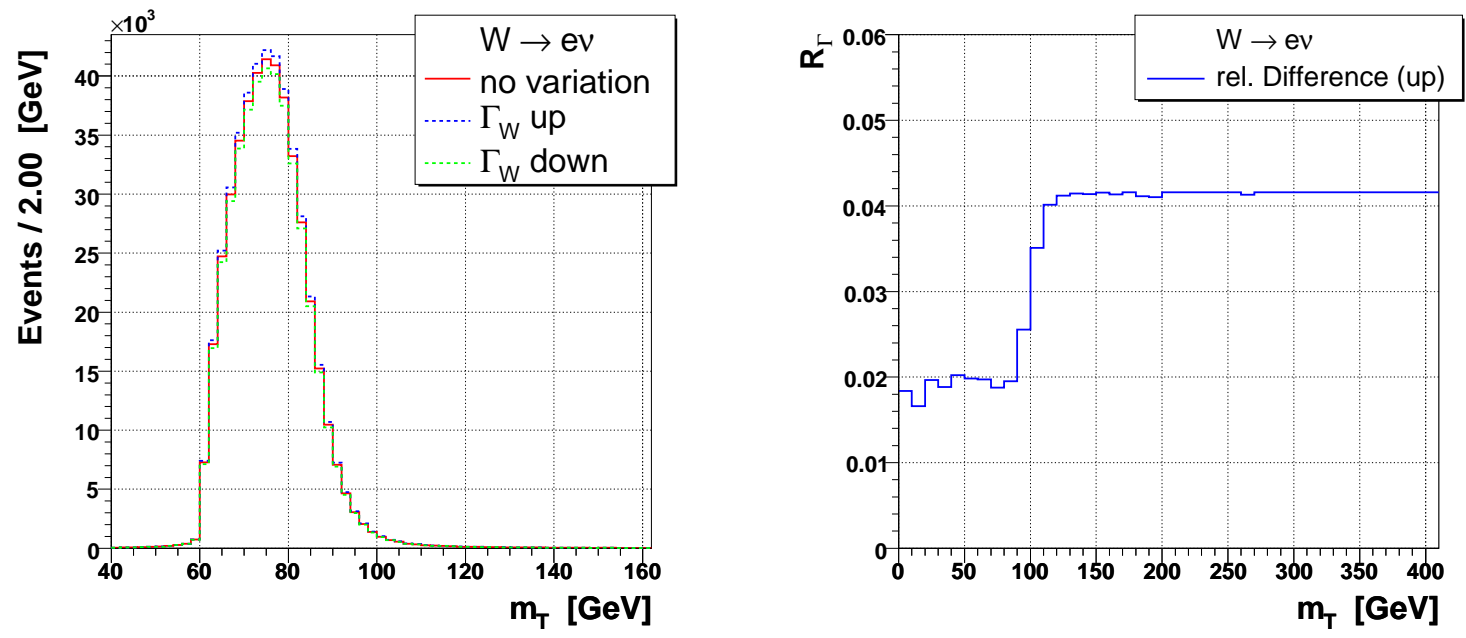

Figure 7.32: Fit and shifted fit functions for the $W p_{T}$ reweighting in events with $n_{\text {jet }}=1,2,3$.
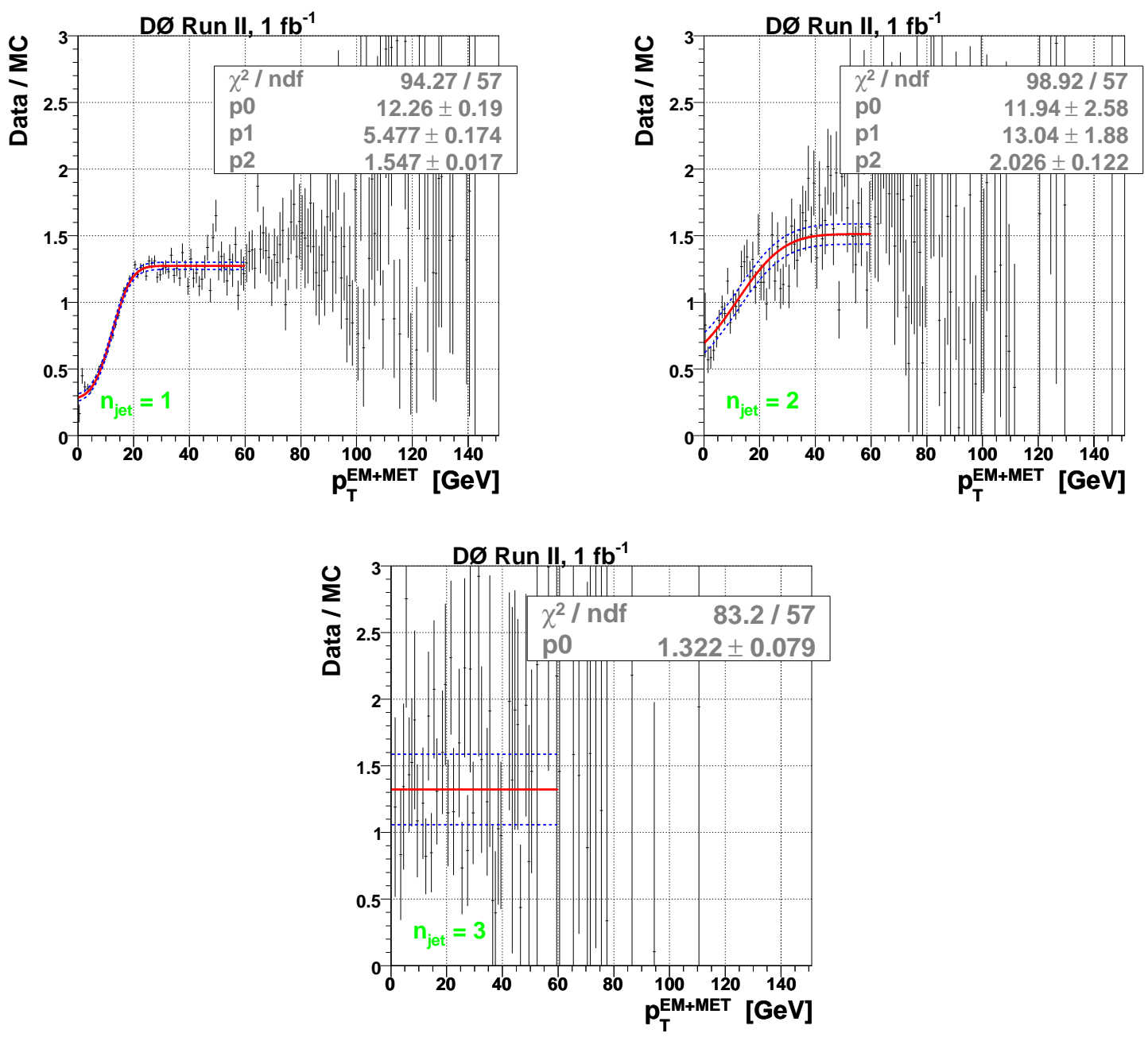


\section{Chapter 8}

\section{Results}

We are all agreed that your theory is crazy. The question which divides us is whether it is crazy enough to have a chance of being correct. My own feeling is that it is not crazy enough.

Niels Bohr

In this chapter, the selected datasets - the $W$ sample and search sample - are compared to the background prediction and to the expectation of a hypothetical $W^{\prime}$ signal for masses from $m_{W^{\prime}}=500 \mathrm{GeV}$ up to $m_{W^{\prime}}=1200 \mathrm{GeV}$. The Pythia Monte Carlo samples are corrected for various effects that are not part of the full simulation. Distributions and numbers for both, the $W$ sample (Sec. 8.1) and the search sample (Sec. 8.2), are discussed. Since no significant excess is found in the data, upper limits on the production cross section times branching fraction, $\sigma_{W^{\prime}} \times \operatorname{Br}\left(W^{\prime} \rightarrow e \nu\right)$, are derived (Sec. 8.3). The binned likelihood method which is used for the limit calculation is briefly reviewed.

\subsection{Distributions and Numbers for the $W$ Sample}

After applying all corrections discussed in the previous section, a very good agreement between data and background prediction (PYTHIA Monte Carlo samples and QCD sample derived from data) can be observed for the $W$ sample defined in Sec. 6.2. In Figs. $8.1-$ 8.4 the following distributions are shown: transverse energy of the electron $E_{T}$; missing transverse energy (MET) $E_{T}$; ratio of electron $E_{T}$ and MET, angular difference between electron and MET, $\Delta \phi(E M, M E T)$; thrust; transverse momentum reconstructed from the electron and MET, $p_{T}^{E M+M E T}$; jet multiplicity $n_{\text {jet }}$; maximal angular differences between jets (if present in the event) and electron/MET, $\Delta \phi(J e t, E M / M E T)$; transverse momentum of the hadronic final state $p_{T}^{H F S}$; jet transverse momenta $p_{T}$ and $\eta$ distributions for the first and second jet in the event, $\phi$ distributions for electron and MET; and finally the electron $\eta$ and $\eta_{\text {det }}$ distributions. The full spectrum of the transverse mass $m_{T}$ reconstructed from electron and MET is displayed in Fig. 8.5. The signal is shown for a hypothetical $W^{\prime}$ boson with mass $m_{W^{\prime}}=500 \mathrm{GeV}$. The corresponding event num- 
bers for the $W$ sample are summarized in Tab. 8.1. The data sample contains 452,984 events, compared to an expected number of $454,056 \pm 478$ (stat) ${ }_{-36,863}^{+35,720}$ (sys) background events. Details of the various contributions to the overall uncertainty for the sum of all backgrounds, the $W$ and the QCD background can be found in Tab. 8.2. The total relative systematic uncertainty of $8 \%$ on the background event numbers in the $W$ sample is dominated by the uncertainties on the electron energy scale, effective luminosity and cross sections ( $4 \%$ each), followed by the uncertainty on the electron reconstruction efficiency correction $(3 \%)$, and on the PDF and the width of the $W$ boson $\Gamma_{W}(2 \%$ each).

Discrepancies exist in distributions related to jet activity, but these affect only a small part of the $W$ sample because of $N\left(n_{\text {jet }}=0\right): N\left(n_{\text {jet }}>0\right) \approx 10: 1$. Other minor differences can be attributed to details of the detector description affecting reconstruction efficiencies which are not perfectly simulated and not covered by corrections.

\section{- Jet activity:}

Higher order QCD contributions are not expected to be described by the (leading order) parton shower Monte Carlo generator PyTHIA. The reweighting of the transverse momentum of the $W$ boson yields a significant improvement, but the transverse momentum distribution of the second jet is still too steep in the Monte Carlo. Furthermore, only small jet multiplicities $\left(n_{\text {jet }} \leq 3\right)$ are fixed by this procedure due to the lack of Monte Carlo statistics at higher multiplicities. However, the agreement between the reweighted $W$ Monte Carlo sample and the data is very good in distributions describing the relative position of jets and the electron and MET, respectively, whereas only the latter ones are important for the present analysis. Nevertheless, the study of distributions of variables related to jet activity serves as an important cross check although any further (kinematical) information from jets is not consulted for the search for $W^{\prime}$ bosons in the inclusive electron + MET final state.

\section{- Details of the detector simulation:}

Fluctuations in the electron $\eta_{\text {det }}$ and $\phi$ distributions can be assigned to a nonuniform response of the calorimeter cells. Although the efficiency correction Sec. 7.2.1 is applied as a function of these two variables, both distributions do not match in every detail. This is due to the fact that the binned $\phi$ correction (16 bins, see Fig. 7.5) does not correspond to the detector geometry with 64 readout cells located on 32 modules. One can clearly see dips in the electron $\phi$ distribution at the boundaries of the $\phi$ modules where the electron reconstruction efficiency is much smaller in data. However, the Monte Carlo samples describe the general tendency. In case of jets, the efficiency corrections do not result in a proper description for the $\eta^{\text {jet } i}$ distributions, especially for jets in the "massless gap" region $0.8<\left|\eta_{\text {det }}\right|<1.4$ (corresponding to approximately $0.6<\left|\eta^{\text {jet }}\right|<1.8$ ) between the central and endcap cryostats, see Fig. 3.18 .

In summary, despite the minor differences, the PYтніa Monte Carlo samples and the QCD multijet background estimated from data are capable to describe the shapes of nearly all distributions. The transverse mass distribution $m_{T}$ of the $W$ sample shown in Fig. 8.5 consists of a small peak at $m_{T} \approx 0 \mathrm{GeV}$ caused by the QCD multijet background, the Jacobian peak around $80 \mathrm{GeV}$ from $W \rightarrow e \nu$ events and a steeply decreasing spectrum 
Figure 8.1: Comparison between data and background prediction for the $W$ sample (1).
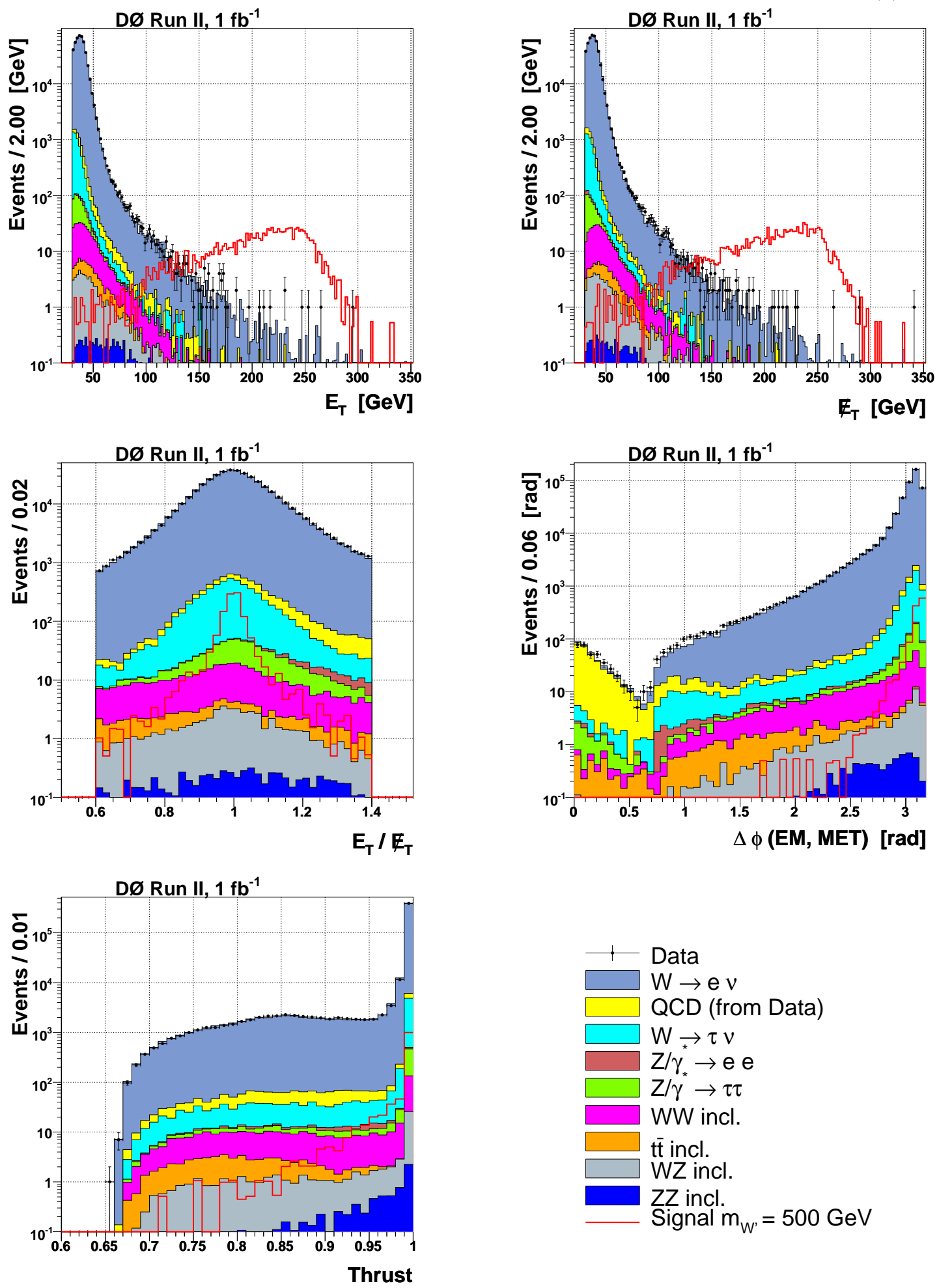
Figure 8.2: Comparison between data and background prediction for the $W$ sample (2).
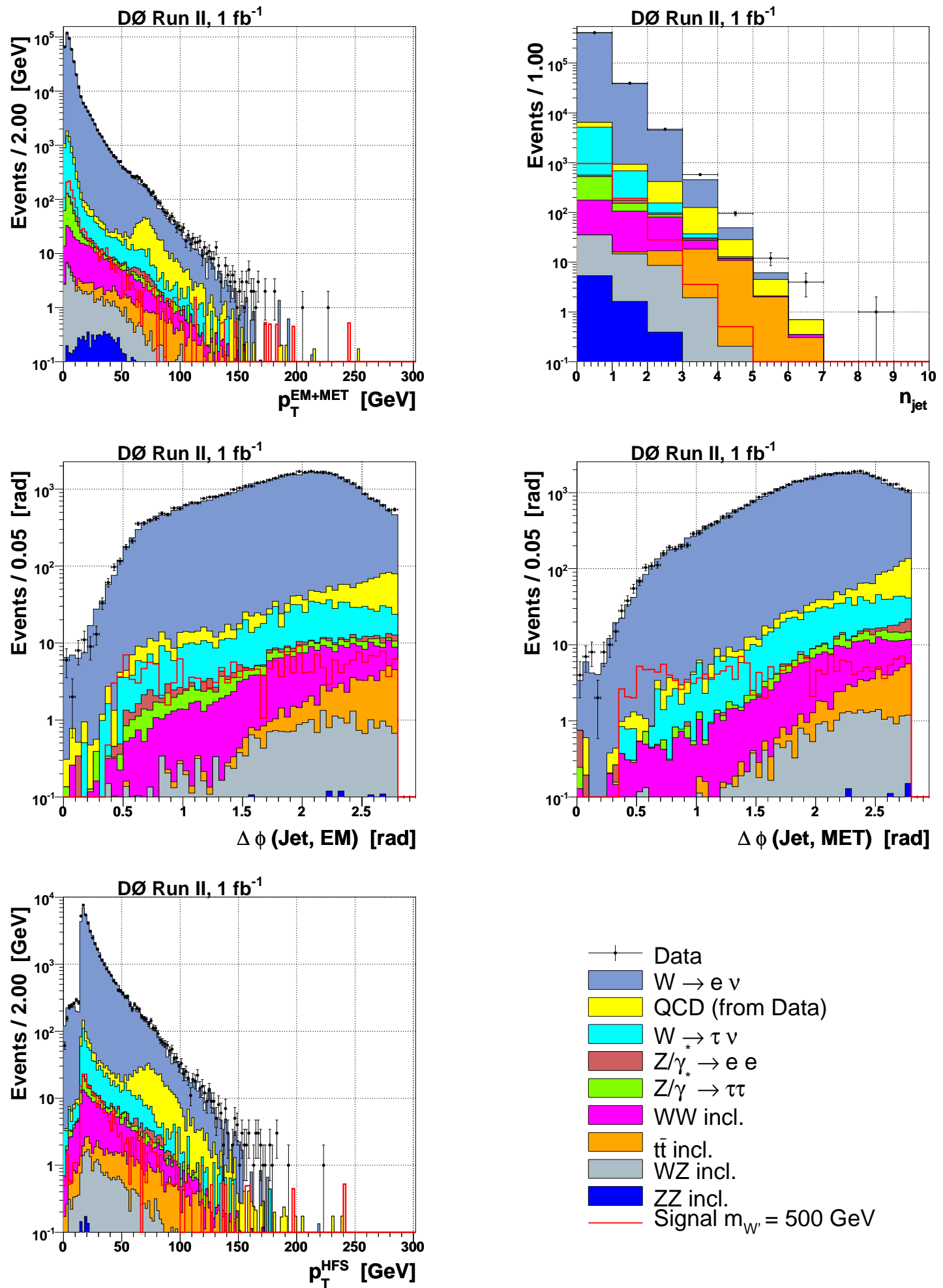
Figure 8.3: Comparison between data and background prediction for the $W$ sample (3).
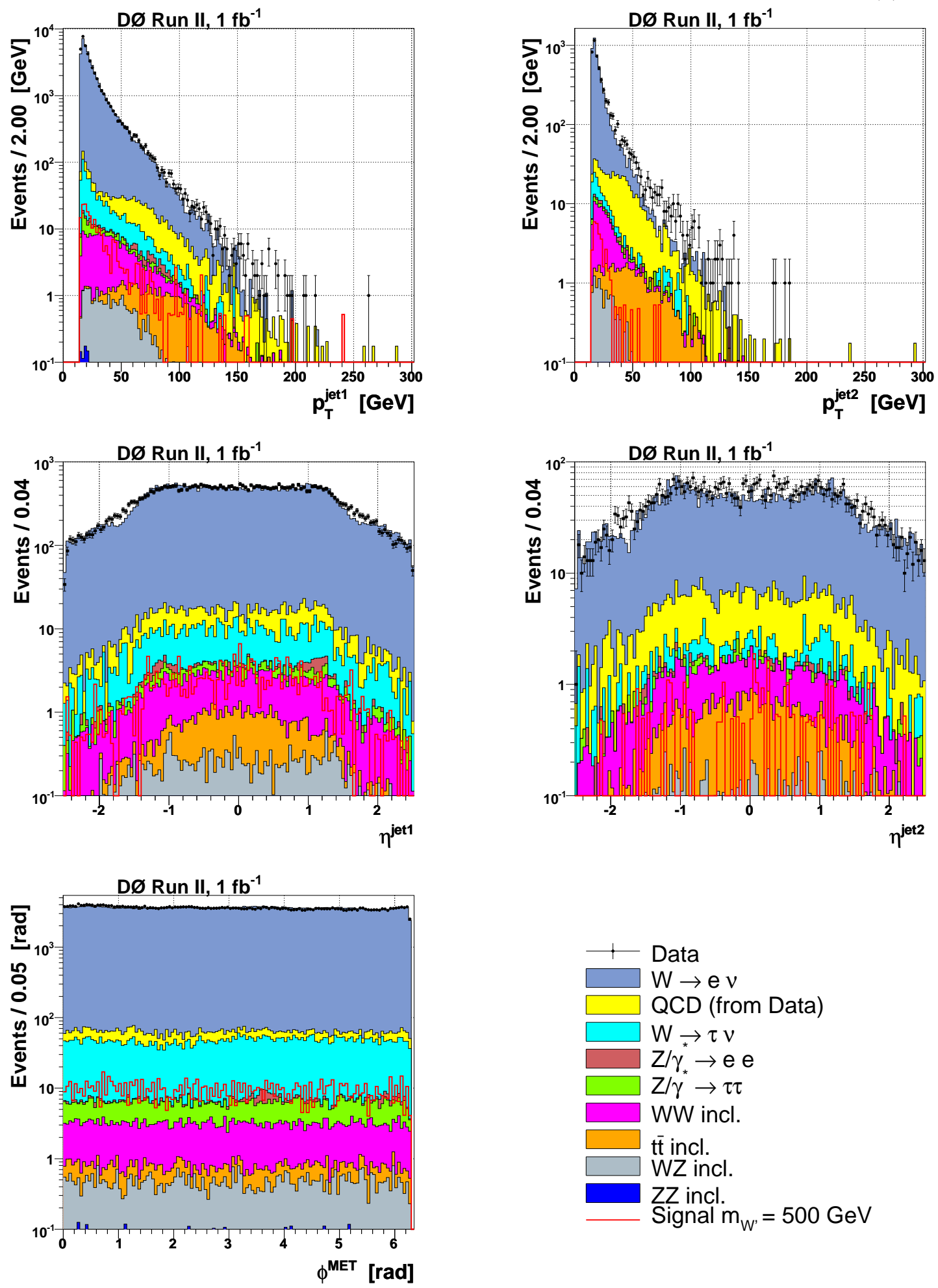
Figure 8.4: Comparison between data and background prediction for the $W$ sample (4).
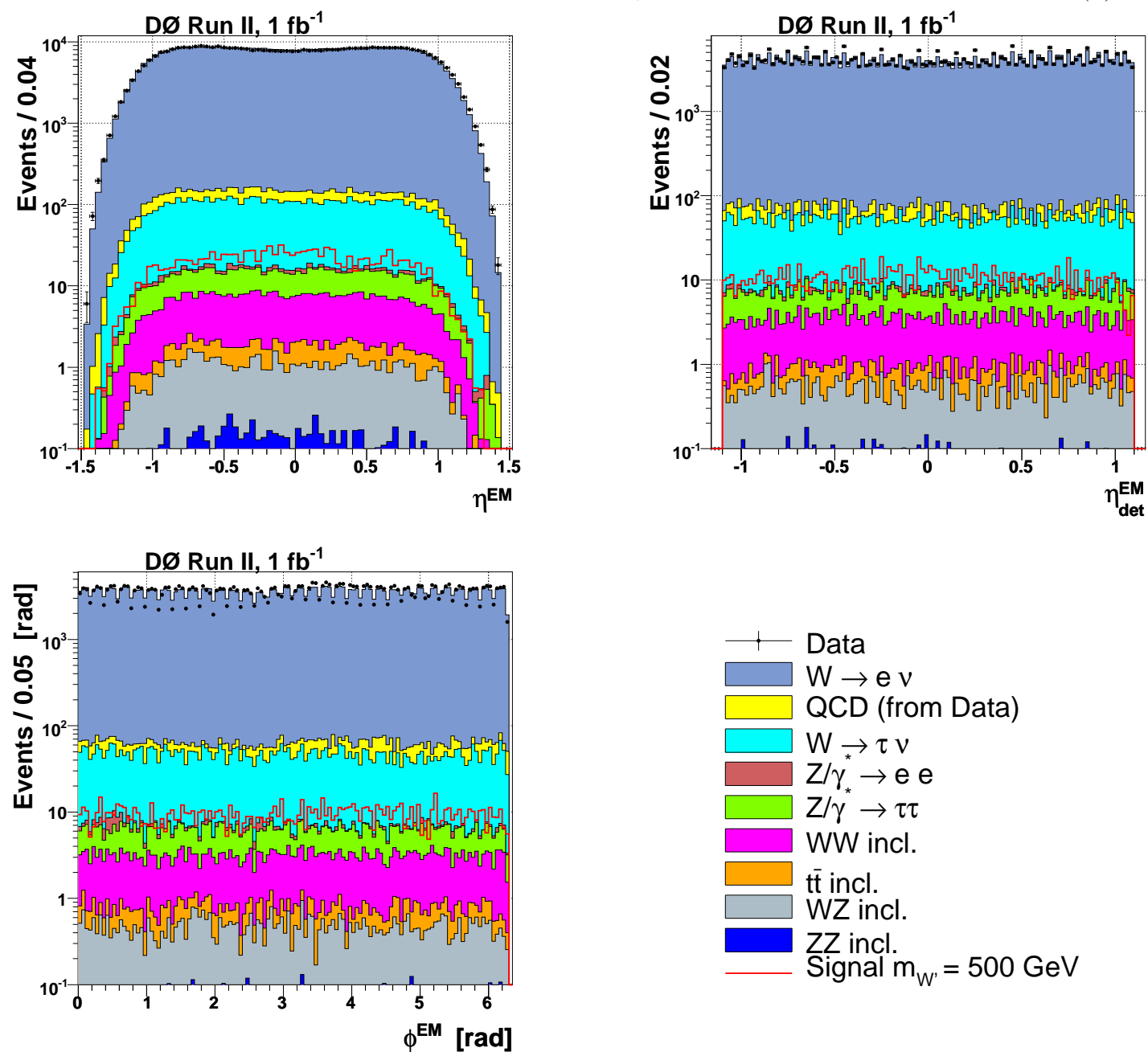

Table 8.1: Events in the $W$ sample compared to the background prediction.

\begin{tabular}{|l|r|r|r|r|}
\hline Process & Events & \multicolumn{3}{|c|}{ Uncertainty } \\
& & statistical & \multicolumn{2}{|c|}{ systematic } \\
& & & & \\
& & & & \\
\hline \hline Data & $\mathbf{4 5 2 9 8 4}$ & & & \\
\hline Sum Backgrounds & 454056 & 478 & 35720 & 36863 \\
\hline$W \rightarrow e \nu$ & 446071 & 475 & 34847 & 35996 \\
$W \rightarrow \tau \nu$ & 5152 & 47 & 802 & 758 \\
$Z \rightarrow e e$ & 74 & 3 & 8 & 7 \\
$Z \rightarrow \tau \tau$ & 406 & 7 & 51 & 50 \\
$W W, W Z, Z Z, t \bar{t}$ (incl.) & 405 & 4 & 35 & 35 \\
$\mathrm{QCD}$ (from data) & 1947 & 29 & 206 & 210 \\
\hline
\end{tabular}


Figure 8.5: Comparison between data and background prediction for the $W$ sample (5). The signal (red line) is shown for a hypothetical $W^{\prime}$ boson with mass $m_{W^{\prime}}=500 \mathrm{GeV}$.

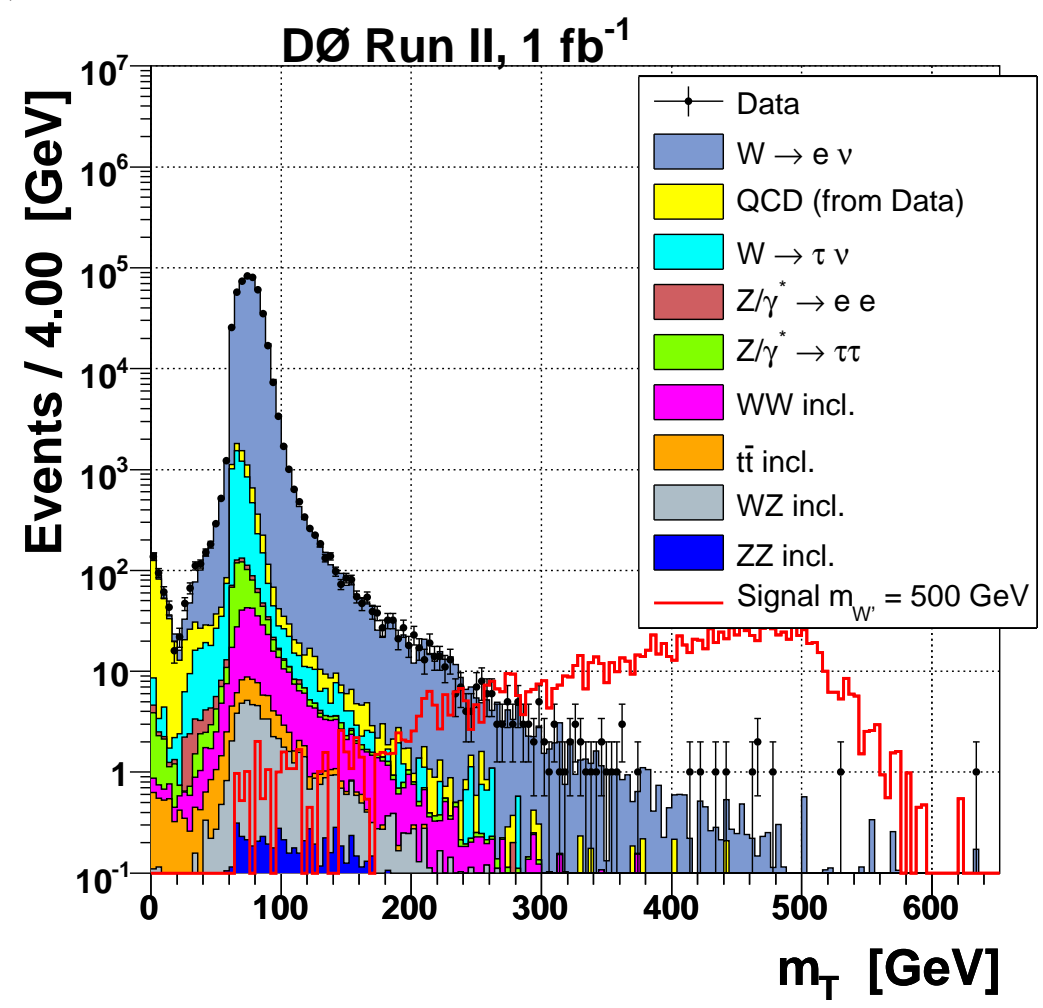

Table 8.2: Relative contributions (in \%) of the various uncertainties on background event numbers in the $W$ sample.

\begin{tabular}{|l|cc|cc|cc|}
\hline Uncertainty & \multicolumn{5}{|c|}{ Relative uncertainty in \% } \\
& Sum backgrounds & \multicolumn{2}{|c|}{$W \rightarrow e \nu$} & QCD (from data) \\
\hline \hline statistical & \multicolumn{2}{|c|}{$\pm \mathbf{0 . 1}$} & \multicolumn{2}{c|}{$\pm \mathbf{0 . 1}$} & \multicolumn{2}{c|}{$\pm \mathbf{1 . 5}$} \\
\hline systematic & $+\mathbf{7 . 7}$ & $-\mathbf{7 . 9}$ & $\mathbf{+ 7 . 8}$ & $\mathbf{- 8 . 1}$ & $+\mathbf{1 0 . 6}$ & $\mathbf{- 1 0 . 8}$ \\
\hline $\mathcal{L}_{W}$ & +3.9 & -3.9 & +4.0 & -4.0 & +0.0 & -0.0 \\
cross section & +3.6 & -3.2 & +3.6 & -3.2 & +3.4 & -3.0 \\
electron reconstruction & +2.8 & -2.8 & +2.8 & -2.8 & \multicolumn{2}{|c|}{$\mathrm{n} / \mathrm{a}$} \\
QCD scaling & +0.0 & -0.0 & \multicolumn{2}{|c|}{$\mathrm{n} / \mathrm{a}$} & +6.0 & -6.0 \\
\hline PDF & +1.4 & -2.3 & +1.4 & -2.4 & +1.5 & -2.3 \\
EM scale & +4.1 & -4.5 & +4.2 & -4.6 & +7.6 & -7.8 \\
EM resolution & +0.3 & -0.3 & +0.3 & -0.3 & +1.1 & -1.3 \\
$p_{T}^{E M+M E T}$ & +0.3 & -0.3 & +0.3 & -0.3 & +0.3 & -0.3 \\
$\Gamma_{W}$ & +1.9 & -1.9 & +2.0 & -2.0 & +1.8 & -1.8 \\
JES & +0.0 & -0.0 & +0.0 & -0.0 & +0.0 & -0.1 \\
\hline
\end{tabular}


for transverse masses $m_{T}>80 \mathrm{GeV}$. The signal of a hypothetical $W^{\prime}$ with mass $m_{W^{\prime}}=$ $500 \mathrm{GeV}$ would appear as a second broader Jacobian Peak at $m_{T} \approx 480 \mathrm{GeV}$.

\subsection{Distributions and Numbers for the Search Sample}

The regions of low and intermediate transverse masses can not be used for the search for the production of heavy charged gauge bosons because these have already been used for the extraction of the scaling factor for the QCD sample $\left(m_{T}<20 \mathrm{GeV}\right)$ and the measurement of the effective luminosity $\mathcal{L}_{W}\left(60 \mathrm{GeV}<m_{T}<120 \mathrm{GeV}\right)$. However, this is not a critical issue due to overwhelming amount of Standard Model background contributions at $m_{T}<$ $140 \mathrm{GeV}$ making a search for such a rare signal process unfeasible. On the other hand, due to the negligible contribution of a possible signal at low transverse masses, this region is perfectly suited for calibration and normalization purposes because of the well-known sources of Standard Model backgrounds.

Therefore, the tail of the spectrum with $m_{T}>140 \mathrm{GeV}$ is considered for the search for heavy charged gauge bosons. The Figs. 8.6 - 8.9 display the following distributions for the search sample: transverse energy of the electron $E_{T}$; missing transverse energy (MET) $E_{T}$; ratio of electron $E_{T}$ and MET; angular difference between electron and MET, $\triangle \phi(E M, M E T)$; thrust; transverse momentum reconstructed from the electron and MET, $p_{T}^{E M+M E T}$; jet multiplicity $n_{\text {jet }}$; maximal angular differences between jets (if present in the event) and electron/MET, $\Delta \phi($ Jet, EM/MET $)$; transverse momentum of the hadronic final state $p_{T}^{H F S}$; jet transverse momenta $p_{T}$ and $\eta$ distributions for the first and second jet in the event; $\phi$ distributions for electron and MET; and finally the electron $\eta$ and $\eta_{\text {det }}$ distributions. In addition, Figs. $8.11-8.14$ show all plots on a linear scale. The full

Table 8.3: Events in the search sample for transverse masses $m_{T}>140 \mathrm{GeV}$.

\begin{tabular}{|l|r|r|r|r|}
\hline Process & Events & \multicolumn{3}{|c|}{ Uncertainty } \\
& & statistical & \multicolumn{2}{|c|}{ systematic } \\
& & & $(+)$ & $(-)$ \\
\hline \hline Data & $\mathbf{9 6 7}$ & & & \\
\hline Sum Backgrounds & 959 & 21 & 89 & 92 \\
\hline$W \rightarrow e \nu$ & 875 & 20 & 89 & 92 \\
$W \rightarrow \tau \nu$ & 20.8 & 3.0 & 2.5 & 3.5 \\
$Z \rightarrow e e$ & 0.28 & 0.17 & 0.04 & 0.13 \\
$Z \rightarrow \tau \tau$ & 2.97 & 0.18 & 0.24 & 0.22 \\
$W W, W Z, Z Z, t \bar{t}($ incl. $)$ & 32.8 & 1.1 & 2.6 & 2.7 \\
$\mathrm{QCD}$ (from data) & 27.4 & 2.5 & 1.7 & 1.7 \\
\hline$W^{\prime} \rightarrow e \nu(500 \mathrm{GeV})$ & 1169 & 24 & 77 & 95 \\
$W^{\prime} \rightarrow e \nu(600 \mathrm{GeV})$ & 393 & 8 & 28 & 36 \\
$W^{\prime} \rightarrow e \nu(700 \mathrm{GeV})$ & 147 & 3 & 12 & 15 \\
$W^{\prime} \rightarrow e \nu(800 \mathrm{GeV})$ & 51.4 & 1.1 & 4.8 & 6.0 \\
$W^{\prime} \rightarrow e \nu(900 \mathrm{GeV})$ & 18.6 & 0.4 & 2.2 & 2.6 \\
$W^{\prime} \rightarrow e \nu(1000 \mathrm{GeV})$ & 7.43 & 0.18 & 1.01 & 1.17 \\
$W^{\prime} \rightarrow e \nu(1100 \mathrm{GeV})$ & 3.39 & 0.08 & 0.46 & 0.53 \\
$W^{\prime} \rightarrow e \nu(1200 \mathrm{GeV})$ & 1.72 & 0.05 & 0.22 & 0.26 \\
\hline
\end{tabular}


Figure 8.6: Comparison between data and background prediction for the search sample (1).
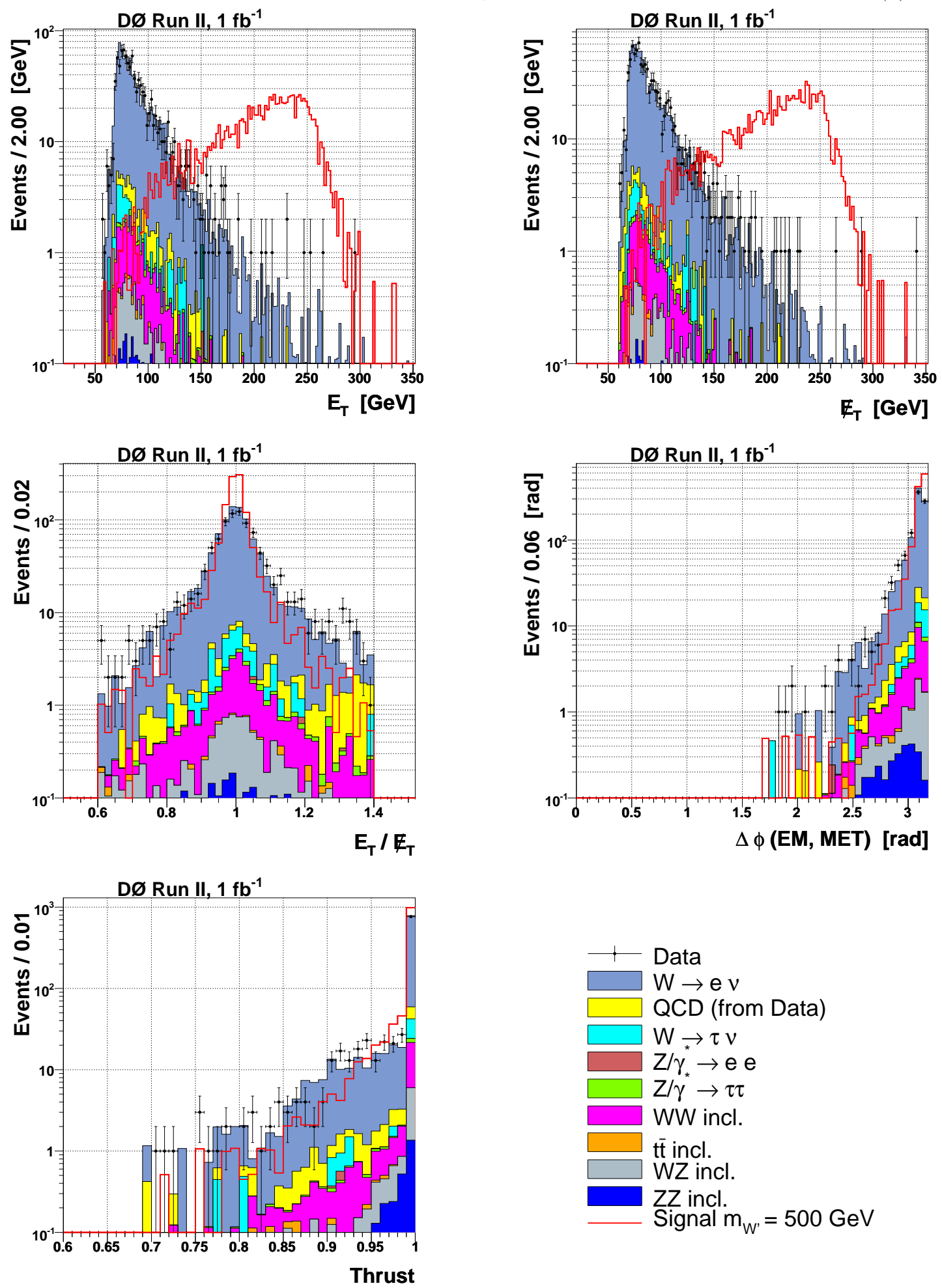
Figure 8.7: Comparison between data and background prediction for the search sample (2).
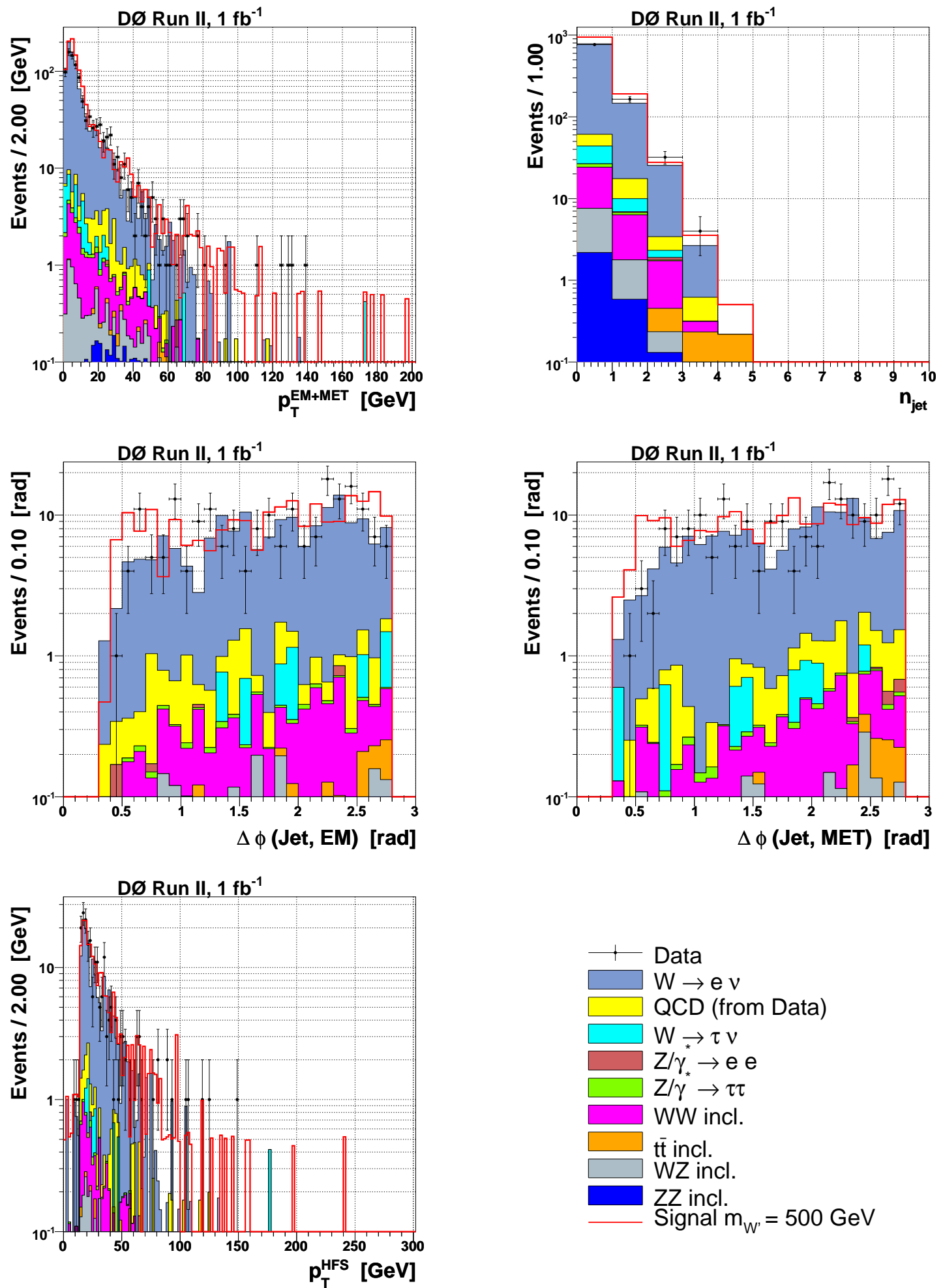
Figure 8.8: Comparison between data and background prediction for the search sample (3).
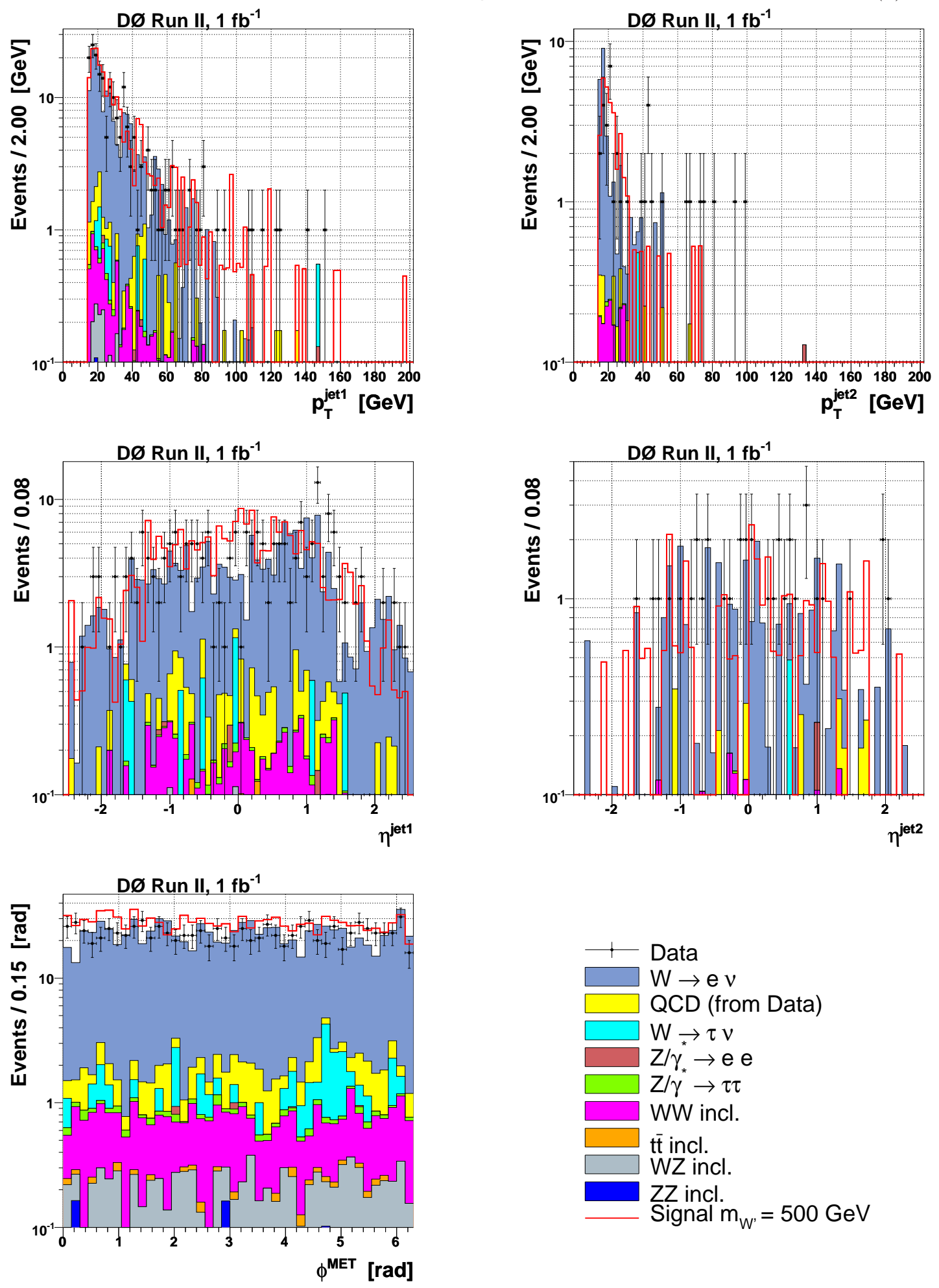
Figure 8.9: Comparison between data and background prediction for the search sample (4).
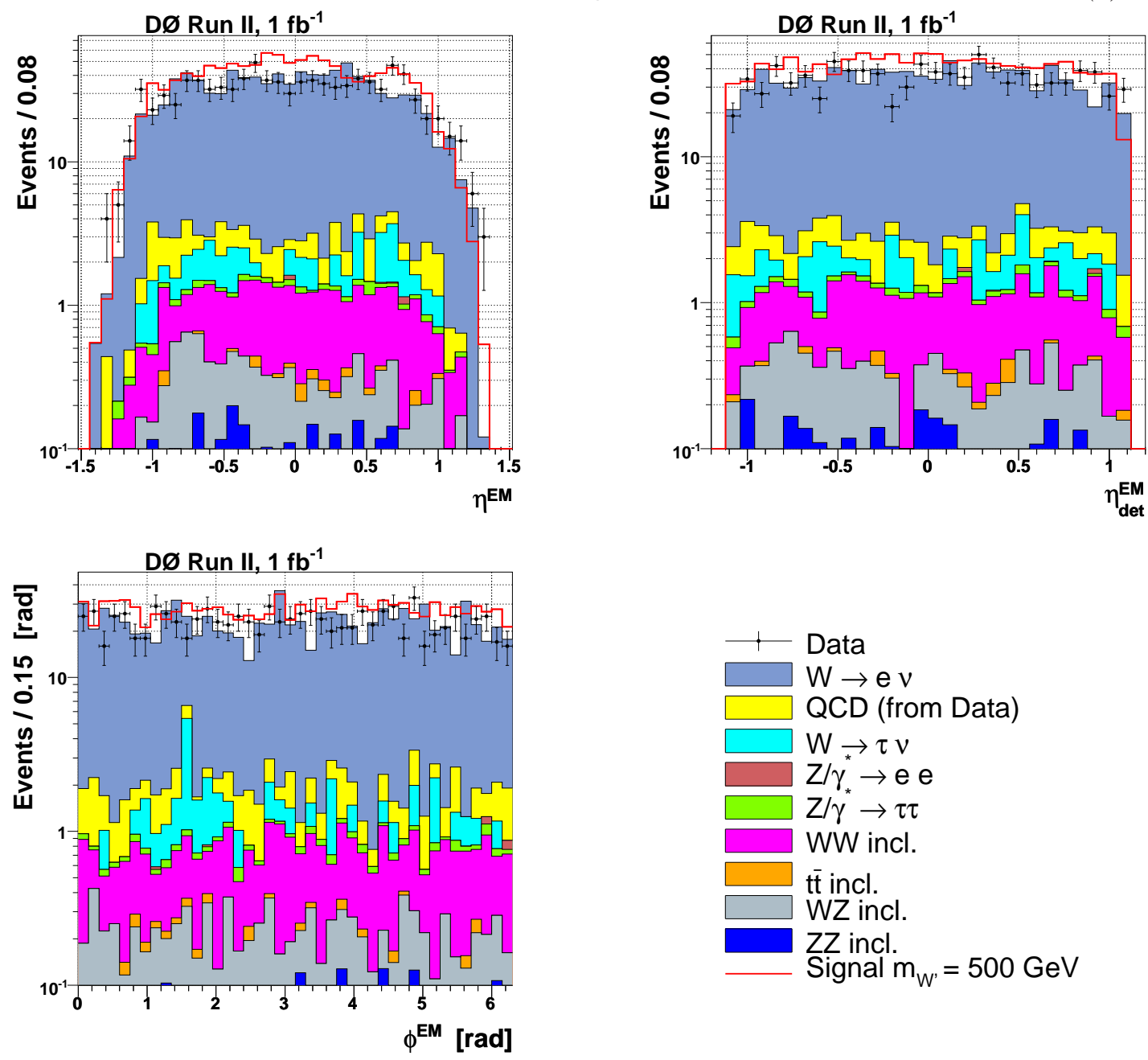

Table 8.4: Relative contributions (in \%) of the various uncertainties on signal and background event numbers in the search sample.

\begin{tabular}{|c|c|c|c|c|c|c|}
\hline \multirow[t]{2}{*}{ Uncertainty } & \multicolumn{6}{|c|}{ Relative uncertainty in \% } \\
\hline & \multicolumn{2}{|c|}{ Sum backgrounds } & \multicolumn{2}{|c|}{$W^{\prime} \rightarrow e \nu(500 \mathrm{GeV})$} & \multicolumn{2}{|c|}{$W^{\prime} \rightarrow e \nu(1200 \mathrm{GeV})$} \\
\hline statistical & \multicolumn{2}{|c|}{ \pm 2.2} & \multicolumn{2}{|c|}{ \pm 2.1} & \multicolumn{2}{|c|}{ \pm 2.6} \\
\hline systematic & +9.3 & -9.6 & +6.6 & -8.2 & +12.8 & -15.1 \\
\hline $\mathcal{L}_{W}$ & +3.3 & -3.3 & +4.0 & -4.0 & +4.0 & -4.0 \\
\hline cross section & +3.0 & -2.7 & +3.4 & -5.8 & +7.7 & -12.2 \\
\hline electron reconstruction & +2.3 & -2.3 & +2.9 & -2.9 & +2.9 & -2.9 \\
\hline QCD scaling & +0.2 & -0.2 & \multicolumn{2}{|c|}{$\mathrm{n} / \mathrm{a}$} & \multicolumn{2}{|c|}{$\mathrm{n} / \mathrm{a}$} \\
\hline $\mathrm{PDF}$ & +3.7 & -2.7 & +2.8 & -2.9 & +9.3 & -7.6 \\
\hline EM scale & +5.9 & -7.0 & +0.0 & -0.0 & +0.4 & -0.9 \\
\hline EM resolution & +0.5 & -0.5 & +0.1 & -0.1 & +0.2 & -0.1 \\
\hline$p_{T}^{E M+M E T}$ & +0.4 & -0.4 & \multirow{2}{*}{\multicolumn{2}{|c|}{$\begin{array}{l}\mathrm{n} / \mathrm{a} \\
\mathrm{n} / \mathrm{a}\end{array}$}} & \multicolumn{2}{|c|}{$\mathrm{n} / \mathrm{a}$} \\
\hline$\Gamma_{W}$ & +3.4 & -3.3 & & & & \\
\hline JES & +0.1 & -0.3 & +0.0 & -0.0 & +0.0 & -0.0 \\
\hline
\end{tabular}


Figure 8.10: Distribution of the transverse mass $m_{T}$ for the search sample.
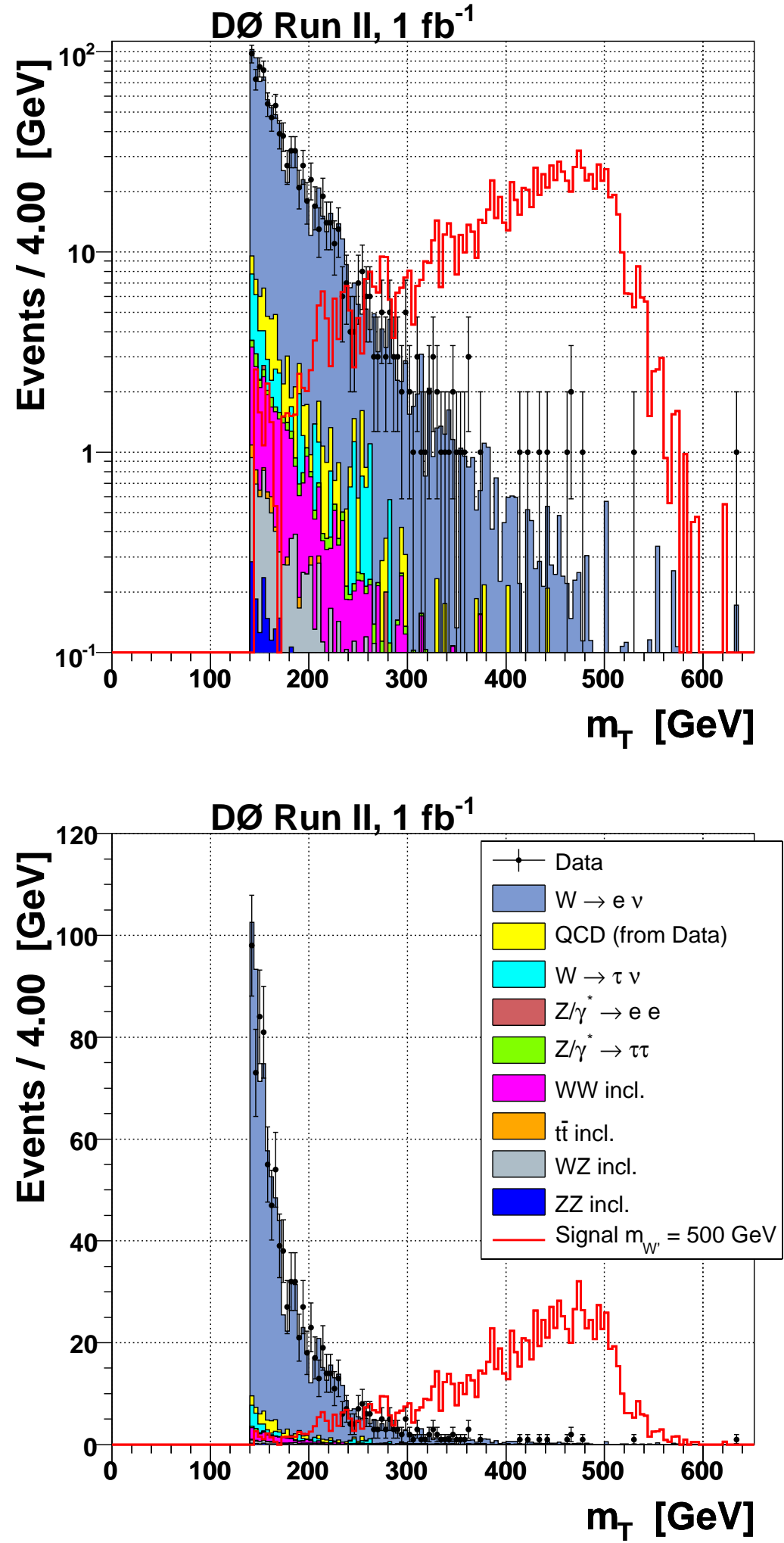
Figure 8.11: Same plots as in Fig. 8.6 on linear scale.
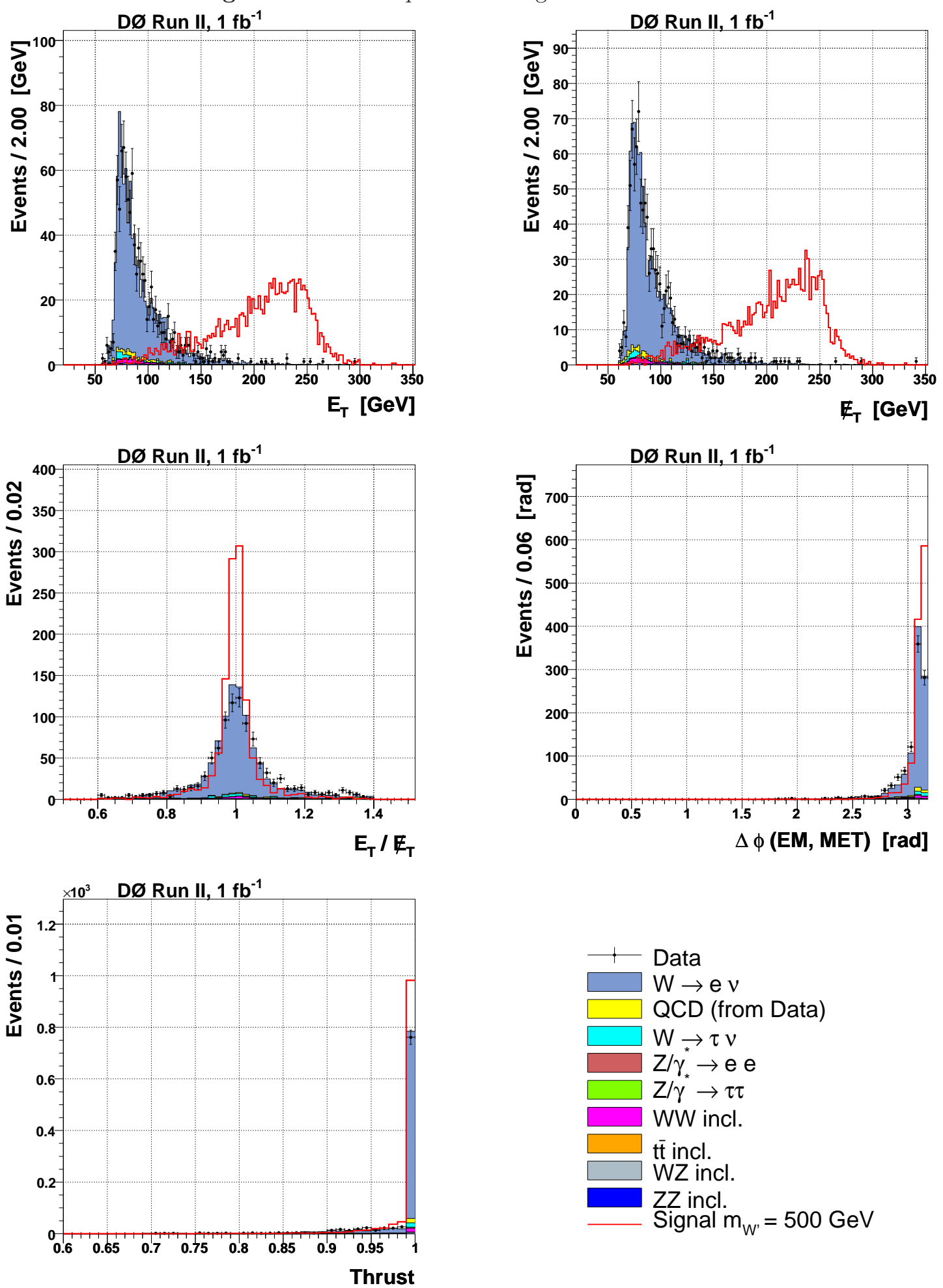
Figure 8.12: Same plots as in Fig. 8.7 on linear scale.
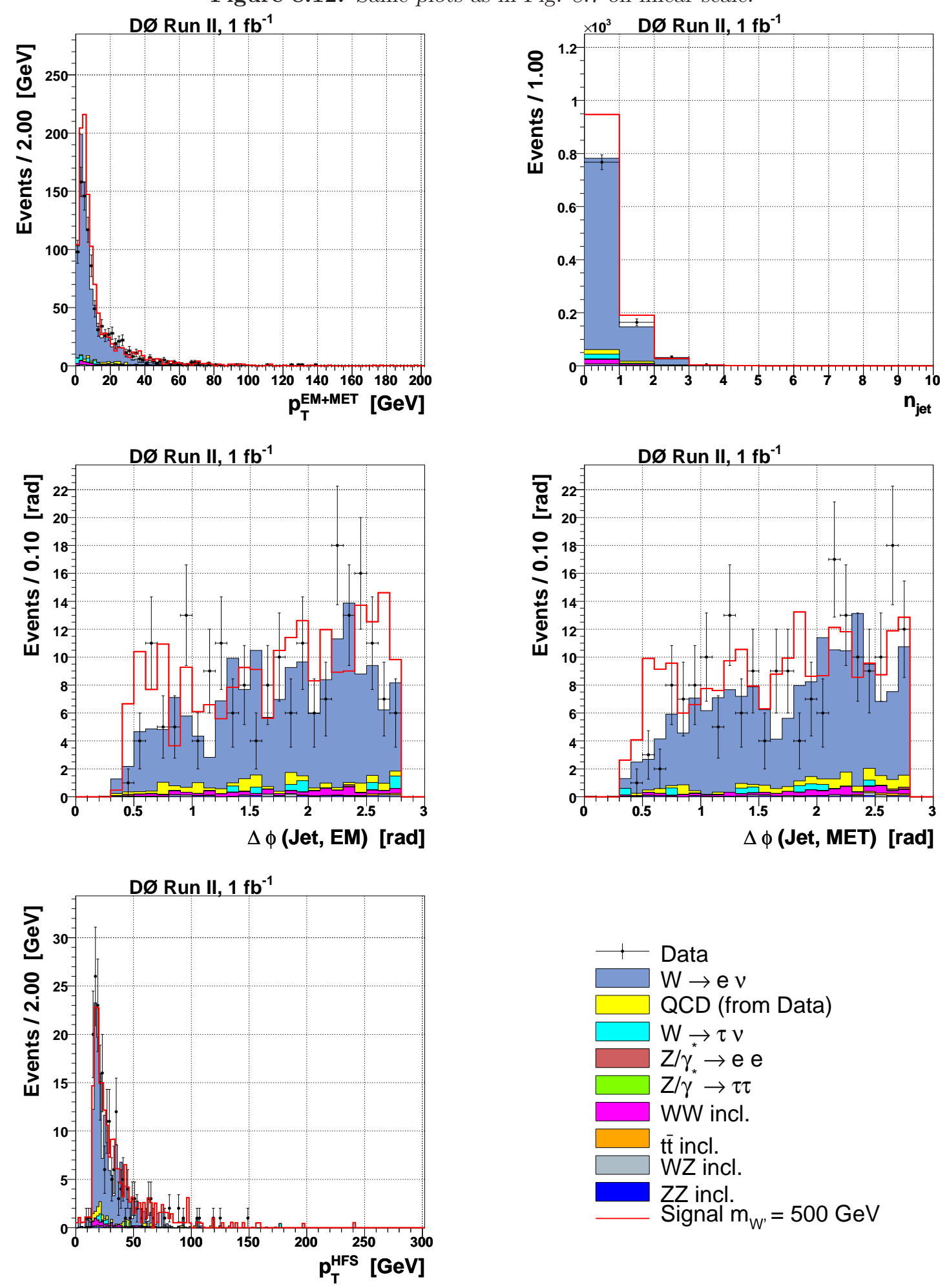
Figure 8.13: Same plots as in Fig. 8.8 on linear scale.
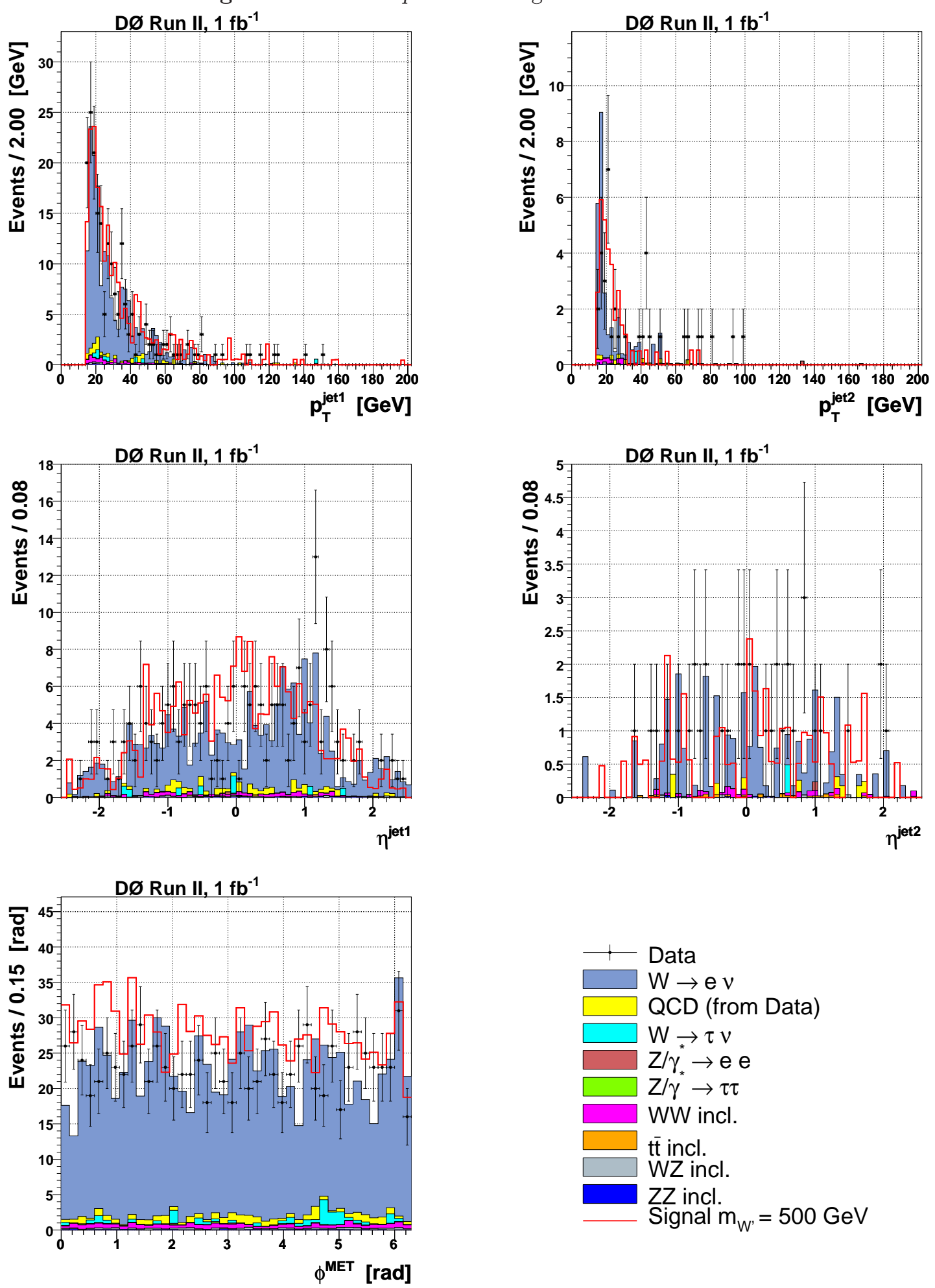
Figure 8.14: Same plots as in Fig. 8.9 on linear scale.
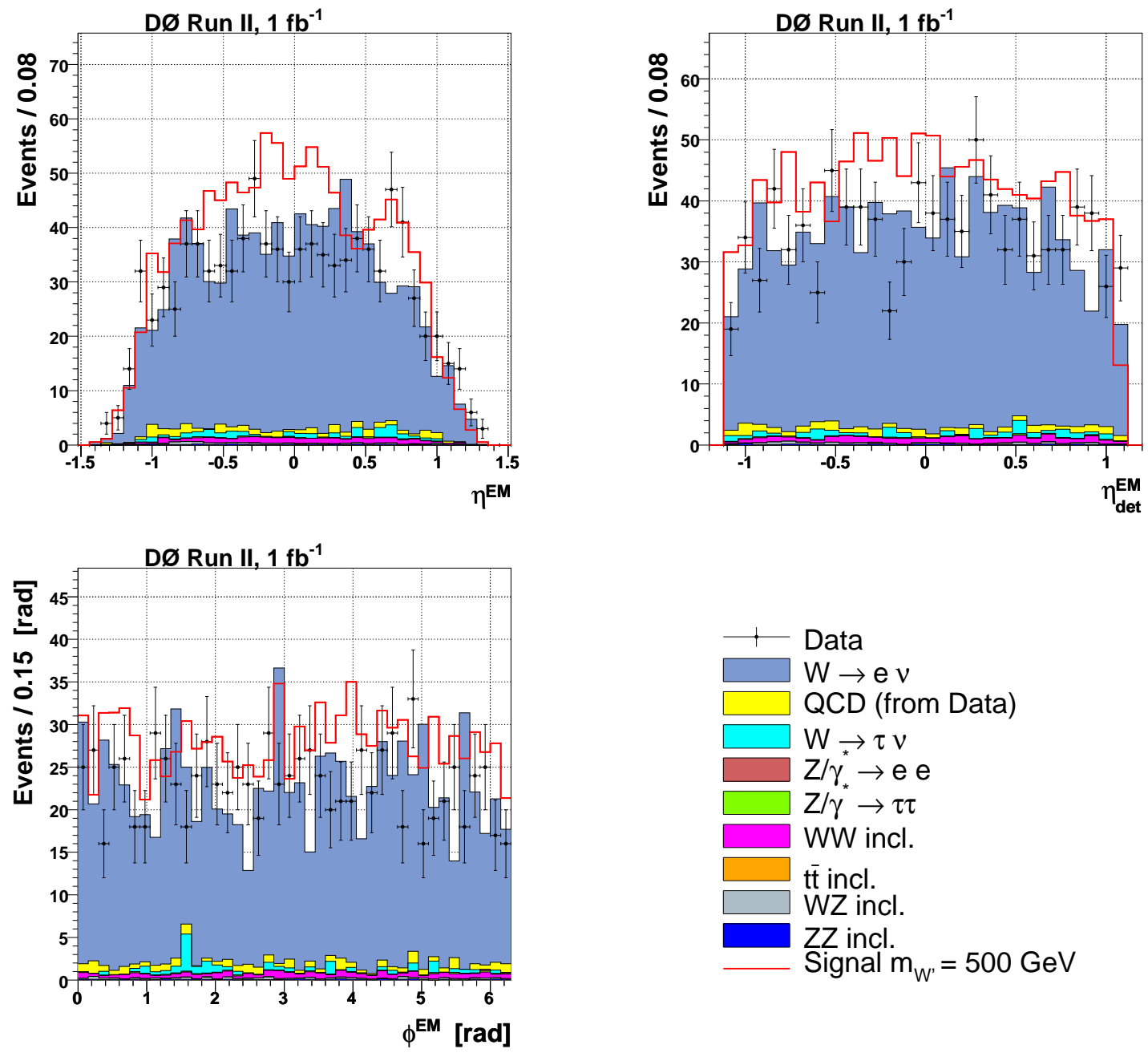
Table 8.5: Events in the search sample for transverse masses $m_{T}>200 \mathrm{GeV}$.

\begin{tabular}{|l|r|r|r|r|}
\hline Process & Events & \multicolumn{3}{|c|}{ Uncertainty } \\
& & statistical & \multicolumn{2}{|c|}{ systematic } \\
& & & $(+)$ & $(-)$ \\
\hline \hline Data & $\mathbf{2 4 1}$ & & & \\
\hline Sum Backgrounds & 242 & 9 & 20 & 17 \\
\hline$W \rightarrow e \nu$ & 219 & 9 & 20 & 17 \\
$W \rightarrow \tau \nu$ & 5.41 & 1.57 & 0.74 & 0.49 \\
$Z \rightarrow e e$ & 0.11 & 0.11 & 0.03 & 0.13 \\
$Z \rightarrow \tau \tau$ & 0.72 & 0.04 & 0.10 & 0.07 \\
$W W, W Z, Z Z, t \bar{t}(\mathrm{incl})$. & 8.64 & 0.56 & 0.88 & 0.78 \\
$\mathrm{QCD}($ from data) & 7.35 & 1.16 & 0.59 & 0.53 \\
\hline$W^{\prime} \rightarrow e \nu(500 \mathrm{GeV})$ & 1147 & 24 & 75 & 93 \\
$W^{\prime} \rightarrow e \nu(600 \mathrm{GeV})$ & 386 & 8 & 27 & 35 \\
$W^{\prime} \rightarrow e \nu(700 \mathrm{GeV})$ & 144 & 3 & 11 & 15 \\
$W^{\prime} \rightarrow e \nu(800 \mathrm{GeV})$ & 50.3 & 1.1 & 4.6 & 5.9 \\
$W^{\prime} \rightarrow e \nu(900 \mathrm{GeV})$ & 17.6 & 0.4 & 2.0 & 2.4 \\
$W^{\prime} \rightarrow e \nu(1000 \mathrm{GeV})$ & 6.88 & 0.17 & 0.94 & 1.07 \\
$W^{\prime} \rightarrow e \nu(1100 \mathrm{GeV})$ & 3.01 & 0.08 & 0.42 & 0.47 \\
$W^{\prime} \rightarrow e \nu(1200 \mathrm{GeV})$ & 1.47 & 0.04 & 0.20 & 0.22 \\
\hline
\end{tabular}

Table 8.6: Events in the search sample for transverse masses $m_{T}>300 \mathrm{GeV}$.

\begin{tabular}{|l|r|r|r|r|}
\hline Process & Events & \multicolumn{3}{|c|}{ Uncertainty } \\
& & statistical & \multicolumn{2}{|c|}{ systematic } \\
& & & $(+)$ & $(-)$ \\
\hline \hline Data & 37 & & & \\
\hline Sum Backgrounds & 37.1 & 2.1 & 6.0 & 3.7 \\
\hline$W \rightarrow e \nu$ & 33.9 & 2.0 & 6.0 & 3.7 \\
$W \rightarrow \tau \nu$ & 0.00 & 0.00 & 0.00 & 0.00 \\
$Z \rightarrow e e$ & 0.00 & 0.00 & 0.00 & 0.00 \\
$Z \rightarrow \tau \tau$ & 0.13 & 0.02 & 0.02 & 0.02 \\
$W W, W Z, Z Z, t \bar{t}($ incl. $)$ & 1.86 & 0.26 & 0.28 & 0.19 \\
$\mathrm{QCD}($ from data $)$ & 1.21 & 0.48 & 0.08 & 0.08 \\
\hline$W^{\prime} \rightarrow e \nu(500 \mathrm{GeV})$ & 1007 & 23 & 67 & 81 \\
$W^{\prime} \rightarrow e \nu(600 \mathrm{GeV})$ & 361 & 8 & 25 & 32 \\
$W^{\prime} \rightarrow e \nu(700 \mathrm{GeV})$ & 138 & 3 & 11 & 14 \\
$W^{\prime} \rightarrow e \nu(800 \mathrm{GeV})$ & 47.4 & 1.1 & 4.3 & 5.4 \\
$W^{\prime} \rightarrow e \nu(900 \mathrm{GeV})$ & 16.2 & 0.4 & 1.9 & 2.2 \\
$W^{\prime} \rightarrow e \nu(1000 \mathrm{GeV})$ & 5.99 & 0.16 & 0.83 & 0.93 \\
$W^{\prime} \rightarrow e \nu(1100 \mathrm{GeV})$ & 2.42 & 0.07 & 0.36 & 0.38 \\
$W^{\prime} \rightarrow e \nu(1200 \mathrm{GeV})$ & 1.10 & 0.04 & 0.16 & 0.17 \\
\hline
\end{tabular}


Table 8.7: Events in the search sample for transverse masses $m_{T}>400 \mathrm{GeV}$.

\begin{tabular}{|l|r|r|r|r|}
\hline Process & Events & \multicolumn{3}{|c|}{ Uncertainty } \\
& & statistical & \multicolumn{2}{|c|}{$\begin{array}{c}\text { systematic } \\
(+)\end{array}$} \\
& & & & \\
& & & & \\
& $\mathbf{1 0}$ & & & \\
\hline \hline Data & 9.28 & 1.05 & 1.53 & 1.34 \\
\hline Sum Backgrounds & 8.27 & 1.00 & 1.52 & 1.34 \\
$W \rightarrow e \nu$ & 0.00 & 0.00 & 0.00 & 0.00 \\
$W \rightarrow \tau \nu$ & 0.00 & 0.00 & 0.00 & 0.00 \\
$Z \rightarrow e e$ & 0.03 & 0.00 & 0.00 & 0.00 \\
$Z \rightarrow \tau \tau$ & 0.46 & 0.13 & 0.06 & 0.06 \\
$W W, W Z, Z Z, t \bar{t}($ incl. $)$ & 0.52 & 0.30 & 0.04 & 0.03 \\
QCD from data) & 711 & 19 & 58 & 67 \\
\hline$W^{\prime} \rightarrow e \nu(500 \mathrm{GeV})$ & 308 & 7 & 22 & 28 \\
$W^{\prime} \rightarrow e \nu(600 \mathrm{GeV})$ & 125 & 3 & 10 & 13 \\
$W^{\prime} \rightarrow e \nu(700 \mathrm{GeV})$ & 44.2 & 1.1 & 4.0 & 5.0 \\
$W^{\prime} \rightarrow e \nu(800 \mathrm{GeV})$ & 15.0 & 0.4 & 1.7 & 2.0 \\
$W^{\prime} \rightarrow e \nu(900 \mathrm{GeV})$ & 5.16 & 0.15 & 0.74 & 0.80 \\
$W^{\prime} \rightarrow e \nu(1000 \mathrm{GeV})$ & 1.96 & 0.06 & 0.31 & 0.32 \\
$W^{\prime} \rightarrow e \nu(1100 \mathrm{GeV})$ & 0.78 & 0.03 & 0.13 & 0.13 \\
$W^{\prime} \rightarrow e \nu(1200 \mathrm{GeV})$ & \multicolumn{3}{|c}{}
\end{tabular}

Table 8.8: Events in the search sample for transverse masses $m_{T}>500 \mathrm{GeV}$.

\begin{tabular}{|l|r|r|r|r|}
\hline Process & Events & \multicolumn{3}{|c|}{ Uncertainty } \\
& & statistical & \multicolumn{2}{|c|}{ systematic } \\
& & & $(+)$ & $(-)$ \\
\hline \hline Data & 2 & & & \\
\hline Sum Backgrounds & 2.32 & 0.53 & 0.50 & 0.68 \\
\hline$W \rightarrow e \nu$ & 2.10 & 0.50 & 0.50 & 0.68 \\
$W \rightarrow \tau \nu$ & 0.00 & 0.00 & 0.00 & 0.00 \\
$Z \rightarrow e e$ & 0.00 & 0.00 & 0.00 & 0.00 \\
$Z \rightarrow \tau \tau$ & 0.01 & 0.00 & 0.00 & 0.00 \\
$W W, W Z, Z Z, t \bar{t}(\mathrm{incl})$. & 0.04 & 0.04 & 0.01 & 0.00 \\
$\mathrm{QCD}($ from data) & 0.17 & 0.17 & 0.01 & 0.01 \\
\hline$W^{\prime} \rightarrow e \nu(500 \mathrm{GeV})$ & 144 & 9 & 61 & 57 \\
$W^{\prime} \rightarrow e \nu(600 \mathrm{GeV})$ & 205 & 6 & 19 & 23 \\
$W^{\prime} \rightarrow e \nu(700 \mathrm{GeV})$ & 101 & 3 & 8 & 10 \\
$W^{\prime} \rightarrow e \nu(800 \mathrm{GeV})$ & 38.1 & 1.0 & 3.5 & 4.4 \\
$W^{\prime} \rightarrow e \nu(900 \mathrm{GeV})$ & 13.5 & 0.4 & 1.6 & 1.8 \\
$W^{\prime} \rightarrow e \nu(1000 \mathrm{GeV})$ & 4.48 & 0.14 & 0.67 & 0.70 \\
$W^{\prime} \rightarrow e \nu(1100 \mathrm{GeV})$ & 1.54 & 0.05 & 0.27 & 0.26 \\
$W^{\prime} \rightarrow e \nu(1200 \mathrm{GeV})$ & 0.59 & 0.03 & 0.11 & 0.10 \\
\hline
\end{tabular}


spectrum of the transverse mass $m_{T}$ reconstructed from electron and MET is shown in Fig. 8.10. The signal is again plotted for a $W^{\prime}$ boson with $m_{W^{\prime}}=500 \mathrm{GeV}$.

A very good agreement between data (967 events) and background prediction (959 \pm 21 (stat) ${ }_{-92}^{+89}$ (sys) events) can be observed. A breakdown of the contributions of the individual background samples, together with expected event numbers for various masses of a hypothetical $W^{\prime}$ boson is given in Tab. 8.3. Tab. 8.4 summarizes the relative contributions of the various uncertainties on event numbers in the search sample for the sum of all backgrounds and two masses of a $W^{\prime}$ boson $\left(m_{W^{\prime}}=500 \mathrm{GeV}, 1200 \mathrm{GeV}\right)$. For the background samples $(9 \%)$ the uncertainty of the electron energy scale is predominant (6\%), whereas the uncertainties of the QCD scaling, electron energy resolution, jet energy scale and reweighting of the $p_{T}$ spectrum of the $W$ boson can be neglected $(<1 \%)$. All other uncertainties are of the order of $2-4 \%$. The total relative systematic uncertainty on the signal samples ranges from $7 \%$ to $14 \%$ with the major fractions stemming from the cross section, PDF and luminosity normalization uncertainties. The Tabs. $8.5-8.8$ show event numbers for higher cuts on the transverse mass $\left(m_{T}>200, \ldots, 500 \mathrm{GeV}\right)$. In summary, data and Standard Model background expectation agree not only at a high level of accuracy in terms of event numbers up to highest transverse masses, but also in the shapes of all distributions.

\subsection{Extraction of the Limit}

Since no significant excess is visible in the data, a statistical evaluation has to be performed in order to quantify at which level the number of data events $d$ is in agreement with the sum of all $N$ background samples $b_{i}$ and a possible (probably small) signal $s$

$$
d=s+\sum_{i=1}^{N} b_{i} .
$$

The upper limit on the cross section for the production of a heavy gauge boson $W^{\prime}$ is calculated as a function of the boson mass, $m_{W^{\prime}}$, using a Bayesian approach, and compared to the theoretical prediction from the Reference Model introduced in Sec. 2.3.2.

Due to the fact that the shapes of the signal distributions significantly depend on the mass of a possible additional gauge boson (see Fig. 5.10), this information is taken into account instead of just using total observed event numbers as quoted in Tab. 8.3. In the following, the binned likelihood method is briefly reviewed. An introduction into statistical data analysis can be found in [158]; details of the method applied in this analysis are summarized in [159] and [160].

\subsubsection{Binned Likelihood Method}

The Poisson distribution specifies the probability to observe $D$ events for a given mean value $d$

$$
P(D \mid d)=\frac{e^{-d} \cdot d^{D}}{D !} .
$$


The mean $d$ is given by the sum of the signal and $N$ background samples

$$
d=s+\sum_{i=1}^{N} b_{i}=\sigma \cdot \mathcal{L} \cdot \mathcal{A}+\sum_{i=1}^{N} b_{i}=\sigma \cdot a+\sum_{i=1}^{N} b_{i} \equiv d(\sigma, a, \vec{b})
$$

with $\vec{b}$ replacing $b_{1}, b_{2}, \ldots, b_{N}$. The number of signal events $s$ can be rewritten using the cross section $\sigma$ and the signal luminosity $a$ which is equal to the product of the luminosity $\mathcal{L}$ and the signal acceptance $\mathcal{A}$.

In general, a conditional probability $P(X \mid Y)$ can be inverted using Bayes' theorem

$$
P(Y \mid X)=\frac{P(X \mid Y) P(Y)}{P(X)} .
$$

The likelihood function $L$ is a conditional probability function considered as a function of its second argument with its first argument held fixed, thus

$$
L \quad: \quad y \mapsto P(X \mid Y=y) \quad \Rightarrow \quad L(y \mid X) \propto P(X \mid Y=y) .
$$

Now Bayes' theorem can be rewritten using the likelihood function

$$
P(Y \mid X) \propto L(y \mid X) P(Y) .
$$

This can be applied in order to derive $P(d \mid D)$ from Eq. 8.2

$$
P(d \mid D)=\frac{L(D \mid d) \cdot \pi(d)}{\mathcal{N}} \equiv P(\sigma, a, \vec{b} \mid D)=\frac{L(D \mid \sigma, a, \vec{b}) \cdot \pi(\sigma, a, \vec{b})}{\mathcal{N}}
$$

with $\mathcal{N}$ taking care of the proper normalization. The posterior probability density $P(\sigma \mid D)$ for the signal cross section $\sigma$ given the observed number of events $D$ is obtained by integration over the parameters $a$ and $\vec{b}$

$$
P(\sigma \mid D)=\frac{1}{\mathcal{N}} \iint \cdots \int L(D \mid \sigma, a, \vec{b}) \cdot \pi(\sigma, a, \vec{b}) \mathrm{d} a \mathrm{~d} \vec{b} \quad \text { with } \quad \mathcal{N}=\int P(\sigma \mid D) \mathrm{d} \sigma .
$$

The prior probability density $\pi(\sigma, a, \vec{b})$ can be disentangled under the assumption that any prior knowledge of $a$ and $\vec{b}$ is independent of the signal cross section

$$
\pi(\sigma, a, \vec{b})=\pi(\sigma) \cdot \pi(a, \vec{b})
$$

The following flat prior is used for the signal cross section

$$
\pi(\sigma)=\left\{\begin{array}{cl}
\frac{1}{\sigma_{\max }} & \text { for } 0<\sigma<\sigma_{\max } \\
0 & \text { otherwise }
\end{array}\right.
$$

The upper bound $\sigma_{\max }$ is chosen such that the posterior probability is negligible for $\sigma>$ $\sigma_{\max }$. Finally, the Bayesian upper limit on the cross section $\sigma_{C L}$ at a CL (Confidence Level) is obtained by solving the following expression (in this analysis: $C L=0.95$ )

$$
C L=\int_{0}^{\sigma_{C L}} P(\sigma \mid D) \mathrm{d} \sigma
$$


using the posterior probability

$$
P(\sigma \mid D) \propto \int L(D \mid \sigma, a, \vec{b}) \cdot \pi(a, \vec{b}) \mathrm{d} a \mathrm{~d} \vec{b}
$$

When considering a kinematic distribution with $N_{\text {bins }}$ bins, the likelihood $L(D \mid d)$ has to be replaced by the product of the likelihood over all bins $i$

$$
L(D \mid d)=\prod_{i=1}^{N_{\text {bins }}} L\left(D_{i} \mid d_{i}\right) .
$$

The technical implementation is realized within the package top_statistics [161], [162]. Monte Carlo importance sampling [163] is used for the numerical integration Eq. 8.12. In this case, a swarm of points $a_{k}, \vec{b}_{k}$ representing the prior density $\pi(a, \vec{b})$ is generated, and the posterior density estimated

$$
\int L(D \mid \sigma, a, \vec{b}) \cdot \pi(a, \vec{b}) \mathrm{d} a \mathrm{~d} \vec{b}=\frac{1}{K} \sum_{k=1}^{K} L\left(D \mid \sigma, a_{k}, \vec{b}_{k}\right) .
$$

The prior $\pi(a, \vec{b})$ contains all relevant information (value and associated uncertainties) of the parameters $a$ and $\vec{b}$. Two kinds of systematic uncertainties (see Sec. 7.4) enter the limit calculation. Uncertainties that affect the normalization are modeled by sampling $a$ and $\vec{b}$ using multivariate Gaussian distributions. The mean of the Gaussian is given by the estimate of the parameter, and the width by a covariance matrix from the associated uncertainties which takes correlations of the systematic uncertainties into account. The shape changing effects (electron energy scale and resolution, PDF uncertainty, uncertainty of the width $\Gamma_{W}$ of the $W$ boson and the uncertainty from the reweighting of the $W p_{T}$ spectrum) are modeled by shifting, one by one, the individual contributions by plus or minus one standard deviation with respect to their nominal value. Fig. 8.15 displays the nominal and the upward/downward shifted distributions for an arbitrary variable. First, the systematic uncertainties that affect the normalization are sampled, then the shape changing systematic uncertainties are taken into account via

Figure 8.15: Example for a shape-changing systematic uncertainty [161].

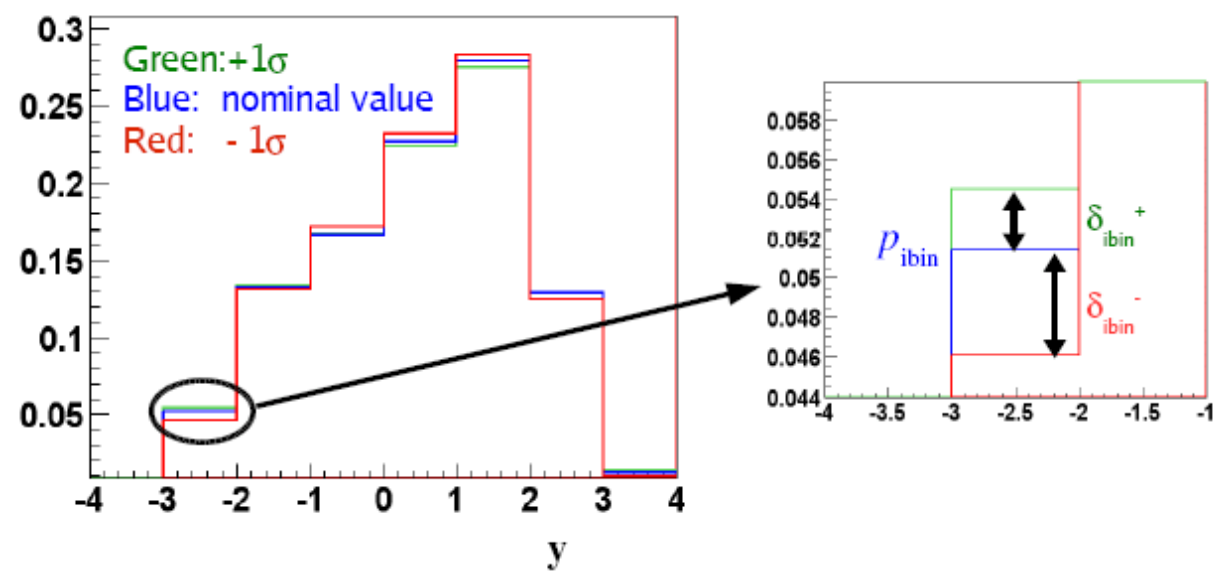




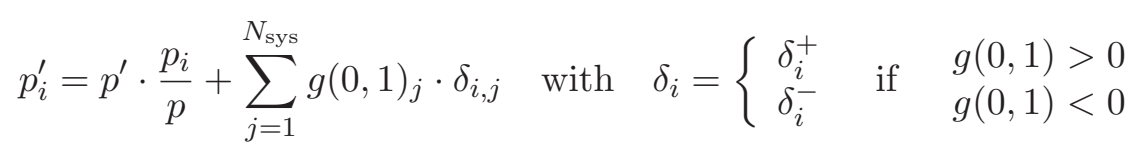

and $p$ being the measured value of the parameter summed over all bins. $p^{\prime}$ denotes the sum over all bins after varying only the uncertainties that affect the overall normalization. The same Gaussian random number $g(0,1)$ is used in one sampling so that correlations of any systematic component across different bins are taken into account.

\subsubsection{Limits on the Production Cross Section and the Mass}

The binned likelihood technique described in the previous section is applied to the entire distribution of the transverse mass $m_{T}$ shown in Fig. 8.10. The systematic uncertainties affecting the shape enter the limit calculation via individual histograms (up and down variation); the normalization uncertainties are contained in a table.

Fig. 8.16 displays the probability density distribution $P(\sigma \mid D)$ (see Eq. 8.12) for two masses $\left(m_{W^{\prime}}=500 \mathrm{GeV}, 1200 \mathrm{GeV}\right)$, and the observed and expected limits derived from the numerical integration of Eq. 8.11 with $C L=0.95$. The observed limit is obtained if $D$ represents the number of observed events in the data. In the calculation of the expected limit, the number of observed data events $D$ is replaced by the sum of all background events $B$ in the posterior probability density

$$
C L^{\exp }=\int_{0}^{\sigma_{C L}^{\exp }} P(\sigma \mid B) \mathrm{d} \sigma \quad \text { with } \quad B=\sum_{i=1}^{N} b_{i} .
$$

All 95\% CL limits on the production cross section times branching fraction, $\sigma_{W^{\prime}} \times$ $\operatorname{Br}\left(W^{\prime} \rightarrow e \nu\right)$ as a function of the mass $m_{W^{\prime}}$ of a hypothetical additional charged gauge boson are shown in Figs. 8.17 and 8.18. The numerical values are quoted in Tab. 8.9. The red curve displays the theoretical expectation from the Reference Model (see Sec. 2.3.2 and 5.3, thus the Manifest Left-Right Symmetric Model with light right-handed neutrinos)

Figure 8.16: Posterior probability distributions for $m_{W^{\prime}}=500 \mathrm{GeV}$ (left) and $m_{W^{\prime}}=1200 \mathrm{GeV}$ (right). The limits (expected and observed) given at the $95 \%$ CL are obtained from the numerical integration of Eq. 8.11.
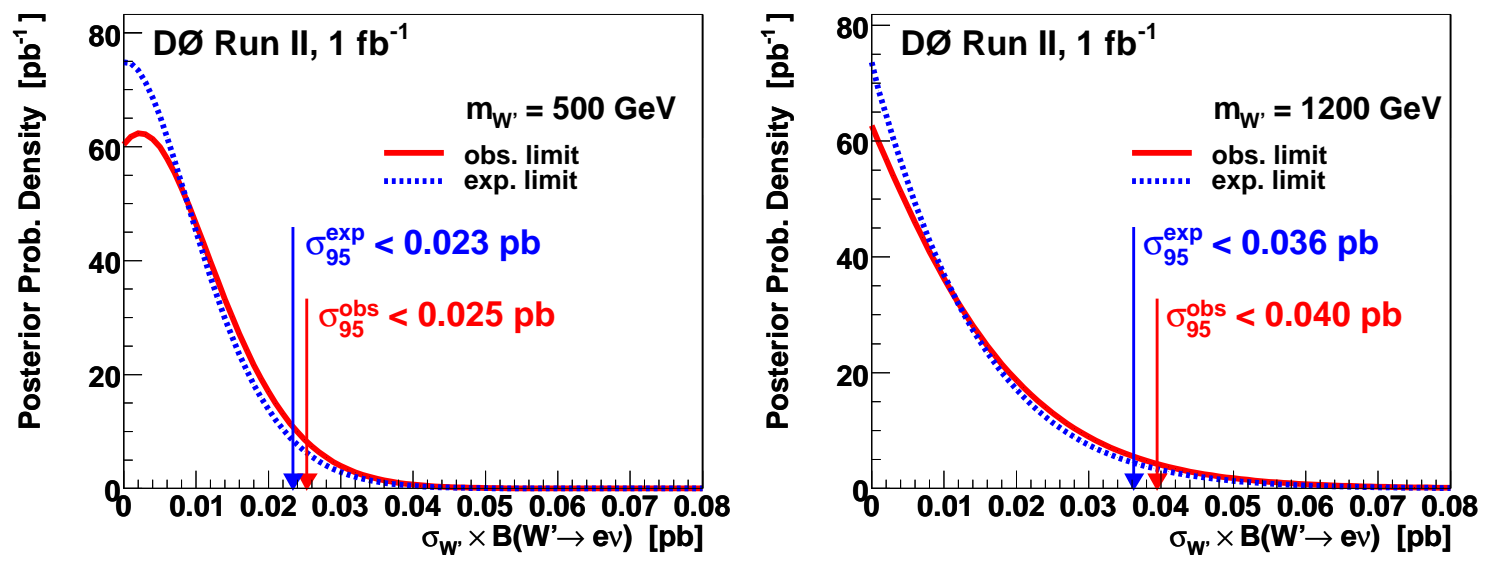
Table 8.9: Limits on the cross section at 95\% CL (see Fig. 8.17).

\begin{tabular}{|c|c|c|c|c|}
\hline$m_{W^{\prime}}[\mathrm{GeV}]$ & \multicolumn{4}{|c|}{ observed (expected) Limit [fb] } \\
& with systematics & \multicolumn{2}{c|}{ without systematics } \\
\hline \hline 500 & 25.4 & $(23.4)$ & 25.3 & $(22.8)$ \\
600 & 18.6 & $(16.3)$ & 18.5 & $(15.6)$ \\
700 & 14.2 & $(12.2)$ & 14.0 & $(11.7)$ \\
800 & 12.6 & $(11.7)$ & 12.4 & $(11.3)$ \\
900 & 12.8 & $(12.3)$ & 13.0 & $(11.9)$ \\
1000 & 16.0 & $(15.8)$ & 16.3 & $(15.1)$ \\
1100 & 24.7 & $(23.5)$ & 23.4 & $(22.1)$ \\
1200 & 39.6 & $(36.3)$ & 37.8 & $(33.2)$ \\
\hline
\end{tabular}

including the uncertainty. The NNLO cross sections are taken from Tab. 5.3. The limit on the mass of the $W^{\prime}$ boson is obtained from the crossover between the lower bound of the theoretical prediction (dashed red line) and the cross section limit curve for the observed limit including systematics. Hence, additional heavy charged gauge bosons with masses below $\sim 1 \mathrm{TeV}$ can be excluded at the $95 \%$ confidence level

$$
m_{W^{\prime}}>1002 \mathrm{GeV} @ 95 \% \mathrm{CL} \text {. }
$$

Fig. 8.17 displays also limits if only statistical uncertainties are taken into account. One can see, that the analysis is limited equally by systematic and statistical uncertainties. This result significantly improves the DØ Run I limit of $800 \mathrm{GeV}$ [33] and the CDF Run II limit of $788 \mathrm{GeV}$ [34], and is the most stringent direct limit to date [164].

The data sample contains several interesting events with extreme high reconstructed transverse masses $m_{T}$. Fig. 8.19 displays a very "clean" data event with a transverse mass of $m_{T}=530 \mathrm{GeV}$. The event properties are summarized in Tab. 8.10. The candidate is compatible with a (SM) $W \rightarrow e \nu$ decay, where the $W$ boson is produced off-shell. In the data 2 events are found with $m_{T}>500 \mathrm{GeV}$, in agreement with an expected number of $2.32 \pm 0.53{ }_{-0.68}^{+0.50}$ background events, see also Tab. 8.8.

The event display shows the tracker with hits (blue points) and reconstructed tracks, and calorimeter towers (64 $\phi$ segments corresponding to $\Delta \phi \approx 0.1$ in the $X Y$ view, $\eta$ is segmented with $\Delta \eta=0.1$ in the $R Z$ view) with energy depositions in the electromagnetic (red) and hadronic layers (blue, not visible). The yellow bar in the $X Y$ view and the lego plot indicates the missing transverse energy $\left(\mathbb{E}_{T}=265 \mathrm{GeV}\right)$. The event contains a well reconstructed electron candidate (small brown bar) with $E_{T}=265 \mathrm{GeV}$. A very straight track $\left(p_{T}=386 \mathrm{GeV}\right)$ is pointing to the electron. According to Eq. 3.9, the uncertainty due to the momentum resolution is $\Delta p_{T} \approx 300 \mathrm{GeV}$, thus compatible with the precise energy measurement. The $\chi^{2}$ probability of the track match including $E / p$ is $P\left(\chi^{2}\right)=0.1$. If only spatial information is taken into account, a much better $\chi^{2}$ probability of the track match is obtained $\left(P\left(\chi_{\text {spatial }}^{2}\right)=0.9\right)$. 
Figure 8.17: Limit on the cross section at 95\% CL as a function of the mass of the $W^{\prime}$ boson. The red curve displays the theoretical expectation with uncertainty. The asterisks and dotted lines show the limit without systematics, whereas the stars and dashed lines show the limit including systematics. The Run I limit is extracted from [33].

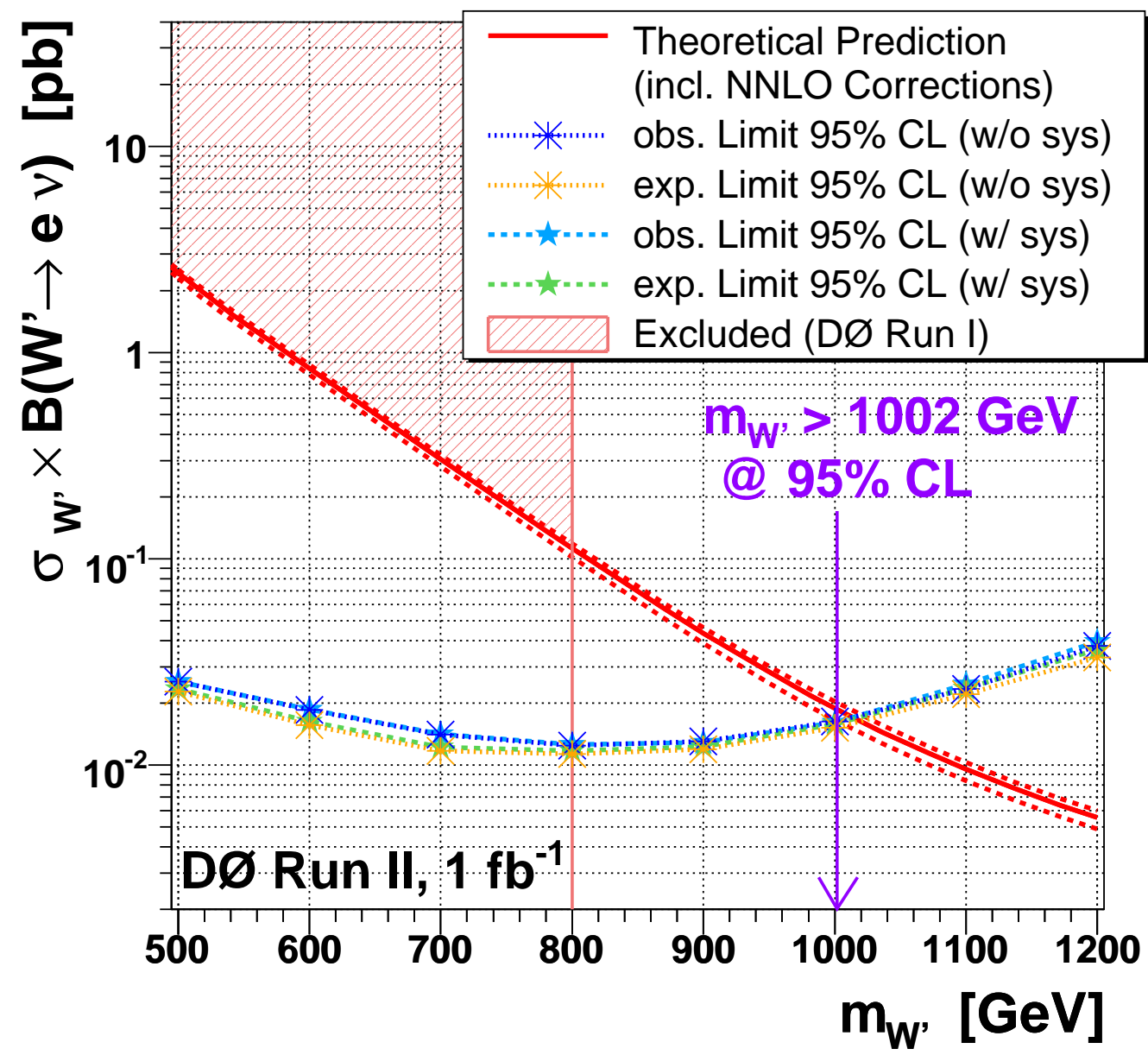


Figure 8.18: Limit on the cross section at $95 \% C L$ as a function of the mass of the $W^{\prime}$ boson, including statistical and systematic errors. The red curve displays the theoretical expectation with uncertainty. The Run I limit is extracted from [33].

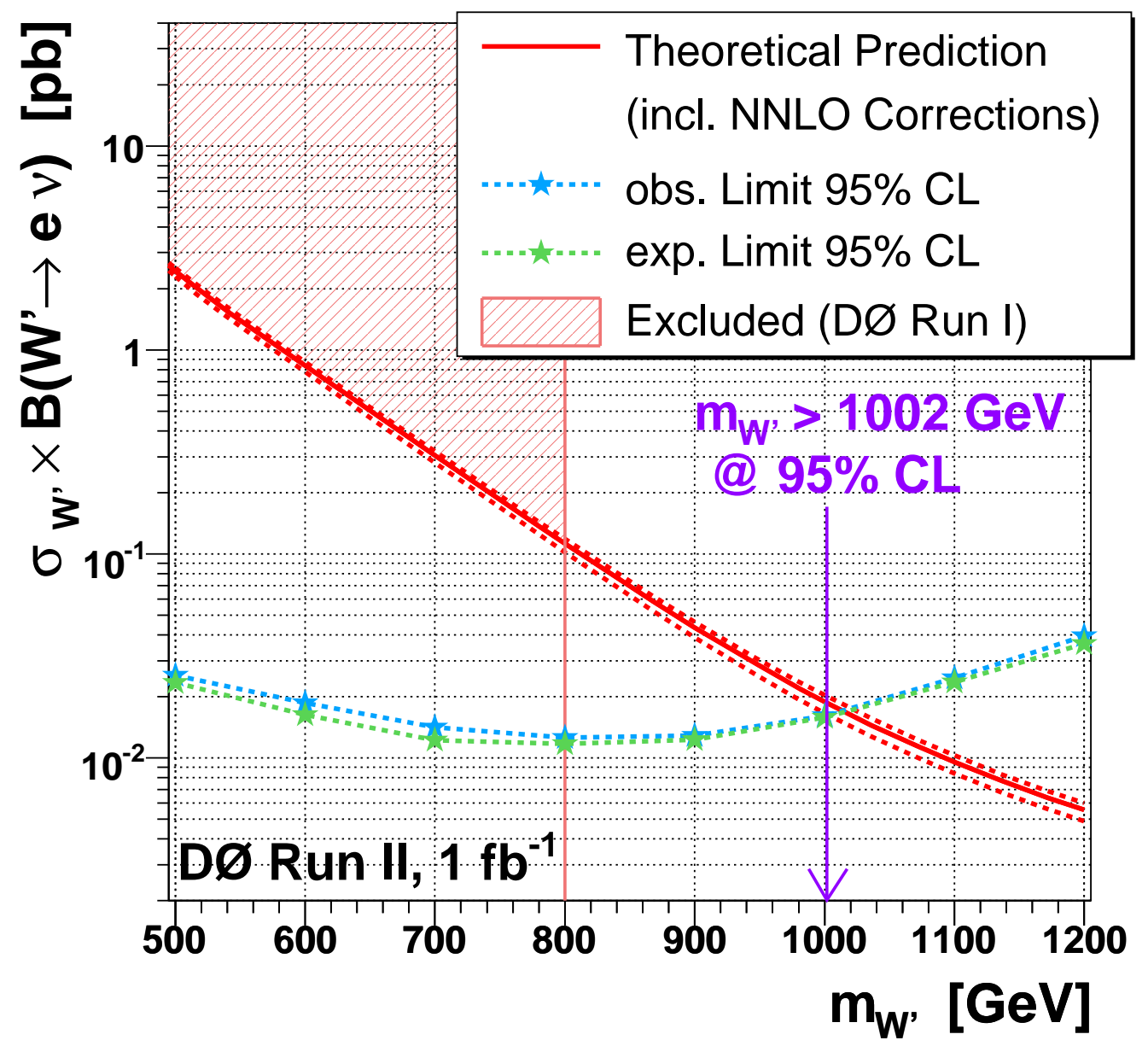


Figure 8.19: Display of an event with high transverse mass: $X Y$ view (top); $R Z$ view (middle); lego plot (bottom).
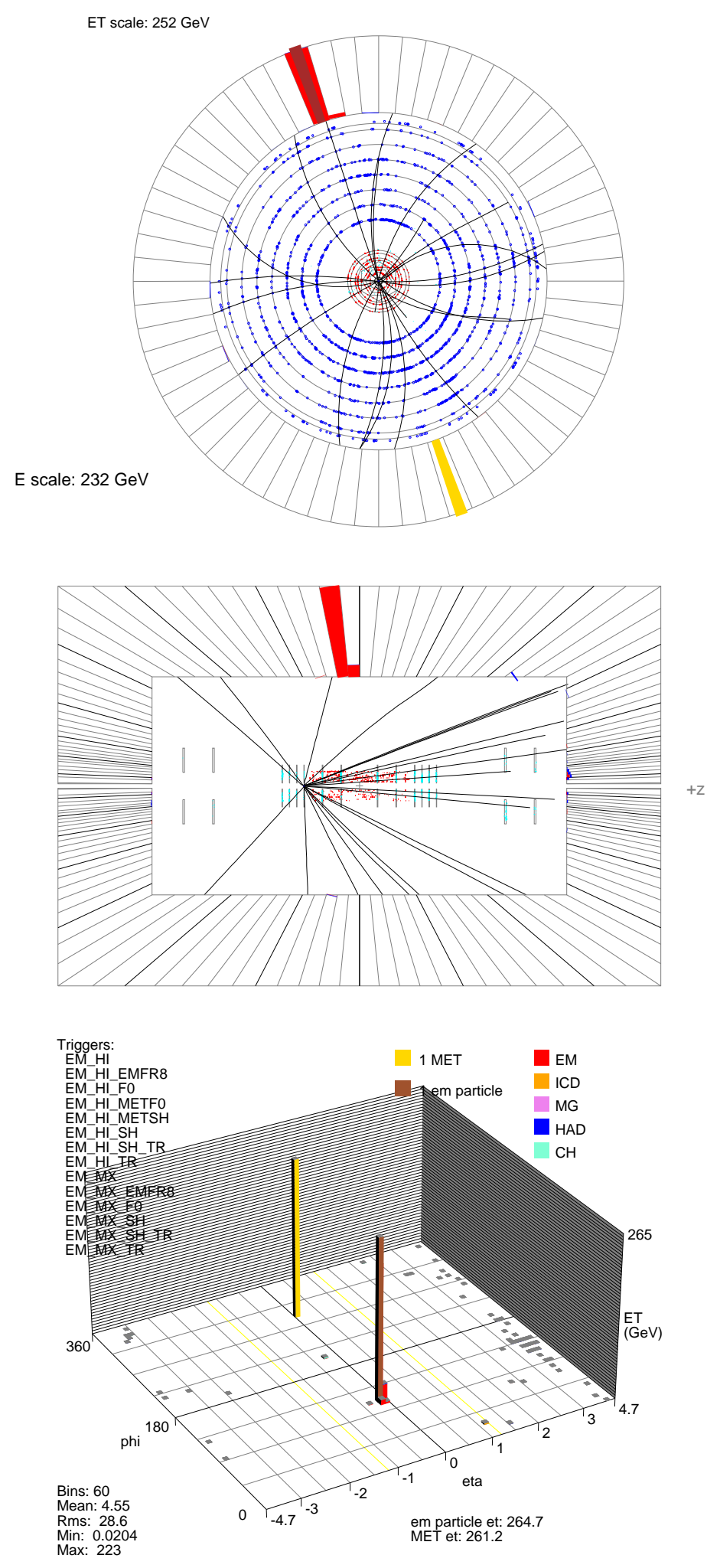
Table 8.10: Properties of the event displayed in Fig. 8.19.

\begin{tabular}{|c|c|}
\hline Variable & Value \\
\hline $\begin{array}{l}\text { Global properties } \\
\text { run number - event number } \\
\text { LBN - tick number } \\
\text { store number } \\
\text { trigger list version } \\
\text { trigger fired } \\
z_{\mathrm{vtx}} \\
n_{\text {tracks }} \text { associated to the vertex }\end{array}$ & $\begin{array}{l}178153-38773035 \\
2437065-10 \\
2692 \\
11.04 \\
\text { all EM_HI and EM_MX } \\
-38.32 \mathrm{~cm} \\
11\end{array}$ \\
\hline $\begin{array}{l}\text { Electron candidate } \\
\left(E, p_{x}, p_{y}, p_{z}\right) \\
E_{T} \\
\chi_{\mathrm{HMx} 7}^{2}, \chi_{\mathrm{HMx} 8}^{2} \\
(\eta, \phi)_{\mathrm{det}} \\
(\eta, \phi) \\
i s o \\
\text { emf } \\
P\left(\chi_{\mathrm{spatial}}^{2}\right) \\
P\left(\chi^{2}\right) \\
\text { electron likelihood } \\
z_{E M} \\
\left|z_{\mathrm{vtx}}-z_{E M}\right|\end{array}$ & $\begin{array}{l}(274,-83,252,69) \mathrm{GeV} \\
265 \mathrm{GeV} \\
0.76,12.1 \\
(-0.13,1.89) \\
(0.26,1.89) \\
0.01 \\
0.99 \\
0.909 \\
0.095 \\
0.998 \\
-38.36 \mathrm{~cm} \\
0.04 \mathrm{~cm}\end{array}$ \\
\hline $\begin{array}{l}\text { Track matched to electron } \\
p_{T} \\
\text { charge } \\
\text { hits in SMT } \\
\text { hits in CFT }\end{array}$ & $\begin{array}{l}386 \mathrm{GeV} \\
+1 \\
8 \\
16\end{array}$ \\
\hline $\begin{array}{l}\text { Jets } \\
n_{\text {jet }}\end{array}$ & 0 \\
\hline $\begin{array}{l}\text { Missing energy } \\
\left(E_{x}, E_{y}\right) \\
\phi^{M E T} \\
E_{T}\end{array}$ & $\begin{array}{l}(85,-251) \mathrm{GeV} \\
5.04 \\
265 \mathrm{GeV}\end{array}$ \\
\hline $\begin{array}{l}\text { Kinematical variables } \\
m_{T} \\
\Delta \phi(\mathrm{EM}, \mathrm{MET}) \\
p_{T}^{E M+M E T} \\
\text { Thrust }\end{array}$ & $\begin{array}{l}530.4 \mathrm{GeV} \\
3.13 \\
2.30 \mathrm{GeV} \\
0.99996\end{array}$ \\
\hline
\end{tabular}




\section{Chapter 9}

\section{Conclusion and Outlook}

Work. Finish. Publish.

Michael Faraday

A search for the production of heavy charged gauge bosons $W^{\prime}$ and the subsequent decay into electron and neutrino has been performed. The new bosons are predicted in many extensions to the Standard Model. In this analysis a general approach (Reference Model, Altarelli et al.) is considered, where the $W^{\prime}$ boson appears as a heavy copy of the Standard Model $W$ boson. It is assumed that the couplings of the $W^{\prime}$ boson to quarks, leptons and gauge bosons of the electroweak interaction are identical to the Standard Model couplings, and that new decay channels (like $W^{\prime} \rightarrow W Z$ ) are suppressed. These assumptions are realized within the Manifest Left-Right Symmetric Model if the $W^{\prime}$ boson is right-handed, and the right-handed neutrinos emerging from the decay are light.

Proton-antiproton collisions at a center-of-mass energy of $\sqrt{s}=1.96 \mathrm{TeV}$ recorded with the $\mathrm{D} \varnothing$ detector have been analyzed. The entire Run II dataset corresponding to an integrated luminosity of about $1 \mathrm{fb}^{-1}$ is investigated for deviations from the Standard Model prediction, but no significant excess is found. Moreover, an excellent agreement between data and background expectation can be stated. The tail of the transverse mass spectrum reconstructed from the electron and the missing transverse energy ( $\sim$ neutrino) is consulted for the statistical interpretation. Since the signal distributions strongly depend on the mass $m_{W^{\prime}}$ of the new gauge boson, the information from the entire distribution is used as input for a binned likelihood. Limits on the production cross section times branching fraction, $\sigma_{W^{\prime}} \times \operatorname{Br}\left(W^{\prime} \rightarrow e \nu\right)$, are of the order of $10-40 \mathrm{fb}$. Comparing the observed limit on the cross section with the theoretical prediction from the Reference Model, additional charged gauge bosons with mass below $1002 \mathrm{GeV}$ can be excluded at the $95 \%$ confidence level. This result significantly improves previous direct limits, and is the most stringent to date.

The current experimental results (including the result of this analysis) do not contradict the predictions of the Standard Model. But with the increasing amount of data taken at the Tevatron collider and with the upcoming collider generation, physics beyond the Standard Model will hopefully come into the experimenters' reach. 
Recently, the production of additional charged gauge bosons in proton-proton collisions at the LHC has been studied using the full CMS detector simulation [165]. Assuming the Reference Model, it will be possible to discover $W^{\prime}$ bosons in the muon + missing transverse energy final state up to masses of $m_{W^{\prime}}=5.5 \mathrm{TeV}$ with a luminosity of $100 \mathrm{fb}^{-1}$, see Fig. 9.1. This amount of data can be taken within one year of LHC operation at the design instantaneous luminosity of $10^{34} \mathrm{~cm}^{-2} \mathrm{~s}^{-1}$.

Figure 9.1: $C M S$ study of the process $W^{\prime} \rightarrow \mu \nu$ in proton-proton collisions at the LHC. The plot on the left-hand side shows the reconstructed transverse mass from the muon and missing transverse energy. The right-hand plot displays the discovery limit as a function of the luminosity and the $W^{\prime}$ mass. In this analysis the Reference Model is considered [165].
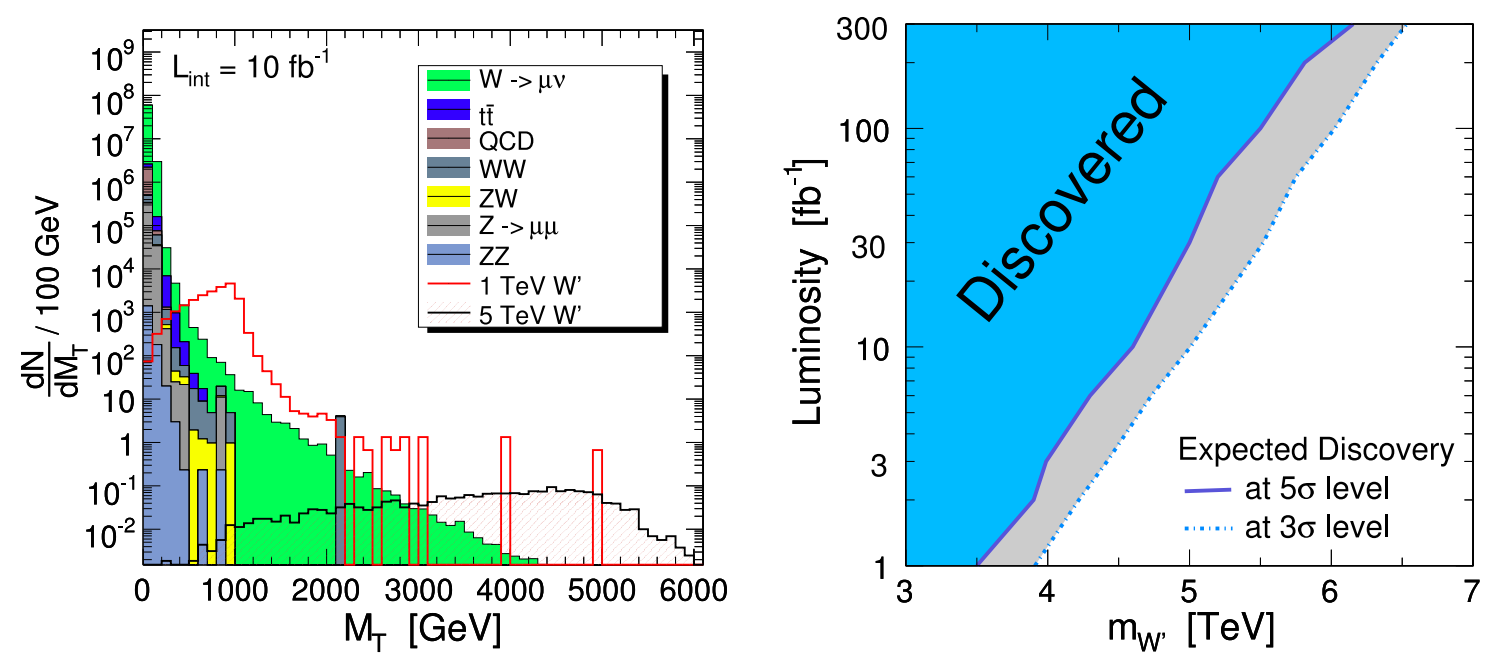


\section{Appendix A}

\section{Abbreviations}

ACNET Accelerator Controls NETwork

ADC Analog to Digital Converter

AFE Analog Front End (board)

BLS BaseLine Subtractor (card; board)

CAF Common Analysis Format

CAFE Common Analysis Format Environment

CC Central Calorimeter

CDF Collider Detector at Fermilab

CERN Conseil Européen pour la Recherche Nucléaire, now: Organisation Européenne pour la Recherche Nucléaire (European Organization for Nuclear Research), Geneva, Switzerland

CFT Central Fiber Tracker

CH Coarse Hadronic (layers of the calorimeter)

CKM Cabbibo-Kobayashi-Maskawa matrix

CL Confidence Level

COOR DØ Run Control system

CPS Central PreShower detector

CSG Common Samples Group

CTEQ Coordinated Theoretical-Experimental Project on QCD

CTT Central Track Trigger

DAQ Data AcQuisition system

DCA Distance of Closest Approach 
DESY Deutsches Elektronen-SYnchroton, Hamburg, Germany

DGLAP Dokshitzer, Gribov, Lipatov, Altarelli and Parisi equations

DLCAT Data Logger event CATataloguer

DLSAM Data Logger SAM interface

DØOM DØ Object Model

EC Endcap Calorimeter

ECN Endcap Calorimeter North

ECS Endcap Calorimeter South

EDM Event Data Model

EM ElectroMagnetic (interaction; layers of the calorimeter)

EMID ElectroMagnetic objects (electron and photon) IDentification

EPICS Experimental Physics and Industrial Control System

EW ElectroWeak (interaction; theory)

FEB Front End Busy

FH Fine Hadronic (layers of the calorimeter)

FNAL Fermi National Accelerator Laboratory, Fermilab, Batavia, Illinois/USA

FPD Forward Proton Detector

FPS Forward PreShower detector

HERA Hadron-Elektron-Ring-Anlage (1992 - 2007), DESY

HFS Hadronic Final State

I/O Input/Output

ICD InterCryostatic Detector

IOC Input/Output Controller

JES Jet Energy Scale

LBN Luminosity Block Number

LEP Large Electron Positron collider (1989 - 2000), CERN

LHC Large Hadron Collider (under construction), CERN

LO Leading Order

MC Monte Carlo 
MDT Mini Drift Tube

MET Missing $\mathbf{E}_{\mathbf{T}}$ (missing transverse energy, $\mathbb{E}_{T}$ )

MNS Maki-Nakagawa-Sakata matrix

MSSM Minimal Supersymmetric Standard Model

N(N)LO Next-to-(Next-to-)Leading Order

PDF Parton Distribution (Density) Function

PDG Particle Data Group

PDT Proportional Drift Tube

QCD Quantum ChromoDynamics

RDC Raw Data Chunk

RF Radio Frequency

RGE Renormalization Group Equation

SAM Sequential Access via Metadata

SBC Single Board Computer

SCA Switched Capacitor Array

SCL Serial Command Link

SDAQ Secondary Data AcQuisition system

SES Significant Event System

SI Système International d'Unités (International System of Units)

SM Standard Model

SMT Silicon Microstrip Tracker

STT Silicon Track Trigger

TFW Trigger FrameWork

VLPC Visible Light Photon Counter

VME VERSAModule Eurocard 



\section{Appendix B}

\section{Rapidity and Pseudorapidity}

The transformation from cartesian coordinates $\{x, y, z\}$ to spherical coordinates $\{r, \theta, \phi\}$ is as follows

$$
\left(\begin{array}{l}
x \\
y \\
z
\end{array}\right)=\left(\begin{array}{c}
r \cos \phi \sin \theta \\
r \sin \phi \sin \theta \\
r \cos \theta
\end{array}\right)
$$

Partons (sea quarks, valence quarks, gluons) which are the constituents of hadrons are involved in the collisions at a hadron collider. They carry different fractions of the momentum of the hadron so that the center-of-mass system of the partons is boosted in the direction of the beam ( $z$-axis) in the laboratory frame.

Thus, the polar angle $\theta$ is not suitable to describe the system because polar angle distributions are not invariant under Lorentz transformations along the beam direction. The rapidity is used instead because the shape of the distribution is invariant under such Lorentz transformations. The rapidity $y$ is calculated from the momentum in $z$-direction $p_{z}$ and the energy $E$ via

$$
y \equiv \frac{1}{2} \ln \left(\frac{E+p_{z}}{E-p_{z}}\right)=\operatorname{artanh} \frac{p_{z}}{E}
$$

The artanh (area hyperbolic tangens) is the inverse function of the tanh (hyperbolic tangens). The last part of Eq. B.2 stems from

$$
\tanh y=\frac{e^{y}-e^{-y}}{e^{y}+e^{-y}}=\frac{\sqrt{\frac{E+p_{z}}{E-p_{z}}}-\sqrt{\frac{E-p_{z}}{E+p_{z}}}}{\sqrt{\frac{E+p_{z}}{E-p_{z}}}+\sqrt{\frac{E-p_{z}}{E+p_{z}}}}=\frac{\left(E+p_{z}\right)-\left(E-p_{z}\right)}{\left(E+p_{z}\right)+\left(E-p_{z}\right)}=\frac{p_{z}}{E}
$$

In the following, a boost along the $z$-axis is performed in order to prove the invariance of the rapidity distribution under Lorentz transformations

$$
\left(\begin{array}{c}
E^{\prime} \\
p_{x}^{\prime} \\
p_{y}^{\prime} \\
p_{z}^{\prime}
\end{array}\right)=\left(\begin{array}{cccc}
\gamma & 0 & 0 & -\beta \gamma \\
0 & 1 & 0 & 0 \\
0 & 0 & 1 & 0 \\
-\beta \gamma & 0 & 0 & \gamma
\end{array}\right)\left(\begin{array}{c}
E \\
p_{x} \\
p_{y} \\
p_{z}
\end{array}\right)
$$


The transverse momentum is invariant under the Lorentz transformation

$$
\vec{p}_{T}^{\prime}=\left(\begin{array}{c}
p_{x}^{\prime} \\
p_{y}^{\prime} \\
0
\end{array}\right)=\left(\begin{array}{c}
p_{x} \\
p_{y} \\
0
\end{array}\right)=\vec{p}_{T} .
$$

The boost affects the rapidity as follows

$$
\begin{aligned}
y^{\prime} & =\frac{1}{2} \ln \left(\frac{E^{\prime}+p_{z}^{\prime}}{E^{\prime}-p_{z}^{\prime}}\right)=\frac{1}{2} \ln \left(\frac{\gamma\left(E-\beta p_{z}-\beta E+p_{z}\right)}{\gamma\left(E-\beta p_{z}+\beta E-p_{z}\right)}\right) \\
& =\frac{1}{2} \ln \left(\frac{\left(E+p_{z}\right)(1-\beta)}{\left(E-p_{z}\right)(1+\beta)}\right)=\frac{1}{2} \ln \left(\frac{E+p_{z}}{E-p_{z}}\right)+\frac{1}{2} \ln \left(\frac{1-\beta}{1+\beta}\right) \\
& =y-\frac{1}{2} \ln \left(\frac{1+\beta}{1-\beta}\right)=y-\operatorname{artanh} \beta .
\end{aligned}
$$

The rapidity distribution is invariant under a boost in $z$-direction because of $\mathrm{d} y^{\prime}=\mathrm{d} y$

$$
\frac{\mathrm{d} N}{\mathrm{~d} y}=\frac{\mathrm{d} N}{\mathrm{~d} y^{\prime}}
$$

The rapidity itself is not invariant $\left(y^{\prime}=y-\operatorname{artanh} \beta\right)$.

In the limit of high energies $\left(p^{2} \gg m^{2} \Rightarrow E^{2}=p^{2}\right)$ one can rewrite the rapidity using $p_{z}=p \cos \theta=E \cos \theta$

$$
\begin{aligned}
y & =\frac{1}{2} \ln \left(\frac{E(1+\cos \theta)}{E(1-\cos \theta)}\right)=-\frac{1}{2} \ln \left(\frac{1-\cos \theta}{1+\cos \theta}\right)=-\frac{1}{2} \ln \left(\frac{1-\cos ^{2} \theta}{(1+\cos \theta)^{2}}\right) \\
& =-\ln \left(\frac{\sin \theta}{1+\cos \theta}\right)=-\ln \left(\frac{2 \sin \frac{\theta}{2} \cos \frac{\theta}{2}}{2 \cos ^{2} \frac{\theta}{2}}\right) \\
& =-\ln \tan \frac{\theta}{2} .
\end{aligned}
$$

This defines the pseudorapidity

$$
\eta \equiv-\ln \tan \frac{\theta}{2}
$$

The pseudorapidity corresponds to the rapidity if the energy is much higher than the rest mass. In this limit the shape of the pseudorapidity distribution is invariant under a Lorentz transformation along the direction of the beam (here: $z$-direction).

Table B.1: Comparison between $\theta$ and $\eta$ values.

\begin{tabular}{|c||c|c|c|c|c|c|c|}
\hline$\theta\left[^{\circ}\right]$ & 0 & 10 & 36.8 & 40.4 & 45 & 90 & 180 \\
\hline$\eta$ & $\infty$ & 2.44 & 1.1 & 1 & 0.88 & 0 & $-\infty$ \\
\hline
\end{tabular}




\section{Appendix C}

\section{Trigger Details}

The following tables summarize the individual Level $1 / 2 / 3$ trigger requirements for the set of inclusive electron triggers [143] used in the analysis. Note, that the same trigger name can have different trigger requirements in different trigger list versions (e.g. E1_SHT20 in trigger list version 12 and 13). The symbol "\%" denotes a wildcard character, e.g. EM_HI_\% stands for EM_HI, EM_HI_EMFR8 or EM_HI_SH.

Table C.1: Level 1 trigger requirements.

\begin{tabular}{|c|c|c|}
\hline Version & Triggername & Level 1 \\
\hline $8-11$ & $\begin{array}{l}\text { EM_HI_\% } \\
\text { EM_MX_\% }\end{array}$ & $\begin{array}{l}\text { one EM trigger tower with } E_{T}>10 \mathrm{GeV} \\
\text { one EM trigger tower with } E_{T}>15 \mathrm{GeV}\end{array}$ \\
\hline $12 / 13$ & $\begin{array}{l}\text { E1_\% } \\
\text { E2_\% } \\
\text { E3_\% }\end{array}$ & $\begin{array}{l}\text { one EM trigger tower with } E_{T}>11 \mathrm{GeV} \\
\text { two EM trigger towers with } E_{T}>6 \mathrm{GeV} \\
\text { one EM trigger tower with } E_{T}>9 \mathrm{GeV} \text { and } \\
\text { two EM trigger towers with } E_{T}>3 \mathrm{GeV}\end{array}$ \\
\hline 13 & E4_\% & one EM trigger tower with $E_{T}>11 \mathrm{GeV}$ \\
\hline 14 & $\begin{array}{l}\text { E1_\%, E3_\% } \\
\text { E4_\% } \\
\text { 2CEM12_\% } \\
\text { 2CEM6_\% }\end{array}$ & $\begin{array}{l}\text { one EM trigger tower with } E_{T}>12 \mathrm{GeV} \\
\text { two EM trigger towers with } E_{T}>6 \mathrm{GeV} \\
\text { one EM trigger tower with } E_{T}>12 \mathrm{GeV} \\
\text { two EM trigger towers with } E_{T}>6 \mathrm{GeV}\end{array}$ \\
\hline
\end{tabular}


Table C.2: Level 2 trigger requirements.

\begin{tabular}{|l|l|l|}
\hline Version & Triggername & Level 2 \\
\hline \hline $8 / 9$ & EM_HI_\%, EM_MX_\% & none \\
\hline $10 / 11$ & $\begin{array}{l}\text { EM_HI_\% } \\
\text { EM_MX_\% }\end{array}$ & $\begin{array}{l}\text { one EM object with } E_{T}>12 \mathrm{GeV} \\
\text { none }\end{array}$ \\
\hline 12 & E1_\%, E2_\%, E3_\% & none \\
\hline 13 & $\begin{array}{l}\text { E1_\%, E2_\%, E3_\% } \\
\text { E4_\% }\end{array}$ & $\begin{array}{l}\text { one EM cluster with } E_{T}>15 \mathrm{GeV} \\
\text { one isolated single tower EM object with } E_{T}>11 \mathrm{GeV}\end{array}$ \\
\hline 14 & $\begin{array}{l}\text { E1_\%,E4_\% } \\
\text { E3_\% } \\
2 \text { CEM\% }\end{array}$ & $\begin{array}{l}\text { one EM cluster with } E_{T}>15 \mathrm{GeV} \\
\text { one isolated single tower EM object with } E_{T}>11 \mathrm{GeV} \\
\text { one EM cluster with } E_{T}>15 \mathrm{GeV}\end{array}$ \\
\hline
\end{tabular}

Table C.3: Level 3 trigger requirements ( $x$ denotes the trigger threshold in $G e V$ ).

\begin{tabular}{|c|c|c|}
\hline Version & Triggername & Level 3 \\
\hline $8-11$ & $\begin{array}{l}\text { EM_HI, EM_MX } \\
\text { EM_\%_EMFR8 } \\
\text { EM_\%_SH }\end{array}$ & $\begin{array}{l}\text { one loose electron with } E_{T}>30 \mathrm{GeV} \\
\text { one very loose electron with } E_{T}>40 \mathrm{GeV} \\
\text { one electron with } E_{T}>20 \mathrm{GeV} \text { satisfying } \\
\text { shower shape requirements }\end{array}$ \\
\hline $12-14$ & $\begin{array}{l}\text { \%_SHT } x \\
\% \_\mathrm{SH} x \\
\text { E1_L } x \\
\text { E1_VL70 } \\
\text { E1_NC90 }\end{array}$ & $\begin{array}{l}\text { one electron with } E_{T}>x \mathrm{GeV} \text { satisfying tight } \\
\text { shower shape requirements } \\
\text { one electron with } E_{T}>x \mathrm{GeV} \text { satisfying loose } \\
\text { shower shape requirements } \\
\text { one loose electron with } E_{T}>x \mathrm{GeV} \\
\text { one very loose electron with } E_{T}>70 \mathrm{GeV} \\
\text { one electron with } E_{T}>90 \mathrm{GeV} \text { without any requirement }\end{array}$ \\
\hline
\end{tabular}




\section{Appendix D}

\section{Modified $W^{\prime}$ Signal}

In this section the cut on the generator mass of the $W^{\prime}$ signal Monte Carlo Eq. 5.5 introduced in Sec. 5.3 is applied. Tabs. D.2 and D.3 show efficiencies and inefficiencies for the various cuts (cf. Tabs. 6.4 and 6.5). All signal samples exhibit an overall cut efficiency of $56 \%$. Major inefficiencies are introduced by the constraint on the centrality $\left(\left|\eta_{\text {det }}\right|<1.1\right)$ and the electron reconstruction.

A comparison between the Monte Carlo samples with and without the cut on the generator mass is shown in Fig. D.1. One can clearly see the impact of the cut, especially at samples with higher $W^{\prime}$ masses. The pronounced Jacobian peak in the samples with the generator cut applied comes along with a significant reduction of event numbers (factor 3.5 for $m_{W^{\prime}}=1100 \mathrm{GeV}$ ). In Tab. D.4 a breakdown of the individual contributions of the various background processes is given, including expected numbers of signal events. The tables Tab. D.5 - D.8 summarize event numbers for higher cuts on the transverse mass.

The 95\% confidence level limit on the production cross section is shown in Figs. D.2 and D.3; the numbers are summarized in Tab. D.1. From this, additional heavy charged gauge bosons with masses below $991 \mathrm{GeV}$ can be excluded

$$
m_{W^{\prime}}>991 \mathrm{GeV} @ 95 \% \mathrm{CL} \text {. }
$$

This limit is very close to the limit of $1002 \mathrm{GeV}$ derived from the whole spectrum, see Eq. 8.17 .

Table D.1: Limits on the cross section at 95\% CL (see Fig. D.2).

\begin{tabular}{|c|c|c|c|c|}
\hline$m_{W^{\prime}}[\mathrm{GeV}]$ & \multicolumn{4}{|c|}{ observed (expected) Limit [fb] } \\
& with systematics & without systematics \\
\hline \hline 500 & 23.1 & $(21.5)$ & 23.2 & $(20.9)$ \\
600 & 16.7 & $(14.5)$ & 16.5 & $(13.9)$ \\
700 & 11.8 & $(10.2)$ & 11.7 & $(9.8)$ \\
800 & 9.2 & $(8.8)$ & 9.1 & $(8.5)$ \\
900 & 8.0 & $(7.7)$ & 7.9 & $(7.5)$ \\
1000 & 7.2 & $(7.1)$ & 7.2 & $(6.9)$ \\
1100 & 7.0 & $(7.0)$ & 6.6 & $(6.7)$ \\
1200 & 6.6 & $(6.8)$ & 6.4 & $(6.4)$ \\
\hline
\end{tabular}


Table D.2: Efficiencies (in \%) for the selection criteria for the signal Monte Carlo samples (with generator mass cut applied).

\begin{tabular}{|l|r|r|r|r|r|r|r|r|}
\hline$m_{W^{\prime}}(\mathrm{GeV})$ & 500 & 600 & 700 & 800 & 900 & 1000 & 1100 & 1200 \\
\hline \hline no cut & 100.0 & 100.0 & 100.0 & 100.0 & 100.0 & 100.0 & 100.0 & 100.0 \\
$+|I D|=10$ or 11 & 93.1 & 92.2 & 92.8 & 93.1 & 92.7 & 92.8 & 93.0 & 91.3 \\
$+E_{T}>30 \mathrm{GeV}$ & 88.6 & 88.3 & 89.4 & 89.3 & 89.1 & 89.4 & 89.5 & 88.6 \\
$+\left|\eta_{\text {det }}\right|<1.1$ & 69.9 & 68.9 & 71.7 & 71.4 & 71.5 & 72.2 & 69.8 & 71.3 \\
$+i s o<0.2$ & 69.9 & 68.9 & 71.7 & 71.4 & 71.5 & 72.2 & 69.8 & 71.3 \\
+ emf $>0.9$ & 69.1 & 68.4 & 71.3 & 71.0 & 70.7 & 71.5 & 69.3 & 70.4 \\
$+\chi_{\text {HMx }}^{2}<12$ & 66.5 & 64.6 & 68.2 & 67.1 & 66.6 & 68.2 & 65.6 & 68.0 \\
$+P\left(\chi_{\text {spatial }}^{2}>0.01\right.$ & 63.4 & 62.0 & 65.0 & 64.4 & 64.1 & 65.7 & 63.4 & 63.2 \\
\hline$+E_{T}>30 \mathrm{GeV}$ & 63.4 & 62.0 & 65.0 & 64.4 & 64.1 & 65.7 & 63.4 & 63.2 \\
\hline$+\left|z_{\text {vtx }}\right|<60 \mathrm{~cm}$ & 62.4 & 61.2 & 64.0 & 63.6 & 63.3 & 64.4 & 62.0 & 61.7 \\
$+n_{\text {tracks }} \geq 3$ & 61.3 & 60.0 & 62.9 & 62.0 & 61.8 & 62.2 & 60.2 & 59.0 \\
\hline$+\left|z_{\text {vtx }}-z_{E M}\right|<5 \mathrm{~cm}$ & 60.4 & 58.9 & 62.1 & 61.5 & 60.9 & 60.4 & 58.5 & 57.8 \\
$+0.6<E_{T} / E_{T}<1.4$ & 59.6 & 58.2 & 62.0 & 61.2 & 60.7 & 60.2 & 58.5 & 57.5 \\
+ jet veto & $\mathbf{5 6 . 1}$ & $\mathbf{5 5 . 0}$ & $\mathbf{5 9 . 0}$ & $\mathbf{5 7 . 8}$ & $\mathbf{5 7 . 4}$ & $\mathbf{5 7 . 8}$ & $\mathbf{5 6 . 3}$ & $\mathbf{5 5 . 7}$ \\
\hline$+m_{T}>140 \mathrm{GeV}$ & $\mathbf{5 6 . 1}$ & $\mathbf{5 5 . 0}$ & $\mathbf{5 9 . 0}$ & $\mathbf{5 7 . 8}$ & $\mathbf{5 7 . 4}$ & $\mathbf{5 7 . 8}$ & $\mathbf{5 6 . 3}$ & $\mathbf{5 5 . 7}$ \\
\hline
\end{tabular}

Table D.3: Inefficiencies (in \%) for the individual selection criteria of the search sample. All selection criteria are applied except for the one under investigation (signal samples with generator mass cut applied).

\begin{tabular}{|c|c|c|c|c|c|c|c|c|}
\hline$m_{W^{\prime}}(\mathrm{GeV})$ & 500 & 600 & 700 & 800 & 900 & 1000 & 1100 & 1200 \\
\hline $\begin{array}{l}\quad|I D|=10 \text { or } 11 \\
+E_{T}>30 \mathrm{GeV} \\
+i s o<0.2 \\
+e m f>0.9 \\
+\chi_{\mathrm{HMx} 7}^{2}<12 \\
+P\left(\chi_{\text {spatial }}^{2}\right)>0.01 \\
\left|\eta_{\text {det }}\right|<1.1\end{array}$ & 14.1 & 15.4 & 14.0 & 15.1 & 15.3 & 15.4 & 16.9 & 18.4 \\
\hline$E_{T}>30 \mathrm{GeV}$ & 0.0 & 0.0 & 0.0 & 0.0 & 0.0 & 0.0 & 0.0 & 0.0 \\
\hline$\left|z_{\mathrm{vtx}}\right|<60 \mathrm{~cm}$ & 1.3 & 0.9 & 1.2 & 1.1 & 0.8 & 1.6 & 2.1 & 1.6 \\
\hline$n_{\text {tracks }} \geq 3$ & 1.6 & 1.8 & 1.4 & 2.3 & 2.1 & 3.1 & 2.9 & 3.6 \\
\hline$\left|z_{\mathrm{vtx}}-z_{E M}\right|<5 \mathrm{~cm}$ & 1.6 & 1.8 & 1.4 & 1.0 & 1.5 & 3.0 & 2.7 & 2.1 \\
\hline $0.6<E_{T} / E_{T}<1.4$ & 0.5 & 0.5 & 0.2 & 0.2 & 0.3 & 0.1 & 0.0 & 0.5 \\
\hline jet veto & 5.9 & 5.5 & 4.9 & 5.6 & 5.4 & 4.1 & 3.8 & 3.1 \\
\hline$m_{T}>140 \mathrm{GeV}$ & 0.1 & 0.0 & 0.0 & 0.0 & 0.0 & 0.0 & 0.0 & 0.0 \\
\hline$\left|\eta_{\text {det }}\right|<1.1 \& m_{T}>140 \mathrm{GeV}$ & 17.0 & 17.8 & 15.6 & 16.4 & 16.2 & 16.0 & 17.7 & 18.4 \\
\hline
\end{tabular}


Figure D.1: Comparison between the two scenarios of the signal Monte Carlo after the cut on the transverse mass $m_{T}>140 \mathrm{GeV}$ (red hatched: no generator mass cut applied, blue: cut on the generator mass is applied).
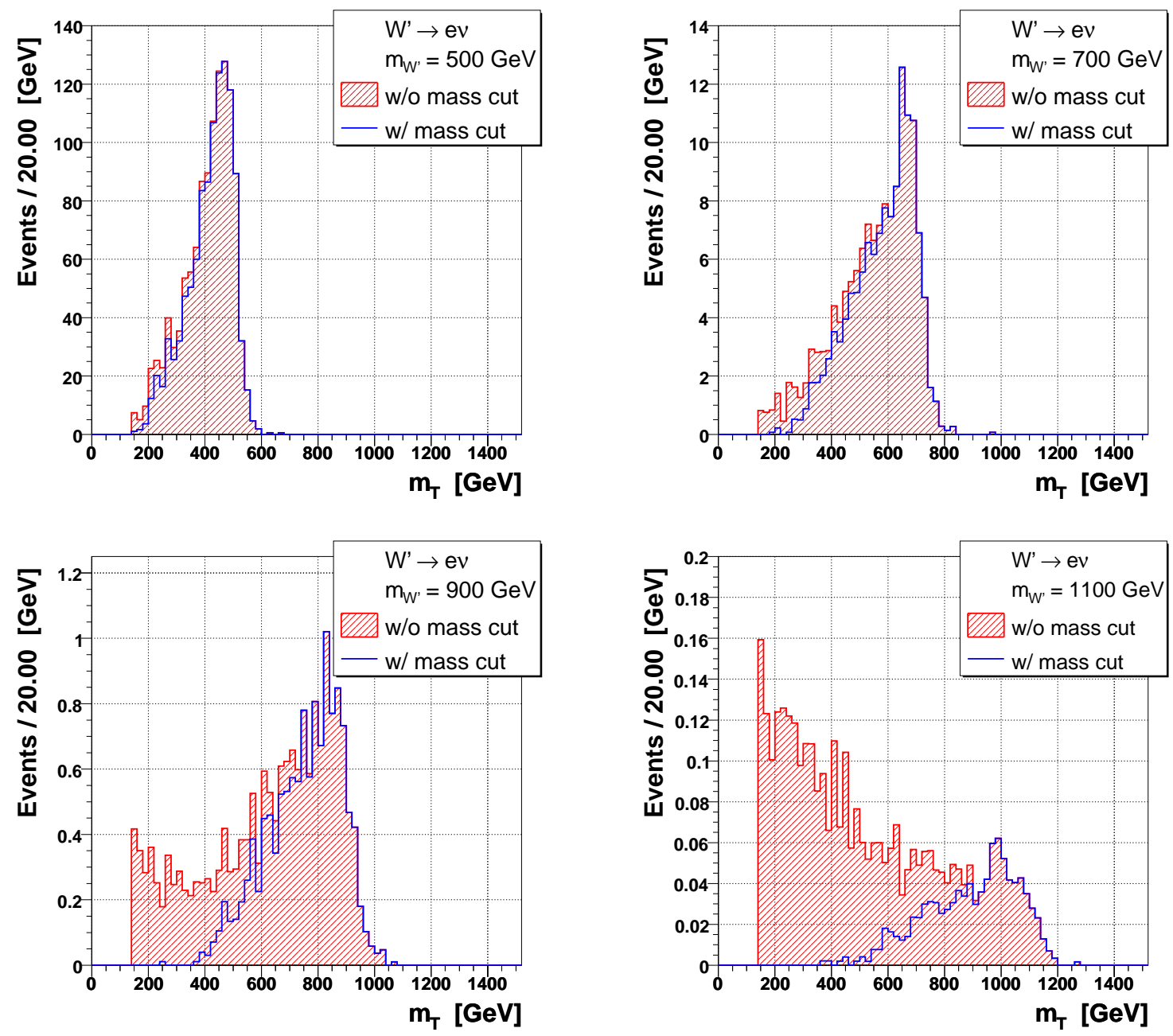
Figure D.2: Limit on the cross section at 95\% CL as a function of the mass of the $W^{\prime}$ boson. The red curve displays the theoretical expectation with uncertainty. The asterisks and dotted lines show the limit without systematics, whereas the stars and dashed lines show the limit including systematics. The Run I limit is extracted from [33].

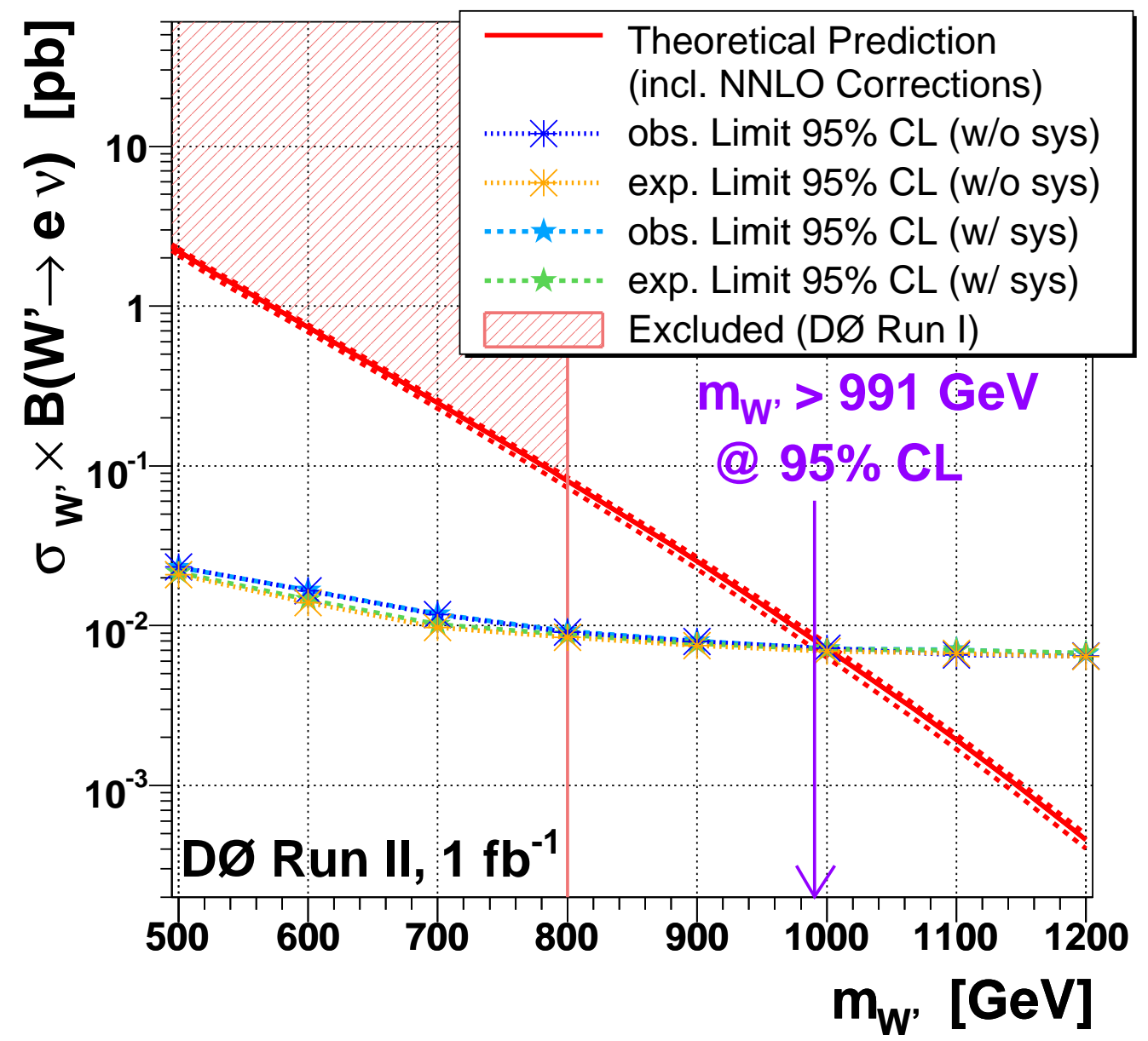


Figure D.3: Limit on the cross section at 95\% CL as a function of the mass of the $W^{\prime}$ boson, including statistical and systematic errors. The red curve displays the theoretical expectation with uncertainty. The Run I limit is extracted from [33].

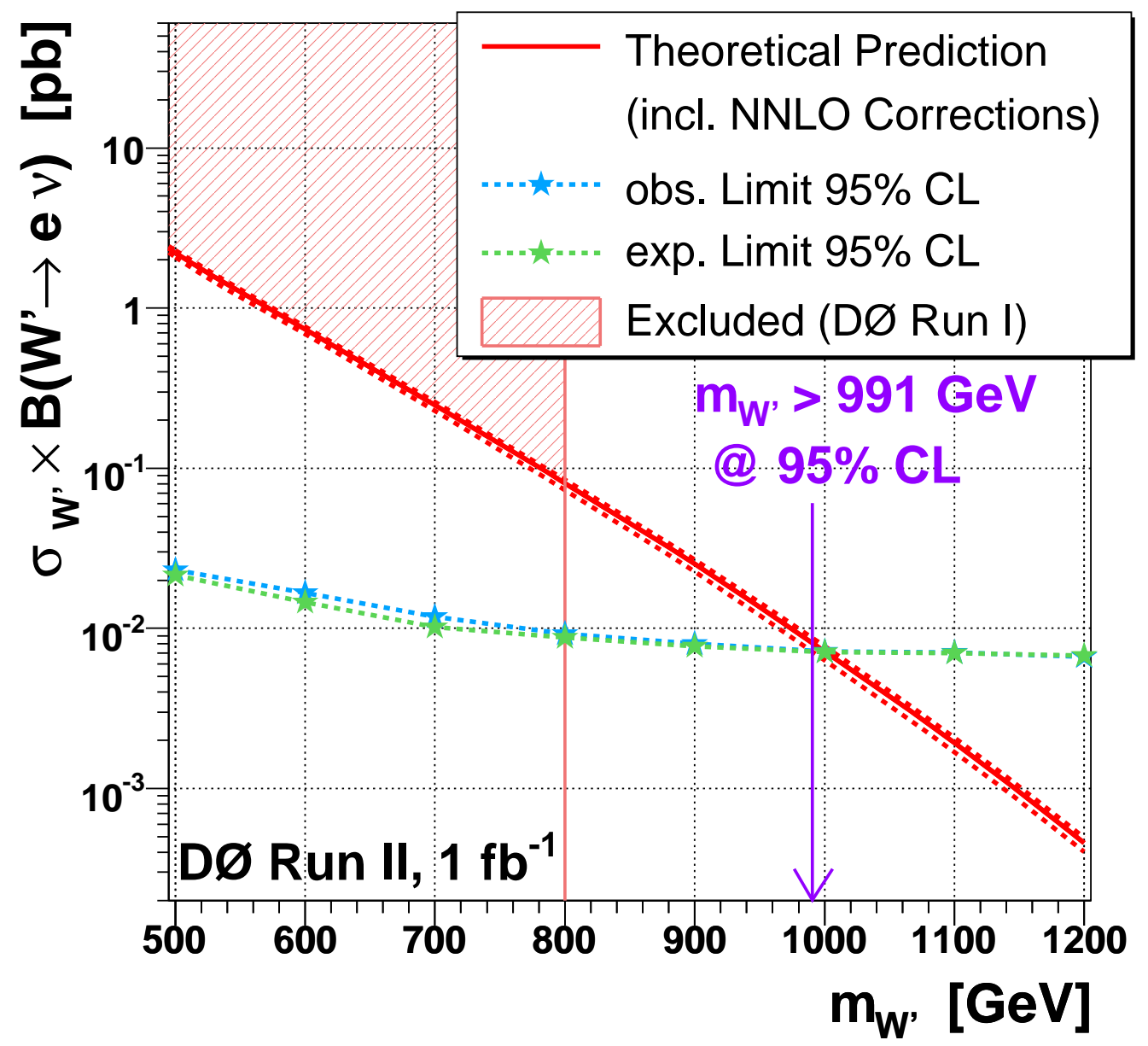


Table D.4: Events in the search sample for transverse masses $m_{T}>140 \mathrm{GeV}$.

\begin{tabular}{|l|r|r|r|r|}
\hline Process & Events & \multicolumn{3}{|c|}{ Uncertainty } \\
& & statistical & \multicolumn{2}{|c|}{ systematic } \\
& & & $(+)$ & $(-)$ \\
\hline \hline Data & $\mathbf{9 6 7}$ & & & \\
\hline Sum Backgrounds & 959 & 21 & 89 & 92 \\
\hline$W \rightarrow e \nu$ & 875 & 20 & 89 & 92 \\
$W \rightarrow \tau \nu$ & 20.8 & 3.0 & 2.5 & 3.5 \\
$Z \rightarrow e e$ & 0.28 & 0.17 & 0.04 & 0.13 \\
$Z \rightarrow \tau \tau$ & 2.97 & 0.18 & 0.24 & 0.22 \\
$W W, W Z, Z Z, t \bar{t}($ incl. $)$ & 32.8 & 1.1 & 2.6 & 2.7 \\
$\mathrm{QCD}($ from data) & 27.4 & 2.5 & 1.7 & 1.7 \\
\hline$W^{\prime} \rightarrow e \nu(500 \mathrm{GeV})$ & 1094 & 24 & 72 & 88 \\
$W^{\prime} \rightarrow e \nu(600 \mathrm{GeV})$ & 358 & 8 & 25 & 32 \\
$W^{\prime} \rightarrow e \nu(700 \mathrm{GeV})$ & 129 & 3 & 10 & 13 \\
$W^{\prime} \rightarrow e \nu(800 \mathrm{GeV})$ & 41.1 & 1.0 & 3.8 & 4.7 \\
$W^{\prime} \rightarrow e \nu(900 \mathrm{GeV})$ & 12.8 & 0.4 & 1.5 & 1.7 \\
$W^{\prime} \rightarrow e \nu(1000 \mathrm{GeV})$ & 3.66 & 0.12 & 0.58 & 0.59 \\
$W^{\prime} \rightarrow e \nu(1100 \mathrm{GeV})$ & 0.96 & 0.04 & 0.20 & 0.18 \\
$W^{\prime} \rightarrow e \nu(1200 \mathrm{GeV})$ & 0.22 & 0.02 & 0.06 & 0.05 \\
\hline
\end{tabular}

Table D.5: Events in the search sample for transverse masses $m_{T}>200 \mathrm{GeV}$.

\begin{tabular}{|c|c|c|c|c|}
\hline \multirow[t]{2}{*}{ Process } & \multirow[t]{2}{*}{ Events } & \multicolumn{3}{|c|}{ Uncertainty } \\
\hline & & statistical & $\begin{array}{l}\text { syst } \\
(+)\end{array}$ & $\begin{array}{l}\text { natic } \\
\qquad(-)\end{array}$ \\
\hline Data & 241 & & & \\
\hline Sum Backgrounds & 242 & 9 & 20 & 17 \\
\hline$W^{\prime} \rightarrow e \nu(500 \mathrm{GeV})$ & 1088 & 24 & 72 & 88 \\
\hline$W^{\prime} \rightarrow e \nu(600 \mathrm{GeV})$ & 358 & 8 & 25 & 32 \\
\hline$W^{\prime} \rightarrow e \nu(700 \mathrm{GeV})$ & 129 & 3 & 10 & 13 \\
\hline$W^{\prime} \rightarrow e \nu(800 \mathrm{GeV})$ & 41.1 & 1.0 & 3.8 & 4.7 \\
\hline$W^{\prime} \rightarrow e \nu(900 \mathrm{GeV})$ & 12.8 & 0.4 & 1.5 & 1.7 \\
\hline$W^{\prime} \rightarrow e \nu(1000 \mathrm{GeV})$ & 3.66 & 0.12 & 0.58 & 0.59 \\
\hline$W^{\prime} \rightarrow e \nu(1100 \mathrm{GeV})$ & 0.96 & 0.04 & 0.20 & 0.18 \\
\hline$W^{\prime} \rightarrow e \nu(1200 \mathrm{GeV})$ & 0.22 & 0.02 & 0.06 & 0.05 \\
\hline
\end{tabular}


Table D.6: Events in the search sample for transverse masses $m_{T}>300 \mathrm{GeV}$.

\begin{tabular}{|l|r|r|r|r|}
\hline Process & Events & \multicolumn{3}{|c|}{ Uncertainty } \\
& & statistical & \multicolumn{2}{|c|}{ systematic } \\
& & & $(+)$ & $(-)$ \\
\hline \hline Data & $\mathbf{3 7}$ & & & \\
\hline Sum Backgrounds & 37.1 & 2.1 & 6.0 & 3.7 \\
\hline$W^{\prime} \rightarrow e \nu(500 \mathrm{GeV})$ & 981 & 22 & 65 & 79 \\
$W^{\prime} \rightarrow e \nu(600 \mathrm{GeV})$ & 344 & 8 & 24 & 31 \\
$W^{\prime} \rightarrow e \nu(700 \mathrm{GeV})$ & 128 & 3 & 10 & 13 \\
$W^{\prime} \rightarrow e \nu(800 \mathrm{GeV})$ & 41.1 & 1.0 & 3.8 & 4.7 \\
$W^{\prime} \rightarrow e \nu(900 \mathrm{GeV})$ & 12.8 & 0.4 & 1.5 & 1.7 \\
$W^{\prime} \rightarrow e \nu(1000 \mathrm{GeV})$ & 3.66 & 0.12 & 0.58 & 0.59 \\
$W^{\prime} \rightarrow e \nu(1100 \mathrm{GeV})$ & 0.96 & 0.04 & 0.20 & 0.18 \\
$W^{\prime} \rightarrow e \nu(1200 \mathrm{GeV})$ & 0.22 & 0.02 & 0.06 & 0.05 \\
\hline
\end{tabular}

Table D.7: Events in the search sample for transverse masses $m_{T}>400 \mathrm{GeV}$.

\begin{tabular}{|l|r|r|r|r|}
\hline Process & Events & \multicolumn{3}{|c|}{ Uncertainty } \\
& & statistical & \multicolumn{2}{|c|}{$\begin{array}{c}\text { systematic } \\
\end{array}$} \\
& & & & $(-)$ \\
\hline \hline Data & $\mathbf{1 0}$ & & & \\
\hline Sum Backgrounds & 9.28 & 1.05 & 1.53 & 1.34 \\
\hline$W^{\prime} \rightarrow e \nu(500 \mathrm{GeV})$ & 707 & 19 & 56 & 66 \\
$W^{\prime} \rightarrow e \nu(600 \mathrm{GeV})$ & 300 & 7 & 21 & 27 \\
$W^{\prime} \rightarrow e \nu(700 \mathrm{GeV})$ & 119 & 3 & 9 & 12 \\
$W^{\prime} \rightarrow e \nu(800 \mathrm{GeV})$ & 39.8 & 1.0 & 3.6 & 4.5 \\
$W^{\prime} \rightarrow e \nu(900 \mathrm{GeV})$ & 12.7 & 0.4 & 1.5 & 1.7 \\
$W^{\prime} \rightarrow e \nu(1000 \mathrm{GeV})$ & 3.64 & 0.12 & 0.57 & 0.59 \\
$W^{\prime} \rightarrow e \nu(1100 \mathrm{GeV})$ & 0.95 & 0.04 & 0.20 & 0.18 \\
$W^{\prime} \rightarrow e \nu(1200 \mathrm{GeV})$ & 0.22 & 0.02 & 0.06 & 0.05 \\
\hline
\end{tabular}

Table D.8: Events in the search sample for transverse masses $m_{T}>500 \mathrm{GeV}$.

\begin{tabular}{|l|r|r|r|r|}
\hline Process & Events & \multicolumn{3}{|c|}{ Uncertainty } \\
& & statistical & \multicolumn{2}{|c|}{$\begin{array}{c}\text { systematic } \\
\end{array}$} \\
& & & $(+)$ & $(-)$ \\
\hline \hline Data & 2.32 & 0.53 & 0.50 & 0.68 \\
\hline Sum Backgrounds & 144 & 9 & 62 & 56 \\
\hline$W^{\prime} \rightarrow e \nu(500 \mathrm{GeV})$ & 205 & 6 & 18 & 23 \\
$W^{\prime} \rightarrow e \nu(600 \mathrm{GeV})$ & 98.3 & 2.6 & 8.2 & 10.1 \\
$W^{\prime} \rightarrow e \nu(700 \mathrm{GeV})$ & 36.2 & 1.0 & 3.3 & 4.2 \\
$W^{\prime} \rightarrow e \nu(800 \mathrm{GeV})$ & 12.2 & 0.3 & 1.4 & 1.6 \\
$W^{\prime} \rightarrow e \nu(900 \mathrm{GeV})$ & 3.55 & 0.12 & 0.56 & 0.57 \\
$W^{\prime} \rightarrow e \nu(1000 \mathrm{GeV})$ & 0.95 & 0.04 & 0.20 & 0.18 \\
$W^{\prime} \rightarrow e \nu(1100 \mathrm{GeV})$ & 0.22 & 0.02 & 0.06 & 0.05 \\
$W^{\prime} \rightarrow e \nu(1200 \mathrm{GeV})$ & 0.22 \\
\hline
\end{tabular}





\section{Bibliography}

[1] The Particle Adventure, http://www.particleadventure.org/

[2] Die kleinsten Teilchen und der Anfang des Universums, http://www.atlas.uni-wuppertal.de/oeffentlichkeit/

[3] S. P. Martin, A Supersymmetry Primer, hep-ph/9709356 (2006)

[4] W.-M. Yao et al., Review of Particle Physics, J. Phys. G33, 1 (2006)

[5] P. Schmüser, Feynman-Graphen und Eichtheorien für Experimentalphysiker, 2. Auflage, Springer, 1995

[6] F. Halzen, A. D. Martin, Quarks and Leptons, Wiley, 1984

[7] Super-Kamiokande Collaboration (Y. Ashie et al.), Evidence for an oscillatory signature in atmospheric neutrino oscillation, Phys. Rev. Lett. 93, 101801 (2004)

[8] D. P. Roy, Solar neutrino oscillation: An Overview, Proceedings of the Symposium: Boston 2004, Particles, strings and cosmology, hep-ph/0409336 (2004)

[9] K2K Collaboration (M. H. Ahn et al.), Measurement of Neutrino Oscillation by the K2K Experiment, Phys. Rev. D74, 072003 (2006)

[10] KamLAND Collaboration (K. Eguchi et al.), First results from KamLAND: Evidence for reactor anti-neutrino disappearance, Phys. Rev. Lett. 90, 021802 (2003)

[11] Quantum Field Theory, http://universe-review.ca/R15-12-QFT.htm

[12] H. Murayama, The origin of neutrino mass, Physics World, 2002

[13] M. Lindner, T. Ohlsson, G. Seidl, See-saw Mechanisms for Dirac and Majorana Neutrino Masses, Phys. Rev. D65, 053014 (2002)

[14] LEP Electroweak Working Group (Winter 2007), http://lepewwg.web.cern.ch/LEPEWWG/plots/winter2007/

[15] G. Altarelli, G. Parisi, Asymptotic Freedom in Parton Language, Nucl. Phys. B126, 298 (1977);

V. N. Gribov, L. N. Lipatov, Deep inelastic ep scattering in perturbation theory, Sov. J. Nucl. Phys. 15, 438 (1972);

Y. L. Dokshitzer, Calculation of the Structure Functions for Deep Inelastic Scattering and $e^{+} e^{-}$Annihilation by Perturbation Theory in Quantum Chromodynamics, Sov. Phys. JETP 46, 641 (1977) 
[16] HERA summary figures for Moriond 2004, http://www-zeus.desy.de/physics/ sfew/PUBLIC/sfew_results/preliminary/moriond04/zeush1.php

[17] J. Pumplin, D. R. Stump, J. Huston, H. L. Lai, P. Nadolsky, W. K. Tung, New Generation of Parton Distributions with Uncertainties from Global QCD Analysis, JHEP 0207, 012 (2002);

D. Stump, J. Huston, J. Pumplin, W. K. Tung, H. L. Lai, S. Kuhlmann, J. F. Owens, Inclusive Jet Production, Parton Distributions, and the Search for New Physics, JHEP 0310, 046 (2003)

[18] The Coordinated Theoretical-Experimental Project on QCD, http://www.phys.psu.edu/ ${ }^{\sim}$ cteq/

[19] V. D. Barger, R. J. N. Phillips, Collider Physics, Addison Wesley, 1993

[20] C. Quigg, Gauge Theories of the Strong, Weak, and Electromagnetic Interactions, Benjamin/Cummings Pub. Co., 1983

[21] T. Nunnemann, NNLO Cross-Sections for Drell-Yan, $Z$ and $W$ Production using Modern Parton Distribution Functions, DØ Note 4476 (2004); http://www-cluedo.fnal.gov/ nunne/cross-sections/nnlo_xsect.html

[22] R. Hamberg, W. L. van Neerven, T. Matsuura, A Complete Calculation of the Order $\alpha_{s}^{2}$ Correction to the Drell-Yan K-Factor, Nucl. Phys. B359, 343 (1991); Erratum-ibid. B644, 403 (2002)

[23] R. N. Mohapatra, Unification and Supersymmetry, Third Edition, Springer, 2002

[24] J. C. Pati, A. Salam, Lepton Number as the fourth "color", Phys. Rev. D10, 275 (1974)

[25] R. N. Mohapatra, J. C. Pati, Left-right gauge symmetry and an "isoconjugate" model of CP violation, Phys. Rev. D11, 566 (1975)

[26] R. N. Mohapatra, J. C. Pati, "Natural" left-right symmetry, Phys. Rev. D11, 2558 (1975)

[27] G. Senjanovic, R. N. Mohapatra, Exact left-right symmetry and spontaneous violation of parity, Phys. Rev. D12, 1502 (1975)

[28] W. Grimus, Introduction to Left-Right Symmetric Models, Lecture given at the 4th Hellenic School on Elementary Particle Physics, Corfu, Greece, 1992

[29] D. Chang, R. N. Mohapatra, Small and Calculable Dirac Neutrino Mass, Phys. Rev. Lett. 58, 1600 (1987);

K. S. Babu and X. G. He, Dirac Neutrino Masses as Two-Loop Radiative Corrections, Mod. Phys. Lett. A4, 61 (1989)

[30] G. Altarelli, B. Mele, M. Ruiz-Altaba, Searching for New Heavy Vector Bosons in $p \bar{p}$ Colliders, Z. Phys. C45, 109 (1989)

[31] UA1 Collaboration (G. Arnison et al.), Further Evidence for Charged Intermediate Vector Bosons at the SPS Collider, Phys. Lett. B129, 273 (1983) 
[32] Particle Data Group, http://pdg.lbl.gov/

[33] DØ Collaboration (V. M. Abazov et al.), Search for new particles in the two-jet decay channel with the DØ detector, Phys. Rev. D69, 111101 (2004)

[34] CDF Collaboration (A. Abulencia et al.), Search for $W^{\prime}$ Boson Decaying to ElectronNeutrino Pairs in p $\bar{p}$ Collisions at $\sqrt{s}=1.96$ TeV, Phys. Rev. D75, 091101 (2007)

[35] J. F. Donoghue, B. R. Holstein, Strong bounds on weak couplings, Phys. Lett. B113, $382(1982)$

[36] G. Beall, M. Bander, A. Soni, Constraint on the Mass Scale of a Left-Right Symmetric Electroweak Theory from the $K_{L}-K_{S}$ Mass Difference, Phys. Rev. Lett. 48, $848(1982)$

[37] R. N. Mohapatra, Limits on the Mass of the Right-handed Majorana Neutrino, Phys. Rev. D34, 909 (1986)

[38] The DØ Experiment, http://www-d0.fnal.gov

[39] The Collider Detector at Fermilab, http://www-cdf.fnal.gov

[40] Fermi National Accelerator Laboratory, http://www.fnal.gov/

[41] U. S. Department of Energy, http://www. energy.gov/

[42] Discoveries at Fermilab, http://www.fnal.gov/pub/inquiring/physics/discoveries/index.html

[43] DØ Collaboration (S. Abachi et al.), Observation of the Top Quark, Phys. Rev. Lett. 74, $2632(1995)$

[44] CDF Collaboration (F. Abe et al.), Observation of Top Quark Production in $\bar{p} p$ Collisions, Phys. Rev. Lett. 94, 2626 (1995)

[45] Google Maps, http://maps.google.com/

[46] Run II Luminosity, http://www-d0.fnal.gov/runcoor/RUN/run2_lumi.html

[47] Accelerator Division Operations Rookie Books, http://www-bdnew.fnal.gov/operations/rookie_books/rbooks.html

[48] Tevatron Run II Handbook, http://www-bd.fnal.gov/runII/

[49] Fermilab's Visual Media Services, http://www-visualmedia.fnal.gov/VMS_Site_2/index.shtml

[50] Fermilab Accelerator Division, http://www-bd.fnal.gov/

[51] FermilaB's Chain of Accelerators, http://www-bd.fnal.gov/public/index.html

[52] C. Rubbia and S. van der Meer, Nobel Prize in Physics 1984 for their decisive contributions to the large project, which led to the discovery of the field particles $W$ and $Z$, communicators of weak interaction, http://nobelprize.org/nobel_prizes/physics/laureates/1984/ 
[53] Fermilab Recycler Department Electron Cooling, http://www-ecool.fnal.gov/

[54] History of the Electron Cooling, http://www-ecool.fnal.gov/default_files/Page702.htm

[55] D. Edmunds, DØ Trigger Framework Tutorial, 2003, http://www.pa.msu.edu/hep/d0/11/framework/tfw_tutorial_march_2003.html

[56] Fermilab Accelerator Controls, http://www-bd.fnal.gov/controls/

[57] S. Klimenko, J. Konigsberg, T. M. Liss, Averaging of the inelastic cross sections measured by the CDF and the E811 experiments, FERMILAB-FN-0741 (2003)

[58] S. Catani, Aspects of QCD, from the Tevatron to the LHC, hep-ph/0005233 (2000)

[59] S. H. Ahn, O. Atramentov, M. Begel, G. Geurkov, J. Hauptman, A. Kupco, S. W. Lee, P. Mal, J. W. Park, R. Partridge, H. Schellman, M. Verzocchi, DØ Luminosity in Run 2: Delivered, DØ Note 3970 (2002), DØ Luminosity in Run 2: Triggered, DØ Note 3971 (2002), DØ Luminosity in Run 2: Recorded, DØ Note 3972 (2002)

[60] Tejinder "Jim" Virdee, Weighty Matters, Inaugural Lecture, 1998, http://cmsinfo.cern.ch/outreach/CMSdocuments/JimInaugural/ JimInaugural_index.html

[61] DØ Collaboration (V. M. Abazov et al.), The Upgraded DØ Detector, Nucl. Instr. and Methods A565, 463 (2006)

[62] Pictures from A. Stone’s D $\varnothing$ web page, http://home.fnal.gov/ alstone/

[63] DØ Central Fiber Tracker Photos, http://d0server1.fnal.gov/projects/SciFi/dons_pictures.html

[64] DØ Collaboration (S. Abachi et al.), The DØ Detector, Nucl. Instr. and Methods A338, 185 (1994)

[65] DØ Collaboration (S. Abachi et al.), The DØ Upgrade: The Detector and its Physics, FERMILAB-PUB-96-357-E (1996)

[66] SVXII Design Group (R. Yarema et al.), A Beginners Guide to the SVXII, FERMILAB-TM-1892 (1994, revised 1996)

[67] DØ Silicon Tracker Drawings and Plots, http://d0server1.fnal.gov/projects/silicon/www/drawings_eng.html

[68] M. D. Petroff, M. G. Stapelbroek, Photon-counting solid-state photomultiplier, IEEE Trans. Nucl. Sci. 36, 158 (1989);

M. D. Petroff, M. Atac, High Energy Particle Tracking Using Scintillating Fibers and Solid State Photomultipliers, IEEE Trans. Nucl. Sci. 36, 163 (1989)

[69] DØ Detector Drawings, http://www-d0.fnal.gov/Run2Physics/WWW/drawings.htm

[70] Picture taken by Jens-Peter Konrath 
[71] D. Chapin, H. Fox, J. Gardner, R. Illingworth, A. Lyon, J. Zhu, Measurement of $Z \rightarrow e e$ and $W \rightarrow e \nu$ Production Cross Sections Using One Tight Central Electron, DØ Note 4897 (2005)

[72] M. Voutilainen, C. Royon, Jet $p_{T}$ resolution using JetCorr v\%.1, DØ Note 5381 (2007)

[73] DØ Luminosity Monitor Detector Homepage, http://www-d0online.fnal.gov/www/groups/lum/

[74] DØ Global Trigger Lists Description, http://www-d0.fnal.gov/trigger_meister/private/www/tl_desc/global.html

[75] M. Souza, ScriptRunner Implementation Notes (1999), http://www-d0.fnal.gov/ moacyr/13/impl.ps

[76] C. Autermann, The Level 3 Isolation Tool, DØ Note 4436 (2004)

[77] C. Magaß, Track Isolation for Electromagnetic Objects at Level 3, DØ Note 4664 (2004)

[78] V. Lesne, Muon isolation tool at L3, DØ Note 4988 (2006)

[79] C. Magaß, Certification of Isolation Tool, Talk given at the L3 Algorithms Meeting, March 29, 2005,

http://www-d0.hef .kun.nl///fullAgenda.php?ida=a05562

[80] DØ Store Summary Plots (Trigger Rates \& Luminosity), http://www-dOonline.fnal.gov/www/groups/tm/daqrates/

[81] C. Magaß, Trigger@DØ - Tools 83 Methods, Talk given at the DØ Germany Meeting, Munich, April 6/7, 2005, http://www-d0.hef.kun.nl///fullAgenda.php?ida=a05459;

C. Magaß, p17 Trigger, Talk given at the D $\varnothing$ Germany Meeting, Aachen, October 12/13, 2006, http://www-d0.hef .kun.nl//fullAgenda.php?ida=a061518

[82] M. Begel, DØ Luminosity in Run 2: ORing triggers, DØ Note 4154 (2003)

[83] T. Gadfort, ORing single muon triggers and one muon+jets trigger, DØ Note 4899 (2005)

[84] A. Naumann, F. Filthaut, Combining Prescaled Triggers, DØ Note 4844 (2005)

[85] J. Hays, V. Kaushik, J. Mitrevski, O. Mundal, C. Schwanenberger, Electron Trigger Efficiencies using Calorimeter Information in p17 Data, DØ Note 5138 (2006)

[86] A. Boehnlein, X. Fan, H. Schellman, M. Souza, Run II Streaming: How to Stream Events, DØ Note 3662 (1999)

[87] DØ SAM Homepage, http://www-d0.fnal.gov/computing/sam/

[88] DØ Global Monitoring, http://www-d0online.fnal.gov/groups/gm/GM.html

[89] DØ Run II Software Algorithms, http://www-d0.fnal.gov/computing/algorithms/ 
[90] S. Protopopescu, S. Baffioni, E. Nagy, ThumbNail: a compact data format, DØ Note $3979(2002)$

[91] D $\varnothing$ Common Samples Group, http://www-d0.fnal.gov/Run2Physics/cs/index.html

[92] Roот System Homepage, http://root.cern.ch/

[93] tmb_analyze package, http://www-d0.fnal.gov/d0dist/dist/packages/tmb_analyze/devel/doc/

[94] DØ Data Format Working Group, Recommendations Regarding Common Analysis Format Content, DØ Note 4647 (2005); http://www-d0.fnal.gov/Run2Physics/cs/caf/

[95] DØ Object Correction: dØcorrect, http://www-d0.fnal.gov/Run2Physics/cs/d0correct/d0correct.html

[96] DØ Online Logbook, https://www-d0online.fnal.gov/crlw/Index.jsp

[97] DØ Online Run Database, http://www-d0online.fnal.gov/run/runQuery.html

[98] DØ Offline Run Quality Database, http://d0db.fnal.gov/qualitygrabber/qualQueries.html

[99] DØ Luminosity Database Operations Report, http://d0db.fnal.gov/lm_db/cgi/lmdb_main.py

[100] P. Verdier, Missing ET Status, Talk given at the All DØ Meeting, October 15, 2004, http://www-d0.hef .kun.nl/fullAgenda.php?ida=a041625

[101] DØ Data Quality Coordination, http://www-d0.fnal.gov/computing/data_quality/d0_private/

[102] L. Duflot, V. Shary, R. Zitoun, I. Torchiani, cal_event_quality package, DØ Note 4614 (2004)

[103] Talks from V. Shary, G. Bernardi, P. Verdier given at the DØ Winter Physics Workshop, February 22 - 24, 2004, http://www-d0.hef .kun.nl/fullAgenda.php?ida=a04273

[104] DØ Top Group, http://www-d0.fnal.gov/Run2Physics/top/index.html

[105] D $\varnothing$ Heavy Flavor ( $b$ and $c$ quark) Jet Identification Group, http://www-d0.fnal.gov/phys_id/bid/d0_private/bid.html

[106] DØ Tau Identification Group, http://www-d0.fnal.gov/〜dOupgrad/d0_private/software/tauid/tauid.html

[107] DØ Muon Identification Group, http://www-d0.fnal.gov/computing/algorithms/muon/muon_algo.html

[108] DØ Tracking/Vertexing, http://www-d0.fnal.gov/global_tracking/ 
[109] DØ Photon Identification Group, http://www-d0.fnal.gov/phys_id/emid/d0_private/photonid.html

[110] Y. Gershtein, Y. Maravin, Search for GMSB SUSY in Diphoton Events with Large Missing $E_{T}$, DØ Note 5425 (2007)

[111] L. Wang, J. Hays, J. Mitrevski, C. Schwanenberger, Electron Likelihood Efficiency in $p 17, \mathrm{D} \varnothing$ Note $5114(2006)$

[112] C. Magaß, Search for $W^{\prime} \rightarrow$ eע using CAF Tools and p17 Data, Talk given at the DØ Winter Physics Workshop, February 20/21, 2006, http://www-d0.hef .kun.nl///fullAgenda.php?ida=a06310

[113] A. Kupco, Jet energy scale and resolution in the DØ calorimeter, Talk given at the CALOR 2004 Conference in Perugia, April 1, 2004, http://www.pg.infn.it/calor2004/

[114] G. C. Blazey et al., Run II Jet Physics, Proceedings of the Workshop: QCD and Weak Boson Physics in Run II, edited by U. Baur, R. K. Ellis, D. Zeppenfeld, FERMILAB-PUB-00/297 (2000)

[115] A. Harel, Jet ID Optimization, DØ Note 4919 (2005)

[116] A. Harel, R. Wagner, Improved L1 Confirmation, DØ Note 4932 (2005)

[117] N. Makovec, J.-F. Grivaz, Shifting, Smearing and Removing Simulated Jets, D Note 4914 (2005)

[118] B. Andrieu, A. Harel, Jet Efficiency Measurement and Monte Carlo Correction Lessons and Options, DØ Note 5169 (2006)

[119] S. Trincaz-Duvoid, P. Verdier, Missing ET Reconstruction in p17, DØ Note 4474 (2004)

[120] S. Calvet, P. Verdier, E. Kajfasz, Towards MissingET Certification and Unclustered Energy Studies, DØ Note 4927 (2005)

[121] D. Käfer, Search for R-parity Violating Supersymmetry in Multilepton Final States with the DØ Detector, Dissertation, III. Physikalisches Institut A, RWTH Aachen (2006),

http://www.physik.rwth-aachen.de/ hebbeker/theses/kaefer_phd.pdf

[122] DØ Run II Simulation,

http://www-d0.fnal.gov/computing/MonteCarlo/MonteCarlo.html

[123] T. Sjostrand, P. Eden, C. Friberg, L. Lonnblad, G. Miu, S. Mrenna and E. Norrbin, High-Energy-Physics Event Generation with PYTHIA 6.1, Comput. Phys. Commun. 135, 238 (2001);

T. Sjostrand, L. Lonnblad, S. Mrenna, P. Skands, Pythia 6.3 Physics and Manual, hep-ph/0308153;

PYTHIA web page, http://www.thep.lu.se/ ${ }^{\sim}$ torbjorn/Pythia.html

[124] B. Andersson, The Lund Model, Nucl. Phys. A461, 513 (1987) 
[125] B. Andersson, G. Gustafson, G. Ingelman, T. Sjostrand, Parton Fragmentation and String Dynamics, Phys. Rept. 97, 31 (1983)

[126] J. Bartels, H. Jung, QCD and Collider Physics III: LHC, Lecture given at the University Hamburg, 2006/2007, https://www.desy.de/ jung/qcd_collider_physics_wise_2006/

[127] Y. Schultz, QCD et la physique auprès des collisionneurs, http://www-subatech.in2p3.fr/ photons/subatech/physics/collisionneurs/

[128] Geant - Detector Description and Simulation Tool, http://wwwasd.web.cern.ch/wwwasd/geant/

[129] DØ Run II Monte Carlo Production web page, http://www-d0.fnal.gov/computing/mcprod/mcc.html

[130] DØ Official Monte Carlo Sample Catalog, http://d0-france.in2p3.fr/WORKING_GROUPS/MONTECARLO/Catalog/current/

[131] S. Eno, D0Raw2Sim, Talk given at the All DØ Meeting, November 7, 2003, http://www-d0.hef .kun.nl//fullAgenda.php?ida=a03560;

S. Eno, Status of Simulation, Talk given at the Joint Algorithm/Physics Conveners Meeting, July 23, 2004, http://www-d0.hef .kun.nl//fullAgenda.php?ida=a041220

[132] R. Field, CDF Run 2 Monte-Carlo Tunes, to appear in the proceedings of the TeV4LHC Workshop, Fermilab, October 21, 2005, http://www.phys.ufl.edu/ ${ }^{\sim}$ rfield/cdf/TeV4LHC_RickField_Proceedings.pdf

[133] DoRaw2sim versus "Normal" Overlay, http://www-d0.fnal.gov/computing/MonteCarlo/d0raw2sim_compare.htm

[134] DØ Generator Level Event Selector: dØ_mess http://www-cluedo.fnal.gov/do_mess/

[135] C. Magaß, Bestimmung des inklusiven Wirkungsquerschnitts für Elektronproduktion bei Proton-Antiproton-Kollisionen, Diplomarbeit, III. Physikalisches Institut A, RWTH Aachen (2003), http://www.physik.rwth-aachen.de/ ${ }^{\sim}$ hebbeker/theses/magass_diploma.pdf

[136] V. Büscher, J.-F. Grivaz, T. Nunnemann, M. Wobisch, Conclusions of MiniWorkshop on PDF uncertainties and related topics, DØ Note 4618 (2004); Mini-Workshop on PDF uncertainties and related topics, September 21, 2004, http://www-d0.hef .kun.nl//fullAgenda.php?ida=a041555

[137] N. Kidonakis, R. Vogt, Theoretical Status of the Top Quark Cross Section, Int. J. Mod. Phys. A20, 3171 (2005)

[138] Private communication with A. Mück and M. Krämer (Institut für Theoretische Physik E, RWTH Aachen) (2007)

[139] Private communication with T. Sjöstrand (2007) 
[140] np_tmb_stream package, http://www-d0.fnal.gov/d0dist/dist/packages/ np_tmb_stream/devel/doc/tmb_streaming.pdf

[141] lm_access package, http://www-d0.fnal.gov/d0dist/dist/packages/lm_access/devel/doc/

[142] S.-J. Park, M. Begel, Efficiency of the Data Quality Calorimeter Flags, DØ Note $5324(2007)$

[143] DØ Single Electron Triggers, https://plone4.fnal.gov/P1/DOWiki/tdaq/tsg/caftrigger/singleetriggers

[144] lm_tools package, http://www-d0.fnal.gov/d0dist/dist/packages/lm_tools/devel/doc/

[145] G. Snow, Adjustments to the Measured Integrated Luminosity in Run IIa, DØ Note $5139(2006)$

[146] T. Kuhl, C. Zeitnitz, DØ Detector Simulation, Talk given at the DØ Workshop, Vancouver, June 17, 2005, http://www-d0.hef .kun.nl//fullAgenda.php?ida=a05987

[147] Private communication with Jan Stark and Jean-Francois Grivaz (2006)

[148] DØ Electron and Photon Identification Group, http://www-do.fnal.gov/phys_id/emid/d0_private/emid.html

[149] J. Hays, J. Mitrevski, C. Schwanenberger, T. Toole, Single Electron Efficiencies in p17 Data and Monte-Carlo Using d0correct from Release p18.05.00, DØ Note 5105 (2006)

[150] B. Tiller, T. Nunnemann, Measurement of the differential $Z^{0}$-boson production crosssection as function of transverse momentum, DØ Note 4660 (2004)

[151] M. L. Mangano, M. Moretti, F. Piccinini, R. Pittau, A. Polosa, ALPGEN, a generator for hard multiparton processes in hadronic collisions, JHEP 0307, 001 (2003)

[152] DØ Common AlPGEn Studies, http://www-d0.fnal.gov/computing/ MonteCarlo/generators/common_alpgen.html

[153] S. Hoche, F. Krauss, N. Lavesson, L. Lonnblad, M. Mangano, A. Schalicke, S. Schumann, Matching Parton Showers and Matrix Elements, hep-ph/0602031 (2006)

[154] M. Begel, L. Christofek, E. Shabalina, Determination of Weighting Factors for ALPGEN Monte Carlo Signal and Background Samples, DØ Note 5016 (2006)

[155] P. Renkel, M. Begel, Correction in inclusive bins cross-sections for matched Alpgen MC samples, DØ Note 5330 (2007)

[156] C. Magaß, EM Energy Scale and Resolution, Talk given at the New Phenomena High pt Lepton Working Group Meeting, May 16, 2007, http://www-d0.hef .kun.nl//fullAgenda.php?ida=a07864 
[157] J. Pumplin, D. Stump, R. Brock, D. Casey, J. Huston, J. Kalk, H. L. Lai, W. K. Tung, Uncertainties of predictions from parton distribution functions II: the Hessian method, Phys. Rev. D65, 014013 (2002)

[158] G. Cowan, Statistical Data Analysis, Clarendon Press (1998)

[159] I. Bertram, G. Landsberg, J. Linnemann, R. Partridge, M. Paterno, H. B. Prosper, A Recipe for the construction of confidence limits, FERMILAB-TM-2104 (2000)

[160] V. Büscher, J.-F. Grivaz, J. Hobbs, A. Kharchilava, G. Landsberg, J. Linnemann, H. Prosper, and S. Söldner-Rembold, Recommendation of the Ad-Hoc Committee on Limit-Setting Procedures to be Used by DØ in Run II, DØ Note 4629 (2004)

[161] S. Jain, D. Kau, H. B. Prosper, B. Vachon, R. Schwienhorst, Computing limits using a Bayesian approach in the package "top_statistics", DØ Note 5123 (2006)

[162] top_statistics package, http://www-d0.fnal.gov/d0dist/dist/releases/ development/top_statistics/doc/top_statistics.html

[163] M. Denny, Introduction to importance sampling in rare-event simulations, Eur. J. Phys. 22, 403 (2001)

[164] DØ Collaboration (V. M. Abazov et al.), Search for $W^{\prime}$ Bosons Decaying to an Electron and a Neutrino with the D0 Detector, FERMILAB-PUB-07/499-E, submitted to Phys. Rev. Lett.

[165] C. Hof, Detection of New Heavy Gauge Bosons $W^{\prime}$ in CMS, Acta Phys. Polon. B38, $443(2006)$ 


\section{List of Figures}

1.1 History of the basics of nature . . . . . . . . . . . . . . 2

1.2 Evolution of the inverse gauge couplings in the SM and MSSM . . . . . . 3

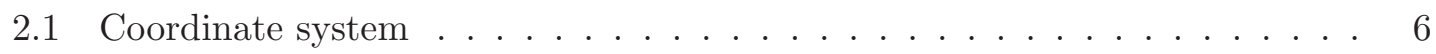

2.2 Kinematics in proton-antiproton collisions . . . . . . . . . . . 7

$2.3 \Delta \chi^{2}$ as a function of the Higgs mass $\ldots \ldots \ldots \ldots$

2.4 Structure function $F_{2}\left(x, Q^{2}\right) \ldots \ldots \ldots \ldots \ldots \ldots \ldots$

2.5 Parton density functions . . . . . . . . . . . . . . . . . 23

2.6 Comparison of measurements of precision observables with Standard Model fits . . . . . . . . . . . . . . . . . . . 26

2.7 Results from the search for the process $W^{\prime} \rightarrow e \nu$ with the CDF detector . . 34

2.8 Contributions from right-handed $W$ bosons in the $K_{L}-K_{S}$ mixing and the neutrinoless double $\beta$ decay . . . . . . . . . . . . . . . . . . . . 34

3.1 Aerial view of the Tevatron collider at the Fermilab site . . . . . . . 36

3.2 Delivered and recorded luminosity in Run II until August 2007 . . . . . . . 36

3.3 Schematic view of the collider complex . . . . . . . . . . . . . 38

3.4 Sketches of the Magnetron and the antiproton source . . . . . . . . . . . . . 39

3.5 Sketch displaying the bunch rotation and stochastic cooling . . . . . . . . . 39

3.6 Tevatron Run IIa beam structure . . . . . . . . . . . . . . . . . . . . . . 41

3.7 Luminosity and number of protons/antiprotons during store \#4571 . . . . . 42

3.8 Peak luminosities during Run II until August 2007 . . . . . . . . . . . . . . 44

3.9 Cross sections and event rates for selected processes; interactions per crossing for different instantaneous luminosities . . . . . . . . . . . . . . . . . 44

3.10 Onion-like structure of a multi-purpose detector; picture of the D $\varnothing$ detector 45

3.11 Schematic view and picture of the $\mathrm{D} \varnothing$ detector . . . . . . . . . . . . . . 46

3.12 Tracking system (cross-sectional view and picture) $\ldots \ldots$. . . . . . . 47 
3.13 Geometry of the SMT . . . . . . . . . . . . . . . . . 48

3.14 Pictures showing the assembly of the SMT . . . . . . . . . . . . 49

3.15 Pictures showing waveguides and AFE boards; calibration spectrum for a

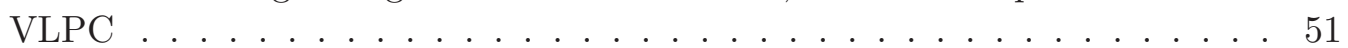

3.16 Calorimeter $($ isometric view and picture $) \ldots \ldots \ldots \ldots$. . . . . . . . 52

3.17 Calorimeter unit cell; readout chain of the calorimeter . . . . . . . . . . 52

3.18 Segmentation of the $\mathrm{D} \varnothing$ calorimeter . . . . . . . . . . . . 53

3.19 Pictures of the calorimeter preamplifiers and BLS boards . . . . . . . . . 54

3.20 Muon detector system: drift tubes and scintillation counters . . . . . . . . 56

3.21 Pictures of the muon detector . . . . . . . . . . . . . . . . . 56

3.22 Pictures of the muon MDTs during the assembly . . . . . . . . . . . . . 57

3.23 Schematic views showing the location and geometry of the luminosity monitors 59

3.24 Pictures of a luminosity monitor . . . . . . . . . . . . . . . . 59

3.25 Distribution of the $z$ position of the reconstructed primary vertex from data 59

3.26 Overview of the trigger and data acquisition system . . . . . . . . 60

3.27 Level 3 execution tree . . . . . . . . . . . . . . . . . . . 61

3.28 Design of the Level 3 isolation tool _ . . . . . . . . . . . . . . . 62

3.29 Performance of the Level 3 isolation tool . . . . . . . . . . . . . . . 63

3.30 Progression of luminosity and trigger rates during store \#4571 . . . . . 64

3.31 Illustration of the data flow through the data acquisition system . . . . . 65

3.32 Physics examine plots for tracks _ . . . . . . . . . . . . . 66

3.33 Pictures of the $\mathrm{D} \varnothing$ control room . . . . . . . . . . . . . 68

3.34 "Ring of fire" noise in the calorimeter; impact of data quality selection criteria on missing transverse energy . . . . . . . . . . . . . . 69

$4.1 \quad$ Measured $t \bar{t} \rightarrow b \bar{b} e \mu \nu \nu$ candidate event . . . . . . . . . . . . . . . . 72

4.2 Definition of isolation for electromagnetic showers . . . . . . . . . . . . 73

4.3 Comparison of track and calorimeter variables . . . . . . . . . . . . . 75

4.4 Width of the electromagnetic shower $\sigma_{\phi}^{E M 3}$ as a function of the electron transverse energy $E_{T} \ldots \ldots \ldots \ldots \ldots \ldots$

4.5 Variable $i s o$ as a function of the electron transverse energy $E_{T} \ldots \ldots$. . . 76

4.6 Variable emf as a function of the electron transverse energy $E_{T} \ldots \ldots$. . 76

4.7 Relative energy fractions in the four EM layers as a function of the electron transverse energy $E_{T} \ldots \ldots \ldots \ldots \ldots \ldots$

4.8 Variable $\chi_{\mathrm{HMx} 7}^{2}$ as a function of the electron transverse energy $E_{T} \ldots \ldots 78$ 
4.9 From partons to jets in the calorimeter . . . . . . . . . . . . . . . . 79

4.10 Seed collinear, $E_{T}$ ordering and infrared sensitivity . . . . . . . . . . 80

4.11 Resolution of the missing transverse energy . . . . . . . . . . . . . . . . . 84

5.1 Cross sections and event rates for the signal and background processes . . . 85

5.2 Simulation Chain . . . . . . . . . . . . . . . . . . . 86

5.3 Fragmentation . . . . . . . . . . . . . . . . . 87

5.4 Event with multiple interactions . . . . . . . . . . . . . . . 88

5.5 Comparison of simulated and measured pile-up events . . . . . . . . . . 88

5.6 Composition of the $Z$ sample; NNLO correction . . . . . . . . . . . . . . . 91

5.7 Signal cross sections as a function of the $W^{\prime}$ mass . . . . . . . . . . . . . . 93

5.8 Mass distributions of the generated $W^{\prime}$ bosons $\ldots \ldots \ldots$. . . . . . 93

5.9 Momentum fractions of the quarks that produce the $W^{\prime}$ boson $\ldots . . .94$

5.10 Distributions of generator variables . . . . . . . . . . . . . . . . 95

5.11 Distributions of variables after reconstruction $(1) \ldots \ldots$. . . . . . . 97

5.12 Distributions of variables after reconstruction $(2) \ldots \ldots \ldots$

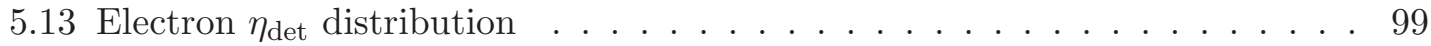

5.14 Electron $\eta_{\text {det }}$ distributions as function of the transverse mass $m_{T} \ldots \ldots$

6.1 Processing chain . . . . . . . . . . . . . . . . . . . . 103

6.2 Trigger efficiency for EM_MX and EM_MX_EMFR8 . . . . . . . . . . . 105

6.3 Overview of the data samples used in the analysis . . . . . . . . . . 106

6.4 Control plots for the $W$ sample . . . . . . . . . . . . . . . . . . . . . . 107

6.5 Motivation for the $E_{T} / E_{T}$ cut $(1) \ldots \ldots \ldots$. . . . . . . . . . . . . . .

6.6 Motivation for the $E_{T} / E_{T}$ cut $(2) \ldots \ldots \ldots$. . . . . . . . . . . . . .

6.7 Motivation for the cuts on the angular differences in events with jets . . . 110

6.8 Consistency check for triggers and luminosity . . . . . . . . . . . . . . . 112

6.9 QCD multijet background (schematic illustration and event display) . . . . 113

$6.10 \chi_{\mathrm{HMx} 7}^{2}$ distribution for data and $W \rightarrow e \nu$ Monte Carlo events; $\Delta \phi(E M, M E T)$ as a function of $m_{T}$ for the QCD sample . . . . . . . . 114

$6.11 m_{T}$ and $\Delta \phi(E M, M E T)$ distributions after scaling the QCD sample $\ldots . .115$

6.12 Distribution of the transverse mass $m_{T}$ for the $W$ sample showing the $W$

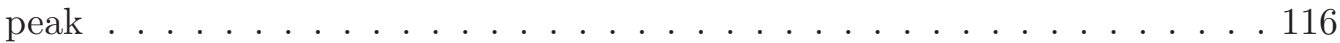

7.1 Electron energy resolution . . . . . . . . . . . . . . . . . . . 118 
7.2 Electron energy resolution study . . . . . . . . . . . . . . . . . . . . 119

7.3 Electron energy scale . . . . . . . . . . . . . . . . . . . . . 120

7.4 Electron reconstruction efficiencies in data and Monte Carlo . . . . . . . . . 123

7.5 Overall efficiency correction factor and uncertainty . . . . . . . . . . . . 124

7.6 Monte Carlo reconstruction efficiencies for signal electrons . . . . . . . . . . 125

7.7 Sketch of a trigger turn-on curve (ideal and real) . . . . . . . . . . . 126

7.8 Trigger efficiencies for the trigger sets used in the analysis . . . . . . . . . 127

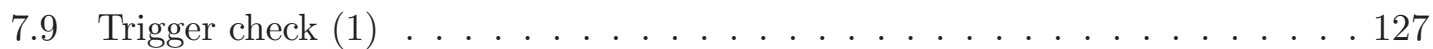

7.10 Trigger check $(2) \ldots \ldots \ldots \ldots \ldots$

7.11 Comparison between data and background prediction after applying all corrections except for the $W p_{T}$ reweighting $(1) \ldots \ldots 130$

7.12 Comparison between data and background prediction after applying all corrections except for the $W p_{T}$ reweighting $(2) \ldots \ldots$. . . . . . . 131

7.13 Higher order contributions to the process $q \bar{q}^{\prime} \rightarrow W \ldots \ldots$. . . . . . 131

7.14 Comparison between data and background prediction in events without any jets after applying all corrections $(1) \ldots \ldots . \ldots \ldots$

7.15 Comparison between data and background prediction in events without any jets after applying all corrections $(2) \ldots \ldots \ldots \ldots$

7.16 Comparison between data and background prediction in events with one jet after applying all corrections except for the $W p_{T}$ reweighting $(1) \ldots 135$

7.17 Comparison between data and background prediction in events with one jet after applying all corrections except for the $W p_{T}$ reweighting $(2) \ldots 136$

7.18 Comparison between data and background prediction in events with one jet after applying the $W p_{T}$ reweighting $(1) \ldots \ldots . \ldots \ldots$. . . . . . . . . 137

7.19 Comparison between data and background prediction in events with one jet after applying the $W p_{T}$ reweighting $(2) \ldots \ldots \ldots \ldots$. . . . . . . . . . 138

7.20 Comparison between data and background prediction in events with two jets after applying all corrections except for the $W p_{T}$ reweighting (1) . . 139

7.21 Comparison between data and background prediction in events with two jets after applying all corrections except for the $W p_{T}$ reweighting (2) $\quad \ldots 140$

7.22 Comparison between data and background prediction in events with two jets after applying the $W p_{T}$ reweighting $(1) \ldots \ldots \ldots$. . . . . . . 141

7.23 Comparison between data and background prediction in events with two jets after applying the $W p_{T}$ reweighting $(2) \ldots \ldots$. . . . . . . . . . 142

7.24 Peak structure in the QCD sample . . . . . . . . . . . . . . 143

7.25 Plots showing the variation of the electron energy resolution . . . . . . . . 145 
7.26 Impact of the electron energy scale variation on the $W$ Monte Carlo sample and one signal point . . . . . . . . . . . . . . . . . . 146

7.27 Hessian method to derive the sets of eigenvector PDFs . . . . . . . . . . 146

7.28 Comparison between CTEQ6L1, CTEQ6.1M.00 and the associated uncer-

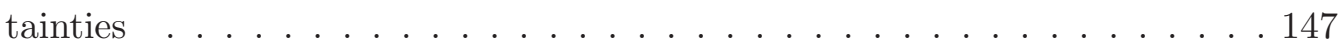

7.29 Impact of the PDF uncertainty on the $W^{\prime}$ signal . . . . . . . . . . . . 148

7.30 Mass distribution for the $W$ Monte Carlo sample . . . . . . . . . . . . . . . 149

7.31 Impact of the uncertainty of the width $\Gamma_{W}$ of the $W$ boson on the transverse mass spectrum . . . . . . . . . . . . . . . 150

7.32 Fit and shifted fit functions for the $W p_{T}$ reweighting $\ldots \ldots \ldots \ldots$

8.1 Comparison between data and background prediction for the $W$ sample (1) 153

8.2 Comparison between data and background prediction for the $W$ sample (2) 154

8.3 Comparison between data and background prediction for the $W$ sample (3) 155

8.4 Comparison between data and background prediction for the $W$ sample (4) 156

8.5 Comparison between data and background prediction for the $W$ sample (5) 157

8.6 Comparison between data and background prediction for the search sample

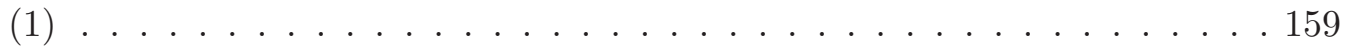

8.7 Comparison between data and background prediction for the search sample

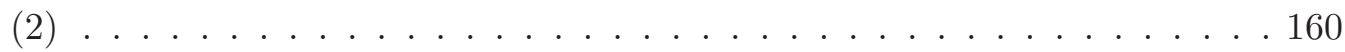

8.8 Comparison between data and background prediction for the search sample

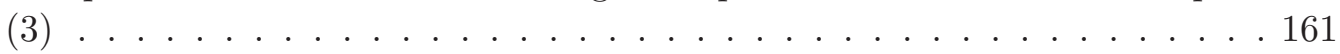

8.9 Comparison between data and background prediction for the search sample

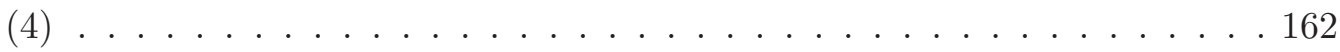

8.10 Distribution of the transverse mass $m_{T}$ for the search sample . . . . . . 163

8.11 Same plots as in Fig. 8.6 on linear scale . . . . . . . . . . . . . . . . . . 164

8.12 Same plots as in Fig. 8.7 on linear scale . . . . . . . . . . . . . . . . 165

8.13 Same plots as in Fig. 8.8 on linear scale . . . . . . . . . . . . . . . . 166

8.14 Same plots as in Fig. 8.9 on linear scale . . . . . . . . . . . . . . . . . . 167

8.15 Example for a shape-changing systematic uncertainty . . . . . . . . . . . 172

8.16 Posterior probability distributions for $m_{W^{\prime}}=500 \mathrm{GeV}$ and $m_{W^{\prime}}=1200 \mathrm{GeV} 173$

8.17 Limit on the cross section at $95 \%$ CL as a function of the mass of the $W^{\prime}$

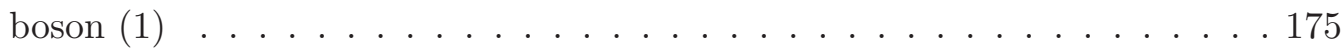

8.18 Limit on the cross section at $95 \% \mathrm{CL}$ as a function of the mass of the $W^{\prime}$ boson $(2) \ldots \ldots \ldots \ldots \ldots \ldots \ldots \ldots$

8.19 Display of an event with high transverse mass . . . . . . . . . . . . . . 177 
9.1 CMS study of the process $W^{\prime} \rightarrow \mu \nu$ in proton-proton collisions at the LHC 180

D.1 Comparison between the two scenarios of the signal Monte Carlo . . . . . 191

D.2 Limit on the cross section at $95 \%$ CL as a function of the mass of the $W^{\prime}$

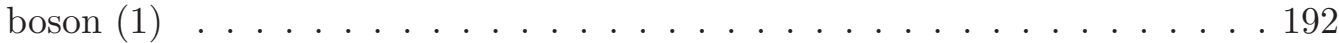

D.3 Limit on the cross section at 95\% CL as a function of the mass of the $W^{\prime}$ boson $(2) \ldots \ldots \ldots 19 \ldots \ldots \ldots$ 


\section{List of Tables}

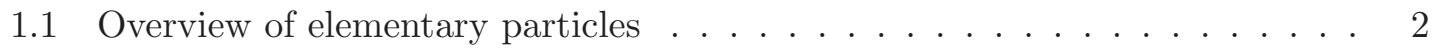

2.1 Properties of the fermions . . . . . . . . . . . . . . . 10

2.2 Quantum numbers for fermions in the Left-Right Symmetric Model . . . . . 28

3.1 Properties of the different accelerators . . . . . . . . . . . . . . 40

3.2 Characteristics of the SMT _ . . . . . . . . . . . . . . . 49

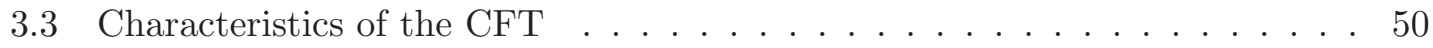

3.4 Characteristics of the calorimeter . . . . . . . . . . . . . . 54

3.5 Energy resolution parameters for the central calorimeter . . . . . . . . . 55

4.1 Identification of Standard Model particles . . . . . . . . . . . . . . . . . 72

5.1 Summary of background Monte Carlo samples used in the analysis . . . . . 90

5.2 Pyтнін parameters for $W^{\prime}$ production . . . . . . . . . . . . . . . 92

5.3 Cross sections and errors due to PDF uncertainties, total width $\Gamma_{W^{\prime}}$ and event numbers for different masses of the $W^{\prime}$ boson . . . . . . . . . . . 93

6.1 Event tags contained in the EMinclusive skim . . . . . . . . . . . . . . . 102

6.2 Data flow during processing . . . . . . . . . . . . . . . . 103

6.3 Trigger selection and luminosity for the trigger list versions and run ranges used in the analysis . . . . . . . . . . . . . . . . . . 104

6.4 Efficiencies for the selection criteria for the signal Monte Carlo samples . . 111

6.5 Inefficiencies for the individual selection criteria of the search sample . . . . 111

7.1 Trigger efficiency and normalization check . . . . . . . . . . . . 128

7.2 Parameters from the fits of $R\left(p_{T}^{E M+M E T}\right) \ldots \ldots \ldots \ldots 134$

7.3 Numbers of the reweighting uncertainty for the different jet multiplicities . 149 
8.1 Events in the $W$ sample compared to the background prediction . . . . . 156

8.2 Relative contributions of the various uncertainties on event numbers in the $W$ sample . . . . . . . . . . . . . . . . . . . . 157

8.3 Events in the search sample for transverse masses $m_{T}>140 \mathrm{GeV} \ldots \ldots 158$

8.4 Relative contributions of the various uncertainties on event numbers in the search sample . . . . . . . . . . . . . . . . . . . . . 162

8.5 Events in the search sample for transverse masses $m_{T}>200 \mathrm{GeV} \ldots \ldots 168$

8.6 Events in the search sample for transverse masses $m_{T}>300 \mathrm{GeV} \ldots \ldots 168$

8.7 Events in the search sample for transverse masses $m_{T}>400 \mathrm{GeV} \ldots \ldots 169$

8.8 Events in the search sample for transverse masses $m_{T}>500 \mathrm{GeV} \ldots \ldots 169$

8.9 Limits on the cross section at $95 \%$ CL . . . . . . . . . . . . . . . . 174

8.10 Properties of the event displayed in Fig. 8.19. . . . . . . . . . . . 178

B.1 Comparison between $\theta$ und $\eta$ values . . . . . . . . . . . . . . 186

C.1 Level 1 trigger requirements . . . . . . . . . . . . . . . . . . . . . . 187

C.2 Level 2 trigger requirements . . . . . . . . . . . . . . . . . . . . 188

C.3 Level 3 trigger requirements . . . . . . . . . . . . . . . . . . . . . . 188

D.1 Limits on the cross section at $95 \%$ CL . . . . . . . . . . . . . . 189

D.2 Efficiencies for the selection criteria for the signal Monte Carlo samples . . 190

D.3 Inefficiencies for the individual selection criteria of the search sample . . . . 190

D.4 Events in the search sample for transverse masses $m_{T}>140 \mathrm{GeV}$. . . . . 194

D.5 Events in the search sample for transverse masses $m_{T}>200 \mathrm{GeV}$. . . . . 194

D.6 Events in the search sample for transverse masses $m_{T}>300 \mathrm{GeV}$. . . . . 195

D.7 Events in the search sample for transverse masses $m_{T}>400 \mathrm{GeV}$. . . . 195

D.8 Events in the search sample for transverse masses $m_{T}>500 \mathrm{GeV} \ldots \ldots . .195$ 


\section{Acknowledgements}

During the last three and a half years I appreciated the help and support of several institutions, groups and individual people who contributed to the successful completion of my $\mathrm{PhD}$ Thesis by some means or other.

First of all, I would like to thank Prof. Dr. Thomas Hebbeker for his willingness to serve as my doctoral advisor. He gave me the opportunity to join the D $\varnothing$ Collaboration as a full member, analyze the data and participate in detector operations. I am glad that I could experience two research stays at Fermilab, the first one from July 2004 to June 2005, and a second shorter one in November 2005 when I only worked on trigger and software related items. He was always appreciative of my extensive trigger work although I sometimes forgot about my own analysis .... Further, I thank Prof. Dr. Achim Stahl for agreeing to be my second referee.

I would like to thank Dr. Martin Wegner who finally convinced me not to perform a CMS analysis based on Monte Carlo "data", but to participate in the famous, running DØ experiment where I could learn a lot about the online system and contribute to further improve its abilities.

I would like to thank Dr. Arnd Meyer for always willing to listen to my problems and to advise me, and for the helpful discussions especially during the last year.

I enjoyed the discussions during our regular D $\varnothing$ Aachen group meetings which contributed to my understanding of physics - in general and in detail. I would especially like to mention Dr. Daniela Käfer and Dr. Christian Autermann. It was a great pleasure to work with you and to profit from your comprehensive knowledge (I am still using Daniela's nice plot macro). I hope I could pass my own experiences to our DØ Diploma Students Oliver Kraff, Philipp Biallass, Lotte Wilke, Patrick Eraerds and Volker Vorwerk. Recently, Prof. Dr. Martin Erdmann took over the responsibility of the weekly meeting. In the discussions with him, Dr. Steffen Kappler, Matthias Kirsch, Daniel Lenz and Jan Steggemann I could learn a lot about top physics at D $\varnothing$.

I really enjoyed the activities within the community of the whole institute (III. Physikalisches Institut A and B, RWTH Aachen), like the christmas parties and "muonchamber-completion" party, and the excursions. I further hope that the annual Seminar in Bad Honnef will always be continued because it was (and still is for me) an important experience to present, discuss and talk about physics and non-physics related things with colleagues at such a nice place outside Aachen.

Concerning my trigger work, I would especially like to thank Prof. Dr. Volker Büscher. He always came up with solutions to problems I had been thinking about for HOURS, and 
lots of new ideas. Most of them could be implemented in the isolation trigger tool which was originally developed by Dr. Christian Autermann. I further appreciate the support within the Level 3 Group (Rick, Daniela, Per). I also enjoyed (yes, I really did !!) to be a member of the DØ Trigger Board and to work with Iain and Marco. I will never forget how we managed to set up trigger list v14. I still miss the stimulating - and sometimes never-ending - discussions and fights ....

Concerning my analysis, I want to thank the New Phenomena Group and the members of the Editorial Board 012 for the valuable discussions. I want especially to mention JeanFrancois Grivaz and Yuri Gershtein (NP Conveners), and Michel Jaffre and Erich Varnes (EB012) for their feedback and for providing inputs. I also appreciate the help of Carsten Hof, Prof. Dr. Michael Krämer, Dr. Alexander Mück and Torbjörn Sjöstrand who contributed to my understanding of $W^{\prime}$ production.

Many people made my stay at Fermilab pleasent: Henrik, Maren, Yvonne, Thorsten, Björn, Cano, Gernot, Thorsten, Arnulf, Christian, Jörg, Oleg, Ingo, Olav, Carsten, Marc, Volker, Su-Jung, Petra, Raimund, Ivor and Jens-Peter. I really enjoyed our regular "Pizza meetings" on Tuesday and all other activities. I also want to thank my roommate MarcAndre who had to bear with me for one year during my research stay at FERMILAB.

I appreciate the financial support of the Deutsche Forschungsgemeinschaft (DFG). From February 2004 to January 2007 I had been granted a scholarship within the Graduate College "Elementarteilchenphysik an der TeV-Skala" at the RWTH Aachen.

I am very thankful that I can always count on my family (my parents, my sister, my brother and my grandmother) - to all intents and purposes.

Last but not least, I am grateful that there is a very important person always standing besides me - my lovely girlfriend Natalie. Thank you so much for your patience and encouragement - just for everything !! 


\title{
Carsten Magaß
}

\author{
Curriculum Vitae
}

\section{Personal Data}

Name:

Address:

Email:

Date of Birth:

Place of Birth:

Nationality:

Merital Status:

Carsten Martin Magaß

Conneallee 37, D-52531 Übach-Palenberg, Germany

magass@physik.rwth-aachen.de

magass@fnal.gov

December 6, 1977

Würselen, Germany

German

unmarried, no children

\section{School Education}

Aug. 1984 - July 1988

Aug. 1988 - June 1997

Catholic Primary School, Übach

Städtisches Gymnasium, Übach-Palenberg

(now: Carolus-Magnus-Gymnasium)

June 1997

Abitur

\section{Alternative Civilian Service}

Oct. 1997 - Sep. 1998 Old People's Home St. Josef, Übach

\section{University Education}

Oct. 1998 - Jan. 2004 Study of physics at the Rheinisch-Westfälische

Oct. 2000

Technische Hochschule (RWTH) Aachen

Oct. 2002 - Dec. 2003

Diplom-Vorprüfung

Diploma Thesis

Bestimmung des inklusiven Wirkungsquerschnitts für

Jan. 2004

Elektronproduktion bei Proton-Antiproton-Kollisionen

Feb. 2004 - Jan. 2007 Diplom, Dipl.-Phys.

Scholarship of the DFG within the Graduate Seminar

"Elementarteilchenphysik an der TeV-Skala"

at the RWTH Aachen

July 2004 - June 2005

Research stay as a guest scientist at Fermilab

Sept. 2004

Aug. 2005

Sept. 2005

Nov. 2005

Aug. 2006

Aug. 2006

Aug. 2007

Feb. 2007 - Oct. 2007

Feb. 2004 - Oct. 2007

Nov. 2007 Annual graduate seminar in Bad Honnef Annual graduate seminar in Bad Honnef "Herbstschule für Hochenergiephysik Maria Laach" Research stay as a guest scientist at FERMILAB "Hadron Collider Physics Summer School" at Fermilab Annual graduate seminar in Bad Honnef Annual graduate seminar in Bad Honnef Research Assistant at the RWTH Aachen Dissertation

Search for New Heavy Charged Gauge Bosons

Graduation, Dr. rer. nat. 



\section{Erklärung}

Hiermit erkläre ich, dass ich die vorliegende Dissertation selbständig erarbeitet und ausschließlich die aufgeführten Referenzen verwendet habe. Alle Quellen und Zitate sind als solche kenntlich gemacht, und die Literaturangaben sind nach meinem besten Wissen und Gewissen korrekt und vollständig wiedergegeben. 
